\title{
39th International Congress of the European Association of Poisons Centres and Clinical Toxicologists (EAPCCT) 21-24 May 2019, Naples, Italy
}

\section{Analysis of 350 consecutive cases of carbon monoxide (CO) poisoning: the Florentine experience}

\author{
Anita Ecolini ${ }^{\mathrm{a}}$, Alessandro Zotto ${ }^{\mathrm{a}}$, Angelo Rotulo ${ }^{\mathrm{a}}$, \\ Alessandra leri ${ }^{b}$, Emanuela Masini ${ }^{a}$, Guido Mannaioni ${ }^{c}$ \\ and Francesco Gambassi ${ }^{\mathrm{b}}$ \\ ${ }^{a}$ Department of Neurofarba, University of Florence, Florence, Italy; \\ ${ }^{b}$ Medical Toxicology Unit and Poison Control Center, Azienda \\ Ospedaliero-Universitaria Careggi, Florence, Italy; 'Neuroscience, \\ Psychology, Drug Research and Child Health, Università degli Studi \\ di Firenze, Florence, Italy
}

Objective: $\mathrm{CO}$ poisoning is a leading cause of morbidity and mortality worldwide. It is mainly caused by heating system malfunction (HSM) or improper use of braziers (IUB). CO binds to heme proteins, reducing blood oxygen transport, blocking mitochondrial adenosine triphosphate (ATP) production, inducing cellular anoxia and damaging the central nervous system, myocardium and muscles. Therapy is supportive, with oxygen as the antidote (normobaric: NBO; hyperbaric: HBO). We present a case series of 350 consecutive patients admitted to the Azienda Ospedaliero-Universitaria Careggi, Florence Emergency Department (ED) from January 2006 to July 2018, focusing on clinical aspects, therapy and follow up (FU).

Case series: Of 350 patients, 195 were female (13 pregnant) and 155 male; mean age was $40 \pm 20$ years (range: 2 months-97 years), 32 were $<18$ years. There were 172 Italians (49.1\%) and 178 migrants (50.9\%). Poisonings were caused mainly by IUB (151, 43.1\%) and $\operatorname{HSM}(149,42.6 \%)$, while 12 (3.4\%) suicidal attempts by engine exhaust and $8(2.3 \%)$ fire-related. At ED admission 335 (95.7\%) presented with Glasgow Coma Score (GCS) $15,10(2.8 \%)$ between $\leq 14$ and $\geq 10$, and $5(1.4 \%) \leq 7$ with seizures and vomiting. Most frequent symptoms were headache $(56 \%)$, loss of consciousness $(43.4 \%)$, dizziness $(18.6 \%)$, nausea (17.7\%), vomiting (15.7\%), rhabdomyolysis (14.3\%), electrocardiogram (ECG) abnormalities (20.9\%, including cardiac ischemia pattern, tachycardia, atrial fibrillation) and troponin increase (9.1\%). Rare events were decreased vision, postural instability, cold sweats, tinnitus, hoarseness and diarrhea. In total 242 (69.1\%) individuals satisfied clinical criteria for $\mathrm{HBO}$ in order to reduce the risk of persistent or delayed neurologic sequelae; 194 (80.1\%) received three or more sessions; 20 could not complete the treatment since no ear equalization could be obtained thus posing a risk of eardrum barotrauma. The others received NBO until resolution of clinical symptoms. In total, 150 (42.9\%) presented for 1month follow-up: 61 (40.7\%) were still symptomatic (headache, dizziness, asthenia, impaired concentration, memory loss and hypoesthesia); 5 in the group that did not complete HBO therapy presented dyspnea, impaired concentration, dizziness, headache and emotional lability.

Conclusion: In this case series $\mathrm{CO}$ poisoning usually occurred accidentally, mainly by IUB or HSM. The most frequent symptoms were headache and loss of consciousness, and $69.1 \%$ of patients needed HBO. Nonetheless, one third of HBO treated patients still showed mild symptoms 1-month after discharge. Major neurological symptoms were present in patients who did not complete $\mathrm{HBO}$ therapy, thus underlining the importance of $\mathrm{HBO}$ in order to avoid neurological sequelae.

\section{Correlation between clinical signs and/or symptoms and blood cyanide concentrations in victims of cyanide poisoning}

\author{
Hanne Herbots, Greet Dieltiens and Kurt Anseeuw \\ Emergency Medicine, ZNA Stuivenberg, Antwerp, Belgium
}

Objective: Diagnosis of cyanide poisoning is difficult [1,2]. We sought to identify key features in decision-making in cyanide poisoning.

Methods: Retrospective chart review of suspected cyanide poisonings at ZNA Hospital, Antwerp, between 1 October 2013 and 1 October 2017. Descriptive statistics were used for demographics, source, clinical characteristics and management. Univariate analysis was performed. Multivariate analysis was unreliable due to small sampling and missing data. We attempted to develop an algorithm with fast and objective parameters.

Results: Overall 156 patients (including 48 emergency responders from Wetteren) were included. Hydrogen cyanide was the most frequent form ( $n=99 ; 63.5 \%)$, followed by nitriles $(n=54 ; 34.6 \%)$ and cyanide salts $(n=3 ; 1.9 \%)$. The most common mechanism was smoke inhalation $(n=98 ; 62.8 \%)$. Descriptive analysis is reported in Table 1. Predictors for elevated cyanide blood concentrations identified by univariate analysis were smoke inhalation $(p=0.005)$, enclosed-space fire $(p=0.005)$, rescued patient $(p=0.02)$, elevated lactate $(p=0.02)$, elevated carboxyhemoglobin $(\mathrm{COHb})$ concentration $(p=0.02)$ and elevated ethanol concentration $(p=0.02)$. Unconsciousness $(p=0.01)$, cardiac arrest $(p=0.045)$, decreased bicarbonate $(p=0.05)$ and elevated anion gap $(p=0.03)$ were predictors for blood cyanide concentrations above $1000 \mu \mathrm{g} / \mathrm{L}$. For smoke inhalation, combining lactate $>2$ $\mathrm{mmol} / \mathrm{L}$ and bicarbonate $<22 \mathrm{mmol} / \mathrm{L}$ OR lactate $>2 \mathrm{mmol} / \mathrm{L}$, bicarbonate $22-26 \mathrm{mmol} / \mathrm{L}$ and $\mathrm{COHb}>10 \%$ could adequately identify cyanide poisoning victims. The respective positive and negative predictive values were $88 \%$ and $95 \%$, and $89 \%$ and $100 \%$.

Conclusion: Classical signs of cyanide poisoning (bitter almond odor, cherry red skin) were absent in these cases. Combination of 
Table 1. Clinical signs and treatment in patients with cyanide poisoning $(n=156)$.

\begin{tabular}{|c|c|c|c|}
\hline Parameter & Yes & No & Unknown \\
\hline \multicolumn{4}{|c|}{ Clinical signs, symptoms and observations } \\
\hline Bitter almond odor detected & $0(0 \%)$ & $156(100 \%)$ & $0(0 \%)$ \\
\hline Red cherry skin & $0(0 \%)$ & $156(100 \%)$ & $0(0 \%)$ \\
\hline Dizziness & $8(5.1 \%)$ & $148(94.9 \%)$ & $0(0 \%)$ \\
\hline Vomiting & $6(3.9 \%)$ & $150(96.2 \%)$ & $0(0 \%)$ \\
\hline Respiratory distress & $29(18.6 \%)$ & $127(81.4 \%)$ & $0(0 \%)$ \\
\hline Unconsciousness/confusion & $23(14.7 \%)$ & $133(85.3 \%)$ & $0(0 \%)$ \\
\hline Seizures & $0(0 \%)$ & $156(100 \%)$ & $0(0 \%)$ \\
\hline Cardiac arrest & $4(2.6 \%)$ & $152(97.4 \%)$ & $0(0 \%)$ \\
\hline Sooty face & $41(26.3 \%)$ & $105(67.3 \%)$ & $2(1.2 \%)$ \\
\hline Burn wounds & $46(29.5 \%)$ & $109(68.9 \%)$ & $1(0.7 \%)$ \\
\hline \multicolumn{4}{|l|}{ Treatments and investigations } \\
\hline Elevated ethanol concentration & $29(18.6 \%)$ & $29(18.6 \%)$ & $98(62.8 \%)$ \\
\hline Hydroxocobalamin given & $60(38.5 \%)$ & $96(61.5 \%)$ & $0(0 \%)$ \\
\hline Intubation/ventilation & $58(37.2 \%)$ & $98(62.8 \%)$ & $0(0 \%)$ \\
\hline Vasopressors given & $29(18.5 \%)$ & $126(80.8 \%)$ & $1(0.7 \%)$ \\
\hline
\end{tabular}

lactate $>2 \mathrm{mmol} / \mathrm{L}$ and bicarbonate $>22 \mathrm{mmol} / \mathrm{L}$ or lactate $>2$ $\mathrm{mmol} / \mathrm{L}$, bicarbonate $22-26 \mathrm{mmol} / \mathrm{L}$ and $\mathrm{COHb}>10 \%$ could be used to diagnose cyanide poisoning.

\section{References}

[1] Anseeuw K, Delvau N, Burillo-Putze G, et al. Cyanide poisoning by fire smoke inhalation: a European expert consensus. Eur J Emerg Med. 2013;20:2-9.

[2] Parker-Cote JL, Rizer J, Vakkalanka JP, et al. Challenges in the diagnosis of acute cyanide poisoning. Clin Toxicol (Phila). 2018;56:609-617.

\section{A consensus statement on the management of patients in outbreaks of methanol poisoning}

\author{
Hossein Hassanian-Moghaddam ${ }^{a}$, Nasim Zamani ${ }^{b}$, \\ Darren Roberts ${ }^{c}$, Jeffrey Brent ${ }^{d}$, Kenneth McMartin ${ }^{e}$, \\ Cynthia Aaron ${ }^{f}$, Michael Eddleston ${ }^{g}$, Kent Olson ${ }^{h}$, \\ Paul I Dargani, Lewis Nelson', Ashish Bhallak, \\ Philippe Hantson', Dag Jacobsen ${ }^{m}$, Bruno Mégarbane ${ }^{n}$, \\ Mahdi Balali-Mood ${ }^{\circ}$, Nicholas Buckley ${ }^{p}$, \\ Sergey Zakharov ${ }^{\mathrm{q}}$, Raido Paasmar ${ }^{r}$, Bhavash Jarwani ${ }^{\mathrm{s}}$, \\ Amirhossein Mirafzal ${ }^{\mathrm{t}}$, Tomas Salek ${ }^{\mathrm{u}}$ and \\ Knut Erik Hovdav \\ ${ }^{a}$ Social Determinants of Health Research Center, Shahid Beheshti \\ University of Medical Sciences, Tehran, Iran; ${ }^{b}$ Clinical Toxicology, \\ Loghman Hakim Hospital, School of Medicine, Shahid Beheshti \\ University of Medical Sciences, Tehran, Iran; 'NSW Poisons

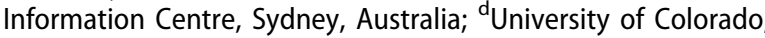 \\ School of Medicine, Aurora, United States; ${ }^{\text {eDepartment of }}$ \\ Pharmacology, Toxicology and Neuroscience, Louisiana State \\ University Health Sciences Center - Shreveport, Shreveport, United \\ States; ${ }^{f}$ Michigan Regional Poison Control Center at Children's \\ Hospital of Michigan, Detroit, United States; ${ }^{9}$ Department of \\ Pharmacology, Toxicology, and Therapeutics, University/BHF \\ Centre for Cardiovascular Science, University of Edinburgh, \\ Edinburgh, United Kingdom; ${ }^{h}$ California Poison Control System, \\ San Francisco Division, University of California, San Francisco, \\ United States; 'Clinical Toxicology, Guy's and St Thomas' NHS \\ Foundation Trust and Faculty of Life Sciences and Medicine, King's \\ College London, London, United Kingdom; 'Department of \\ Emergency Medicine, Rutgers Medical School, Newark,
}

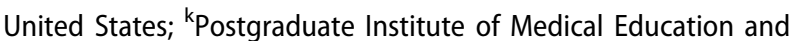
Research, Chandigarh, India; 'Department of Intensive Care, Cliniques Universitaires Saint Luc, Université Catholique de Louvain, Brussels, Belgium; ${ }^{\mathrm{m}}$ Department of Acute Medicine, Ullevaal University Hospital, Oslo, Norway; ${ }^{\text {nDepartment of Medical }}$ and Toxicological Critical Care, Lariboisière Hospital, INSERM UMRS 1144, Paris-Diderot University, Paris, France; ${ }^{\circ}$ Medical Toxicology Research Center, School of Medicine, Mashhad University of Medical Sciences, Mashhad, Iran; ${ }^{\mathrm{P} D e p a r t m e n t}$ of Pharmacology, University of Sydney, NSW Poison Information Center, Sydney, Australia; 'Occupational Medicine, 1st Faculty of Medicine, Charles University and General University Hospital, Toxicological Information Centre, Prague, Czech Republic; ' ${ }^{\prime}$ Anesthesiology and ICU, Pärnu County Hospital, Pärnu, Estonia; 'Emergency Medicine, VSGH, Ahmedabad, India; ${ }^{\mathrm{t} E m e r g e n c y ~ M e d i c i n e, ~ K e r m a n ~ U n i v e r s i t y ~}$

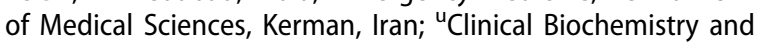
Pharmacology, Tomas Bata Hospital, Zlín, Czech Republic; ${ }^{\vee}$ Acute Medicine, The Norwegian CBRNE Centre of Medicine, Oslo University Hospital, Oslo, Norway

Objective: The aim of our study was to develop a strategy for prioritising patients, who are being treated with alcohol dehydrogenase inhibitors, for haemodialysis during methanol poisoning outbreaks.

Methods: Twenty-two international experts on methanol poisoning were invited to join the process; consensus statements were made by a Delphi method. A simple risk-assessment algorithm was developed by merging similar data from two outbreaks $[1,2]$. Based on six different levels of risk in the algorithm, four separate clinical scenarios were used to assess expert opinion on decisionmaking:

Group 1: $\mathrm{pH}<6.74$ and coma

Group 2: $\mathrm{pH}<6.74$ and alert or $\mathrm{pH}$ 6-74-6.99 and comatose Group 3: $\mathrm{pH}$ 6.74-6.99 and alert Group 4: $\mathrm{pH} \geq 7$ and alert

Results: The experts agreed that the severity of the poisoning and prognosis should be used as the basis for triaging of patients. All experts prioritised Group 2 for hemodialysis with Group 4 the lowest priority, particularly in those without visual disturbance. Thereafter, 14 experts (64\%) felt that Group 1 should be prioritised due to their severe clinical condition and lower chance of survival without hemodialysis. However, eight (36\%) felt Group 3 should be prioritised above Group 1, as 17\% of survivors in Group 1 were likely to suffer marked/irreversible neurological sequelae, while Group 3 had a reasonable chance of 
survival without sequelae if haemodialysis was performed immediately.

Conclusion: We recommend that severely poisoned patients with a high chance of beneficial outcome - those with a $\mathrm{pH}<6.74$ who are alert or those with $\mathrm{pH}$ 6.74-6.99 who are comatose - are the highest priority for haemodialysis; and that those with a moderate acidosis, particularly when no visual disturbance is present, are the lowest priority. There were fewer consensuses on the priority in intermediate patients and so the remaining patients should be prioritised based on the number of patients and local expertise and resources. This strategy can be used as a decision support in outbreaks of methanol poisoning when resources do not meet demand.

\section{References}

[1] Paasma R, Hovda KE, Hassanian-Moghaddam H, et al. Risk factors related to poor outcome after methanol poisoning and the relation between outcome and antidotes - a multicenter study. Clin Toxicol (Phila). 2012;50:823-831.

[2] Zakharov S, Pelclova D, Urban P, et al. Czech mass methanol outbreak 2012: epidemiology, challenges and clinical features. Clin Toxicol (Phila). 2014;52:1013-1024.

\section{Poisons Centres need to prepare for a new product notification procedure}

\author{
Ronald De Groot, Pieter Brekelmans and \\ Dylan W De Lange \\ Dutch Poisons Information Center, University Medical Center \\ Utrecht, Utrecht, Netherlands
}

\begin{abstract}
Objective: Companies placing hazardous mixtures on the EU market have a legal obligation to provide product information to Poisons Centres (PC) or equivalent "appointed bodies". Regulation (EU) No 2017/452 will harmonise information requirements and electronic format in EU Member States (MS). The new rules come into effect from 2020. Appointed bodies need to be ready to receive this information.
\end{abstract}

Implementation: The European Chemicals Agency (ECHA) is responsible for developing a variety of tools. Companies will be able to generate the format by use of an offline or online tool and submit information through an online Poisons Centres Notification (PCN) portal. Companies with advanced information technology (IT) systems could directly prepare the format and link to the PCN portal by system to system integration. Appointed bodies can download the product information from the portal. To prepare for processing the format in national databases, a "developer's guide" and example files are available from ECHA [1]. Alternatively, appointed bodies that do not want to develop or adapt their national IT system can access product information online via a searchable database. The Dutch PC favours a combination: downloading consumer/professional mixture product information to retain the current practice of linking product information to exposure cases and online access to information on (the foreseen millions of) industrial mixtures with low exposure incidence rates. Either way, the portal could replace national procedures to directly receive information from companies, reducing national costs e.g. on personnel and IT tools. Use of the portal is not a legal requirement and individual MS could decide to maintain a national procedure, as an additional or required submission route. To prevent administrative burden for companies it would be beneficial for all MS to at least allow the PCN portal as an option. Ultimately, information should become available to Poison Centres enabling them to provide rapid medical advice in an emergency. In some MS the PC is officially appointed to receive product information, but in most MS (approximately 75\%) a governmental authority is appointed. PCs should discuss with their national authority how the new procedures might affect their access to product information.

Conclusion: Appointed bodies/Poisons Centres should prepare to implement the EU harmonised product information requirements and format. They should decide whether to make use of ECHA's searchable database or have product information locally available. In this last case, national databases need to be adapted in order to process the new format.

\section{Reference}

[1] ECHA Poisons Centres website [cited 2018 Sep 25]. Available from: https://poisoncentres.echa.europa.eu

\section{Avoiding emergency ambulance dispatch for suspected non- intentional poisoning by prior telephone consultation with the UK National Poisons Information Service}

\author{
David A James ${ }^{a}$, Nathan George ${ }^{a}$, Sally M Bradberry ${ }^{b}$, \\ Euan A Sandilands ${ }^{c}$, John P Thompson ${ }^{d}$ and \\ Simon HL Thomas ${ }^{\mathrm{a}, \mathrm{e}}$ \\ ${ }^{a}$ National Poisons Information Service (Newcastle Unit), Regional \\ Drug and Therapeutics Centre, Newcastle Hospitals NHS \\ Foundation Trust, Newcastle, United Kingdom; ${ }^{b}$ National Poisons \\ Information Service (Birmingham Unit), City Hospital, Birmingham, \\ United Kingdom; ' National Poisons Information Service (Edinburgh \\ Unit), Royal Infirmary, Edinburgh, United Kingdom; ${ }^{\mathrm{d}}$ National \\ Poisons Information Service (Cardiff Unit), University Hospital \\ Llandough, Cardiff, United Kingdom; ${ }^{\mathrm{e}}$ Medical Toxicology Centre, \\ Institute of Cellular Medicine, Newcastle University, Newcastle, \\ United Kingdom
}

Objective: To investigate the potential for the National Poisons Information Service (NPIS) to prevent unnecessary Ambulance Service emergency vehicle and crew callouts, by provision of predispatch advice in cases of suspected non-intentional poisoning.

Methods: Enquiries to the NPIS (Newcastle) Unit from Ambulance Services were analysed prospectively from 1 October 2017 to 31 March 2018. Only "first point of contact", crew onscene, non-intentional poisoning enquiries were included and it was also established if the crew were attending solely in response to potential poisoning. Enquiries in which NPIS advised "home care" were scrutinised and a judgement made by two authors as to whether the presence of or assessment by an onscene crew may have reassured NPIS staff and influenced this decision. A corresponding financial year (2017-18) of national NPIS data was analysed to enable estimation of national impact by extrapolation of local results.

Results: In this 6 month period NPIS (Newcastle) answered 628 ambulance enquiries from across the UK, with a large variation in rates of enquiry between Ambulance Services. Of these, 274 (44\%) concerned non-intentional poisoning, all of which were included in the study. Crew were already on-scene in 193 cases $(70 \%, 95 \% \mathrm{Cl} 65-76 \%)$ and the NPIS advised home care or no action for 105 of these. There were 7 cases where there was at least one other non-poisoning reason for attendance and 7 further cases where it was judged that the crew on-scene presence and/or assessment may have influenced the NPIS staff making a 
home care decision. Therefore, advance consultation with the NPIS may have avoided an emergency vehicle and crew call-out in the remaining $91(47 \%, 95 \% \mathrm{Cl} 40-54 \%)$ of crew on-scene, non-intentional poisonings. Analysis of NPIS national data identified 2,031 non-intentional poisoning enquiries from Ambulance Services in 2017-18. If 70\% were on scene (as in this study) an estimated national annual total of 1,442 crew on-scenes, nonintentional poisoning enquiries are received. If $47 \%$ of these were avoided by prior telephone consultation, this would prevent 668 ambulance dispatches annually.

Conclusion: Ambulance service resources could be saved if advice was obtained from NPIS before dispatch in cases of nonintentional poisoning. Further work is needed to explore reasons for variation in use of NPIS between Ambulance Services.

\section{Evaluation by telephone-follow-up of mild intoxications managed at home by the Poison Control Centre's medical doctors}

\author{
Elena Brambilla, Valeria M Petrolini, Giulia Scaravaggi, \\ Eleonora Buscaglia, Maria Di Primo and \\ Carlo A Locatelli \\ Pavia Poison Control Centre - National Toxicology Information \\ Centre - Clinical and Experimental Lab, Toxicology Unit, Maugeri \\ Clinical and Scientific Institutes IRCCS and University of Pavia, \\ Pavia, Italy
}

Objective: Poison Control Centers (PCC) avoid unnecessary hospital admission and reduce healthcare public spending when intoxications with low risk for patients are treated at home. Follow-up calls can be efficiently used to evaluate the appropriateness and effectiveness of the intoxications managed at home since they allow for repeated and rapid assessment of patients' clinical outcome.

Methods: A prospective observational study over a seven-month period (October 2017 to April 2018) on all cases involving patients managed at home was performed. Cases for which hospitalization was recommended or toxicity was ruled out, were excluded. Therefore, all included patients had present or potential toxic effects. After the first toxicological expert advice, followup calls were performed 1-3 days later to collect information about the patient's condition (unless the patient/caregiver had not called before for worsening symptoms), and to verify compliance to the treatment advice given. At the same time, patients were asked questions to investigate awareness about PCC services and which healthcare service they would have called in the absence of the PCC.

Results: Among 3123 calls received by the general public during the study period, 570 patients met the inclusion criteria. Most patients (59\%) were children aged 1-5 years. Drugs were the most common cause of intoxication (33\%, especially antibiotics and non-steroidal anti-inflammatory drugs [NSAIDs]), followed by household products (29\%, especially detergent and bleach), cosmetics (11\%) and plants (5\%). At the first call, 490/570 (86\%) patients were asymptomatic, while $80 / 570$ (14\%) presented symptoms (Poison Severity Score [PSS] 1-2). Ninety-one patients (15.9\%) were lost to follow-up (after at least two recalls). Among the responders, all symptomatic patients $(n=71)$ had recovered with therapy prescribed by the toxicologist; among asymptomatic patients $(n=408), 26(22 \%)$ developed the expected symptoms, which regressed within a few days with the prescribed therapy. Thirteen patients went to the hospital anyway, but only in 4 cases due to worsening of symptoms. In $78 \%$ of cases, patients were compliant to prescribed therapies. Overall $45 \%$ of patients obtained the Pavia PCC emergency number from emergency departments, and $72 \%$ of patients would have gone to hospital without the toxicological consultation given by the PCC.

Conclusion: The possibility to perform follow-up calls within 2 days provides PCC doctors a valid instrument to monitor homecare patients. This method allows management at home in cases of mild intoxications, avoiding hospital admission. Follow-up calls, which are largely appreciated by the patient/carer, are also an efficient tool to collect epidemiological data.

\section{Cocaine poisoning in the intensive care unit: are there differences between cocaine hydrochloride- and crack-related toxicity?}

\author{
Lisa Catherine ${ }^{\mathrm{a}}$, Marion Soichot ${ }^{\mathrm{b}}$, Sebastian Voicu ${ }^{\mathrm{a}}$, \\ Laurence Labat ${ }^{\mathrm{b}}$ and Bruno Mégarbane ${ }^{a}$ \\ ${ }^{a}$ Department of Medical and Toxicological Critical Care, Lariboisière \\ Hospital, Paris-Diderot University, INSERM UMRS-1144, Paris, \\ France; ${ }^{b}$ Laboratory of Toxicology, Lariboisière Hospital, Paris- \\ Diderot University, INSERM UMRS-1144, Paris, France
}

Objective: Cocaine is the most frequently used psychostimulant illicit drug worldwide. In France, its use has significantly increased during the last ten years, with two main modalities: the recreational snorting of cocaine hydrochloride and the inhalation of smoked "crack" (free-base form of cocaine, obtained by cocaine salification using common baking soda or ammoniac) in highly dependent and dissocialized users. The neurological, cardiovascular, respiratory and metabolic complications of cocaine are relatively well-known; however, differences between cocaine hydrochloride and crack-related toxicities have been poorly investigated. Our objectives were to describe and compare the circumstances and the resulting complications of cocaine use in the patients admitted to the intensive care unit (ICU) in relation to the route of exposure, i.e. snorting versus inhalation.

Methods: We conducted a retrospective single-centre observational study including all cocaine-poisoned patients admitted to the intensive care unit over an 8-year period (2011-2018). Comparisons between the two routes of cocaine exposure were performed using an univariate analysis (chi-squared and MannWhitney tests, as required).

Results: Seventy-two patients (age, 35 years [30; 46] (median, [percentiles $25 ; 75])$ ) were included. Toxicity mainly resulted from multidrug use with benzodiazepines (79\%), ethanol (32\%), methadone (29\%), tetrahydrocannabinol (23\%) and amphetamines (21\%). The Glasgow Coma Score (GCS) on admission was 10 [3; 15]. Complications included cardiac arrest $(n=9)$, aspiration pneumonia $(n=19)$, acute renal failure $(n=15)$, malignant hypertension $(n=7)$, ischemia/thrombosis events $(n=5)$, seizures $(n=$ $5)$, hyperthermia $(n=5)$, myocardial infarction $(n=1)$ and death $(n=5)$. Users by snorting $(n=34)$ presented significantly more severe symptoms than users by inhalation $(n=25)$, with lower $\operatorname{GCS}(p=0.02)$, more intense adrenergic syndrome $(p=0.009$ for tachycardia), more elevated plasma lactate concentration $(p=$ $0.002)$, lower platelet count $(p<0.0001)$ and more marked rhabdomyolysis $(p=0.02)$. Snorting cocaine was less frequently associated with methadone use $(p<0.0001)$. Cocaine snorting patients more frequently developed cardiac arrest $(p=0.02)$, aspiration pneumonia $(p=0.04)$ and required mechanical ventilation $(p=0.0002)$

Conclusion: Cocaine use may lead to severe complications requiring ICU admission. Our findings suggest that complications attributed to cocaine hydrochloride snorting are more severe than the complications attributed to crack inhalation. Experimental investigations may interestingly complement our 
study to allow better understanding of the mechanistic reasons supporting these observed differences.

\section{A case of etizolam withdrawal syndrome}

\author{
Anthony Scoccimarro, Ryan Marino and \\ Joshua Shulman \\ University of Pittsburgh School of Medicine, Pittsburgh, United \\ States
}

Objective: Etizolam is a thienodiazepine known to interact with the benzodiazepine receptor site. There have been no reported cases of etizolam withdrawal.

Case report: A 27-year-old man presented after witnessed seizure activity at home. The patient had been taking etizolam for several weeks and decided he wanted to self-discontinue the medication 4 days prior to arrival, at which time he had taken a dose of $70 \mathrm{mg}$. On the day of presentation, the patient's girlfriend had stated he collapsed onto a table with generalized limb jerking followed by a period of confusion that improved gradually. On initial examination, he was notably tremulous and tachycardic (up to 142 beats per minute). The serum ethanol concentration was $35 \mathrm{mg} / \mathrm{dL}$, although he denied daily ethanol use. He was given $20 \mathrm{mg}$ intravenous diazepam with improvement. A qualitative urine gas chromatography/mass spectrometry (GC/MS) screen was significant for diazepam, hydrocodone, hydrocodone metabolite, nicotine, carisoprodol metabolite or meprobamate, caffeine, doxylamine, mitragynine, and dextromethorphan. His vital signs and tremor improved throughout his stay through the administration of symptom-triggered diazepam. He was ultimately discharged with a diazepam taper without incident.

Conclusion: Although etizolam has a similar mechanism of action as benzodiazepines, there are no reported cases of withdrawal secondary to etizolam use. Etizolam dependence has been reported, but that patient did not develop symptoms consistent with withdrawal. Our patient had a previous history of sedativehypnotic withdrawal due to a variety of benzodiazepines and had reported a previous seizure in the setting of GABAergic withdrawal. The reported elimination half-life of etizolam is 3.4 hours. However, our patient took an excessive amount of etizolam on his last day of use, which may have prolonged its half-life although its toxicokinetics are unknown. This patient's presentation is furthermore confounded by the presence of several other xenobiotics detected on GC/MS, although the patient had reported taking these substances as a means of staving off withdrawal. From a management perspective, withdrawal from etizolam is similar to withdrawal from sedative hypnotic xenobiotics and can likely be treated in a similar fashion to benzodiazepine withdrawal.

\section{Analytical confirmation of benzodiazepine use in $\mathbf{5 0 0}$ patients presenting with acute recreational drug toxicity to a Central London Emergency Department}

\author{
Joanna C White ${ }^{a}$, Paul I Dargan ${ }^{b}$, Simon Hudson ${ }^{c}$, \\ Rachelle Abouchedid ${ }^{a}$, Alison M Dines ${ }^{a}$, \\ John RH Archer ${ }^{\mathrm{b}}$ and David M Wood ${ }^{\mathrm{b}}$ \\ ${ }^{a}$ Clinical Toxicology, Guy's and St Thomas' NHS Foundation Trust,

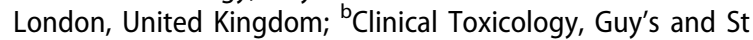 \\ Thomas' NHS Foundation Trust; Faculty of Life Sciences and \\ Medicine, King's College London, London, United Kingdom;
}

'Laboratory and Managed Services, LGC Ltd, Fordham, United Kingdom

Objective: There is increasing concern about the use of benzodiazepines - both classical benzodiazepines available as regulated medicines and novel benzodiazepines available as new psychoactive substances (NPS) - in recreational drug users. The aim of this study was to determine how often benzodiazepines were detected in patients presenting to the emergency department (ED) with acute recreational drug toxicity.

Methods: We conducted a prospective study enrolling 500 consecutive adult patients presenting to a central London ED with acute recreational drug toxicity from March-December 2016. Ethical approval was granted for surplus serum samples taken as part of routine clinical care to be anonymised and analysed for benzodiazepines by high-resolution accurate mass-spectrometry with liquid-chromatography (HRAM-LCMSMS). Data was not available on the proportion of patients prescribed benzodiazepines in the ED prior to venepuncture or that were on regular prescribed benzodiazepines as opposed to taking them recreationally as part of the presentation.

Results: Overall 233 (46.6\%) of the 500 patients had a benzodiazepine detected, mean \pm SD age $34.9 \pm 9.2$ years, 198 male. The maximum number of different benzodiazepines detected in one patient was 7; only 62 patients had a single benzodiazepine detected. Classical benzodiazepines detected were: diazepam $(n=159)$, nordazepam $(n=145)$, oxazepam $(n=96)$, temazepam $(n=85)$, clonazepam $(n=44)$, lorazepam $(n=31)$, demoxapam $(n=25)$, alprazolam $(n=24)$, chlordiazepoxide $(n=20)$, midazolam $(n=13)$, delorazepam $(n=2)$, flurazepam $(n=2)$, lormetazepam $(n=1)$, bromazepam $(n=1)$ and prazepam $(n=1)$. Novel benzodiazepines were only detected in 3 patients $(1.3 \%$ of those in whom a benzodiazepine was detected): flubromazepam, diclazepam and etizolam each detected in one patient. Only 14 patients $(2.8 \%$ of the whole cohort, $6.0 \%$ of those in whom a benzodiazepine was detected) self-reported that they had used a benzodiazepine as part of their presentation. There was very poor correlation between the benzodiazepines reported to be taken and those detected.

Conclusion: Benzodiazepines were detected in almost half of this large cohort of patients with acute recreational drug toxicity, although less than $3 \%$ self-reported use suggesting that the use of benzodiazepines in patients presenting to the ED with acute recreational drug toxicity is underestimated. Greater awareness of the use of benzodiazepines in these patients is important as this can impact both on clinical presentation and management.

\section{Comparison of the hepatocellular toxicity of para-halogenated amphetamines and methcathinones}

\author{
Xun Zhou, Melanie Walter, Matthias E Liechti, \\ Stephan Krähenbühl and Dino Luethi \\ Division of Clinical Pharmacology and Toxicology, Department of \\ Biomedicine, University Hospital Basel and University of Basel, \\ Basel, Switzerland
}

Objective: Halogenated derivatives of amphetamine-type stimulants have repeatedly appeared on the drug market, often with altered pharmacological profile and/or legal status compared to the non-halogenated substances. The effect of halogenation on toxicity of new designer stimulants is, however, not well studied and the aim of the present study was therefore to investigate the hepatocellular toxicity of para-fluorinated and para-chlorinated amphetamines and methcathinones (beta-keto $\mathrm{N}$ methylamphetamines). 
Methods: We measured cell membrane integrity, adenosine triphosphate (ATP) content, and superoxide levels in human hepatoma HepG2 cells after exposure to amphetamine, 4fluoroamphetamine, 4-chloroamphetamine, methcathinone, 4-fluoromethcathinone, and 4-chloromethcathinone for 24 hours. In addition, we measured the oxygen consumption rate (OCR) with a Seahorse XF analyzer. Differences between vehicle control and test drugs were calculated with ANOVA followed by Dunnett's test. P-values below 0.05 were considered to be statistically significant.

Results: All substances depleted cellular ATP content at concentrations lower ( 0.25 to $2 \mathrm{mM}$ ) than cell membrane integrity loss occurred $(\geq 0.5 \mathrm{mM})$, suggesting mitochondrial toxicity. All amphetamines impaired basal respiration and the halogenated derivatives additionally impaired the maximal respiratory capacity of the cells in the range of $0.1-2.0 \mathrm{mM}$, confirming mitochondrial toxicity. Methcathinone and 4-fluoromethcathinone did not disrupt cellular respiration in the investigated concentration range ( $\leq 2 \mathrm{mM}$ ) but 4-chloromethcathinone decreased the maximal respiration and uncoupled oxidative phosphorylation at $1 \mathrm{mM}$. We observed an increase in mitochondrial superoxide at concentrations equal to concentrations that induced cell membrane damage $(0.5-2.0 \mathrm{mM})$. For amphetamines as well as methcathinones, we observed the following toxicity rank order for the para-substituents: chloride $>$ fluoride $>$ hydrogen. Furthermore, the investigated amphetamines proved to be more toxic compared to the corresponding methcathinones.

Conclusion: In addition to an altered pharmacological profile, the halogenation of stimulants may substantially increase their toxicity. Amphetamine, 4-fluoroamphetamine, 4-chloroamphetamine, and 4-chloromethcathinone are mitochondrial toxicants impairing the function of the electron transport chain and depleting cellular ATP stores. The beta-keto group and $\mathrm{N}$-methylation of the methcathinones seems to decrease the toxicity compared to the corresponding amphetamines. However, since liver injury is rare in users of these drugs, affected persons likely have susceptibility factors rendering them more sensitive for these drugs.

\section{A chloromethcathinone-like new psychoactive drug (NPS): epidemiological features and clinical characteristics of intoxication}

\author{
Emilio Salgado ${ }^{a}$, Marina Parra ${ }^{b}$, Oscar Pozoc, \\ Josep Marcos ${ }^{\mathrm{d}}$, Jordi To-Figueras ${ }^{\mathrm{b}}$ and \\ Santiago Nogué ${ }^{2}$ \\ ${ }^{a}$ Clinical Toxicology Unit, Emergency Department, Hospital Clínic, \\ Barcelona, Spain; ${ }^{b}$ Biochemistry and Molecular Genetics Unit, \\ Hospital Clínic, Barcelona, Spain; 'Integrative Pharmacology and \\ Systems Neuroscience Group, Hospital de Mar, Barcelona, Spain; \\ Experimental and Health Sciences Department, Universitat \\ Pompeu Fabra, Barcelona, Spain
}

Objective: To describe the main clinical and epidemiological characteristics of poisoning by a new chloromethcathinone-like compound.

Methods: Spot urine samples were obtained from patients admitted to the Emergency Department (ED) of the Hospital Clinic, Barcelona, due to drug abuse. A preliminary drug screening by immunoassay (IA) was performed by Dimension EXL analyzer (Siemens Healthineers), including the analysis of amphetamines, benzodiazepines, cannabis, cocaine, methadone and opioids. Positive results were confirmed by gas chromatography-mass spectrometry (GC-MS). Internal standard was added to each urine sample and drugs were extracted by solid phase extraction (SPE, Bond Elut Certify). After derivatization, identification of compounds and metabolites was performed on an Agilent 7890A gas chromatograph (GC) system coupled to an Agilent 5975C mass selective detector (Agilent Technologies, Santa Clara, CA, USA).

Results: Between August 2017 and May 2018, 7 cases of intoxications by a new chloromethcathinone-like compound were detected. All the cases were male, between 25 and 38 years old (average 33 years), the majority were occasional abusers (85.7\%), with chronic human immunodeficiency virus (HIV) infection in $57.1 \%$, and $42.8 \%$ in a "chemsex" context. Most (85.7\%) of the intoxications were mixed, and the main substances found were gamma-hydroxybutyrate acid (GHB) in $85.7 \%$, amphetamine derivatives in $71.4 \%(3,4$ methylenedioxymethamphetamine [MDMA] 42.8\%, amphetamine $28.5 \%$ and methamphetamine $28.5 \%$ ), cocaine in $42.8 \%$ and ethanol in $42.8 \%$. In terms of clinical presentation, the sedative effect of GHB predominated, with coma as the main complaint in $57.1 \%$. In the only recorded case of non-combined poisoning, the chief complaint was palpitation, and hypertension and tachycardia were the main clinical features. All patients were treated with supportive measures and discharged home, with an average stay of 7 hours in the emergency department.

Conclusion: This is the first description in the literature to date of the clinical effect of this group of new substances of abuse $[1,2]$. Usage of this new cathinone compound is characterized by recreational use in seropositive young men and their association with other drugs of abuse, mainly GHB and amphetamine derivatives. In the only case of a non-combined poisoning, its clinical manifestations were compatible with a sympathomimetic toxidrome. All patients recovered without sequelae with only supportive measures.

\section{References}

[1] Grifell M, Ventura M, Carbón X, et al. Patterns of use and toxicity of new para-halogenated substituted cathinones: 4-CMC (clephedrone), 4-CEC (4-chloroethcatinone) and 4-BMC (brephedrone). Hum Psychopharmacol. 2017;32:e2621.

[2] Taschwer M, Weiß JA, Kunert O, et al. Analysis and characterization of the novel psychoactive drug 4-chloromethcathinone (clephedrone). Forensic Sci Int. 2014;244:e56-e59.

\section{Methadone poisonings in France: comparison of suicide attempts versus abuse during a seven-year experience of the French Poison Control Center Network}

\author{
Romain Torrents ${ }^{\mathrm{a}}$, Mathieu Glaizal ${ }^{\mathrm{b}}$, \\ Katharina Von Fabeck ${ }^{\mathrm{b}}$, Bastien Domangéa \\ Corinne Schmitt ${ }^{\mathrm{b}}$, Julien Reynoard ${ }^{\mathrm{b}}$, \\ Audrey Boulamery ${ }^{\mathrm{a}}$, Luc De Haro ${ }^{\mathrm{b}}$ and \\ Nicolas Simon ${ }^{\mathrm{C}}$

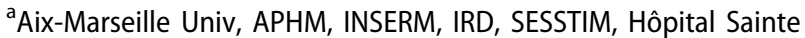 \\ Marguerite, Service de Pharmacologie Clinique, Centre Antipoison

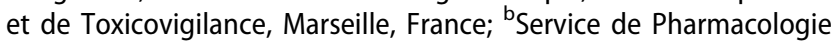 \\ Clinique, Centre Antipoison et de Toxicovigilance, Hôpital Sainte \\ Marguerite, APHM, Marseille, France; 'Marseille Univ, APHM, \\ INSERM, IRD, SESSTIM, Hôpital Sainte Marguerite, Service de \\ Pharmacologie Clinique, Centre Antipoison et de Toxicovigilance, \\ Marseille, France
}

Objective: Methadone is an opioid agonist prescribed in France for the treatment of opioid dependency. In order to evaluate the 
clinical toxicity of methadone, the authors present seven years' experience of the French Poison Control Center (FPCC) Network at the national level.

Methods: This study is a retrospective analysis of methadone exposures reported to the FPCC between 15 October 2010 and 15 October 2017. The two pharmaceutical forms (capsule and syrup) were considered. Childhood accidental poisonings were excluded. Results: Overall 1415 cases of methadone poisoning were included (29\% female, $71 \%$ male, average age $34 \pm 10$ years); $90 \%$ of patients had a history of illicit addiction. Methadone treatment was prescribed for $69 \%$ of the patients $(31 \%$ were naïve patients). The two main circumstances of exposure were abuse (47\% of the cases) and suicide attempts (41\%). Methadone capsules were taken in $45 \%$ of the cases, the syrup in $35 \%$, and unknown for $20 \%$. The ingested methadone dose was significantly higher for suicide attempts (average dose $250 \mathrm{mg}$ ) than in cases of abuse (average dose $140 \mathrm{mg}$ ). In total 126 deaths were observed representing $7 \%$ of the global lethal medicine poisonings observed during the studied period at the national level for the FPCC. Comparison of the abuse cases versus the suicide attempts emphasizes differences: female patients were more commonly implicated in suicide attempts with ingestion of their own treatment, mainly capsules at home with co-ingestion of benzodiazepines; while abuse cases concerned mainly males who ingested syrup, frequently associated with other narcotics, and with a higher morbidity and mortality (4 times more lethal outcomes with abuse cases compared to suicide attempts).

Conclusion: Despite lower ingested quantities of methadone with abuse, this study observed higher morbidity and mortality in this circumstance versus suicide attempts. Many reasons can explain this result (high percentage of naïve patients during abuse, positive image of methadone in the addicted population). Undoubtedly, for clinical toxicologists, methadone must be considered as a highly toxic medicine.

\section{Trends in synthetic cathinone use in poisoned patients in Italy from the National Alert System observatory}

\author{
Carlo A Locatellia, Eleonora Buscaglia ${ }^{a}$, \\ Giulia Scaravaggi ${ }^{a}$, Azzurra Schicchi ${ }^{a}$, Pietro Papa ${ }^{b}$ \\ and Davide Lonati ${ }^{\mathrm{a}}$ \\ apavia Poison Control Centre - National Toxicology Information \\ Centre - Clinical and Experimental Lab, Toxicology Unit, Maugeri \\ Clinical and Scientific Institutes IRCCS and University of Pavia, \\ Pavia, Italy; ${ }^{b}$ Laboratory of Analytical Toxicology, Fondazione IRCCS \\ Policlinico San Matteo-Pavia, Pavia, Italy
}

Objective: Synthetic cathinones are the second largest group of new substances monitored by the European Monitoring Centre for Drugs and Drug Addiction (EMCDDA). This study analyses the variability of compounds involved in patients identified by our Poison Control Centre (PCC) (coordinating centre for the Italian Alert System for NPS), the clinical manifestations and outcome.

Methods: All cases referred to our PCC with history of abuse of synthetic cathinones were prospectively collected during an 8year period (2011-September 2018). Patients in which the compound was analytically identified (biological sample/consumed product) were studied. All cases were assessed for demographic data, circumstances of consumption, clinical course and outcome. Results: Overall 33 cases were included (88\% males; age 18-54 years-old). Main clinical manifestations were: severe agitation (75\%), tachycardia (53\%), hallucinations (36\%), and mydriasis (22\%). During the last two years $50 \%$ of patients consumed the substance in the context of chemsex parties (mainly involving men who have sex with men). Multiple traumatic lesions and self-injury wounds were frequently observed. All cases required
Table 1. Trends in synthetic cathinones in poisoned patients in Italy from the National Alert System observatory (2011-2018).

\begin{tabular}{|c|c|c|}
\hline Year & Identified cathinone & $\begin{array}{l}\text { Number } \\
\text { of cases }\end{array}$ \\
\hline \multirow[t]{3}{*}{2011} & Butylone + methylenedioxypyrovalerone (MDPV) & 1 \\
\hline & Butylone & 1 \\
\hline & 4-Methylethcathinone (4-MEC) & 1 \\
\hline \multirow[t]{3}{*}{2012} & 4-MEC* & 2 \\
\hline & Mephedrone + pentedrone & 1 \\
\hline & MDPV & 1 \\
\hline \multirow[t]{2}{*}{2013} & MDPV & 2 \\
\hline & Mephedrone + MDPV & 1 \\
\hline \multirow[t]{2}{*}{2014} & Methylone & 2 \\
\hline & MDPV & 1 \\
\hline \multirow[t]{4}{*}{2015} & a-pyrrolidinovalerophenone (a-PVP) & 1 \\
\hline & MDPV & 3 \\
\hline & Methylone & 1 \\
\hline & Mephedrone + a-PVP & 1 \\
\hline \multirow[t]{3}{*}{2016} & Methylone & 2 \\
\hline & $a-P V P$ & 1 \\
\hline & bk-2C-B* & 1 \\
\hline \multirow[t]{4}{*}{2017} & 4-Methyl-N-ethyl-pentedrone (4-MEAP) & 1 \\
\hline & $\mathrm{N}$-Ethyl-pentylone & 1 \\
\hline & Mephedrone & 1 \\
\hline & New compound not yet recognized & 1 \\
\hline \multirow[t]{5}{*}{2018} & N-Ethyl-pentylone & 2 \\
\hline & Methylenedioxypyrrolidinohexiophenone (MDPHP) & 1 \\
\hline & Ethylcathinone & 1 \\
\hline & Chloroethcathinone & 1 \\
\hline & $\begin{array}{l}\text { Methylethylcathinone }+ \text { methylmethcathinone }+\mathrm{N} \text {-Ethyl- } \\
\text { pentedrone* }\end{array}$ & 1 \\
\hline
\end{tabular}

*Also identified in products.

sedation (benzodiazepines/propofol). There were no fatal cases. In 3 cases analysis was also performed on the consumed products. Seventeen different synthetic cathinones (also in association in 5 cases) were identified (Table 1).

Conclusion: Our experience shows an increase in the identification of new synthetic cathinones during the last two years, compared to the previous period. In particular, 4/9 synthetic cathinones found in the last two years (4-MEAP, N-ethyl-pentylone, MDPHP and chloroethcathinone) had never been identified in Italy previously (in biological samples or drug seizures). Despite the variability in compounds detected, no significant changes in the clinical picture at hospital admission was registered. Synthetic cathinone abuse should be suspected when history of a chemsex party is reported.

\section{Acknowledgement}

Supported by a grant of the Antidrug Department-Presidency of the Council of Ministers, Italy.

\section{Cytochrome P450 enzymes contribute to the metabolism of LSD to nor-LSD and 2-oxo-3-hydroxy-LSD: implications for clinical LSD use}

\author{
Dino Luethi, Stephan Krähenbühl, Matthias E Liechti \\ and Urs Duthaler \\ Division of Clinical Pharmacology and Toxicology, Department of \\ Biomedicine, University Hospital Basel and University of Basel, \\ Basel, Switzerland
}

Objective: In recent years, experimental research on lysergic acid diethylamide (LSD) in healthy subjects and patients with anxiety 
associated with a life-threatening disease has gained new momentum [1]. In humans, LSD is rapidly metabolized into several structurally similar metabolites but firm evidence of the involved metabolizing enzymes is currently lacking. To unravel potential interactions of enzyme polymorphisms and prescription drugs with LSD use, the aim of the current study was to identify the human cytochrome P450 (CYP450) isoforms involved in the metabolism of LSD to nor-LSD and 2-oxo-3-hydroxy-LSD.

Methods: LSD and its metabolites, nor-LSD and 2-oxo-3-hydroxyLSD, were analyzed by liquid chromatography tandem mass spectrometry. LSD was incubated with human liver microsomes over 4 hours and the production of nor-LSD and 2-oxo-3-hydroxy-LSD was quantified. Metabolism was inhibited by the addition of specific CYP450 inhibitors (isoform: 1A2, 2B6, 2C8, 2C9, 2C19, 2D6, $2 \mathrm{E} 1$, and $3 \mathrm{~A} 4$ ). The performance of the microsomal assay was assessed for each experiment based on the metabolic turnover of specific CYP450 substrates with and without inhibitors. Moreover, results obtained with microsomes were verified using recombinant CYP450 isoforms. Analysis of covariance was used to assess the involvement of the CYP450 isoforms in the formation of the metabolites ( $P<0.05$ was considered statistically significant).

Results: Human liver microsomes displayed a minor formation $(<1 \%)$ of nor-LSD and 2-oxo-3-hydroxy-LSD over 4 hours. Still, CYP450 isoforms 2D6, 2E1, and 3A4 significantly contributed to the formation of nor-LSD. Moreover, CYP450 isoforms 1A2, 2C9, $2 E 1$, and $3 A 4$ were significantly involved in the formation of 2oxo-3-hydroxy-LSD. These findings could be verified using recombinant CYP450 isoforms.

Conclusion: Highly relevant CYP450 isoforms are responsible for LSD metabolism. Therefore, genetic polymorphisms and drug interactions could potentially influence the elimination of LSD and may consequently impact drug safety and efficacy. Drugdrug interaction studies are however required to assess the clinical relevance of these findings.

\section{Reference}

[1] Liechti ME. Modern clinical research on LSD. Neuropsychopharmacology. 2017;42:2114-2127.

\section{Changes in the new psychoactive substances (NPS) involved in acute drug toxicity Emergency Department presentations reported to Euro-DEN Plus Network between 2014 and 2017}

David M Wood ${ }^{a}$, Alison M Dines ${ }^{a}$, Knut Erik Hovda ${ }^{b}$, Fridtjof Heyerdahl $l^{b}$, Christopher Yates ${ }^{c}$, Isabelle Giraudon ${ }^{d}$ and Paul I Dargan ${ }^{a}$; on behalf of the Euro-Den Plus Research Group

${ }^{a}$ Clinical Toxicology, Guy's and St Thomas' NHS Foundation Trust, London, United Kingdom; ${ }^{b}$ Norwegian National Unit for CBRNe Medicine, Oslo University Hospital, Oslo, Norway; 'Emergency Department and Clinical Toxicology Unit, Hospital Universitari Son Espases, Mallorca, Spain; ${ }^{d}$ European Monitoring Centre for Drugs and Drug Addiction (EMCDDA), Lisbon, Portugal

Objective: Data from user surveys and analysis of seizures reported to the European Monitoring Centre for Drugs and Drug Addiction (EMCDDA) suggests that there has been decreased use and availability of cathinones mirrored by increased use and availability of synthetic cannabinoids across Europe in the last
Table 1. Number of Euro-DEN Plus presentations per year and percentage of annual presentations involving each class of new psychoactive substance (NPS).

\begin{tabular}{lcccc}
\hline & 2014 & 2015 & 2016 & 2017 \\
\hline $\begin{array}{l}\text { Total number of presenta- } \\
\text { tions involving an NPS }\end{array}$ & $520(9.6 \%)$ & $654(12.4 \%)$ & $532(10.0 \%)$ & $476(6.0 \%)$ \\
$\quad \begin{array}{l}\text { (\% of total Euro-DEN } \\
\quad \text { Plus presentations) }\end{array}$ & & & & \\
Cathinones & $76.9 \%$ & $51.2 \%$ & $30.1 \%$ & $17.2 \%$ \\
Synthetic cannabinoids & $5.4 \%$ & $14.8 \%$ & $53.0 \%$ & $69.5 \%$ \\
Branded/unknown NPS & $13.7 \%$ & $31.2 \%$ & $14.1 \%$ & $8.6 \%$ \\
Phenylethylamine & $3.3 \%$ & $2.9 \%$ & $2.6 \%$ & $2.1 \%$ \\
Other NPS & $0 \%$ & $0.5 \%$ & $0.2 \%$ & $0.6 \%$ \\
Tryptamine & $1.5 \%$ & $1.2 \%$ & $0.9 \%$ & $0.2 \%$ \\
Piperidine & $1.2 \%$ & $0.6 \%$ & $0 \%$ & $0 \%$ \\
Arylcyclohexamine & $0.6 \%$ & $0.3 \%$ & $0.6 \%$ & $0.8 \%$ \\
Novel benzodiazepine & $0.2 \%$ & $0.5 \%$ & $0.4 \%$ & $0.4 \%$ \\
Novel opioid & $0.2 \%$ & $0 \%$ & $0.6 \%$ & $1.7 \%$ \\
\hline
\end{tabular}

few years. This study aimed to determine whether there have been similar trends in the reported NPS associated with acute toxicity Emergency Department presentations across Europe.

Methods: The Euro-DEN Plus project collects longitudinal data currently from 32 Emergency Departments in 22 European countries on presentations with acute recreational drug and NPS toxicity. The Euro-DEN database was searched for presentations involving the use of one or more NPS from 1 January 2014 to 31 December 2017. Data was extracted on the reported NPS involved in each presentation. Analysis was undertaken to determine the percentage of total annual NPS presentations that involved the following classes of NPS: i) cathinones; ii) synthetic cannabinoids; iii) phenylethylamines; iv) branded/unknown NPS; v) tryptamines; vi) piperidines; vii) arylcyclohexamines; viii) novel benzodiazepines; ix) novel opioids and $x$ ) other NPS.

Results: The proportion of Euro-DEN Plus presentations involving an NPS remained stable over the 4 year study period $(6.0-12.4 \%$ of annual Euro-DEN Plus presentations). The percentage of EuroDEN Plus presentations involving the different classes of NPS is shown in Table 1. The most striking change was the shift in the dominant NPS reported in Euro-DEN Plus presentations between 2014 and 2017 (2014: cathinones 76.9\%, synthetic cannabinoids 5.4\%; 2017: cathinones $17.2 \%$, synthetic cannabinoids $69.5 \%$ ).

Conclusion: The reported changes in the predominant NPS being used across Europe from population/sub-population level surveys and seizures over the last few years are also seen in the most commonly reported NPS classes involved in NPS toxicity presentations to the Emergency Department.

\section{Emergency department presentations related to acute toxicity following recreational use of cannabis products in Switzerland}

\author{
Evangelia Liakoni ${ }^{\mathrm{a}}$, Yasmin Schmid ${ }^{\mathrm{b}}$, Irene Scholz ${ }^{\mathrm{a}}$, \\ Laura Mueller ${ }^{\mathrm{c}}$, Aristomenis K Exadaktylos ${ }^{\mathrm{d}}$, \\ Alessandro Ceschi ${ }^{\mathrm{C}}$ and Matthias E Liechti ${ }^{\mathrm{b}}$ \\ ${ }^{a}$ Clinical Pharmacology and Toxicology, Department of General \\ Internal Medicine; Institute of Pharmacology, Inselspital, Bern \\ University Hospital, University of Bern, Bern, Switzerland; 'bivision \\ of Clinical Pharmacology and Toxicology, Basel University Hospital \\ and University of Basel, Basel, Switzerland; 'Division of Clinical \\ Pharmacology and Toxicology, Institute of Pharmacological \\ Sciences of Southern Switzerland, Ente Ospedaliero Cantonale,

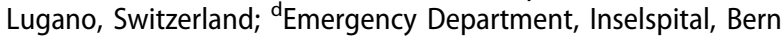 \\ University Hospital, University of Bern, Bern, Switzerland
}


Objective: Cannabis is the most commonly used psychoactive substance worldwide. Although the recreational use of cannabis is usually associated with low toxicity, severe cases including fatalities have been reported. Since co-use of alcohol or other psychoactive substances is very common, it is often difficult to differentiate the acute effects of lone cannabis intoxication from those of other substances. We reviewed clinical effects and outcome in patients presenting to hospital after recreational use of cannabis.

Methods: Retrospective analysis of all cases presenting at the emergency departments of three large hospitals in Switzerland (University Hospital Bern, University Hospital Basel, Ente Ospedaliero Cantonale of Southern Switzerland) due to acute toxicity related to recreational use of cannabis alone or in combination with other substances.

Results: During the study period (Bern: May 2012 - March 2018; Basel: October 2013 - March 2018; Southern Switzerland: January 2017 - March 2018), 717 attendances were related to acute toxicity of cannabis used recreationally, with 112 cases due to cannabis alone (16\%). The mean patient age of the lone cannabis intoxications was 27 years (range 15-68 years), and 78\% $(n=87)$ were male. The route of administration was inhaled/smoked in 61 cases $(54 \%)$, oral in 16 cases $(14 \%)$, and not recorded in 35 cases (31\%). The most commonly reported clinical symptoms/ signs were palpitations $(n=24,21 \%)$, impaired consciousness (i.e. Glasgow Coma Score (GCS) 7-14; $n=22,20 \%$ ), nausea/vomiting $(n=21,19 \%)$, and anxiety $(n=19,17 \%)$. Treatment was required in 78 cases $(70 \%)$, including intubation in one case $(1 \%)$ and sedation in 25 cases (22\%). Most intoxications were of minor severity $(n=67,60 \%)$ and there were no fatalities. Severe complications included coma $(n=9,8 \%)$, psychosis $(n=7,6 \%)$, hallucinations $(n=6,5 \%)$, seizures $(n=5,4 \%)$, supraventricular arrhythmia $(n=$ $1,1 \%)$, and respiratory depression $(n=1,1 \%)$. Most patients $(n=$ $92,82 \%)$ were medically discharged from the emergency department, $12(11 \%)$ were referred to psychiatric care and one $(1 \%)$ to the intensive care unit.

Conclusion: In most cases lone cannabis intoxication was associated with minor toxicity. Nevertheless, severe complications including coma were also reported, and there were cases requiring intubation and admission to intensive or psychiatric care, indicating that lone cannabis intoxication does not exclude considerable health risks.

\section{The trend in the use of substances of abuse over the past three and a half years in Greece}

\author{
Vassiliki Sofidiotou, Myrto Bonataki, Eleni Basanou, \\ Vassiliki Touloupaki, Nikoleta Oikonomou, \\ Angeliki Kalostou, Dimitrios Kolovatsios and \\ Polyxeni Neou \\ Greek Poison Information Center, "P \& A Kyriakou" Children's \\ Hospital, Athens, Greece
}

Objective: The purpose of this study is to record the trend in the use of substances abuse in Greece and to highlight the increasing trend in the use of new psychoactive substances and the prevalence of polydrug use and drug misuse in addicted subjects.

Methods: An analysis of enquiries to the Greek Poison Centre on symptomatic patients presenting to emergency departments (ED) across Greece from 2015 to June 2018. Information was collected on age, gender, symptoms, type of substances and combinations. Results: Among 710 patients aged 11-75 years, most were males (78\%). Mild to severe central nervous system and cardiovascular symptoms occurred in $52 \%$ and in $17 \%$, respectively. In addition,
$16 \%$ of patients had renal failure, respiratory distress or fluid and electrolyte disorders. Patients were divided according to selfreported drug use at admission to the ED into one of two groups, either isolated use of a single drug of abuse or reported polydrug use. In the isolated use of older substances throughout the study there was a progressive increase in the use of opioids and cannabis and almost constant cocaine use, with the exception of the decrease in the use of cocaine in 2016. As regards the new psychoactive substances of abuse (e.g. synthetic cannabinoids, cathinones and opiates, phenethylamines, ketamine, gamma-hydroxybutyrate (GHB), gamma-butyrolactone (GBL), volatile nitrites and salvia), there was a gradual increase in use $(6,10$, 18, 13 respectively in 2015, 2016, 2017 and the first half of 2018). One death and six serious cases occurred among the 47 cases of this group. Polydrug use had a frequency of $42 \%(298 / 710$ patients). Opioids dominated multiple drug usage with $55 \%$, while cocaine/3,4-methylenedioxymethamphetamine (MDMA) occured in $27.8 \%$ and cannabinoids at $17.1 \%$. During the study period, $17 \%$ of cases $(121 / 710)$ of drug misuse were recorded.

Conclusion: Drug use, and the associated problems, is a complex and multifaceted phenomenon which changes over time. Polydrug use and drug misuse is very common among addicts $[1,2]$ in Greece, as in other countries. In Greece cocaine-MDMA and opioids continue to be the main cause of morbidity, while new psychoactive substances have an upward trend with risk of severe symptoms.

\section{References}

[1] Jones JD, Mogali S, Come SD. Polydrug abuse: A review of opioid and benzodiazepine combination use. Drug Alcohol Depend. 2012;125:8-18.

[2] Bouvier BA, Waye KM, Elston B, et al. Prevalence and correlates of benzodiazepine use and misuse among adults who use prescription opioids non-medically. Drug Alcohol Depend. 2018;183:73-77.

\section{Unintentional clandestine opioid (U-47700) overdose in an adolescent after counterfeit alprazolam use}

\author{
Brittany P Chapman ${ }^{\mathrm{a}}$, Jeffrey T Lai ${ }^{\mathrm{a}}$, Alex J Krotulski ${ }^{\mathrm{b}}$, \\ Melissa F Fogarty ${ }^{\mathrm{b}}$, Matthew K Griswold ${ }^{\mathrm{c}}$, \\ Barry K Logan ${ }^{\mathrm{b}}$ and Kavita M Babu ${ }^{\mathrm{a}}$ \\ a University of Massachusetts Medical School, Worcester, United \\ States; ${ }^{\text {b } C e n t e r ~ f o r ~ F o r e n s i c ~ S c i e n c e ~ R e s e a r c h ~ a n d ~ E d u c a t i o n ~ a t ~ t h e ~}$ \\ Fredric Rieders Family Foundation, Willow Grove, United States; \\ 'Hartford Hospital, Hartford, United States
}

Objective: To describe the case of an adolescent who intended to use alprazolam but experienced a near-fatal overdose resulting from unintended exposure to the clandestine opioid, U-47700.

Case report: A 17-year-old male with a history of daily marijuana use and previous hallucinogen use became apneic and unresponsive en route to school. School staff administered $4 \mathrm{mg}$ of intranasal naloxone, reversing his altered mental status and respiratory depression. Upon arrival to the emergency department (ED), the patient's vital signs were heart rate $125 \mathrm{bpm}$, respiratory rate 18 , and pulse oximetry $100 \%$ (room air). His pupils were $4 \mathrm{~mm}$ in diameter, equal and reactive to light, and a neurologic exam was normal. The attending physician suspected opioid overdose and questioned the patient regarding substance use. The patient reported insufflating a white powder he had been led to believe was "crushed Xanax," but adamantly denied ever using opioids. Due to disparity between his reported exposure and observed clinical effects, blood and urine specimens 
were obtained. All blood specimens and half of the urine specimen were sent to the Center for Forensic Science Research and Education (CFSRE) for analysis using quadrupole time-of-flight mass spectrometry coupled with ultra-high performance liquid chromatography (LC-QTOF-MS). The remaining urine was sent to the hospital lab for a comprehensive drug screen using gas chromatography/mass spectrometry (GC/MS). The hospital urine drug screen detected caffeine only; however, directed analysis with LCQTOF-MS confirmed the presence of the parent compound U47700 at $47.5 \mathrm{ng} / \mathrm{mL}$, and the metabolites N-desmethyl-U-47700 and $\mathrm{N}, \mathrm{N}$-didesmethyl-U-47700. The same instrumental analysis demonstrated congruent results in all blood specimens. Additionally, U-47700 was quantitated in the pair of blood specimens collected 4.5 hours apart, revealing concentrations of 282.4 $\mathrm{ng} / \mathrm{mL}$ (collected 1 hour after ED presentation) and $18.7 \mathrm{ng} / \mathrm{mL}$, respectively. These findings implicate $\mathrm{U}-47700$ as the culprit of the toxic effects experienced by the patient.

Conclusion: Increased availability of high-potency opioid agonists necessitates heightened suspicion for unintentional exposure to these agents. In this case, an adolescent who intended to use alprazolam encountered U-47700 with life-threatening effect. Clinicians should remain vigilant for symptoms consistent with opioid overdose, have a low threshold for empiric naloxone treatment, and consider obtaining specimens for high-end analytical testing (e.g. LC-QTOF-MS) to improve surveillance and facilitate public health interventions.

\section{Analytically confirmed use of 3- methoxy-phencyclidine (3-MeO-PCP) in acute non-fatal polysubstance poisonings in Ibiza}

\author{
María A Leciñena ${ }^{a}$, Emilia Moreno ${ }^{b}$, Laura Sahuquillo ${ }^{b}$, \\ Jordi Puiguriguer Ferrando ${ }^{c}$, Christopher Yates ${ }^{c}$, \\ Isabel Gomila ${ }^{\mathrm{d}}$, Miguel Angel Elorza ${ }^{\mathrm{e}}$ and \\ Bernardino Barceló Martin \\ aEmergency Department, Hospital Can Misses, Ibiza, Spain; \\ ${ }^{b}$ Clinical Analysis Department, Hospital Can Misses, Ibiza, Spain; \\ 'Emergency Department and Clinical Toxicology Unit, Hospital \\ Universitari Son Espases, Palma de Mallorca, Spain; ${ }^{d}$ Clinical \\ Analysis Department, Hospital Universitari Son Llàtzer; Research \\ Institute of Health Sciences (IdISBa), Palma de Mallorca, Spain; \\ eLaboratory of Clinical Toxicology, Clinical Analysis Department, \\ Hospital Universitari Son Espases; Research Institute of Health \\ Sciences (IdISBa), Palma de Mallorca, Spain
}

Objective: 3-MeO-PCP is a dissociative psychoactive drug sold as a legal alternative to phencyclidine (PCP). Documented poisonings associated with its consumption are scarce [1]. We present two cases of analytically confirmed use of 3-MeO-PCP in acute multiple poisonings.

Case series: Case 1: A 32-year-old woman was admitted to the Emergency Department (ED) due to suspected ketamine, cocaine and ecstasy poisoning. She was unconscious, hypertensive, and tachycardic, with periods of oxygen desaturation. She presented sialorrhea, upper limb rigidity, trismus and mydriasis. She was acidotic ( $\mathrm{pH} 7.251)$ with high serum lactate $(4.81 \mathrm{mmol} / \mathrm{L})$ and creatine kinase (CK) $(940 \mathrm{U} / \mathrm{L})$. She received biperiden $(5 \mathrm{mg})$, diazepam (5 mg), ondansetron (4 mg) and midazolam (3 mg), with clinical improvement. At 7 hours post-admission, she was agitated with a decreased level of consciousness and required sedation. She was discharged after 13 hours. Case 2: A 24-yearold man was admitted to the ED due to a suspected ketamine, cocaine and amphetamine poisoning, hypertensive, but conscious, oriented and hemodynamically stable. Captopril (25 mg) and diazepam $(10 \mathrm{mg}$ ) were administered. He was discharged 4 hours later. The urine drug immunoassays were positive for $\mathrm{PCP}$, amphetamines and cocaine in both patients, and also for cannabis in case 2. Confirmatory results by gas chromatography-mass spectrometry ruled out the presence of $P C P$ and methamphetamine, however, 3-MeO-PCP and MDMA/MDA were detected in both patients, as well as cocaine and metabolites, and ketamine in case 2 (Table 1). Serum concentration of 3-MeO-PCP in case 1 was $46.6 \mathrm{ng} / \mathrm{mL}$.

Conclusion: Detection of 3-MeO-PCP in these patients represents the first identifications performed in Spain. Concentrations reported are comparable to those documented in non-fatal poisonings. A positive PCP result raises suspicion of possible detection of 3-MeO-PCP.

\section{Reference}

[1] Bertol E, Pascali J, Palumbo D, et al. 3-MeO-PCP intoxication in two young men: First detection in Italy. Forensic Sci Int. 2017;274:7-12.

\section{Substance abuse-related Emergency Room visits in Ramathibodi Hospital, Bangkok, Thailand}

\author{
Satariya Trakulsrichai ${ }^{\mathrm{a}}$, Wipada Pispan ${ }^{\mathrm{a}}$ and \\ Umaporn Udomsubpayakul ${ }^{\mathrm{b}}$

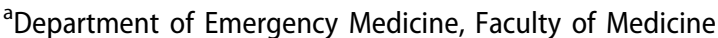 \\ Ramathibodi Hospital, Mahidol University, Bangkok, Thailand; \\ ${ }^{\mathrm{b}}$ Section for Clinical Epidemiology and Biostatistics, Research \\ Center, Faculty of Medicine Ramathibodi Hospital, Mahidol \\ University, Bangkok, Thailand
}

Objective: To describe and analyze clinical characteristics, toxicological laboratory findings, life-saving procedures required and outcomes of substance abuse patients who visited the Emergency Room (ER), Ramathibodi Hospital, Bangkok, Thailand.

Methods: A retrospective study of hospital medical records of substance abuse patients who visited the ER during a 9-year period (2009-2017).

Table 1. Toxicological findings in urine samples collected at admission in two patients admitted with polysubstance poisoning in Ibiza.

\begin{tabular}{|c|c|c|}
\hline \multirow[b]{2}{*}{ Drug } & \multicolumn{2}{|c|}{ Concentration, ng/mL (ng/mg creatinine) } \\
\hline & Case 1 & Case 2 \\
\hline 3-Methoxy-phencyclidine (3-MeO-PCP) & $9645.3(22588.6)$ & $560.52(870.37)$ \\
\hline Benzoylecgonine & $15623.5(36589.1)$ & 49838.49 (77149.36) \\
\hline Cocaine & Not detected & $362.6(563.09)$ \\
\hline 3,4-methylenedioxymethamphetamine (MDMA) & $580(1358.3)$ & $27356.97(42479.77)$ \\
\hline Methylenedioxyamphetamine (MDA) & Not detected & 2430.67 (3774.33) \\
\hline Ketamine & Not detected & $2087.23(3241.03)$ \\
\hline Norketamine & Not detected & $1250.72(1942.11)$ \\
\hline
\end{tabular}


Results: Seventy-four patients presented to the ER with a history and clinical symptoms of substance abuse. The mean age was $28.2 \pm 12.8$ years; most were male $(55 \%)$ and students (34.7\%). Some patients arrived by ambulance (15.8\%). The most common groups of single substances reported were amphetamines (27\%), opioids including tramadol and dextromethorphan (23\%), sedatives (10.8\%) and kratom (10.8\%). Nine patients reported multiple drug use. The common clinical presentations were confusion/agitation (31.6\%), depressed level of consciousness (19.3\%) and seizure (14\%). Abnormal vital signs and cardiovascular system effects were found in $62.5 \%$ and $54.4 \%$ of patients, respectively. Mass spectrometry blood or urine toxicology tests reported methamphetamine $(20.3 \%)$, tramadol $(14.8 \%)$, codeine $(9.4 \%)$, mitragynine/kratom (6.8\%), morphine, dextromethorphan, alprazolam, heroin, cannabinoids (5.4\% each), 3,4-methylenedioxymethamphetamine (MDMA), ketamine, toluene, trihexyphenidyl (4\% each), promethazine and clonazepam (2.7\% each). Five substances were detected in the urine of one patient including mitragynine, codeine, tramadol, dextromethorphan and clonazepam. The history of substance use was consistent with the findings from the confirmation tests in $71 \%$ of patients, while in $37.5 \%$ of these patients more substances were detected than they self-reported. The groups of substances from history taking were not statistically significantly different from confirmation laboratory findings. Most patients received supportive treatment while life-saving procedures including endotracheal intubation, central catheter insertion, and cardiopulmonary resuscitation were performed in 8 patients. Fourteen patients received naloxone. The median ER stay was 9 (1-48) hours. Some patients $(41.2 \%)$ were admitted to the hospital. The median hospital stay was 4 (1-25) days. One patient died due to opioid overdose. We performed subgroup analysis between the patients who needed and did not need lifesaving procedures. We found that age, gender, visiting ER by ambulance and types or groups of substances used were not statistically significantly different between the two groups.

Conclusion: Amphetamines, opioids including tramadol and dextromethorphan, sedatives and kratom were common substances of abuse related to ER visits in Thailand. The history of substances used might be helpful if laboratory analysis is unavailable. Although some patients require life-saving procedures, the mortality from substance abuse was quite low.

\section{Recreational food: if legal highs are in the kitchen}

\author{
Diletta Sabatini, Paolo Boldrini, Viola Mazzucco and \\ Maria Caterina Grassi \\ Clinical Toxicology - Poison Control Centre and Drug Dependence Unit, \\ Policlinico Umberto I - "Sapienza", University of Rome, Rome, Italy
}

Objective: Nutmeg is commonly used as a spice but for its psychotomimetic properties is also considered as a legal high [1]. Nitrous oxide has gained popularity among adolescents and young adults and its misuse is alarming widespread [2]. Hence, we report two cases of teenagers who easily gained access to these two legal highs for recreational and self-medication purposes.

Case reports: Case 1. The Poison Control Centre (PCC) of Umberto I Hospital of Rome was contacted at 3:00 a.m. about a 16-year-old boy, $50 \mathrm{Kg}$, who had ingested about $7 \mathrm{~g}$ of nutmeg 14 hours before. He presented a clinical history of depressive mood and social isolation. Six hours after ingestion he developed emesis, abdominal pain and headache. In particular, emesis was repeated and persistent. No psychotomimetic symptoms were reported. The PCC suggested admission to the local Emergency Department (ED) to treat the emesis and to assess his suicidal risk. The boy revealed that he tried the nutmeg for its euphoriainducing properties following some reports read on the Internet. Case 2. A 14-year-old girl was admitted at the local ED as the result of inhaling nitrous oxide contained in a dairy cream spray for recreational use. She reported nausea, dizziness, tachycardia (heart rate $106 \mathrm{bpm}$ ) and anterograde amnesia. The PCC was contacted and suggested monitoring of vital signs and to perform an arterial blood gas test to check for methemoglobinemia. No abnormalities were detected and the patient was discharged without symptoms 6 hours later. She revealed she had inhaled the nitrous oxide with friends after reading about the procedure on the Internet.

Conclusion: Although these two cases have low clinical relevance because of the transient and minor symptoms presented they allow some considerations: i) the lasting trend among adolescents to search for information on the Internet about substances usually sold legally and easily available, ii) the use of these substances even as self-medication, iii) easy availability may lead to underestimation of the risks associated with their abuse, and iv) the variability in the psychopharmacological response as well as the concentrations of its active components does not ensure a "safe" level of exposure in the context of nutmeg intoxication [1].

\section{References}

[1] Sangalli BC, Chiang W. Toxicology of nutmeg abuse. J Toxicol Clin Toxicol. 2000;38:671-678.

[2] Thompson AG, Leite MI, Lunn MP, et al. Whippits, nitrous oxide and the dangers of legal highs. Pract Neurol. 2015;15:207-209.

\section{Increasing enquiries to the UK National Poisons Information Service (NPIS) concerning alprazolam}

\author{
Leonard Hawkins ${ }^{a}$, David Lupton ${ }^{b}$, Gillian Jackson ${ }^{b}$, \\ Sally M Bradberry ${ }^{c}$, Euan A Sandilands ${ }^{b}$ and \\ Simon HL Thomas ${ }^{a}$ \\ ${ }^{a}$ National Poisons Information Service (Newcastle Unit), Newcastle \\ Hospitals NHS Foundation Trust, Newcastle, United Kingdom; \\ ${ }^{b}$ National Poisons Information Service (Edinburgh Unit), Royal \\ Infirmary, Edinburgh, United Kingdom; 'National Poisons \\ Information Service (Birmingham Unit), City Hospital, Birmingham, \\ United Kingdom
}

Objective: Alprazolam $\left(\mathrm{Xanax}^{\circledast}\right)$ is a benzodiazepine tranquilizer first authorized in the UK in 1982 and indicated for the shortterm treatment of moderate or severe anxiety states and anxiety associated with depression. We studied trends in enquiries to the UK NPIS and accesses to the poisons information database TOXBASE $^{\circledR}$ relating to alprazolam because of increasing reports of alprazolam abuse in the UK.

Methods: A search was performed of all telephone enquiries to the NPIS and TOXBASE accesses concerning alprazolam for the period April 2013 to September 2018 (inclusive).

Results: During the 5.5 years studied there were 494 NPIS telephone enquiries involving alprazolam (females $51 \%$, males $47 \%$, sex unknown 2\%) and 13,383 accesses to the alprazolam entry on TOXBASE (including those made via the TOXBASE App). Telephone enquiries most often came from hospitals (67\%), primary care (14\%) and ambulance services (11\%). Exposure was most commonly classified as intentional overdose (57\%), recreational abuse (20\%), unknown (7\%) therapeutic error $(6 \%)$ or accidental (5\%). Peak age groups were 20-29 years in males and 3039 years in females. At the time of the enquiry, poisoning severity score (PSS) was recorded as 0 (no toxicity) in 34\%, 1 (minor toxicity) in 46\%, 2 (moderate toxicity) in $11 \%, 3$ (severe toxicity) in $5 \%$ and was not recorded in $3 \%$, but other substances were also commonly involved and are likely to have contributed to the 
Table 1. Annual trends in telephone enquiries and TOXBASE accesses relating to alprazolam.

\begin{tabular}{lcc}
\hline Year (April-March) & Telephone enquiries & TOXBASE accesses \\
\hline $2013-14$ & 70 & 768 \\
$2014-15$ & 53 & 840 \\
$2015-16$ & 70 & 944 \\
$2016-17$ & 69 & 1823 \\
$2017-18$ & 160 & 5624 \\
$2018-19$ (half year to September) & 72 & 3384 \\
\hline
\end{tabular}

effects seen. Annual enquiry and TOXBASE access numbers demonstrate substantial increases since 2016 (Table 1).

Conclusion: Alprazolam-related telephone enquiries and TOXBASE accesses have increased substantially in the UK since 2016. This may reflect increasing availability and misuse of alprazolam obtained via the Internet or dealers.

\section{Internet availability of modafinil and methylphenidate}

\author{
Joanna Hockenhull, David M Wood and \\ Paul I Dargan \\ Clinical Toxicology, Guy's and St Thomas' NHS Foundation Trust, \\ London, United Kingdom
}

Objective: There are reports of prescription stimulants being purchased online for use as cognitive enhancers or "smart drugs". The aim of this study was to investigate availability of modafinil and methylphenidate from Internet suppliers from the perspective of a typical UK-based customer.

Methods: Using the European Monitoring Centre for Drugs and Drug Addiction (EMCDDA) Internet snapshot methodology, this study was conducted July to August 2018, using Internet search engines: "bing.co.uk", "google.co.uk" and "yahoo.co.uk" and the search terms: "buy modafinil", "buy provigil", "buy methylphenidate" and "buy ritalin". Websites were excluded based on being unresponsive, not selling the desired drug, not shipping to the UK or requiring a prescription to purchase.

Results: A total of 104 modafinil and 138 methylphenidate websites were identified from which the drug could be purchased without a prescription from 67 (64.4\%) and 22 (19.8\%) websites, respectively. The majority of websites excluded were due to the desired drug not being available to purchase while only five modafinil (4.8\%) and six methylphenidate (5.4\%) websites were excluded on the basis of requiring a prescription or licence. It was specifically stated that no prescription was required on 27 modafinil (40.3\%) and 16 methylphenidate (72.7\%) websites. Minimum purchase quantities ranged from 10-90 tablets for modafinil and 1-1,005 tablets for methylphenidate with no apparent upper limit to the number that could be purchased. The price per tablet varied from $£ 0.38-5.31$ for modafinil and $£ 0.15-5.70$ for methylphenidate. Free shipping was offered if more than a certain amount was spent on 54 modafinil (80.4\%) and ten methylphenidate $(45.5 \%)$ websites and discounts were offered on 52 modafinil (77.6\%) and six methylphenidate (27.3\%) websites.

Conclusion: Modafinil and methylphenidate are widely available to purchase on the Internet in the UK without a prescription. The pricing on websites encourages users to buy greater quantities to qualify for discounts and free shipping. The quantities available suggest these purchases may be misused by individuals in greater amounts than would be legitimately prescribed or diverted to other individuals increasing the risk of non-medical use of the drugs. This survey methodology, developed by the EMCDDA, has previous provided useful information regarding the availability of new psychoactive substances and we have shown here that it can also be applied to monitoring Internet markets for prescription drugs. Continual monitoring using this methodology will enable law enforcement and policy makers to understand availability of these substances to potential users.

\section{Regional variation in non-medical use of benzodiazepines in the UK}

\author{
Joanna Hockenhull ${ }^{a}$, David M Wood ${ }^{a}$, \\ Colleen M Haynes ${ }^{\mathrm{b}}$, Joshua C Black ${ }^{\mathrm{b}}$, \\ Karilynn Rockhill ${ }^{\mathrm{b}}$, Richard C Dart ${ }^{\mathrm{b}}$ and Paul I Dargan ${ }^{\mathrm{a}}$ \\ ${ }^{a}$ Clinical Toxicology, Guy's and St Thomas' NHS Foundation Trust, \\ London, United Kingdom; ${ }^{\mathrm{b}}$ Rocky Mountain Poison \& Drug Center, \\ Denver Health, Denver, United States
}

Objective: Recent investigations by regulatory authorities in the UK have confirmed increasing diversion and non-medical use (NMU) of prescription medicines, particularly benzodiazepines. There is limited published data on the prevalence of NMU of benzodiazepines in UK, however, and whether there are regional differences in this activity.

Methods: Data collected in the third quarter of 2017 in the UK by online Survey of Non-Medical Use of Prescription Drugs were analysed. Post-stratification weights were applied to responses from 10,019 individuals to represent distribution of age, gender, and region of 52,927,659 adults living in the UK. This online survey collects data on the prevalence, motivations and behaviours for NMU of prescription drugs. The prevalence estimates of last 12 month NMU were calculated of any benzodiazepines (alprazolam, bromazepam, chlordiazepoxide, clobazam, clonazepam, diazepam, flurazepam, lorazepam, nitrazepam, oxazepam, or temazepam) and the five most commonly non-medically used benzodiazepines (diazepam, temazepam, lorazepam, clonazepam, and alprazolam) for the following regions: London, England (except London), Northern Ireland (NI), Scotland, and Wales.

Results: London had the highest prevalence for any benzodiazepine NMU (1.86\%, 95\% Confidence Interval (Cl) 1.15-2.58) while the lowest prevalence was seen in the rest of England $(0.67 \%, 95 \% \mathrm{Cl} 0.47-0.87)$ (Table 1$)$. While the prevalence of NMU of benzodiazepines is low, there are differences by type.

Table 1. Prevalence of last 12 month non-medical use of benzodiazepines in the UK.

\begin{tabular}{|c|c|c|c|c|c|c|}
\hline \multirow[b]{2}{*}{ Region } & \multicolumn{6}{|c|}{ Last 12 month non-medical use $\%(95 \% \mathrm{Cl})$} \\
\hline & Any Benzodiazepine & Alprazolam & Clonazepam & Diazepam & Lorazepam & Temazepam \\
\hline London & $\begin{array}{c}1.86 \\
(1.15-2.58)\end{array}$ & $\begin{array}{c}0.42 \\
(0.08-0.76)\end{array}$ & $\begin{array}{c}0.51 \\
(0.13-0.89)\end{array}$ & $\begin{array}{c}1.08 \\
(0.53-1.63)\end{array}$ & $\begin{array}{c}0.63 \\
(0.22-1.04)\end{array}$ & $\begin{array}{c}0.42 \\
(0.08-0.76)\end{array}$ \\
\hline England (except London) & $\begin{array}{c}0.67 \\
(0.47-0.87)\end{array}$ & $\begin{array}{c}0.07 \\
(0.01-0.14)\end{array}$ & $\begin{array}{c}0.14 \\
(0.03-0.24)\end{array}$ & $\begin{array}{c}0.54 \\
(0.37-0.71)\end{array}$ & $\begin{array}{c}0.14 \\
(0.05-0.22)\end{array}$ & $\begin{array}{c}0.19 \\
(0.09-0.29)\end{array}$ \\
\hline Northern Ireland & $\begin{array}{c}0.95 \\
(0.00-2.79)\end{array}$ & $\begin{array}{c}0.95 \\
(0.00-2.79)\end{array}$ & 0 & 0 & 0 & 0 \\
\hline Scotland & $\begin{array}{c}0.91 \\
(0.26-1.56)\end{array}$ & $\begin{array}{c}0.42 \\
(0.00-0.90)\end{array}$ & 0 & $\begin{array}{c}0.82 \\
(0.19-1.45)\end{array}$ & 0 & $\begin{array}{c}0.09 \\
(0.00-0.25)\end{array}$ \\
\hline Wales & $\begin{array}{c}1.33 \\
(0.00-2.77)\end{array}$ & 0 & $\begin{array}{c}0.29 \\
(0.00-0.86)\end{array}$ & $\begin{array}{c}1.33 \\
(0.00-2.77)\end{array}$ & $\begin{array}{c}0.29 \\
(0.00-0.86)\end{array}$ & 0 \\
\hline
\end{tabular}


Diazepam showed the highest prevalence of non-medical use in London, England (except London), Scotland, and Wales, while alprazolam showed the highest prevalence in NI.

Conclusion: There is regional variation in the NMU of benzodiazepines within the UK, despite similar regional prescribing policies. Further research is needed to investigate factors responsible for the regional variation observed and understand the motivations of benzodiazepine NMU to inform the design of public health interventions.

\section{Differences in the pattern and prevalence of non-medical use of prescription benzodiazepines, gamma- aminobutyric acid (GABA) analogues and stimulants in Europe}

\author{
Joanna Hockenhull ${ }^{\mathrm{a}}$, Paul I Dargan ${ }^{\mathrm{a}}$, \\ Colleen M Haynes ${ }^{\mathrm{b}}$, Joshua C Black ${ }^{\mathrm{b}}$, Richard C Dart ${ }^{\mathrm{b}}$ \\ and David M Wood ${ }^{\mathrm{a}}$ \\ ${ }^{a}$ Clinical Toxicology, Guy's and St Thomas' NHS Foundation Trust,

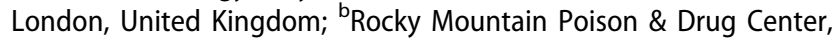 \\ Denver Health, Denver, United States
}

Objective: There is increasing concern across Europe on the non-medical use (NMU) of prescription medicines. The aim of this study was to determine the prevalence of last 12 month NMU of benzodiazepines, GABA analogues, and stimulants through data collected in five European countries via the Survey of Non-Medical Use of Prescription Drugs (NMURx) Program.

Methods: Data from the NMURx Program from the $4^{\text {th }}$ quarter of 2017 from France $(n=10,072)$, Germany $(n=15,051)$, Italy, $(n=$ $10,019)$, Spain $(n=10,062)$, and the 3rd quarter of 2017 from the UK $(n=10,019)$ were analysed. Post-stratification weights for gender, region, and age were applied to represent distribution of the general population of adults in each country. For each survey, the estimated prevalence and 95\% confidence interval of respondents reporting NMU within the last 12 months were calculated and compared. NMU was defined as "using a medication without a doctor's prescription or for any reason other than what was recommended by their doctor".

Results: Spain, Italy and France had higher prevalence of last 12 month NMU of benzodiazepines than GABA analogues (Table 1). Germany had the highest prevalence of recent NMU of GABA analogues and the lowest prevalence for benzodiazepines; NMU of GABA analogues in Germany was at least double that seen in other countries. The rate of NMU for stimulants were low in all countries compared to the other drug groups.

Conclusion: Differences were observed in last 12 month NMU of different drug classes across five countries in Europe. Further work is required to understand the reasons for these differences in NMU and whether this relates to national prescribing practices, ease of diversion between countries or other reasons.

Table 1. Prevalence of last 12 month non-medical use of prescription drugs in Europe.

\begin{tabular}{lccc}
\hline & \multicolumn{3}{c}{ Last 12 Month Non-Medical Use, Prevalence \% $(95 \% \mathrm{Cl})$} \\
\cline { 2 - 4 } Country & Benzodiazepines & GABA analogues & Stimulants \\
\hline France & $1.7(1.5-2.0)$ & $1.4(1.2-1.6)$ & $0.6(0.4-0.7)$ \\
Germany & $1.0(0.9-1.2)$ & $3.2(2.9-3.5)$ & $0.7(0.6-0.9)$ \\
Italy & $2.5(2.1-2.8)$ & $1.1(0.8-1.3)$ & $0.6(0.4-0.7)$ \\
Spain & $4.0(3.6-4.4)$ & $1.5(1.2-1.7)$ & $1.0(0.8-1.2)$ \\
UK & $1.0(0.8-1.2)$ & $1.3(1.1-1.6)$ & $0.5(0.4-0.7)$ \\
\hline
\end{tabular}

Understanding patterns in NMU can inform strategies and interventions to prevent NMU more appropriately.

\section{Gender differences in benzodiazepine-addicted patients}

\author{
Gabija Valauskaite ${ }^{a}$, Deima Eitmontaite ${ }^{a}$ and \\ Robertas Badaras ${ }^{\mathrm{b}}$ \\ ${ }^{a}$ Vilnius University, Faculty of Medicine, Vilnius, Lithuania; ${ }^{b}$ Vilnius \\ University Emergency Hospital, Centre of Toxicology, Faculty of \\ Medicine, Vilnius University, Vilnius, Lithuania
}

Objective: The aim of this study was to establish different addiction patterns between genders in benzodiazepine (BZD)-addicted patients.

Methods: We retrospectively evaluated patients, who were diagnosed with BZD dependence and had undergone benzodiazepine detoxification treatment at Vilnius University Emergency Hospital 2012 to 2018. Patients admitted for benzodiazepine overdose and those who had been using benzodiazepines for $<6$ months were excluded from the study. Since patients had used different kinds of benzodiazepines, the doses were converted into diazepam equivalent (DE) [1]. Data was processed by MS Excel and SPSS 22.0 software.

Results: Overall 54 patients were included, 33 females and 21 males. The age median was 48.5 years (27-74 years), female age median was 53 years (34-74 years), male age median was 46 years (27-65 years). Age was not statistically significantly different between genders, $p=0.136$. $D E$ dose median was $56 \mathrm{mg}$ (15-450 $\mathrm{mg}$ ). Men used statistically significantly higher $\mathrm{DE}$ doses than women, $p=0.037$. The median $\mathrm{DE}$ in men was $68 \mathrm{mg}(20-450$ $\mathrm{mg}$ ) and in women $38 \mathrm{mg}(15-329 \mathrm{mg})$. The median duration of hospitalization was 9 days (3-44 days). Men spent statistically significantly less time in hospital, $p=0.018$. The median hospitalization time in men was 7 days (3-38 days) and in women 10 days (3-44 days). Men were more frequently addicted to smoking than women, $38.1 \%$ versus $6.1 \%, p=0.003$. Men were also more frequently addicted to alcohol than women, $52.4 \%$ versus $21.2 \%$, $\mathrm{p}=0.018$.

Conclusion: Men were more prone to be addicted to alcohol or cigarettes in comparison to women. Men were also using higher doses of benzodiazepines and spent less time in treatment.

\section{Reference}

[1] Farinde A. Benzodiazepine Equivalency Table: Benzodiazepine Equivalency. 2017 April 28 [cited 2018 October 5]. Available from: https://emedicine.medscape.com/article/2172250-overview

\section{Fatality associated with therapeutic antidepressant and NBOMe recreational drug use}

\author{
William J Meggs ${ }^{\mathrm{a}}$, Susan N Miller ${ }^{\mathrm{a}}$, \\ Jennifer L Parker-Cote ${ }^{a}$ and Mohan Punja ${ }^{b}$ \\ ${ }^{a}$ Emergency Medicine, East Carolina University, Greenville, United \\ States; ${ }^{b}$ Emergency Medicine, Augusta University, Marietta, United \\ States
}

Objective: Many psychotropic drugs sold for recreational purposes enhance serotonin effects. Serotonin reuptake inhibitors such as fluoxetine have the potential to interact with recreational drugs including the NBOMe serotonergic compounds. Lithium 
carbonate also has potential adverse reactions with serotonin agents. A case is presented of a fatality that occurred immediately after an individual prescribed fluoxetine and lithium for depression used the street drug 25I-NBOMe.

Case report: A 25-year-old woman in a good state of health had a history of anxiety and depression treated with fluoxetine and lithium. She had no adverse effects from her antidepressant treatments. She snorted a drug obtained by her boyfriend for recreational use and developed altered mental status that was documented in four videos recorded by her boyfriend. Officials investigating the death requested Medical Toxicology review of the videos and autopsy report. In the first videos she was alert but non-verbal and ataxic. She became prone, staring, with tachypnea and jerking respirations. The boyfriend activated the emergency medicine services system when she suffered a cardiopulmonary arrest approximately 8 hours after the exposure. Cardiopulmonary resuscitation (CPR) was unsuccessful. Four unused capsules at the scene that were identical to the capsule snorted were analyzed and contained the psychotropic drug 25INBOMe. At autopsy, a therapeutic concentration of fluoxetine was found. Lithium concentration was not reported. Urine levels of $25 \mathrm{I}-\mathrm{NBOMe}$ were below the detection limit of $0.062 \mathrm{mg} / \mathrm{L}$.

Conclusion: 25I-NBOMe and related compounds can have severe and even fatal consequences [1]. Fatal drug interactions can occur between drugs that enhance serotonin and other neurotransmitters, including serotonin syndrome [2]. This patient was taking fluoxetine and possibly lithium, putting her at risk for serotonin syndrome. Death occurred eight hours after using 25INBOMe, suggesting serotonergic street drugs can be lethal in those taking therapeutic serotonergic drugs.

\section{References}

[1] Kriakoui C, Marinelli E, Frati P, et al. NBOMe: new potent hallucinogens - pharmacology, analytical methods, toxicities, fatalities: a review. Eur Rev Med Pharmacol Sci. 2015;19:3270-3281.

[2] Boyer EW, Shannon M. The serotonin syndrome. N Engl J Med. 2005;352:1112-1120.

\section{Acute recreational drug toxicity in women: a case series from Oslo, Norway}

\author{
Victoria L Syse ${ }^{a}$, Mette Brekke ${ }^{b}$, Marit M Grimsrud ${ }^{a}$, \\ Per Sverre Persett ${ }^{c}$, Fridtjof Heyerdahl ${ }^{d}$, \\ Knut Erik Hovda ${ }^{e}$ and Odd Martin Vallersnes ${ }^{\dagger}$ \\ ${ }^{\mathrm{a}}$ Faculty of Medicine, University of Oslo, Oslo, Norway; ${ }^{\mathrm{b}} \mathrm{General}$ \\ Practice Research Unit, University of Oslo, Oslo, Norway; \\ 'Department of Acute Medicine, Oslo University Hospital, Oslo, \\ Norway; ${ }^{\mathrm{d}}$ Department of Prehospital Medicine, Oslo University \\ Hospital, Oslo, Norway; ${ }^{\mathrm{T} T h e}$ Norwegian CBRNe Centre of \\ Medicine, Oslo University Hospital, Oslo, Norway; ${ }^{f}$ Department of \\ General Practice, University of Oslo, Oslo, Norway
}

Objective: Female drug users report poorer physical and mental health than males. To see whether this impacts on presentations to emergency medical services, we describe female patients treated for acute recreational drug toxicity, and compare them with male patients as to their clinical state and toxic agents taken.

Methods: Case series from a primary care emergency outpatient clinic and a hospital emergency department in Oslo, Norway. All patients treated for acute recreational drug toxicity from October 2013 through March 2015 were included, except patients with lone alcohol intoxication. We collected data on gender, age, toxic agents taken, clinical state, length of stay, treatment, and disposition. Patients were grouped according to whether they had taken opioids or not, as a proxy differentiation between heavy drug users and party drug users. Data from the two clinical settings were analyzed separately.

Results: In the opioid group, there were $229 / 1215$ (18.8\%) female cases at the outpatient clinic and 21/92 (22.8\%) at the hospital, while in the non-opioid group there were $270 / 1003(26.9 \%)$ at the outpatient clinic and 47/185 (25.4\%) at the hospital. Female patients were younger than males, median 31 versus 34 years ( $p$ $<0.001)$. In the outpatient opioid group, more women required treatment other than observation $(37.6 \%$ versus $29.6 \%, p=$ 0.024). In the hospital opioid group, no women were intubated versus $21.1 \%$ among men ( $p=0.019)$, and median length of stay was shorter, 14:56 hours versus $21: 25$ hours $(p=0.040)$. In the outpatient non-opioid group, psychosis was less common among women $(8.5 \%$ versus $13.6 \%, p=0.037)$. In the hospital non-opioid group, more women presented with anxiety $(61.7 \%$ versus $42.0 \%$, $\mathrm{p}=0.030)$ and seizures $(17.0 \%$ versus $5.8 \%, \mathrm{p}=0.031)$, and fewer women were intubated $(6.4 \%$ versus $21.0 \%, p=0.039)$. Hospital median peak creatine kinase levels were lower among women both in the opioid group ( $124 \mathrm{IU} / \mathrm{L}$ versus $366 \mathrm{IU} / \mathrm{L}, \mathrm{p}=0.005$ ) and the non-opioid group ( $129 \mathrm{IU} / \mathrm{L}$ versus $233 \mathrm{IU} / \mathrm{L}, \mathrm{p}=0.022$ ). Gamma-hydroxybutyrate was more common among women both at the outpatient clinic $(14.4 \%$ versus $8.6 \%, p<0.001)$ and at the hospital $(60.3 \%$ versus $36.4 \%, p=0.001)$, while heroin was less common among women at the outpatient clinic $37.1 \%$ versus $47.0 \%, p<0.001)$. On most variables there were no significant differences between genders.

Conclusion: One in four patients treated for acute recreational drug toxicity were women. Women were younger, had shorter duration of stay, were less frequently intubated and had lower creatine kinase levels. Otherwise, the differences between genders in terms of clinical state and treatment were small.

\section{High-dose propranolol for significant clenbuterol toxicity refractory to esmolol and phenylephrine}

\author{
Jessica V Rivera ${ }^{\mathrm{a}}$, Justin Arnold ${ }^{\mathrm{b}}$ and \\ William F Rushton ${ }^{\text {b }}$ \\ ${ }^{a}$ Department of Pharmacy, University of Alabama at Birmingham, \\ Birmingham, United States; ${ }^{\mathrm{b}}$ Department of Emergency Medicine, \\ Office of Medical Toxicology, University of Alabama at \\ Birmingham, Birmingham, United States
}

Objective: Clenbuterol is a potent, non-selective beta-adrenergic agonist. Exposures in the US most commonly result from use of adulterated illicit substances or abuse for anabolic effects. Toxicity resembles that of other beta-adrenergic agonists with symptoms including: tachycardia, hypotension, hypokalemia, hypomagnesemia, hyperglycemia, tremor, muscle fasciculations, and myocardial ischemia. Clenbuterol's potency and long duration of action make management of acute toxicity particularly challenging. Short acting beta-adrenergic antagonists, such as esmolol and metoprolol, are utilized in reported cases; yet, patients commonly remain symptomatic despite therapy [1].

Case report: A 45-year-old male presented as a transfer from an outside hospital for significant clenbuterol toxicity. The patient reported using approximately $80 \mu \mathrm{g}$ of clenbuterol four times per week to facilitate weight loss and muscle building. The morning of presentation, he ingested the remainder of his tablets $(100 \mu \mathrm{g})$ in addition to an unknown amount of resin from the bottom of the bottle. He immediately experienced severe light-headedness, diaphoresis, and palpitations. Upon presentation, he exhibited tachycardia, hypotension, acute kidney injury, elevated anion gap 
metabolic acidosis, and diffuse ST-segment depressions on his electrocardiogram. In the emergency department, he was aggressively treated with potassium supplementation and intravenous lorazepam. He subsequently developed a narrow-complex tachydysrhythmia persisting with rates up to 200 beats per minute and hypotension requiring phenylephrine. Due to refractory tachydysrhythmia, esmolol was aggressively titrated up to 300 $\mu \mathrm{g} / \mathrm{kg} / \mathrm{min}$. The patient developed worsening anxiety, tremulousness, and severe encephalopathy. Toxicology consultation recommended enteral propranolol 12 hours after the initial presentation. Propranolol $60 \mathrm{mg}$, followed by a second dose just under 6 hours later resulted in rapid improvement of encephalopathy, tachycardia, and hypotension. Esmolol and phenylephrine infusions were titrated off and the patient was transferred out of the intensive care unit on day 2. Presence of clenbuterol was confirmed on a urine anabolic steroid screen.

Conclusion: This case is noteworthy for refractory shock and tachydysrhythmia, despite use of a beta- 1 antagonist and an alpha-1 agonist. Propranolol has previously been described for refractory methylxanthine toxicity due to its non-selective inhibition of all beta receptors. Toxicity from clenbuterol, a non-selective agonist, appeared to rapidly improve with the addition of propranolol. The longer duration and lipophilic properties of propranolol may prevent further rebound toxicity. Clinicians may consider the adjunctive use of propranolol for refractory clenbuterol toxicity.

\section{Reference}

[1] Hoffman RJ, Hoffman RS, Freyberg CL, et al. Clenbuterol ingestion causing prolonged tachycardia, hypokalemia, and hypophosphatemia with confirmation by quantitative levels. Clin Toxicol (Phila). 2001;39:339-344.

\section{Opioid overdoses admitted to the intensive care unit over a 10-year period: clinical features and opioids involved}

\author{
Rhianna Willems ${ }^{a}$, Ahmed S Gouda ${ }^{b}$, Karim Jaffal ${ }^{\mathrm{a}}$, \\ Anne Buisine ${ }^{c}$, Laurence Labat ${ }^{c}$ and \\ Bruno Mégarbane ${ }^{a}$ \\ ${ }^{\mathrm{a} D e p a r t m e n t}$ of Medical and Toxicological Critical Care, Lariboisière \\ Hospital, Paris-Diderot University, INSERM UMRS-1144, Paris, \\ France; ${ }^{b}$ National Egyptian Center of Environmental and \\ Toxicological Research (NECTR), Cairo University, Cairo, Egypt; \\ 'Laboratory of Toxicology, Lariboisière Hospital, Paris-Diderot \\ University, INSERM UMRS-1144, Paris, France
}

Objective: An opioid overdose crisis is currently ongoing in North America in relation to the misuse of opioid analgesics and the spread of new fentanyl analogues on the recreational scene. Data regarding opioid overdoses in France remains scarce. Our objectives were to describe opioid overdose cases admitted to the intensive care unit (ICU) and characterize the compounds involved, clinical features, complications and patient management. Methods: We conducted a retrospective single-centre observational study including all patients admitted in the ICU during a 10 year period (2008-2018) for opioid overdose evidenced by the typical opioid toxidrome (coma, pinpoint pupils and bradypnea) associated with a compatible blood and urine toxicological screen.

Results: In 10 years, 231 patients ( 147 males and 84 females; age 38 years (33-46) [median (percentiles 25-75)]) were admitted the ICU for opioid intoxication. The patients presented consciousness impairment [Glasgow Coma Score 6 (3-10)], respiratory rate of $11 /$ min (8-15) and pinpoint pupils (90\%). Naloxone (64\%) and flumazenil (20\%) were administered to avoid tracheal intubation; but mechanical ventilation was required in $36 \%$ of patients [duration 20 hours (11-72)]. Duration of ICU stay was 41 hours (24-72). The observed complications included aspiration pneumonia (35\%), rhabdomyolysis (26\%), acute respiratory distress syndrome $(13 \%)$, cardiovascular failure $(11 \%)$, withdrawal syndrome $(8 \%)$, hospital-acquired infection (4\%) and death (1\%). Toxicity was related to opioid misuse (52\%) versus opioid overdose (48\%). Multidrug exposure was found in $100 \%$ of cases; the opioids involved were as follows: morphine $(44 \%)$, heroin (32\%), buprenorphine $(30 \%)$, methadone $(26 \%)$, codeine $(24 \%)$, tramadol $(6 \%)$, dextropropoxyphene $(6 \%)$, fentanyl $(1 \%)$ and oxycodone $(0.5 \%)$. Prevalence of the different opioids was mainly influenced by the banning of dextropropoxyphene from the market in 2011 and the consecutive increase in tramadol prescriptions.

Conclusion: Our large cohort of opioid poisoning cases clearly supports the risks of life-threatening consequences in case of misuse or overdose. Mixed opioid use and combination with other legal and illegal drugs are frequent, supporting the role of drug-drug interactions in the onset of toxicity in these multidrug poisonings.

\section{Acute health problems due to recreational drug use in Slovakia}

\section{Blažena Cagáňová and Silvia Plačková}

National Toxicological Information Centre, Department of Occupational Medicine and Toxicology, University Hospital Bratislava, Faculty of Medicine Comenius University, Bratislava, Slovakia

Objective: To analyse the epidemiological and clinical characteristics of patients with acute recreational drug toxicity in Slovakia, focussing on novel psychoactive substances (NPS).

Methods: Retrospective analysis of patients with acute recreational drug toxicity presenting to a hospital and reported to the National Toxicological Information Centre (NTIC) in Slovakia, 2015-2017. Intoxications with ethanol alone were excluded.

Results: During the study period 438 of the 14,726 cases were directly related to acute toxicity of substances used recreationally. The mean patient age was 27 years (range 13 to 62 ) and $54 \%$ were male. Alcohol co-ingestion was reported in $27 \%$ of the cases. Use of more than one recreational drug occurred in $46 \%$. Most presentations were related to amphetamines $(44 \%)$, of which methamphetamine was the most frequently involved (60\%). Presentations related to cannabis accounted for $22 \%$, volatile substances (mostly toluene) $12 \%$, followed by opiates ( $7 \%$, psychoactive plants/mushrooms (5\%) and cocaine (4\%). The most common recreationally used prescription drugs were dextromethorphan $(7 \%)$ and benzodiazepines $(5 \%)$. There were six intoxications with novel psychoactive substances (1.4\%): a-pyrrolidinovalerophenone (a-PVP), 5-methoxy-N-methyl-N-isopropyltryptamine (5-MeO-MiPT), 4-methoxyphencyclidine (4-MeO-PCP), and three cases with synthetic cannabinoids receptor agonists. All the cases related to NPS were of minor to moderate severity. The most frequently reported clinical symptoms were tachycardia $(52 \%)$, agitation $(41 \%)$ and anxiety (27\%). Overall $36 \%$ of patients had impaired consciousness (Glasgow Coma Score $[\mathrm{GCS}]<15$ ), among them, $9 \%$ were unconscious (GCS <8) at presentation or pre-hospital. We recorded two fatalities related to dextromethorphan abuse in men aged 22 and 55 years (laboratory confirmed). All the cases related to NPS were of minor to moderate severity.

Conclusion: In Slovakia, most medical problems regarding recreational drug use were related to methamphetamine (domestically produced "pervitin") and were mainly characterized by sympathomimetic toxicity and mental disorders. Cases with acute toxicity 
linked to NPS appeared to be uncommon and were of minor to moderate severity.

\section{Appearances can be deceiving: a case of an infant with unexpected intoxication}

\author{
Elena Bellelli ${ }^{\mathrm{a}}$, Mara Pisani ${ }^{\mathrm{a}}$, Carla Olita ${ }^{\mathrm{a}}$, \\ Tatiana Federici ${ }^{a}$, Andrea Deidda ${ }^{a}$, Francesco P Rossi ${ }^{a}$, \\ Davide Lonati ${ }^{\mathrm{b}}$, Marta Crevani ${ }^{\mathrm{b}}$, Antonino Reale ${ }^{\mathrm{a}}$ and \\ Marco Marano ${ }^{c}$ \\ 'amergency Department, Bambino Gesù Children Hospital, Rome,

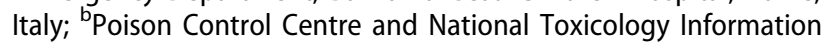 \\ Centre, Toxicology Unit, IRCCS Maugeri Foundation, Pavia, Italy; \\ ${ }^{\circ}$ Emergency Department PICU, Bambino Gesù Children Hospital, \\ Rome, Italy
}

Objective: To describe the case of an infant with cannabinoid and cocaine intoxication presenting with an atypical clinical history and symptoms requiring a complex differential diagnosis approach. Initial diagnosis was head injury.

Case report: An 11-month-old female child was transported to the Emergency Department by ambulance with an history of afebrile seizure after a head trauma. She was accompanied by a police officer and two adults who claimed to be her neighbors. Vital signs and physical examination were unremarkable. On neurologic examination the patient was alert with normal pupillary diameter and reflexes, episodes of staring eyes and nystagmus, mild axial hypertonia, frequent crying and irritability. Blood gas analysis and routine exams were normal. Brain computerised tomography (CT) scan showed diffuse hemispherical hypodensity of the white matter. In view of the poorly detailed history, and the child's clinical condition, it was suspected that the baby had been abused, and a complete screen consisting of skeletal surveys, ophthalmologic examination, urinary drug screening, psychiatric and psychosocial evaluation of the family was planned. Urinary toxicological screening was positive for benzoylecgonine and tetrahydrocannabinol (THC) with values of $341.35 \mathrm{ng} / \mathrm{mL}$ and $95.81 \mathrm{ng} / \mathrm{mL}$, respectively. During hospitalization the patient's mother reported that the baby was at home with her boyfriend during the episode. She denied her boyfriend's use of recreational drugs. The patient's clinical condition gradually improved and she was discharged in good general conditions after 5 days of hospitalization. The hospital's social pediatrics department was consulted, and it was determined that the baby lived in a hazardous home environment, in terms of negligence and abuse. So urgent protection measures were taken and the baby was placed in a state care nursery and taken into the state's protection.

Conclusion: In this case the patient presented with an atypical syndrome due to drug intoxication, consisting of an afebrile seizure. Seizures represent a possible but uncommon symptom of drug intoxication. Caregivers referred to head trauma and this lead clinicians to suspect a head injury. Considering the implausible history and the unusual social context, an abuse protocol was initiated and the diagnosis of intoxication emerged. In conclusion, we want to emphasize the importance of considering potential drug intoxication, especially in cases of ambiguous family or social contexts.

\section{An ETH-LAD trip with unfavourable consequences: a case report}

\author{
Michaela Sommer ${ }^{a}$, Christina Grumann ${ }^{a}$, \\ Volker Auwärter ${ }^{\mathrm{a}}$, Anne Stürzebecher ${ }^{\mathrm{b}}$ and \\ Maren Hermanns-Clausen ${ }^{c}$
}

${ }^{a}$ Forensic Toxicology, Institute of Forensic Medicine, Medical Center - University of Freiburg, Freiburg, Germany; ${ }^{\text {b Poisons }}$ Information Center, Helios Klinikum Erfurt GmbH, Erfurt, Germany; 'Poisons Information Center, Department of General Pediatrics, Adolescent Medicine and Neonatology, Center for Pediatrics, Medical Center - University of Freiburg, Freiburg, Germany

Objective: Lysergic acid diethylamide (LSD) is among the most popular hallucinogenic drugs. In recent years, several LSD analogues have emerged on the new psychoactive substances market, one of them being 6-ethyl-6-nor-lysergic acid diethylamide (ETH-LAD). So far, there are only limited data about the hallucinogenic properties of ETH-LAD and clinical intoxications. We present a case with analytically confirmed ETH-LAD intoxication from a prospective study on intoxication cases from emergency departments involving new psychoactive substances.

Case report: Just before midnight, a 17-year-old boy took three stamps of an LSD derivative and consumed cannabis. Shortly after the consumption he became aggressive, got into a dispute with his girlfriend and rioted. Four hours later, he was found unconscious (Glasgow Coma Score 10) and was brought to an emergency department. On arrival at the hospital the patient was increasingly aggressive and severely agitated. High doses of midazolam were given. In addition, he was diagnosed with pulmonary contusion, subsequently intubated and ventilated. Furthermore, injuries of rotator cuff and subgaleal hemorrhage were diagnosed. The patient was transferred to the intensive care unit. Laboratory results revealed signs of disseminated intravascular coagulation (DIC), minor metabolic acidosis and rhabdomyolysis (creatine kinase 3,990 $\mu \mathrm{mol} / \mathrm{s}^{*}$, myoglobin $83,300 \mu \mathrm{g} / \mathrm{L}$ ). In blood samples obtained approximately 8 hours after intake, ETHLAD $(38 \mathrm{ng} / \mathrm{mL})$ was identified using liquid chromatography-electrospray ionization-tandem mass spectrometry (LC-ESI-MS/MS). The further clinical course was complicated by acute renal injury (creatinine $153 \mu \mathrm{mol} / \mathrm{L}$ ). Over the next few days most symptoms resolved, but following extubation psychotic symptoms and agitation persisted. Additionally, a hearing impairment was noted. Dexmedetomidine, diazepam and lorazepam were used to control severe ongoing agitation. After consultation with a psychiatrist, antipsychotic therapy was started with olanzapine. He was transferred to a psychiatric clinic for treatment of psychosis 17 days after ingestion.

Conclusion: Little is known about the clinical effects of high doses of ETH-LAD. The compound was shown to produce hallucinogenic effects in humans at slightly lower doses than LSD. In addition, like LSD, a stimulation of $D_{1}$ and $D_{2}$ receptors has been described. We present a case with an analytically confirmed intake of ETH-LAD. Clinical course was characterised by combativeness and extreme agitation resulting in muscular injuries. Furthermore, a persistent psychotic disorder resulted. The reported hearing impairment in the context of ETH-LAD consumption is a rather unexpected finding.

\section{Maculopathy after use of isopropyl nitrate poppers documented with optical coherence tomography (OCT)}

Massimo Trombini ${ }^{\mathrm{a}}$, Vittoria Murro ${ }^{\mathrm{b}}$, Alessandro Zotto ${ }^{\mathrm{a}}$, Angelo Rotulo ${ }^{a}$, Francesco Gambassi ${ }^{c}$, Cecilia Lanzi ${ }^{c}$, Guido Mannaioni ${ }^{\mathrm{d}}$ and Alessandra Pistelli ${ }^{\mathrm{c}}$

${ }^{\mathrm{a}}$ Department of Neurofarba, Università degli Studi di Firenze, Florence, Italy; ${ }^{b}$ Ophthalmology Unit, Azienda OspedalieroUniversitaria Careggi, Florence, Italy; 'Poison Control Centre, Toxicology Unit, Azienda Ospedaliero-Universitaria Careggi, 
Florence, Italy; ${ }^{d}$ Neuroscience, Psychology, Drug Research and Child Health, Università degli Studi di Firenze, Florence, Italy

Objective: Poppers are common recreational drugs containing alkyl nitrates utilized by inhalation to facilitate or to enhance sexual experience mostly in the gay male community. Their more common side effects are represented by orthostatic hypotension with reflex tachycardia, flushing and headache while visual loss and maculopathy are rarely reported. Reports of poppers-induced maculopathy are described both in chronic use and more rarely after a single or sporadic exposure. We present a case of a patient with acute monolateral maculopathy after "jungle juice" poppers (isopropyl nitrate) use documented with optical coherence tomography (OCT) findings.

Case report: A 38-year-old male who was human immunodeficiency virus (HIV) positive and receiving antiretroviral therapy, was admitted to the hospital due to a central vision impairment of the left eye. The patient complained of a yellow/blue halo that had lasted 5 days and started immediately after the inhalation of "jungle juice" poppers. After excluding a possible side effect induced by pharmacological treatment with highly active antiretroviral therapy (elvitegravir/cobicistat/emtricitabine/tenofovir alafenamide), the ophthalmologist performed optical coherence tomography (OCT) imaging upon the suggestion of a toxicologist. This exam showed disruption of the inner/outer retinal segment junction. The patient was followed up with other OCT examinations and four months later did not show any residual sign of disruption.

Conclusion: Use of poppers can cause ocular damage. In 2007 the chemical structure of the Jungle Juice poppers brand was modified from isobutyl nitrite to isopropyl nitrite and this change has been considered as one possible cause of macular damage, together with a possible direct or indirect effect of nitric oxide on the macula [1]. The pathogenesis of the adverse ophthalmic effect, however, remains unclear and further studies are needed in order to better understand the exact mechanism of poppers toxicity.

\section{Reference}

[1] Audo I, El Sanharawi M, Vignal-Clermont C, et al. Foveal damage in habitual poppers users. Arch Ophthalmol. 2011;129:703-708.

\section{Fatality from a synthetic cannabinoid adulterated with brodifacoum}

\author{
Alfred Aleguas ${ }^{\mathrm{a}}$, Julia M Pearson ${ }^{\mathrm{b}}$, Tamas Peredy ${ }^{\mathrm{a}}$ \\ and Dina Swanson ${ }^{\mathrm{b}}$ \\ ${ }^{a}$ Florida Poison Information Center Tampa, Tampa General \\ Hospital, Tampa, United States; ${ }^{\mathrm{b}}$ Medical Examiner Department, \\ Hillsborough County, Tampa, United States
}

Objective: Brodifacoum is one of the long-acting anticoagulant rodenticides (LAARs), producing a prolonged and profound coagulopathy [1]. On 8 March 2018 the first cases of coagulopathy associated with use of synthetic cannabinoids containing brodifacoum were reported to US poison centers and state health departments. Between that date and 28 September, there have been 318 cases and 10 fatalities in 11 states [2]. The current epidemic suggests intentional contamination, but the root cause is unclear. One theory is that the addition of brodifacoum increases the high experienced by the user. We report a fatality from the use of a synthetic cannabinoid adulterated with brodifacoum.
Case report: A 37-year-old male who had dyspnea and hemoptysis the previous evening was found dead by his father the next morning sitting on the couch where he had slept. He was a known chronic marijuana and synthetic cannabinoid user but was not suspected of suicidality. Blood was noted on the face and chest. Efforts by first responders to resuscitate were unsuccessful and he was pronounced dead on scene. Post-mortem examination revealed pulmonary congestion with bloody, frothy sputum. The diaphragm and psoas muscles were hemorrhagic. The abdominal cavity contained approximately 1-2 liters of gross, bloody fluid with areas of the upper gastrointestinal tract showing focal hemorrhagic areas. The genitourinary tract contained frank blood and blood clots in the ureters. The bladder demonstrated areas of hemorrhage in the mucosa. Based upon similarities to recently reported cases, blood was sent to a reference lab for analysis. The synthetic cannabinoid 5F-ADB and tetrahydrocannabinol (THC) metabolite were detected, along with a positive result for brodifacoum. The cause of death was hemorrhagic diathesis due to brodifacoum poisoning.

Conclusion: A high index of suspicion of brodifacoum-related poisoning should be maintained with patients presenting with unexpected hematuria, hemoptysis, hematemesis, melena, and wounds that fail to stop bleeding. These patients will typically have a high or even unmeasurable prothrombin time (INR). The coagulopathy responds to high-dose oral phytonadione (vitamin $\mathrm{K}_{1}$ ), up to $80 \mathrm{mg}$ twice daily. Therapy may be needed for months.

\section{References}

[1] King N, Tran Minh-Ha. Long-acting anticoagulant rodenticide (superwarfarin) poisoning: A review of its historical development, epidemiology, and clinical management. Transfus Med Rev. 2015;29:250-258.

[2] Centers for Disease Control and Prevention. Outbreak Alert: Potential life-threatening vitamin $\mathrm{k}$ dependent antagonist coagulopathy associated with synthetic cannabinoid use [cited 2018 Sep 18]. Available from: https://content.govdelivery.com/accounts/ USCDC/bulletins/1e6dac3

\section{Clinical toxicity from analytically confirmed exposure to the synthetic cathinone methylenedioxy pyrrolidinohexiophenone (MDPHP) in the UK}

\author{
Gareth Hardy ${ }^{a}$, Emma Stoddard ${ }^{b}$, Michael Dunn ${ }^{c}$, \\ Simon L Hill ${ }^{d}$ and Simon HL Thomas ${ }^{c}$ \\ ${ }^{a}$ Emergency Department, Blackpool Teaching Hospitals NHS

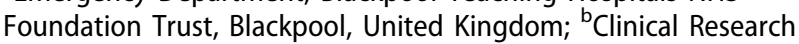 \\ Centre, Blackpool Teaching Hospitals NHS Foundation Trust, \\ Blackpool, United Kingdom; ' Medical Toxicology Centre, Newcastle \\ University, Newcastle, United Kingdom; ${ }^{\mathrm{d} C l i n i c a l}$ Toxicology, \\ Newcastle Hospitals NHS Foundation Trust, Newcastle, United \\ Kingdom
}

Objective: Methylenedioxypyrrolidinohexiophenone (MDPHP) is a synthetic cathinone stimulant with structural similarities to methylenedioxypyrovalerone (MDPV). There have been several media reports in the UK of extreme behavioural disturbances following use of "monkey dust", reported to contain MDPHP, many from the Stoke-on-Trent area. The limited clinical data available indicate that the clinical effects of MDPHP are similar to those of other cathinones [1]. Here we report analytical prevalence of MDPHP in adults recruited to the UK Identification Of Novel 
psychoActive substances (IONA) study, which is linking clinical features with analytical findings in consenting adults with severe toxicity from suspected use of novel psychoactive substances (NPS) [2], together with clinical features in one patient with isolated MDPHP exposure.

Case report: Clinical and analytical information were available for 415 IONA participants recruited in the UK between March 2015 and May 2018. MDPHP was found in samples from only one, a 21-year-old male who presented to a hospital in Blackpool in February 2017 with extreme agitation, distress, hallucinations, paranoid ideation including the belief that people were trying to kill him, sinus tachycardia (140 beats/min), and mydriasis. He did not admit to use of any substance apart from alcohol but claimed that something may have been put into a drink he had consumed approximately 10.5 hours earlier. Laboratory results were unremarkable other than an elevated white cell count $(23.3$ x $10^{9} / \mathrm{L}$ ) and creatine kinase (initially 732 , increasing to $1815 \mathrm{IU} / \mathrm{L}$ before improving). He received intravenous fluids but no other specific therapy. He remained agitated for more than 8 hours after presentation and needed a 2 day in-patient stay. Blood and urine samples taken within 30 minutes of presentation were analysed by liquid chromatography-tandem mass spectrometry as previously described [2]. MDPHP was identified in both samples but no other drugs of misuse were found.

Conclusion: MDPHP causes stimulant clinical features, sometimes including prominent effects on mental state. MDPHP use appears infrequent in populations served by the 28 research sites across the UK

\section{References}

[1] Beck O, Bäckberg $M$, Signell $P$, et al. Intoxications in the STRIDA project involving a panorama of psychostimulant pyrovalerone derivatives, MDPV copycats. Clin Toxicol (Phila). 2018;56:256-263.

[2] Hill SL, Dunn M, Cano C, et al. Human toxicity caused by indole and indazole carboxylate synthetic cannabinoid receptor agonists: From horizon scanning to notification. Clin Chem. 2018;64: 346-354.

\section{Loperamide-related enquiries to the UK National Poisons Information Service (NPIS)}

\section{Rebecca ML Waugh ${ }^{a}$, Yasmin H Peacock ${ }^{a}$, Leonard Hawkins ${ }^{a}$, Sally M Bradberry, Euan A Sandilands' ${ }^{c}$, John P Thompson ${ }^{d}$ and Simon HL Thomas \\ ${ }^{a}$ National Poisons Information Service (Newcastle) Unit, Newcastle Hospitals NHS Foundation Trust, Newcastle, United Kingdom; ${ }^{\mathrm{b}}$ National Poisons Information Service (Birmingham Unit), City Hospital, Birmingham, United Kingdom; 'National Poisons Information Service (Edinburgh Unit), Royal Infirmary, Edinburgh, United Kingdom; ${ }^{d}$ National Poisons Information Service (Cardiff Unit), University Hospital Llandough, Cardiff, United Kingdom; ${ }^{\mathrm{e}}$ Medical Toxicology Centre, Newcastle University, Newcastle, United Kingdom}

Objective: Loperamide, a $\mu$-opioid receptor agonist with low oral bioavailability, is used widely to treat diarrhoea. Serious toxicity is unlikely unless very large quantities are taken. Abuse of loperamide is increasing in some countries and there is an association between high dosage and cardiotoxicity. This study assessed annual trends in loperamide-related enquiries to the UK National Poisons Information Service (NPIS).
Table 1. Annual loperamide-related NPIS telephone enquiries and TOXBASE accesses.

\begin{tabular}{lcc}
\hline Year & NPIS enquiries & TOXBASE accesses \\
\hline 2009 & 60 & 923 \\
2010 & 62 & 1057 \\
2011 & 57 & 1043 \\
2012 & 67 & 1164 \\
2013 & 64 & 1420 \\
2014 & 73 & 1159 \\
2015 & 59 & 1258 \\
2016 & 39 & 1159 \\
2017 & $34^{*}$ & 1274 \\
TOTAL & 515 & 10457 \\
\hline
\end{tabular}

*to 21 November 2017

Methods: Telephone enquiry records involving loperamide were reviewed from 1 January 2009 to 21 November 2017. Accesses to TOXBASE $^{\circledR}$, the NPIS online poisons information database were quantified from 1 January 2009 to 31 December 2017.

Results: Over the period of the study there were 515 NPIS telephone enquiries related to loperamide exposure, but recreational abuse was suspected in only 3 of these $(0.6 \%)$. Annual numbers of enquiries discussed by telephone did not increase over the period of study, while modest increases in TOXBASE accesses are consistent with increasing overall use of the database for all substances over that period (Table 1). Peak age groups for loperamide enquiries overall were $20-29$ years $(n=62)$ in adults and $<5$ years $(n=173)$ in children. When recorded $(n=499)$ most patients had no $(n=359)$ or minor $(n=112)$ features of toxicity; moderate $(n=17)$ or severe $(n=11)$ toxicity was uncommon in adults and all but 2 of these patients had co-ingested other substances that might account for their features. In children $<5$ years, there were no cases of moderate or severe poisoning. QT prolongation was recorded in two adults following chronic misuse of large doses of loperamide, with one developing torsades de pointes.

Conclusion: These results suggest that loperamide misuse is uncommon and not increasing in the UK. Nevertheless, clinical toxicologists and poisons centres should be aware of loperamide abuse and its associated clinical complications.

\section{Oxycodone abuse during pain- relief prescription: an unusual but important cause}

\section{Eleonora Buscaglia ${ }^{\mathrm{a}}$, Davide Lonati ${ }^{\mathrm{a}}$, Marta Crevani ${ }^{\mathrm{a}}$, Giulia Scaravaggi ${ }^{\mathrm{a}}$, Pietro Papa ${ }^{\mathrm{b}}$ and Carlo A Locatelli ${ }^{\mathrm{a}}$

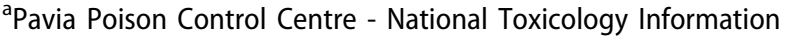 Centre - Clinical and Experimental Lab, Toxicology Unit, Maugeri Clinical and Scientific Institutes IRCCS and University of Pavia,

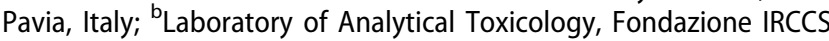 Policlinico San Matteo-Pavia, Pavia, Italy}

Objective: Oxycodone is a widely used $\mu$-opioid receptor agonist for the treatment of acute and chronic pain. $\mathrm{N}$-demethylation via CYP3A4/A5 is quantitatively the most important route of oxycodone metabolism: noroxycodone ( $80 \%$ circulating metabolite) has a weak antinociceptive effect. Moreover, CYP2D6 catalyzes the O-demethylation to oxymorphone (approximately 13\% of the metabolism), that has a $\mu$-opioid receptor affinity 10-40 times higher than oxycodone and is itself a very potent antinociceptive drug. Polymorphism of cytochrome P450 isoenzymes (CYPs) gives rise to important interindividual and interethnic variability in metabolism and in clinical response to these drugs. We describe the mechanisms of oxycodone abuse in an adult treated for pain. Case report: A 53-year-old Caucasian male was evaluated in our unit for oxycodone abuse started following a prescription for 
control of chronic lower back pain (scoliosis and spondylolisthesis L5-S1). History was positive for human immunodeficiency virus (HIV) (treated with emtricitabine-tenofovir $200 \mathrm{mg} / 245 \mathrm{mg}$, atazanavir $300 \mathrm{mg}$ and ritonavir $100 \mathrm{mg}$, all once daily) and hypertension (treated with losartan $12.5 \mathrm{mg}$, once daily). He progressively increased the oxycodone dosage to obtain analgesic effect. At his first admission he was taking $80 \mathrm{mg}$ in association with acetaminophen ( $3 \mathrm{~g} /$ day). Despite this dosage, he experienced withdrawal symptoms (sweating, nausea, agitation and diarrhea). At admission, blood oxycodone, oxymorphone, noroxycodone and noroxymorphone concentrations were 70.42, 0.0, 9.4, and 0.67 $\mathrm{ng} / \mathrm{mL}$, respectively. Oxycodone concentration decreased progressively once he stopped the drug. During hospitalization the addiction symptoms were successfully controlled with clonidine and benzodiazepines. To obtain pain control, he underwent two peridural injections of methylprednisolone $80 \mathrm{mg}$ and ropivacaine. A surgical treatment of spondylolisthesis was finally suggested. Genotyping for CYP2D6 was performed showing that our patient was a poor metabolizer (PM); CYP3A4 activity was normal.

Conclusion: In our case, CYP polymorphism (CYP2D6 PM) was the cause of the lack of response to the analgesic therapy that led the patient to progressively increase the oxycodone dosage. The oxycodone concentration was higher than usually detected in treated patients, even if subtoxic. Undetectable concentrations of the main analgesic metabolite (oxymorphone) resulted in lack of clinical response in this case. Clinicians should be aware that the lack of response to prescribed therapy can be a subtle cause of drug abuse. Evaluation of potential drug interactions and CYP polymorphism genotyping is advisable to choose the best therapy and the targeted dose for each patient.

\section{Non-medical use of prescription stimulants in Europe in the Non- Medical Use of Prescription Drug (NMURx) National Surveys}

\author{
Janetta L Iwanicki ${ }^{\mathrm{a}}$, Colleen M Haynes ${ }^{\mathrm{a}}$, \\ Joshua C Black ${ }^{a}$, Marc Auriacombe ${ }^{b}$, Paul I Darganc, \\ Maria Francina Fonseca ${ }^{d}$, Marilena Guareschi ${ }^{\mathrm{e}}$, \\ Norbert Scherbaum ${ }^{\mathrm{f}}$, David M Wood ${ }^{\mathrm{C}}$ and \\ Richard C Dart ${ }^{\mathrm{a}}$
}

${ }^{a}$ Rocky Mountain Poison \& Drug Center, Denver, United States; ${ }^{b}$ Department of Psychiatry and Addiction, Victor Pachon Medical School, Université Victor Segalen, Bordeaux, France; ${ }^{\mathrm{C} C l i n i c a l}$ Toxicology, Guy's and St Thomas' NHS Foundation Trust; Faculty of Life Sciences and Medicine, King's College London, London, United Kingdom; ${ }^{\mathrm{d}}$ Institut de Neuropsiquiatria i Addiccions-INAD, Hospital del Mar; Institut Hospital del Mar d'Investigacions Mèdiques (IMIM), Barcelona, Spain; ${ }^{\mathrm{e}}$ Associazione per I'Utilizzo delle Conoscenze Neuroscientifiche a fini Sociali (AU-CNS), Pietrasanta; Department of Neurosciences, Santa Chiara University Hospital, Pisa, Italy; ${ }^{\mathrm{f}}$ LVR-Klinikum Essen, Essen, Germany

Objective: Non-medical use (NMU) of prescription stimulants has been described in Europe; however, cross-country comparisons can be difficult. Using a harmonized questionnaire and sampling methodology, we estimated the prevalence of prescription stimulant NMU in five European countries.

Methods: NMURx is a series of cross-sectional online surveys. NMURx collects data from the adult general population on NMU of prescription drugs, as well as demographics and behaviors. NMU was defined as using a medication without a doctor's prescription or for any reason other than what was recommended by one's doctor. NMURx data from France $(2 \mathrm{Q} 2017, \mathrm{n}=10,012)$, Germany (4Q2017, $\mathrm{n}=15,051)$, Italy (2Q2017, $\mathrm{n}=10,084)$, Spain (4Q2017, $\mathrm{n}=10,062)$, and the UK $(1 \mathrm{Q} 2017, \mathrm{n}=10,004)$ were analyzed. Poststratification weights were applied to represent national adult population distributions. National rates of past 12 month NMU were calculated by country. Demographics and other risk factors were calculated among those who reported last year NMU.

Results: Estimated rates of stimulant NMU were highest in Spain (960.2 per 100,000 population, $95 \% \mathrm{Cl} 772.3-1148.1$ ), followed by Germany $(668.2,95 \% \mathrm{Cl} 522-814.4)$, Italy $(563.2,95 \% \mathrm{Cl} 409.1-$ 717.4), France $(585.7,95 \% \mathrm{Cl} 428.6-742.7)$, and the UK $(397.6,95 \%$ CI 209.2-586). In Spain, Italy, Germany, and France, a majority of those who non-medically used stimulants were young and male, and more likely to be current students compared to the general population (Table 1). Illicit drug use, previous treatment for substance abuse, and substantial or severe Drug Abuse Screening Test (DAST-10) scores were also more common among those who non-medically used. These subgroup estimates were not calculated for the UK because too few respondents endorsed stimulant NMU to perform additional analyses.

Conclusion: In four of five European countries, NMURx respondents with prescription stimulant NMU were more likely to be young, male, current students, have used illicit drugs, have

Table 1. Percent of NMURx respondents with stimulant nonmedical use (NMU) in the past 12 months versus percent of all respondents (95\% CI).

\begin{tabular}{|c|c|c|c|c|c|c|c|c|}
\hline \multirow[b]{2}{*}{$\begin{array}{l}\text { Respondent } \\
\text { parameters }\end{array}$} & \multicolumn{2}{|c|}{ France } & \multicolumn{2}{|c|}{ Germany } & \multicolumn{2}{|c|}{ Italy } & \multicolumn{2}{|c|}{ Spain } \\
\hline & Stimulant NMU & $\begin{array}{c}\text { General } \\
\text { Population }\end{array}$ & Stimulant NMU & $\begin{array}{c}\text { General } \\
\text { Population }\end{array}$ & Stimulant NMU & $\begin{array}{c}\text { General } \\
\text { Population }\end{array}$ & Stimulant NMU & $\begin{array}{c}\text { General } \\
\text { Population }\end{array}$ \\
\hline Aged $<25$ years & $\begin{array}{c}20.6 \% \\
(7.3-34.0)\end{array}$ & $\begin{array}{c}10.3 \% \\
(9.6-11.0)\end{array}$ & $\begin{array}{c}38.1 \% \\
(26.5-49.8)\end{array}$ & $\begin{array}{c}8.9 \% \\
(8.4-9.5)\end{array}$ & $\begin{array}{c}18.3 \% \\
(8.2-28.3)\end{array}$ & $\begin{array}{c}8.3 \% \\
(7.7-8.8)\end{array}$ & $\begin{array}{c}33.9 \% \\
(23.2-44.6)\end{array}$ & $\begin{array}{c}11.4 \% \\
(10.7-12.0)\end{array}$ \\
\hline Aged $25-34$ years & $\begin{array}{c}58.5 \% \\
(43.5-73.5)\end{array}$ & $\begin{array}{c}15.5 \% \\
(14.8-16.3)\end{array}$ & $\begin{array}{c}27.7 \% \\
(17.4-37.9)\end{array}$ & $\begin{array}{c}15.4 \%(14.8- \\
16.0)\end{array}$ & $\begin{array}{c}32.3 \% \\
(20.9-43.7)\end{array}$ & $\begin{array}{c}13.4 \% \\
(12.9-14.0)\end{array}$ & $\begin{array}{c}27.4 \% \\
(18.3-36.5)\end{array}$ & $\begin{array}{c}14.4 \% \\
(13.8-15.0)\end{array}$ \\
\hline Male & $\begin{array}{c}83.8 \% \\
(72.4-95.1)\end{array}$ & $\begin{array}{c}47.8 \% \\
(46.7-48.8)\end{array}$ & $\begin{array}{c}76.8 \% \\
(68.0-85.5)\end{array}$ & $\begin{array}{c}48.8 \% \\
(47.9-49.6)\end{array}$ & $\begin{array}{c}52.3 \% \\
(38.4-66.2)\end{array}$ & $\begin{array}{c}48.0 \% \\
(46.9-49.1)\end{array}$ & $\begin{array}{c}69.3 \% \\
(58.8-79.8)\end{array}$ & $\begin{array}{c}48.8 \% \\
(47.6-49.9)\end{array}$ \\
\hline Current student & $\begin{array}{c}40.2 \% \\
(25.2-55.3)\end{array}$ & $\begin{array}{c}7.8 \% \\
(7.3-8.4)\end{array}$ & $\begin{array}{c}28.5 \% \\
(17.7-39.2)\end{array}$ & $\begin{array}{c}8.0 \% \\
(7.5-8.5)\end{array}$ & $\begin{array}{c}32.8 \% \\
(19.8-45.8)\end{array}$ & $\begin{array}{c}8.6 \% \\
(8.1-9.1)\end{array}$ & $\begin{array}{c}44.8 \% \\
(34.0-55.7)\end{array}$ & $\begin{array}{c}13.6 \% \\
(12.8-14.3)\end{array}$ \\
\hline $\begin{array}{l}\text { Lifetime illicit } \\
\text { drug use }\end{array}$ & $\begin{array}{c}50.8 \% \\
(35.6-65.9)\end{array}$ & $\begin{array}{c}18.4 \% \\
(17.6-19.2)\end{array}$ & $\begin{array}{c}68.2 \% \\
(58.0-78.5)\end{array}$ & $\begin{array}{c}25.5 \% \\
(24.8-26.2)\end{array}$ & $\begin{array}{c}53.7 \% \\
(40.1-67.3)\end{array}$ & $\begin{array}{c}20.8 \% \\
(19.9-21.6)\end{array}$ & $\begin{array}{c}61.5 \% \\
(51.2-71.8)\end{array}$ & $\begin{array}{c}24.5 \% \\
(23.6-25.5)\end{array}$ \\
\hline $\begin{array}{l}\text { Previous sub- } \\
\text { stance abuse } \\
\text { treatment }\end{array}$ & $32.1 \%$ & $1.9 \%$ & $28.7 \%$ & $1.9 \%$ & $9.4 \%$ & $0.7 \%$ & $25.1 \%$ & $2.3 \%$ \\
\hline & $(18.0-46.2)$ & $(1.6-2.1)$ & $(17.9-39.4)$ & $(1.7-2.2)$ & $(2.5-16.4)$ & $(0.5-0.9)$ & $(16.0-34.1)$ & $(2.0-2.6)$ \\
\hline DAST-10 & $11.9 \%$ & $0.9 \%$ & $8.6 \%$ & $0.9 \%$ & $6.8 \%$ & $0.6 \%$ & $12.4 \%$ & $1.2 \%$ \\
\hline Substantial (6-8) & $(1.9-21.9)$ & $(0.7-1.1)$ & $(1.9-15.4)$ & $(0.8-1.1)$ & $(0.8-12.7)$ & $(0.4-0.7)$ & $(5.3-19.5)$ & $(1.0-1.5)$ \\
\hline DAST-10 & $8.0 \%$ & $0.9 \%$ & $1.6 \%$ & $0.4 \%$ & $1.3 \%$ & $0.4 \%$ & $4.6 \%$ & $0.6 \%$ \\
\hline Severe $(9-10)$ & $(0.0-16.8)$ & $(0.7-1.1)$ & $(0.0-3.9)$ & $(0.3-0.5)$ & $(0.0-3.8)$ & $(0.3-0.6)$ & $(0.1-9.2)$ & $(0.4-0.7)$ \\
\hline
\end{tabular}


sought substance abuse treatment, and have higher DAST-10 scores than the general population.

\section{Recreational ingestion of a compounded topical analgesic presenting with coma and cardiotoxicity}

\author{
Ryan Marino, Alexander Sidlak and Michael Lynch \\ University of Pittsburgh School of Medicine, Pittsburgh, United States
}

Objective: Compounded topical analgesics have historically included nonsteroidal anti-inflammatory medications or local anesthetics, however, newer compounded formulas are made with a range of pharmaceuticals, despite scant evidence to support their use and no Food and Drug Administration (FDA) regulation. Few reported cases of overdose exist in the literature. We describe a novel overdose involving a compounded topical analgesic with subsequent development of mixed toxidrome reflecting the constituent ingredients.

Case report: A 45-year-old man presented to the emergency department (ED) after he ingested his compounded topical analgesic. He was found unresponsive with the empty container and large amounts of the medication in his oropharynx. The medicine consumed by the patient contained $6 \%$ gabapentin, $2 \%$ baclofen, $2 \%$ amitriptyline, $1.75 \%$ lidocaine, $1.75 \%$ prilocaine, and $0.5 \%$ meloxicam with $360 \mathrm{~g}$ dispensed. Initial Glasgow Coma Score (GCS) was 3 and he required immediate intubation for respiratory failure. Electrocardiogram showed mild, new QRS interval prolongation of $104 \mathrm{~ms}$ and urine drug screen was positive for tricyclic antidepressants, which he was not otherwise prescribed. QRS narrowed to 94 ms after sodium bicarbonate, and he was admitted to the intensive care unit on a bicarbonate infusion. He was unresponsive to all stimuli for 24 hours without iatrogenic sedation. His mental status slowly improved and he was extubated on hospital day three. The patient left against medical advice on day four.

Conclusion: This patient's clinical presentation was consistent with a mixed toxidrome reflecting the constituent pharmaceuticals in the compounded product, which contained $21600 \mathrm{mg}$ gabapentin, $7200 \mathrm{mg}$ baclofen, $7200 \mathrm{mg}$ amitriptyline, $6300 \mathrm{mg}$ lidocaine, 6300 mg prilocaine, and $1800 \mathrm{mg}$ meloxicam. The patient was comatose, with respiratory failure requiring mechanical ventilation. Central nervous system depression resulted from a combination of high dose gabapentin and baclofen. The patient additionally manifested signs of sodium channel blockade related to amitriptyline toxicity. This case describes a novel overdose on a compounded topical analgesic composed of gabapentin, baclofen, amitriptyline, lidocaine, prilocaine, and meloxicam. Given the ease of ingestion of multiple drugs with diverse mechanisms of action in concentrations vastly exceeding those of individual oral doses, these compounded agents have potential for high morbidity and mortality.

\section{Pyoderma gangrenosum from the cocaine adulterant levamisole}

\author{
Charlotte Goldfine ${ }^{\mathrm{a}}$, Jeffrey $\mathrm{T} \mathrm{Lai}^{\mathrm{b}}$, Kelli Hickle ${ }^{\mathrm{b}}$ and \\ Kavita M Babu ${ }^{\text {b }}$ \\ ${ }^{a}$ Division of Medical Toxicology, Department of Emergency \\ Medicine, University of Massachusetts Medical Center, Worcester,

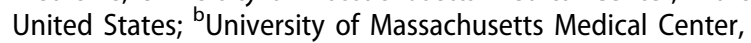 \\ Worcester, United States
}

Objective: To present the case of a patient who developed the unusual cutaneous manifestation of pyoderma gangrenosum, attributed to the cocaine adulterant levamisole.
Case report: A 40-year-old female with history of heroin and cocaine use presented to the emergency department for evaluation of multiple wounds on her right forearm that had developed during the preceding week. She also noted black areas on her fingertips and ear that had been present for 1 week. She denied any fever, chills, numbness, or sensory changes. She denied ongoing injection drug use but reported insufflating cocaine 1 week ago. Vital signs showed temperature $36.6^{\circ} \mathrm{C}$ blood pressure $110 / 62 \mathrm{mmHg}$, pulse 64 , and respiratory rate 16 . Examination revealed an extensive area of increased warmth, erythema and induration on the dorsum of her right forearm that was tender to palpation, with 3 large, discrete, non-healing ulcers covered in fibrinous granulation tissue. There was no expressible purulent drainage. Small necrotic nodules were noted on the fingertips of her bilateral hands. A serum drug of abuse screen (DOA9) was positive for benzodiazepines and cocaine metabolites. Hepatitis C antibody was positive, but viral RNA was not detected. Human immunodeficiency virus (HIV) testing was negative. Serologic testing was notable for positive antinuclear antibodies (ANA), positive perinuclear anti-neutrophil cytoplasmic antibodies ( $p$-ANCA) $>1: 640$, and chromatin antibody concentration $5.6 \mathrm{IU} / \mathrm{mL}$, which was consistent with levamisole-induced vasculitis. She was evaluated by plastic surgery and dermatology who concurred with the diagnosis of levamisole-induced vasculitis with resultant pyoderma gangrenosum. Her ulcerations improved with supportive care during her hospitalization, but she was lost to follow-up after discharge.

Conclusion: Cocaine abuse has known associated morbidity and mortality. Levamisole has been seen as an adulterant in cocaine since 2003 and has been found in up to $70 \%$ of cocaine in the US [1]. Levamisole is as an anthelminthic medication, but its use was discontinued in 1999 because of associated leukopenia, agranulocytosis, and cutaneous vasculitis [2]. Due to widespread adulteration of cocaine with levamisole, it is important to be aware of adverse effects related to its use.

\section{References}

[1] Abdul-Karim R, Ryan C, Rangel C, et al. Levamisole-induced vasculitis. Proc (Baylor Univ Med Center). 2013;26:163-165.

[2] Ghias AAP, Brine P. Vanishing vasculitis: a case of acute necrotic skin findings without pathologic features of vasculitis from adulterated cocaine. J Community Hosp Intern Med Perspect. 2017; 7:321-324.

\section{Self-inflicted severe genital friction burns secondary to hypersexual response from synthetic cathinone and cocaine use}

\author{
Shirley Shao, Ryan Marino, Joshua Shulman and \\ Michael Abesamis \\ University of Pittsburgh School of Medicine, Pittsburgh, United \\ States
}

Objective: Synthetic cathinones, known colloquially as "bath salts", are sympathomimetic substances chemically similar to cathinone, a naturally occurring substance found in the khat plant (Catha edulis), which are used recreationally for stimulant and euphoric effects. Hypersexuality is a complex behavioral response that has been described as sequelae of sympathomimetic abuse in other phenylethylamine compounds. We present a unique case of intravenous synthetic cathinone abuse leading to hypersexual reaction causing self-inflicted genital friction burns. 
Case report: A 52-year-old man was transferred from an outside hospital for evaluation of genital burns. He said he had been using "bath salts" intravenously for 4 days in addition to cocaine. He stated that for the subsequent 3 days of use, he experienced insomnia and masturbated continuously. He eventually ran out of his cathinone, and tried a synthetic cannabinoid, which caused hallucinations and prompted him to call the emergency medical service (EMS). He required intravenous midazolam for agitation prior to transfer. Exam showed diffuse, self-inflicted, partial thickness burns of the entire shaft of the penis and the entire scrotum, with large areas of full thickness friction burns, lichenification, blisters and erosions. He complained of dysuria and was unable to void spontaneously, necessitating placement of a urinary catheter for bladder decompression. He was admitted to the burn unit, where he exhibited rapid improvement in degree of agitation. Local wound care was performed and his urinary retention improved. He was discharged home on hospital day 5. Qualitative urine gas chromatography/mass spectroscopy evaluation was positive for pyrrolidinopropiophenone (PPP) and ethyl pentylone, as well as midazolam, cocaine, ecgonine methyl ester, nicotine metabolite and caffeine.

Conclusion: Synthetic cathinones are sympathomimetic substances that inhibit reuptake of dopamine, serotonin and norepinephrine and induce stimulant effects similar to amphetamines, cocaine and 3,4-methylenedioxy-N-methylamphetamine (MDMA). Sympathomimetics have been associated with hypersexual behaviors and self-injurious activity, although self-inflicted genital burns secondary to cathinone use have not previously been reported. This case highlights an unusual circumstance where synthetic cathinone use caused a hypersexual reaction leading to severe self-inflicted friction burns to the genitalia resulting from prolonged masturbation, which necessitated admission to a burn center.

\section{Recreational use of cough syrup: report of four cases in adolescents}

\author{
Jorge G Perez Tuñon, Rocío Martiñón Ríos, \\ Magaly Figueroa, Daniela Mandujano Meneses and \\ Mireille Arango Mathieu \\ Centro Toxicológico, Hospital Angeles Lomas, Huixquilucan, \\ Mexico
}

Objective: In Mexico there are no cough relievers with codeine. "Purple Drank" or "lean drink" originally contained codeine and promethazine, which are not available in Mexico. It now contains diphenhydramine and dextromethorphan and is added to a soft drink or caramel to improve the flavor and used recreationally among adolescents due to their low cost and availability without prescription. Diphenhydramine overdose causes sedation, anticholinergic syndrome, delirium and hallucinations; dextromethorphan causes euphoria and dissociative effects. Other possible complications include cardiac arrhythmias and seizures. We describe the clinical manifestations of recreational use of diphenhydramine and dextromethorphan.

Case reports: Case 1. A 15-year-old male ingested "purple drank" (diphenhydramine), presenting with BP $130 / 90 \mathrm{mmHg}, \mathrm{HR} 105$ bpm, RR $18 \mathrm{rpm}$, temperature $37.5^{\circ} \mathrm{C}$, palpitations, anxiety, myoclonus and delirium. He received crystalloids and diazepam 10 mg IV 3 doses, improving anticholinergic symptoms, but delirium persisted for 48 hours, so he was transferred to a psychiatric hospital. Case 2. A 13-year-old male ingested "four loko" (ethanol with caffeine) and diphenhydramine, presented with BP 150/80 $\mathrm{mmHg}, \mathrm{HR} 108 \mathrm{bpm}, \mathrm{RR} 30 \mathrm{rpm}$, temperature $37^{\circ} \mathrm{C}$, palpitations, anxiety, dilated pupils with no response, dry mucous membranes and flushing. An arterial blood sample showed metabolic acidosis $\left(\mathrm{pH}\right.$ 7.23, $\mathrm{PCO}_{2} 24$, bicarbonate 13, base excess -8.3). Metabolic acidosis was corrected with crystalloids. Diazepam $10 \mathrm{mg}$ IV was administered and he was discharged 18 hours after exposure. Case 3. A 16-year-old female with history of cocaine and marijuana consumption, ingested diphenhydramine and dextromethorphan. She presented with BP 140/70 mmHg, HR 103 bpm, RR $18 \mathrm{rpm}$, temperature $36^{\circ} \mathrm{C}$, anxiety, dilated pupils with diminished reflexes, dry mucous membranes, flushing, absent bowel sounds and urinary retention. Urine toxicology screen was positive for phencyclidine (PCP). Crystalloids were administered and $10 \mathrm{mg}$ of diazepam twice; symptoms resolved 24 hours later. Case 4 . A $15-$ year-old female ingested an undisclosed amount of vodka and diphenhydramine, presenting with BP $110 / 60 \mathrm{mmHg}, \mathrm{HR} 100$ bpm, RR $24 \mathrm{rpm}$, temperature $36.2^{\circ} \mathrm{C}$, anxiety, delirium and hallucinations. Serum ethanol was $119 \mathrm{mg} / \mathrm{dL}$, two hours after the last drink. Diazepam $10 \mathrm{mg}$ was administered twice and symptoms resolved 14 hours later.

Conclusion: Patients 1 and 4 presented diphenhydramine-related delirium and which was persistent in patient 1 . Cases 2 and 4 consumed ethanol, developing metabolic acidosis in case 2 . Treatment was based on fluid replacement, benzodiazepines and management of complications. Easy access of adolescents to cough syrups favors their consumption for recreational purposes, which can cause life-threatening complications.

\section{Analytical prevalence of novel synthetic opioids including fentanyls in samples from patients presenting with apparent heroin overdose in the UK}

Michael Dunn ${ }^{\mathrm{a}}$, Simon L Hill ${ }^{\mathrm{b}}$, Gareth Hardy, Jamie Cooper ${ }^{d}$, Paul I Dargan ${ }^{\mathrm{e}}$, David M Wood ${ }^{\mathrm{e}}$, Johann Grundlingh ${ }^{f}$, Richard Parris ${ }^{g}$, Himanshu Kataria ${ }^{h}$, Michael Eddleston ${ }^{i}$, Jane Officer ${ }^{j}$ and Simon HL Thomas ${ }^{\mathrm{a}}$

${ }^{a}$ Medical Toxicology Centre, Newcastle University, Newcastle, United Kingdom; ${ }^{b}$ Clinical Toxicology, Newcastle Hospitals NHS Foundation Trust, Newcastle, United Kingdom; ' ${ }^{\mathrm{E}}$ Emergency Department, Blackpool Teaching Hospitals NHS Foundation Trust, Blackpool, United Kingdom; ${ }^{d}$ Emergency Department, Aberdeen Royal Infirmary, Aberdeen, United Kingdom; ' $\mathrm{C}$ Cinical Toxicology, Guy's and St Thomas' NHS Foundation Trust and Faculty of Life Sciences and Medicine King's College London, London, United

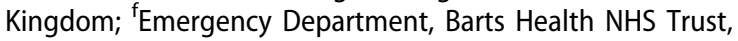
London, United Kingdom; ' ${ }^{9}$ Emergency Department, Bolton NHS Foundation Trust, Bolton, United Kingdom; ${ }^{\text {hEmergency }}$ Department, St Helens and Knowsley Teaching Hospitals NHS Foundation Trust, Prescot, United Kingdom; 'Clinical Toxicology, Royal Infirmary, Edinburgh, United Kingdom; 'Scottish Police Authority Forensic Services, Edinburgh, United Kingdom

Objective: The Identification Of Novel psychoActive substances (IONA) study is linking clinical features with analytical findings in adults with severe toxicity from suspected use of novel psychoactive substances (NPS) or non-pharmaceutical opioids including heroin. The latter group was added because of the increasing contribution of novel synthetic opioids (NSO) including fentanyls to fatal apparent heroin overdose in North America. Here we report analytical prevalence of NSO in study participants presenting with apparent non-pharmaceutical opioid overdose.

Methods: With ethical approval, adults presenting to 28 participating British hospitals with severe acute toxicity after suspected non-pharmaceutical opioid exposure were recruited. Clinical features were recorded using a structured data collection sheet. Blood and urine samples were analysed using liquid chromatography-tandem mass spectrometry [1]. 
Results: Clinical and analytical data are available for 53 adults recruited from November 2015 to May 2018 (median age 38 years, range $20-62$ years; $81 \%$ male). Heroin use was reported in 46 (intravenous 32, smoked 21, snorted 1, route not known 4, multiple routes 12) and suspected in the remaining 7 patients because of clinical features or naloxone response. Acute co-use of other drugs of misuse was reported in 32 cases (60\%). When known $(n=23)$, median estimated time following exposure was 3.76 hours (range 0.15-12.3 hours). Common clinical features were reduced level of consciousness $(n=45,85 \%)$, miosis $(n=29$, $55 \%)$, agitation $(n=26,49 \%)$, acidosis $(n=22,41 \%)$ and seizures $(n=20,38 \%)$. Naloxone use was reported in 33 (62\%). Conventional opioids most commonly identified were methadone $(n=31,58 \%)$, diacetylmorphine metabolites $(n=21,40 \%)$ and codeine $(n=22,41 \%)$. The only NSO detected was alfentanil, identified in samples from one patient (1.8\%). NSO were also detected in samples from 22 of 362 (6.1\%) separate IONA participants with suspected NPS rather than non-pharmaceutical opioid use. These were ${ }^{*}$ fentanyl $(n=15,4.1 \%)$, *alfentanil $(n=5,1.4 \%)$, *sufentanil $(n=3,0.8 \%){ }^{*}$ remifentanil, carfentanil and furanylfentanil (1 each, $0.3 \%$ ). However, therapeutic use of licensed fentanyls (marked *) prior to blood or urine sampling has not been excluded in all these cases.

Conclusion: NSO were uncommonly detected in this small sample of UK patients presenting with suspected heroin overdose. However, continuing vigilance, including ongoing analysis of patient samples, remains essential because of the ongoing risk of NSO misuse, including occult use for heroin fortification.

\section{Reference}

[1] Hill SL, Dunn M, Cano C, et al. Human toxicity caused by indole and indazole carboxylate synthetic cannabinoid receptor agonists: From horizon scanning to notification. Clin Chem. 2018;64:346-354.

\section{Fatal serotonin syndrome after multi-drug use}

\author{
Amelia Curtis ${ }^{a}$, Jeffrey $T$ Lai $^{\mathrm{b}}$, Mark Neavyn ${ }^{\mathrm{b}}$ and \\ Brittany P Chapman ${ }^{\mathrm{b}}$ \\ ${ }^{a}$ Department of Emergency Medicine, University of Massachusetts, \\ Worcester, United States; 'bniversity of Massachusetts, Worcester, \\ United States
}

Objective: To describe a patient who presented to the emergency department (ED) following an opioid overdose and rapidly developed autonomic instability and psychomotor agitation suggestive of serotonin syndrome, ultimately resulting in cardiac arrest and death.

Case report: A 36-year-old male was brought to the ED by emergency medical services (EMS), who had been called to his residence for suspected overdose. The patient was initially agitated but alert, with tachycardia, tactile hyperthermia, and spontaneous clonus in the extremities. En route to the ED, he had a generalized tonic-clonic seizure, treated with midazolam and naloxone. This was followed by cardiac arrest. Transient return of spontaneous circulation (ROSC) was obtained several times during transport followed by recurrent arrest. The patient was endotracheally intubated, and received epinephrine, atropine, dopamine, amiodarone, and ultimately transcutaneous pacing by EMS. On arrival to the ED, he had a heart rate of $44 \mathrm{bpm}$, blood pressure 55/43 $\mathrm{mmHg}$, and rectal temperature $42{ }^{\circ} \mathrm{C}$. He was manually ventilated by EMS with $\mathrm{FiO} 2100 \%$ at a rate of 26 ; pulse oximetry was $98 \%$. Resuscitative efforts in the ED included active cooling, paralysis, multiple vasopressors, calcium chloride, and sodium bicarbonate.
With active cooling, the patient's core body temperature reduced from a maximum of $43^{\circ} \mathrm{C}$ to $36.2^{\circ} \mathrm{C}$. Laboratory analyses showed sodium $137 \mathrm{mmol} / \mathrm{L}$, potassium $9.0 \mathrm{mmol} / \mathrm{L}$, chloride $89 \mathrm{mmol} / \mathrm{L}$, bicarbonate $10 \mathrm{mmol} / \mathrm{L}$, blood urea nitrogen $46 \mathrm{mg} / \mathrm{dL}$, creatinine $7.84 \mathrm{mg} / \mathrm{dL}$, glucose $421 \mathrm{mg} / \mathrm{dL}$, creatine kinase 35,520 U/L, venous $\mathrm{pH}$ 6.75, and lactate $19.8 \mathrm{mmol} / \mathrm{L}$. Despite aggressive supportive care, the patient suffered asystolic cardiac arrest soon after arrival to the intensive care unit and expired. Blood samples obtained in the ED were sent to the Center for Forensic Science Research and Education, and confirmed the presence of tetrahydrofuranyl fentanyl (THF fentanyl), fentanyl, norfentanyl, 4-anilino$\mathrm{N}$-phenethylpiperidine, naloxone, methadone, tramadol, methamphetamine, amphetamine, and midazolam. Severe serotonin syndrome is the likely cause of death in the setting of hyperthermia, autonomic instability, and spontaneous clonus progressing to muscle rigidity, and documented exposure to serotonergic agents.

Conclusion: THF fentanyl is a synthetic fentanyl analog uncommonly implicated in fatal opioid overdose, with only two confirmed cases in the US. We present the case of a patient who developed rapidly progressive autonomic dysregulation and neuromuscular hyperactivity consistent with serotonin syndrome, with laboratory testing confirming exposure to THF fentanyl.

\section{Overview of the Italian black market of performance and image enhancing drugs (PIED)}

\author{
Sara Odoardi, Serena Mestria, Valeria Valentini and \\ Sabina Strano Rossi \\ Institute of Public Health, Section of Legal Medicine, Università \\ Cattolica del S. Cuore, Rome, Italy
}

Objective: Recently the black market of counterfeit medicines has rapidly increased; these products are easily sold through the Internet, where they can be purchased without any medical examination or prescription. In addition, these products are often contaminated with other substances or mislabeled, which poses a public health risk. We give an overview of analysis of over 100 black market preparations seized in Italy, mainly sold through the Internet.

Methods: We analyzed over 120 medicaments, in different pharmaceutical forms (e.g. tablets, capsules and oily solutions for injection). Many had packaging and labels which appeared to be home-printed, with logos of non-existent pharmaceutical companies, fake batch numbers and no expiry dates. Other medicaments had no label at all. We analyzed the samples by liquid chromatography-tandem mass spectrometry (LC-HRMS) in order to identify anabolic steroids and esters, hormone-modulators, phosphodiesterase type 5 inhibitors (PDE5I), stimulants and anorectic drugs. The ease of sample preparation together with a fast run time of only 16 minutes permitted rapid identification of over 100 substances [1,2].

Results: The analytes identified were mainly mixture of testosterone esters, nandrolone decanoate, oxandrolone, stanozolol and methandienone. In some samples hormone-modulators, like tamoxifen, clomiphene, letrozole and anastrozole, melatonin stimulators, were identified and others contained stimulants such as ephedrine and yohimbine, whereas capsules and tablets contained substances used to enhance sexual performance, such as sildenafil, thiosildenafil, vardenafil and dapoxetine. In many cases the actual composition was different from that declared on the labels. In other cases known substances were not identified.

Conclusion: The results underline the dangers for human health due to the use of substances obtained via illegal and uncontrolled sources. Furthermore, mislabeling or label absence can 
lead to a high risk to health due to the inadvertent use of drugs that can act synergically with others. These data can be useful to clinical toxicologists in order to evaluate possible intoxications due to unlabeled or mislabeled preparations.

\section{References}

[1] Odoardi S, Castrignanò E, Martello S, et al. Determination of anabolic agents in dietary supplements by liquid chromatographyhigh-resolution mass spectrometry. Food Addit Contam Part A Chem Anal Control Expo Risk Assess. 2015;32:635-647.

[2] Strano-Rossi S, Odoardi S, Castrignanò E, et al. Liquid chromatography-high resolution mass spectrometry (LC-HRMS) determination of stimulants, anorectic drugs and phosphodiesterase 5 inhibitors (PDE5I) in food supplements. J Pharm Biomed Anal. 2015;106:144-152.

\section{Seizures resulting from ethanol withdrawal: management and prognosis in the intensive care unit}

\author{
Camille Tacquin ${ }^{a}$, Isabelle Malissin ${ }^{a}$, Nicolas Deye ${ }^{a}$, \\ Nathalie Kubis ${ }^{b}$ and Bruno Mégarbane ${ }^{a}$ \\ ${ }^{a}$ Department of Medical and Toxicological Critical Care, Lariboisière \\ Hospital, Paris-Diderot University, INSERM UMRS-1144, Paris, \\ France; ${ }^{b}$ Laboratory of Neurophysiology, Lariboisière Hospital, \\ Paris-Diderot University, Paris, France
}

Objective: In France, excessive ethanol use represents a major public health problem with resulting morbidities and social and medical expenses. Chronic alcohol drinking induces dependence and tolerance and may thus be responsible for withdrawal syndrome complicated by seizures due to ethanol-induced alterations in brain neurotransmitters including gamma-aminobutyric acid/glutamine imbalance. Our objective was to describe patient characteristics, morbidities, management and final outcome of the patients admitted to the intensive care unit (ICU) due to ethanol withdrawal complicated by seizures.

Methods: We conducted a retrospective single-center observational study including all presumed chronic alcoholic patients admitted to the ICU from 2014 to 2018 for ethanol withdrawalinduced seizures. The diagnosis was considered by the physicians in charge based on the results of all useful clinical, biological, imaging and electroencephalogram (EEG) data usually required to rule out alternative diagnoses.

Results: Fifty-three patients [45 males (85\%) and 8 females (15\%); age 49 years (12) (median (interquartile range)] were included in the study. The patients were homeless (44\%) and jobless (52\%). They had previously presented ethanol withdrawal syndrome $(62 \%)$ including seizures (53\%). Patients developed status epilepticus (70\%) associated with delirium tremens (13\%). Complications included aspiration pneumonia (49\%), cardiovascular failure (18\%, mainly vasoplegic shock requiring norepinephrine infusion), neurological deficits (8\%) and skin compression lesions (19\%). Patients required mechanical ventilation (53\%) for 3 days (12). All patients were treated with anticonvulsive drugs of which the two mostly commonly used were levetiracetam (80\%) and clonazepam (72\%). Two patients (4\%) died. Readmission to the emergency department (49\%) and re-hospitalization in an ICU or medical ward (38\%) were remarkable during the first year following their ICU admission, while only $13 \%$ had psychological and addictological follow-up.

Conclusion: Ethanol withdrawal may be responsible for seizures in the chronic alcoholic requiring ICU admission with life-threatening complications and death. Our data support once again the absolute necessity to strengthen the fight against excessive ethanol drinking in our society.

\section{Ethylene glycol poisoning and the development of delayed neurological sequelae}

\author{
Therese Becker, Betty S Chan and Angela L Chiew \\ Toxicology, Prince of Wales Hospital, Randwick, Australia
}

Objective: We describe a patient with non-convulsive status epilepticus on day 11 after ingestion of ethylene glycol.

Case report: A 59-year-old man with a history of depression and opiate dependence was brought to hospital unconscious. He had no prior history of seizures. Fifteen hours prior to admission his family observed that he was ataxic with slurred speech. On arrival he had a Glasgow Coma Score (GCS) of 3, heart rate 60 bpm, blood pressure $146 / 79 \mathrm{mmHg}$, and oxygen saturations $95 \%$ (on oxygen). His initial venous blood gas showed a raised anion gap metabolic acidosis with $\mathrm{pH} 6.922, \mathrm{pCO}_{2} 37.9 \mathrm{mmHg}$, bicarbonate $7.4 \mathrm{mmol} / \mathrm{L}$, and anion gap of 28.6. He had acute kidney injury (creatinine 303 $\mu \mathrm{mol} / \mathrm{L}$ ). Osmolar gap was $17.6 \mathrm{mmol} / \mathrm{kg}$. Calcium oxalate crystals were detectable in urine and his family found an empty container of ethylene glycol he later admitted ingesting. Magnetic resonance imaging (MRI) on day 2 showed swelling in the posterior brainstem with extension to the thalami and basal ganglia, consistent with ethylene glycol toxicity. He was intubated, loaded with ethanol, given a bolus of sodium bicarbonate and started on intermittent haemodialysis (IHD). Just prior to starting dialysis he had two tonicclonic seizures that ceased with benzodiazepines. IHD was continued for 5.5 hours followed by continuous veno-venous haemodiafiltration (CVVHDF) for 11 hours. His acidosis resolved. He was extubated on day 6 and remained delirious but was improving daily. On day 11 he deteriorated; GCS dropped from 14 to 9 and he was non-verbal and not following commands but would open eyes to voice and make purposeful movements. He had no increased tone. An urgent brain computerised tomography (CT) scan showed no pathology. An electroencephalogram confirmed non-convulsive status epilepticus. He was given benzodiazepines and levetiracetam. The next morning he returned to baseline of two days prior. He was discharged 6 weeks later with cognitive impairment that was improving. He had no further seizures despite non-compliance with anticonvulsants.

Conclusion: Ethylene glycol toxicity is known to cause seizures in the acute setting. Furthermore, there are reports of delayed onset neurological symptoms and brain stem dysfunction, including rapid onset Parkinson's disease typically occurring 5-20 days post-ingestion [1,2], however, sudden onset of a non-convulsive status epilepticus has not previously been reported.

\section{References}

[1] Reddy NJ, Sudini M, Lewis LD. Delayed neurological sequalae from ethylene glycol, diethylene glycol and methanol poisoning. Clin Toxicol (Phila). 2010;48:967-973.

[2] Morgan BW, Ford MD, Follmer R. Ethylene glycol ingestion resulting in brainstem and midbrain dysfunction. Clin Toxicol (Phila). 2000;38:445-451.

\section{Hyperlactatemia in the acutely ethanol-poisoned patient: does ethanol play a direct role?}

\author{
Elmire Chauvière ${ }^{\mathrm{a}}$, Pierre Mora ${ }^{\mathrm{a}}{ }^{\text {, Hervé Gourlain }}{ }^{\mathrm{b}}$ and \\ Bruno Mégarbane ${ }^{a}$
}


${ }^{a}$ Department of Medical and Toxicological Critical Care, Lariboisière Hospital, Paris-Diderot University, INSERM UMRS-1144, Paris, France; ${ }^{b}$ Laboratory of Toxicology, Lariboisière Hospital, ParisDiderot University, INSERM UMRS-1144, Paris, France

Objective: Acute ethanol poisoning represents a frequent cause of admission to the emergency department and intensive care unit (ICU) in France. Ethanol is theoretically able to increase the serum lactate concentration, however, hyperlactatemia in the acutely ethanol-poisoned patient has been reported rarely and its exact mechanism is still debated. We aimed to determine the prevalence and describe the etiologies of lactate elevation following excessive ethanol ingestion.

Methods: We conducted a retrospective single-centre observational study including all patients admitted to the intensive care unit (ICU) during 7 years (2011-2018) for acute drunkenness (defined by ethanol concentration $>1 \mathrm{~g} / \mathrm{L}$, in the absence of significant drug co-ingestions) and who presented hyperlactatemia (defined by lactate concentration $>2 \mathrm{mmol} / \mathrm{L}$ ).

Results: Fifty-four patients (16 females and 38 males; median age, 41 years [inter-quartile interval, 26] with past history of chronic alcoholism, 52\%) were included, representing 54\% of the patients admitted for acute drunkenness to the ICU during the same period. Patients were comatose (Glasgow Coma Score 3 [4]) requiring tracheal intubation (81\%). Complications included aspiration pneumonia $(41 \%)$, sepsis $(26 \%)$, cardiovascular failure $(13 \%)$, vomiting $(9 \%)$, seizures $(6 \%)$, hospital-acquired infection $(2 \%)$ and death (6\%). Blood ethanol concentration was $3.4 \mathrm{~g} / \mathrm{L} \mathrm{(2.3)} \mathrm{on}$ admission and plasma lactate concentration $2.5 \mathrm{mmol} / \mathrm{L}(0.9)$ on admission, peaking at $2.9 \mathrm{mmol} / \mathrm{L}(1.1)$, with no significant correlations between both parameters $\left(R^{2}=0.006\right.$ and 0.03 , respectively). The main reasons for increase in lactate concentration were sepsis (26\%), hypovolemia (19\%) and trauma (9\%). The existence of underlying alcoholic cirrhosis explained $2 \%$ of the hyperlactatemia cases. In $30 \%$ of the cases, no etiology except ethanol was found to explain hyperlactatemia onset. In this patient subset, peak lactate concentration was significantly less elevated $(2.4 \mathrm{mmol} / \mathrm{L}$ [0.9] versus $3.0 \mathrm{mmol} / \mathrm{L}[1.6], \mathrm{p}=0.02)$ and decrease in serum bicarbonate more limited $(23.4 \mathrm{mmol} / \mathrm{L}$ [1.6] versus $22.0 \mathrm{mmol} / \mathrm{L}$ [4.5], $\mathrm{p}=0.05$ ).

Conclusion: Acute drunkenness can be complicated by hyperlactatemia. In approximately $30 \%$ of the cases, elevation in serum lactate can only be attributed to the presence of ethanol in the body. Metabolic investigations should be conducted to better characterize the involved mechanism, one hypothesis being the disequilibrium induced by ethanol in the intracellular ratio of reduced $(\mathrm{NADH})$ and oxidized $\left(\mathrm{NAD}^{+}\right)$nicotinamide adenine dinucleotide.

\section{Pre-existing anemia: a risk factor in children's acute toxic methemoglobinemia}

\author{
Viorela Nitescua , Dora Boghitoiu ${ }^{b}$, Radu Nicolaescuc, \\ Catalin Alexandru Paslaru ${ }^{b}$ and Coriolan Ulmeanu ${ }^{c}$ \\ ${ }^{a}$ Toxicology and Intensive Care Unit, Pediatric Poisoning Centre, \\ Emergency Clinical Hospital for Children "Grigore Alexandrescu", \\ Bucharest, Romania; ${ }^{b}$ University of Medicine and Pharmacy "Carol \\ Davila", Bucharest, Romania; 'Pediatric Poisoning Centre, \\ Emergency Clinical Hospital for Children "Grigore Alexandrescu", \\ Bucharest, Romania
}

Objective: To evaluate the influence of pre-existing anemia on the clinical picture and evolution in children with acute toxic methemoglobinemia.
Table 1. Severity of methemoglobinemia in children with and without preexisting anemia.

\begin{tabular}{lcc}
\hline $\begin{array}{l}\text { Serum } \\
\text { methemoglobin (\%) }\end{array}$ & $\begin{array}{c}\text { Group 1 with pre-existing } \\
\text { anemia }(\mathrm{n}=50)\end{array}$ & $\begin{array}{c}\text { Group 2 without } \\
\text { anemia }(\mathrm{n}=21)\end{array}$ \\
\hline $5-20 \%$ mild & 5 & 7 \\
$20-45 \%$ moderate & 22 & 0 \\
$45-70 \%$ severe & 22 & 4 \\
$>70 \%$ very severe & 1 & 0 \\
\hline
\end{tabular}

Methods: We performed a 5 year retrospective study of children with acute toxic methemoglobinemia admitted (typically with generalised cyanosis) to our department, between 2013 and 2017. Patients aged over 2 years and those with other comorbidities, except anemia were excluded. All the children were from rural areas and the cause of the methemoglobinemia in all cases was nitrate-contaminated water. The patients were divided into two groups: group 1 with pre-existing anemia (hemoglobin $<11$ $\mathrm{g} / \mathrm{dL}$ ) and group 2 with normal hemoglobin concentration. The two groups were analyzed taking into consideration the serum methemoglobin concentration as this is the main indicator of clinical severity [1], number of antidote doses required and length of hospitalization.

Results: A total of 71 children, aged less than 2 years, were included in the study; group 1 comprised 50 cases with 21 children in group 2. The number of patients with moderate and severe clinical picture was higher in group $1(n=44 / 71,60.2 \%)$, compared to group $2(\mathrm{n}=14,19.7 \%, \mathrm{p}=0.03)$. The median value of antidote doses required was greater in anemic patients then in those without anemia (1.48 versus 1.24 ) but the difference was not statistically significant $(p=0.17)$. The median length of hospitalization was higher in group 1 compared to group 2, the difference being statistically significant (2.98 versus 1.66 days, $p=$ 0.01 ).

Conclusion: Pre-existing anemia represents a risk factor for a more severe clinical picture in children with acute toxic methemoglobinemia. The recovery is slower in anemic children with acute toxic methemoglobinemia, even though the need for additional doses of methylene blue was not significantly higher for this category of patients.

\section{Reference}

[1] Osterhoudth KC. Methemoglobinemia. In: Erickson TB, Ahrens WR, Aks SE, et al. Pediatric Toxicology: Diagnosis and Management of the Poisoned Child. McGraw-Hill; 2005: p.492-500.

\section{Triage of patients with venlafaxine overdose: setting a dose threshold for hospital referral}

\author{
Saskia J Rietjens, Laura Hondebrink, \\ Fabian KJ Van Hoof and Irma De Vries \\ Dutch Poisons Information Center, University Medical Center \\ Utrecht, Utrecht, Netherlands
}

Objective: The serotonin-norepinephrine reuptake inhibitor venlafaxine is widely used for several indications such as depression and anxiety disorder. Convulsions and cardiotoxicity are wellknown complications of venlafaxine overdose. We aimed to refine our dose threshold for hospital referral to improve triage of patients exposed to venlafaxine.

Methods: We performed a two-year prospective follow-up study of venlafaxine mono-intoxications reported to the Dutch Poisons Information Center. Data were collected on patient characteristics, 
the exposure, clinical course and treatment using telephone surveys with patients and physicians. The severity of the intoxication was classified using the Poisoning Severity Score. A literature search was performed focusing on the clinical effects after venlafaxine poisoning, related to dose.

Results: We investigated 52 venlafaxine mono-exposures (12 children and 40 adults; range: 1-71 years). The median self-reported dose was $12.8 \mathrm{mg} / \mathrm{kg}$ (range: $0.9-84 \mathrm{mg} / \mathrm{kg}$ ), most often involving extended-release preparations (92\%). The most common reported effects were: sleepiness (54\%), dizziness (33\%), headache (25\%), agitation (23\%), tachycardia (35\%), hypertension (27\%), nausea (35\%), diaphoresis (29\%) and mydriasis (25\%). Overall $12 \%$ of patients remained asymptomatic, $71 \%$ developed mild symptoms, and $13 \%$ moderate symptoms. Severe symptoms occurred in two patients. One patient developed signs of serotonin syndrome and another patient was unresponsive. Convulsions or severe cardiotoxicity were not reported. To study the dose-effect relationship, we excluded patients with spontaneous emesis or in whom gastrointestinal decontamination was applied, and patients with multiple venlafaxine ingestions ( $>1$ hour between separate exposures). In this subset of patients $(n=21)$, the lowest self-reported dose causing a moderate intoxication was $11.5 \mathrm{mg} / \mathrm{kg}$, involving a 39-year-old woman who developed pronounced hypertension $(164 / 107 \mathrm{mmHg})$, chest pain, tremor and sleepiness. In the literature, convulsions ( $\geq 18.6 \mathrm{mg} / \mathrm{kg}$; estimated dose using bodyweight of $70 \mathrm{~kg}$ [1]) and acute heart failure ( $\geq 51.9 \mathrm{mg} / \mathrm{kg}$ [2]) are described following large overdose.

Conclusion: Hospital referral of intoxicated patients is indicated when moderate or severe symptoms could occur. Therefore, based on literature and our data, a venlafaxine dose threshold for hospital referral of approximately $12 \mathrm{mg} / \mathrm{kg}$ appears safe. Application of such a dose threshold can aid in decision-making in triaging patients exposed to venlafaxine and prevent unnecessary hospital admissions.

\section{References}

[1] Whyte I, Dawson A, Buckley N. Relative toxicity of venlafaxine and selective serotonin reuptake inhibitors in overdose compared to tricyclic antidepressants. Q J Med. 2003;96:369-374.

[2] Batista $M$, Dugernier $T$, Simon $M$, et al. The spectrum of acute heart failure after venlafaxine overdose. Clin Toxicol (Phila). 2013;51:92-95.

\section{Ocular exposures reported to US Poison Control Centers from 2011 to 2015}

\author{
Craig W Heise ${ }^{a}$ and Sumit Agarwal ${ }^{b}$ \\ ${ }^{a}$ Division of Medical Toxicology and Precision Medicine, University \\ of Arizona College of Medicine - Phoenix, Phoenix, United States; \\ bivision of Care Transformation, Banner - University Medical \\ Center Phoenix, Phoenix, United States
}

Objective: Identify and characterize the ocular exposures reported to poison control centers over a five-year period from 2011 to 2015 in the US.

Methods: We retrospectively analyzed data from 477,274 calls for ocular exposure to the National Poison Data System. Abstracted data included case age and gender, generic and product codes, exposure reason, exposure route, clinical effects, treatments given, and medical outcomes. Those cases with exposure other than the eye and skin were excluded, including any with parenteral or oral exposure. Major effect cases exhibited signs or symptoms as a result of the exposure that were life-threatening or resulted in significant residual disability or disfigurement.

Results: A mean volume of 95,454 calls per year were reported to poison control centers, with most exposures occurring unintentionally, at home and predominantly in children under five years of age. Children under the age of ten had 25 (0.02\%) major outcomes reported, all initially reported to the poison control center from home and were then sent to a healthcare facility (from which final outcome was obtained by poison control), 20 from laundry detergents and 5 from bleach. There were 709 $(0.15 \%)$ reported major outcomes, suggesting a permanent significant visual disability. Most serious adult exposures occurred at work due to alkali exposures. Major outcomes occurred more commonly in men than women $(n=347[0.35 \%]$ versus $n=229$ [0.16\%], p-value $<0.001$ ). Men also had higher incidence of major outcomes from occupational exposure compared to women $(\mathrm{n}=$ $174[0.96 \%]$ ) versus $n=18$ [0.20\%], $p$-value $<0.001)$. There was an increasing incidence in exposures in those over 64-years-old. The most common treatment provided was irrigation and wash for the affected eye.

Conclusion: Children under five are most susceptible, may have permanent disability due to laundry detergent exposure, and concerted intervention is needed in this age group. Many serious adult exposures occurred at work due to alkali exposures

\section{An analysis of adder bite referrals to the UK National Poisons Information Service (NPIS) requiring} antivenom

\author{
David Stewart ${ }^{a}$, Sally M Bradberry ${ }^{b}$, \\ Simon HL Thomasc, John P Thompson ${ }^{\text {, }}$, \\ Euan A Sandilands ${ }^{a}$ and Michael Eddleston ${ }^{a}$ \\ ${ }^{a}$ National Poisons Information Service, (Edinburgh Unit), Royal \\ Infirmary, Edinburgh, United Kingdom; ${ }^{\text {b National Poisons }}$ \\ Information Service (Birmingham Unit), City Hospital, Birmingham, \\ United Kingdom; 'National Poisons Information Service, (Newcastle \\ Unit), Regional Drug and Therapeutics Centre, Newcastle, United

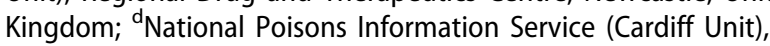 \\ University Hospital Llandough, Cardiff, United Kingdom
}

Objective: The European adder (Vipera berus) is the only native venomous snake in the UK. Adders can be found throughout the UK (except Ireland) and are generally seasonally active between the months of April and September. Bites are infrequently reported to the UK National Poisons Information Service (NPIS). Our objective was to analyse the epidemiology of adder bites requiring antivenom administration referred to the NPIS.

Methods: We analysed all telephone enquiries to the NPIS involving adder bites where antivenom was administered over a 2.5 year period (March 2016 - August 2018). Data was analysed with respect to patient gender, age, time of year and geographical location. We also recorded the time taken from bite to antivenom administration and the number of doses of antivenom given.

Results: Over the study period, the NPIS received 95 telephone enquiries regarding adder envenoming requiring administration of antivenom. The majority of cases involved adults $(n=78$, $82.1 \%$; age $>13$ years) with males being more commonly involved $(n=61,64.2 \%)$. While the earliest case was reported in March and latest in September, the majority occurred between the months of May and August $(n=83,87.4 \%)$. Most bites occurred in South England and Wales $(n=77,81.1 \%)$ with only 6 $(6.3 \%)$ cases reported in Scotland. In the majority of cases $(n=$ $66,69.5 \%)$ only one dose of antivenom was required. The median time (range) from adder bite to antivenom administration was 
4.5 hours (1.2 to 51.5 hours). Twenty-nine (30.5\%) patients required an additional dose of antivenom due to ongoing features of envenomation and three patients (3.2\%) required a third dose of antivenom.

Conclusion: The UK NPIS receives approximately 30 enquiries per year concerning envenomed patients requiring antivenom following an adder bite. Most cases occur in the summer months and in southern England and Wales. While the majority of cases achieve sufficient resolution of symptoms with one dose of antivenom, a proportion required additional doses of antivenom. Further analysis is required to determine the relationship between time of antivenom administration, severity of symptoms and length of hospital stay.

\section{A review of recommendations for and use of single dose activated charcoal in enquiries to the UK National Poisons Information Service (2017 to mid 2018)}

\author{
Emma J Moyns ${ }^{a}$, Muhammad EMO Elamin ${ }^{a}$, \\ Euan A Sandilands $\mathrm{s}^{\mathrm{b}}$, John P Thompson ${ }^{\mathrm{c}}$, \\ Simon HL Thomas ${ }^{d}$ and Sally M Bradberry ${ }^{a}$ \\ ${ }^{a}$ National Poisons Information Service (Birmingham Unit), City \\ Hospital, Birmingham, United Kingdom; ${ }^{b}$ National Poisons \\ Information Service (Edinburgh Unit), Royal Infirmary, Edinburgh, \\ United Kingdom; 'National Poisons Information Service (Cardiff \\ Unit), University Hospital Llandough, Cardiff, United Kingdom; \\ ${ }^{\mathrm{d}}$ National Poisons Information Service (Newcastle Unit), Regional \\ Drug and Therapeutics Centre, Newcastle, United Kingdom
}

Objective: To review enquiries to the UK National Poisons Information Service (NPIS) discussing the use of single dose activated charcoal (SDAC).

Methods: A retrospective analysis of UK NPIS enquiry records between 1 January 2017 and 30 June 2018 was undertaken for enquiries containing the terms "charcoal" or "SDAC", and/or where pre-defined fields for "charcoal given" or "charcoal recommended" had been selected.

Results: There were 972 relevant enquiries of which 898 involved SDAC, representing $1.6 \%$ of all enquiries involving ingestion received during this period. SDAC administration was recommended in 395 enquiries with recommended use within 1 hour post-ingestion in 347 enquiries (source: ambulance $62.5 \%$, prison $30.0 \%$, hospital $7.2 \%$ and police $0.3 \%$ ). The shortest time post-ingestion to SDAC recommendation was 5 minutes. SDAC was recommended beyond 1 hour post-ingestion in 31 cases ( 2 hours $n=16$, 3 hours $n=6,4$ hours $n=2,5$ hours $n=2$ and $>5$ hours $n=5$ ), in 14 enquiries with unknown time of ingestion and in 3 staggered overdose cases. Reasons for recommending late administration included large overdose $(n=11)$, ingestion of agents associated with potentially severe toxicity for which TOXBASE advises considering SDAC $>1$ hour (i.e. colchicine, foxglove, opiates, tricyclic antidepressants) $(n=8)$ and sustained release drugs $(n=7)$. The longest time post-ingestion for SDAC recommendation was 36 hours (computerised tomography scan demonstrated unknown tablets in the stomach). In 131 enquiries SDAC had already been administered at the time of the enquiry (source: hospital $46.6 \%$, prison $28.2 \%$, ambulance $24.4 \%$ and National Health Service (NHS) walk in centre $0.8 \%)$. SDAC was known to have been administered $<1$ hour postingestion in $58 \%$ of these 131 enquiries. Vomiting $(n=10,7.6 \%)$ was the only adverse effect reported. In 21 cases, SDAC was offered but declined by the patient. In a further 21 enquiries, the patient was referred to hospital from primary care for SDAC administration. SDAC was recommended but unavailable in 62 enquiries (ambulance $51.6 \%$ and prison $48.4 \%$ ). The use of SDAC was discussed but not recommended in 268 enquiries; reasons included $>1$ hour postingestion $(n=153)$, below toxic dose $(n=51)$, charcoal not indicated/effective $(n=23)$, combination of reasons $(n=16)$, staggered overdose $(n=10)$ and patient's airway unsupported $(n=5)$.

Conclusion: The NPIS makes an important contribution to the use of SDAC in the poisoned patient. There may be a role for wider availability of charcoal to ambulance and prison services. Assessment of SDAC efficacy would require detailed enquiry follow up which is limited by resource availability. Incidence of adverse effects reported to the NPIS from SDAC administration is low.

\section{Does the use of extensive monographs influence the duration of telephone calls at a poison center? A pilot study}

\author{
Iris Venster, Claudine $C$ Hunault, \\ Agnes G Van Velzen, Gerard A Zoelen and \\ Irma De Vries \\ Dutch Poisons Information Center, University Medical Center \\ Utrecht, Utrecht, Netherlands
}

Objective: Providing uniform information at a qualitative high level by a large group of Specialists in Poison Information (SPIs) is challenging. The Dutch Poisons Information Center (DPIC) has developed a robust digital information system in which DPIC monographs are integrated. These monographs are written at the DPIC, based on the scientific literature and DPIC experience. Products containing specific substances are linked to the appropriate monograph in the DPIC information system. Next to information on physical/chemical properties, kinetics, symptoms, therapy etc., these monographs contain information on dose-effect levels whenever possible. Having predetermined levels for establishing the severity of a poisoning (none, mild, moderate or severe) for many compounds greatly adds to a uniform triage and information supply by SPIs. However, these monographs contain much information, and the availability of more data to discuss may result in a prolonged duration of calls. In this pilot study, our aim was to investigate the median length of calls before and after release of a monograph.

Methods: We selected monographs that were released in 20132014 , with at least 20 calls before and after the release. Only calls from GPs or veterinarians concerning single exposures were considered. The primary outcome was call duration in seconds. A power calculation was performed before analyzing any data. A Non-Parametric Wilcoxon Signed Rank Test for related samples was used to test the difference in duration of calls between "before" and "after" release of a monograph.

Results: In total 182 pairs of calls were included, concerning 7 monographs: metronidazole, fusidic acid, galantamine, duloxetine, levocetirizine, blue-green algae, and cyanogenic glycoside-containing plants. The median duration of calls before release of a monograph was 231 seconds (IQR $=184$ ), and after release 203 seconds $(\mathrm{IQR}=178)$, resulting in a significant difference of 28 seconds ( $p<$ 0.001 ). However, the effect of using a monograph largely differed between the selected monographs, and there was a high variability in the duration of calls. For example, a decrease of 111 seconds and low variability in the duration of calls were observed for levocetirizine after release of the monograph. On the other hand, an increase of 23 seconds was observed for the blue-green algae monograph, with a high variability in call duration.

Conclusion: An overall decrease in the median duration of telephone calls was observed when using extensive monographs. However, more research is needed to establish why the effect of using a monograph differs so largely between the different monographs. 


\section{Increasing rates of serious and fatal 2,4-dinitrophenol poisoning in the UK}

Leonard Hawkins ${ }^{\mathrm{a}}$, David J Lupton ${ }^{\mathrm{b}}$, Gillian Jackson ${ }^{\mathrm{b}}$, Sally M Bradberryc, Euan A Sandilands ${ }^{\mathrm{b}}$, John P Thompson ${ }^{d}$ and Simon HL Thomas ${ }^{a}$

${ }^{a}$ National Poisons Information Service (Newcastle Unit), Newcastle Hospitals NHS Foundation Trust, Newcastle, United Kingdom; ${ }^{\mathrm{b}}$ National Poisons Information Service (Edinburgh Unit), Royal Infirmary, Edinburgh, United Kingdom; ' National Poisons Information Service (Birmingham Unit), City Hospital, Birmingham, United Kingdom; ${ }^{d}$ National Poisons Information Service (Cardiff Unit), Llandough Hospital, Cardiff, United Kingdom

Objective: 2,4-Dinitrophenol (DNP) is sometimes taken for weight reduction or "body sculpting", but can cause life-threatening poisoning. Here we report trends in enquiries to the UK National Poisons Information Service (NPIS) related to systemic DNP exposure, including fatal cases.

Methods: NPIS enquiries concerning systemic DNP exposure were identified between January 2007 and September 2018 (inclusive); separate enquiries about the same case were consolidated into a single record. Fatality data were triangulated with information on DNP-related deaths held by the UK Food Standards Agency (FSA) and Office for National Statistics (ONS).

Results: The 115 individuals with systemic DNP exposure discussed with the NPIS included $74(64 \%)$ males and 41 (36\%) females. Of these, $20(17.4 \%, 95 \%$ Confidence Intervals [Cl] $11.2 \%$, $25.8 \%$ ) are known to have died, with no significant difference in mortality between males $(12 / 74,16.2 \%, 95 \% \mathrm{Cl} 9.0 \%, 27.0 \%)$ and females $(8 / 41,19.5 \%, 95 \% \mathrm{Cl} 9.4 \%, 35.3 \%, \mathrm{P}>0.05)$. Fatalities were more common when the mode of exposure was unknown (4/10, 40\%, $\mathrm{P}=0.0051)$, acute $(12 / 42,28 \%, \mathrm{P}=0.0011)$ or acuteon-chronic $(2 / 12(18 \%, \mathrm{P}>0.05)$ compared with chronic exposure $(2 / 51,3.9 \%)$. There were 6 further DNP-related deaths that were not discussed with NPIS but which were known to the Food Standards Agency (FSA, 2012-2018) or the Office for National Statistics (ONS, 2007-2016), demonstrating at least 26 DNP-related deaths in the UK since 2007. Numbers of cases have increased substantially since 2012, with peaks in 2013, 2015 and 2018 (Table 1).

Table 1. Enquiries and fatalities recorded by the National Poisons Information Service (NPIS), the Food Standards Agency (FSA) and Office for National Statistics (ONS) relating to 2,4-dinitrophenol exposure in the UK.

\begin{tabular}{|c|c|c|c|c|c|}
\hline \multirow[b]{2}{*}{ Year } & \multicolumn{3}{|c|}{ All systemic NPIS cases } & \multicolumn{2}{|c|}{ Fatal cases } \\
\hline & Males & Females & Total & $\begin{array}{c}\text { NPIS } \\
\text { data only }\end{array}$ & $\begin{array}{l}\text { Combined } \\
\text { NPIS, FSA } \\
\text { and ONS data }\end{array}$ \\
\hline 2007 & 1 & 1 & 2 & 0 & 1 \\
\hline 2008 & 1 & 0 & 1 & 1 & 1 \\
\hline 2009 & 0 & 0 & 0 & 0 & 0 \\
\hline 2010 & 0 & 0 & 0 & 0 & 0 \\
\hline 2011 & 0 & 0 & 0 & 0 & 0 \\
\hline 2012 & 5 & 0 & 5 & 1 & 2 \\
\hline 2013 & 19 & 2 & 21 & 3 & 4 \\
\hline 2014 & 3 & 6 & 9 & 0 & 0 \\
\hline 2015 & 16 & 19 & 35 & 6 & 7 \\
\hline 2016 & 7 & 6 & 13 & 1 & 2 \\
\hline 2017 & 7 & 5 & 12 & 2 & 3 \\
\hline 2018 (Jan-Sept) & 15 & 2 & 17 & 6 & 6 \\
\hline Total & 74 & 41 & 115 & 20 & 26 \\
\hline
\end{tabular}

Conclusion: DNP-related enquiries and fatalities have increased substantially in recent years. Measures taken since 2013 to educate potential users and restrict sales have had partial but unsustained impact. Further work is needed to reduce availability and human use of this highly toxic substance.

\section{A review of enquiries received by the UK National Poisons Information Service (NPIS) involving colchicine exposure (2008 to 31 July 2018)}

\author{
Gillian A Cooper ${ }^{\mathrm{a}}$, John P Thompson ${ }^{\mathrm{a}}$, \\ Sally M Bradberry ${ }^{b}$, Euan A Sandilands ${ }^{c}$, \\ Simon HL Thomas ${ }^{d}$ and James M Coulson ${ }^{a}$ \\ ${ }^{a}$ National Poisons Information Service (Cardiff Unit), University \\ Hospital Llandough, Cardiff, United Kingdom; ${ }^{b}$ National Poisons \\ Information Service (Birmingham Unit), City Hospital, Birmingham, \\ United Kingdom; 'National Poisons Information Service (Edinburgh \\ Unit), Royal Infirmary, Edinburgh, United Kingdom; ${ }^{\text {d} N a t i o n a l ~}$ \\ Poisons Information Service (Newcastle Unit), Regional Drug and \\ Therapeutics Centre, Newcastle, United Kingdom
}

Objective: Primarily used in the treatment of gout, colchicine has a narrow therapeutic index, the reported fatal dose varies widely and there is no specific antidote. We report on the pattern of enquiries received by the NPIS in England and Wales.

Methods: Records of telephone enquiries received from England and Wales by the NPIS between 1 January 2008 and 31 July 2018 were reviewed and reported.

Results: The NPIS received a total of 248 enquiries regarding 202 cases during the study period. Enquiry rates demonstrate a statistically significant consistently upward trend from $0.01 \%$ of total enquiries in 2008 to $0.14 \%$ in 2017 (Mann-Kendall Trend Test, p < $0.05)$. Peak incidence, $33 \%$, occurred in the $70+$ age group and in males (65\%). Therapeutic error was reported for $46 \%$, intentional exposure $28 \%$, accidental $19 \%$, with unknown and other accounting for $7 \%$ of cases. Analysis of Poisoning Severity Score (PSS) indicated an increased risk of severity with colchicine exposure when compared with all enquiries; none $42 \%$ versus $67 \%$; minor $40 \%$ versus $25 \%$; moderate $10 \%$ versus $3 \%$; severe $6 \%$ versus $1 \%$ (chi-squared with Yates correlation, $\mathrm{p}<0.05)$. The proportion of intentional moderate/severe cases (25 of 33) was greater than for the less severe (31 of 169) (chi-squared with Yates correlation, $p$ $<0.05)$. Thirteen cases (39\%) involved multiple agent exposure. The ingested dose (calculated on average weight when not documented) involving acute ingestion ranged from 0.09 to $1.1 \mathrm{mg} /$ $\mathrm{kg}$. Outcome was reported in 22 cases. In those where recovery was reported $(n=11)$ the highest acute dose was $75 \mathrm{mg}$ (approximately $1.1 \mathrm{mg} / \mathrm{kg}$ ). In this case, symptoms included vomiting, abdominal pain, diarrhoea and increased transaminase activity; no details on late complications were recorded. Eleven deaths were reported in $2008(n=1) ; 2012(n=1) ; 2014(n=1)$; $2016(n=3) ; 2017(n=3)$ and $2018(n=2)$, all of which related to acute or staggered intentional exposure. Seven cases were reported to involve multiple drug exposure. The lowest dose in which death was reported with no other medication involved was $15 \mathrm{mg}$ (estimated $0.21 \mathrm{mg} / \mathrm{kg}$ ).

Conclusion: The annual incidence of colchicine enquiries increased over the study period. This trend is of concern, given that the proportion of moderate or severe PSS cases is higher than the average, compared to all NPIS enquiries during the same period. All reported deaths resulted from intentional exposure, which itself accounts for almost a third of the enquiries. Further analysis with prescription data is warranted to identify potential strategies to reduce the risk of poisoning with colchicine. 


\section{Handling public health and media concerns over magnetic putty: a case study by the UK National Poisons Information Service}

\author{
Ho MR Lee ${ }^{a}$, Muhammad EMO Elamin ${ }^{\text {, }}$ \\ Nicola Barlow ${ }^{\mathrm{b}}$, Euan A Sandilands, \\ John P Thompson ${ }^{d}$, Simon HL Thomas ${ }^{\mathrm{e}}$, \\ J Allister Vale ${ }^{a}$ and Sally M Bradberry ${ }^{a}$ \\ ${ }^{a}$ National Poisons Information Service (Birmingham Unit), City \\ Hospital, Birmingham, United Kingdom; ${ }^{\mathrm{b}}$ Trace Elements \\ Laboratory, Sandwell and West Birmingham Hospitals NHS Trust,

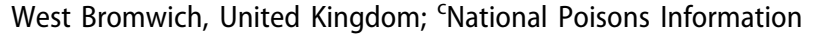 \\ Service (Edinburgh Unit), Royal Infirmary, Edinburgh, United \\ Kingdom; ${ }^{d}$ National Poisons Information Service (Cardiff Unit), \\ University Hospital Llandough, Cardiff, United Kingdom; ${ }^{\text {NNational }}$ \\ Poisons Information Service (Newcastle Unit), Regional Drug and \\ Therapeutics Centre, Newcastle, United Kingdom
}

Objective: In January 2018 a magnetic putty toy was found to contain seven times the permitted EU limit for arsenic. We review exposures to magnetic putty reported to the UK National Poisons Information Service (NPIS) following media publicity and describe how the service dealt with these public concerns.

Methods: Enquiries to the NPIS from 1 January 2018 to 1 July 2018 were correlated with media reports driving public interest in magnetic putty.

Results: During the study period, there were 174 enquiries from doctors and other healthcare workers concerning 184 patients. Enquiry frequency peaked on 20 February 2018 ( $n=57)$, coinciding with multiple national news stories and social media posts. Exposures were most commonly reported in patients aged 6-10 years (53.3\%). Exposures were reported following skin contact alone $(n=173)$ or ingestion $(n=11)$. The majority of patients were asymptomatic $(n=142,76.6 \%)$. The remaining 42 patients $(22.8 \%)$ reported minor symptoms, most commonly abdominal pain $(n=25)$, followed by nausea $(n=8)$, headache $(n=8)$ and diarrhoea $(n=8)$ and were the subjects of focussed follow-up. On 20 February 2018 an alert was placed on TOXBASE ${ }^{\oplus}$, the NPIS online information database, and the NPIS advised measurement of arsenic in a spot urine sample in symptomatic patients. An attempt was also made to measure the arsenic concentration in the putty used by those symptomatic. Urine arsenic concentrations (upper limit of total arsenic for an unexposed population is $15 \mathrm{nmol} / \mathrm{mmol}$ creatinine) were obtained in 9 of the 42 symptomatic patients and ranged from 20 to $430 \mathrm{nmol} / \mathrm{mmol}$ [median $181 \mathrm{nmol} / \mathrm{mmol}$; IQR $45-298 \mathrm{nmol} / \mathrm{mmol}$. To mitigate for nontoxic dietary arsenobetaine, repeat urine samples were recommended after abstention from seafood but, despite substantial effort, a second urine arsenic concentration was obtained in only 4 of these 9 cases: $8 \mathrm{nmol} / \mathrm{mmol}$ (previously $181 \mathrm{nmol} / \mathrm{mmol}$ ); 19 $\mathrm{nmol} / \mathrm{mmol}$ (previously $293 \mathrm{nmol} / \mathrm{mmol}$ ); $49 \mathrm{nmol} / \mathrm{mmol}$ (previously $39 \mathrm{nmol} / \mathrm{mmol}$ ); $57 \mathrm{nmol} / \mathrm{mmol}$ (previously $51 \mathrm{nmol} / \mathrm{mmol}$ ). In at least one of these cases (repeat urine arsenic $49 \mathrm{nmol} /$ $\mathrm{mmol}$ ), the patient had continued to eat fish fingers despite advice. No arsenic was detected in the single magnetic putty sample submitted for analysis and used by the child with the highest recorded urine concentration $(430 \mathrm{nmol} / \mathrm{mmol})$. Although no repeat urine sample was received from this child, it is probable that the arsenic concentration reflected dietary intake.

Conclusion: Poisons information services need to respond rapidly to public health concerns of this kind and interpret, often limited, toxicological data accurately and correctly.

\section{Accidental chemical poisonings in nursing homes}

\author{
Anna-Mariia Termälä, Terhi Lampinen and \\ Leena Soininen \\ Poison Information Center, University of Helsinki and Department \\ of Emergency Medicine and Services, Helsinki University Hospital, \\ Helsinki, Finland
}

Objective: A preliminary study to describe accidental chemical poisonings in special adult groups, i.e. those with memory disorders and intellectual disability, living in nursing homes.

Methods: Retrospective review of Finnish Poison Information Centeŕs (FPIC) call records and one case report.

Results: During the study period, from 1 January to 31 July 2018, FPIC received 113 inquiries (from a total of 21463) regarding chemical exposure in disabled or dementia patients living in nursing homes. The age distribution was $15-50$ years $(n=21)$, 51-70 years $(n=18)$ and $71-100$ years $(n=53)$. Age was not recorded in 21 calls. FPIC received calls from nursing staff $(n=74)$, physicians $(n=18)$, first responders $(n=14)$ and the public $(n=7)$. Substances involved were detergents $(n=59)$, cosmetics for daily hygiene $(n=35)$, alcohols (mainly hand disinfection products) $(n=13)$, and others $(n=6)$. In total $40 / 113$ patients were symptomatic. No data of patient records and patient follow-up was available. The index case for this survey was a 54-year-old patient with Down's syndrome who drank an unknown amount of liquid laundry detergent ( $\mathrm{pH}$ 9.5), while taking a shower in a nursing home. The patient was initially asymptomatic, except for coughing while eating. Within 2 hours he developed decreased level of consciousness and wheezing and the emergency services were contacted. When the first responders arrived, less than 3 hours after ingestion, the patient's blood pressure was $101 / 81 \mathrm{mmHg}$, pulse $62 / \mathrm{min}$, oxygen saturation with room air was $73-92 \%$ and Glasgow Coma Score (GCS) 9/15. The patient was brought to the Emergency Department where he had severe breathing difficulties, coma and metabolic acidosis $(\mathrm{pH} 7.1$, base excess -13.6), elevated lactate $(11.4 \mathrm{mmol} / \mathrm{L})$ and was intubated. Blood pressure was $88 / 50 \mathrm{mmHg}$ despite norepinephrine infusion $0.87 \mu \mathrm{g} / \mathrm{kg} / \mathrm{min}$. Computed tomography scan and bronchoscopy was performed and findings indicated aspiration. In the intensive care unit, the patient had severe metabolic acidosis and hemodynamic collapse that did not response to aggressive treatment. He died the next day due to multi-organ failure. The main finding in forensic autopsy was aspiration.

Conclusion: This index case shows that ingestion of laundry detergent can be fatal. Our follow-up survey indicates that more patients are at risk of exposure. Further study is needed to investigate chemical poisonings in nursing homes to ensure quality of care for those patients living where they are dependent on healthcare professionals.

\section{Valpromide overdose: how to interpret the plasma concentration of valproic acid?}

Sophie Delhumeau, Gaël Le Roux, Géraldine Meyer, Ali Touré, Marion Legeay and Marie Deguigne

Centre Antipoison et Toxicovigilance Grand Ouest, CHU d'Angers, Angers, France 
Objective: This study is to examine the relationship between the severity of valpromide poisoning and plasma valproic acid concentration.

Methods: A retrospective and observational study of valpromide exposures reported to the Angers Poison Control Center (PCC), France, over a sixteen year period and for which a valproic acid quantitation was performed. The severity was assessed according to the Poisoning Severity Score (PSS).

Results: A total of 163 cases were included, of which 161 were deliberate overdoses. The median age was 41 years and the sexratio was 0.73 . The median presumed dose intake was $6 \mathrm{~g}(0.3-$ $63 \mathrm{~g})$. The main signs observed were: somnolence $(n=54)$, coma (Glasgow Coma Score $[\mathrm{GCS}] \leq 8)(\mathrm{n}=43)$, hyperammonemia $(\mathrm{n}=$ $42)$, hypotension $(n=22)$, including two cases of circulatory shock, cardiac arrest $(n=6)$, metabolic acidosis $(n=19)$, tachycardia $<140$ bpm $(n=17)$, vomiting $(n=15)$, and hyperlactatemia $(>5 \mathrm{mmol} / \mathrm{L}, \mathrm{n}=10$ ). Severe poisoning (PSS3 and 4) accounted for almost one third of cases $(n=51)$. Among the 5 fatal cases, valproic acid quantitation was performed post-mortem in 4 cases (concentrations of 179 and $335 \mathrm{mg} / \mathrm{L}$ in single exposures and 270 and $820 \mathrm{mg} / \mathrm{L}$ in multiple exposures). For 32 patients with at least 2 dosages of valproic acid, the maximum concentration was observed at $16 \pm 11$ hours after ingestion. Severity was correlated with plasma valproic acid concentration $(r=0.67, p<0.00001)$. Median concentrations of patients with PSS1 and PSS3 were significantly different $(p=0.001)$. The risk of severe intoxication (PSS3) was greatly increased when the plasma concentration was greater than $200 \mathrm{mg} / \mathrm{L}$ (OR 19 [2.9-239], $\mathrm{p}=0.0002$ ).

Conclusion: In case of overdose with valpromide, the concentration of valproic acid associated with severe intoxication is much lower than in the case of overdose with valproic acid $(200 \mathrm{mg} / \mathrm{L}$ versus $850 \mathrm{mg} / \mathrm{L})$ [1].

\section{Reference}

[1] Spiller HA, Krenzelok EP, Klein-Schwartz W, et al. Multicenter case series of valproic acid ingestion: serum concentrations and toxicity. J Toxicol Clin Toxicol. 2000;38:755-760.

\section{Surgical removal of quetiapine bezoars}

\author{
Hilda MH Phan and Mark Personne \\ Swedish Poisons Information Centre, Swedish Medical Products \\ Agency, Stockholm, Sweden
}

Objective: Quetiapine is an atypical antipsychotic drug. In large overdoses, pharmacobezoar formations (mainly seen with sustained-release preparations) can complicate patient management due to delayed onset of severe symptoms and reduced effectiveness of decontamination procedures [1]. Fatalities have been reported after ingestion of more than $10 \mathrm{~g}$. Three cases of slowrelease quetiapine overdose are presented where pharmacobezoar formations occurred and different surgical procedures were deemed necessary.

Case reports: Case 1. A 57-year-old man presented at the hospital after a multidrug overdose including quetiapine $33 \mathrm{~g}(110$ tablets $\times 300 \mathrm{mg}$ ) and unknown amounts of oxazepam, lamotrigine and alcohol. His level of consciousness declined and an abdominal computerised tomography (CT) scan showed a vast amount of tablets in the ventricle and duodenum. Gastroscopy was initiated, but difficulties in removing the sticky conglomerate (the size of a tennis ball) lead to the decision to execute an acute laparotomy with manual removal of the bezoar followed by whole bowel irrigation (WBI). The patient continued to be circulatory stable and was taken off the ventilator the following day. Case 2. A 50-year-old woman ingested quetiapine $18 \mathrm{~g}$ (90 tablets $\times 200 \mathrm{mg}$ ) and an unknown amount of methylphenidate and zopiclone. She showed signs of central nervous system depression, nystagmus, tachycardia and twitching of the extremities. A CT scan revealed a lump of tablets resembling a cauliflower. Gastroscopic removal was time-consuming and technically difficult. A broad basket was the best, but not an optimal tool. Aspiration pneumonia ensued but otherwise the course was uneventful. Case 3. A 36-year-old man presented at the hospital shortly after a massive overdose of $80 \mathrm{~g}$ (200 tablets x 400 $\mathrm{mg}$ ). WBI ( 2 liter/hour) was initiated but proved to be ineffective. A CT scan showed a conglomerate $(8 \times 5 \mathrm{~cm})$ in the ventricle, 20 tablets in the duodenum and 10 tablets in the jejunum. The bezoar was impossible to remove with gastroscopic methods, and laparoscopic extraction through the abdominal cavity was initiated. Technical difficulties were evident but most of the bezoar could be removed. The post-operative period was uneventful.

Conclusion: Slow-release formulations and the risks of bezoar formation add extra dimensions to patient management after a large overdose. A swift decontamination plan sometimes involving $\mathrm{WBI}$ and various surgical procedures can be vital for a good prognosis. Slow-release formulations without the propensity of forming bezoars are to be preferred.

\section{Reference}

[1] Rauber-Lüthy C, Hofer KE, Bodmer M, et al. Gastric pharmacobezoars in quetiapine extended-release overdose: a case series. Clin Toxicol (Phila). 2013;51:937-940.

\section{Regional variability in intentional exposures to prescription medications in France and Italy}

\author{
Samantha J Leroy ${ }^{\text {a }}$, Zachary R Margolin ${ }^{\text {a }}$ \\ James J Lagrotteria ${ }^{a}$, Geoff Severtson ${ }^{a}$, \\ Bruno Mégarbane ${ }^{\mathrm{b}}$, Antoine Villa ${ }^{\mathrm{b}}$, Fabrizio Sesanac, \\ Janetta L Iwanicki ${ }^{\mathrm{a}}$ and Richard C Dart ${ }^{\mathrm{a}}$ \\ ${ }^{a}$ Rocky Mountain Poison \& Drug Center, Denver, United States; \\ ${ }^{b}$ Centre Antipoison et de Toxicovigilance de Paris, Paris, France; \\ 'Poison Control Centre of Milan, Milan, Italy
}

Objective: To assess regional differences in rates of intentional exposures involving benzodiazepines, GABA analogs, prescription opioids, and Z-drugs within France and Italy.

Methods: Data from the Researched Abuse, Diversion and Addiction-Related Surveillance (RADARS ${ }^{\circledR}$ ) System Global Toxicosurveillance Network (GTNet) were used. Rates of intentional exposures per 100,000 population were calculated by Nomenclature of Territorial Units for Statistics (NUTS) regions using data collected from participating poison centres in Italy (Milan) and France (Paris). The Milan Poison Centre receives approximately $67.5 \%$ of the total human exposure calls to Italian centres. The Paris Poison Centre reports coverage of $18 \%$ of the national population. Intentional exposures include exposures where the patient was attempting to gain a euphoric effect (abuse), attempting self-harm (suicide), or intentionally improperly used a medication for reasons other than to gain a euphoric effect or for self-harm (misuse). Data collected on exposures involving select prescription opioids, benzodiazepines, GABA analogues, and Z-drugs from first quarter of 2012 through fourth quarter of 2016 were analyzed. Exposures where postal code information could be linked to a NUTS level-3 region were used 
Table 1. Regional variability in intentional exposures to prescription medications in France and Italy, showing regions with the highest rate of intentional exposures per 100,000 population by country and drug group in descending order.

\begin{tabular}{|c|c|c|c|c|}
\hline Country & $\begin{array}{c}\text { Benzodiazepines } \\
\mathrm{n} \text { (rate per } 100,000 \text { population) }\end{array}$ & $\begin{array}{c}\text { GABA analogs } \\
\mathrm{n} \text { (rate per } 100,000 \text { population) }\end{array}$ & $\begin{array}{c}\text { Prescription opioids } \\
\mathrm{n} \text { (rate per } 100,000 \text { population) }\end{array}$ & $\begin{array}{c}\text { Z-drugs } \\
\mathrm{n} \text { (rate per } 100,000 \text { population) }\end{array}$ \\
\hline Italy & $\begin{array}{l}\text { Varese } \\
\mathrm{n}=426(47.9) \\
\text { Enna } \\
\mathrm{n}=58 \text { (34.5) } \\
\text { Rovigo } \\
\mathrm{n}=77(32.3)\end{array}$ & $\begin{array}{l}\text { Varese } \\
\mathrm{n}=40(4.5) \\
\text { Perugia } \\
\mathrm{n}=21(3.2) \\
\text { Cremona } \\
\mathrm{n}=10(2.8)\end{array}$ & $\begin{array}{l}\text { Varese } \\
\mathrm{n}=39(4.4) \\
\text { Livorno } \\
\mathrm{n}=13(3.9) \\
\text { Grosseto } \\
\mathrm{n}=8(3.6)\end{array}$ & $\begin{array}{l}\text { Varese } \\
\mathrm{n}=35(3.9) \\
\text { Enna } \\
\mathrm{n}=5(3) \\
\text { Ascoli Piceno } \\
\mathrm{n}=6(2.9)\end{array}$ \\
\hline
\end{tabular}

in the numerator. Population estimates by region from Eurostat [1] were used as the denominator.

Results: In France, Paris had the highest rate of intentional exposures involving benzodiazepines and exposures involving prescription opioids per 100,000 population (Table 1). Meurtheet-Moselle had the highest rate of intentional exposures involving GABA analogs, and Haute-Marne had the highest rate per population for Z-drugs. In Italy, Varese had the highest rate of intentional exposures for all drug groups.

Conclusion: There is substantial regional variation in intentional exposures involving prescription medications. Considering regional rates per population is important in developing targeted interventions. Results are limited by population coverage and medications comprising drug groups vary between countries.

\section{Reference}

[1] EUROSTAT website. Population on 1 January by age group, sex and NUTS 3 region [cited 2018 Sep 28]. Available from: http://appsso.eurostat.ec.europa.eu/nui/show.do?dataset=demo_r_pjangrp3\&lang=en

\section{Button battery exposures in Australian children: a prospective observational study highlighting the role of poisons information centres}

\author{
Rose Cairns ${ }^{\mathrm{a}}$, Jared A Brown ${ }^{\mathrm{a}}$, Kishen Lachireddy, \\ Carol J Wylie ${ }^{c}$, Jeff Robinson ${ }^{d}$, Andrew H Dawson ${ }^{a}$ \\ and Nicholas A Buckley ${ }^{a}$ \\ ${ }^{a}$ New South Wales Poisons Information Centre, The Children's \\ Hospital at Westmead, Sydney Pharmacy School, The University of \\ Sydney, Sydney, Australia; ${ }^{b}$ Environmental Health Branch, Health \\ Protection NSW, Sydney, Australia; ' $Q$ Queensland Poisons \\ Information Centre, Lady Cilento Children's Hospital, Brisbane, \\ Australia; ${ }^{d}$ Victorian Poisons Information Centre, Austin Hospital, \\ Melbourne, Australia
}

Objective: Button battery ingestion is a worldwide problem, with evidence of increasing harm and deaths in recent decades. In the last 5 years, two Australian children have died following ingestion of button batteries. In the 2015 coronial inquest into one case, a number of health system problems were identified, and the coroner recommended that Australian Poisons Information Centres (PICs) are the first point of contact following button battery exposures. Australian PIC experience includes cases of treatment delay due to lack of healthcare professional recognition of risks, and/or lack of local resources. Consequently, New South Wales PIC
(NSWPIC) introduced a protocol for button battery exposures, whereby PIC staff call ahead to the hospital a child is to present at. The PIC (i) confirms the hospital's ability to perform an X-ray (if not, callers are diverted to another hospital to minimise delay), and (ii) discusses the risk of severe injury to ensure the child is given priority for X-ray. This study aims to characterise Australian button battery exposures, focusing on exposure circumstances and preventable health system shortcomings.

Methods: A prospective observational study of button battery exposure calls to NSWPIC, November 2015-May 2017, using a followup survey to obtain outcome data and additional details. Survey data was combined with nationwide PIC data over the same period.

Results: Australian PICs were consulted on 578 exposures over the 19-month study period, including 506 paediatric cases. The median (IQR) age for the pediatric cases was 23 months (14-36 months). Where the source was identified, batteries came from toys in $26 \%$ of cases, with hearing aids, watches, and remote controls being other common sources. Children in outer regional, remote and very remote areas were overrepresented, and 15 cases were referred to a different hospital due to X-ray facilities being unavailable at their nearest hospital. We identified inconsistent triage from a range of first responders, and knowledge gaps regarding button battery dangers amongst some healthcare professionals.

Conclusion: Button battery exposures are a common call to Australian PICs. This study highlights a potential role of education campaigns, professional guidelines, and child-resistant battery compartments in toys and household devices. PICs calling ahead to ensure X-ray availability/diversion to a different hospital likely reduced delays for this time-critical exposure. Data collected by PICs can provide useful information for public health and product safety initiatives. A PIC-led protocol to direct initial medical management of button battery exposures could reduce delays and improve outcomes.

\section{Review of enquiries to the UK National Poisons Information Service (NPIS) Birmingham Unit originating from NHS 111, NHS 24 and NHS Direct advice services}

Pardeep S Jagpal ${ }^{\mathrm{a}}$, Hayley A Williams ${ }^{\mathrm{a}}$, Muhammad EMO Elamin ${ }^{\mathrm{a}}$, Euan A Sandilands ${ }^{\mathrm{b}}$, Simon HL Thomas', John P Thompson ${ }^{\mathrm{d}}$ and Sally M Bradberry ${ }^{\mathrm{a}}$

${ }^{a}$ National Poisons Information Service (Birmingham Unit), City Hospital, Birmingham, United Kingdom; ${ }^{\text {b}}$ National Poisons Information Service (Edinburgh Unit), Royal Infirmary, Edinburgh, United Kingdom; 'National Poisons Information Service (Newcastle Unit), Regional Drug and Therapeutics Centre, Newcastle, United 
Kingdom; ${ }^{\mathrm{d}}$ National Poisons Information Service (Cardiff Unit), University Hospital Llandough, Cardiff, United Kingdom

Objective: The UK National Poisons Information Service (NPIS) Birmingham Unit received 12,017 telephone enquiries over a one year period (April 2017 to March 2018), of which 36\% $(n=4,412)$ originated from services providing telephone advice to members of the public in England, Scotland and Wales via NHS 111, NHS 24 and NHS Direct respectively. These call centres are thus the largest NPIS telephone service user. The objectives of the study were to review reasons for these enquiries and consider if they identified any service modification needs.

Methods: A prospective survey was undertaken of enquiries originating from NHS 111, 24 and Direct services to the Birmingham Unit over 3 weeks 23 April to 13 May 2018. Enquirers were asked the reason for their call and these were reviewed.

Results: During the study period the Birmingham Unit manned the NPIS telephone line for 168 hours and handled 685 enquiries, of which $253(37 \%)$ fulfilled the inclusion criteria. The majority originated from NHS $111(\mathrm{n}=241,95 \%)$ followed by NHS Direct $(\mathrm{n}=7,3 \%)$ and NHS $24(\mathrm{n}=5,2 \%)$. Enquirers included nurses $(n=219,87 \%)$, paramedics $(n=23,9 \%)$ and doctors $(n=10,4 \%)$ The most common reasons for enquiries were: subject of enquiry did not have an entry on the UK's primary clinical toxicology database, TOXBASE $^{\oplus}(n=83,33 \%)$, enquirers seeking assistance with calculations $(n=42,16 \%)$ or seeking assistance with using TOXBASE $^{\oplus}(n=36,14 \%)$. Of the 83 enquiries with no specific TOXBASE ${ }^{\circledR}$ entry, agents most commonly involved home and non-pesticide garden products $(41 \%, n=34)$, personal care products $(18 \%, n=15)$, nutritional supplements and over-the-counter remedies $(13 \%, n=11)$. Despite absence of a specific TOXBASE entry, in 55 of 83 enquiries there was a general entry providing advice on the type of product involved. Of the 36 enquiries where assistance was sought with using TOXBASE ${ }^{\oplus}$, enquirers required help with interpreting the risk assessment "alert box" $(44 \%, \mathrm{n}=16)$; interpreting other TOXBASE $^{\circledR}$ information $(22 \%$, $\mathrm{n}=8)$; navigating $\operatorname{TOXBASE}^{\otimes}(20 \%, \mathrm{n}=7)$ and where enquirers felt there was insufficient information on TOXBASE $^{\circledR}$ to answer their specific question $(14 \%, n=5)$.

Conclusion: NHS telephone advice line operators are the largest users of the NPIS telephone service. Despite measures to make TOXBASE $^{\oplus}$ as comprehensive as possible: general entries for agents where the vast number of products makes specific entries impossible; a continuous process of revision of existing and production of new entries and a vigilance system for agents not on TOXBASE ${ }^{\oplus}$, the NPIS telephone service remains essential to provide a comprehensive 24-hour poisons information service. End users may benefit from further TOXBASE ${ }^{\circledR}$ training.

\section{Can poisons centre data inform safer prescribing? A pilot review of propranolol exposures reported to the UK National Poisons Information Service (NPIS)}

\author{
Hayley A Williams ${ }^{a}$, Des Henke ${ }^{a}$, \\ Muhammad EMO Elamin ${ }^{\mathrm{a}}$, Euan A Sandilands $\mathrm{s}^{\mathrm{b}}$, \\ Simon HL Thomasc, John P Thompson ${ }^{\mathrm{d}}$ and \\ Sally M Bradberry ${ }^{a}$ \\ ${ }^{a}$ National Poisons Information Service (Birmingham Unit), City \\ Hospital, Birmingham, United Kingdom; ${ }^{b}$ National Poisons \\ Information Service (Edinburgh Unit), Royal Infirmary, Edinburgh, \\ United Kingdom; 'National Poisons Information Service (Newcastle \\ Unit), Regional Drug and Therapeutics Centre, Newcastle, United

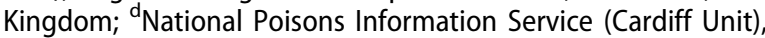 \\ University Hospital Llandough, Cardiff, United Kingdom
}

Objective: NHS Digital prescription data demonstrate a substantial rise in the number of primary care prescriptions for propranolol in England, from 2,540,362 in 2005 to 4,349,504 in 2015 [1]. Amidst media concern surrounding deaths from propranolol overdose in those for whom it is prescribed for anxiety, we undertook a pilot review of one year's NPIS propranolol exposure data, including an assessment of when it was recorded if propranolol was the patient's own medication and if so, the indication for the prescription.

Methods: A retrospective analysis of NPIS enquiries 1 April 2017 to 31 March 2018.

Results: We received 354 enquiries regarding 333 individuals involved in 339 exposures. For all exposures, enquiries originated from: hospital $(n=107,31.6 \%)$, ambulance $(n=103,30.4 \%)$, general practice $(n=44,13.0 \%)$, NHS telephone advice services $(n=$ $42,12.4 \%)$, prison $(n=38,11.2 \%)$ and other sources $(n=5,1.4 \%)$. Although most exposures occurred at home $(n=284,83.8 \%)$, a substantial number occurred in prison $(n=38,11.2 \%)$. Forty percent of exposures (137/339) involved only propranolol, though alcohol was ingested in 7 of these. There were 250 cases of deliberate ingestion with a dose stated in 208. The median reported dose was $560 \mathrm{mg}$ (IQR 280-1280 mg) and maximum reported was $7680 \mathrm{mg}$. The maximum Poisoning Severity Score (PSS) [2] was known for 328 exposures; none $(n=163,48.1 \%)$, minor $(n=100$, $29.5 \%)$, moderate $(n=28,8.3 \%)$, severe $(n=30,8.8 \%)$ and fatal $(n=7,2.1 \%)$. The seven patients who died were aged 17-46 years and three ingested propranolol only (dose known in one case: $3360 \mathrm{mg}$, indication known in one case: migraine). Propranolol was the patient's own medication in 147 of 339 $(43.4 \%)$ cases, with the specific indication known in only 36 of these. Anxiety was the most common indication $(n=20)$.

Conclusion: In the year 2017-2018 the UK NPIS received approximately one enquiry per day regarding propranolol. Propranolol was known to be the patient's own medication in at least $43 \%$ of exposures though the indication for prescription was not routinely recorded. A prospective poison centre study to better clarify the circumstances surrounding propranolol overdose amongst those for whom it is prescribed could inform safer prescribing.

\section{References}

[1] Prescribing and Medicines Team. Prescriptions dispensed in the community: England 2005-2015. Health and Social Care Information Centre. 2016 [cited 2018 Oct 17]. Available from: https://digital.nhs.uk/data-and-information/publications/statistical/ prescriptions-dispensed-in-the-community/prescriptions-dispensedin-the-community-statistics-for-england-2005-2015

[2] Persson $\mathrm{HE}$, Sjöberg GK, Haines JA, et al. Poisoning severity score. Grading of acute poisoning. J Toxicol Clin Toxicol. 1998;36:205-213.

\section{Poison centre data on botulism: results from an EAPCCT survey}

\author{
Davide Lonati ${ }^{\mathrm{a}}$, Maria Caterina Grassi ${ }^{\mathrm{b}}$, \\ Helena Lindal Baldvinsdottir ${ }^{c}$, Polyxeni Neou ${ }^{d}$, \\ Jonas Moens ${ }^{\mathrm{e}}$, Piotr M Kabata ${ }^{\mathrm{f}}$, Luc De Haro ${ }^{\mathrm{g}}$, \\ Mare Oder ${ }^{\text {h }}$, Miran Bvari, Christine Rauber-Lüthy', \\ Sergey Zacharov', Helmut Schiel', Dieter Genser', \\ Fabrizio Anniballi ${ }^{\mathrm{m}}$ and Carlo A Locatelli ${ }^{\mathrm{a}}$ \\ apavia Poison Control Centre - National Toxicology Information \\ Centre - Clinical and Experimental Lab, Toxicology Unit, Maugeri \\ Clinical and Scientific Institutes IRCCS and University of Pavia, \\ Pavia, Italy; ${ }^{b}$ Clinical Toxicology, Poison Control Centre and Drug \\ Dependence Unit, Policlinico Umberto I and Department of \\ Physiology and Pharmacology "Erspamer" - "Sapienza" University
}


of Rome, Rome, Italy; 'Icelandic National Hospital, Landspítali Háskólasjúkrahús, Reykjavik, Iceland; ' ${ }^{d}$ Greek Poison Centre, "P \& A Kyriakou" Children's Hospital, Athens, Greece; ${ }^{\text {eBelgian Poison }}$ Control Centre, Brussels, Belgium; 'Department of Clinical Toxicology, Medical University Gdansk, Gdansk, Poland; ${ }^{9}$ Centre Antipoison, Hôpital Sainte Marguerite, Marseille, France; hPoisoning Information Center, Estonian Health Board, Tallin, Estonia; 'University Medical Centre Ljubljana, Ljubljana, Slovenia; jNational Poisons Centre, Tox Info Suisse, Associated Institute of the University of Zurich, Zurich, Switzerland; ${ }^{\text {TToxicological }}$ Information Centre, General University Hospital, Prague, Czech Republic; 'Poisons Information Centre, Vienna, Austria; ${ }^{m}$ National Reference Centre for Botulism (NRCB), Department of Veterinary Public Health and Food Safety, National Institute of Health (ISS), Rome, Italy

Objective: To collect epidemiological data and information on the clinical management, diagnostic capability and antidote availability in cases of botulism in poison control centers/poisoning treating facilities (PCCs) located in different countries.

Methods: An electronic survey was sent to EAPCCT members in March 2018. The survey included 19 questions on (i) epidemiological data (registered by PCCs during 2015-2017) as well as questions on (ii) availability/location of specific laboratory, (iii) clinical management, (iv) type of antitoxin availability (including dosage/adverse drug reaction) and (v) its location. A reminder email was sent after 3 weeks.

Results: Fourteen PCCs completed the survey (Austria, Belgium, Czech Republic, Estonia, France, Germany, Greece, Iceland, Ireland, Italy, Poland, Slovenia, South Africa and Switzerland). Ireland, Estonia, Slovenia and Poland PCCs declared no experience with botulism because cases were managed by Infectious Diseases Services. Therefore 10 questionnaires were analyzed. Cases of foodborne botulism, infant and adult intestinal botulism, and wound botulism were registered by PCCs. Specific laboratories for diagnosis are available in 7 countries (70\%), all located in government services (in 2 countries operative 24 hours). The detection of botulinum/botulinum producing clostridia is carried out by polymerase chain reaction (PCR) and in vivo tests. Turnaround time (TAT) varies from 2 to 72 hours for PCR and from 12 hours to 7 days for in vivo testing. All PCCs, except two, prescribe antidote before laboratory confirmation. Trivalent Equine Antitoxin is the unique formulation available, and the dosage varies from 1 to 4 bottles. No severe acute adverse reactions have been reported. Antitoxin is stocked in PCCs/hospitals/pharmacies and in 6 countries in strategic stockpiles. Conclusion: PCC experience on botulism varied greatly: some services manage all cases occurring in the country as reference centers, while others refer to Infectious Diseases Services. During the study period (3 years), all forms of botulism have been observed by PCCs (including rare forms such as wound and intestinal botulism). PCR diagnosing testing is not routinely available, and in vivo tests remain the gold standard, even if, accordingly, TAT is too long to be useful in the first phase of clinical management. Trivalent Equine Antitoxin is available, and administration is safe. On the contrary, the recommended dose varies significantly among countries. Antidote storage in strategic stockpiles may be useful to manage public health emergencies or unconventional events. A harmonization of management of botulism between PCCs would seem appropriate for the future.

\section{Fatal colchicine poisoning in babies due to sound-alike drugs: a call for a common European poisoning database}

\author{
Mojca Dobaja Borak ${ }^{\mathrm{a}}$, Damjan Grenc ${ }^{\mathrm{a}}$, Davide Lonati ${ }^{\mathrm{b}}$, \\ Carlo A Locatelli ${ }^{\mathrm{b}}$, Valeria M Petrolini ${ }^{\mathrm{b}}$ and \\ Miran Brvar ${ }^{\mathrm{a}}$
}

${ }^{a}$ Centre for Clinical Toxicology and Pharmacology, University Medical Centre Ljubljana, Ljubljana, Slovenia; ${ }^{\text {b Pavia Poison }}$ Control Centre - National Toxicology Information Centre - Clinical and Experimental Lab, Toxicology Unit, Maugeri Clinical and Scientific Institutes IRCCS and University of Pavia, Pavia, Italy

Objective: Sound-alike drugs can lead to serious dispensing medication errors that in some cases can even lead to death. We present two cases of poisonings that happened as a result of dispensing colchicine prescription drug instead of a homeopathic medicine containing colocynthis recommended for treating abdominal cramps.

Case reports: Case 1: In 2000, a 1-month-old Italian newborn female was given 3 tablets of colchicine $(0.85 \mathrm{mg} / \mathrm{kg})$ due to a mistake by a pharmacist, since colchicine was sold in place of a homeopathic remedy containing colocynthis. She was admitted at the emergency department (ED) due to vomiting, diarrhoea, hypotension and cardiac failure. Echocardiography demonstrated diffuse ventricular hypokinesia and laboratory results revealed leucocytosis that was followed by leukopenia and thrombocytopenia. Computerised tomography (CT) scans showed hypodense brain lesions. She was discharged with severe cerebral sequelae. Subsequent toxicological analysis confirmed colchicine in urine samples collected on ED admission. Case 2: In 2017, 3-week-old Slovenian newborn female was given 4 tablets of colchicine (1 $\mathrm{mg} / \mathrm{kg}$ ) dissolved in water on early Sunday afternoon. Soon after ingestion, she started to vomit and developed diarrhoea. Her parents called a family health nurse, who had advised them earlier to buy colocynthis to treat baby's abdominal cramps (3-5 granules of colocynthis/dose). At this point the dispensing error was not realised, but the nurse advised them to take baby to a doctor as soon as possible, since newborns can dehydrate very easily. In the evening, the baby calmed down so the parents decided to wait until the following working day but she subsequently developed marmorated skin and they took her to the ED at 5 am on Monday. On arrival she was unconscious with mydriasis, unmeasurable blood oxygen saturation, respiratory rate 12 bpm and pulse $80 \mathrm{bpm}$. Apnoea and asystole appeared after an episode of vomiting. Laboratory results revealed lactic acidosis $(\mathrm{pH} 6.5$, lactate $16 \mathrm{mmol} / \mathrm{L})$, hyperkalaemia $(9.0 \mathrm{mmol} / \mathrm{L})$ and anaemia (haemoglobin $58 \mathrm{~g} / \mathrm{L}$ ). Resuscitation was unsuccessful and she died 19 hours post-ingestion. Liquid chromatographytandem mass spectrometry (LC-MS/MS) confirmed colchicine in blood samples.

Conclusion: Dispensing medication errors caused unimaginable damage and could have been prevented by harmonisation of European poisoning data and development of a surveillance system with early alerts on such events. Use of very similar names of a homeopathic medicine and a drug with a narrow therapeutic index should be banned. For the future, harmonisation of European poisoning databases is advisable to prevent unnecessary deaths and the EAPCCT may play a key role in this mission.

\section{Ranolazine-related toxicity after intentional overdose as reported to the UK National Poisons Information Service}

\author{
Dacia Jones ${ }^{\mathrm{a}}$, Sally M Bradberry ${ }^{\mathrm{b}}$, Euan A Sandilands ${ }^{c}$ \\ John P Thompson ${ }^{d}$ and Simon HL Thomas ${ }^{a}$ \\ ${ }^{a}$ National Poisons Information Service (Newcastle Unit), Newcastle \\ Hospitals NHS Foundation Trust, Newcastle, United Kingdom; \\ ${ }^{\mathrm{b}}$ National Poisons Information Service (Birmingham Unit), City \\ Hospital, Birmingham, United Kingdom; ${ }^{C}$ National Poisons \\ Information Service (Edinburgh Unit), Royal Infirmary, Edinburgh,
}


United Kingdom; ${ }^{d}$ National Poisons Information Service (Cardiff Unit), University Hospital Llandough, Cardiff, United Kingdom

Objective: Ranolazine is a piperazine derivative that acts as an inhibitor of the late sodium channel current and is used in the management of stable angina pectoris. Data regarding toxicity after overdose are limited, although there is a single case report of seizures in a 15-year-old male following ingestion of an unknown amount of ranolazine [1]. This analysis was performed to describe the clinical features of hospital inpatients with intentional ranolazine overdose as discussed during enquiries to the UK National Poisons Information Service (NPIS).

Methods: Telephone enquiry records involving ranolazine were reviewed for the period 1 January 2010 to 1 July 2018. Patient demographic data and details of severity of poisoning and outcome were extracted.

Results: Over the study period there were 47 telephone enquiries relating to 46 individual presentations, most concerning unintentional or therapeutic excess. Of 11 cases of intentional overdose, 9 were managed in hospital (age range 23-65 years, 6 male, 3 female). Median reported dose was $7 \mathrm{~g}$ (range 1.5-22.5 g), other co-ingestants were reported in 8 cases as follows: nicorandil $(n=3)$, diltiazem $(n=3)$, propranolol $(n=1)$ and ivabradine $(n=1)$. Clinical features reported included cardiac arrhythmias ( $n=6$; bradycardia 2, "tachycardia" 1, ventricular tachycardia 1, cardiac arrest 2$)$, hypotension $(n=3)$, seizures $(n=3)$, QT interval prolongation $(n=2)$ and metabolic acidosis $(n=2)$. In patients with a poisoning severity score (PSS) of 2 or higher, the onset of toxicity from time of ingestion ranged from 6 to 26 hours. One patient is known to have died 12 hours after a reported ingestion of $22.5 \mathrm{~g}$ ranolazine. As yet, however, follow-up information is incomplete for 7 of the 9 patients.

Conclusion: Intentional ranolazine overdose is uncommon. Cases often involve co-ingestion of other cardiotoxic medicines. Severe toxicity occurs in a high proportion of cases overall and may be delayed. Therefore prolonged in-patient observation is appropriate.

\section{Reference}

[1] Akil N, Bottei E, Kamath S. Ranolazine overdose-induced seizures. Am J Emerg Med. 2015;33:e5-6.

\section{Usability of the EAN-Code on product labels for product identification in poisons centres with a view to the upcoming Unique Formula Identifier (UFI)}

\author{
Andreas Stürer ${ }^{\mathrm{a}}$, Verena Kauth ${ }^{\mathrm{b}}$, Oliver Sauer ${ }^{\mathrm{b}}$, \\ Daniela Acquarone ${ }^{c}$, Andreas Schaper $^{\mathrm{d}}$, Carola Seidel ${ }^{\mathrm{e}}$, \\ Angelika Holzer ${ }^{f}$, Simone Just ${ }^{g}$, Uwe Stedtler ${ }^{h}$, \\ Colette Degrandi', Erol Tutdibi and Florian Eyer ${ }^{k}$ \\ ${ }^{a}$ Poisons Information Centre, University Medical Centre, Mainz, \\ Germany \& Society for Clinical Toxicology (GfKT), Germany, \\ Austria, Switzerland; ${ }^{b}$ Poisons Information Centre, University

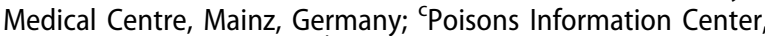 \\ Charité, Berlin, Germany; ${ }^{\mathrm{d}} \mathrm{GIZ}$-Nord Poisons Centre, University \\ Medical Centre Göttingen, Göttingen, Germany; ${ }^{\text {ePoisons }}$ \\ Information Centre, University Hospital, Bonn, Germany; ${ }^{\text {fPoisons }}$ \\ Information Centre, Gesundheit Österreich GmbH, Wien, Austria; \\ 9Poisons Information Centre, HELIOS Klinikum, Erfurt, Germany; \\ ${ }^{\mathrm{h}}$ Poisons Information Center, Center for Pediatrics, Medical Center \\ - University of Freiburg, Freiburg, Germany; 'National Poisons
}

Information Centre, Tox Info Suisse, Associated Institute of the University of Zurich, Zurich, Switzerland; 'Poisons Information Centre, University Hospital, Homburg, Germany; ${ }^{\text {KPoisons }}$ Information Centre, Klinikum rechts der Isar, Technical University of Munich, Munich, Germany

Objective: Product identification (PI) in poisons centres (PC) after exposure to a chemical agent is the first important step in providing poisons information. Since PI by trade name is often inconclusive, the European Commission (EC) included the UFI in CLP Regulation (Classification Labelling Packaging EC-No 1272/ 2008) [1]. The German Society for Clinical Toxicology (GfKT) together with all 10 German, Austrian und Swiss PCs currently investigates $\mathrm{PI}$ in a multinational study monitoring exposures to liquid laundry detergent packets (LDPs) with weekly reports of quality-controlled cases to the manufacturer from August 2017 until July 2019 (MAPOG-study). One of the aims is to analyse the usability of the European Article Number (EAN-Code) for PI in emergency settings.

Methods: Prospective data compilation of all exposures to LDPs from one manufacturer from 1 August 2017 to 31 January 2018 in the web-based GfKT database by 10 PCs. The caller was asked for the EAN-code, both at incoming calls, and during a structured follow-up interview 1-3 days later. For exact allocation a list of trade names with corresponding EAN-codes provided by the manufacturer was embedded in the PC/GfKT databases.

Results: Overall 395 calls were received during the first 6 months; 282 cases $(71 \%)$ had successful follow-up. Callers represented: $51 \%$ general public, $41 \%$ clinicians, 3\% general practitioners, $3 \%$ emergency physicians, $2 \%$ others. PI during the first call to PC $(n=395)$ succeeded in $65 \%$ with EAN-code (high level of $\mathrm{Pl}$ ), $14 \%$ with product type (e.g. "all-purpose ..."; medium level of $\mathrm{PI}$ ), and in $21 \%$ with brand name only (low level of $\mathrm{PI}$ ). In the subgroup with follow-up $(n=282)$ a total of $54 \%(n=152)$ were identified with EAN-code initially. In 130 cases with follow-up where an EAN-code was not available during the first contact, $23 \%(n=64)$ were identified with EAN-code, 19\% $(n=55)$ with product type, and $4 \%(n=11)$ with brand name during follow up. The EAN-codes were not available due to unavailable packaging, or overload of calls in PCs.

Conclusion: PI using EAN-code was feasible in $65 \%$ of LDP exposures during emergency calls to PCs and could be increased to $77 \%$ with a subsequent follow-up interview. PI takes time but is essential for correct advice and of additional scientific value for case recording by PCs. With this experience we expect that the UFI will improve PI in PCs.

\section{Reference}

[1] Desel $H$. The CLP Unique Formula Identifier (UFI) for hazardous mixtures will help poisons centres (PC) give the best advice and will increase the value of $P C$ case records for regulatory risk assessment under REACH. Clin Toxicol (Phila). 2017;55:408.

\section{Estonian Poisons Information Centre: slowly but surely - experience of the first 10 years}

\author{
Mare Oder and Ruth Kastanje \\ Poison Information Centre, Estonian Health Board, Tallinn, Estonia
}

Objective: The Estonian Poisons Information Centre (EPIC) and its call centre have been working since 6 October 2008 when our emergency medicine nurses with toxicology training first started handling enquiries from colleagues and the general population. 
Besides consulting on poisoning calls, we are actively teaching and training hospital staff, ambulance teams, rescue departments and emergency call centre staff as well as the general population. A large part of the EPICs activities has been media work in different channels. We give an overview of the impact of the first 10 years of EPIC on the Estonian healthcare system and general population awareness.

Methods: All calls answered by the EPIC, published articles, lectures about poisoning and prevention were analysed via timeline from 1 October 2008 to 30 September 2018.

Results: The PIC hotline worked 24/7 from 2012-2016 and from May 2018. In 10 years 16,200 calls have been consulted, more than 200 courses and at least one media event a week were provided (2008-2015 and from 2018). The number of hospitalized patients with poisoning has decreased 8\% every year, e.g. 5064 in 2016 and 4656 in 2017. The number of ambulance visits due to poisoning has also decreased every year (from $1 \%$ of all calls to $0.6 \%$ ) and in $70 \%$ of all cases home observation was advised so reducing needless emergency room (ER) and ambulance visits. The number of calls to the PIC was correlated with provided lectures (number of calls decreased 2016-2017 10.6\% compared with 2015). The knowledge of the general population about the hotline (accessed by polls) has been rising 2008-2015 (20\% of all population) but needs further improvement.

Conclusion: In the first 10 years the EPIC has found its place in the Estonian healthcare system and proved itself a trustworthy partner to doctors, nurses and ambulance staff as well as the general population. The impact of poison information consultations has seen a decrease in ambulance calls and hospital visits due to poisoning. Systematic education increases awareness of the population [1] without an expensive media campaign, and has a positive impact on the volume of poisoning calls handled by the centre. Educational programmes have an immediate effect on call volume through population knowledge about the PIC's telephone number, but have not had an immediate effect on the number of patients hospitalized for poisoning.

\section{Reference}

[1] Põld K, Oder M. The effect of active poison information education on the call volume and structure. Clin Toxicol (Phila). 2011;49:240.

\section{Attempted suicides: the American experience}

\author{
Saumitra Rege ${ }^{\mathrm{a}}$, Duc Anh $\mathrm{Ngo}^{\mathrm{b}}$, Heather Borek ${ }^{\mathrm{a}}$ and \\ Christopher P Holstege ${ }^{a}$ \\ ${ }^{a}$ Division of Medical Toxicology, Department of Emergency \\ Medicine, University of Virginia, Charlottesville, United States; \\ ${ }^{\mathrm{b}}$ Division of Student Affairs, Department of Student Health, \\ University of Virginia, Charlottesville, United States
}

Objective: Suicide is a leading cause of death in the US, and has increased by $28 \%$ since 1999 [1]. In Europe, self-harm attempts in 2015 exceeded 50,000. This study aims to characterize the suspected suicide attempts (SS) that are reported to US Poison Centers (PCs).

Methods: We identified SS reported to PCs from 2011 to 2017. Key demographic and clinical aspects of SS were descriptively assessed. Calls from acute care hospitals and emergency departments $(\mathrm{ACH})$, and cases involving opioids were studied. Poisson regression models were used to evaluate the trends in the number and rates (per 100,000 human exposures) of SS. The percentage changes during the study period were reported.
Results: Overall, there were 1.7 million SS cases reported to US PCs during the study period. Among these, $77 \%$ were directly reported by $\mathrm{ACH}$ and $12.4 \%$ involved an opioid. Patients aged between 20 and 39 years (39.3\%) constituted the most common age group. The proportion of teenagers among SS cases increased during the study period $(21.2 \%$ to $30.3 \%)$. Females accounted for $65.4 \%$ cases. Most exposures occurred in a residence (94.6\%). More than one substance was reported for $60.4 \%$ cases. Major clinical effects were demonstrated in $5.2 \%$ of exposures and the case fatality rate was $0.4 \%$. Major effects were more common in cases where an opioid was reported (8.7\%). Among cases, $26.7 \%$ were admitted to a critical care unit (CCU). The proportion of cases from $\mathrm{ACH}$ increased during the study period (73\% versus $83 \%$ ). Benzodiazepines were the most common exposure substance reported for SS (19.5\%), while hydrocodone $(4.3 \%)$ was the most common opioid. The most frequent clinical effect demonstrated was drowsiness (17.2\%). Charcoal therapy was reported for $16 \%$ cases while naloxone was used in $4.9 \%$ cases. In approximately a quarter of the cases, these therapies were used after recommendations from a PC. SS increased by $21.9 \%$ (95\% Cl: $21.2 \%, 22.6 \%, \mathrm{p}<0.001)$ while the SS rate also increased by $34.7 \%$ ( $95 \% \mathrm{Cl}: 31.1 \%, 38.2 \%, \mathrm{p}<0.001)$.

Conclusion: The number of SS cases handled by the PCS increased significantly. However, there was a low fatality rate. PCs should play a significant role in the care of this patient population and become involved earlier in the case.

\section{Reference}

[1] Hedegaard H, Curtin SC, Warner M. Suicide rates in the United States continue to increase. NCHS Data Brief, no 309. Hyattsville, MD: National Center for Health Statistics. 2018 [cited 2018 Oct 18]. Available from: https://www.cdc.gov/nchs/data/databriefs/db309.pdf

\section{Evaluation of the quality of data submitted by industry to the Belgian Poison Centre}

\author{
Christina Tobback and Martine Mostin \\ Poison Centre, Brussels, Belgium
}

Objective: The Poison Centre $(\mathrm{PC})$ is the appointed body for Belgium and Luxemburg in accordance with Article 45(1) of Regulation (EC) No 1272/2008 to receive information on hazardous mixtures placed on the market. The current notification systems, data formats, etc. differ depending on the Member States. Therefore the European Commission added an Annex on harmonized information relating to emergency health response in Regulation EU 2017/542, which shall apply from 1 January 2020. In Belgium the importer or downstream user placing a hazardous mixture on the market needs to provide the following data to the Poison Centre: a safety data sheet (SDS), the mixture composition (C) and label (L). The goal of this study is to evaluate the baseline quality of the data as they are provided today by the importer or downstream user to the Belgian Poison Centre in order to identify potential pitfalls to be addressed by the guidance working group.

Methods: All importers and downstream users who placed hazardous mixtures, except for pesticides and biocides, on the Belgian market and who introduced their data to the Poison Centre in 2017 were selected. For each of them the first submitted mixture classified as hazardous on the basis of an health effect was then selected and the data were evaluated. 
Results: In 2017, 246 importers or downstream users notified hazardous mixtures; 15 were not selected since they notified exclusively hazardous mixtures without an health effect. Data of 231 mixtures were included. In total, 231 SDS, 193 C and $193 \mathrm{~L}$ were evaluated. Items such as product identification, ingredients and their percentage, classification and labelling were checked. Overall, 75/231 (32.5\%) SDS, 120/193 (62.2\%) C and 122/193 (63.2\%) L fully complied with the current legislation. A complete dataset (SDS, C and L) was provided for 172/231 (74.5\%) mixtures. There was some inconsistency between these different documents regarding product identification, ingredients and their percentage, classification and labelling in half of the cases. Most of the information was sufficient to deal with an emergency, however, because of data inconsistency time could be lost during risk assessment.

Conclusion: In the guidance working group attention should be paid both to data completeness and consistency.

\section{Surveillance of hydrocodone overdoses using a National Real-time Data System}

\author{
Saumitra Rege ${ }^{\mathrm{a}}$, Duc Anh $\mathrm{Ngo}^{\mathrm{b}}$ and \\ Christopher $\mathrm{P}$ Holstege ${ }^{\mathrm{a}}$ \\ ${ }^{a}$ Division of Medical Toxicology, Department of Emergency \\ Medicine, University of Virginia, Charlottesville, United States; \\ bivision of Student Affairs, Department of Student Health, \\ University of Virginia, Charlottesville, United States
}

Objective: According to the US Drug Enforcement Administration, over 136 million hydrocodone prescriptions were dispensed in 2013, with approximately 24.4 million people over the age of 12 years using it for non-medical purposes [1]. This study aims to examine the national trends in hydrocodone exposures reported to US poison centers (PCS).

Methods: The National Poison Data System (NPDS) was queried for all closed, human exposures to hydrocodone from 2011 to 2017 using the American Association of Poison Control Center (AAPCC) generic code identifiers. Key demographic and clinical characteristics were descriptively assessed. Hydrocodone reports from acute care hospitals and emergency departments $(\mathrm{ACH})$ were analyzed. Trends in hydrocodone frequencies and rates (per 100,000 human exposures) were analyzed using Poisson regression. Percent changes during the study period were reported.

Results: There were 168,361 hydrocodone exposures reported to PCs, with calls declining $(30,615$ to 17,033$)$ during the study period. Among the overall hydrocodone calls, the proportion of calls from ACH increased from 50.2\% to 59.2\% from 2011 to 2017. Multiple substance exposures accounted for $55.8 \%$ of the overall hydrocodone calls and $71.1 \%$ of the calls from $\mathrm{ACH}$. Residence was the most common site of exposure (95.5\%). Demographically, $54.8 \%$ cases were females and the most frequent age group was 20-39 years (33.0\%). Suspected suicides (43.3\%) and therapeutic errors (17.6\%) were commonly observed reasons for exposure. The proportion of suspected suicides $(43.3 \%$ versus $67.6 \%)$ was higher in cases reported by $\mathrm{ACH}$. The most frequent co-occurring substances reported were benzodiazepines (19.8\%) and alcohol (9.8\%). Approximately 19\% of the patients reporting hydrocodone exposures were admitted to the critical care unit (CCU). Tachycardia and drowsiness were the most frequently demonstrated clinical effects. Naloxone was a reported therapy for $13.4 \%$ cases and administered prior to PC contact in most cases. Major effects were seen in $4.7 \%$ cases and the case fatality rate for hydrocodone was $0.8 \%$. There were 709 deaths reported within $\mathrm{ACH}$ during the study period. The frequency of hydrocodone exposures decreased by $44.3 \%(95 \% \mathrm{Cl}$ :
$-45.4 \%,-43.4 \% ; \mathrm{p}<0.001)$ and the rate decreased by $38.6 \%(95 \%$ $\mathrm{Cl}:-43.8 \%,-33.1 \% ; \mathrm{p}=0.002)$.

Conclusion: PC data demonstrated a decreasing trend of hydrocodone exposures, which may in part be attributed to the federal rescheduling of combination hydrocodone products in 2014. However, the increase in the proportion of calls from the $\mathrm{ACH}$ indicates higher severity of such exposures.

\section{Reference}

[1] Drug Enforcement Administration, Office of Diversion Control Drug \& Chemical Evaluation Section. Hydrocodone; 2014 [cited 2018 Sep 19]. Available from: https://www.deadiversion.usdoj.gov/drug_chem_ info/hydrocodone.pdf.

\section{Retinoid use during pregnancy and lactation in Finland: analysis of Teratology Information Centre data}

\author{
Emma Sipari ${ }^{a}$, Heli Malm ${ }^{b}$, Michael Stigson ${ }^{c}$ and \\ Justus Vasama ${ }^{a}$ \\ ${ }^{a}$ University of Helsinki and Department of Emergency Medicine \\ and Services, Helsinki University Hospital, Poison Information \\ Centre, Helsinki, Finland; ${ }^{b}$ University of Helsinki and Helsinki \\ University Hospital, Department of Emergency Medicine and \\ Services, Teratology Information Service, Helsinki, Finland; \\ 'Department of Toxicology, Faculty of Pharmacy, Uppsala \\ University, Uppsala, Sweden
}

Objective: Retinoids are a group of substances containing vita$\min A$ and its natural and synthetic derivatives. Retinoids are important during embryonic development and they regulate many physiological processes. Embryonal exposure to retinoid medications can cause a $25 \%$ risk for major malformations with a characteristic pattern of malformations. Retinoids are frequently used medications in Finland, in 2015, almost 16,000 patients received systemic retinoids for acne. Pregnancy prevention programs have been established globally to prevent retinoid exposure during pregnancy because of teratogenicity. In Finland, the prescribing restrictions were updated in 2005. The purpose of this study was to assess the frequency of retinoid exposure during pregnancy and lactation in Finland, and to study the number of retinoid exposures during pregnancy.

Methods: All calls recorded in the Helsinki Teratology Information Service database from 1 June 2006 to 31 December 2016 concerning retinoids were analyzed individually to confirm the exposure and relevance. Data were processed in Microsoft Excel.

Results: During the study period the total number of calls to the Finnish Teratology Information Service was 60,121. In total, 368 enquiries concerned synthetic retinoids, and 33 of these concerned retinoid exposures during the first trimester of pregnancy or the wash-out period (recommended time interval between cessation of retinoid use and conception). Exposures during the first trimester or the wash-out period occurred each year during the study period. The most commonly used retinoid during the study period was isotretinoin $(n=151)$

Conclusion: Despite an established pregnancy prevention program, exposures to synthetic retinoids during pregnancy still occur; the number of annual exposures has remained stable and not decreased. These exposures are potentially avoidable, and more strict measures to prevent inadvertent exposure are needed. These should include more strict policies in prescribing, and increasing awareness in healthcare professionals as well as in patients. 


\section{Exposures to stomatological preparations, dental products, and exposures in the context of toothache reported to the Poisons Information Centre Erfurt, 1997-2017}

\author{
Beate Budenz, Michael Deters, Dagmar Prasa and \\ Helmut Hentschel \\ Poisons Information Centre, c/o HELIOS Klinikum Erfurt GmbH, \\ Erfurt, Germany
}

Objective: We sought to examine cases of exposure to stomatological preparations (SP), dental products (DP), and all human exposures in the context of toothache (ECTA) as information on this subject is lacking.

Methods: Retrospective study of cases registered by the Poisons Information Centre (PIC) Erfurt from 1997 to 2017.

Results: In total 184 cases of exposures to DP (153 cases with different tooth filling materials), 1235 cases of exposures to SP (114 cases with fluoride containing products), and 1620 cases of ECTA were registered. While no clear tendency in the frequency of exposures to SP and DP could be observed, there was an almost tenfold increase of cases in the context of toothache from 16 in 1997 to 169 in 2017. Symptom severity in exposures to DP and SP were asymptomatic or mild. In ECTA, however, 51 cases with moderate $(5$ cases with prolonged paracetamol overdose) and at least 5 cases with severe symptoms ( 3 cases with prolonged paracetamol overdose) were detected. The two other cases with severe symptoms are described. Case 1. A 41 year-old man under phenprocoumon therapy for chronic ischemic heart disease treated himself with unknown amounts of acetylsalicylic acid and metamizole over two days because of toothache. Severe bleeding occurred following extraction of two teeth by a dentist. The International Normalized Ratio (INR) could not be measured. Massive haematoma of the tongue, pharynx and hypopharynx required intubation and a tracheostomy. Coagulopathy was treated by administration of fresh frozen plasma and oral vitamin $\mathrm{K}$. The serum metamizole concentration was approximately ten-fold the therapeutic range. After treatment for 13 days in an intensive care unit the patient was discharged to a peripheral unit. Case 2. Gingival injection of lidocaine plus adrenaline in a 37-year old healthy woman by a dentist resulted in severe bradycardia and cardiac arrest. Intravenous administration of Akrinor (cafedrine/theodrenaline) restored cardiac function.

Conclusion: Acute toxicity of stomatological preparations and dental products appears to be low. In ECTA, however, prolonged paracetamol overdose by the patient [1], and lack of awareness of an increased bleeding risk with concomitant intake of vitamin $\mathrm{K}$ antagonists and non-steroidal anti-inflammatory drugs [2] can result in severe symptoms.

\section{Reference}

[1] Vogel J, Heard KJ, Carlson C, et al. Dental pain as a risk factor for accidental acetaminophen overdose: a case-control study. Am J Emerg Med. 2011;29:1125-1129.

[2] Cascorbi I. Drug interactions - principles, examples and clinical consequences. Dtsch Arztebl Int. 2012;109:546-555.

\section{The Icelandic Poisons Information Centre: evaluation of the range and nature of inquiries}

\author{
G Svanhvít Michelsen ${ }^{\mathrm{a}}$, Helena Lindal Baldvinsdottir ${ }^{\mathrm{b}}$, \\ Curtis P Snook ${ }^{c}$, Gudborg A Gudjonsdottir ${ }^{\mathrm{a}}$ and \\ Freyja Jonsdottir ${ }^{d}$ \\ ${ }^{a}$ Faculty of Pharmaceutical Sciences, University of Iceland, \\ Reykjavik, Iceland; ${ }^{b}$ Poison Information Centre, Icelandic National \\ Hospital, Reykjavik, Iceland; 'Emergency Department, National \\ University Hospital, Reykjavik, Iceland; 'University Hospital of \\ Iceland, Faculty of Pharmaceutical Sciences, University of Iceland, \\ Pharmacy of the National University Hospital of Iceland, Reykjavik, \\ Iceland
}

Objective: To analyse telephone inquiries made to the Icelandic Poisons Information Centre (PIC). To evaluate range and nature of inquiries and assess opportunities to enhance poison prevention in Iceland.

Methods: Registrations from January 2010-December 2017 [1] were retrieved from the PIC-database and analysed with regards to variables such as age, gender, time of day, reason and route of exposure. Data processing and analysis were performed in Microsoft Excel and R Studio.

Results: The average annual rate of recorded inquiries to the PIC was $2.57 / 1000$ of the population in 2010-2017. Overall 6747 inquiries were recorded; $30.8 \%$ involved children aged 1-3 years. The adult age group involved in most inquiries were aged 20-29 years. No increase in pharmaceutical inquiries was observed in the older age groups despite the increased use of pharmaceutical products and decrease in cognitive skills in the elderly. For inquiries involving children $\leq 12$ years a male predominance was seen, but for those involving teenagers and adults, a female predominance was observed. Inquiries involving chemicals were more frequent in children $\leq 6$ years, but pharmaceutical inquiries were predominant in all older age groups. The highest number of inquiries occurred between 16:00 - 20:00. This resembles data from the annual report of the Swedish Poisons Information Centre in 2016 [2]. Overall $60.9 \%$ of inquiries occurred following unintentional exposure and $86.4 \%$ of events happened at home. Unintentional exposure, abuse and accidents in the workplace were more common in males, but self-harm was more common in females. The most common cause of chemical inquiries were household cleaning products $(33.5 \%)$, but the most common pharmaceuticals were psycholeptics and anticonvulsants (13.9\%).

Conclusion: The results are descriptive for the status of registration over the past 8 years. However, it is known that registration was incomplete. The results indicate that preventative measures for child poisoning need to be directed at the home environment, with emphasis on storage and handling of chemicals that can be harmful to children. Data analysis suggests that the range and nature of exposures and poisonings in Iceland is similar to other western countries [3].

\section{References}

[1] Annual Reports of the Poison Information Centre 2010-2017 [cited 2018 Oct 18]. Available from: https://www.landspitali.is/sjuklingaradstandendur/deildir-og-thjonusta/eitrunarmidstod/

[2] Giftinformationscentralen. (2016). About the Swedish Poisons Information Centre [cited 2018 Oct 1]. Available from: https://giftinformation.se/servicemeny/in-english/about-the-swedish-poisonsinformation-centre/

[3] Bogevig S, Hogberg LC, Dalhoff KP, et al. Status and trends in poisonings in Denmark 2007-2009. Dan Med Bull. 2011;58:A4268. 


\section{Antidote logistics in Slovakia}

\section{Silvia Plačkováa , Blažena Cagáňováa , Olga Otrubova ${ }^{a}$, Jaroslav Kresanek ${ }^{b}$ and Igor Batora ${ }^{a}$ \\ ${ }^{a}$ National Toxicological Information Centre, Department of Occupational Medicine and Toxicology, University Hospital Bratislava, Faculty of Medicine Comenius University, Bratislava, Slovakia; 'bSlovak Medical University, Bratislava, Slovakia}

Objective: Fast and cost-effective antidote distribution is important to guarantee efficacy in the treatment of some acute poisonings. The National Toxicological Information Centre (NTIC) elaborated the actual "List of antidotes" according to the World Health Organization (WHO), which is used in our country. All antidotes must be imported and many are not registered. The aim of the present study was to investigate antidote availability in Slovak hospitals.

Methods: In January 2018 NTIC established a national online database providing current information about types and quantities of antidotes, expiration data, locations of storage in hospitals, e.g. emergency departments (ED) or intensive care units (ICU) and hospital pharmacies. This information is updated continuously by NTIC in close cooperation with the hospital pharmacies. Results: The NTIC regularly elaborates and publishes national antidote recommendations, which include type of antidote and stock quantities. In our country, there are three different levels of antidote storage: 1. Local, in all hospitals - antidotes intended for immediate access and commonly used. 2. Regional, in larger hospitals - often expensive antidotes which can preferably be co-ordinated within the region. 3. National, with a 24-hour service - very expensive and rarely used antidotes. Group 1 and 2 antidotes are kept mostly in hospital pharmacies in Slovakia and in ED or ICU. The Slovak national storage is handled by the NTIC and some regional hospitals. Emergency transport is operated by ambulances or delivery firms. The costs of antidote use and distribution in Slovakia are divided between the Ministry of Health and the hospitals. Expensive or rarely used antidotes (botulinum antitoxin, digoxin antibodies, succimer [2,3-dimercaptosuccinic acid], fomepizole, potassium hexacyanoferrate, unithiol [sodium 2,3-dimercaptopropane sulphonate]) are funded by the Ministry of Health.

Conclusion: The development of the national coordinated system and the establishment of the online database improved the access to information about antidote stock quantities in Slovakia and resulted in $60 \%$ increase in effective cooperation among hospital pharmacies in Slovakia. In case of a massive intoxication, the NTIC can coordinate and provide information about the availability and stock quantities of the required antidotes.

\section{Towards optimization of antidote availability in Belgian hospitals}

\author{
Jonas Moens ${ }^{\mathrm{a}}$, Jonas Van Baelen ${ }^{\mathrm{a}}$, Martine Mostin ${ }^{\mathrm{a}}$, \\ Dominique Vandijck ${ }^{b}$ and Anne-Marie K Descamps ${ }^{a}$ \\ ${ }^{a}$ Belgian Poison Centre, Brussels, Belgium; ${ }^{\mathrm{b}}$ Faculty of Medicine \\ and Health Sciences, Ghent University, Ghent, Belgium
}

Objective: The aim of this study was to investigate the availability of 22 antidotes in all Belgian hospitals with an emergency service and to optimize the stockpile of antidotes of the Belgian Poison Centre (BPC). A third objective was to investigate at hospital level the maintenance cost to hold a selection of antidotes. Furthermore, to explore the added value of an online platform developed by the BPC with shared information on the real-time availability of antidotes in Belgian hospitals. In the context of preparedness for chemical incidents, a last objective was to verify if hospitals met the government's requirement to maintain a stock of atropine equal to twice their annual consumption.

Methods: An online questionnaire was sent between February and April 2018 to all 126 hospitals with an emergency service. The selection of 22 antidotes, of which 12 were marked on account of evidence-based proof of efficacy, was based on the most frequent intoxications in Belgium. For each hospital the list of available antidotes was compared with the 12 marked antidotes in order to calculate the expense of an optimal availability to treat a $75 \mathrm{~kg}$ patient for the first 24 hours.

Results: Response rate was $29 \%$, of which 4 were university hospitals and 33 were non-university hospitals. Methylene blue, deferoxamine, hydroxocobalamin, calcium gluconate and intravenous lipid emulsion were available in more than $60 \%$ of the hospitals and $42 \%$ of hospitals held an atropine stock that met the imposed quantity. Digoxin immune Fab fragments (DIF) was ordered most frequently in urgent cases and was considered to be the most important antidote to be available via the depot of the BPC. The availability of the 12 marked antidotes in the hospitals could be realized with an average extra budget of less than $€ 1,000 /$ year without DIF or $€ 3,500 /$ year with DIF (based on the cost of six vials of Digifab ${ }^{\circledR}$ ). Most respondents were interested in an online platform sharing the stock of other hospitals in real-time, developed and controlled by the BPC.

Conclusion: Not all antidotes commonly used in toxicology are available in the Belgian hospitals. Furthermore, the storage of atropine in most hospital pharmacies does not comply with the government's requirement. A minor investment will improve availability of the selected antidotes. Mutual cooperation between hospitals and the BPC, together with the development of a real-time online platform by the BPC, could boost the availability of antidotes.

\section{Availability of antidotes in the hospitals of the National Health Care System of Greece}

\author{
Nikoleta Oikonomou, Myrto Bonataki, \\ Vassiliki Papathanassiou, Dimitrios Demenagas, \\ Angeliki Kalostou, Konstantinos Fountas, \\ Spyridoula Zoniou and Polyxeni Neou \\ Greek Poison Information Center, Children's Hospital “P \& A \\ Kyriakou", Athens, Greece
}

Objective: The Greek Poison Information Center is unique in our country. In cooperation with the Greek Ministry of Health it determines and ensures the availability of antidotes by upgrading and redefining their stock in all hospitals every two years. The objective of this study is to define the availability of antidotes to fulfil local necessities in a country with great geographical heterogeneity to save resources.

Methods: We categorized the antidotes in groups according to the time of onset of the desirable therapy because of the variability in the urgency of administration of each antidote. There are a limited number of medicines used in Primary Health Care Units for immediate administration. These Units are not included in our study. The first group of antidotes $(n=14)$ includes those which should be available in all hospitals. The second group $(n=10)$ those which are administered less frequently and there is usually time for them to be transferred from selected hospitals. The third includes those, which are rarely administered, very expensive and the need of their administration is not so urgent. We considered adequate availability for each antidote to be the amount required for the treatment of at least one patient for 48 hours and as partial adequacy the existence of the half of this amount. 
Results: According to the defined categories, we received reports from 82 out of 101 hospitals on the availability of the three groups of antidotes. For the group of 14 antidotes that should be available in all hospitals $(\mathrm{n}=82)$, there was adequate availability for $70.4 \%$ (range $50.4-80.5 \%$ ), partial adequacy for $7.7 \%$ (range $2.4-22 \%$ ) and inadequacy for $21.9 \%$ (range 14.6-34.1\%). Concerning the ten antidotes that are held in selected hospitals $(n=25)$ we found adequate availability for $42 \%$ (range $16-68 \%$ ), partial adequacy for $7.6 \%$ (range 4-32\%) and inadequacy for $49.6 \%$ (range: $32-68 \%$ ). For the last four antidotes that are required rarely, which are held in one hospital in each region of the country $(n=11)$, we found adequate availability for $27.3 \%$ (range: 9.1-63.6\%), partial adequacy for 2.3\% (range: 1.1-9.1\%) and inadequacy for $70.5 \%$ (range: $36.4-90.9 \%$ ).

Conclusion: Despite our systematic vigilance, there are hospitals that do not have sufficient antidote stocks. This is either due to economic reasons or negligence. For these reasons, we need to continue and strengthen our efforts for more regular communication with all health units and those responsible for solving the problem.

\section{Prehospital antidote administration in patients with acute poisoning in Baku, Azerbaijan}

\author{
Ismayil Afandiyev ${ }^{\mathrm{a}}$ and Murad Mirzazade ${ }^{\mathrm{b}}$ \\ ${ }^{a}$ Toxicology, Clinical Medical Center, Baku, Azerbaijan; ${ }^{\text {bC Central }}$ \\ Ambulance Station, Baku, Azerbaijan
}

Objective: Depending on the substance involved, the course and prognosis of acute intoxication may depend on timely and appropriate antidote therapy. The aim of this study was to analyze antidote therapy in the prehospital setting in acutely poisoned patients in Baku, Azerbaijan and to determine methods of optimization.

Methods: We analyzed the prehospital medical care of 10,964 patients with acute poisoning brought by ambulance service and admitted to the Poison Center in Baku in 2008-2016.

Results: The most common agents were pharmaceuticals $(\mathrm{n}=$ $4725,43.1 \%)$, carbon monoxide $(n=2675,24.4 \%)$, corrosives $(n=1184,10.8 \%$, particularly acetic acid $[n=809,68.3 \%$ of this group]), alcohol $(n=625,5.7 \%)$, envenomation $(n=417,3.8 \%)$, pesticides $(n=384,3.5 \%)$ and narcotics $(n=351,3.2 \%)$. The use of available antidotes in the prehospital setting for a number of toxicology nosologies was insufficient (Table 1). Atropine was used in only $32.7 \%$ of cases $(n=168)$ of organophosphorus insecticide poisoning, and pyridoxine in $14.5 \%$ of cases $(n=76)$ of intoxication with antituberculous agents. In the prehospital setting specific antivenom was administered in $34.6 \%$ cases of snakebite $(n=231)$. Antidotes were not used by ambulance teams because of insufficient drug supply, lack of clinical protocols for the treatment of acute poisoning and lack of training. The administration of a significant number of important antidote drugs (e.g. glucagon, deferoxamine, oximes, succimer, dicobalt EDTA, potassium hexacyanoferrate, penicillamine, sodium calcium edetate, methylene blue, fomepizole and others) was impossible because they are not available due to the lack of production in Azerbaijan and no exports from abroad.

Conclusion: The current toxic-epidemiological situation in Azerbaijan calls for improvements in the supply of antidotes as outlined in the World Health Organization (WHO) Essential Medicines List in all public health facilities. Also, given the possibility of mass poisonings and chemical incidents it is necessary to create a permanent and sufficient stock of antidote agents in Azerbaijan.
Table 1. Prehospital antidote administration in patients with acute poisoning in Baku, Azerbaijan, 2008-2016.

\begin{tabular}{lcc}
\hline Antidote/drug & $\begin{array}{c}\text { Number of } \\
\text { patients where } \\
\text { therapy was } \\
\text { appropriate }(\mathrm{n})\end{array}$ & $\begin{array}{c}\text { Frequency } \\
\text { of use } \\
\text { (n; percentage) }\end{array}$ \\
\hline Activated charcoal & 4996 & $221 ; 4.4 \%$ \\
Acetylcysteine & 170 & 0 \\
Atropine & 168 & $55 ; 32.7 \%$ \\
Pyridoxine & 76 & $11 ; 14.5 \%$ \\
Phytonadione (Vitamin K1) & 136 & $4 ; 2.9 \%$ \\
Glucagon & 98 & 0 \\
Deferoxamine & 23 & 0 \\
Digoxin-specific antibody (Fab) & 10 & 0 \\
Succimer & 22 & 0 \\
Dimercaprol & 22 & 0 \\
Calcium gluconate & 338 & $16 ; 4.7 \%$ \\
Methylene blue & 11 & 0 \\
Sodium thiosulfate & 37 & $1 ; 2.7 \%$ \\
Naloxone & 391 & $0 ; 0.0 \%$ \\
Pralidoxime (oximes) & 168 & $0 ; 0.0 \%$ \\
Snake antivenom & 231 & $80 ; 34.6 \%$ \\
Scorpion antivenom & 12 & 0 \\
Black widow spider antivenom & 30 & 0 \\
Flumazenil & 489 & 0 \\
Fomepizole & 6 & 0 \\
\hline & &
\end{tabular}

\section{Antidote use in Hungary: use of recommended antidotes}

\section{Csaba Pap}

Department of Emergency Medicine and Clinical Toxicology, Péterfy Hospital, Budapest, Hungary

Objective: According to an expert consensus guideline (ECoG) based on a comprehensive summary of medical literature [1] 45 antidotes are recommended for stocking, and one $100 \mathrm{~kg}$ patient is considered as the basis for calculating the amount of antidote to stock. We determined the availability and the need of antidotes in our department in light of this ECoG to optimize the cost-effectiveness and efficiency of stocking and administering antidotes.

Methods: A retrospective record of the weekly orders of medicaments and a retrospective analysis of cases treated at our department involving the administration of antidotes recommended by the ECoG were performed over an almost 7 year period (January 2012-September 2018).

Results: During this period we used 18 of the 45 recommended antidotes (Table 1). Of the remaining 27 we have 5 (viper antivenin, lipid emulsion, protamine, 4-factor prothrombin complex concentrate [4-PCC], pyridoxine), which we did not use in the last 7 years and we do not stock 22 for various reasons. All the antidotes in stock are available immediately. As far as the stored amount of antidotes is concerned, currently we have amounts of atropine, calcium gluconate, cyproheptadine, dextrose, flumazenil, lipid emulsion, naloxone and sodium bicarbonate for more than 5 patients; acetylcysteine IV, ethanol IV, physostigmine, phytonadione, pyridoxine and thiamine for 2-5 patients; deferoxamine, hydroxocobalamin, leucovorin, methylene blue, protamine and PCC-4 for one patient. We have an insufficient amount of viper antivenin and DigiFab. There have been no deaths because of insufficient supply or delay.

Conclusion: Use of acetylcysteine, pralidoxime and atropine has dramatically decreased over the past few years. Regarding our 
Table 1. Antidotes recommended by Expert Consensus Guidelines and used at our department in Hungary, 2012-2018.

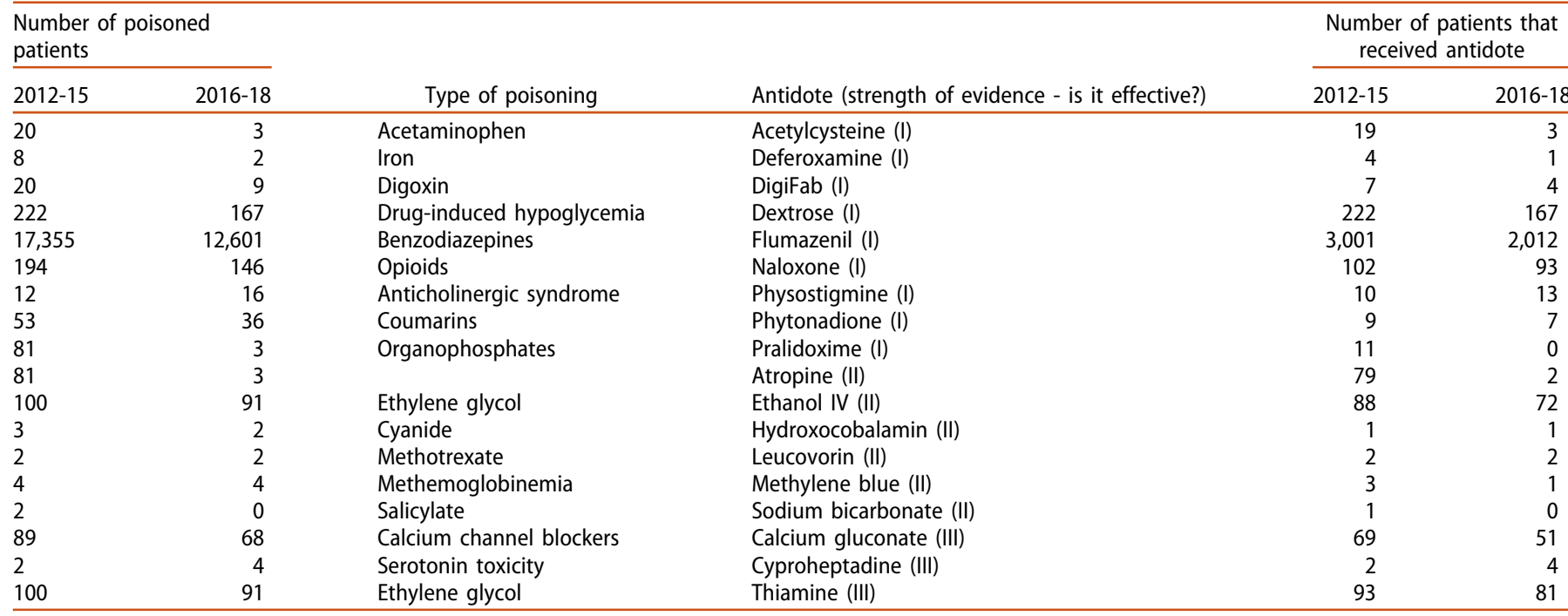

antidote policy, our treatment protocols have been partly consistent with the recommendations of the ECoG.

\section{Reference}

[1] Dart RC, Goldfrank LR, Erstad BL, et al. Expert Consensus Guidelines for stocking of antidotes in hospitals that provide emergency care. Ann Emerg Med. 2018;71:314-325.

\section{The impact of unavailability of three antidotes on patient management}

Giulia Scaravaggi, Giulia Lodrini, Valeria M Petrolini, Eleonora Buscaglia, Olha Maystrova, Carlo A Locatelli, Marta Crevani, Sarah Vecchio and Francesca Chiara

Pavia Poison Control Centre - National Toxicology Information Centre - Clinical and Experimental Lab, Toxicology Unit, Maugeri Clinical and Scientific Institutes IRCCS and University of Pavia, Pavia, Italy

Objective: Surveys conducted in different European [1,2] and non-European countries [3] show the lack of antidotes, even those considered to be of high priority use, is a widespread problem. The aim of this study was to evaluate the impact of unavailability of three antidotes on patient management.

Methods: A retrospective analysis of cases referred to our Poison Control Center in a four-year period (2014-2017). We considered all cases that, according to the toxicological evaluation, needed treatment with one of these three high-priority antidotes: digoxin Fab fragments, methylene blue and physostigmine. For each case, we evaluated clinical indication, availability of antidote onsite and, in case of deficiency, the solution adopted.

Results: We evaluated 278 cases from all over Italy (Table 1). Methylene blue was indicated for toxic methemoglobinemia (24/ 40), ifosfamide encephalopathy (5/40) and vasoplegic shock (11/ 40). Indications for digoxin Fab fragments were drug overdose (26/121), intoxication due to drug accumulation (72/121), and intentional oleander ingestion (23/121). Physostigmine was indicated for drug intoxication (17/117), abuse of anticholinergic plants (7/117) and ingestion of toxic plants confused with edible vegetables $(93 / 117)$.
Table 1. The impact of unavailability of three antidotes on patient management.

\begin{tabular}{lccc}
\hline & $\begin{array}{c}\text { Digoxin } \\
\text { Fab-fragments }\end{array}$ & $\begin{array}{c}\text { Methylene } \\
\text { blue }\end{array}$ & Physostigmine \\
\hline $\begin{array}{l}\text { Patients requiring } \\
\text { antidote treatment }\end{array}$ & 121 & 40 & 117 \\
$\begin{array}{l}\text { Median age (range) } \\
\text { M/F }\end{array}$ & $\begin{array}{c}(1-1 \pm 22.6 \\
(1-97)\end{array}$ & $\begin{array}{c}48.6 \pm 19.6 \\
(0-87)\end{array}$ & $\begin{array}{c}44.4 \pm 21.4 \\
(3-88)\end{array}$ \\
$\begin{array}{l}\text { Number of cases of } \\
\quad \text { lack of antidote }\end{array}$ & 0.53 & 1.5 & 0.98 \\
$\begin{array}{l}\text { Solution adopted } \\
\text { Antidote received } \\
\quad \text { from another hospital }\end{array}$ & $57 / 121$ & $5 / 40$ & $67 / 117$ \\
$\begin{array}{c}\text { Transfer of patient to } \\
\text { another hospital }\end{array}$ & $42 / 57$ & $3 / 5$ & \\
$\begin{array}{l}\text { Patients not treated } \\
\text { with antidote }\end{array}$ & $11 / 57$ & $2 / 5$ & $15 / 67$ \\
\hline
\end{tabular}

Conclusion: We recorded numerous cases where antidote was unavailable. The clinical consequences of this was unknown, but the availability of life-saving antidotes is essential for appropriate management of intoxicated patients. When antidotes are missing, the solutions involve a delay or an inadequacy in care and an increase in costs.

\section{References}

[1] Locatelli C, Petrolini V, Lonati $D$, et al. [Antidotes availability in Emergency Departments of the Italian National Health System and development of a national data-bank on antidotes]. Ann Ist Super Sanita. 2006:42:298-309. Italian.

[2] Thanacoody RH, Aldridge G, Laing W, et al. National audit of antidote stocking in acute hospitals in the UK. Emerg Med J. 2013;30:393-396.

[3] Juurlink DN, McGuigan MA, Paton TW, et al. Availability of antidotes at acute care hospitals in Ontario. CMAJ. 2001;165:27-30.

\section{A survey of the antidote stocking in Norway}

Yvonne Lao ${ }^{\mathrm{a}}$, Dag Jacobsen ${ }^{\mathrm{b}}$, Barbro J Spillum Espen R Nakstad and Knut Erik Hovda ${ }^{a}$ 


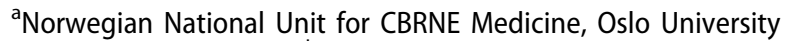
Hospital, Oslo, Norway; ${ }^{b}$ Department of Acute Medicine, Oslo University Hospital, Oslo, Norway; ${ }^{C}$ Norwegian Poisons Information Centre, National Institute of Public Health, Oslo, Norway

Objective: Inadequate stockpiling of antidotes is a well-known problem. International recommendations are typically based on the urgency of availability for each antidote [1-3]. Simplified, the World Health Organization (WHO), US and UK recommend antidotes to be immediately available, available within 1-2 hours or within 6 hours/supra-regional storage. The present Norwegian national recommendations differ from these, as hospitals are grouped in small, large and regionals units with different stockpiling recommendations. We conducted a survey to describe the current antidote preparedness in Norway, and evaluate whether hospitals follow stockpiling recommendations or not.

Methods: An electronic survey about antidote preparedness was sent to all hospitals in Norway $(n=50)$ with somatic emergency function in 2016. We defined "regional hospital" as the largest university hospital in each health region and a "large hospital" as the one with the largest number of people in each health trust. Thirty-one hospitals were categorized as small, 15 as large and 4 as regional hospitals. Each hospital was asked which antidotes were available from a total list of 35 .

Results: The response rate to the survey was $100 \%$. Only 11 of the 50 hospitals stockpiled all the antidotes recommended for their hospital size. Regional hospitals had the highest percentage (100\%) of availability and large hospitals the lowest (7\%). Among the 17 antidotes recommended to be stockpiled in all hospitals, only acetylcysteine, atropine, charcoal, flumazenil, phytomenadione, and naloxone were stockpiled at all hospitals. Sodium sulphate for barium poisoning was the antidote with lowest availability (36\%).

Conclusion: A large proportion of Norwegian hospitals do not follow national recommendations for antidote stockpiling. The results of our study indicate that the classification based on hospital size should be reconsidered as one measure to enhance current antidote preparedness.

\section{References}

[1] Pronczuk de Garbino J, Haines JA, et al. Evaluation of antidotes: activities of the International Programme on Chemical Safety. J Toxicol Clin Toxicol. 1997;35:333-343.

[2] Dart RC, Goldfrank LR, Erstad BL, et al. Expert Consensus Guidelines for stocking of antidotes in hospitals that provide emergency care. Ann Emerg Med. 2018;71:314-25.e1.

[3] Royal College of Emergency Medicine and National Poisons Information Service. Guideline on Antidote Availability for Emergency Departments (January 2017) [cited 2018 Oct 4]. Available from: www.rcem.ac.uk/docs/College\%20Guidelines/

\section{Efficacy of dimethyl trisulfide in cyanide-poisoned swine}

\author{
Ophir Lavon $^{\mathrm{a}}$ and Gary Rockwood ${ }^{\mathrm{b}}$ \\ ${ }^{a}$ Clinical Pharmacology and Toxicology Unit, Carmel Medical \\ Center, Haifa, Israel; ${ }^{b}$ US Army Medical Research Institute of \\ Chemical Defense, Aberdeen Proving Ground, United States
}

Objective: To evaluate the efficacy of intramuscular (IM) dimethyl trisulfide (DMTS) in cyanide-poisoned swine.

Methods: A comparative animal study using 9 swine, randomized into 2 study groups. Animals (while alert) were poisoned intravenously with potassium cyanide $(4 \mathrm{mg} / \mathrm{kg})$. The first group (controls) were not treated further. The second group were treated with DMTS 100 milligrams/kg IM within 1 minute of cyanide administration. The measured outcomes included survival rate, a clinical score $(0=$ dead to $5=$ fully alert and mobile), mean blood pressure, pulse, blood $\mathrm{pH}$, lactate and methemoglobin levels. The animals were observed continuously up to 30 minutes after cyanide dosing.

Results: All animals collapsed within 20 seconds after cyanide injection and developed signs of severe poisoning including respiratory distress, generalized convulsions, loss of consciousness, a marked decrease in blood pressure and pulse and profound lactic metabolic acidosis. Three of 4 untreated animals died during the observation period ( $25 \%$ survival), while 2 of the treated animals died (60\% survival). Clinical scores of the surviving animals at 30 minutes after poisoning were better in the treated animals ( 1 versus $3,4,5)$. Blood lactate concentrations and $\mathrm{pH}$ were significantly better in the treated swine. Methemoglobin (MetHb) levels remained low in 4 of 5 of the DMTS animals $(1.7 \%, 1 \%, 0.9 \%, 1.4 \%)$ and the fifth reached a peak of $12.7 \%$.

Conclusion: DMTS given IM demonstrated clinical efficacy in cyanide-poisoned swine. Several limitations exist: (1) a sub-optimal formulation with potentially low bioavailability (low MetHb may indicate the low bioavailability); (2) small group size and high variability among swine; (3) lack of DMTS pharmacokinetic data to correlate and validate the clinical results. Improved formulation and a more comprehensive study design are recommended for future research. DMTS shows potential as a novel cyanide antidote.

\section{Don't play with fire! House fire- related smoke injuries in Hungary}

\author{
Csaba Pap and István Elek \\ Department of Emergency Medicine and Clinical Toxicology, \\ Péterfy Hospital, Budapest, Hungary
}

Objective: Smoke inhalation commonly occurs in fire victims and can be associated with several sequelae such as asphyxia, thermal injury, chemical injury and systemic toxicity from inhaling carbon monoxide, cyanide, hydrogen sulfide, chlorinated pulmonary agents or hydrocarbons. The aim of this study was to assess the incidence and severity of house fire-related smoke inhalation in civilians (non-firefighters) in Hungary.

Methods: Data of patients hospitalized after suspected fire-related smoke inhalation without airway burning at our department between January 2013 and December 2017 were analyzed retrospectively. The following data were recorded: patients' age, gender, sources of poisoning, duration of exposure, clinical and laboratory findings, therapeutic measures, and outcome of poisoning.

Results: Overall 325 patients were evaluated, 174 of the patients were male and 151 were female. The mean age was 51.2 years. The three main sources of poisoning were: food left on the stove or in the oven that caught fire, smoking in bed and failure of electrical systems. The frequency of complaints was as follows: cough $68 \%$, dyspnea $27 \%$, headache $23 \%$, dizziness $14 \%$, palpitation $10 \%$, and chest pain $5 \%$. Abnormal vital signs included: hypotension $6 \%$, hypertension $27 \%$, tachycardia $39 \%$ and tachypnea $24 \%$. Abnormal clinical findings were: smoky odor $96 \%$, singed nasal hair $39 \%$, singed skin and/or hair $18 \%$, carbonaceous deposits in the nose, pharynx and sputum $42 \%, 22 \%$ and $29 \%$, respectively, wheezing $34 \%$, hoarseness $23 \%$, stridor $9 \%$, confusion $29 \%$ and coma $4 \%$. About half the patients were regular smokers and $41 \%$ of them consumed alcohol. Mean carboxyhemoglobin and methemoglobin values was $8.3 \%$ and $0.9 \%$, respectively. Cyanide concentrations were not available during the study period. There were only 4 cases with $\mathrm{pO}_{2}$ more than $60 \mathrm{mmHg}$ from a venous blood sample referring to cyanide 
exposure. As for therapy: humidified oxygen $81 \%$, expectorants $71 \%$, bronchodilators $36 \%$, steroids $13 \%$, and nebulized racemic epinephrine $6 \%$. Bronchoalveolar lavage was performed in 14 patients and 13 patients required mechanical ventilation. Of 325 patients 38 had carbon monoxide poisoning, 4 had suspected mild cyanide poisoning and there were no cases with methemoglobinemia or suspected inhalation of hydrogen sulfide. Seventeen patients developed pneumonia and 8 myocardial injury and there was no noncardiogenic pulmonary edema or pneumonitis. Overall 318 patients were discharged, 14 were transferred to departments of pulmonology or cardiology and 3 patients died.

Conclusion: House fire-related smoke inhalation without airway burning in Hungary is mainly accidental and generally has a good prognosis without severe systemic toxicity.

\section{The predictive impact of plasma neutrophil gelatinase-associated lipocalin (NGAL) in acute carbon monoxide poisoning from charcoal burning}

\author{
Jeong Mi Moon ${ }^{\mathrm{a}}$, Byeongjo Chun ${ }^{\mathrm{b}}$, SD Lee ${ }^{\mathrm{a}}$ and \\ MH Shin ${ }^{\mathrm{C}}$ \\ aDepartment of Emergency Medicine, Chonnam National \\ University Medical School, Gwangju, Republic of South Korea; \\ ${ }^{b}$ Chonnam National University Medical School, Gwangju, Republic \\ of South Korea; ${ }^{C}$ Department of Preventive Medicine, Chonnam \\ National University Medical School, Hwasun, Republic of South Korea
}

Objective: Neutrophil gelatinase-associated lipocalin (NGAL) is a promising biomarker of acute renal disease [1]. The aim of this study was to assess the feasibility of using the plasma concentration of NGAL at the time of presentation in the emergency department (ED) to predict acute kidney injury (AKI) during hospitalization and the long-term neurological outcomes of acute carbon monoxide $(\mathrm{CO})$ poisoning from charcoal-burning.

Methods: This retrospective study included 260 patients who suffered acute CO poisoning from charcoal-burning. Data were collected on patient demographic characteristics, plasma NGAL concentrations at the time of presentation at the ED, the in-hospital occurrence of AKI as defined by the Kidney Disease: Improving Global Outcomes (KDIGO) guidelines, and long-term neurological outcomes.

Results: The median plasma NGAL concentration at the time of presentation in the ED after acute $\mathrm{CO}$ poisoning from charcoalburning was 78 (54-115) $\mathrm{ng} / \mathrm{mL}$. The NGAL concentration was an independent predictor of the development of AKI (aOR $2.195(95 \%$ $\mathrm{Cl} 1.244-3.873)$ ) and could be used to stratify the severity of AKI (aOR 2.802 (95\% Cl 1.782-4.406)). The area under the receiver operating characteristic curve (AUC) of the predictive model for AKI that included both the plasma NGAL concentration and clinical parameters $(0.934(95 \% \mathrm{Cl} 0.896-0.961))$ was comparable to that of the predictive model including only the clinical parameters $(0.915(95 \% \mathrm{Cl}$ $0.874-0.947)$, $p$ value $=0.320$ ). The plasma NGAL concentration at the time of presentation in the ER was an independent factor predicting long-term neurological outcomes in patients who did not develop AKI during hospitalization (aOR 10.683 (95\% Cl 1.91359.643)). In patients who did not develop AKI, the plasma NGAL concentration significantly improved the predictive accuracy of the model when used in combination with clinical parameters (AUC = 0.959 (95\% Cl 0.919-0.982) in the model with plasma NGAL and clinical parameters versus AUC $=0.919(95 \% \mathrm{Cl} 0.869-0.953)$ in the model with clinical parameters only, $p$ value $=0.033$ ). In contrast, the plasma NGAL concentration was not associated with long-term neurological outcomes in patients who developed AKI.

Conclusion: The plasma NGAL concentration at the time of presentation in the ED can significantly improve the accuracy of the prediction of long-term neurological outcomes when used in combination with other clinical parameters in patients who do not develop AKI after acute charcoal-burning $\mathrm{CO}$ poisoning.

\section{Reference}

[1] Haase M, Haase-Fielitz A, Bellomo R, et al. Neutrophil gelatinaseassociated lipocalin as a marker of acute renal disease. Curr Opin Hematol. 2011;18:11-18.

\section{The predictive value of scores based on peripheral complete blood cell count for long-term neurological outcome in acute carbon monoxide intoxication}

\author{
Byeongjo Chun, Jeong Mi Moon and YS Cho \\ Chonnam National University Medical School, Gwangju, Republic \\ of South Korea
}

Objective: Recently, several scores based on the peripheral complete blood count (CBC) calculated from the counts of different leukocytes or platelets have been reported to be useful for predicting the outcome of diseases related to systemic inflammation $[1,2]$. This study aimed to investigate whether these scores can help predict the long-term neurological outcome of acute carbon monoxide (CO) poisoning.

Methods: This retrospective study included 279 patients with acute $\mathrm{CO}$ poisoning. Data on the demographic details, serial peripheral $C B C$ and scores based on peripheral $C B C$ (neutrophillymphocyte ratio (NLR), monocyte-lymphocyte ratio (MLR), platelet-lymphocyte ratio (PLR), systemic immune inflammation index (SII)) over the first 12 hours after presentation together with the clinical course during hospitalization and long-term neurological outcomes were collected.

Results: Among 279 patients, 33 (11.8\%) developed severe persistent neurological sequelae. Patients with a poor long-term neurological outcome had higher neutrophil and monocyte counts and lower lymphocyte counts over the first 12 hours after admission than patients with a good outcome. The diagnostic performance of the NLR, MLR and SIl over the first 12 hours for predicting longterm neurological outcome was acceptable (receiver operating characteristic (ROC)-area under the curves (AUC) $>0.7$ ). These scores at presentation were independently associated with the long-term neurological outcome (log NLR 12.7 (2.3-69.7), log NLR 9.3 (1.946.0), log PLR 22.6 (2.0-260.5), and log SII 15.8 (3.0-82.1)). The multivariate model including log SII at presentation (0.939 (0.903-0.965)) had significantly better prognostic accuracy than the model including log white blood cell count $(0.902(0.860-0.935), p$ value $=0.048$. The optimal cut-off value for log SII at presentation was 3, with $97 \%$ (84.2-99.9) sensitivity and $64.2 \%$ (57.8-70.2) specificity.

Conclusion: The SII at presentation could significantly improve the prognostic accuracy for predicting the long-term neurological outcome in patients with acute CO poisoning. The SII is an inexpensive and easily measurable parameter, and might be used as a prognostic tool in clinical fields.

\section{References}

[1] Zahorec R. Ratio of neutrophil to lymphocyte counts - rapid and simple parameter of systemic inflammation and stress in critically ill. Bratisl Lek Listy. 2001;102:5-14. 
[2] Tao C, Wang J, Hu X, et al. Clinical value of neutrophil to lymphocyte and platelet to lymphocyte ratio after aneurysmal subarachnoid hemorrhage. Neurocrit Care. 2017;26:393-401.

\section{Murder by cyanide injection}

\author{
Samuel C Holstege ${ }^{\mathrm{a}}$, Benjamin J Holstege ${ }^{\mathrm{a}}$ and \\ Christopher $\mathrm{P}$ Holstege $\mathrm{b}^{\mathrm{b}}$ \\ ${ }^{a}$ Chemistry, Calvin College, Grand Rapids, United States; \\ ${ }^{b}$ Emergency Medicine/Medical Toxicology, University of Virginia, \\ Charlottesville, United States
}

Objective: Homicidal poisonings are uncommon. We present the unique murder of a woman by cyanide injection and her progression as documented by video recording, telephone recording and medical personnel documentation.

Case report: On Valentine's Day evening, a 35-year-old woman was accosted in her driveway by her disguised ex-boyfriend and given a forceful injection of cyanide (later considered to be potassium cyanide) in the left buttock from a vintage steel syringe. The attack was captured on home video surveillance at 20:06. She was able to make it into her home where she and her mother called emergency medical services (EMS) at 20:08. On the recording, while talking with dispatch personnel, she frantically requested help, pleading: "Someone shot something in my body. I feel like I can't walk. My leg hurts bad... My leg is totally numb." While talking with the operator throughout her course, prior to EMS arrival, her mental status declined. EMS arrived at 20:17 with initial recorded vital signs (20:25): blood pressure 108/ $62 \mathrm{mmHg}$; pulse 96 beats per minute; respirations 14 breaths per minute; and Glasgow Coma Score (GCS) 10. By 20:31, her vital signs were blood pressure $99 / 31 \mathrm{mmHg}$, pulse 91 beats per minute, respiratory rate 4 breaths per minute and GCS 3 . She arrived to the emergency department and received advanced medical care, was placed on life support and was found to have a $\mathrm{pH}$ of $6.945, \mathrm{pCO}_{2} 18.9 \mathrm{mmHg}, \mathrm{pO}_{2} 76 \mathrm{mmHg}$, bicarbonate 4.2 $\mathrm{mmol} / \mathrm{L}$ and lactate $25 \mathrm{mmol} / \mathrm{L}$. Despite aggressive measures, including hydroxocobalamin, the patient was later declared brain dead. The patient's cyanide concentration was later determined to be markedly elevated on multiple health system blood draws.

Conclusion: There are few past cases of murder accomplished by forced cyanide intramuscular injection. This case provides a precise timeline from the video footage of the injection, the 911 call, the EMS transport and the subsequent hospital care. Potassium cyanide is miscible in water, with $1 \mathrm{~mL}$ of water able to hold approximately $700 \mathrm{mg}$ of potassium cyanide, well above the lethal dose. Cyanide stability in water while in a syringe and the potential diffusion of cyanide through tissues once injected are highlighted. This case highlights the unique time course and clinical characteristics associated with a direct intramuscular injection of cyanide.

\section{Pitfalls of differential diagnosis: deep vein thrombosis or snake bite?}

\author{
Anne Stürzebecher ${ }^{a}$, Beate Machold ${ }^{b}$ and \\ Dagmar Prasa ${ }^{a}$

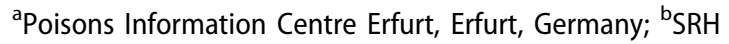 \\ Zentralklinikum Suhl, Suhl, Germany
}

Objective: Vipera berus (European adder) distribution in Germany seems to have increased in recent years, and bites by this species have become a common enquiry to the Poisons Information Centre (PIC) Erfurt. Snake bites occur regularly in our PIĆs area of responsibility, especially in rather warm summers, but most citizens are still not aware of (any) snakes living nearby. We report a case that initially presented as deep vein thrombosis but most probably was a Vipera berus bite.

Case report: A 57-year-old male presented to his family physician with swelling and livid discolouration on his right leg, and was promptly referred to hospital under suspicion of deep vein thrombosis. Diagnostic measures could not confirm the suspected diagnosis. Further examination of the leg revealed a bite mark above the ankle. When the patient was consequently interviewed again, he informed the doctor that he had been to the woods the previous day, and may have been bitten by "something". He remembered to have felt a "sting" and a "sharp pain" above his right ankle, and found a red spot of about 2 to $3 \mathrm{~cm}$ in diameter but he did not clearly see an animal. He then walked home (a long distance). In the evening, the whole leg began to feel tender, and the patient felt dizzy and queasy. The following morning, he went to see his doctor. In hospital, the leg was cooled and elevated, and symptoms resolved over the next three days. Besides a mild and transient elevation of C-reactive protein and creatine kinase, laboratory findings were within normal limits, and no coagulopathy was detected. Antivenom was not administered.

Conclusion: Although Vipera berus bites often remain asymptomatic or cause only mild local symptoms, severity of symptoms can increase in cases when immobilisation is not applied. The PIC Erfurt has observed more significant symptoms after Vipera berus bites in several cases, when patients either had to use the bitten limb or did not follow the doctoŕs recommendation of immobilisation. These patients often developed extensive swelling and livid discolouration of the extremity, but administration of antivenom was not necessary. This case report emphasises the benefit of (early) immobilisation for the prevention of more pronounced symptoms. Unless unavoidable, patients should be strongly discouraged to use the bitten limb. It also shows the importance of differential diagnosis, especially in cases that initially seem obvious.

\section{Preclinical assessment of the neutralizing ability of a monospecific antivenom in the treatment of Bothrops lanceolatus in Martinique}

\author{
Dabor Resiere ${ }^{a}$, Ana Sylvia Arias ${ }^{b}$, Maureen Villalta ${ }^{b}$, \\ Alexandra Ruvado ${ }^{b}$, Yanick Brouste ${ }^{a}$, Andre Cabie ${ }^{a}$, \\ Remi Neviere ${ }^{a}$, Raymond Cesaire ${ }^{a}$, Hatem Kallel ${ }^{c}$, \\ Hossein Mehdaoui ${ }^{a}$, Bruno Mégarbane ${ }^{d}$ and \\ José Maria Gutiérrez ${ }^{\mathrm{b}}$ \\ aService des Urgences et de Reanimation Polyvalente, Centre \\ Hospitalier Universitaire, Fort-de-France, Martinique, France; \\ ${ }^{b}$ Instituto Clodomiro Picado, Facultad de Microbiología, \\ Universidad de Costa Rica, San Jose, Costa Rica; 'Intensive Care \\ Unit, Cayenne General Hospital, Cayenne, French Guiana; \\ ${ }^{\mathrm{d}}$ Department of Medical and Toxicological Critical Care, \\ Lariboisiere Hospital, Paris-Diderot University, INSERM 1144, Paris, \\ France
}

Objective: Bothrops lanceolatus is an endemic viperid species in the Lesser Caribbean island of Martinique. Envenoming by this species is characterized by local and systemic effects, among which the development of thrombosis in various organs is the most severe complication. We aimed to experimentally characterize the different venom activities using in vivo mouse tests and in vitro assays and to assess the efficacy of a new antivenom Fab2 variety.

Methods: A challenge dose of venom was selected for each effect. A fixed concentration of venom was incubated with varying dilutions of antivenoms during 30 minutes at $37^{\circ} \mathrm{C}$. Aliquots 
of the mixtures, containing the corresponding "challenge dose" for each effect, was tested in the corresponding experimental systems. The preclinical efficacy of a batch of monospecific Bothrofav ${ }^{\circledast}$ antivenom currently in use in Martinique was assessed. Results: The venom induced lethal, local and systemic hemorrhagic, edema-forming, myotoxic, in vivo coagulant, defibrinogenating, thrombocytopenic, proteinase and phospholipase A2 activities. The antivenom was highly effective in neutralization of all activities tested, in agreement with its described clinical efficacy. This batch of antivenom showed significantly higher preclinical efficacy as compared to a previous batch used in the past.

Conclusion: The assessment of the preclinical efficacy of the Bothrofav $^{\circledast}$ antivenom batch currently in use in Martinique demonstrated its high efficacy, which is superior to that of the first batch of this monospecific antivenom used in previous decades. No thrombotic effects were detected.

\section{A snake in the house is worth 14 in the bush: estimating the price of antivenin treatment for exotic snake envenomation in Sweden}

\author{
Peter Hultén, Mikael Julin, Stefan Arvidsson and \\ Erik Lindeman \\ Swedish Poisons Information Centre, Stockholm, Sweden
}

Objective: Exotic snake envenomation (ESEs) came to the Swedish poisons centre's (PC) attention in the 1990s. The popularity of keeping these animals as pets surged after Sweden's entry into the European Union, with annual rates of 30-35 bites of venomous exotic snakes. At least 130 different species of exotic snakes are estimated to be kept in public and private herpetaria in Sweden. Some of these snakes can cause severe envenomation and require the administration of antivenin. The Scheele National Pharmacy (SNP) in Stockholm stocks 17 different polyvalent antivenins for ESEs. The price of individual antivenins is notoriously high, but total annual costs in maintaining an intact stock, with procurement and maintenance costs included, is unknown. In this report we use PC data and information from the SNP to assess the scope and costs of antivenin use in ESEs in Sweden.

Methods: PC records from January 2000-September 2018 were searched for cases of exotic snake bites. Hospital cases were examined in detail, including examination of hospital records sent to the PC, focussing on type of snake and use of antivenin. Data on the costs of antivenin stock-maintenance for 2016 as a sample year was calculated by the SNP at our request.

Results: Overall 158 bites involving 70 different snake species were identified. Trimeresurus albolabris was the most common $(n=26)$, followed by Agkistrodon contortrix, Crotalus atrox $(n=11$ each) and Naja kaouthia $(n=9)$. Most patients $(98 \%)$ were male and most bites occurred in fingers/hands. Most patients developed either no or mild-moderate symptoms. There were no lethal cases. Antivenin was administered in 46 cases. SNP costs for antivenin in 2016 was $220,000 €(100,000 €$ in treatment costs and the rest in replacement costs for expired product).

Conclusion: There has been a substantial decline in annual Swedish PC cases of venomous exotic snake bites since the 1990s. During the last 19 years there has been a large interannual variation with 4-15 cases/year, with no discernible overall trend up or down. The number of ESEs receiving antivenin was $0-6 /$ year, meaning roughly $1 / 3$ of bites received treatment. During 2016 six ESEs were treated with antivenin at a total cost of $220,000 €$. Cassation was the largest expenditure item in the 2016 budget for the antivenin stockpile. As a comparison, the 2016 cost of maintaining antivenin supply and treating 81 envenomations from Vipera berus, the only indigenous venomous snake in Sweden, was also $220,000 €$.

\section{Envenomation by a Western green mamba (Dendroaspis viridis): a report of three episodes in the same patient}

\author{
Joan Fuchs ${ }^{a}$, Stefan Weiler ${ }^{\mathrm{a}}$, Roger Aeberhard ${ }^{\mathrm{b}}$ and \\ Jürg Meier ${ }^{c}$

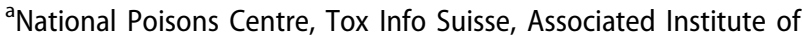 \\ the University of Zurich, Zurich, Switzerland; ${ }^{b}$ Snakeparadise.ch, \\ Eschlikon, Switzerland; 'Department of Environmental Sciences, \\ University of Basel, Basel, Switzerland
}

Objective: There are apparently many people holding venomous snakes in Switzerland. Throughout the last decade, about 80 exotic snakebite cases were recorded at the Swiss Poisons Information Centre. The African elapid snake genus Dendroaspis comprises four species. The severity of bites by Western green mambas ( $D$. viridis) is estimated to be intermediate between the highly dangerous, neurotoxic black mamba (D. polylepis) and the possibly least venomous Eastern green mamba ( $D$. angusticeps) [1]. With $D$. viridis, neurological symptoms are supposed to be more pronounced than with $D$. angusticeps [2], and more comparable to Jameson's mamba (D. jamesoni) [3]. We present three episodes of bites by $D$. viridis in the same patient.

Case series: One of the authors, a 52-year-old snake-breeder with a history of a bite by a Calloselasma rhodostoma and subsequent antivenom therapy was bitten in 2001, 2008 and 2015 by nonrelated $D$. viridis snakes bred in Switzerland. While episode one only resulted in minor local pain and a tightness of the throat, massive local swelling was the main clinical symptom in episodes two and three, with only minimal neurologic symptoms (despite a probable double bite, i.e. four fang marks, in episode two) such as a tingling sensation of the body and a tightness of his throat, resulting in mild, spontaneously resolving dyspnoea. Compartment syndrome during episode two required fasciotomy, albeit without prior administration of antivenom, which the patient had refused. In the third episode two vials of antivenom (SAIMR polyvalent snake antivenom, SA Vaccine Producers (Pty) Ltd) showed good efficacy. The patient developed an exanthema of his whole body after antivenom, despite prior administration of steroids and antihistamines.

Conclusion: After three individual bites by a supposedly highly neurotoxic snake, the patient only suffered from considerable local swelling, without marked neurologic symptoms such as ptosis, muscle weakness, or respiratory depression.

\section{References}

[1] Warrell DA. Clinical toxicology of snakebite in Africa and the Middle East/ Arabian Peninsula. In: Meier J, White J, eds. Handbook of clinical toxicology of animal venoms and poisons. Boca Raton: CRC Press, 1995:463.

[2] Leclerc T, Debien B, Perez JP, et al. [Mamba envenomation in mainland France: management of exotic envenomations needs rethinking]. Ann Fr Anesth Reanim. 2008;27:323-325. French.

[3] Chippaux JP, Courtois B, Roumet D, et al. [Envenoming by mamba bites (Dendroaspis viridis). Report of a case with good recovery]. Med Trop. 1977;37:545-549. French.

\section{Complex regional pain syndrome following a centipede bite: a case report}

Satariya Trakulsrichai ${ }^{\mathrm{a}}$, Suthimon Thumtecho ${ }^{\mathrm{b}}$, Charuwan Sriapha ${ }^{c}$ and Winai Wananukul ${ }^{b}$ 


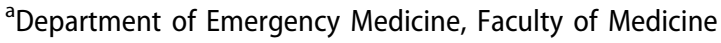
Ramathibodi Hospital, Mahidol University, Bangkok, Thailand; ${ }^{\mathrm{b}}$ Department of Medicine, Faculty of Medicine Ramathibodi Hospital, Mahidol University, Bangkok, Thailand; 'Ramathibodi Poison Center, Faculty of Medicine Ramathibodi Hospital, Mahidol University, Bangkok, Thailand

Objective: Complex regional pain syndrome (CRPS) is a chronic pain condition that usually affects one limb and typically develops after an injury such as fracture or trauma. CRPS after snake bite has been described in few case reports [1,2]. To our knowledge, development of CRPS following centipede bite has not been reported in the literature. We describe the development of CRPS in a woman following a centipede bite.

Case report: A 31-year-old female was bitten by an unidentified centipede on her right 2nd toe. Her underlying disease was von Willebrand disease and was followed-up at a hematology clinic. After the bite, she visited the nearby hospital and was treated. After about 1-2 weeks, she developed severe pain and increased sensitivity (allodynia) in her right foot. She also complained of a skin color change of her right 2 nd toe and swelling of her whole right foot. She had normal vital signs. Her right foot was warm, erythematous and edematous with hyperpigmented skin on the 2nd toe. The capillary refill and peripheral pulses were all normal. Her blood chemistries and X-ray were unremarkable. Ultrasound of the right foot showed no hematoma. She had normal nerve conduction velocity studies. She visited our hospital Emergency Room and outpatient clinics on multiple occasions and received both antibiotics and analgesics for about 1 month. Nonetheless, her clinical signs and symptoms did not improve. Finally, she fulfilled the Budapest criteria and was diagnosed with CRPS type 1. After she was treated with gabapentin and referred for rehabilitation, her clinical signs and symptoms were significantly improved. Although, she received treatment within the first few months of onset of symptoms, her clinical course lasted more than 6 months and has not completely resolved.

Conclusion: Centipede bite might cause and be an eliciting event for CRPS. Awareness of this condition is important and necessary for early diagnosis and proper management, thereby avoiding unnecessary investigations and treatment.

\section{References}

[1] Seo YH, Park MR, Yoo SH. Development of complex regional pain syndrome after a snake bite: a case report. Korean J Pain. 2014;27:68-71.

[2] Kleggetveit IP, Skulberg PK, Jørum E. Complex regional pain syndrome following viper-bite. Scand J Pain. 2016;10:15-18.

\section{A confirmed Beautiful Pit Viper (Trimeresurus venustus) bite resulting in local symptoms}

\author{
Joan Fuchs ${ }^{\mathrm{a}}$, Karine Bessire ${ }^{\mathrm{b}}$ and Stefan Weiler ${ }^{\mathrm{a}}$ \\ ${ }^{a}$ National Poisons Centre, Tox Info Suisse, Associated Institute of \\ the University of Zurich, Zurich, Switzerland; ${ }^{b}$ Department of \\ Emergency Medicine, Centre Hospitalier Universitaire Vaudois \\ (CHUV), Lausanne, Switzerland
}

Objective: Trimeresurus venustus (or Cryptelytrops venustus) is a nocturnal, mainly arboreal green pit viper native to Thailand and Malaysia. It measures up to $70 \mathrm{~cm}$ in length and feeds mainly on frogs, lizards and small mammals. Related species such as $T$. albolabris and T. trigonocephalus have been shown to cause considerable hematologic disorders, such as coagulopathy and bleeding or disseminated intravascular coagulation, as well as local tissue injury, sometimes resulting in skin necrosis $[1,2]$. Reports in the literature of bites by $T$. venustus are scarce [3]. We present a case of a bite that resulted only in local symptoms.

Case report: A 60-year-old snake owner of 70 snakes, with no prior bite event in 35 years, nor any relevant medical history, was bitten by an adult $T$. venustus on the third finger of his right hand while taking it from a box. The bite was painful and swelling progressed to include his whole hand within an hour after the bite. He was treated symptomatically with pre-emptive antibiotics and analgesics but did not develop any hematological disorders such as coagulopathy and bleeding or disseminated intravascular coagulation. He was discharged 24 hours after the bite with some residual slight hypoesthesia at the bite site, which persisted until 5 weeks after the bite.

Conclusion: This patient developed only local swelling, as was seen with two other well-documented cases reported to our Poisons Center, and one case presented as a poster elsewhere [3]. He recovered with symptomatic therapy and did not require antivenom. Therefore, it seems that $T$. venustus bites present with less severe symptoms compared to other Trimeresurus species.

\section{References}

[1] Greene S, Galdamez LA, Tomasheski R. White-Lipped Tree Viper (Cryptelytrops albolabris) envenomation in an American viper keeper. J Emerg Med. 2017;53:e115-e118.

[2] Namal Rathnayaka RM, Kularatne SA, Ranathunga PE. Coagulopathy and extensive local swelling following Green pit viper (Trimeresurus trigonocephalus) envenoming in Sri Lanka. Toxicon. 2017;129:95-99.

[3] Suteparuk S, Vasaruchapong T. A report of a confirmed case of beautiful pit viper (Trimeresurus venustus) bite. Poster presented at: A Toxicologist's Rendez-vous - a meeting of the disciplines. The 15th International Scientific Conference of the Asia Pacific Association of Medical Toxicology (APAMT), November 17-20, 2016, Singapore.

\section{Ten years' experience of the National Serum Depot in the Netherlands}

\section{Marieke A Dijkman and Irma De Vries}

Dutch Poisons Information Center, University Medical Center Utrecht, Utrecht, Netherlands

Objective: A National Serum Depot (NSD) is operational in the Netherlands, guaranteeing rapid antivenom supply during medical emergencies. The NSD was established in 2008 at the National Institute for Public Health and the Environment (RIVM). The Dutch Poisons Information Centre (DPIC) advises on the contents of the Depot, and on antivenom delivery to physicians treating bite and sting victims. All exotic venomous bites and stings consultations in this 10 year period were analysed in order to identify potential gaps in the NSD content.

Methods: A retrospective analysis of cases from the DPIC database from April 2008 to April 2018.

Results: In ten years, 59 bite or spitting incidents by 27 different exotic snake species were reported to the DPIC. Most bites were inflicted by Naja kaouthia $(n=11)$, Crotalus atrox $(n=10)$ and Trimeresurus albolabris $(n=4)$. The snake bite victims (51 males / 5 females, all adults) were predominantly bitten in the upper extremities $(n=54)$, a leg $(n=1)$, and the face $(n=1)$. Two men were spat at by Naja nigricollis and a Naja pallida. One female patient had a splash incident in a laboratory resulting in freezedried snake venom in the eye. In 32 bite incidents antivenom was indicated and in 21 cases the antivenom was administered 
to the patient. Crofab was most often distributed $(n=10)$ and administered $(n=10)$, followed by Thai Red Cross Cobra antivenin ( $\mathrm{n}=8$ distributed and 4 administered). In this same period 35 scorpion stings were reported, involving adults as well as children. Twenty-three incidents took place in the Netherlands; $57 \%$ by stowaways in luggage and imported plants and $43 \%$ by scorpions kept in a terrarium. It was possible to identify most of the stowaway scorpions (72\%), which is necessary in case antivenom treatment would be needed. However in none of these patients were envenomation signs and symptoms severe enough to justify antivenom treatment. This was also the case for the spider bites and fish stings reported to the DPIC.

Conclusion: This analysis confirms the assumptions made when the NSD was started, that all species of especially venomous snakes and scorpions can be encountered in the Netherlands. No adjustments were needed in the range of antivenoms available at the NSD, however adjustments were made in relation to the number of vials of Crofab and Thai Red Cross Cobra antivenin kept in stock, ensuring adequate treatment of more than one patient at the same time.

\section{Impact of the media on the Poison Control Centres: the false outbreak of Loxosceles rufescens spider bite in Italy}

\author{
Diletta Sabatini ${ }^{\mathrm{a}}$, Paolo Boldrini ${ }^{\mathrm{a}}$, Michele S Milella ${ }^{\mathrm{a}}$, \\ Viola Mazzucco ${ }^{a}$, Susanna M Signoretti ${ }^{a}$, \\ Manuela Graziani ${ }^{\mathrm{a}}$, Emanuela Lanni ${ }^{\mathrm{a}}$ and \\ Maria Caterina Grassi ${ }^{\mathrm{b}}$ \\ ${ }^{a}$ Clinical Toxicology - Poison Control Centre and Drug Dependence \\ Unit, Policlinico Umberto I - "Sapienza" University of Rome, Rome, \\ Italy; 'Department of Physiology and Pharmacology "V. Erspamer" \\ - Clinical Toxicology - Poison Control Centre and Drug \\ Dependence Unit, Policlinico Umberto I - "Sapienza" University of \\ Rome, Rome, Italy
}

Objective: To describe the increase in telephone calls to the Poison Control Centre (PCC) of Umberto I Hospital of Rome related to Loxosceles rufescens (LR) spider bites following an alarming awareness campaign in the media.

Methods: Telephone calls to the PCC concerning alleged LR bites from April 2016 to September 2018 were reviewed. The month of April 2018, when the lay press published the first alarming article, was identified as an index. Data were normalized for the increase in overall consultations registered in 2018.

Results: The recorded enquiries regarding LR bites were as follows: i) 22 calls over the two year period April 2016-March 2018, of which 4 were from Emergency Departments (ED) and 18 from private citizens; ii) 79 calls from April 2018 to September 2018, of which 18 were from ED and 61 from private citizens. The mean age of subjects was 49 years (range: 12 months - 86 years). Only 2 subjects presented a skin lesion suggestive of a LR bite. A 3year-old child was hospitalized for 1 day for a necrotic skin lesion and low-grade fever and a 61-year-old man who presented an ulcerative skin lesion and had already been treated at home with oral antibiotic therapy.

Conclusion: Irrational fears about insects and other arthropods are very common. LR rarely bites humans and, contrary to what is often stated or claimed, its bite causes minor, transient effects. Currently, there no fatal cases are known [1]. The "lethal" reputation of LR is mostly predicated by the lay press. It is well-known that the increase in mass media coverage is associated with an increase in ED admissions [2]. This report allows the following considerations: i) the mass media play a pivotal role in public health behavior and may influence healthcare utilization, ii) medical news reports may cause alarm, often unjustified, and may have significant costs either in terms of public health or in terms of people's quality of life, iii) diagnoses of LR bite are mostly incorrect or refer at least to non-verified bites [1].

\section{References}

[1] Nentwig W, Pantini P, Vetter RS. Distribution and medical aspects of Loxosceles rufescens, one of the most invasive spiders of the world (Araneae: Sicariidae). Toxicon. 2017;132:19-28.

[2] Codish S, Novack L, Dreiher J, et al. Impact of mass media on public behavior and physicians: an ecological study of the H1N1 influenza pandemic. Infect Control Hosp Epidemiol. 2014;35:709-716.

\section{The spotted weever (Trachinus araneus): a little-known danger for the diver}

\author{
Bastien Domangé ${ }^{a}$, Valentin Pruvot ${ }^{b}$, Julien Reynoard ${ }^{c}$, \\ Romain Torrents ${ }^{d}$, Corinne Schmittc, Luc De Haro ${ }^{c}$ \\ and Nicolas Simon ${ }^{\mathrm{d}}$ \\ ${ }^{a}$ Clinical Pharmacology, Poison Control Center, Aix-Marseille Univ, \\ APHM, Marseille, France; ${ }^{b}$ Department of Pediatrics, Bastia \\ Hospital, Bastia, France; ${ }^{C}$ Clinical Pharmacology, Poison Control \\ Center, APHM, Marseille, France; ${ }^{\mathrm{d} C l i n i c a l}$ Pharmacology, Poison \\ Control Center, Aix-Marseille Univ, APHM, INSERM, IRD, SESSTIM, \\ Marseille, France
}

Objective: During the summer 2018, two unusual cases of weever fish stings were reported to the Marseille Poison Control Center. Those cases are exceptional due to the circumstances of envenomation and the site of puncture (chest) with severe complications.

Case reports: In July 2018, a 52-year-old tourist was taking videos while diving in Milos (Cyclades, Greece) when he was stung in the chest by a fish. The pain was immediately excruciating with local skin erythema. He reported being unwell for 10 hours with mild relief with paracetamol and aspirin. Twenty days later, he reported a local granuloma-like persistent lesion. Analysis of the video footage confirmed the fish involved was a spotted weever ( $T$. araneus). A 16-year-old who was snorkeling in the region of Calvi (Corsica, France) in August 2018 was also videorecording his diving trip. When near a fish lying on the sea bed, it immediately rushed him and stung him twice under the right armpit. Suffering from local pain, he consulted the nearest emergency department and was discharged with painkillers, but returned a day later with severe abdominal, lumbar and thoracic pain, vomiting, asthenia and dyspnea. A computerised tomography $(\mathrm{CT})$ scan showed a right pleural effusion and he was transferred to the nearest pediatrics department (Bastia). He was polypneic, sweating, and tachycardic (122 bpm) and venous blood gas showed a respiratory acidosis ( $\left.\mathrm{pH} 7.3, \mathrm{pCO}_{2} 48 \mathrm{mmHg}\right)$. On examination, the erythema was extensive and painful, and suggestive of a dermohypodermatitis. Two wounds of about 5 $\mathrm{mm}$ each were reported. Biological assessment confirmed a major inflammatory syndrome. A pleural puncture was performed to remove $760 \mathrm{~mL}$ of serohematic fluid and improve his respiratory status. The next day, clinical examination raised suspicion of a return of the effusion, confirmed by echotomography and CT scan. There was infiltration of the subcutaneous tissue and he was sent to surgery for chest tube insertion and wound exploration. Thoracic drainage removed 1 litre of a serous fluid and he received three antibiotics. The patient was discharged 10 days 
after the sting. The video footage confirmed the culprit was $T$. araneus.

Conclusion: These cases confirm what only experienced marine naturalists know: when disturbed, especially during the breeding season (May to August), the spotted weever fish can be aggressive and sting the unwary. Divers of the Mediterranean sea should be aware of the potential life-threatening danger of stings from this fish.

\section{Coagulopathy and the use of rotational thromboelastometry $\left(\right.$ ROTEM $\left.^{\oplus}\right)$ after a Bothrops asper bite}

\author{
Johanna Nordmark Grass ${ }^{\mathrm{a}}$, Erik Gremo ${ }^{\mathrm{b}}$ and \\ Anna Ågren ${ }^{c}$ \\ ${ }^{\text {a }}$ Swedish Poisons Information Centre, Stockholm, Sweden; \\ ${ }^{b}$ Department of Anaesthesiology and Intensive Care, \\ Mälarsjukhuset, Esiklstuna, Sweden; 'Division of Cardiovascular \\ Medicine, Karolinska Institutet, Stockholm, Sweden
}

Objective: Bothrops asper venom causes local tissue damage and affects blood coagulation in several different ways with both procoagulant and anticoagulant effects [1]. A case of severe coagulopathy after a Bothrops asper bite is presented. Rotational thromboelastometry (ROTEM ${ }^{\oplus}$ ) was used to monitor coagulation.

Case report: A previously healthy man was bitten in his left thumb by his pet Bothrops asper. He arrived at hospital 40 minutes later. Vital signs were stable but the finger was swollen with a small (3-4 mm) necrotic area at the bite site. Laboratory tests on admission showed normal platelet count, international normalized ratio (INR) and activated partial thromboplastin time (aPTT), but no detectable fibrinogen. ROTEM ${ }^{\oplus}$ showed prolonged clotting time and reduced clot firmness in INTEM and EXTEM and no fibrinogen clot component. Three hours after the bite 10 vials of Polyvalent Antivenom Lyophilised (Instituto Clodomiro Picado, Universidad de Costa Rica) were administered. Another two hours later there was a total absence of clot formation in INTEM, EXTEM and FIBTEM, indicating severe coagulopathy. The patient received 10 additional vials of antivenom 7.5 hours post-bite. Ten hours post-bite discrete clot formation could be observed together with an ongoing massive hyperfibrinolysis that was present until 48 hours post-bite. At 66 hours there was still a slightly abnormal ROTEM ${ }^{\circledast}$ with low fibrinogen clot component. In spite of the findings in the ROTEM ${ }^{\circledast}$ analysis his platelet count, INR and aPTT were normal or near normal throughout the course. Plasma fibrinogen was not detectable until after 21 hours post-bite and was $1.42 \mathrm{~g} / \mathrm{L}$ (reference value $>2 \mathrm{~g} / \mathrm{L}$ ) at 66 hours. Except for the local symptoms, which improved after antivenom, the patient remained clinically stable. He had no signs of bleeding except for microscopic hematuria. No blood products or coagulation factors were administered.

Conclusion: In this case severe coagulopathy and fibrinolysis were detected with ROTEM $^{\oplus}$ analysis in spite of normal or nearnormal thrombocyte count, INR and aPTTs (the latter remarkable as plasma fibrinogen was low or not even detectable). This illustrates that with conventional laboratory tests there might be a risk of missing severe coagulation abnormalities after snakebite. ROTEM $^{\oplus}$ may prove useful in diagnosing and following snake venom-induced coagulopathies.

\section{Reference}

[1] Alape-Grion A, Sanz L, Escolano J, et al. Snake venomics of the lancehead pitviper Bothrops asper: geographic, individual and ontogenic variations. J Proteome Res. 2008;7:3556-3571.

\section{Unique clinical effects of dwarf adder (Bitis species) envenoming: a case series}

\author{
Cherylynn A Wium, Catharina E Du Plessis, \\ Carine J Marks and Gert J Muller \\ Clinical Pharmacology, Stellenbosch University, Cape Town, South \\ Africa
}

Objective: It is known that the venom of the berg adder (Bitis atropos), a dwarf or minor adder, is both cytotoxic and neurotoxic. The bites of other southern African minor adders have thus far not been associated with neurotoxicity [1]. This is a report of five minor adder bites, other than berg adder, causing neurotoxic effects.

Case series: Three of the five patients were bitten by Bitis xeropaga (36, 21 and 31/2-years-old, all male), and two by Bitis peringueyi (9-year-old male and 5-year-old female). All the cases presented with local cytotoxic effects and all developed ophthalmoplegia with prominent mydriasis. The two older patients in the $B$. xeropaga group complained of changes in their sense of taste and smell (a common feature of berg adder envenoming). They also experienced difficulty in walking. The youngest patient had difficulty in breathing and was ventilated. This patient presented with convulsions due to hyponatraemia on day two. Hyponatremia is a common complication of berg adder bite [2]. It has, however, never been documented in other minor adder bites. The cause of the hyponatremia is probably due to the presence of a natriuretic polypeptide in the venom. Both patients in the $B$. peringueyi group had an unsteady gait and the 5-year-old experienced a bitter taste in the mouth. It took four weeks for the 9-year-old patient's eye symptoms and signs to resolve, but the 5 -year-old female's pupils continued to be dilated two years after the bite.

Conclusion: It has now been shown for the first time that other minor adder bites (excluding berg adder) can also induce serious neurotoxic effects.

\section{References}

[1] Marais J. Adders or Vipers. In: Marais J, ed. A complete guide to the snakes of southern Africa. 2nd ed. Cape Town, South Africa: Struik Publishers, 2004:62-87.

[2] Müller GJ, Modler $H$, Veale $D J H$, et al. Snake bite in southern Africa: diagnosis and management. CME. 2012;30:362-381.

\section{Treatment of serotonin syndrome due to 5-hydroxytryptophan intoxication in a Catahoula Leopard Dog}

Francesca Morello ${ }^{a}$, Caterina Veronesi ${ }^{a}$, Silvia Bertocchi ${ }^{\mathrm{a}}$, Silva Rubini ${ }^{\mathrm{b}}$ and Carlo A Locatelli ${ }^{\mathrm{c}}$

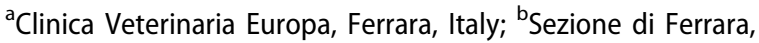
Istituto Zooprofilattico Sperimentale della Lombardia e dell'Emilia Romagna, Ferrara, Italy; 'Pavia Poison Control Centre - National Toxicology Information Centre, Pavia, Italy

Objective: Tryptophan is an essential amino acid that is not normally synthesized by the body, but necessary for the 
accomplishment of numerous biological functions, some of which pertain to the central nervous system. Serotonin (5-hydroxytryptamine) is a molecule with several effects on central and peripheral nervous system. The purpose of this paper is to describe a case of accidental intoxication of a dog with 5-hydroxytryptophan. An excess of tryptophan, and therefore of serotonin, contributes to activate the mechanisms of norepinephrine, dopamine and glutamine release at the anterior hypothalamus; these substances will determine important alterations such as arrhythmias, vomiting, diarrhea, disseminated intravascular coagulation (DIC), rhabdomyolysis.

Case report: A female 1-year-old Catahoula Leopard dog $(21 \mathrm{~kg})$ was taken to the veterinary clinic with gastrointestinal symptoms (vomiting and profuse diarrhea) and sensory abatement. The owners reported accidental ingestion of 16 tablets of Relaxigen Pet 20 (NBF LANES Milano), which contains, among other ingredients, $62 \mathrm{mg}$ 5-hydroxytryptophan (5-HTP). This drug was intended for a cohabiting dog, an adult Doberman, with a behavioral problem. During the visit the dog initially developed hyperthermia, mucous membrane congestion (capillary refill less than 2 seconds), tachypnea, altered sensory status (dysphoria, hyperexcitation, vocalization), miosis, salivation, muscle tremors and motor incoordination. Heart rate was normal. In the following hours periods of ataxia and drowsiness alternated with hyperexcitation and dysphoria and she developed marked systemic hypertension (maximum 220/100 $\mathrm{mmHg}$ ) and tachycardia with TP prolongation on the electrocardiogram. She was stabilized with diazepam $(0.5 \mathrm{mg} / \mathrm{kg} \mathrm{IV})$, and given a gastric lavage under general anesthesia, activated charcoal, lactated Ringer's, esmolol (bolus of $200 \mu \mathrm{g} / \mathrm{kg}$ IV followed by $25 \mu \mathrm{g} / \mathrm{kg} / \mathrm{min}$ IV), until remission of tachycardia, acepromazine $(0.25 \mathrm{mg} / \mathrm{kg} \mathrm{IM})$, and furosemide (1 mg/kg IM). The dog recovered completely; two weeks after discharge, hemato-biochemical profile and electrocardiogram were normal.

Conclusion: This intoxication manifests with non-specific symptoms and only a correct and thorough anamnestic investigation can help the clinician in the formulation of the correct diagnosis and appropriate treatment.

\section{Coral snake bite: a single snake, two consecutive envenomations}

\author{
Fabio Bucaretchi ${ }^{\mathrm{a}}$, Carla F Borrasca-Fernandes ${ }^{\mathrm{a}}$, \\ Eduardo M De Capitani ${ }^{\mathrm{a}}$ and Stephen Hyslop ${ }^{\mathrm{b}}$ \\ ${ }^{a}$ Campinas Poison Control Center and Department of Pediatrics, \\ School of Medical Sciences, State University of Campinas \\ (UNICAMP), Campinas, Brazil; ${ }^{\mathrm{b}}$ Department of Pharmacology and \\ Campinas Poison Control Center, School of Medical Sciences, State \\ University of Campinas (UNICAMP), Campinas, Brazil
}

Objective: To describe two patients envenomed by the same coral snake (Micrurus corallinus).

Case details: Two previously healthy men (32 and 34-years-old) found a coral snake in a woodpile while collecting wood for a barbecue. During the barbecue, both men became drunk and "played" with the snake believing that they were handling a false coral snake; both were bitten within a few minutes of each other. The clinical features of envenomation started 8-10 hours postbite, with both patients complaining of palpebral ptosis and muscle weakness; they were admitted to a primary care hospital 10 and 14 hours post-bite, respectively. After admission of the first case, the Poison Control Center was contacted and, because of suspected coral snake envenomation, the patient was transferred to our referral Emergency Department (ED, $175 \mathrm{~km}$ away). Table 1 summarizes the main features of both cases. In case 1, a test with anticholinesterase drug (neostigmine $1 \mathrm{mg}$ ) was also done at our ED, with partial improvement within 5 minutes ("could open the eyes", patient's comment); however, no objective measurement of muscle strength was performed. Both patients were successfully treated with coral snake antivenom $\left(\mathrm{Fab}_{2} ; 1 \mathrm{~mL}\right.$ of antivenom neutralizes $1.5 \mathrm{mg}$ of $M$. frontalis reference venom in mice; 1 vial $=10 \mathrm{~mL}$ ). Neither patient developed signs of respiratory failure; both were discharged one day postadmission, with improvement of myasthenia, but still showing palpebral ptosis. Both cases reported a transient loss of taste during follow-up. The offending snake, brought by the brother of Case 2, was identified as M. corallinus ( $42 \mathrm{~cm}$ long).

Conclusion: Bites by Micrurus spp. are rare. Systemic envenomation is characterized by a myasthenic syndrome caused by presynaptic and/or postsynaptic neurotoxins acting at the neuromuscular junction. This report highlights that a single coral snake can cause envenomation in more than one patient.

\section{Thrombotic microangiopathy and acute renal failure following Echis coloratus bite in Israel}

\author{
Yael Lurie ${ }^{\mathrm{a}}$, Ilan Rahmani Tzvi-Ran ${ }^{\mathrm{b}}$, Taysir Nasasra ${ }^{\mathrm{b}}$, \\ Lior Fuchs ${ }^{c}$, Yaniv Almog ${ }^{c}$, Ori Galante ${ }^{c}$ and \\ Yedidia Bentur ${ }^{\mathrm{a}}$ \\ ${ }^{a}$ Israel Poison Information Center, Rambam Health Care Campus, \\ Haifa, Israel; ${ }^{\mathrm{b}}$ Department of Medicine, Soroka University Medical \\ Center, Beer-Sheva, Israel; ' $M e d i c a l$ Intensive Care Unit, Soroka \\ University Medical Center, Beer-Sheva, Israel
}

Objective: Envenomation-induced thrombotic microangiopathy is a known but uncommon complication of various elapids and viper snake bites. It is characterized by thrombocytopenia, microangiopathic hemolytic anemia, and acute kidney injury (AKI) which continues beyond the resolution of venom-induced coagulopathy. Echis coloratus belongs to the viperidae family, and is the most common venomous snake in southern Israel. It can cause local signs, coagulopathy with prothrombin time (PT) and partial thromboplastin time (PTT) prolongation, thrombocytopenia and low fibrinogen. Azotemia and renal failure were also described. Well documented full-blown thrombotic microangiopathy has not been reported. The treatment of Echis coloratus bite includes supportive measures and specific antivenom, which is usually associated with resolution of the coagulopathy. The

Table 1. Main features observed in two patients envenomed by the same coral snake (Micurus corallinus) and bitten within a few minutes of each other

\begin{tabular}{|c|c|c|}
\hline & Case 1 & Case 2 \\
\hline Bite site & Left wrist and forearm & Right hand \\
\hline Admission to the referral hospital & Approximately 16 hours post-bite & Approximately 19 hours post-bite \\
\hline Clinical manifestations & $\begin{array}{l}\text { Palpebral ptosis, mandibular ptosis, ophthalmoplegia, } \\
\text { muscle weakness, dysphagia, abdominal pain and } \\
\text { generalized myalgia }\end{array}$ & $\begin{array}{l}\text { Local numbness, headache, palpebral ptosis, muscle } \\
\text { weakness, dysphagia, difficulty in speaking and } \\
\text { sleepiness }\end{array}$ \\
\hline Creatine kinase (reference <171 U/L) & 302 & 256 \\
\hline Use of neostigmine & Yes & No \\
\hline Antivenom (mL) & 90 (approximately 19 hours post-bite) & 90 (approximately 22 hours post-bite) \\
\hline Transitory loss of taste (salty and sweet) & 3-4 weeks & 2-3 weeks \\
\hline
\end{tabular}


treatment of thrombotic microangiopathy is supportive, including renal replacement therapy and blood products as needed. As the mechanism is probably different from anti-a disintegrin and metalloproteinase with thrombospondin motifs (ADAMTS)-13 antibodies mediated thrombotic thrombocytopenic purpura the role of plasmapheresis is questionable. We report two cases of thrombotic microangiopathy associated with severe coagulopathy, hemolysis and AKI following Echis coloratus snake-bite.

Case reports: Two male patients aged 39 and 70 years, were brought to the Emergency Department after being bitten by Echis coloratus in the desert area of southern Israel. On admission, both had normal vital signs, local pain and mild local swelling, without active bleeding. Laboratory evaluation demonstrated unclotted blood, hemoglobin 15.5 and $12.6 \mathrm{~g} / \mathrm{dL}$, platelet counts 170,000 and $101,000 / \mathrm{mcL}$, and creatinine 0.96 and $1.74 \mathrm{mg} / \mathrm{dL}$. They were treated with fresh frozen plasma, cryoprecipitate (one patient) and $50 \mathrm{~mL}$ of Echis coloratus antivenom (monovalent, unfractionated Equine source antivenom, Kamada Pharmaceuticals, Israel). Coagulation tests normalized but during the following days they developed hemolysis (lowest hemoglobin 6.4 and $8.2 \mathrm{~g} / \mathrm{dL}$ ) with elevated lactate dehydrogenase (LDH) and schistocytes on peripheral blood smear, severe thrombocytopenia $(26,000$ and $30,000 / \mathrm{mcL}$ ) and oliguric renal failure (creatinine 10.4 and $7.6 \mathrm{mg} / \mathrm{dL}$ ). ADAMST13 activity was normal and antiADAMST13 antibodies were negative in one patient and not technically measurable due to hemolysis in the other. They underwent hemodialysis and plasmapheresis (four and six courses). During the following days, hemolysis ceased with gradual recovery of renal function. On follow up examination 60 days after the bite, all clinical and laboratory abnormalities had fully recovered.

Conclusion: The clinical course and laboratory findings in these patients are compatible with Echis coloratus-induced thrombotic microangiopathy with AKI. Repeated evaluation of coagulation tests, complete blood count, signs of hemolysis and renal function is crucial in every case of Echis coloratus bite.

\section{Epidemiology of Viperidae snake envenoming in central and south- eastern Europe: CEE Viper Study}

Mojca DobajaBorak ${ }^{a}$, Željka Babićb $^{b}$ Niko Bekjarovski ${ }^{c}$,

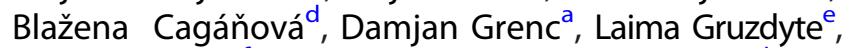
Piotr M Kabata ${ }^{\mathrm{f}}$, Ruth Kastanje ${ }^{\mathrm{g}}$, Zuzana Kolpach ${ }^{\mathrm{h}}$, Anna Krakowiaki, Csaba Papj, Julia Radenkova-Saevak, Jacek Sein-Anand', Slavica Vučinićm ${ }^{m}$, Sergey Zacharov ${ }^{h}$, Michael Eddleston ${ }^{\mathrm{n}}$ and Miran Brvar ${ }^{\mathrm{a}}$

${ }^{a}$ Centre for Clinical Toxicology and Pharmacology, University Medical Centre Ljubljana, Ljubljana, Slovenia; ${ }^{b}$ Poison Control Centre, Institute for Medical Research and Occupational Health, Zagreb, Croatia; ' University Clinic for Toxicology, Skopje, Macedonia; ${ }^{d}$ National Toxicological Information Centre, University

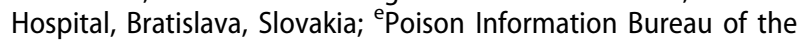
Health Emergency Situations Centre of the Ministry of Health,

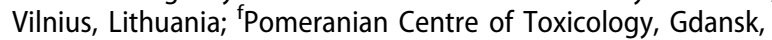
Poland; ${ }^{9}$ Estonian Poisoning Information Centre, Tallinn, Estonia; ${ }^{h_{T}}$ Toxicological Information Centre, Department of Occupational Medicine, 1st Faculty of Medicine, Charles University and General University Hospital in Prague, Prague, Czech Republic; 'Nofer Institute of Occupational Medicine, Łódź, Poland; 'Department of Toxicology, Péterfy Hospital, Budapest, Hungary; ${ }^{\mathrm{k} C l i n i c}$ of Toxicology, University Hospital for Emergency Medicine "N.I. Pirogov", Sofia, Bulgaria; 'Pomeranian Centre of Toxicology; Department of Clinical Toxicology, Medical University of Gdańsk, Gdańsk, Poland; ${ }^{m}$ National Poison Control Centre, Military Medical Academy, Belgrade, Serbia; ${ }^{n}$ Department of Pharmacology, Toxicology, and Therapeutics, University/BHF Centre for
Cardiovascular Science, University of Edinburgh, Edinburgh, United Kingdom

Objective: Across Europe, snakebites affect 4-11 people per million population annually [1]. In central and south-eastern Europe, snakes of the Viperidae family are common: Vipera berus, $V$. aspis, $V$. ammodytes, and V. ursinii. However, very little is known about the epidemiology of snakebite in this region. Our aim was to evaluate the epidemiology of Viperidae bites and antivenom use in central and south-eastern Europe over the summer of 2018 (April-September).

Methods: A prospective multicentre survey of Viperidae snakebites and use of antivenoms in poison control centres and toxicology departments in Estonia, Lithuania, Poland, Czech Republic, Slovakia, Hungary, Slovenia, Croatia, Macedonia and Bulgaria. Data reported included the number of confirmed Viperidae family snakebites, clinical picture, laboratory results, and antivenom treatment. Data from other invited countries (Latvia, Belarus, Russia, Serbia and Montenegro and Romania) was not available by October 2018. Audebert's clinical severity grading was used [2].

Results: Eleven poison control centres and toxicology departments in 10 countries completed the survey; covering a population of 43 million $(58 \%$ of entire population of included countries). Overall 126 Viperidae bites were recorded (2.9/million); antivenoms were administered to 20 patients $(0.47 / \mathrm{million})$. Ninety-three patients $(73.8 \%)$ were bitten by $V$. berus in 7 countries with median severity score $1 / 3 ; 12 / 93$ (12.9\%) were treated with antivenom (5 ViperaTab, 5 Biomed, 2 unknown). Thirteen patients were bitten by $V$. ammodytes (10\%) in 3 countries with median severity score $2 / 3 ; 7 / 13(53.8 \%)$ were treated with antivenom (3 ViperaTab, 4 Bulbio). In 20 cases, the species was unidentified and 1 was treated with ViperaTab. No bite by $V$. aspis and $V$. ursinii was reported. Antivenom adverse reactions and lethal outcome did not occur.

Conclusion: The incidence of Viperidae snakebites in central and south-eastern Europe is at least 2.9/million populations; but is likely more, since not all snakebites would have been treated in toxicology departments or reported to local poison control centers. $V$. berus bites were most common, and although $V$. ammodytes bites had a higher median severity score it was not significant ( 2 compared to 1 for $V$. berus bites; $p=0.18$ ). Antivenom was used in half of the reported $V$. ammodytes bites (compared to $13 \%$ of $V$. berus bites). Three different antiViperidae antivenoms were used, none of which is European Medicines Agency (EMA)-approved. Only ViperaTab was used in both $V$. berus and $V$. ammodytes bites.

\section{References}

[1] Chippaux JP. Epidemiology of snakebites in Europe. Toxicon. 2012;59:86-99.

[2] Audebert F. Envenoming by viper bites in France: clinical gradation and biological quantification by ELISA. Toxicon. 1992;30:599-609.

\section{Assessing potential health threats by monitoring poison control centre data: a new alert in Italy concerning toxic concentrations of ricin in fertilizers}

\author{
Franca Davanzo ${ }^{a}$, Andrea Bolognesi ${ }^{\mathrm{b}}$ and \\ Laura Settimi ${ }^{\mathrm{C}}$

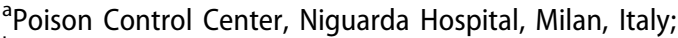 \\ ${ }^{\mathrm{b}}$ Department of Experimental Diagnostic and Specialty Medicine,
}


Bologna University, Bologna, Italy; 'Department of Environment and Health, National Institute of Health, Rome, Italy

Objective: Since the early 1980s, the Milan National Poison Control Centre (MNPCC) has implemented a surveillance and alerting system (MNPCC-SAS) to characterize hazardous exposures, identify unexpected events, support prevention and legislative acts and evaluate their impact [1]. The MNPCC-SAS recently generated an alert concerning organic nitrogen fertilizers (ONFs) containing ricin. We describe how this alert was generated and the resulting preventive actions in Italy.

Methods: A review of animal cases with severe or lethal poisoning associated with ingestion of organic nitrogen fertilizers (ONFs) occurred between May 2017 and August 2018. Laboratory detection of ricin in ONF samples and in vitro toxicity assays to measure functional activity of the detected ricin.

Results: In May 2017, the MNPCC was asked to provide toxicological advice for a dog that developed severe gastric effects within 6 hours following ingestion of an ONF (nitrogen 5.5\%). The dog died 24 hours after exposure due to internal hemorrhage. The MNPCC-SAS immediately generated an alert since this was unexpected for this type of product. The labelled contents of the ONF was $90-100 \%$ unspecified seed cake. Subsequently, it was determined that this was castor oil bean (Ricinus communis) cake and, therefore, suspected to contain ricin. Four lethal and five severe canine poisonings due to ingestion of castor oil cake ONFs were identified by the MNPCC-SAS over the next few months and promptly notified to the Italian Ministry of Health. Laboratory analyses detected more than $69 \mathrm{mg}$ ricin $/ 100 \mathrm{~g}$ of fertilizer. In October 2018 the Italian Ministry of Health removed these products from the market.

Conclusion: Ricin is a potent phytotoxin. The oral $\mathrm{LD}_{50}$ dose in humans has been estimated to be $1-20 \mathrm{mg} / \mathrm{kg}$ [2]. It can be easily extracted and used in terrorism and bio-crime [3]. In view of this and the canine cases reported in Italy and other countries [2], ricin in ONFs should be urgently regulated by European and national legislations and subject to stringent control.

\section{References}

[1] Settimi L, Davanzo F, Bacis G, et al. National information system for the surveillance of dangerous exposures and intoxication: cases detected in 2014. Ninth annual report. Rapporti ISTISAN 2018 [cited 2018 Oct 9]. Available from: http://old.iss.it/publ/index.php? lang $=1 \&$ anno $=2018 \&$ tipo $=5$

[2] Worbs S, Köhler K, Pauly D, et al. Ricinus communis intoxications in human and veterinary medicine - a summary of real cases. Toxins (Basel). 2011;3:1332-1372.

[3] Roxas-Duncan VU, Smith LA. Of beans and beads: ricin and abrin in bioterrorism and biocrime. J Bioterror Biodef. 2012;S2-S002.

\section{Once upon a time there was a frog ... toxicity from the kambô ritual}

\author{
Simone Lippmann, Christian Lakotta and \\ Daniela Acquarone \\ Poisons Information Center, Charité Universitätsmedizin Berlin, \\ Berlin, Germany
}

Objective: For many centuries South American shamans have traditionally used the poison of Phyllomedusa bicolor (giant leaf frog or kambô) as part of a cleansing ritual for healing a hunter's body and mind [1]. A shaman healer applies the frog's poisonous secretion into freshly burned skin. Subsequently, the subject experiences a kind of flash and has to vomit violently. This represents the process of cleansing. The frog's secretion contains peptides such as dermophin and deltorphin, which are potent opioid receptor agonists. There are case reports in the literature of liver toxicity [2], triggering a persistent psychosis [3] and even death due to cardiotoxicity [4]. In addition, there is a certain risk of dependency and addiction. The kambô ritual is becoming increasingly popular in Europe and we report such a case.

Case report: A young woman was admitted to hospital with persistent nausea, frontal stabbing headache and acute visual disturbances five hours after a kambô ritual. Three circular burn marks about one $\mathrm{cm}$ in size were found on the left upper arm. Immediately after the procedure, the patient suffered cramps in the hands and feet and vomited twice. There were no changes in consciousness. During examination, she had no neurological abnormalities or cardiovascular symptoms. All clinical symptoms could be attributed to the absorption of active peptides easily absorbed through the freshly wounded skin. She recovered without treatment within 12 hours.

Conclusion: From its origin in the tribes of the Amazon, the frog venom as well as various other shaman rituals, have spread throughout Europe and enjoy growing popularity as a spirituallyinspired medical practice. The result is an increase in medical emergencies that confront doctors in the emergency department with new challenges.

\section{References}

[1] Erspamer V, Erspamer GF, Severini C, et al. Pharmacological studies of 'sapo' from the frog Phyllomedusa bicolor skin: a drug used by the Peruvian Matses Indians in shamanic hunting practices. Toxicon. 1993;31:1099-1111.

[2] Pogorzelska J, Łapiński TW. Toxic hepatitis caused by the excretions of the Phyllomedusa bicolor frog - a case report. Clin Exp Hepatol. 2017;3:33-34.

[3] Roy R, Baranwal A, Espiridion ED. Can overuse of Kambô cause psychosis? Cureus. 2018;10:e2770.

[4] Aquila I, Gratteri S, Sacco MA, et al. The biological effects of kambo: is there a relationship between its administration and sudden death? J Forensic Sci. 2018;63:965-968.

\section{Severe thrombocytopenia after confirmed Crotalus horridus envenomation refractory to repeat bolus dosing of Crotalidae polyvalent immune fab}

\author{
Matthew K Griswold ${ }^{a}$, Dayne Laskey ${ }^{b}$, Patrick Filkins ${ }^{b}$ \\ and Lynn Farrugia ${ }^{a}$ \\ ${ }^{a}$ Emergency Medicine, Hartford Hospital, Hartford, United States; \\ ${ }^{b}$ Pharmacy Practice, University of Saint Joseph School of \\ Pharmacy, Hartford, United States
}

Objective: To describe a case of a patient who suffered rattlesnake envenomation and developed latent thrombocytopenia refractory to repeat bolus dosing of Crotalidae polyvalent immune fab (CPIF).

Case report: A 59-year-old man presented to the emergency department after an envenomation between the 1st and 2nd digits of his left hand by a known, geo-tagged 19-year-old timber rattlesnake (Crotalus horridus). He developed increased swelling of his forearm and received two loading doses of 6 vials CPIF with three subsequent 2 vial maintenance doses over the first 24 hours. After loading with CPIF, platelets were $202 \mathrm{k} / \mu \mathrm{L}$. This declined over subsequent days and became undetectable 96 hours after presentation (Table 1). The hemoglobin achieved a 
Table 1. Platelet counts in a patient bitten by Crotalus horridus and treated with antivenom in the first 24 hours and on day 7.

\begin{tabular}{lccccccccccccccc}
\hline & Day 1 & Day 2 & Day 3 & Day 4 & Day 5 & Day 6 & Day 7 & Day 8 & Day 9 & Day 10 & Day 11 & Day 12 & Day 13 & Day 14 \\
\hline Platelets $(\mathrm{k} / \mu \mathrm{L})$ & 202 & 172 & 124 & 19 & $<5$ & $<5$ & 5 & 13 & 7 & 6 & 8 & 20 & 24 & 43 \\
\hline
\end{tabular}

nadir of $5.6 \mathrm{~g} / \mathrm{dL}$ on day 6 and he received two red blood cell transfusions. CPIF was reloaded on day 7 with 8 vials given in two divided doses 6 hours apart based on success in other case reports [1]. The patient had a transient increase in platelet level to $13 \mathrm{k} / \mu \mathrm{L}$ which fell to $7 \mathrm{k} / \mu \mathrm{L}$ over the next day. On hospital day 14 the platelets rose above $40 \mathrm{k} / \mu \mathrm{L}$, at which point he was discharged to home. At follow-up 2 months later the platelets were $166 \mathrm{k} / \mu \mathrm{L}$ and swelling had improved significantly, however he continued to experience moderate neuropraxia of his $2 \mathrm{nd}$ and 3rd digits.

Conclusion: Envenomation by $C$. horridus is well associated with cases of severe thrombocytopenia refractory to CPIF administration. Prior publications have suggested that delayed repeat bolus dosing of CPIF may be beneficial in treated $C$. horridus venom induced thrombocytopenia [1]. We present a case where delayed repeat bolus dosing has no obvious effect on the patient's thrombocytopenia.

\section{Reference}

[1] Clark RF, O'Connell CW, Villano JH, et al. Severe recurrent coagulopathy following crotaline envenomation refractory to maintenance dosing of antivenom. Am J Emerg Med. 2015;33:856.e3-5.

\section{Gamma-hydroxybutyrate (GHB) and gamma-butyrolactone (GBL) poisonings admitted to the ICU: features and usefulness of plasma GHB concentration measurement}

\author{
Charlotte Héliodore ${ }^{a}$, Isabelle Malissin ${ }^{a}$, \\ Hervé Gourlain ${ }^{\mathrm{b}}$, Laurence Labat ${ }^{\mathrm{b}}$ and \\ Bruno Mégarbane ${ }^{a}$ \\ aDepartment of Medical and Toxicological Critical Care, Lariboisière \\ Hospital, Paris-Diderot University, INSERM UMRS-1144, Paris, \\ France; ${ }^{b}$ Laboratory of Toxicology, Lariboisière Hospital, Paris- \\ Diderot University, INSERM UMRS-1144, Paris, France
}

Objective: Gamma-hydroxybutyrate (GHB) and gamma-butyrolactone $(\mathrm{GBL})$ poisoning has reportedly increased recently in France. GHB, an anesthetic compound, has been classified as Schedule III controlled substance due to its recreational abuse potential and use for sexual assault. GBL, a GHB precursor, is legally marketed as a solvent although responsible for the same neurological toxicity and abuse liability. Our objectives were to describe the clinical features of acute $\mathrm{GHB} / \mathrm{GBL}$ poisoning, to investigate the predictive value of the plasma GHB concentration on admission and to study the relationships between the plasma GHB concentration and coma depth.

Methods: We conducted a retrospective single-center observational study including all GHB/GBL-poisoned patients admitted to the intensive care unit (ICU) over 7 years (January 2011 to July 2018). Plasma GHB concentration was measured using liquid chromatography coupled to mass spectrometry. We determined the GHB pharmacokinetic parameters, studied the correlation between the plasma GHB concentration and coma depth on admission, and modeled the relationships between the plasma
GHB concentrations and the Glasgow Coma Score (GCS) in each patient when possible.

Results: One hundred and thirteen GHB/GBL-poisoned patients (14 females/99 males, aged 33 years [16] (median, inter-quartile interval) were included. An increasing trend was observed in the number of GHB/GBL and mainly GBL poisonings. On ICU admission, the patients presented deep consciousness impairment (GCS 3 [4]), with marked hypotonia (36\%), osteotendinous reflex abolition $(47 \%)$, myosis $(48 \%)$, marked hypothermia $\left(35.5^{\circ} \mathrm{C}[1,4]\right)$ and increase in plasma creatine phosphokinase (235 UI/L $[903,25])$. Treatment was supportive including mechanical ventilation (76\%). Outcome was favorable with rapid wake-up and short hospitalization (17 hours [21]). Three major complications were noted including aspiration pneumonia (36\%), rhabdomyolysis (9\%) and withdrawal syndrome (2\%). No significant correlation between coma depth and plasma GHB concentration were observed on admission $\left(R^{2}=0.015\right)$. Individual modeling of the GCS/plasma GHB concentration relationships allowed the role of two major confounders, i.e. ethanol co-ingestion and tolerance to GHB due to chronic use. These relationships were well-fitted with the sigmoidal $\mathrm{E}_{\max }$ model, where a maximal toxic effect, suggesting the saturation of the drug receptors, was associated with a wide range of GHB concentrations. The high values of the Hill coefficients showed that a small decrease in plasma GHB concentrations near its $\mathrm{EC}_{50}$ resulted in a dramatic improvement in the consciousness level.

Conclusion: GHB/GBL poisonings are increasing in the ICU with life-threatening presentations. Despite the well-fitted sigmoidal relationships between the GCS and GHB concentration at each individual level, inter-individual variability in the expression of GHB-induced neurotoxicity exists, mainly related to ethanol coingestion and tolerance.

\section{Butanediol conversion to gamma-hydroxybutyrate markedly reduced by the alcohol dehydrogenase blocker fomepizole}

\author{
Evangelia Liakoni ${ }^{\mathrm{a}, \mathrm{b}}$, Hallam Gugelmann ${ }^{\mathrm{c}}$, \\ Delia A Dempsey ${ }^{a}$, Timothy J Wiegand ${ }^{d}$, \\ Christopher Havel ${ }^{a}$, Peyton Jacob ${ }^{a}$ and \\ Neal L Benowitz ${ }^{\mathrm{a}, \mathrm{c}, \mathrm{e}}$
}

aDivision of Clinical Pharmacology and Experimental Therapeutics, Department of Medicine, University of California, San Francisco, United States; ${ }^{\mathrm{b}}$ Clinical Pharmacology and Toxicology, Department of General Internal Medicine Inselspital, Bern University Hospital, University of Bern, Bern, Switzerland; 'California Poison Control Center, University of California, San Francisco, United States; dUniversity of Rochester Medical Center, Rochester, United States; e Department of Bioengineering and Therapeutic Sciences, University of California, San Francisco, United States

Objective: 1,4-Butanediol, a readily available industrial solvent, is rapidly and extensively converted to gamma-hydroxybutyrate (GHB) in vivo by the enzyme alcohol dehydrogenase (ADH), and is therefore abused for its euphoric effects. This study investigated the potential role of the ADH inhibitor fomepizole in moderating the conversion of butanediol to GHB in humans. Secondary outcomes included assessing the clinical effects of butanediol. 
Methods: Double-blind, placebo-controlled crossover study with six healthy volunteers. The participants received, on two different days, either intravenous placebo or $15 \mathrm{mg} / \mathrm{kg}$ fomepizole, followed by oral administration of $25 \mathrm{mg} / \mathrm{kg}$ butanediol. Blood samples for GHB and butanediol concentration analysis were collected up to 36 hours after butanediol dosing; vital signs were continuously monitored, and subjective drug effects were assessed by visual analog scale (VAS) questionnaires up to six hours after butanediol dosing.

Results: Pretreatment with fomepizole resulted in significantly higher butanediol maximal plasma concentrations (mean $\mathrm{Cmax}$ 29.8 versus $3.6 \mu \mathrm{g} / \mathrm{mL}, \mathrm{p}=0.001$ ) and area under the plasma concentration-time curve (median AUC last 6110.0 versus 179.4 $\min ^{*} \mu \mathrm{g} / \mathrm{m}, \mathrm{p}=0.028$ ) compared to the placebo arm. Conversely, GHB concentrations were low in the fomepizole arm, and high in the placebo arm (mean Cmax 10.9 versus $50.4 \mu \mathrm{g} / \mathrm{mL}, \mathrm{p}=0.001$ ). There were no significant differences in subjective responses as assessed by VAS scores between the fomepizole and placebo arm, and also no significant differences regarding the heart rate and oxygen saturation between the two arms or compared to the baseline. With fomepizole, the mean arterial pressure was significantly lower $(p<0.05)$ at 45 and 90 minutes compared to placebo and at 90, 105 and 120 minutes compared to baseline, while no significant differences to baseline were seen in the placebo arm.

Conclusion: Pretreatment with fomepizole resulted in significantly higher butanediol and lower GHB plasma concentrations and $A U C$, thus confirming that $A D H$ is the primary pathway for the conversion. Furthermore, blood pressure was significantly lower after fomepizole compared to placebo, which could indicate an intrinsic effect of butanediol. Based on our findings, further investigation of the utility of fomepizole as an antidote in cases of butanediol intoxication to prevent GHB toxicity might be warranted.

\section{Acute beta-blocker overdose: factors associated with cardiovascular mortality and management in the intensive care unit}

\author{
Dabor Resiere ${ }^{a}$, Bruno Mégarbane ${ }^{b}$, Bruno Sánchez ${ }^{c}$, \\ Julian Fabre ${ }^{\mathrm{d}}$ and Hossein Mehdaoui ${ }^{\mathrm{a}}$ \\ ${ }^{a}$ Critical Care Unit, University Hospital of Martinique, Martinique, \\ France; ${ }^{b}$ Medical and Toxicological ICU, Lariboisière Hospital, Paris, \\ France; 'Department of Cardiovascular Surgery, University Hospital \\ of Martinique, Martinique, France; ${ }^{\mathrm{d}}$ Department of Cardiology, \\ University Hospital of Martinique, Martinique, France
}

Objective: Beta-adrenergic antagonists are commonly used worldwide to treat hypertension, tremor, migraines, ischemic heart disease, heart failure, arrhythmias, portal hypertension, angina and panic attacks. Propranolol, a beta-adrenergic antagonist with membrane stabilizing properties, is the most common toxicant used in suicide attempts in Martinique. Although respiratory depression, bronchospasm, bradycardia, severe hypotension and seizures may result from beta-blocker intoxication, wide QRS intervals and cardiovascular depression represent the most common cause of morbidity and mortality. Our objective was to determine factors associated with the development of cardiovascular mortality [1].

Methods: We conducted a prospective study over 10 years (January 2005 to December 2015), including all poisoned patients admitted to the intensive care unit. Beta-blocker-poisoned patients were identified and clinical and biological parameters associated with the onset of death were analyzed based on univariate analysis.
Results: Overall 308 poisoned patients (173 males/135 females; aged 47 years [16-79] (median [percentiles 25-75]; Simplified Acute Physiology Score (SAPS) II of 120 [49-94]) were admitted to the intensive care unit during the study period. Among these patients, 100 had ingested presumed elevated doses of cardiotoxicants (class-I anti-arrhythmic drugs $40 \%$, beta-blockers $15 \%$, calcium-channel blockers 10\%). Overall 50 of the 100 patients survived. The mortality rate attributed to beta-blocker poisoning was $32 \%$. Eighteen patients with prolonged cardiac arrest treated with extracorporeal membrane oxygenation (ECMO) survived. Prognosticators in beta-blocker poisoning were $(p<0.05)$ : QRS widening on admission, arterial $\mathrm{pH}$ and lactate concentration, SAPS II score on admission and ECMO performance under massage.

Conclusion: The most important factor associated with increased risk of cardiovascular mortality in beta-blocker poisoning is exposure to a beta-blocker with stabilizing activity. ECMO appears to be an efficient salvage technique in case of refractory toxic cardiac failure or arrest.

\section{Reference}

[1] Jeffrey NL, John MH, Toby LL, et al. Acute beta blocker overdose: factors associated with the development of cardiovascular mortality. Clin Toxicol (Phila). 2000;38:275-281.

\section{Access and use of bystander naloxone among emergency department patients with opioid abuse in the era of adulterated heroin with fentanyl}

\author{
Hannah Bach ${ }^{a}$, Siamak Moayedi ${ }^{b}$, Brenten R Hurt ${ }^{c}$, \\ Atizaz Hussain ${ }^{c}$, Valerie R Jenkins ${ }^{c}$, Ilya J Lazzeri ${ }^{c}$, \\ Stephen Schenkel ${ }^{\mathrm{b}}$ and Hong $\mathrm{K} \mathrm{Kim}^{\mathrm{b}}$ \\ ${ }^{a}$ Emergency Medicine, University of Maryland Medical Center, \\ Baltimore, United States; ${ }^{\mathrm{b}}$ Emergency Medicine, University of \\ Maryland School of Medicine, Baltimore, United States; 'University \\ of Maryland School of Medicine, Baltimore, United States
}

Objective: Increase in opioid-related deaths has prompted the expansion of bystander naloxone (BNAL) programs. Despite efforts to increase access to BNAL, it is unknown if opioid abusers are able to obtain and use BNAL. Therefore, we conducted a study to determine the access and use of BNAL among emergency department (ED) patients with opioid abuse.

Methods: Cross-sectional study conducted at an urban hospital in Baltimore, Maryland, from 20 May to 30 July 2018. Research assistants screened ED patients from 12:00 to 22:00, 7 days/week. Consenting adult patients with active opioid abuse (use within the last 7 days) were enrolled. Survey was performed to obtain demographic data, substance abuse history, and patients' access and use of BNAL. Rapid Response ${ }^{\text {TM }}$ Single Drug Test Strips (BTNX Inc. ON, Canada) were used to detect fentanyl in urine samples. Our primary outcome was possession of BNAL; secondary outcomes included prevalence of fentanyl, knowledge and use of BNAL, and barriers to obtaining and using BNAL. Descriptive and subgroup analysis were performed.

Results: Overall 451 ED patients were identified with substance abuse during our study period; of these, 208 reported opioid abuse and 165 patients consented to participate. The mean age was 46.4 years and $127(77.0 \%)$ were male. Survey results are summarized in Table 1. A large proportion of patients (69.7\%; $\mathrm{n}=115$ ) obtained BNAL through a distribution program or with a 
Table 1. Survey questions and results regarding bystander naloxone (BNAL) among opioid users presenting to an emergency department.

\begin{tabular}{|c|c|c|c|c|c|c|}
\hline \multirow[b]{3}{*}{ Questions } & \multirow{2}{*}{\multicolumn{2}{|c|}{$\begin{array}{l}\text { All patients } \\
(n=165)\end{array}$}} & \multirow{2}{*}{\multicolumn{2}{|c|}{$\begin{array}{l}\text { Fentanyl positive } \\
\qquad(\mathrm{n}=104)\end{array}$}} & \multirow{2}{*}{\multicolumn{2}{|c|}{$\begin{array}{c}\text { Overdose patients } \\
(n=42)\end{array}$}} \\
\hline & & & & & & \\
\hline & Yes & No & Yes & No & Yes & No \\
\hline 1. Do you know of BNAL & $118(71.5 \%)$ & $47(28.5 \%)$ & $78(75.0 \%)$ & $26(25.0 \%)$ & $24(57.1 \%)$ & $18(42.9 \%)$ \\
\hline 2. Did you receive BNAL Rx? & $39(23.6)$ & $126(76.3 \%)$ & $26(25.0 \%)$ & $78(75.0 \%)$ & $6(14.3 \%)$ & $36(85.7 \%)$ \\
\hline $\begin{array}{l}\text { a) If Yes, you received a Rx, did you fill it? } \\
\text { b) If No, what were barriers to obtaining BNAL? }\end{array}$ & $25(64.1 \%)$ & $14(35.9 \%)$ & $18(69.3 \%)$ & $8(30.8 \%)$ & $2(33.3 \%)$ & $4(66.7 \%)$ \\
\hline - Cost & $3(21.4 \%)$ & NA & 1 & NA & 2 & NA \\
\hline - Lost the Rx & $2(14.3 \%)$ & NA & 1 & NA & 1 & NA \\
\hline - Did not attempt to fill & $5(35.7 \%)$ & NA & 4 & NA & 2 & NA \\
\hline - Pharmacy did not have BNAL & $1(7.1 \%)$ & NA & 1 & NA & 0 & NA \\
\hline - Unknown & $3(21.4 \%)$ & NA & 1 & NA & 1 & NA \\
\hline 3. Did you receive BNAL kit? & $90(54.6 \%)$ & $75(45.4 \%)$ & $60(57.7 \%)$ & $44(42.3 \%)$ & $15(35.7 \%)$ & $27(64.3 \%)$ \\
\hline a) If yes, you received a kit, did you receive BNAL training? & $78(86.7 \%)$ & $12(13.3 \%)$ & $53(88.3 \%)$ & $7(6.7 \%)$ & $14(93.3 \%)$ & $1(6.7 \%)$ \\
\hline b) If yes, did you use your BNAL? & $42(46.7 \%)$ & $48(53.3 \%)$ & $29(48.3 \%)$ & $31(51.7 \%)$ & $4(26.7 \%)$ & $11(73.3 \%)$ \\
\hline $\begin{array}{l}\text { 4. Do you know how to administer BNAL? } \\
\text { a) If no, why not? }\end{array}$ & $99(60.4 \%)$ & $65(39.6 \%)$ & $66(63.5 \%)$ & 37 (35.6\%) & $21(50.0 \%)$ & $20(47.6 \%)$ \\
\hline - Did not receive training & $63(96.9 \%)$ & NA & $35(94.6 \%)$ & NA & $20(100.0 \%)$ & NA \\
\hline - Trained but forgot & $2(3.1 \%)$ & NA & $2(5.4 \%)$ & NA & NA & NA \\
\hline 5. Do you use opioid with someone who has access to BNAL? & $61(37.0 \%)$ & $103(62.4 \%)$ & $40(38.5 \%)$ & $64(61.5 \%)$ & $12(28.6 \%)$ & $29(69.1 \%)$ \\
\hline
\end{tabular}

prescription. Although up to $63.5 \%$ of the patients knew how to administer BNAL, lack of training was the main reason for patients' inability to administer BNAL. ED patients with acute overdose were less familiar with BNAL, including its access and use, compared to other groups.

Conclusion: The majority of the patients obtained BNAL and training through BNAL distribution programs. However, knowledge, access and use of BNAL by ED patients with acute overdose were low.

\section{Prevalence of fentanyl exposure among emergency department patients with history of opioid abuse}

\author{
Hannah Bach ${ }^{\mathrm{a}}$, Siamak Moayedi ${ }^{\mathrm{b}}$, Brenten R Hurt ${ }^{\mathrm{c}}$, \\ Hussain Atizaz', Valerie R Jenkins ${ }^{c}$, llya J Lazzeri', \\ Stephen Schenkel ${ }^{\mathrm{b}}$ and Hong K Kim ${ }^{\mathrm{b}}$ \\ ${ }^{a}$ Emergency Medicine, University of Maryland Medical Center, \\ Baltimore, United States; ${ }^{b}$ Emergency Medicine, University of \\ Maryland School of Medicine, Baltimore, United States; 'University \\ of Maryland School of Medicine, Baltimore, United States
}

Objective: There has been a dramatic increase in the production and distribution of illicitly manufactured fentanyl over the past 5 years. Consequently, overdose fatalities due to fentanyl and its analogs from the use of adulterated heroin has spiked significantly. It is unknown how much heroin supply is adulterated with fentanyl and what proportion of heroin users have been exposed to fentanyl. Therefore, the purpose of our study was to determine the prevalence of fentanyl exposure in Emergency Department (ED) patients with active opioid abuse.

Methods: We conducted a cross-sectional study at an urban hospital in Baltimore, Maryland, from 20 May 2018 to 30 July 2018. Research assistants identified eligible ED patients from 12:00 to 22:00, 7 days a week. Consenting adult patients with active opioid abuse, defined as use within the last 7 days, were enrolled. A survey was performed to obtain information on demographic, substance abuse history, and knowledge of fentanyl. Rapid Response $^{\text {TM }}$ Single Drug Test Strip (BTNX Inc. ON, Canada) was used to detect fentanyl in urine samples. Our primary outcome was the prevalence of fentanyl exposure; secondary outcomes included patient's knowledge regarding strength and risk of overdose death from opioids and the prevalence of intentional abuse of fentanyl. Descriptive and subgroup analysis were performed.
Results: Overall 451 ED patients reported substance abuse of any kind during the study period. Of these patients, 208 reported active opioid abuse; 165 patients consented to the study. The mean age was 46.4 years and $77.0 \%(n=127)$ were male. Acute opioid overdose occurred in 42 patients. The majority of the patients $(89.7 \%)$ identified heroin as their preferred opioid of abuse via the intranasal route $(64.2 \%)$. Cocaine $(57.6 \%)$ and marijuana $(54.5 \%)$ were most commonly reported concurrent drugs of abuse. Most patients $(84.2 \%, \mathrm{n}=139)$ identified fentanyl as a high potency opioid while $85.5 \%(n=141)$ reported highest risk of death in fentanyl overdose. Yet, 47 (28.5\%) intentionally purchased fentanyl for abuse. Of the 134 urine samples obtained, fentanyl was detected in 104 patients $(77.6 \%) ; 72.1 \%(n=74)$ reported no history of intentional purchase of fentanyl for abuse. Most overdose patients (85.7\%) tested positive for fentanyl.

Conclusion: The majority of ED patients with active opioid use were exposed to fentanyl. Despite the knowledge of fentanyl's potency and high risk of death from overdose, a significant proportion of ED patients intentionally purchased fentanyl for abuse. Future studies are needed to further characterize those at risk for fentanyl exposure.

\section{Towards real-time opioid abuse surveillance: machine learning for automatic characterization of opioid- related tweets}

\author{
Abeed Sarker $^{a}$, Graciela Gonzalez-Hernandez ${ }^{b}$, \\ Francis De Roos ${ }^{c}$ and Jeanmarie Perrone ${ }^{c}$ \\ aiostatistics, Epidemiology and Informatics, University of \\ Pennsylvania, Philadelphia, United States; ${ }^{\text {b University of }}$ \\ Pennsylvania, Philadelphia, United States; ' ${ }^{\circ}$ Emergency Medicine, \\ University of Pennsylvania, Philadelphia, United States
}

Objective: Social media may serve as an important platform for the surveillance of population-level opioid abuse in near realtime. Our objectives for this study were to (i) manually characterize a small number of opioid-mentioning Twitter posts, and (ii) to implement and evaluate the performance of a supervised machine learning algorithm for the automatic characterization of opioid-related chatter to improve the current state of opioid toxicosurveillance.

Methods: We collected data from Twitter via the public streaming application program interface (API) using prescription and 
illicit opioid keywords (e.g. Percocet ${ }^{\circledast}$ and heroin), including street names (e.g. China white) and common misspellings (e.g. percoset). We developed annotation guidelines using the Grounded Theory approach [1]; we first grouped tweets into topics and then into broad categories. Two annotators manually categorized a random sample of the tweets into four classes: self-reported abuse, information sharing, non-English, and unrelated. We applied natural language processing methods to preprocess the tweet texts and generate feature vectors. We then trained and evaluated a Random Forest classifier via 10 -fold cross validation, a process in which $90 \%$ of the data are used for training and $10 \%$ is used for evaluation in 10 cycles.

Results: We annotated a total of 5979 tweets, with 550 overlapping tweets between the two annotators to compute agreement. Inter-annotator agreement was $\mathrm{K}=0.75$ (Cohen's kappa), representing moderate agreement. Agreement was hampered by the lack of context in many tweets, for which the categorization depended on subjective assessment of the annotator. Disagreements were resolved by a third annotator. The final data set consisted of 1108 (18.53\%) abuse tweets, 1382 (23.11\%) information tweets, 3206 (53.62\%) unrelated tweets, and 283 (4.73\%) non-English tweets. The classifier obtained an accuracy of $68.7 \%$. Analysis of the errors revealed that lack of context in tweets frequently caused the classifier to classify tweets wrongly to the unrelated class.

Conclusion: Our manual categorization efforts revealed the difficulty of annotating tweets with high annotator agreement. The automatic classification experiments produced promising results, despite the small amount of annotated data, suggesting that automated, real-time opioid toxicosurveillance may be a possibility with more annotated data.

\section{Acknowledgments}

This study is funded in part by the NIDA/NIH (R01DA046619) and by a grant from the Pennsylvania Department of Health. The content is solely the responsibility of the authors.

\section{Reference}

[1] Martin PY, Turner BA. Grounded theory and organizational research. J Appl Behav Sci. 1986;22:141-157.

\section{Characterizing opioid-related tweets: a comparison between prescription and illicit opioid chatter}

\author{
Abeed Sarker ${ }^{a}$, Graciela Gonzalez-Hernandez ${ }^{b}$, \\ Francis De Roos ${ }^{c}$ and Jeanmarie Perrone ${ }^{c}$ \\ ${ }^{a}$ Biostatistics, Epidemiology and Informatics, University of \\ Pennsylvania, Philadelphia, United States; ${ }^{\text {bUniversity of }}$ \\ Pennsylvania, Philadelphia, United States; 'Emergency Medicine, \\ University of Pennsylvania, Philadelphia, United States
}

Objective: Social media data capture an abundance of information about substance abuse, including opioid abuse, in near real-time. Our objectives were to manually characterize a sample of posts from Twitter (tweets) mentioning opioids and to compare these characterizations between illicit opioids (IO) and prescription opioids (PO).

Methods: We collected data from Twitter using PO and IO keywords (e.g. Percocet ${ }^{\oplus}$ and heroin), including street names (e.g. China white) and common misspellings (e.g. percocit). Two annotators manually categorized a random sample of the tweets into three classes: self-reported abuse, information sharing and unrelated. We compared the volumes and proportions of tweets belonging to these categories for POs and IOs.

Results: We annotated a total of 8153 tweets, with 550 overlapping tweets between the two annotators to compute agreement. Inter-annotator agreement was moderate [1] $(\mathrm{k}=0.75$; Cohen's kappa). The majority of the tweets mentioned IOs rather than POs (6094 versus 2059). However, the proportions of abuserelated and information-sharing tweets were significantly higher for PO tweets, and the proportion of unrelated tweets was much higher for 10 tweets (Table 1). Our analysis of the tweets revealed that 10 tweets were highly noisy, with references to songs or movie dialogues, leading to a large proportion to be categorized as unrelated.

Conclusion: There is significant variation in the contents of tweets mentioning POs and IOs. Future methods attempting to utilize social media big data for opioid toxicosurveillance should take into account the low signal-to-noise ratio, particularly for IOs, and devise automatic filtering strategies.

\section{Acknowledgments}

This study is funded in part by the NIDA/NIH (R01DA046619) and by a grant from the Pennsylvania Department of Health. The content is solely the responsibility of the authors.

\section{Reference}

[1] Landis JR, Koch GG. The measurement of observer agreement for categorical data. Biometrics. 1977;33:159-174.

\section{Influence of genetic polymorphisms within the serotonin system on the acute effects of 3,4- methylenedioxymethamphetamine (MDMA)}

\author{
Patrick Vizeli ${ }^{\mathrm{a}}$, Henriette E Meyer Zu Schwabedissen ${ }^{\mathrm{b}}$ \\ and Matthias E Liechti ${ }^{\mathrm{a}}$ \\ ${ }^{a}$ Clinical Pharmacology and Toxicology, University Hospital Basel,

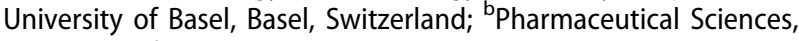 \\ University of Basel, Basel, Switzerland
}

Objective: 3,4-Methylenedioxymethamphetamine (MDMA, ecstasy) use can lead to adverse events such as sympathomimetic toxicity or hyperthermia. Most of the acute effects of MDMA

Table 1. Distributions of tweets mentioning illicit and prescription opioids, their annotated categories, proportions within each category and $95 \%$ confidence intervals $(\mathrm{Cl})$.

\begin{tabular}{|c|c|c|c|c|c|c|}
\hline \multirow[b]{2}{*}{ Tweet category } & \multicolumn{3}{|c|}{ Illicit opioids } & \multicolumn{3}{|c|}{ Prescription opioids } \\
\hline & Count & Proportion & $95 \% \mathrm{Cl}$ & Count & Proportion & $95 \% \mathrm{Cl}$ \\
\hline Abuse & 1212 & 0.199 & $0.19-0.21$ & 555 & 0.270 & $0.25-0.29$ \\
\hline Information & 1348 & 0.221 & $0.21-0.23$ & 659 & 0.320 & $0.30-0.34$ \\
\hline
\end{tabular}


including its mood, cardiostimulant, and thermogenic effects can be attributed to the release of serotonin (5-hydroxytryptamine, 5HT) [1]. Accordingly, we hypothesized that genetic variants including single-nucleotide polymorphisms and polymorphic regions in the 5 -HT system genes influence the acute responses to MDMA in humans.

Methods: We characterized the effect of common genetic variants within genes coding for the $5-\mathrm{HT}$ system (TPH1 rs1800532

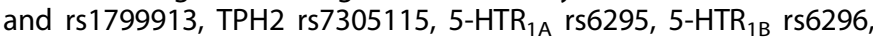
$5-\mathrm{HTR}_{2 \mathrm{~A}}$ rs6313, and SLC6A4 5-HTTLPR and rs25531) on the acute physiological and subjective response to $125 \mathrm{mg}$ MDMA compared to placebo in 124 healthy subjects (64 women) using data derived from eight similar randomized, double-blind, placebocontrolled crossover studies from the same laboratory Differences in maximal effects (MDMA-placebo) were analyzed using one-way analyses of variance (ANOVAs) with genotype as between-subject factor. The area under the MDMA plasma concentration-time curve from 0-6 hours was included as covariate in the ANOVAs. Results were additionally corrected for multiple testing using the Nyholt method.

Results: Carriers of the 5-HTR $2 \mathrm{~A}$ rs6313 A-allele displayed higher scores of "good drug effect" compared to homozygous G-carriers (mean \pm SD increase: $80 \pm 25$ versus $66 \pm 29 ; F_{1,121}=6.93, p=0.01$ ). Individuals with a short-allele of the SLC6A4 5-HTTLPR had higher ratings for "good drug effect", "drug liking", and lower ratings for "bad drug effect" compared to homozygous long-allele carriers ( $79 \pm 27$ versus $70 \pm 27,80 \pm 26$ versus $72 \pm 27$, and $13 \pm 25$ versus $25 \pm 25$, respectively; $F_{1,121}=6.51, p=0.01, F_{1,121}=5.06, p=0.03$ and $F_{1,121}=4.94, p=0.03$, respectively). MDMA produced a higher peak increase in body temperature in G-allele carriers of the TPH2 rs7305115 compared to homozygous A-allele individuals $\left(0.3 \pm 0.5\right.$ versus $\left.-0.1 \pm 0.6^{\circ} C_{;} F_{1,121}=4.84, p=0.01\right)$. There was no difference in the cardiostimulant effect of MDMA associated with any of the genetic variants tested. After correction for multiple testing, none of the above-mentioned effects of genetic polymorphisms within the 5-HT system that altered the response to MDMA remained statistically significant.

Conclusion: The findings suggest that variations in genes coding for 5-HT system targets do not significantly influence the effects of MDMA in healthy subjects and are unlikely to play a role in MDMA intoxications despite the clear role of $5-\mathrm{HT}$ as a mediator of MDMA's effects and toxicity.

\section{Reference}

[1] Liechti ME, Baumann C, Gamma A, et al. Acute psychological effects of 3,4-methylenedioxymethamphetamine (MDMA, "Ecstasy") are attenuated by the serotonin uptake inhibitor citalopram. Neuropsychopharmacology. 2000;22:513-521.

\section{The time-course of the serotoninergic syndrome in relation to plasma 3,4-methylenedioxymetham- phetamine (MDMA) concentrations in severely poisoned patients}

\author{
Mathieu Bouthemy ${ }^{\mathrm{a}}$, Isabelle Malissin ${ }^{\mathrm{a}}$, \\ Marion Soichot ${ }^{b}$, Laurence Labat ${ }^{\mathrm{b}}$ and \\ Bruno Mégarbane ${ }^{a}$ \\ a Department of Medical and Toxicological Critical Care, Lariboisière \\ Hospital, Paris-Diderot University, INSERM UMRS-1144, Paris, \\ France; ${ }^{b}$ Laboratory of Toxicology, Lariboisière Hospital, Paris- \\ Diderot University, INSERM UMRS-1144, Paris, France
}

Objective: The recreational use of 3,4-methylenedioxymethamphetamine (MDMA) has become common since the late 1990s and the number of poisonings has significantly increased during recent years. MDMA is responsible for serotoninergic toxicity leading to the onset of hyperthermia and multiorgan failure. Our objectives were to describe the time course of body temperature changes, serotoninergic signs and biological parameters in severely MDMA-poisoned patients in order to understand the evolution of these parameters in relation to the plasma concentration of MDMA and its main metabolite, methylenedioxyamphetamine (MDA).

Methods: We conducted a retrospective single-centre observational study including all MDMA-poisoned patients admitted to the intensive care unit (ICU) and who developed fever (body temperature $>38.5^{\circ} \mathrm{C}$ ) and symptoms/signs of serotoninergic syndrome (according to Sternbach's criteria). Plasma MDMA and MDA concentrations were determined using liquid chromatography coupled to mass spectrometry.

Results: Sixteen MDMA-poisoned patients (out of 58 MDMAexposed patients admitted to the ICU over a 10-year period) who presented serotoninergic syndrome with fever $\left(>38.5^{\circ} \mathrm{C}\right)$ attributed to MDMA exposure and treated with supportive care, external cooling and cyproheptadine (a non-specific serotonin receptor antagonist) were included in this study. The patients were 6 females and 10 males, aged 22-years-old [20; 26] (median [percentiles $25 ; 75$ ] and had used recreational MDMA in a nightclub $(75 \%)$, in the street $(19 \%)$ or at home $(6 \%)$. Body temperature reached $39.7^{\circ} \mathrm{C}[38.8 ; 40.9]$ and the presentation was complicated by cardiac arrest (19\%), cardiovascular failure (44\%), aspiration pneumonia (56\%), hospital-acquired infection (25\%) and fatality $(6 \%)$. In four patients $(25 \%)$, worsening in the clinical (heart rate, pyramidal syndrome, electroencephalogram encephalopathy) and biological parameters (transaminases, prothrombin index, creatine kinase) included in the serotoninergic syndrome was surprisingly observed in parallel to the increase in body temperature (due to a concomitant infectious or inflammatory non-toxic event). The observed worsening occurred in the absence of any increase in the plasma MDMA and MDA concentrations $(n=2)$ and even in the presence of undetectable concentrations $(n=2)$.

Conclusion: MDMA use results in serotonin syndrome possibly leading to the onset of hyperthermia, organ failure and death, despite optimal supportive care, cooling and cyproheptadine administration. Worsening in the serotonin syndrome during the ICU stay of severely MDMA-poisoned patients, without increase in plasma MDMA concentration was observed in $25 \%$ of patients. The exact molecular mechanisms involved in this "serotoninergic memory" remain to be clarified.

\section{Efficacy and safety of dantrolene in toxic hyperthermia: a systematic review}

\author{
Alexandre Doucet ${ }^{\mathrm{a}}$, Hugo Langlois ${ }^{\mathrm{b}}$, Eric Villeneuve ${ }^{\mathrm{b}}$, \\ Camille Tétreault ${ }^{c}$, Josh J Wang ${ }^{d}$ and Sophie Gosselin ${ }^{e}$ \\ ${ }^{a}$ Faculty of Medicine, McGill University, Montréal, Canada; \\ ${ }^{\mathrm{b}}$ Pharmacy Department, McGill University Health Centre, Montréal, \\ Canada; 'Faculty of Medicine, Sherbrooke University, Sherbrooke, \\ Canada; ${ }^{\mathrm{d} D e p a r t m e n t}$ of Emergency Medicine, Division of Medical \\ Toxicology, New York University School of Medicine, New York,

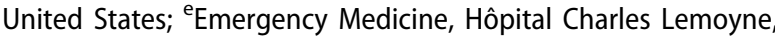 \\ Longueuil, Canada
}

Objective: Assess the efficacy and safety of dantrolene treatment for hyperthermia with a focus on toxin-induced hyperthermia. 
Table 1. Aggregate data of case reports (164 total cases from 153 case reports) of dantrolene use in toxic hyperthermia.

\begin{tabular}{|c|c|c|c|c|c|}
\hline & $\mathrm{N}$ & $\begin{array}{c}\text { Dantrolene dose leading } \\
\text { to symptoms improve- } \\
\text { ment }(\mathrm{mg} / \mathrm{kg}) \text {, median } \\
\text { (IQR) }\end{array}$ & Mortality (\%) & $\begin{array}{c}\text { Patients with reported } \\
\text { temperature decrease post } \\
\text { dantrolene }(\%)\end{array}$ & $\begin{array}{c}\text { Maximal temperature } \\
\text { reported (Celsius), mean } \\
(\mathrm{SD})\end{array}$ \\
\hline Miscellaneous anesthetics & 98 & $2.5(2-4.25)$ & 7.1 & 92 & $39.4(1.30)$ \\
\hline $\begin{array}{l}\text { Neuroleptic malignant } \\
\text { syndrome (NMS) }\end{array}$ & 45 & $2(1-7.75)$ & 2 & 86 & $39.6(0.98)$ \\
\hline Miscellaneous toxins & 20 & $1.5(1-2.63)$ & 20 & 90 & $41.1(1.46)$ \\
\hline Heatstroke & 1 & 24 & 0 & 100 & 42.2 \\
\hline
\end{tabular}

Methods: Multiple publication databases were systematically searched from inception to 31 December 2017 to identify human studies in English, French and Spanish. Randomized control trials (RCT), observational studies and case reports were included. Article selection followed the Preferred Reporting Items for Systematic Reviews and Meta-Analyses (PRISMA) system using predefined criteria for the use of dantrolene in the acute treatment of hyperthermia.

Results: Of 757 full-text articles, 157 met our study criteria. No randomised controlled trial (RCT) on toxin-induced hyperthermia but two on heatstroke were found. One reported a 19.5 minutes difference in cooling time for patients treated with dantrolene $2.5 \mathrm{mg} / \mathrm{kg}$. None reported improved neurological outcomes, survival or length of stay in patients treated with a single dose of dantrolene $2-4 \mathrm{mg} / \mathrm{kg}$ compared to conventional cooling measures. One observational study on malignant hyperthermia (MH) reported an average $2.4{ }^{\circ} \mathrm{C}$ decrease, normalization of heart rate and rigidity after one dose of dantrolene $2.5 \mathrm{mg} / \mathrm{kg}$. Another observational study reported a vague significant improvement in temperature and rigidity with dantrolene $2.5 \mathrm{mg} / \mathrm{kg}$ in patients with neuroleptic malignant syndrome compared to treatment with other medications. Table 1 presents aggregate data on 153 case reports. Precise chronology and magnitude of reported improvements is lacking. The overall evidence is very low with only one RCT achieving moderate Grading of Recommendations Assessment, Development and Evaluation (GRADE). No major adverse reactions were identified.

Conclusion: The use of dantrolene outside of $\mathrm{MH}$ is not supported by high quality evidence. While dantrolene seems efficacious to treat toxin-induced rigidity and hyperthermia or heatstroke, its use is mainly anecdotal with very poor reporting of essential data. Standardized reporting of essential criteria to assess dantrolene treatment for toxin-induced hyperthermia would improve evaluation of its potential efficacy.

\section{Pollyanna in an age of fentanyls}

\author{
Erik Lindeman and Jenny Westerbergh \\ Swedish Poisons Information Center, Stockholm, Sweden
}

Objective: Swedish poison centre (PC) enquiries concerning new psychoactive substances (NPS) have decreased by $70 \%$ since 2014. Declining numbers of NPS notified to the EU-Early Warning system (EWS) and a decline in self-reported NPS-use in Swedish high school surveys suggest that the PC data reflects a true reduction in prevalence. Factors related to the societal normalization of NPS, including increasing physician familiarity with the phenomenon and less active research from the PC, may bias PC data however. Furthermore, a large contemporary increase in fatal NPS-poisonings in Sweden has been established by the National Board of Forensic Medicine. According to the Swedish police this increase in NPS-fatalities is almost entirely caused by fentanyls disseminated via the Internet, mainly as nasal sprays. In 2016-17 deaths from these drugs outnumbered heroin deaths in Sweden. Fentanyl also occurs as diverted medicinal patches, but no seizures of fentanyls sold as or admixed to heroin or other street drugs have been made in Sweden [1]. The purpose of this study was to determine if the epidemic of fatal fentanyl poisoning was discernible in PC call data.

Methods: Suspected fentanyl/fentanyl analog exposures from 2012-17 were retrieved from the PC data base and compared to published records of analytically confirmed fentanyl/fentanyl analog deaths [1].

Results: PC data: Fentanyl patch abuse calls were stable at around 30/year. Internet fentanyls appeared in PC records from 2014 with 6 calls, followed by 36, 86 and 75 in 2015-17. No deaths were explicitly recorded and clinical details suggest the vast majority of patients survived. Published data: Investigations have established that 90 analytically confirmed fentanyl deaths 2014-16 were caused by fentanyl sold as fentanyl analogs over the Internet. Deaths from fentanyl originating from medicinal products have been stable at around 30/year since 2011. Fentanyl analogs appear in the forensic data in 2015 with 34 deaths, followed by 74 and 101 in 2016-17 [1].

Conclusion: The opposing contemporary Swedish trends of falling PC call rates and rising death rates related to NPS illustrate the weakness of PC data in detecting poisoning fatalities. No deaths from fentanyls, the main forensic culprits, were recorded in PC records, implying insignificant overlap between cohorts. Although not causally connected, the ratio of PC cases to forensic deaths during the period is remarkably small; $1: 1$ for fentanyl patch abuse and 1:1.5 for Internet fentanyls, suggesting that the mere appearance of fentanyls in PC calls should be a cause for concern.

\section{Reference}

[1] Swedish Police Forces: National status report on fentanyl analogs 2018. ref A503.217/2017 v02.00.

\section{Epidemiology of fentanyl exposures reported to US Poison Centers, 2014-2017}

\author{
Saumitra Rege ${ }^{a}$, Duc Anh Ngo ${ }^{b}$, Asaad I Alsufyani ${ }^{a}$ \\ and Christopher $\mathrm{P}$ Holstege ${ }^{\mathrm{a}}$ \\ ${ }^{a}$ Division of Medical Toxicology, Department of Emergency \\ Medicine, University of Virginia, Charlottesville, United States; \\ ${ }^{b}$ Division of Student Affairs, Department of Student Health, \\ University of Virginia, Charlottesville, United States
}

Objective: There were approximately 63,000 drug overdose deaths reported in the US in 2016, with approximately $66 \%$ involving an opioid. Synthetic opioids, including fentanyl, accounted for nearly half of these deaths, an increase of $14 \%$ from 2010 [1]. This study examines the epidemiology of fentanyl exposures reported to US poison centers (PCS).

Methods: The National Poison Data System (NPDS) was queried for all closed, human exposures to fentanyl from 2014 to 2017 using generic code identifiers. Key demographic and clinical 
characteristics were descriptively assessed. Reports from acute care hospitals and emergency departments $(\mathrm{ACH})$ were analyzed. Trends in fentanyl frequencies and rates (per 100,000 human exposures) were analyzed using Poisson regression with percent changes being reported.

Results: There were 6,021 fentanyl exposures reported to PCs, with calls increasing $(1,421$ to 1,783$)$ during the study period. Among the overall fentanyl calls, $64.5 \%$ calls were received from ACH. Multiple substance exposures accounted for $50.6 \%$ of calls and $55.1 \%$ of calls from ACH. Residence was the most common site of exposure (84.8\%). Among cases, $51.9 \%$ were males, and $39.5 \%$ were aged between $20-39$ years. Intentional abuse $(36.7 \%)$ and suspected suicides (18.8\%) were commonly observed reasons for exposure. The proportion of intentional abuse $(40.1 \%)$ and suspected suicides (25.5\%) was higher in cases reported by $\mathrm{ACH}$. The most frequent co-occurring substance reported was benzodiazepines (18.0\%). Approximately $29 \%$ of the cases were admitted to the critical care unit (CCU). Respiratory depression and drowsiness were the most frequently demonstrated clinical effects. Naloxone was a reported therapy for $44.2 \%$ of cases and performed in the majority of cases prior to PC contact. Major $(14.7 \%)$ and moderate $(30.4 \%)$ effects were most frequent and the case fatality rate was $8.1 \%$, with the number of deaths sharply increasing during the study period (34 to 305). There were 134 deaths reported within $\mathrm{ACH}$. The frequency of fentanyl exposures increased by $25.4 \%$ (95\% Cl: $17.1 \%, 34.5 \%$; $p<0.001)$, and the rate increased by $28.6 \%(95 \% \mathrm{Cl}: 7.5 \%, 77.5 \% ; \mathrm{p}<0.001)$. Conclusion: PC data demonstrated an increasing trend of reported fentanyl exposures. Such increases are most likely a result of the increasing availability of fentanyl due to lower costs and easier manufacturing. In those cases where fentanyl was reported, patient outcome (e.g., death, major outcomes) was worse compared to other opioids.

\section{Reference}

[1] Hedegaard $H$, Curtin SC, Warner M. Suicide rates in the United States continue to increase. NCHS Data Brief, no 309. Hyattsville, MD: National Center for Health Statistics. 2018 [cited 2018 Oct 18]. Available from: https://www.cdc.gov/nchs/data/databriefs/db309.pdf

\section{Characterization of Chemical, Biological, Radiological, Nuclear (CBRN) Incidents Worldwide From 1970 to 2016 as reported by the Global Terrorism Database (GTD)}

\section{Katie Myers and Cynthia Santos}

Department of Emergency Medicine, Rutgers New Jersey Medical School, Newark, United States

Objective: The Global Terrorism Database (GTD) is an opensource, unclassified database that has catalogued over 170,000 terrorism events worldwide from 1970 to 2016. It is maintained by the National Consortium for the Study of Terrorism and Responses to Terrorism, a Department of Homeland Security Center of Excellence, in an effort to enhance understanding of terrorism and facilitate its defeat. It is the most extensive unclassified database on terrorism events worldwide and contains information on the date and location of each incident, the weapons used, the nature of the target, the number of casualties, and the group or individual responsible. We analyzed all Chemical, Biological, Radiological and Nuclear (CBRN) terrorism incidents reported by the GTD.
Methods: Data analysis was performed to determine incidence over time, mortality, morbidity, and geographical distribution for each weapon type. We included all CBRN incidents reported by the GTD and excluded other non-CRBN incidents.

Results: There were a total of 170,350 reported terrorism attacks between 1970 and 2016 in the database, of which $398(0.23 \%)$ used CBRN weapons. There were 349 chemical (87.7\%), 36 biological (9.0\%), 13 radiological (3.3\%), and 0 nuclear attacks. There was a $538 \%$ increase in the annual incidence in chemical attacks after 2011. Throughout the study period, chemical weapons resulted in a mean fatality of 6.6 deaths per incident (range 0 1300 , median 1) and mean morbidity of 51.8 injuries per incident (range 0-5500, median 1). Biological weapons had a mean fatality of 0.3 deaths per incident (range $0-2$, median 0 ) and mean morbidity of 26.0 injuries per incident (range $0-751$, median 0 ). There were no fatalities or injuries associated with radiological attacks. The majority of events occurred in South Asia ( $n=101,25.4 \%)$, the Middle East and North Africa $(n=69,17.3 \%)$, Western Europe $(\mathrm{n}=55,13.8 \%)$, and North America $(\mathrm{n}=54,13.6 \%)$.

Conclusion: This data analysis is our attempt to characterize trends in CBRN terrorism attacks worldwide using the GBD, an open-access, unclassified database of terrorist events from 1970 to 2016. Chemicals were the most commonly reported weapons in these events. There was a five-fold increase in chemical attacks in the last 5 years of the study period, implicating the need for adequate chemical terrorism education and disaster preparedness among first responders and healthcare providers. Particularly in regions with the highest incidence of CBRN attacks, efforts to both prevent further attacks and prepare to respond to attacks should take a priority in the global fight against terrorism.

\section{Valproic acid poisoning: modeling of the pharmacokinetic/ pharmacodynamic relationships and analysis of usefulness of L-carnitine infusion}

Philippe Nguyen ${ }^{a}$, Lucie Chevillard ${ }^{a}$, Hervé Gourlain ${ }^{b}$, Isabelle Malissin ${ }^{\mathrm{a}}$ and Bruno Mégarbane ${ }^{\mathrm{a}}$

${ }^{a}$ Department of Medical and Toxicological Critical Care, Lariboisière Hospital, Paris-Diderot University, INSERM UMRS-1144, Paris, France; ${ }^{b}$ Laboratory of Toxicology, Lariboisière Hospital, ParisDiderot University, INSERM UMRS-1144, Paris, France

Objective: Valproic acid (VPA) is commonly used as an anticonvulsive and antipsychotic drug. Acute VPA poisoning causes dysfunction in fatty acid metabolism resulting in life-threatening neurological, cardiovascular and metabolic disorders including hyperammonemia and hyperlactatemia. L-carnitine has been used as an antidote to reverse VPA toxicity without clinical evidence of effectiveness. We aimed to describe the features and outcome of VPA-poisoned patients admitted to the intensive care unit (ICU) and to investigate the effects of L-carnitine treatment on VPA pharmacokinetics (PK) and its contribution to patient prognosis.

Methods: We conducted a retrospective single-center cohort study. All consecutive patients admitted to the ICU for acute VPA intoxication (defined as presence of compatible symptoms with plasma VPA concentration $>90 \mathrm{mg} / \mathrm{L}$ ) between February 1998 and December 2016 were included. L-carnitine was administered according to the physician in charge. VPA elimination half-lives were determined using a non-compartmental approach. Population-based models for VPA PK and individual pharmacodynamics (PD) were obtained. The effects of L-carnitine were investigated using multivariate analysis in a propensity score matching study. 
Results: Sixty-nine patients were included. The most frequent presentation was consciousness impairment (70\%) with multiorgan failure (Sequential Organ Failure Assessment [SOFA] score, 4 [1-6] (median [percentiles 25-75]). The most frequent biological impairment was increase in lactate concentration $(2.9 \mathrm{mmol} / \mathrm{L}$ [1.8-4.2]). Patients treated with L-carnitine presented significantly higher peak lactate concentration $(4.6 \mathrm{mmol} / \mathrm{L}$ [3.0-6.1] versus 3.5 $\mathrm{mmol} / \mathrm{L}[2.5-4.9], \mathrm{p}=0.002$ ) and more severe neurological disorders $(79 \%$ versus $34 \%, p=0.001)$ but not significantly different VPA concentrations. However, based on the propensity score approach, the only difference associated with L-carnitine administration was plasma lactate on admission $(p=0.001)$. When comparing matched L-carnitine-treated and non-treated patients, no significant benefit of L-carnitine was demonstrated using the difference between the worse and the admission values of the SOFA score, the delay in plasma lactate normalization and the VPA half-life. The pharmacokinetic parameters of the wellfitted population-based model of VPA PK in overdose were $\mathrm{K}_{\mathrm{a}}$ of $0.5 \mathrm{~h}^{-1}, \mathrm{~K}_{\mathrm{e}}$ of $0.03 \mathrm{~h}^{-1}$, and half-life of 4.2 hours.

Conclusion: VPA poisoning is responsible for life-threatening features including hyperlactatemia. Treatment using L-carnitine did not alter VPA elimination or patient outcome in the ICU. Our PK and PD models appear helpful to improve the management of the VPA-poisoned patients by allowing the identification of the conditions of inter-individual variability.

\section{In vitro pharmacological profiles as predictors of effects and clinical potency of monoaminergic new psychoactive substances}

\section{Dino Luethi and Matthias E Liechti}

Division of Clinical Pharmacology and Toxicology, Department of Biomedicine, University Hospital Basel and University of Basel, Basel, Switzerland

Objective: Pharmacological profiles of new psychoactive substances (NPS) can be established rapidly in vitro and provide information on newly emerged drugs. In the present study, we investigated whether in vitro monoamine uptake inhibition assays can predict effects and effective psychoactive doses of stimulants, and whether interactions with serotonin 5-hydroxytryptamine $2 \mathrm{~A}$ $\left(5-\mathrm{HT}_{2 \mathrm{~A}}\right)$ receptors can predict effective psychoactive doses of psychedelics in humans.

Methods: Using Spearman rank-order correlation, we correlated in vitro pharmacological data of 75 stimulants and 45 psychedelics with human doses that were available on Internet information websites and in books. We have determined all pharmacological data using radiolabeled neurotransmitters to assess for transporter inhibition, radiolabeled ketanserin displacement to determine $5-\mathrm{HT}_{2 \mathrm{~A}}$ receptor affinity, and a calcium mobilization assay to investigate $5-\mathrm{HT}_{2 \mathrm{~A}}$ receptor activity [1].

Results: Based on the reported information, we could make dose estimates for 54 of 75 stimulants and 35 of 45 psychedelics that we have profiled in our laboratory. For stimulants, dopamine and norepinephrine transporter inhibition potency of stimulants positively correlated with human doses $(P<0.001)$, whereas serotonin transporter inhibition potency inversely correlated with human doses $(\mathrm{P}<0.01)$. Serotonin $5-\mathrm{HT}_{2 \mathrm{~A}}$ receptor affinity significantly correlated with doses for psychedelics $(P<0.01)$, but 5 $\mathrm{HT}_{2 \mathrm{~A}}$ receptor activation potency did not $(\mathrm{P}=0.6)$.

Conclusion: For stimulants, the dopaminergic versus serotonergic activity is a marker of the reinforcing effects and abuse liability of a substance [2]. Furthermore, the results of the present study demonstrate that potent dopamine and norepinephrine transporter inhibition correlate with lower pharmacological doses for stimulants in humans. In contrast, higher serotonin transporter inhibition potency was an indicator of lower stimulant potency and higher human doses. The calcium mobilization assay used to determine $5-\mathrm{HT}_{2 \mathrm{~A}}$ receptor activation did not predict the clinical potency of psychedelics but it was necessary to determine whether a substance has psychedelic properties. The clinical potency of psychedelics, however, was predicted by ketanserin displacement assays. These findings have important implications as they indicate that relatively fast and simple in vitro screenings are useful to characterize and legally schedule new psychoactive substances.

\section{References}

[1] Luethi D, Trachsel D, Hoener MC, et al. Monoamine receptor interaction profiles of 4-thio-substituted phenethylamines (2C-T drugs). Neuropharmacology 2018;134:141-148.

[2] Baumann MH, Ayestas MA, Dersch CM, et al. Effects of phentermine and fenfluramine on extracellular dopamine and serotonin in rat nucleus accumbens: therapeutic implications. Synapse. 2000;36:102-113.

\section{Impact of a new legislation in Germany on synthetic cannabinoids analytically identified in intoxication cases}

Michaela Sommer ${ }^{\mathrm{a}}$, Verena Angerer ${ }^{\mathrm{a}}$, Volker Auwärter ${ }^{\mathrm{a}}$, Florian Eyer $^{b}$ and Maren Hermanns-Clausen ${ }^{c}$

${ }^{a}$ Forensic Toxicology, Institute of Forensic Medicine, Medical Center - University of Freiburg, Freiburg, Germany; ${ }^{b}$ Department of Clinical Toxicology, Klinikum rechts der Isar, Technical University of Munich, Munich, Germany; ' Poisons Information Center, Department of General Pediatrics, Adolescent Medicine and Neonatology, Center for Pediatrics, Medical Center - University of Freiburg, Freiburg, Germany

Objective: In recent years, an increasing number of new psychoactive substances (NPS) have become available through marketing on the Internet. To prevent the emergence and spread of further chemical variations of known scheduled drugs, a new German law, the Neue-psychoaktive-Stoffe-Gesetz (NPSG, NPS Law), entered into force on 26 November 2016. This study presents an overview of analytically confirmed synthetic cannabinoid (SC) intoxications, during a 3-year period (July 2015 - June 2018), because SCs represent the group of NPS with the highest prevalence in Germany.

Methods: Prospective observational study of patients treated in hospitals after intake of NPS. Clinical and analytical data were combined and reported to a Poison Center. Blood and urine samples were collected from enrolled patients and comprehensive drug analyses were performed by liquid-liquid extraction prior to liquid chromatography-tandem mass spectrometry (LC-MS/MS). In order to determine the effect of the NPSG, the results of 18months before and after the introduction of the new law were compared.

Results: In the period considered, SC intake was verified in 32 patients in the 18-month period before and in 21 patients after the legislative change. Twenty different SCs were detected. Two or more SCs were detected in $34 \%$ of cases before the law change, and in $81 \%$ afterwards. In addition, the median age of patients increased slightly from 19.5 to 24.5 years. Severity of poisoning was moderate or severe in $81 \%$ of patients before and $71 \%$ after introduction of the NPSG. SCs not covered by the NPSG were detected from February 2017 in nine patients, particularly Cumyl-PEGACLONE in 6 patients, 5F-MDMB-P7AICA in 2 
patients, EG-018 and 5F-Cumyl-P7AICA, once each. In both periods the most prevalent SC detected in the samples was 5F-ADB (7 patients in the first, 15 patients in the second period).

Conclusion: In 2017, there were significantly less documented intoxications compared to 2016 and the second half of 2015, but in the beginning of 2018 the number of verified SC intoxications began increasing again. Since the law was amended, a number of SCs currently not covered by the NPSG were detected. It is assumed that these SCs were deliberately designed to bypass the NPSG. Nevertheless, the decreasing number of cases suggests an effect of the NPSG, at least temporarily. Furthermore, it is feared that the increase in the first 6 months of 2018 is not only growth by chance but rather the end of a short-term positive impact of the new law.

\section{Reported and analytically detected substances in cases of acute recreational drug toxicity: a multicenter European comparison of immunoassay and additional analytical methods}

\section{Evangelia Liakoni ${ }^{a}$, Alison M Dines ${ }^{b}$, Yasmin Schmid ${ }^{c}$, Knut Erik Hovda ${ }^{d}$, Fridtjof Heyerdahl ${ }^{d}$, Christopher Yates ${ }^{e}$, David M Wood ${ }^{f}$, Paul I Dargan ${ }^{f}$ and Matthias E Liechti ${ }^{\mathrm{C}}$; on behalf of the Euro-Den Plus Research Group \\ ${ }^{a}$ Clinical Pharmacology and Toxicology, Department of General Internal Medicine, Inselspital, Bern University Hospital; Institute of Pharmacology, University of Bern, Bern, Switzerland; ${ }^{b}$ Clinical Toxicology, Guy's and St Thomas' NHS Foundation Trust and King's Health Partners, London, United Kingdom; 'Division of}

Clinical Pharmacology and Toxicology, Basel University Hospital and University of Basel, Basel, Switzerland; ${ }^{\mathrm{d}}$ The Norwegian CBRNe Centre of Medicine, Department of Acute Medicine, Oslo University Hospital, Oslo, Norway; ${ }^{\text {}}$ Clinical Toxicology Unit, Emergency Department, Hospital Universitari Son Espases, Research Institute of Health Sciences (IdISBa), Palma de Mallorca, Spain; 'Clinical Toxicology, Guy's and St Thomas' NHS Foundation Trust and King's Health Partners; Clinical Toxicology, Faculty of Life Sciences and Medicine, King's College London, London, United Kingdom

Objective: To describe reported and analytically detected substances in cases of acute recreational drug/novel psychoactive substance (NPS) toxicity in centres using only immunoassay and centres where confirmatory analytical methods are available.

Methods: Retrospective analysis within the European Drug Emergencies Network (Euro-DEN Plus) project of emergency department presentations with acute recreational drug/NPS toxicity from October 2015 to December 2017. Only cases with available analytical results were included and grouped into those with only immunoassy (IA) and those with additional analytical tests (e.g. liquid or gas chromatography-mass spectrometry (MS)) available $(I A+M S)$ as part of routine clinical care (regardless of whether additional methods were used in all or in a proportion of the cases).

Results: Overall 2,668 (18.6\%) of the 14,330 Euro-DEN Plus presentations had documented analytical tests; 547 using IA (from 6 centres) and 2,121 using IA + MS (from 11 centres). The most commonly reported and/or detected substances were amphetamine-type drugs $(n=1,122)$ and tetrahydrocannabinol (THC) $(n=1,102)$. See Table 1. Reported but not detected substances included gamma-hydroxybutyrate (GHB)/gamma-butyrolactone (GBL), lysergic acid diethylamide (LSD), NPS, methylphenidate, and hallucinogenic mushrooms in the IA group, and caffeine/ energy drinks, steroids, and plants in the IA + MS group. Mephedrone was the most commonly reported NPS $(n=13$; detected in two IA + MS cases). Excluding synthetic cannabinoids,

Table 1. Reported and analytical results for some of the most common substance categories in patients presenting with acute recreational drug/novel psychoactive substance (NPS) toxicity.

\begin{tabular}{|c|c|c|c|c|c|c|c|c|}
\hline \multirow[b]{2}{*}{$\begin{array}{l}\text { Substance/ sub- } \\
\text { stance group }\end{array}$} & \multicolumn{4}{|c|}{ Immunoassy only used for detection (IA) } & \multicolumn{4}{|c|}{$\begin{array}{l}\text { Substances detected by immunoassy, liquid or gas chromatography-mass } \\
\text { spectrometry or both (IA + MS) }\end{array}$} \\
\hline & $\begin{array}{l}\text { Only } \\
\text { reported }\end{array}$ & $\begin{array}{l}\text { Reported } \\
\text { and } \\
\text { detected }\end{array}$ & $\begin{array}{c}\text { Only } \\
\text { detected }\end{array}$ & $\begin{array}{l}\text { \% detected among } \\
\text { total reported, i.e. } \\
\text { "reported and } \\
\text { detected"/(“only } \\
\text { reported" + } \\
\text { "reported and } \\
\text { detected") }\end{array}$ & $\begin{array}{l}\text { Only } \\
\text { reported }\end{array}$ & $\begin{array}{l}\text { Reported } \\
\text { and } \\
\text { detected }\end{array}$ & $\begin{array}{c}\text { Only } \\
\text { detected }\end{array}$ & $\begin{array}{l}\text { \% detected among } \\
\text { total reported, i.e. } \\
\text { "reported and } \\
\text { detected"/("only } \\
\text { reported" + } \\
\text { "reported and } \\
\text { detected") }\end{array}$ \\
\hline Cocaine & 10 & 171 & 34 & $95 \%$ & 76 & 562 & 154 & $88 \%$ \\
\hline $\begin{array}{l}\text { Tetrahydrocannabin- } \\
\text { ol }\end{array}$ & 18 & 141 & 91 & $89 \%$ & 73 & 498 & 281 & $87 \%$ \\
\hline Heroin & 8 & 79 & 0 & $91 \%$ & 22 & 99 & 5 & $82 \%$ \\
\hline $\begin{array}{l}\text { Gamma-hydroxybu- } \\
\text { tyrate/gamma- } \\
\text { butyrolactone } \\
\text { (GHB/GBL) }\end{array}$ & 15 & 0 & 0 & $0 \%$ & 131 & 128 & 22 & $49 \%$ \\
\hline \multicolumn{9}{|l|}{ NPS } \\
\hline $\begin{array}{l}\text { NPS other than syn- } \\
\text { thetic } \\
\text { cannabinoids* }\end{array}$ & 9 & 0 & 0 & $0 \%$ & 42 & 27 & 14 & $39 \%$ \\
\hline $\begin{array}{l}\text { Synthetic } \\
\text { cannabinoids* }\end{array}$ & 0 & 0 & 0 & Not applicable & 13 & 21 & 8 & $62 \%$ \\
\hline
\end{tabular}

*IA test currently not readily available for these substances 
the NPS most commonly detected (IA + MS) were amfepramone $(\mathrm{n}=7$; three patients with no specified NPS use, two no NPS, and two with alpha-pyrrolidinovalerophenone [alpha-PVP] reported use), and methylenedioxypyrovalerone (MDPV) $(n=5$; four with reported use).

Conclusion: While $>75 \%$ detection was seen in both groups in cases with reported cocaine, THC, heroin or sedatives use, amphetamine-type substances had a detection of $<75 \%$ in the IA group, and substances such as NPS and GHB/GBL were detected only in centres with additional methods available.

\section{Acute poisoning by chemicals in Spain: results of the Spanish Toxic Surveillance System (STSS) 2017}

\author{
Ana Ferrer Dufol ${ }^{\mathrm{a}}$, Santiago Nogue Xarau ${ }^{\mathrm{b}}$, \\ Francisco Ruiz Ruiz ${ }^{c}$, Laura Rejas Morras ${ }^{d}$ and \\ Agustin Garcia Urdangarin ; on behalf of the Spanish \\ Foundation of Clinical Toxicology (FETOC) \\ aUnit of Clinical Toxicology, Clinic University Hospital, Zaragoza, \\ Spain; ${ }^{b}$ Unit of Clinical Toxicology, Hospital Clinic, Barcelona, \\ Spain; ${ }^{C} E D$, Clinic University Hospital, Zaragoza, Spain; ${ }^{\mathrm{d} C l i n i c}$ \\ University Hospital, Zaragoza, Spain; 'Zaragoza University, \\ Zaragoza, Spain
}

Objective: STSS, designed in 1999 between the Spanish Ministry of Health and a group of Clinical Toxicologists working in the Emergency Department (ED) of a public hospital, aims to report cases of acute poisoning by chemical products in their ED to evaluate the risks of exposure to these substances under the current EU regulations. This Program has been managed since 2010 by the Spanish Foundation of Clinical Toxicology (FETOC). We present here the results of the Program in 2017.

Methods: The participating hospitals report all cases of intoxication due to household, agricultural or industrial chemicals treated in their ED. An online questionnaire is accessible through the FETOC website around the clock by means of an encrypted system. The files are downloaded on a regular basis to a database (File Maker $9.0^{\circledR}$ ) to allow a final yearly report to be presented to the Health Ministry.

Results: In 2017 the Program collected 1277 cases from 23 Hospitals covering a population of about 10 million people. Median patient age is $38 \pm 24$ years. The distribution by gender is even: 641 males (age $36 \pm 23$ years) and 618 females (age $39 \pm 25$ years). Young patients under 16 years of age comprised $21 \%$. Domestic accidents were significantly prevalent (78\%) followed by suicide gestures (9\%) and occupational accidents ( $8 \%)$ ( $p<$ $0.05)$. The main chemicals involved were as follows: toxic gases $(45 \%)$, caustics $(16 \%)$, irritant gases $(12 \%)$, solvents $(8 \%)$ and detergents $(8 \%)$ and pesticides $(6 \%)$. The main routes of entry were respiratory $(61 \%)$ and oral (32\%). Ocular (7\%) and cutaneous contact $(2 \%)$ are much less frequent $(p<0.05)$. Overall $71 \%$ of the patients were symptomatic at admission presenting with digestive (31\%), neurological (28\%), respiratory (23\%) and ocular (8\%) symptoms, most of them mild. Only 230 cases were defined as severe. Most patients (77\%) received some treatment, mainly symptomatic (44\%). In $40 \%$ of the cases, an antidote was used: oxygen in 484 carbon monoxide exposures (8 also received hydroxocobalamin), ethanol was used in 4 cases, and atropine in 8 cases of organophosphates poisoning (with oximes in 5 patients). Only $12 \%$ required hospital admission for 24 hour observation and $17(1 \%)$ were admitted to an intensive care unit. The mortality rate was $0.86 \%$ : 7 cases due to suicide gestures and 4 due to domestic accidents.

Conclusion: Acute poisoning by chemicals in Spain are low-risk events, caused mainly by domestic accidents mostly due to toxic gases (CO) and caustic cleaning agents.

\section{National poisons centre data collection: pilot study on Pesticide Poisoning Monitoring in Germany (PIMONT-PES)}

\author{
Andreas Stürer ${ }^{\mathrm{a}}$, Esther Feistkorn ${ }^{\mathrm{b}}$, Kathrin Begemann ${ }^{\mathrm{b}}$, \\ Nina Glaser ${ }^{b}$, Daniela Acquaronec, Dagmar Prasa ${ }^{\mathrm{d}}$, \\ Martin Ebbecke ${ }^{e}$, Florian Eyer ${ }^{f}$, \\ Maren Hermanns-Clausen ${ }^{g}$, Carola Seidel ${ }^{\mathrm{h}}$, \\ Erol Tutdibi ${ }^{i}$ and Herbert Desel ${ }^{\mathrm{b}}$

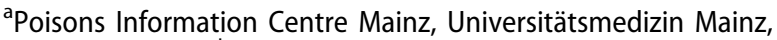 \\ Mainz, Germany; ${ }^{b}$ Poisoning and Product Documentation Centre, \\ German Federal Institute for Risk Assessment (BfR), Berlin, \\ Germany; ' Poisons Information Centre, Charité Universitätsmedizin

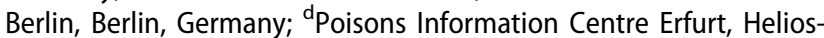 \\ Klinikum, Erfurt, Germany; ${ }^{\mathrm{e}} \mathrm{GIZ}$-Nord Poisons Center, University \\ Medical Center Göttingen, Göttingen, Germany; ${ }^{\text {fPoisons }}$ \\ Information Centre Munich, Klinikum rechts der Isar, Munich, \\ Germany; ${ }^{9}$ Poisons Information Centre VIZ-Freiburg, \\ Universitätskinderklinik Freiburg, Freiburg, Germany; ${ }^{\text {hPoisons }}$ \\ Information Centre Bonn, Universitätskinderklinik Bonn, Bonn, \\ Germany; 'Poisons Information Centre Homburg, \\ Universitätskinderklinik des Saarlandes, Homburg, Germany
}

Objective: Compilation of poisons centre (PC) data facilitates health reporting and sensitive detection of unusual case series, but is challenging if only partially harmonized case databases are used. As a model for case data collection in Europe all eight German PC, the German Society for Clinical Toxicology (GfKT) and the German Federal Institute for Risk Assessment have started a national case data collection on pesticide poisonings, mainly to fulfil legal reporting needs. The task is a subproject of a pilot study to establish a more general National Monitoring of Poisonings in Germany sponsored by the Federal Government. We report the data from the first 4 months of collection.

Methods: Continuous and prospective case data collection started on 1 May 2018, scheduled until 30 April 2019. All calls to any PC in Germany reporting a human exposure to a pesticide product are included. A basic dataset is collected for all cases. For a subgroup of exposures to repellent products (according to Biocides Regulation (EC) No 528/2012, Annex V) clinical data, if available with medical follow-up, is added. Toxic agents were selected according to the German TKS product category system [1]. Compilation of data was performed via the Web-based GfKT Case Database.

Results: In total 1,602 pesticide exposures were collected within the 4-month study period; $55.4 \%$ were oral exposures, $23.8 \%$ inhalations and $18.7 \%$ dermal contacts. Children aged $<1$ year were involved in $22.9 \%$ of cases, $1-6$ years in $42.8 \%$ and schoolaged children in 5.9\%. Most cases (94.5\%) were accidental (including $2.5 \%$ occupational) while $3.8 \%$ were intentional (mainly suicidal, in $0.6 \%$ the product being supplied by another person). Clinical data were collected for 163 of 251 repellent exposures. Patients developed either no symptoms $(68.1 \%)$ or minor poisoning (31.9\%).

Conclusion: The PIMONT-PES dataset provides a reliable quantitative overview of pesticide poisoning events reported to PCs in Germany, facilitating legal European and national reporting obligations. In the subset of cases with complete follow-up no exposure to repellents (like DEET) leading to more than minor symptoms is recorded so far, despite a high concern for acute toxic effects of repellents derived from animal studies. Data of heterogeneous PC case databases could be collated successfully via Web-based GfKT Case Database. Thus, PIMONT may serve as a technical model for a Europe-wide PC case data collection. 


\section{Reference}

[1] Stürer A, Hüller G, Reinecke HJ, et al. Harmonization of categorization systems for agents: First data from German Poisons Centers. Clin Toxicol (Phila). 2007;45:337.

\section{Tak: the computational toxicological machine}

\author{
Michael Chary ${ }^{\mathrm{a}}$, Michele Burns ${ }^{\mathrm{a}}$ and Edward Boyer ${ }^{\mathrm{b}}$ \\ ${ }^{a}$ Harvard Medical Toxicology Fellowship, Boston Children's

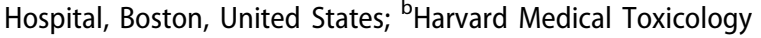 \\ Fellowship, Brigham and Women's Hospital, Boston, United States
}

Objective: To expand access to toxicologic consultation in resource-limited areas by using artificial intelligence.

Methods: Tak is an artificial intelligence machine constructed using a network of first-order logic statements (axioms) weighted by probabilities. We derived, by committee consensus, 90 axioms to capture essential features of the six classic toxidromes: sympathomimetic, anticholinergic, cholinergic, sedative-hypnotic, serotonin syndrome, and opioid intoxication. Each of these six toxidromes was characterized by five abnormal clinical findings (e.g. tachycardic, bradypneic, delirium). We trained Tak on 1200 synthetic poisoned patients, 200 "classic" presentations for each toxidrome and evaluated Tak on another independent group of 250 synthetic poisoned patients. Each synthetic poisoned patient was generated by choosing three signs or symptoms from the intended toxidrome and between 0-2 symptoms from a "distracting" toxidrome. We compared Tak's evaluation of these synthetic poisoned patients to the evaluation of two blinded medical toxicologists.

Results: Tak correctly identified the toxidrome in 62\% (628/1000) in the internal validation data set, most commonly confusing anticholinergic with sympathomimetic presentations and opioid with sedative-hypnotic presentations. Tak correctly identified the toxidrome in $13 \%(16 / 119)$ of the cases in the external validation data set. Toxicologists identified the toxidrome in $16 \%(19 / 119)$ of the cases. The inter-rater reliability between the two raters was 0.81 . This difference was not statistically significant $(p=0.46$; chi-square test). Toxicologists and Tak agreed with each other on 39\% (45/ 118) of the cases. The most common sources of disagreement were over anticholinergic versus sympathomimetic toxidromes.

Conclusion: Our study is the first demonstration that toxicological acumen can be digitized using probabilistic logic. This digitization performs as well as clinicians in identifying at a single point in time toxidromes represented by five vital sign abnormalities. Our approach holds promise to scale up medical reasoning to match the scope of modern medical knowledge and pace of research and digitize toxicological acumen. Limitations of our study include a low accuracy rate for both Tak and human raters, which may reflect the use of synthetic instead of actual patients. This study did not consider other elements of the patient presentation, such as the history, or laboratory findings, which can help identify the toxidrome. Our analysis only considered the most likely toxidrome, although Tak can generate a ranked list of toxidromes for each patient.

\section{Poppers poisoning cases admitted to the intensive care unit: Is there a methemoglobinemia threshold responsible for tissue dysoxia?}

\author{
May Yaker ${ }^{\mathrm{a}}$, Eya Hamdi ${ }^{\mathrm{b}}$, Karim Jaffal ${ }^{\mathrm{a}}$ and \\ Bruno Mégarbane
}

${ }^{a}$ Department of Medical and Toxicological Critical Care, Lariboisière Hospital, Paris-Diderot University, INSERM UMRS-1144, Paris, France; 'baboratory of Biochemistry, Lariboisière Hospital, ParisDiderot University, INSERM UMRS-1144, Paris, France

Objective: The recreational use of poppers has been increasing in France since the last judgement of the National Council in June 2013 re-authorizing their marketing. Poppers contain various alkyl nitrites which are highly oxidant compounds able to induce methemoglobinemia (MetHb) with deleterious and even lifethreatening consequences. Our objective was to describe the circumstances, complications and outcome of patients admitted to the intensive care unit (ICU) for MetHb onset attributed to exposure to poppers and to investigate the relationship between the serum lactate concentration and the MetHb before treatment.

Methods: We conducted a retrospective single-center observational study including all patients admitted to the ICU with increased blood MetHb ( $>0.7 \%$ ) following exposure to poppers. We investigated the relationships between oxygen saturation, serum lactate concentration and MetHb on ICU admission by calculating the Pearson's coefficients and using the Bartlett's sphericity test.

Results: Twenty-six patients (24 males and 2 females; aged 42 years $(35 ; 48)$ [median (percentiles 25;75)]) were included. The poppers had been snorted (77\%), ingested (19\%) or snorted + ingested (4\%), in a recreational multi-drug exposure $(65 \%$; mostly accompanied by gamma-hydroxybutyrate use). On admission, MetHb was $20.0 \%(1.5 ; 44.0)$. The patients received methylene blue infusion (62\%), mechanical ventilation (35\%), catecholamine infusion (12\%) and blood transfusion (4\%). On admission, the oxygen saturation was $91 \%(83 ; 94)$ and weakly correlated to the MetHb $\left(R^{2}=0.3 ; p=0.01\right)$ while the serum lactate concentration was $2.2 \mathrm{mmol} / \mathrm{L}(1.3 ; 3.9)$ and highly correlated to $\mathrm{MeHb}\left(\mathrm{R}^{2}=\right.$ $0.7 ; \mathrm{p}<0.0001)$. A plasma lactate concentration of $>2 \mathrm{mmol} / \mathrm{L}$ was highly predictive of MetHb $>20 \%$, with $91.7 \%$ sensitivity, $90.9 \%$, specificity, $91.7 \%$ positive predictive value and $90.9 \%$ negative predictive value.

Conclusion: The use of poppers is responsible for life-threatening consequences attributed to MetHb. Our data clearly support the recommendation by international and French guidelines to administer methylene blue in patients developing MetHb $>20 \%$ by evidencing that tissue hypoxia is almost consistently present above this threshold.

\section{Clinical outcomes from early use of digoxin-specific antibodies versus observation in chronic digoxin poisoning (ATOM-4)}

\author{
Betty S Chan ${ }^{a}$, Geoffrey K Isbister ${ }^{b}$, Colin B Page ${ }^{c}$, \\ Katherine Z Isoardi ${ }^{c}$, Angela L Chiew ${ }^{a}$, \\ Katharine A Kirby ${ }^{d}$ and Nicholas A Buckley ${ }^{d}$ \\ ${ }^{a}$ Emergency Medicine \& Clinical Toxicology Unit, Prince of Wales \\ Hospital, Sydney, Australia; ${ }^{\mathrm{b}}$ Clinical Toxicology Research Group, \\ University of Newcastle, Newcastle, Australia; 'Clinical Toxicology \\ Unit, Princess Alexandra Hospital, Brisbane, Australia; ${ }^{\mathrm{d}}$ Discipline of \\ Pharmacology, University of Sydney, Sydney, Australia
}

Objective: From our previous studies [1], we hypothesised patients with elevated digoxin concentrations may derive little benefit from digoxin-specific antibodies (digoxin-Fab) because their presenting complaint was more closely related to multiple co-morbidities. We compared the outcome of patients who were initially treated with digoxin-Fab with those receiving supportive care. 
Table 1. Outcome of patients initially treated with digoxin-Fab versus those initially observed (abstract 129).

\begin{tabular}{|c|c|c|c|}
\hline Chronic digoxin poisoning & Initial digoxin-Fab $(\mathrm{n}=78)$ & Initial observation $(n=50)$ & P-value \\
\hline Mean heart rate change within 4 hours (bpm) & $8 \pm 1$ & $7 \pm 3$ & 0.54 \\
\hline Mean potassium concentration change (mmol/L) & $-0.5 \pm 0.1$ & $-0.4 \pm 0.1$ & 0.85 \\
\hline Median Length of Stay (days) & 6 (IQR 3-11; range $1-43$ ) & 6 (IQR 3-10; range 1-32) & 0.51 \\
\hline Fatality (\%) & $9(11 \%)$ & $7(14 \%)$ & 0.68 \\
\hline
\end{tabular}

\pm Standard error of the mean, IQR: interquartile range.

Methods: Patients were prospectively recruited if they had an elevated digoxin concentration or signs/symptoms of toxicity thought to be from digoxin. Patients who were initially managed with digoxin-Fab were compared with those not initially receiving digoxin-Fab (observation group). Patients presenting with ventricular arrhythmias before initial assessment were excluded. Primary outcome was mortality. Secondary outcomes were length of stay (LOS), change in heart rate and potassium concentration.

Results: From September 2013 to January 2018, 128 patients were recruited; 78 (61\%) received initial digoxin-Fab. Digoxin-Fab and supportive care groups had an initial median heart rate of 46 (range 20-120) versus $52 \mathrm{bpm}$ (range 29-91), systolic blood pressure $110 \mathrm{mmHg}$ (range 65-180) versus $125 \mathrm{mmHg}$ (range 90184), respectively. Digoxin concentrations were $4.4 \mathrm{nmol} / \mathrm{L}$ (range 3.3-9.0) versus $4.2 \mathrm{nmol} / \mathrm{L}$ (range 2.0-11.2) and potassium concentrations $5.4 \mathrm{mmol} / \mathrm{L}$ (range 3-11) versus $5.1 \mathrm{mmol} / \mathrm{L}$ (range $3.5-8.2$ ) were similar. Median dose of digoxin-Fab used was 1.5 vials (IQR $1-2)$. There were 9 deaths (12\%) in the Fab group compared to 7 (14\%) in patients given supportive care (risk difference $-2.5 \%$; 95\% Cl:-14 to $9 \% ; \mathrm{p}=0.68$ ). The median LOS, mean changes in potassium concentration and heart rate within 4 hours were similar in both groups (Table 1).

Conclusion: There did not appear to be any benefit from the routine use of digoxin-Fab in patients thought to have chronic digoxin poisoning. These patients have multiple co-morbidities that may be contributing to their clinical features; other treatments are often equally effective.

\section{Reference}

[1] Chan BS, Isbister GK, O'Leary M, et al. Efficacy and effectiveness of anti-digoxin antibodies in chronic digoxin poisonings from the DORA study (ATOM-1). Clin Toxicol (Phila). 2016;54:488-494.

\section{Can severe digoxin poisoning be treated without using antibody fragments?}

\section{Csaba Pap}

Department of Emergency Medicine and Clinical Toxicology, Péterfy Hospital, Budapest, Hungary

Objective: Digoxin immune Fab is a safe and effective antidote for life-threatening digoxin intoxication. Guidelines [1] suggest that it should be administered to all patients with life-threatening tachy-bradyarrhythmias, hyperkalaemia $(>6 \mathrm{mmol} / \mathrm{L})$ or haemodynamic instability with an elevated serum digoxin concentration ( $>2 \mu \mathrm{g} / \mathrm{L}$ ). Different guidelines suggest stocking 10-15 vials [2]. Digoxin immune Fab, however, is very expensive and can be hard to obtain, consequently it is not available even in tertiary care hospitals worldwide. Here we present 6 cases in which severely poisoned patients with markedly elevated serum digoxin concentrations were not given the antidote but recovered with supportive care and were discharged without sequelae.

Case series: Table 1 summarizes the clinical course of patients. All patients were administered $4-7 \mathrm{~g}$ of magnesium sulfate IV and atropine as required IV or IM for 1-4 days. The dose of magnesium depended on their renal function. The 61-year-old male also received a transient pacemaker.

Conclusion: We raise the hypotheses that in our patients, highdose magnesium [3] and the permanently decreased heart rate [4] may have had a protective effect on the toxicity of digoxin. There is a need to develop an internationally accepted treatment protocol for cases where digoxin immune Fab is not available.

Table 1. Summary of clinical course of six patients with severe acute digoxin intoxication managed without digoxin immune Fab (abstract 130).

\begin{tabular}{|c|c|c|c|c|c|c|}
\hline Patient & Age/ gender & $\begin{array}{l}\text { Ingested dose of } \\
\text { digoxin (mg) }\end{array}$ & Coingestants & $\begin{array}{l}\text { Time from ingestion } \\
\text { to sample blood } \\
\text { (hours) }\end{array}$ & $\begin{array}{l}\text { Serum digoxin } \\
\text { concentration } \\
(\mathrm{ng} / \mathrm{mL})\end{array}$ & Main clinical findings \\
\hline 1. & $45 \mathrm{~F}$ & 10 & $\begin{array}{c}\text { Nitroglycerin, } \\
\text { diazepam }\end{array}$ & 12 & 8.0 & $\begin{array}{l}\text { Mobitz II 2nd degree } \\
\text { atrioventricular block } \\
\text { (heart rate } 25-40 \mathrm{bpm} \text { ) }\end{array}$ \\
\hline 2. & $61 \mathrm{M}$ & 12.5 & None & 12 & 8.61 & $\begin{array}{l}\text { 3rd degree atrioventricu- } \\
\text { lar block (heart rate } \\
24-53 \mathrm{bpm})+ \text { mild } \\
\text { hypotension }\end{array}$ \\
\hline 4. & $81 \mathrm{~F}$ & 12.5 & None & 12 & 11.15 & $\begin{array}{l}\text { 3rd degree atrioventricu- } \\
\text { lar block (heart rate } \\
32-59 \mathrm{bpm})+ \text { mild } \\
\text { hypotension }\end{array}$ \\
\hline 6. & $79 M$ & 15 & Clonazepam & 14 & 16.12 & $\begin{array}{l}\text { Atrial fibrillation (heart } \\
\text { rate } 29-62 \mathrm{bpm})+ \\
\text { moderate hypotension }\end{array}$ \\
\hline
\end{tabular}




\section{References}

[1] Chan BS, Buckley NA. Digoxin-specific antibody fragments in the treatment of digoxin toxicity. Clin Toxicol (Phila). 2014;52:824-836.

[2] Dart RC, Goldfrank LR, Erstad BL, et al. Expert Consensus Guidelines for stocking of antidotes in hospitals that provide emergency care. Ann Emerg Med. 2018;71: 314-325.

[3] Cohen L, Kitzes R. Magnesium sulfate and digitalis-toxic arrhythmias. JAMA. 1983;249:2808-2810.

[4] Xie JT, Cunningham PM, January CT. Digoxin-induced delayed afterdepolarizations: biphasic effects of digoxin on action potential duration and the Q-T interval in cardiac Purkinje fibers. Methods Find Exp Clin Pharmacol. 1995;17:113-120.

\section{Self-harm with Nerium oleander ingestion in Italy}

\author{
William Brambilla ${ }^{a}$, llaria Melara ${ }^{a}$, Valeria M Petrolini ${ }^{b}$, \\ Francesca Chiarab, Marta Crevani ${ }^{b}$, Sarah Vecchio ${ }^{b}$ \\ and Carlo A Locatelli ${ }^{\text {b }}$ \\ a'Emergency Medicine Fellowship Program, University of Pavia,

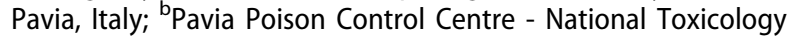 \\ Information Centre - Clinical and Experimental Lab, Toxicology \\ Unit, Maugeri Clinical and Scientific Institutes IRCCS and University \\ of Pavia, Pavia, Italy
}

Objective: Nerium oleander is native to the Mediterranean regions of Europe and Africa. As with yellow oleander (Cascabela thevetia, previously Thevetia peruviana), all parts of the plant contain cardiac glycosides (mainly oleandrin) which, like digoxin, inhibit the $\mathrm{Na}^{+} / \mathrm{K}^{+}$-ATPase dependent pump. Cardioactive glycosides can be detected in blood because of cross-reactivity with the digoxin immunoassays. Similarly, they can be bound by digoxin-specific antibody fragments (Fab) [1]. Deliberate ingestion may occur as a means of suicide as described for yellow oleander in countries outside Europe [2]. We describe a European case series of self-harm intoxication by Nerium oleander.

Methods: All cases of oleander ingestion for suicidal purpose, referred to our Poison Control Centre from January 2008 to September 2018, were retrospectively evaluated for exposure modalities, clinical manifestations, analytical determinations and treatment.

Results: Eighty-four patients (mean age $42 \pm 17$ years; M/F 42/42) were studied. The most frequent method of intoxication was the ingestion of raw leaves (58.2\%), followed by infusions or decoctions of leaves (34.6\%). Gastrointestinal symptoms occurred in $70.2 \%$, cardiovascular in $56.0 \%$ and neurological in $16.7 \%$. There were no deaths. Nineteen patients were treated with digoxin Fab fragments (80 to $760 \mathrm{mg}, 2-19$ vials). Six patients needed a temporary pacemaker. Digoxin was measured in 64 cases, and was positive in 51/64 (mean $0.87 \pm 0.85 \mathrm{ng} / \mathrm{mL}$. As expected, the correlation between positive digoxinemia and the presence of cardiac abnormalities was not statistically significant $(p=0.0703)$; conversely, its negativity value was statistically significant in excluding the manifesting of serious cardiac symptoms (defined as the presence of heart rate $<40$ beats/minute and/or ventricular arrhythmia) $(p=0.0047)$. Moreover, the cross-reactive digoxin concentration did not seem to be related to the severity of symptoms $(\mathrm{p}=0.0988)$.

Conclusion: Self-harm intoxication by Nerium oleander is an increasingly frequent event in our country. Digoxinemia can confirm the diagnosis of oleander poisoning and in our cases, the negativity of the test allowed exclusion of serious complications, but not the potential of developing cardiac symptoms or alterations. As previously described, the digoxinemia concentration did not correlate with symptom severity.

\section{References}

[1] Bandara V, Weinstein SA, White J, et al. A review of the natural history, toxinology, diagnosis and clinical management of Nerium oleander (common oleander) and Thevetia peruviana (yellow oleander) poisoning. Toxicon. 2010;56:273-281.

[2] Eddleston M, Rajapakse S, Rajakanthan, et al. Anti-digoxin Fab fragments in cardiotoxicity induced by ingestion of yellow oleander: a randomised controlled trial. Lancet. 2000;355:967-972.

\section{Baclofen-attributed withdrawal syndrome: clinical and electroencephalographic features and reversal in the rat}

\author{
Solène Palmiéri ${ }^{a}$, Pierre-Franois Rogliano ${ }^{a}$, \\ Marion Soichot ${ }^{b}$, Lucie Chevillard ${ }^{a}$, Patricia Risède ${ }^{a}$, \\ Laurence Labat ${ }^{\mathrm{b}}$ and Bruno Mégarbane ${ }^{\mathrm{a}}$ \\ ${ }^{a}$ Department of Medical and Toxicological Critical Care, Lariboisière \\ Hospital, Paris-Diderot University, INSERM UMRS-1144, Paris, \\ France; ${ }^{b}$ Laboratory of Toxicology, Lariboisière Hospital, Paris- \\ Diderot University, INSERM UMRS-1144, Paris, France
}

Objective: Baclofen, a gamma-aminobutyric acid $\left(G_{A B A}\right)$ receptor agonist, is increasingly used to manage alcohol dependence, leading to increasing poisonings. Preclinical and clinical studies describe the onset of withdrawal syndrome following baclofen cessation. In the baclofen-poisoned patient admitted to the intensive care unit, withdrawal syndrome rapidly follows toxicity with intricate features and difficult diagnosis. Our objectives were to characterize baclofen withdrawal in the rat and study its reversal using different common drugs.

Methods: The study was conducted in a Sprague-Dawley rat model, pretreated with repeated and increasing doses of baclofen from 5 to $15 \mathrm{mg} / \mathrm{kg} 3$ times daily during 15 days, using neurobehavioral (locomotion, anxiety, and memory tests) and electroencephalography (EEG), (using Markand's scale [1]) investigations to evidence withdrawal syndrome and the effectiveness of the different treatments (topiramate, diazepam, gammahydroxybutyrate [GHB] and baclofen, daily administered at pharmacological doses from day 5 , as evidenced to be the peak of withdrawal syndrome) used to reverse its deleterious effects.

Results: Baclofen withdrawal resulted in marked encephalography (peaking at grade 3 of Markand's scale), anxiety ( $<<0.05$ ); but no significant effects on locomotion and memory were observed. Topiramate and diazepam administration led to the reversion of baclofen withdrawal-induced encephalopathy. GHB administration at the tested dose resulted in improvement of withdrawal features but onset of additional signs of toxicity.

Conclusion: Baclofen withdrawal is responsible in the rat for marked encephalopathy with possible beneficial reversion using topiramate and diazepam. However, the exact molecular mechanisms involved remain to be investigated.

\section{Reference}

[1] Markand ON. Electroencephalography in diffuse encephalopathies. J Clin Neurophysiol. 1984;1:357-407. 


\section{Susceptibility to the metamizole metabolite N-methyl-4- aminoantipyrine (MAA) depends on the differentiation state of myeloid progenitor cells}

\author{
Deborah Rudin ${ }^{a}$, Stephan Krähenbühl ${ }^{\mathrm{a}}$ and \\ Manuel Haschke ${ }^{b}$ \\ ${ }^{a}$ Clinical Pharmacology \& Toxicology, University Hospital Basel, \\ University Basel, Basel, Switzerland; ${ }^{b}$ Clinical Pharmacology and \\ Toxicology, University Hospital Bern, University of Bern, Bern, \\ Switzerland
}

Objective: Metamizole, an analgesic and antipyretic drug widely used due to its good tolerability and overall favorable safety profile, can cause life-threatening neutropenia in susceptible patients $[1,2]$. Since previous results indicated that metamizole cytotoxicity may be limited to neutrophil precursor cells (unpublished data), our objective was to investigate the change in sensitivity of neutrophil precursors during differentiation and the accompanying enzymatic changes.

Methods: We initiated differentiation of promyelocytic HL60 cells into neutrophils and assessed the change in susceptibility of the cells to the cytotoxic combination of the main metamizole metabolite N-methyl-4-aminoantipyrine (MAA) with the hemoglobin break down product hemin. We monitored the differentiation progress morphologically by light microscopy and by expression of the cellular surface marker CD66a. We assessed the expression of antioxidative and heme degrading enzymes during neutrophil differentiation by polymerase chain reaction (PCR) and Western blots. Accompanying susceptibility to cytotoxicity elicited by MAA and hemin was determined by detection of apoptosis and necrosis by Annexin V/PI staining.

Results: HL60 cells differentiated by around 45\% into band and mature neutrophils within 5 days. At day 3 of the differentiation process, the cells consisted predominately of metamyelocytes and became significantly resistant to the combination of MAA and hemin, which was cytotoxic for less differentiated promyelocytic HL60 cells. The expression of the antioxidative enzyme superoxide dismutase-2 (located in the mitochondrial matrix) increased significantly during differentiation, whereas the superoxide dismutase-1 expression (located in the mitochondrial intermembrane space and cytosol) decreased. Heme oxygenase-1, which degrades hemin, was progressively expressed during differentiation. Interestingly, the expression of cytochrome c reductase, which is needed for heme oxygenase- 1 function, increased to a maximum at day 3 and decreased again with further differentiation. Further, the cellular glutathione content, an important antioxidant, increased during differentiation.

Conclusion: We showed that HL60 cells become resistant to the cytotoxic combination MAA/hemin on the metamyelocyte state of differentiation. We suppose that the increasing cellular antioxidative capacity and/or the ability to degrade hemin are responsible for the observed change in susceptibility.

\section{References}

[1] Andrès E, Maloisel F. Idiosyncratic drug-induced agranulocytosis or acute neutropenia. Curr Opin Hematol. 2008;15:15-21.

[2] Huber M, Andersohn F, Sarganas G, et al. Metamizole-induced agranulocytosis revisited: results from the prospective Berlin CaseControl Surveillance Study. Eur J Clin Pharmacol. 2015;71:219-227.

\section{Reactive carbonyl compounds in the mechanisms of neuroinflammation in acute methanol
poisoning}

\author{
Jiri Hlusicka ${ }^{a}$, Lucie Lischkova ${ }^{b}$, Marian Regenda ${ }^{b}$, \\ Petr Kacer ${ }^{c}$ and Sergey Zakharov ${ }^{b}$ \\ ${ }^{a}$ Toxicological Information Centre, Charles University in Prague, \\ Prague, Czech Republic; ${ }^{b}$ Charles University in Prague, Prague, \\ Czech Republic; ' Institute of Chemical Technology, Prague, Czech \\ Republic
}

Objective: Methanol poisonings are characterized by high lethality rate, and long-term visual sequelae and brain damage in survivors. Leukotriene-mediated neuroinflammation plays an important role in the mechanisms of methanol toxicity [1]. In toxic brain damage, the reactive carbonyl compounds are produced due to neuronal membrane lipids peroxidation. We measured acute concentrations of markers of carbonyl stress and the follow-up concentrations in patients with acute methanol poisoning.

Methods: Blood serum samples were collected from 28 patients hospitalized with acute intoxication and from 36 survivors two years after discharge. In these samples, concentrations of $\mathrm{C} 6, \mathrm{C} 7$, $\mathrm{C} 8, \mathrm{C} 9, \mathrm{C} 10, \mathrm{C} 11$ and $\mathrm{C} 12$ reactive aldehydes measured using the method of liquid chromatography-electrospray ionization-tandem mass spectrometry.

Results: The acute serum concentrations of all measured reactive aldehydes were higher than the follow-up concentrations: C6 $36.4 \pm 4.8$ versus $21.6 \pm 5.2 \mathrm{ng} / \mathrm{mL}$; C7 $38.9 \pm 5$ versus $17.0 \pm 2.0$ $\mathrm{ng} / \mathrm{mL}$; C8 $18.8 \pm 3.9$ versus $4 \pm 0 \mathrm{ng} / \mathrm{mL}$; C9 $36.5 \pm 3.9$ versus 19.0 $\pm 3.0 \mathrm{ng} / \mathrm{mL} ; \mathrm{C} 106.1 \pm 0.4$ versus $4.0 \pm 0.5 \mathrm{ng} / \mathrm{mL} ; \mathrm{C} 11 \quad 13.6 \pm 3.0$ versus $3.7 \pm 0.6 \mathrm{ng} / \mathrm{mL}$; and $\mathrm{C} 127.8 \pm 0.4$ versus $4.7 \pm 0.4 \mathrm{ng} / \mathrm{mL}$ (all $\mathrm{p}<0.001$ ). The patients who survived had higher serum concentrations than those who died: C6 $38.6 \pm 5.9$ versus $28.3 \pm 1.7$ $\mathrm{ng} / \mathrm{mL} \quad(\mathrm{p}=0.002) ; C 8 \quad 20.7 \pm 4.7$ versus $11.8 \pm 1.2 \mathrm{ng} / \mathrm{mL} \quad(\mathrm{p}=$ $0.001)$; C9 $37.7 \pm 4.8$ versus $31.8 \pm 3.8 \mathrm{ng} / \mathrm{mL}(p=0.042)$; and C12 $7.9 \pm 0.6$ versus $7.3 \pm 0.5 \mathrm{ng} / \mathrm{mL}(p=0.022)$. The association was present between severity of metabolic acidosis, anion gap, and the acute concentrations of measured biomarkers: C6 $(r=-0.39$; $\mathrm{p}=0.046) ; \mathrm{C} 7(\mathrm{r}=-0.42 ; \mathrm{p}=0.035) ; \mathrm{C} 8(\mathrm{r}=-0.48 ; \mathrm{p}=0.012) ; \mathrm{C} 9$ $(r=-0.39 ; p=0.046) ; C 11(r=-0.47 ; p=0.015)$. All measured concentrations of acute biomarkers positively correlated with acute serum concentrations of leukotrienes (all $p<0.05$ ).

Conclusion: Acute elevation of serum concentrations of reactive carbonyl compounds in methanol-poisoned patients suggests its involvement in the neuroinflammatory response to toxic brain damage.

\section{Acknowledgements}

Supported by the Ministry of Health of the Czech Republic (AZV), grant No. 16-27075A, the Project 43/16/RPZP, and the Projects PROGRES Q25 and Q29 of Charles University in Prague.

\section{Reference}

[1] Zakharov S, Kotikova K, Nurieva O, et al. Leukotriene-mediated neuroinflammation, toxic brain damage, and neurodegeneration in acute methanol poisoning. Clin Toxicol (Phila). 2017;55:249-259. 


\section{Identification of toxic inhalation hazards using the in vitro CULTEX ${ }^{\circledR}$ Radial Flow System (RFS)}

\author{
Amelie Tsoutsoulopoulos ${ }^{\mathrm{a}}$, Katrin Gohlsch ${ }^{\mathrm{b}}$, \\ Niklas Möhle ${ }^{c}$, Andreas Breit ${ }^{\mathrm{b}}$, Harald Mückter ${ }^{\mathrm{b}}$, \\ Thomas Gudermann ${ }^{\mathrm{b}}$, Olaf Krischenowski ${ }^{\mathrm{c}}$, \\ Sebastian Hoffmann ${ }^{d}$, Horst Thiermann ${ }^{\text {a }}$, \\ Michaela Aufderheide ${ }^{c}$ and Dirk Steinritz ${ }^{a}$ \\ a Bundeswehr Institute of Pharmacology and Toxicology, Munich,

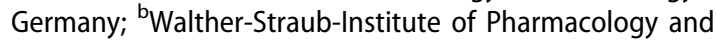 \\ Toxicology, Munich, Germany; ${ }^{C}$ Cultex ${ }^{\circledast}$ Laboratories $\mathrm{GmbH}$, \\ Hannover, Germany; ${ }^{d}$ seh consulting + services, Paderborn, \\ Germany
}

Objective: Inhalation of airborne particles with subsequent poisoning accounts for approximately $5 \%$ of cases reported to poison control centers (PCC). However, reliable data on human pulmonary toxicity are very limited. Thus, animal experiments are frequently performed to provide industry, healthcare systems and PCC with the required information. Over the past few years, animal experiments have been criticized from an animal welfare perspective. Therefore, alternatives for the assessment of pulmonary toxicity that focus on animal-free in vitro methods in order to replace, reduce or refine animal experiments (the " $3 \mathrm{R}^{\prime}$ principle of Russell and Burch) are necessary. However, no alternative in vitro methods have been sufficiently validated so far. We investigated the CULTEX ${ }^{\circledast}$ Radial Flow System (RFS) in vitro exposure system that enables a homogenous exposure of cultivated cells to airborne particles at the air-liquid interface (ALI), showing substantial similarity to the physiological conditions in the human alveolar region and the inhalation situation. A former pre-validation study (BMBF 0315710) successfully demonstrated the general applicability of the CULTEX ${ }^{\oplus}$ RFS, its transferability, stability and reproducibility. Based on these results, the aim of the present study was optimization and validation of the CULTEX ${ }^{\oplus}$ RFS and the generation of a prediction model for acute inhalation toxicity testing.

Methods: In three independent laboratories, human lung epithelial cells were exposed under ALI conditions to 20 coded test substances with available in vivo reference data. These substances were selected based on pre-defined criteria by screening Registration, Evaluation, Authorisation and Restriction of Chemicals (REACH) registration dossiers and the literature. Cytotoxicity, used as an indicator of toxicity, was compared to the respective incubator controls. Substances were considered to pose an acute inhalation hazard when viability decreased below $50 \%$ (prediction model (PM) 50\%) or $75 \%$ (PM 75\%) at any of the three exposure times (15, 30 or 60 minutes).

Results: Results of the three laboratories were compared to existing in vivo animal data and revealed an overall concordance of $85 \%(17 / 20)$, with a specificity of $83 \%(10 / 12)$ and a sensitivity of $88 \%$ (7/8). Depending on the applied PM, the within-laboratory and between-laboratory reproducibility ranged from $90-100 \%$. The low rate of non-valid experiments $(7 / 138=5 \%)$ is strong evidence of the robustness and transferability of the CULTEX ${ }^{\oplus}$ RFS.

Conclusion: The CULTEX ${ }^{\circledR}$ RFS is an appropriate in vitro screening method for the assessment of substances regarding their acute toxic inhalation potential. It is a very powerful tool that helps to support PCC in the assessment of the toxicity of airborne particles.

\section{The molecular imaging contrast agent, cationic ferritin, does not result in toxicity in a murine model of chronic kidney disease}

\author{
Asaad I Alsufyani ${ }^{a}$, Kimberly A Deronde ${ }^{b}$, \\ Nathan P Charlton ${ }^{a}$, Helen P Cathro ${ }^{c}$, \\ Kevin M Bennett ${ }^{\mathrm{d}}$ and Jennifer R Charlton ${ }^{\mathrm{e}}$ \\ ${ }^{a}$ Emergency Medicine, Division of Medical Toxicology, University of

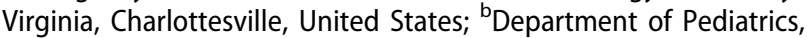 \\ University of Virginia, Charlottesville, United States; 'Department \\ of Pathology, University of Virginia, Charlottesville, United States; \\ ${ }^{\mathrm{d}}$ Mallinckrodt Institute of Radiology, Washington University, St. \\ Louis, United States; ${ }^{\text {DDepartment }}$ of Pediatrics, Division of \\ Nephrology, University of Virginia, Charlottesville, United States
}

Objective: Cationic ferritin (CF) has been used as a targeted magnetic resonance contrast agent to measure nephron endowment within the kidneys of healthy animal models and in ex vivo human kidneys. Ferritin is a highly conserved protein that forms an iron oxide core, abundantly derived from the spleen of horses. To translate CF-enhanced magnetic resonance imaging (MRI) to use in humans, however, the toxicity of the agent must be assessed in models of chronic kidney disease (CKD). We hypothesize that horse-based CF will not induce renal injury, iron toxicity, or immunologic reaction in a mouse model of CKD.

Methods: CKD was induced in adult mice $(n=22) 4$ weeks after intraperitoneal injection of folic acid $(125 \mathrm{mg})$. Animals received intravenous CF $(0.0575 \mathrm{mg} / \mathrm{g}$ body weight) or saline, were monitored for a week then euthanized (five males, six females/group). Serum was analyzed. Kidneys, liver and spleen tissues were weighed and normalized to body weight. Tissues were stained with Prussian Blue to semi-quantitatively score iron content (0-4). Periodic acid Schiff highlighted wedge-shaped areas of kidney damage to assess CKD severity.

Results: CKD severity, based on the number of wedge-shaped lesions (median CF: 4 versus saline: $4.5, \mathrm{p}=0.90$ ), and weight change (median CF: -1.2 versus saline: $2.5 \%, p=0.08$ ) were not different between the CF-treated and saline controls. There was no kidney specific toxicity associated with CF: no difference in kidney weight/body weight (median CF: 9.8 versus saline: $8.64 \mathrm{mg} / \mathrm{g}, \mathrm{p}=$ 0.89 ) or renal function (creatinine, median CF: 0.2 versus saline: 0.2 $\mathrm{mg} / \mathrm{dL}, \mathrm{p}=0.38$ or blood urea nitrogen (BUN), median CF: 42 versus saline: $29 \mathrm{mg} / \mathrm{dL}, \mathrm{p}=0.14)$. The animals receiving CF exhibited no iron toxicity with no difference in liver weight/body weight (median CF: 51 versus saline: $49 \mathrm{mg} / \mathrm{g}, \mathrm{p}=0.56$ ), liver function tests (AST median CF: 129 versus saline: $54 \mathrm{U} / \mathrm{L}, \mathrm{p}=0.46$ and ALT median CF: 29 versus saline: $47 \mathrm{U} / \mathrm{L}, \mathrm{p}=0.71$ ), or serum iron (median $\mathrm{CF}$ : 117 versus saline: $106 \mu \mathrm{g} / \mathrm{dL}, \mathrm{p}=0.86$ ). There was no difference in white blood cell counts (median CF: and saline: $1.5 \mathrm{k} / \mu \mathrm{L}, \mathrm{p}=0.89$ ), however, there was more iron deposited in the liver (median CF: 2 versus saline: $0, p=0.0001$ ) and lung (median CF: 1 versus saline: 0 , $\mathrm{p}=0.034$ ) of animals receiving CF than saline.

Conclusion: Horse-based CF does not induce renal or iron toxicity or an immune reaction in MRI-detectable doses in a murine model of CKD. These data provide further support for the use of $\mathrm{CF}$ in patients. 


\section{New formulations of endomorphin-2 peptide analogue to reduce opioid-like respiratory toxicity: an experimental investigation}

\author{
Christophe Camillerapp ${ }^{a}$, Lucie Chevillard ${ }^{a}$, \\ Charlotte Martin $^{b}$, Patricia Risède ${ }^{a}$, Steven Ballet ${ }^{b}$ and \\ Bruno Mégarbane ${ }^{a}$ \\ a Department of Medical and Toxicological Critical Care, Lariboisière \\ Hospital, Paris-Diderot University, INSERM UMRS-1144, Paris, \\ France; ${ }^{b}$ Research Group of Organic Chemistry, Vrije Universiteit \\ Brussel, Brussels, Belgium
}

Objective: Fatal overdoses secondary to opioid analgesic misuse have been exponentially increasing for 10 years, representing a major public health issue. Therefore, pharmacological research has focused on synthetic derivatives of endogenous opioid peptides and development of innovative extended release formulations to achieve better analgesic control with a lower risk of neurorespiratory toxicity.

Methods: The antinociceptive (using hot plate) and respiratory (using whole body plethysmography) effects of an endomorphin2 peptide analogue, $\mathrm{CM}-80$, its co-formulation with hydrogel and CM-57 biogel (two distinct injectable amphipathic peptide-based Hydrogels [1]) were investigated in the Sprague-Dawley rat and their respective $\mathrm{ED}_{50}$ measured. Drugs were administered by subcutaneous route. Areas under the curve of the time-course of the different parameters were calculated and comparisons between the different subgroups were performed using ANOVA for one factor followed by Dunnett's tests for multiple comparisons.

Results: In comparison to the control, only the co-formulation induced marked and prolonged antinociceptive effects $(P<$ $0.001)$. By contrast, all three products triggered significant dosedependent increase in inspiratory time $(P<0.01)$ and respiratory depression $(P<0.05)$. Based on analgesia/ventilation $E_{50}$ ratios (0.6 versus 1.2 versus 1.6$)$ and on the therapeutic indexes (2.3 versus 1.3 versus 2.1 ), only the $C M-80$ peptide presented a favorable benefit, however with a limited index.

Conclusion: The tested formulations were unable to reduce $C M$ 80 -attributed opioid-mediated respiratory toxicity in the rat. CM80 peptide, its coformulation and biogel, have thus limited potential therapeutic benefit. Establishing new formulations with less toxicity for analgesic opioids remains a major but difficult aim for future research.

\section{Reference}

[1] Oyen E, Martin C, Caveliers V, et al. In vivo imaging of the stability and sustained cargo release of an injectable amphipathic peptidebased hydrogel. Biomacromolecules. 2017;18:994-1001.

\section{Use of a new model, Ex vivo Eye Irritation Test (EVEIT), to evaluate bromide toxicity}

\author{
Adeline Navarro $^{a}$, Norbert Schrage ${ }^{b}$, Hervé Coudouel ${ }^{c}$ \\ and Laurence Mathieu ${ }^{\mathrm{C}}$ \\ ${ }^{\text {a S}}$ cientific Communication, PREVOR, Angleur, Belgium; ${ }^{b}$ University \\ Hospital Aachen, Aachen, Germany; 'PREVOR, Valmondois, France
}

Objective: Bromine is a highly corrosive, powerful oxidant, whose vapors can cause pulmonary edema. Bromine is very volatile and can provoke delayed skin or eye lesions [1]. These lesions appear slowly, first with a brown discoloration, then tissue necrosis and finally formation of slow-healing ulcers [2]. Little knowledge exists about the action of bromide in direct contact with corneas. So the aim of this study was to evaluate the ocular toxicity of bromide.

Methods: The EVEIT model replaces live animal experiments and uses eyes recovered from butcheries. Eyes from freshly slaughtered rabbits were enucleated no more than 4 hours after death and were kept at $4{ }^{\circ} \mathrm{C}$. After enucleation the eyes were examined, no epithelial damage or opacification were seen. We exposed the eyes for 20 seconds with a $10 \mathrm{~mm}$ diameter filter paper (Macherey and Nagel) containing $20 \mu \mathrm{L}$ of bromine. After exposure $5 \mathrm{~mL}$ Ringer's solution was used to remove excess fluid from the surface. All eyes were evaluated by photographic documentation after one hour with a fluorescein stain. Corneas were then excised and fixed in 3.7\% formaldehyde, prepared for histology and stained with hematoxylin-eosin for microscopic evaluation.

Results: With $0.05 \%$ bromide, histology revealed a small stratification visible in the epithelium with no stromal or deep stromal reaction. The cornea and endothelium showed no structural changes. With $0.75 \%$ bromide, little erosions on the corneal surface were observed. Histology showed a slight superficial vacuolisation and distinct degeneration of the epithelium. The stroma and endothelium were completely normal. With 3\% bromide, fluorescein stain detected cornea damage. Histology showed a severely altered epithelium with large stratification with vacuoles and lysis of the superficial cellular layer. The epithelium was completely flattened but the anterior stroma appeared unchanged.

Conclusion: No deep changes were observed in the cornea in these concentrations (0.05-3\%). Endothelium and stroma stayed intact but the epithelium was severely altered with increasing concentrations, from 0.75 to $3 \%$ with only 20 seconds of exposure. We will evaluate higher concentrations to further investigate the effects of bromine on the eye.

\section{Reference}

[1] Kim IH, Seo SH. Occupational chemical burns caused by bromine. Contact Dermatitis. 1999:41:4.

[2] Price JA, Rogers JV, McDougal JN, et al. Gene expression analysis of bromine-induced burns in porcine skin. Toxicol Lett. 2008;182:69-78.

\section{Verification of diazinon poisoning of a 5-year-old boy}

\author{
Markus Siegert ${ }^{\mathrm{a}}$, Johannes Finter ${ }^{\mathrm{b}}$, Marianne Koller ${ }^{\mathrm{a}}$, \\ Horst Thiermann ${ }^{\mathrm{a}}$ and Harald John ${ }^{\mathrm{a}}$ \\ a Bundeswehr Institute of Pharmacology and Toxicology, Munich, \\ Germany; ${ }^{b}$ University Medical Center Hamburg-Eppendorf, \\ Hamburg, Germany
}

Objective: To describe the process and results of an investigation of ingestion of a suspected organophosphorus (OP) pesticide in a child.

Case report: During vacation in Western Asia, a 5-year-old boy swallowed an unknown liquid and developed signs and symptoms of a mild cholinergic crisis (no laboratory parameters available). For medical supervision he was transferred to the University Medical Center Hamburg-Eppendorf. Inhibition of butyrylcholinesterase (BChE) was detected. He was monitored but symptoms had resolved and he required no therapeutic 
intervention. However, the parents were highly concerned and wanted clarification. Therefore, blood and urine samples were sent to the Bundeswehr Institute of Pharmacology and Toxicology for the identification of the poison. At day 5 after ingestion he was in good physical shape and was discharged. As the BChE was inhibited on arrival in Hamburg, an anticholinesterase poisoning presumably by an OP pesticide was assumed. No standard methods for poison analysis were available. To identify the agent plasma samples were investigated for the presence of protein adducts with BChE and human serum albumin (HSA) by liquid-chromatography-tandem-mass spectrometry (LC-MS/MS). $\mathrm{BChE}$ was extracted from plasma using immunomagnetic separation followed by proteolysis using pepsin to produce a nonapeptide containing the poison-modified serine ${ }^{198}$ residue. To yield poison-modified tyrosine residues derived from HSA, plasma samples were directly treated with pronase. For the identification of the poison, it's leaving group as well as other biotransformation products, plasma proteins were precipitated with acetonitrile and the supernatant was analyzed by LC-MS/MS. In addition, the diethyl ether layer obtained after liquid-liquid extraction of the plasma samples was analyzed by gas chromatography-mass spectrometry (GC-MS). Furthermore, urine was monitored for hydrolysis products of OP pesticides by LC-MS/MS. BChE-adducts of diethyl phosphate as well as HSA-adducts of diethyl phosphate and diethyl thiophosphate were detected in plasma. Furthermore, diethyl phosphoric acid and diethyl thiophosphoric acid were found in urine. These compounds are typical biomarkers of diethyl phosphorothioate poisoning indicating biotransformation of a thiono pesticide into its more toxic oxono derivative. Diazinon, its oxono variant diazoxon, as well as their leaving group 2-isopropyl-6-methyl-4-pyrimidinol were identified in the supernatant after plasma precipitation. In addition, diazinon was also found by GC-MS.

Conclusion: Despite only mild poisoning the combination of diverse modern mass spectrometric techniques used for analysis of plasma and urine allowed identification of the unknown poison. Protein-adducts, the pesticide, as well as its biotransformation products proved ingestion of diazinon. Therefore, bioanalytical investigations confirmed intoxication and provided clarification of the parent's concerns.

\section{Homicidal poisonings in the US: an analysis of the Federal Bureau of Investigation's Uniform Crime Reports from 1976-2015}

\author{
Christopher P Holstege, Micaela E Saathoff, \\ Samuel C Holstege, Duc Anh Ngo and Saumitra Rege \\ Emergency Medicine/Medical Toxicology, University of Virginia, \\ Charlottesville, United States
}

Objective: Homicidal poisonings are rare in society. This study was conducted to determine the trends in homicidal poisoning cases in the US and the characteristics of those poisonings.

Methods: The US Federal Bureau of Investigation's Uniformed Crime Reports (UCRs) was queried for all Homicidal Poisonings from 1976 to 2015. We descriptively assessed the demographic characteristics of each case, including age/race of victim and offender, relationship of offender to victim, and circumstances. Trends in homicidal poisoning were determined based on each decade.

Results: There were a total of 632,328 US homicides reported to the UCRs from 1976 to 2015 . Of those, 1,932 were defined as Homicidal Poisonings (0.30\%). Single victim/single offender $(1,239)$ was the largest category, followed by single victim/multiple offender (342) and single victim/unknown offenders (274). Of the multiple victim category, multiple victims/single offender
(79) was the largest. In regards to relationship to victim, the most common was acquaintance (441), followed by friend (182), son (181), daughter (141), wife (141), husband (78) and mother (41). The offender was more commonly male (999) followed by female (657), with the remaining cases unknown. The victim was more commonly male $(1,077)$ followed by female $(851)$, with the remaining cases unknown. By offender race, white $(1,359)$, black (240), and Asian (25) were the most common. By victim race, white $(1,611)$, black $(251)$, and Asian (29) were the most common. The offender age was as follows: 10-19 $(n=10), 20-29(n=504)$, 30-39 $(n=490), 40-49(n=270), 50-59(n=151), 60-69(n=49)$, and the remaining cases were over 70 or unknown. Victims by years of age were as follows: 0-9 $(n=343), 10-19(n=193), 20-29$ $(n=475), 30-39(n=321), 40-49(n=221), 50-59(n=122), 60-69$ $(n=94)$, and the remainder were over 70 or unknown. The average number of poisonings per year has been increasing with each decade: 1976-1985 $(n=41), 1986-1995(n=43), 1996-2005$ $(n=53)$, and 2006-2015 $(n=75)$.

Conclusion: This study of the UCRs data demonstrates a rising number of homicidal poisonings reported each year in the US despite a decreasing trend in overall US homicides. Contrary to other reports, males comprised the highest number of offenders and of victims. In regards to relationship with victim, homicidal poisoners are most often acquaintances or friends of the victim. It is important to note that the UCRs only documents detected poisonings; a significant number of these crimes potentially go undetected/unreported.

\section{Octodrine adulteration in sports supplements: two cases of adverse effects in healthy young men}

\author{
Chantal CJ Roelen ${ }^{a}$, Antoinette JHP Van Riel ${ }^{a}$, \\ Bastiaan J Venhuis ${ }^{b}$, Dana Ohana ${ }^{\mathrm{b}}$, \\ Pauline M Verputten ${ }^{a}$ and Irma De Vries ${ }^{a}$
}

${ }^{a}$ Dutch Poisons Information Center, University Medical Center,

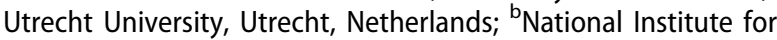
Public Health and the Environment (RIVM), Bilthoven, Netherlands

Objective: The Dutch Poisons Information Center (DPIC) monitors cases regarding dietary supplements. Sports supplements can contain amphetamine-type stimulants $[1,2]$ and if serious symptoms are reported after supplement use, we try to obtain a sample of the product for analysis to determine the cause-effect relationship. Samples were analyzed by the National Institute for Public Health and the Environment (RIVM) using ultra-high performance liquid chromatography-quadrupole time-of-flight mass spectrometry (UPLC-QTOF-MS/MS) with octodrine (2-amino-6methylheptane) and caffeine as reference standards. Here we describe two cases where a relatively new experimental stimulant was demonstrated in sports supplements.

Case reports: Case 1: A 33-year-old male took one capsule of a sports supplement called T6 before his workout. During the exercise he experienced palpitations, tachycardia, chest pains and dyspnea. Symptoms lasted for several hours before he decided to attend the emergency department (ED). He was monitored for several hours, no abnormalities were detected and he was discharged without sequelae. Case 2 concerned a 27-year-old male who took one "scoop" ( $4.5 \mathrm{~g}$ ) of a pre-workout powder called Black Booster, prior to his workout. A few hours after training, he developed agitation and restlessness and presented at the ED with electrocardiogram (ECG) abnormalities (peaked T-waves) and mildly elevated creatine kinase (CK $7300 \mathrm{U} / \mathrm{L}$ ). He was hospitalized because of rhabdomyolysis with rising CK levels (23699 U/L), despite IV fluid therapy (4 $\mathrm{L}$ saline $0.9 \%)$. After 5 days he left the hospital against medical advice; his CK levels were still elevated $(18605 \mathrm{U} / \mathrm{L})$. Both products had 1,3-dimethylamylamine 
(1,3-DMAA) listed on their label, however, analysis demonstrated the experimental stimulant octodrine in both products; $19 \mathrm{mg} /$ capsule in T6 and $30.4 \mathrm{mg} / \mathrm{scoop}(4.5 \mathrm{~g})$ in Black Booster. Therapeutic oral doses of octodrine (obsolete) ranged from 8 to $33 \mathrm{mg}[1]$.

Conclusion: Two sports supplements declaring the banned ingredient 1,3-DMAA on their label, actually contained the unregulated stimulant octodrine. To our knowledge, this is the first reported adulteration with octodrine in sports supplements in Europe. Currently the Dutch Inspectorate for Health and Youth (IGJ) is considering an enforcement level of $100 \mathrm{mg}$ of octodrine daily.

\section{References}

[1] Cohen PA, Travis JC, Keizers PHJ, et al. Four experimental stimulants found in sports and weight loss supplements: 2-amino-6methylheptane (octodrine), 1,4-dimethylamylamine (1,4-DMAA), 1,3-dimethylamylamine (1,3-DMAA) and 1,3-dimethylbutylamine (1,3-DMBA). Clin Toxicol (Phila). 2018;56:421-426.

[2] Catalani V, Prilutskaya M, Al-Imam A, et al. Octodrine: New questions and challenges in sport supplements. Brain Sci. 2018;8:E34.

\section{2. "Roar again": analysis of a sexual enhancer bought on the Internet}

\author{
Xavier Duval ${ }^{\mathrm{a}}$, Patricia Compagnon ${ }^{\mathrm{b}}, \mathrm{M}$ Palayer $^{\mathrm{b}}$, \\ Marie Deguigne ${ }^{a}$ and Gaël Le Roux ${ }^{a}$ \\ ${ }^{a}$ Centre Antipoison et Toxicovigilance Grand Ouest, $\mathrm{CHU}$ d'Angers, \\ Angers, France; ${ }^{b}$ Laboratoire de Pharmacologie-Toxicologie, CHU \\ d'Angers, Angers, France
}

Objective: A man contacted the western France Poison Center surprised by the amazing effectiveness of a product called Stimulion ${ }^{\circledR}$ (dry extract of Lepidium meyenii tuber, Rhodiola rosea root, Panax ginseng leaf, Astragalus membranaceus root, Vitis vinifera seed, Cinnamomum verum bark, zinc, and vitamins E, B6, B1, B9, B12). The Poison Control Center sent two capsules to the laboratory for qualitative and quantitative analysis.

Methods: The content of each capsule $(0.45 \mathrm{~g})$ was dissolved in $20 \mathrm{~mL}$ of a water/methanol mixture and analyzed by high-performance liquid chromatography with diode-array detection (HPLC-DAD). The identification was performed by spectral comparison using a spectrum library. Quantification was performed against a calibration range with the reference molecule.

Results: The capsules analyzed contained $87.2 \mathrm{mg}$ and $87.6 \mathrm{mg}$ of sildenafil, respectively. The patient ingested 3 capsules (about $270 \mathrm{mg}$ ) which represent 2.7 times the maximum recommended dose of sildenafil in the treatment of erectile dysfunction. The patient did not present any symptoms, especially priapism. The analysis bulletin was sent to the French Drug Agency (ANSM) to draft a health policy Decision against the operator of the website that markets the product. The alert was sent to the Health Security Agency (ANSES) to alert the French PCC network and the National Nutrivigilance Scheme.

Conclusion: The use of such substances without knowledge, or when misinformed, may have serious health consequences. Similar cases have already been described in the EU. Indeed, other 5-phosphodiesterase inhibitors have been found in such dietary supplements, at different doses. The regulatory uncertainty surrounding Internet sales of dietary supplements and the wide variety of products offered require special monitoring.

\section{Fatal 2,4-dinitrophenol (2,4-DNP) ingestion and development of a novel laboratory testing methodology for post-mortem quantification}

\author{
Varun Vohra ${ }^{a}$, Joseph O Jones ${ }^{b}$, Jeanna M Marraffa ${ }^{a}$, \\ Michael G Holland ${ }^{\mathrm{a}}$, Christine Giffin ${ }^{c}$, Carolyn Kappen ${ }^{c}$ \\ and Jeffrey $\mathrm{H}$ Moran ${ }^{\mathrm{b}}$ \\ ${ }^{a}$ SUNY Upstate Medical University, Upstate New York Poison \\ Center, Syracuse, United States; ${ }^{b}$ PinPoint Testing LLC, Little Rock, \\ United States; 'Erie County Medical Examiner's Office, Buffalo, \\ United States
}

Objective: 2,4-Dinitrophenol (2,4-DNP) is advertised as an unapproved weight loss compound. The mechanism of action involves the production of a hypermetabolic state by uncoupling oxidative phosphorylation [1]. Although reports of severe toxicity and death following 2,4-DNP exposure are documented, laboratory testing of concentrations is currently limited. We describe a case of a 27-year-old male inmate found unresponsive and pulseless after a reported ingestion of $4-8 \mathrm{~g}$ of 2,4-DNP. On arrival to the Emergency Department (ED), the patient was asystolic and return of spontaneous circulation (ROSC) was never achieved. Our objective is to highlight a fatal 2,4-DNP ingestion and the subsequent development of a novel testing methodology to quantify concentrations.

Methods: Quantification of 2,4-DNP concentrations was performed using blood specimens using a custom ToxBox ${ }^{\circledast}$ liquid chromatography tandem mass spectrometry (LC-MS-MS) method. Sample extracts were analyzed using an Agilent 1260 quaternary liquid chromatography system coupled to an Agilent 6420 LC-MS/ MS. Standards were manufactured in a 96wellplate format to deliver precise concentrations. The final internal standard concentration in $250 \mu \mathrm{L}$ blood samples was $50 \mathrm{ng} / \mathrm{mL}$ for 2,4-dinitrophenol-d3. The custom DNP ToxBox ${ }^{\oplus}$ LC-MS/MS method specified in the package insert utilized $5 \mu \mathrm{L}$ injections on a $2.6 \mu \mathrm{m}$ Phenomenex ${ }^{\circledR}$ Kinetex $^{\circledR}$ Phenyl-Hexyl (50 $x 4.6 \mathrm{~mm}$ ) LC column heated to $35^{\circ} \mathrm{C}$. The level of detection is $0.001 \mu \mathrm{g} / \mathrm{mL}$.

Results: The post-mortem blood specimen concentration 2,4DNP was $70.0 \mu \mathrm{g} / \mathrm{mL}$. The autopsy revealed the cause of death due to cardiac arrest.

Conclusion: We report a fatal 2,4-dinitrophenol ingestion and subsequent post-mortem quantification using a novel analytical testing methodology. Previous fatalities due to acute oral overdose of 2,4-DNP have been reported in literature with blood concentrations ranging between $12-99 \mathrm{mg} / \mathrm{L}$ [3]. Despite federal legislation banning the use of DNP for weight loss, its purchase and use continue to be reported [2]. Limited capability exists to confirm the presence of 2,4-DNP in laboratory testing and is therefore seldom employed. This study highlights the development of a novel testing method to quantify blood 2,4-DNP concentrations in post-mortem samples.

\section{References}

[1] Grundlingh J, Dargan P, El-Zanfaly $M$, et al. 2,4-Dinitrophenol (DNP): A weight loss agent with significant acute toxicity and risk of death. J Med Toxicol. 2011;7:205-212. 
[2] Zack F, Blaas V, Goos C, et al. Death within 44 days of 2,4-dinitrophenol intake. Int J Legal Med. 2016;130:1237-1241.

[3] Baselt RC. Disposition of Toxic Drugs and Chemicals in Man Biomedical Publications, 11th ed. Biomedical Publications, Seal Beach, CA. 2008, p711.

\section{Quetiapine overdose causing false positive bedside urine toxicology immunoassay for tricyclic antidepressants}

\author{
Wail Lam Yip \\ Accident and Emergency, Queen Mary Hospital, Hong Kong, China
}

\begin{abstract}
Objective: Quetiapine is an antipsychotic becoming more commonly prescribed in Hong Kong. We present a case of quetiapine overdose resulting in a false positive tricyclic antidepressants (TCA) screen in bedside urine toxicology immunoassay. Such false positive results may potentially cause confusion in clinical management.
\end{abstract}

Case report: A 58-year-old gentleman with bipolar affective disorder (BAD) was found sleepy and confused in a minibus and brought to the emergency department. He was tachycardic (118 bpm) and noted to have pinpoint pupils. His bedside urine toxicology immunoassay (Abon Biopharm, Hangzhou, China) was positive for benzodiazepine and TCA. However, his electrocardiogram (ECG) did not demonstrate any features of sodium-channel blockade such as interventricular conduction delay or right axis deviation of the terminal $40 \mathrm{~ms}$ of the QRS complex. A complete blood count and basic metabolic profile were unremarkable, while ethanol, salicylate and acetaminophen were undetectable in serum. A comprehensive high-performance liquid chromatography with diode-array detection (HPLC-DAD) conducted in our clinical biochemistry laboratory later confirmed quetiapine and benzodiazepine, but was negative for TCA. He was admitted to an emergency medicine ward for observation with clinical improvement without specific interventions over two days. He was finally discharged from the ward after psychiatric assessment.

Conclusion: We present a case of quetiapine overdose resulting in false positive for TCA on a bedside urine toxicology immunoassay drug screen, likely due to structural similarity $[1,2]$. No other known cross-reactive substances were detected on our HPLCDAD testing. Such cross-reactivity may potentially cause confusion in clinical management. As quetiapine is becoming more commonly prescribed in our locality, medical practitioners should be aware of this pitfall. They should not rely solely on this false positive immunoassay result in clinical management. Drug history of the patient should be clarified, while other investigations like ECG should be considered to assist in making appropriate diagnosis and guiding prompt management.

\section{References}

[1] Saitman A, Park HD, Fitzgerald RL. False-positive interferences of common urine drug screen immunoassays: a review. J Anal Toxicol. 2014;38:387-396.

[2] Chathanchirayil SJ. False positive urine drug screening for tricyclic antidepressants in patients taking quetiapine. Aust NZJ Psychiatry. 2011;45:792.

\section{Intoxication events in Ferrara's Emergency Department in 2017: Emilia-Romagna Antidotes Reference Department}

\author{
Davide Sighinolfi ${ }^{a}$, Roberto Zoppellari ${ }^{\mathrm{b}}$, \\ Riccardo Fontanac, Brunella Quartac, \\ Paola Scanavaccac, Antonella Tallarico ${ }^{\mathrm{a}}$ and \\ Angela Ricci Frabattista ${ }^{c}$ \\ aEmergenza-Urgenza, Azienda Ospedaliero-Universitaria Ferrara, \\ Ferrara, Italy; ${ }^{\text {b} A n e s t e s i a ~ e ~ R i a n i m a z i o n e, ~ A z i e n d a ~ O s p e d a l i e r o-~}$ \\ Universitaria Ferrara, Ferrara, Italy; 'Biotecnologie, Trasfusionali e \\ di Laboratorio - Farmacia Ospedaliera, Azienda Ospedaliero- \\ Universitaria Ferrara, Ferrara, Italy
}

Objective: Since 2015 there has been activate monitoring of patients accessing the Emergency Department in Ferrara with intoxication diagnosis. Clinicians, closing the medical report, can flag the "Intoxication" option, and information is sent to the hospital pharmacist. The aim is to implement an antidote database accessible via the Internet (Antidotes Internet Portal, AIP) in order to properly manage intoxication-related patients. The aim of the project is to analyze medical reports received via AIP in 2017 in order to understand which intoxications and antidotes have a special relevance for acute medicine.

Methods: Medical reports from 2017 involving intoxication were analyzed. Reported intoxications included poisoning involving drugs, chemicals, plants, fungi, animal bites, medical devices, nutritional supplements and pharmaceutical drugs; focus was on the context of the intoxication (accidental, intentional or due to a history of abuse with overdose, according to the E-ICD-9-CM). Clinical cases were divided by age, gender, toxicant, antidote and context.

Results: Overall 71 cases were evaluated with median age 50.7 years; $59.2 \%$ were female. In $47.8 \%$ the intent was self-harm, $28.2 \%$ accidental and $23.9 \%$ due to abuse. Responsible toxicants were pharmaceutical drugs, used alone or in combination (64.8\%), chemicals $(18.3 \%)$, ethanol (8.5\%), psychotropic drugs (7.0\%) and plants (1.4\%). Of pharmaceutical drugs, most cases involved benzodiazepines (45.7\%), followed by non-opioid analgesics-antipyretics-antirheumatic drugs (15.2\%), dabigatran-idarucizumab (8.7\%), antidepressants (4.3\%), opioids (4.4\%), and antidiabetic medications, diuretics, beta-blockers, anti-hypertensive medications (2.2\%). In $59.2 \%$ of cases an antidote was used (flumazenil $33.3 \%$, activated charcoal $26.2 \%$, idarucizumab $9.5 \%, \mathrm{~N}$ acetylcysteine $9.5 \%$, naloxone $9.5 \%$, fomepizole $4.8 \%$, and sodium bicarbonate, diazepam and vitamin $\mathrm{K} 2.4 \%$ each). In $21.1 \%$ of cases pharmacological treatment without antidotal therapy was required; of these, drugs to treat symptoms were used in 13 cases, including gastroprotectants (8.5\%), benzodiazepines for psychomotor activity (4.2\%), and aerosolized cortisone after toxic gas inhalation (2.8\%). In the remaining $19.7 \%$ of cases, the use of an antidote or other drug therapy was not necessary. The second largest group comprised a broad variety of different chemical products: dishwashing agents, shampoo, but also corrosive drain cleaners and toxic alcohols such as ethylene glycol.

Conclusion: The monitoring of Emergency Department accesses and data collection in the AIP database helped maintain appropriate clinical practice and ensure proper management of intoxicated patients. Data collection is important at a clinical epidemiologic level and even more in the logistic management of antidote activities (rare antidotes, expiry dates, budget impact) 
and in order to build a stronger connection between clinicians, pharmacists, and chemical analytical toxicological laboratories, improving the efficiency of the regional health system.

\section{The effect of minimum unit alcohol pricing on toxicology presentations in Scotland}

Karen Osinski ${ }^{\mathrm{a}}$, Thomas Erlandsen ${ }^{\mathrm{b}}$, Janice Pettie ${ }^{\mathrm{a}}$, James W Dear ${ }^{\mathrm{a}}$ and Euan A Sandilands ${ }^{\mathrm{a}}$

${ }^{a}$ National Poisons Information Service (Edinburgh Unit), Royal Infirmary, Edinburgh, United Kingdom; 'bniversity of Edinburgh, Edinburgh, United Kingdom

Objective: On the 1 May 2018 Scotland became the first country worldwide to introduce Minimum Unit Alcohol Pricing (MUAP), aspiring to tackle the public health burden associated with alcohol misuse. This legislation prohibits sale of alcohol below a minimum price ( 50 pence) per unit. Although introduced primarily to target alcohol misuse the legislation may have wider public health implications. A correlation has previously been demonstrated between alcohol consumption and overdose [1]. Our objective was to analyse the effect of MUAP legislation on the number of patients presenting to hospital following drug overdose.

Methods: Data were collected on all patients presenting to the Emergency Department of the Royal Infirmary of Edinburgh following drug overdose for four months before and four months after legislation change (1 January to 30 April and 1 May to 31 August 2018). Data are presented as the total number and percentage of relevant presentations. Significance was determined by two-tailed Fisher's exact test.

Results: A total of 2679 toxicology presentations occurred during the eight month study. There was no significant difference in the number of presentations pre- and post-MUAP (pre-MUAP: 1351, $50.5 \%$ of eight month total; post-MUAP: $1328,49.5 \%$ of eight month total, $\mathrm{p}=0.55$ ). The demographics of both groups were similar, with females accounting for a greater proportion of presentations (pre-MUAP: $740,54.8 \%$ of pre-MUAP group; postMUAP: $714,53.8 \%$ of post-MUAP group, $p=0.61$ ). Similar age distributions were observed between groups with the peak age of presentation being 20-29 years. Comparing each age category revealed no statistical difference pre- and post-MUAP ( $p>0.05$ for all comparisons). No significant difference in intent was recorded following legislation (pre-MUAP: self-harm $809,59.8 \%$ of pre-MUAP group, recreational 420 (31.1\%), accidental 122 (9.1\%); post-MUAP: self-harm $838,62.3 \%$ of post-MUAP group, recreational $369(27.8 \%)$, accidental 122 (9.2\%); p > 0.05 for all comparisons). Similarly, no difference was observed in the proportion of patients reporting co-ingestion of alcohol (pre-MUAP: 447, $33.1 \%$ of pre-MUAP group; post-MUAP: $458,34.5 \%$ of post-MUAP group, $\mathrm{p}=0.46$ ).

Conclusion: This study demonstrated no significant change in frequency or demographics of toxicology presentations following the introduction of MUAP legislation in Scotland. Furthermore, there was no difference in the proportion of patients who coingested alcohol. A nationwide study is required to analyse the full public health impact of MUAP on toxicology presentations.

\section{Reference}

[1] Berman ME, Fanning JR, Guillot CR, et al. Effect of alcohol dose on deliberate self-harm in men and women. J Consult Clin Psychol. 2017;85:854-861.

\section{Increased number of consultations on opioid analgesics at the Dutch Poisons Information Center: highlighting oxycodone}

\author{
Corine C Visser, Chris Oerlemans, \\ Agnes G Van Velzen, Henneke N Mulder-Spijkerboer, \\ Dylan W De Lange, Antoinette JHP Van Riel and \\ Irma De Vries \\ Dutch Poisons Information Center, University Medical Center, \\ Utrecht University, Utrecht, Netherlands
}

Objective: From the "opioid crisis" in the US and Canada, we learned that a liberal prescription of opioid analgesics increases the risk of addiction and overdose on a large scale. In 2017, an estimated 1 in 15 adults in the Netherlands were prescribed an opioid analgesic at least once. The Dutch Poisons Information Center (DPIC) is closely monitoring exposures to opioid analgesics.

Methods: A retrospective analysis of DPIC cases involving opioid analgesics between 2007 and 2017, focussing on tramadol, morphine, oxycodone, and fentanyl. Information about patients using prescription opioids was derived from the Dutch National Health Care Institute and the Dutch Foundation for Pharmaceutical Statistics.

Results: In 2007, the DPIC received a total of 315 enquiries about single-compound formulations of opioid analgesics. This increased to 671 enquiries in 2017. A remarkable increase concerned oxycodone (258 consultations in 2017, a $732 \%$ increase compared to 2007; Table 1). This is proportionally higher than the increase in the number of patients using oxycodone (479\%). A detailed analysis of cases between 2013 and 2017 showed 15 fatal oxycodone intoxications. While $79 \%$ of patients using oxycodone are above 45 years of age, our results showed that intoxications occur across all age groups. Sixteen percent of patients in the DPIC cases were younger than $25,35 \%$ were between 25 and 44 , and $49 \%$ were 45 years or older.

Conclusion: In the past decade, the increase in opioid analgesic prescriptions (especially oxycodone) has led to a disproportional increase in consultations to the DPIC. It is encouraging that of all oxycodone users, $60 \%$ used it short-term (1 or 2 prescriptions), limiting the risk for addiction. Nonetheless, a more restrictive

Table 1. Number of patients for whom the Dutch Poisons Information Center (DPIC) was consulted and the number of patients using opioid analgesics per year.

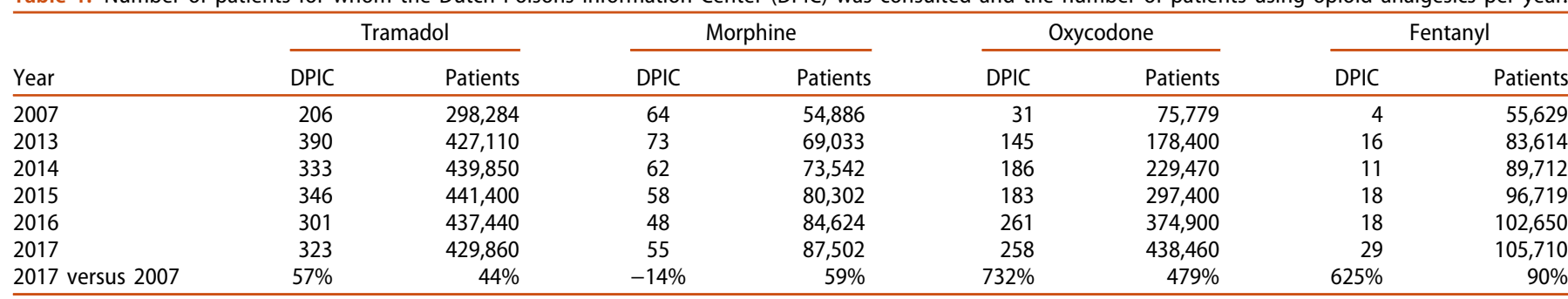


prescription policy and more attention to reduce prolonged use of opioid medication remains necessary in order to prevent an opioid crisis in the Netherlands.

\section{Two years of experience implementing an Emergency Department protocol for management of chemical submission cases}

\author{
Jordi Puiguriguer Ferrando ${ }^{a}$, Christopher Yates $^{a}$, \\ Bernardino Barcelo Martin ${ }^{b}$, Miguel Angel Elorza ${ }^{b}$, \\ Juan Ortega Perez ${ }^{b}$, Isabel Gomila Muñiz ${ }^{c}$ and \\ Catalina Homar Amengual ${ }^{\mathrm{b}}$ \\ ${ }^{a}$ Emergency Department, Clinical Toxicology Unit, Hospital \\ Universitari Son Espases, Palma de Mallorca, Spain; ${ }^{\text {bHospital }}$ \\ Universitari Son Espases, Palma de Mallorca, Spain; ' ${ }^{C}$ ospital Son \\ Llatzer, Palma de Mallorca, Spain
}

Objective: Chemical submission (CS) is the surreptitious administration of psychoactive substances with criminal intent. In 2016 a multidisciplinary emergency department (ED) protocol was created to systematise care of patients with suspected CS, sometimes related to drug-facilitated sexual assault (DFSA). The aim was to analyse clinical and toxicological characteristics of cases in which this protocol was activated during the first two years of implementation.

Methods: Patients presenting to the ED with suspected CS from 1 May 2016 to 15 August 2018 were included. Age, gender, residence, substances admitted to have been taken, CS location, arrival to ED, toxins detected (ethanol in serum; cannabis, cocaine, benzodiazepines, opiates, amphetamines and ecstasy in urine by immunoassay; gamma-hydroxybutyrate (GHB), scopolamine, ketamine, general drug screening and confirmation of positive immunoassays in urine by gas chromatography-mass spectrometry [GC/MS]) and initial clinical situation were analysed. Results: Overall 111 patients were treated (Table 1), 0.03\% of ED presentations over the period; 81 women $(72.9 \%)$, with a mean age of 27.7 (7-66) years, median 23 years. There were 58 local residents (52.2\%). Ethanol ingestion was self-reported in $62.3 \%$ cases $(n=71$, of which 30 were DFSA and 41 non-DFSA); most events occurred in a bar $(n=44,39.6 \%)$ or nightclub $(n=27$, $24.3 \%)$. The most frequent symptoms were amnesia (77.4\%), confusion (57.6\%) and decreased level of consciousness (28.8\%). In terms of laboratory confirmation, after alcohol $(n=94,82.4 \%)$, the most common toxins were cannabis $(n=27,24.3 \%)$ and cocaine $(n=23,20.7 \%)$. Comparing CS in patients with DFSA $(n=45,40.5 \%)$ and without DFSA $(n=56,59.5 \%)$, there were differences in mean age, gender (female, $p<0.001$ ), and non-residents $(p=0.05)$.

Conclusion: A CS protocol standardises the response to management of these presentations. Ethanol was the most common toxin detected, followed by cannabis and cocaine. DFSA-CS victims were younger, more likely female, and non-residents, with amnesia and confusion the predominant symptoms.

\section{In-flight opioid overdose and the availability of onboard naloxone: an international survey of commercial airlines}

\section{Josh J Wang ${ }^{a}$, Vincent Poirier ${ }^{b}$, Anna-Maria Carvalho ${ }^{c}$ Rana Biary ${ }^{\mathrm{a}}$ and Mark K Su ${ }^{\mathrm{d}}$ \\ ${ }^{a}$ Emergency Medicine, Division of Medical Toxicology, New York University School of Medicine, New York, United States; ${ }^{b}$ Emergency Medicine, McGill University Health Centre, Montréal, Canada; 'Emergency Medicine, University of British Columbia, Vancouver, Canada; ${ }^{\mathrm{d}} \mathrm{New}$ York City Poison Control Center, New York City Department of Health and Mental Hygiene, New York, United States}

Objective: Opioid overdose resulted in 42,000 deaths in the US in 2016 [1]. Published cases of in-flight opioid overdose are rare but these reports provide an incomplete view of the problem when publicly reported statistics are lacking [2]. The hypoxic, resource-austere cabin environment complicates the management of such patients even further. This study is the first to report on the occurrence and impact of in-flight opioid overdose

Table 1. Characteristics of patients with suspected chemical submission with and without drug-facilitated sexual assault (DFSA).

\begin{tabular}{|c|c|c|c|}
\hline $\begin{array}{l}\text { Variable } \\
\mathrm{N}\end{array}$ & $\begin{array}{l}\text { Drug-facilitated sexual assault (DFSA) } \\
\qquad \mathrm{n}=45(40.5 \%)\end{array}$ & $\begin{array}{l}\text { No drug-facilitated sexual assault } \\
n=66(59.5 \%)\end{array}$ & $\begin{array}{c}\text { P value* } \\
-\end{array}$ \\
\hline Gender & $43(95.5 \%)$ & $38(57.7 \%)$ & 0.001 \\
\hline \multicolumn{4}{|l|}{ Females } \\
\hline Local resident (Balearic Islands) & $18(40.0 \%)$ & $40(60.6 \%)$ & 0.05 \\
\hline Foreigner or tourist & $27(60.0 \%)$ & $26(39.4 \%)$ & \\
\hline Alcohol ingestion self-reported & $30(66.6 \%)$ & $41(63.1 \%)$ & 0.62 \\
\hline Nightclub & $10(22.2 \%)$ & 17 (25.7\%) & 0.66 \\
\hline Home & $10(22.2 \%)$ & $10(15.1 \%)$ & 0.34 \\
\hline \multicolumn{4}{|l|}{ Symptoms } \\
\hline Amnesia & 37 (82.2\%) & 49 (74.2\%) & 0.32 \\
\hline Confusion & 34 (75.5\%) & $30(45.5 \%)$ & 0.01 \\
\hline Decrease in consciousness & $8(17.7 \%)$ & $24(36.3 \%)$ & 0.03 \\
\hline \multicolumn{4}{|l|}{ Toxicological screening } \\
\hline Ketamine & $1(2.2 \%)$ & $1(1.5 \%)$ & 0.78 \\
\hline Average stay (hours) & $6: 02$ & $5: 51$ & - \\
\hline Arrival by ambulance & $10(22.2 \%)$ & $20(30.3 \%)$ & 0.34 \\
\hline
\end{tabular}


and the availability of naloxone within a large international sample of commercial airlines.

Methods: A survey was administered to all airline health and safety representatives attending the 2018 Aviation Health Conference on 25 September 2018 in London, England. Each representative answered on behalf of a single, unique commercial airline. No personal identifying data were collected. The study protocol was reviewed and exempted by the New York City Department of Health and Mental Hygiene institutional review board.

Results: The survey response rate was $88 \%(21 / 24)$ including one partial response. Carriers of all sizes from Asia, Australasia, Europe, and North America were represented. Thirty-three percent of airlines (7/21) reported at least one in-flight opioid overdose in the past 12 months, including 3/5 North American airlines. Ten percent of airlines $(2 / 21)$ reported a flight diversion due to suspected opioid-overdose in the past 12-months. Sixtytwo percent of airlines (13/20) had no naloxone available in the emergency medical kit. No airlines permitted flight crew to administer naloxone without a medical order. Interestingly, thirtyfive percent of airlines (7/20) carried opioid analgesics in the emergency medical kit and $45 \%$ of airlines (9/20) reported at least one in-flight opioid administration in the past 12-months.

Conclusion: In-flight opioid overdose on commercial airlines is common. In contrast, most airlines do not carry naloxone on board. Further efforts must be made to combat opioid overdose in the skies. We believe that making naloxone a staple component of emergency medicine kits and reducing barriers to flight crew administration would be a step in this direction.

\section{References}

[1] Seth P, Scholl L, Rudd RA, Bacon S. Overdose deaths involving opioids, cocaine, and psychostimulants-United States, 2015-2016. Am J Transplant. 2018;18:1556-1568.

[2] Hinkelbein J. Significant more research required: no further progress without sound medical data and valid denominators for inflight medical emergencies. J Trav Med. 2015;22:355-356.

\section{Most intoxications with macrolide antibiotics are the result of a medication error}

\author{
Agnes G Van Velzen, Irma De Vries and \\ Dylan W De Lange \\ Dutch Poisons Information Center, University Medical Center \\ Utrecht, Utrecht, Netherlands
}

Objective: Macrolide antibiotics are frequently prescribed to treat upper airway, skin, ear, and other infections. The number of acute intoxications with macrolide antibiotics that result from medication errors seems to be relatively high compared to other drugs. Our objective was to analyse the incidence and cause of medication errors with macrolides, with the aim to prevent overdosage in the future.

Methods: Enquiries to the Dutch Poisons Information Center (DPIC) from 2015 to 2017 involving human exposure to macrolide antibiotics were selected from the database. Corresponding telephone calls were replayed, and data on patient characteristics, exposure circumstances, and symptoms were analysed.

Results: From 2015 to 2017 the DPIC was consulted about 97 patients exposed to macrolide antibiotics. In 68 cases (70\%) overdosage was the result of a medication error. Macrolides involved in these medication errors were azithromycin $(n=59)$, clarithromycin $(n=7)$, and erythromycin $(n=2)$. Products involved were drug suspensions $(n=44)$, tablets $(n=22)$, or unknown $(n=2)$. Medication errors were made by caretakers $(n=41)$, physicians $(n=12)$, patients $(n=11)$, and pharmacists $(n=2)$. In two cases it was unclear who caused the error. Medication errors by patients and caretakers resulted from ingesting the wrong volume, taking an extra dose, or taking the drug three times a day instead of once daily for three days. In several cases of ingesting the wrong volume, the entire content of a $15 \mathrm{~mL}$ bottle (azithromycin $40 \mathrm{mg} / \mathrm{mL}$ ) was ingested at once, or the bottle was emptied when ingesting the last dose of a treatment course. Interestingly, the scenario in which the drug was taken three times a day instead of once daily for three days caused almost a third of all medication errors $(n=20)$. In many of these cases, patients/caretakers misunderstood the physician's instructions about the dosing regimen, even when the drug was prescribed and labelled correctly. In four cases however, this scenario was the result of a prescription or labelling error. Fourteen patients showed symptoms when the DPIC was contacted. Most frequently reported were gastrointestinal effects. No serious symptoms occurred.

Conclusion: Medication errors with macrolide antibiotics occur frequently, but do not result in serious toxicity. Nevertheless, medication errors should be prevented. When prescribing a suspension, it is advisable to provide patients with the smallest possible quantity of the drug, to avoid overdosing. In addition, physicians should pay attention to clear communication when instructing a patient on how to take a drug, to avoid misunderstandings about frequency and duration of dosing.

\section{Gender and age-specific increases in suicide attempts by self- poisoning in the US among youths and young adults from 2000 to 2018}

\author{
Henry A Spiller ${ }^{\mathrm{a}}$, John P Ackerman ${ }^{\mathrm{b}}$, Natalie E Spiller ${ }^{\mathrm{c}}$ \\ and Marcel J Casavant ${ }^{\mathrm{a}}$ \\ ${ }^{a}$ Central Ohio Poison Center, NationWide Children's Hospital, \\ Columbus, United States; ${ }^{\mathrm{b}}$ Center for Suicide Prevention \& \\ Research, Behavioral Health Services, NationWide Children's \\ Hospital, Columbus, United States; 'College of Medicine, University \\ of Louisville, Louisville, United States
}

Objective: Evaluate the incidence and outcomes from intentional suspected-suicide self-poisoning in children and young adults ages 10 to 27 years old from 2000 to 2018 .

Methods: Review of intentional suspected suicide cases reported to the National Poison Data System from US poison centers from 2000 to 2018 for patients 10 to 27 years old. For comparison of annual rates, population data by year of age was obtained from the US Census Bureau. We evaluated changes in 1) annual incidence, 2) annual rate/100,000 population and 3) medical outcome by patient age and gender.

Results: There were $1,888,112$ intentional suspected suicide selfpoisoning exposures of which 1,312,465 (70\%) were female. From 2000 to 2010 there was a $1.2 \%$ annual increase in the number of cases of intentional suspected suicide exposures, followed by a 6.3\% annual increase from 2011 to 2018. This increase occurred disproportionately in females and children aged 11-17 years $(p<$ 0.05). From 2000 to 2010 in 10 to 15 year olds there was a decline in the annual number and rate that ranged from 5 to $33 \%$ and 13 to $26 \%$, respectively. From 2011 to 2018 there was a dramatic increase in number and rate that ranged from $131 \%$ to $301 \%$ and $126 \%$ to $229 \%$, respectively. In the age groups 10 years old to 18 years old the increase from 2011 to 2018 was driven almost exclusively by females. In 9 to 27 years olds, in contrast to the younger age groups, there was a flattening of the curve with a decrease in slope after 2007. There were 404,837 
moderate outcomes, 57,242 major outcomes and 1,974 deaths. The largest increases occurred in the 10 to 15 year old age groups. However, these increases occurred in all age groups, both males and females, and were primarily influenced by increases in moderate outcomes. The highest number of exposures with a serious outcome occurred among ages 14 to 19 years. The percentage of cases per age group with a serious outcome, major effect or death increased with age and significantly over time $(p<0.05)$.

Conclusion: The temporal increase in the 10 to 15 year age groups and in females after 2011 coincides with the expansion and influence of social media in adolescence. The temporal increase in severity may be from acquired capability, with increased ability to search online for methods of self-harm and increase of what is shared on social media networks.

\section{Suicidal pharmaceutical overdose: evolution over 10 years}

\author{
Jordi Puiguriguer Ferrando ${ }^{a}$, Christopher Yates ${ }^{b}$, \\ Sandra García Antich ${ }^{\mathrm{b}}$, Carolina Roset Ferrer ${ }^{\mathrm{b}}$, \\ Elena Gervilla Garciac, Rafael Jimenez Lopez ${ }^{c}$ and \\ Juan Ortega Perez ${ }^{\mathrm{b}}$ \\ aEmergency Department, Toxicology Unit, Hospital Universitari Son \\ Espases, Palma de Mallorca, Spain; ${ }^{b}$ Hospital Universitari Son \\ Espases, Palma de Mallorca, Spain; ' Universitat Illes Balears, Palma \\ de Mallorca, Spain
}

Objective: Suicide is the 10th cause of death worldwide, and the second most common in people aged 15-29 years of age. In Spain, it has been the principal cause of unnatural death since 2007. We present an epidemiological study to characterise suicidal pharmaceutical overdose (SPO) comparing two years separated by a 10 year period treated at an Emergency Department (ED).

Methods: Retrospective observational analysis of SPO comparing 2007 cases with 2017. Variables analysed were age, gender, type of medication (grouped into benzodiazepines [including Z-drugs], antidepressants, antipsychotics, anticonvulsants, analgesics), number of medications implicated in the suicide attempt, coingested toxins and treatment measures. Patients younger than 15 years were excluded.

Results: Overall 451 SPO cases were seen, 64.3\% from 2007 and $35.7 \%$ from 2017 . In 2007 , they made up $26.8 \%$ of total poisoning cases and $0.23 \%$ of total ED presentations. In 2017, they were $8.7 \%$ of total poisoning cases and $0.11 \%$ of total ED presentations. Mean age was 40 years (2007) and 41.4 years (2017). Females were predominant in both periods $(p=0.011)$. The commonest pharmacological group were benzodiazepines $(63.7 \%)$ with no differences between the periods $(p=0.89)$. Polysubstance exposures were common in both periods, especially in 2007, particularly medication plus cocaine $(p=0.008)$ and medication plus ethanol $(p=0.049)$; in 2017 medication with other recreational drugs was common $(p=0.011)$. Less gastrointestinal decontamination was performed in 2017 ( $p<0.001$ ), and less antidotes were used, though the difference was not significant $(p=0.723)$. There were less admissions in $2017(p=$ 0.093). In 2007 there were three SPO deaths, and none in 2017 $(p=0.195)$.
Conclusion: A significant trend in less suicidal pharmaceutical overdoses was seen over the 10 year period. Also significant was the reduced use of gastrointestinal decontamination, as well as lower hospital admissions. There was a non-significant decrease in antidote use and deaths. Benzodiazepines were the most frequent group, with a trend towards polysubstance exposures.

\section{Poisoning in the UK: estimating incidence and circumstance}

\author{
Gillian Jackson ${ }^{\mathrm{a}}$, Simon HL Thomas ${ }^{\mathrm{b}}$, \\ John P Thompson ${ }^{c}$, Sally M Bradberry ${ }^{\mathrm{d}}$ and \\ Euan A Sandilands ${ }^{a}$ \\ ${ }^{a}$ National Poisons Information Service (Edinburgh Unit), Royal \\ Infirmary, Edinburgh, United Kingdom; ${ }^{\text {b National Poisons }}$ \\ Information Service (Newcastle Unit), Regional Drug and \\ Therapeutics Centre, Newcastle, United Kingdom; 'National \\ Poisons Information Service (Cardiff Unit), University Hospital \\ Llandough, Cardiff, United Kingdom; ${ }^{\mathrm{d}}$ National Poisons Information \\ Service (Birmingham Unit), City Hospital, Birmingham, United \\ Kingdom
}

Objective: Poisoning is a common cause of hospital presentation each year in the UK. Establishing the true incidence of poisoning is challenging however as many patients are assessed in the community avoiding hospital presentation. TOXBASE is the primary information resource for UK healthcare professionals managing poisoned patients and TOXBASE access data has previously been used as an indication of the incidence of poisoning. We have built on previous work through analysing TOXBASE access data to provide a clearer indication of the incidence and type of poisoning in the UK.

Methods: TOXBASE users were surveyed over two separate weeks (9-16 July 2018 and 20-27 August 2018) using an online voluntary questionnaire appearing every time an entry was accessed. Users were asked if their enquiry was directly patientrelated and if so, whether this was their first access to TOXBASE with respect to that patient. Users were then questioned about the circumstances of the poisoning episode (self-harm, accidental, therapeutic excess or recreational misuse).

Results: During the study period there were a total of 48,467 TOXBASE accesses, of which 19,024 (39.2\%) generated completed surveys. The data demonstrate that 17,467 (91.8\%) accesses were patient-related and in 11,477 (65.7\%) of these, it was the user's first access to the database. Of the remaining accesses for which surveys were completed, 1061 (5.6\%) were for educational reasons and in $496(2.6 \%)$ cases no reason was provided. When firsttime patient-related accesses were interrogated further, the data demonstrate that $5325(46.4 \%)$ related to an episode of selfharm, 4180 users (36.4\%) reported the poisoning was accidental, 1090 (9.5\%) reported therapeutic excess and 706 accesses (6.2\%) were regarding recreational misuse. In 176 cases (1.5\%), the user did not provide information about circumstances.

Conclusion: We have shown how TOXBASE access data can be analysed to provide a measure of the epidemiology of poisoning the UK. Accesses most commonly represented episodes of selfpoisoning, although a significant proportion (45.9\%) were due to accidental poisoning or therapeutic excess. Extrapolating this data over a year would indicate an annual incidence of approximately 766,000 cases of poisoning in the UK. Further analysis 
over a longer period is required to further delineate the true incidence of poisoning in the UK.

\section{Pack size restriction of non- opioid analgesics sold over-the- counter in Danish pharmacies: a national cohort study using nationwide register and biochemical data}

\author{
Britt Morthorst ${ }^{\mathrm{a}}$, Annette Erlangsen ${ }^{\mathrm{b}}$, Manon Chaine ${ }^{\mathrm{c}}$, \\ Frank Eriksson ${ }^{d}$, Keith Hawton ${ }^{\mathrm{e}}$, Kim P Dalhoff ${ }^{f}$ and \\ Merete Nordentoft ${ }^{\mathrm{a}}$ \\ ${ }^{a}$ Research Unit Mental Health Center Copenhagen, Danish \\ Research Institute for Suicide Prevention, Hellerup, Denmark; \\ ${ }^{\mathrm{b}}$ Danish Research Institute for Suicide Prevention, Hellerup, \\ Denmark; 'Statens Serum Institut, Copenhagen, Denmark; 'Section \\ of Biostatistics, Department of Public Health, University of \\ Copenhagen, Copenhagen, Denmark; ' Department of Psychiatry, \\ Centre for Suicide Research, Oxford University, Oxford, United \\ Kingdom; ${ }^{\mathrm{f} C l i n i c a l}$ Toxicology, Department of Clinical \\ Pharmacology, Bispebjerg and Frederiksberg University Hospital, \\ Copenhagen, Denmark
}

Objective: Paracetamol is reported to be the most frequently used drug for overdoses in Europe [1,2]. To prevent self-poisoning, pack size restriction of over-the-counter (OTC) medication has been put forward. Promising British findings [3] encouraged the Danish Minister of Health to implement such efforts. We assessed the impact of pack size restriction of non-opioid analgesics sold over-the-counter in Danish pharmacies implemented in 2013.

Methods: We obtained hospital data from nationwide registers and biochemical parameters from laboratory databases with national coverage. Specific outcomes of interest were IDC-10 codes T39 and X60 registered in the National Patient Register and the Psychiatric Central Research Register and biomarkers taken routinely during treatment for non-opioid analgesics poisonings in Denmark. We applied a before and after design using time-trend-analysis to examine the impact of the intervention.

Results: During 2002-2015 an 18.5\% reduction was noted in non-opioid analgesic poisonings treated in hospitals with an instant effect change of RR $0.815(95 \% \mathrm{Cl} 0.729$ to $0.912 ; \mathrm{p}<$ 0.000 ) after the restriction. In the age group 10-17 years intentional non-opioid analgesic poisonings decreased by $38 \%$ instantly following the intervention RR $0.622(95 \% \mathrm{Cl} 0.439$ to $0.880 ; p=0.007)$. Also, a level change in the results of liver function tests were observed. The number of tests with ALT activity $\geq 210 \mathrm{U} / \mathrm{L}$ significantly decreased at the exact time of the intervention RR $0.734(95 \% \mathrm{Cl} 0.579$ to $0.931 ; \mathrm{p}=0.011)$ showing a $27 \%$ reduced risk of liver impairment. The risk of hepatotoxicity significantly decreased by $31 \%$ with a level change in ALT $\geq 1000$ $\mathrm{U} / \mathrm{L}$ RR $0.686(95 \% \mathrm{Cl} 0.494$ to $0.953, \mathrm{p}=0.025)$ with a $40 \%$ decrease in combined tests indicative of fatal outcome.

Conclusion: The pack size restriction applied to OTC drugs of non-opioid analgesics reduced the number of poisonings treated in hospital. As noted through biomarkers the number of tests suggesting severe liver injury significantly decreased post-intervention. Evidence is provided for pack size restriction of non-opioid analgesics as an effective means restriction strategy.

\section{References}

[1] Morthorst BR, Soegaard B, Erlangsen A. Incidence rates of deliberate self-harm in Denmark 1994-2011. Crisis. 2016;37:256-264.
[2] Carroll R, Benger J, Potokar J, et al. Epidemiology, management and outcome of paracetamol poisoning in an inner city emergency department. Emerg Med J. 2015;32:155-160.

[3] Hawton K, Bergen $\mathrm{H}$, Bernal W, et al. Long term effect of reduced pack sizes of paracetamol on poisoning deaths and liver transplant activity in England and Wales: interrupted time series analyses. BMJ. 2013;346:f403.

\section{Poisoning mortality in Spain: the EXITOX study}

\author{
Jordi Puiguriguer Ferrando ${ }^{a}$, Santiago Nogue ${ }^{b}$, \\ Ana Ferrer Dufol ${ }^{\mathrm{C}}$, Lidia Garcia ${ }^{\mathrm{d}}$, August Supervía \\ and Francisca Cordoba ${ }^{f}$ \\ ${ }^{a}$ Hospital Universitari Son Espases, Palma de Mallorca, Spain; \\ ${ }^{\mathrm{b}}$ Hospital Clinic de Barcelona, Barcelona, Spain; ${ }^{{ }^{2} H o s p i t a l}$ Clinico \\ Universitario Lozano Blesa, Zaragoza, Spain; ${ }^{d}$ Hospital Parc Tauli, \\ Sabadell, Spain; ${ }^{\mathrm{e}}$ Hospital del Mar, Barcelona, Spain; ${ }^{\mathrm{f}}$ Hospital \\ Moisses Broggi, Sant Just Desvern, Spain
}

Objective: The Spanish Foundation of Clinical Toxicology (FETOC) created the EXITOX study to improve knowledge of intrahospital deaths due to poisoning. The data from the first six years of experience are presented.

Methods: Epidemiological data registry of clinical and treatment variables of poisoning-related deaths in sentinel Spanish public hospitals from 2012 to 2017 using an encrypted online data entry form. Analyzed variables were gender, mean age, single or multiple intoxication, toxin(s), intention, route of exposure, therapies (Gl decontamination, antidotes, extracorporeal treatments), cause of death and strength of relationship between poisoning and death according to a clinical toxicologist's judgement.

Results: Overall 114 cases from 111 hospitals were collected; 67 (58.7\%) were males, mean age 59.4 years (range 19-97). Suicide was the intention in 49 cases (42.3\%) and drug overdose in 31 (27.2\%). In 87 cases (76.3\%) only one poison was present, with household products $(n=37,32.4 \%)$ and medications $(n=35$, $30.7 \%$ ) the most frequently involved. In multiple ingestions, ethanol and benzodiazepines were the most common combination. The most common toxin was hydrochloric acid $(n=20,17.5 \%)$ (Table 1). Oral exposure was the most common route $(n=87$, $76.3 \%)$. Gut decontamination was used in 5 cases (4.3\%), antidotes in $36(31.5 \%)$ and extracorporeal elimination in 7 patients (6.1\%).

Table 1. Substances involved in cases of hospital death in Spain due to intoxication, 2012 to 2017 (EXITOX). Substances only registered in one case have been omitted.

\begin{tabular}{lc}
\hline Substance involved & Number of cases \\
\hline Hydrochloric acid & 20 \\
Benzodiazepines & 15 \\
Cocaine & 12 \\
Miscellaneous stimulants and street drugs & 9 \\
Ethanol & 9 \\
Methanol & 8 \\
Digoxin & 7 \\
Fumes & 7 \\
Metformin & 7 \\
Opioids & 7 \\
Acetaminophen & 7 \\
Tricyclic antidepressants & 4 \\
Cannabis & 4 \\
Sodium hypochlorite & 4 \\
Carbon monoxide & 4 \\
Ammonia & 2 \\
Heroin & 2 \\
Sodium hydroxide & 2 \\
Selective serotonin reuptake inhibitors (SSRI) & 2
\end{tabular}


The primary cause of death was multiorgan failure in 24 (21.1\%), followed by anoxic encephalopathy and corrosive damage both 22 (19.3\%). The relationship between poisoning and death was considered to be without a doubt in 73 patients (64\%).

Conclusion: FETOC coordinated the first analysis of intrahospital mortality due to poisoning in Spain over a six year period. There were more deaths among males, patients were older as compared to total poisonings and suicidal motivation was the most common cause.

\section{Acute poisoning in the elderly: substances involved and predictive factors of death}

\author{
Anne Fuster ${ }^{a}$, Ahmed S Gouda ${ }^{b}$, Karim Jaffal ${ }^{a}$, \\ Nicolas Péron ${ }^{a}$, Pierre Mora ${ }^{a}$, Hélène Migueres ${ }^{a}$, \\ Isabelle Malissin ${ }^{a}$, Sebastian Voicu ${ }^{a}$, Nicolas Deye ${ }^{a}$ \\ and Bruno Mégarbane ${ }^{a}$ \\ ${ }^{a}$ Department of Medical and Toxicological Critical Care, Lariboisière \\ Hospital, Paris-Diderot University, INSERM UMRS-1144, Paris, \\ France; ${ }^{b}$ National Egyptian Center of Environmental and \\ Toxicological Research (NECTR), Cairo University, Cairo, Egypt
}

Objective: The French population is getting older and approximately $18.8 \%$ of the population is $>65$ years of age, representing an increase of 3.7 points in twenty years. With the increase in life expectancy, the prevalence of chronic cardiovascular, metabolic and neurodegenerative diseases has increased. Our objective was to describe the critical poisonings admitted to the intensive care unit (ICU), identify the toxicants involved and determine the prognosis.

Methods: We conducted a retrospective single-center observational study including all the poisoned patients of $>65$ years of age admitted during a 12-year period (January 2007 to July 2018). Using an univariate analysis, we studied the predictive factors associated with death.

Results: Overall 287 patients (approximately 7\% of the poisoned patients admitted during the same period of time; age 74 years [69; 82] (median [percentiles 25; 75]); 62\% females and 38\% males) were included. These patients had cardiovascular (75\%), psychiatric (23\%), locomotor (23\%), neurodegenerative (15\%) morbidities and were dependent $(18 \%)$. Toxicity resulted from a multidrug ingestion [2 toxicants [1; 3], including benzodiazepines (40\%), ethanol (18\%), beta-blockers (17\%) and antidepressants (15\%)]. The main presentation on admission was impaired consciousness (Glasgow Coma Score $12[7 ; 15])$. The patients were intubated and mechanically ventilated (46\%), received antidote $(56 \%)$ and catecholamines (39\%). Complications included aspiration pneumonia (37\%), acute renal failure (26\%), hospital acquired-infection (21\%) and death (11\%). Limitation of the active therapeutics was decided in 12 patients (representing 38\% of the fatalities). The length of hospital stay was 4 days $[2 ; 8]$ with secondary transfer to another medical ward $(81 \%)$. On admission, rather than the clinical factors, elevated serum lactate $(p<0.0001)$, creatinine $(p<0.0001)$, transaminases $(p$ $<0.0001)$ and white blood cell count $(0.02)$ and decreased arterial $\mathrm{pH}(\mathrm{p}=0.002)$ and prothrombin time $(\mathrm{p}<0.0001)$ were associated with a fatal outcome.

Conclusion: Poisoning in patients aged $>65$ years admitted to the ICU are not rare, related to a higher rate of cardiovascular drugs and responsible for important morbidities and elevated mortality (11\%).

\section{Prescription versus over-the- counter (OTC) in Swedish paracetamol poisoning}

\author{
Erik Lindeman, Paula Hernandez, Helene Salmonson \\ and Johanna Nordmark Grass
}

Swedish Poisons Information Center, Stockholm, Sweden

Objective: OTC sales of paracetamol in Sweden have been stable at around 40-45 million Defined Daily Doses (DDD i.e. $3 \mathrm{~g} /$ day for paracetamol) since the turn of the millennium (data obtained from the Swedish eHealth Agency), while there has been a marked increase in prescription paracetamol (PP) sales during the same time period [1]. PP and OTC sales volumes were similar in 2000 but by 2017 PP accounted for $77 \%$ of paracetamol sales (128 million DDD). Of note, this threefold rise in PP sales occurred in parallel with a fourfold increase in paracetamol poisonings reported to the Swedish Poison Center (PC). PP is covered by public health insurance and therefore cheaper than OTC, and is sometimes free of charge. PP pack sizes typically contain 100 pills (500 $\mathrm{mg}, 1 \mathrm{~g}$ or extended release $665 \mathrm{mg}$ ) while OTC pack sizes are limited to twenty $500 \mathrm{mg}$ pills. In 2017 PP was issued to 1.2 million Swedes aged $\geq 15-85$ years (total age group size 8.2 million) [1]. In this report we endeavor to test the hypothesis that the greater volumes, low cost and large pack sizes of PP may be reflected in a greater proportion of PC cases of paracetamol poisoning.

Methods: Hospital calls to the PC concerning deliberate overdoses of paracetamol in patients older than 15 years during January and February 2018 were included. Callers were asked to find out if the pills the patient had taken were OTC or PP. A subgroup was identified as massive ingestions defined as ingestions over $30 \mathrm{~g}$ or a measured serum concentration at $2 \mathrm{x}$ nomogram level for NAC-treatment (e.g. $300 \mathrm{mg} / \mathrm{L}$ at 4 hours).

Results: Overall 214 cases were identified. In 60 (28\%) the origin of the ingested pills could not be established. In 112 (52\%) PP was the culprit and in $42(20 \%)$ OTC paracetamol was ingested. Also, 31 cases met the criteria for massive ingestion; 20 PP (64\%), 10 OTC (32\%) and one of unknown origin.

Conclusion: PP poisonings accounted for at least $52 \%$ of the total caseload and $64 \%$ of massive ingestions while prescriptions were issued to $15 \%$ of the population under study. The data fits the notion that the dissemination of high volumes of low-priced paracetamol in large packs via prescriptions increases the risk of poisoning. The short time-frame and small sample size pose significant limitations to the generalizability of our findings and further study is needed.

\section{Reference}

[1] Swedish National Board of Health and Welfare. Statistical database [cited 2018 Sep 27]. Available from: http://www.socialstyrelsen.se/ statistik/statistikdatabas/lakemedel Swedish.

\section{Adherence of care givers of children to advice provided by a National Poison Information Center}

\author{
Gal Neuman ${ }^{\mathrm{a}, \mathrm{b}}$, Adi N Miller-Barmak ${ }^{\mathrm{b}}$, Lana Khoury ${ }^{\mathrm{b}}$, \\ Iris Levdov-Avital ${ }^{\mathrm{a}}$, Maisar Salama ${ }^{\mathrm{a}}$, Taleb Moed ${ }^{\mathrm{a}}$ and \\ Yedidia Bentur ${ }^{\mathrm{a}, \mathrm{c}}$ \\ ${ }^{a}$ Clinical Pharmacology and Toxicology Section, Israel Poison \\ Information Center, Rambam Health Care Campus, Haifa, Israel;
}


${ }^{b}$ Department of Pediatrics, Rambam Health Care Campus, Haifa, Israel; 'The Rappaport Faculty of Medicine, Technion-Israel Institute of Technology, Haifa, Israel

Objective: One of the major roles of poison information centers is prevention of unnecessary visits to emergency departments (ED) due to non-toxic or minimally toxic exposures. To date, there are no published up to date data on the adherence rate of the public to the Israel Poison Information Center consultation, or factors associated with non-adherence. This study's objective was to evaluate the extent of adherence of non-medical caregivers of children to consultations provided by the Israel Poison Information Center in cases involving children 6 years old and younger, and assess the number of ED referrals that are prevented by the Poison Center.

Methods: A prospective cohort study based on a telephone survey. Calls from the public regarding children 6 years old and younger were included. The research was conducted over a year (2017/2018), evaluating calls being made during three different months representing different seasons and holidays.

Results: A total of 1785 callers completed the telephone survey during the study period. When asked whether they followed the Israel Poison Information Center advice regarding referral to the $\mathrm{ED}$, ambulatory care or home observation, $93 \%$ of the callers said they adhered to the advice provided. Most callers (1450, 81\%) were advised to remain at home and were not referred to additional medical care. Of the callers who were advised to stay home, 496 (34\%) said that they would have gone to the ED had the Israel Poison Information Center been unavailable.

Conclusion: The vast majority of pediatric care givers adhered to the Israel Poison Information Center advice regarding poisonings and poison exposures involving children 6 years and younger. The Poison Center prevented almost 500 unnecessary referrals of young children to the ED in a period of three months. This is the first prospective study to evaluate the effect of advice given by the Israel Poison Information Center in the pediatric population.

\section{Teenage oxycodone exposures reported to the US National Poison Data System}

\author{
Saumitra Rege ${ }^{a}$, Duc Anh $\mathrm{Ngo}^{b}$, Marissa Kopatic ${ }^{\mathrm{a}}$ and \\ Christopher P Holstege \\ aDivision of Medical Toxicology, Department of Emergency \\ Medicine, University of Virginia, Charlottesville, United States; \\ ${ }^{\mathrm{b}}$ Division of Student Affairs, Department of Student Health, \\ University of Virginia, Charlottesville, United States
}

Objective: According to the Substance Abuse and Health Services Administration (SAMSHA), there were 182,748 visits to emergency departments (ED) related to oxycodone products in 2010 [1]. In 2016, 3.6\% adolescents aged 12-17 years reported misusing opioids over the past year. This study examines the trends in oxycodone exposures among teenagers reported to US poison centers (PCs).

Methods: The National Poison Data System (NPDS) was queried for all oxycodone exposures in patients aged 13-19 years from 2011 to 2017. We descriptively assessed the demographic and clinical characteristics. Trends in oxycodone frequencies and rates (per 100,000 teenage exposures) were analyzed using Poisson regression. Percent changes during the study period were reported.

Results: There were 9,374 teenage exposures to oxycodone reported to the PCs from 2011 to 2017. Among these calls, the proportion of calls from acute care hospitals and EDs (ACH) increased from $47.8 \%$ to $70.2 \%$ from 2011 to 2017 . Multiple substance exposures accounted for $56.7 \%$ and $69.2 \%$ of the overall and $\mathrm{ACH}$ calls, respectively. Approximately $15.8 \%$ of the cases were admitted to the critical care unit, with $18 \%$ being admitted to a psychiatric facility. Residence was the most common site of exposure (90.4\%). Females were more frequent $(57.1 \%)$. Suspected suicide $(51.2 \%)$ was the most common reason for exposure, with intentional abuse accounting for $21.3 \%$. The proportion of suspected suicides (66.8\%) was higher among cases reported by $\mathrm{ACH}$ and increased during the study period (57.9\% to $71.9 \%)$. Minor effects $(33.2 \%)$ were the most prevalent among cases. There were 34 teenage deaths due to oxycodone. The most frequent cooccurring substance reported was benzodiazepines (7.6\%). Tachycardia and drowsiness were the most frequently demonstrated clinical effects. Naloxone was utilized as therapy for $15.6 \%$ cases and administered prior to PC recommendation in most cases. Overall, teenage oxycodone exposure calls decreased by $21.3 \%$ (95\% Cl: $-27.9 \%,-15.3 \%$; $p<0.001)$, while the rate of such exposures decreased by $13.1 \%$ ( $95 \% \mathrm{Cl}:-38.9 \%,-7.3 \%$; $\mathrm{p}<0.001$ ).

Conclusion: PC data demonstrated a decreasing trend of oxycodone exposures among teenagers, which may be attributed to the changing policies and opioid prescribing practices. However, the increase in the proportion of calls from $\mathrm{ACH}$ indicates higher severity of such exposures, especially when multiple substances were involved.

\section{Reference}

[1] Substance Abuse and Mental Health Services Administration, Center for Behavioral Health Statistics and Quality. (July 2, 2012). The DAWN Report: Highlights of the 2010 Drug Abuse Warning Network (DAWN) Findings on Drug-Related Emergency Department Visits. Rockville, MD [cited 2018 Oct 23]. Available from: https://www.samhsa.gov/data/ sites/default/files/DAWN096/DAWN096/SR096EDHighlights2010.htm

\section{Demographic trends in injury with alcohol intoxication associated with emergency department visits among students in a US public university}

\author{
Duc Anh Ngo ${ }^{a}$, Rege Saumitra ${ }^{b}$ and \\ Christopher P Holstege ${ }^{b}$ \\ ${ }^{\text {a} D i v i s i o n ~ o f ~ S t u d e n t ~ A f f a i r s, ~ D e p a r t m e n t ~ o f ~ S t u d e n t ~ H e a l t h, ~}$ \\ University of Virginia, Charlottesville, United States; ${ }^{b}$ Division of \\ Medical Toxicology, Department of Emergency Medicine, \\ University of Virginia, Charlottesville, United States
}

Objective: Few studies have explored the epidemiology of student injury presenting to the emergency department (ED) as a consequence of hazardous drinking. This study examined demographic trends in ED injury visits following alcohol intoxication among students presenting to a major US university health system.

Methods: The ED electronic medical records from 8 academic years 2010 to 2017 were queried for student visits and their records were linked to the university's student admission datasets. Student ED visits with both injury and alcohol intoxication were identified based on International Classification of Diseases (ICD) codes. Student characteristics and trends in the rate of injury ED visits associated with alcohol intoxication per 100 ED student visits were analyzed.

Results: There were 12,450 student ED visits (49\% males) to the ED of which 1,274 (10.2\%) visits involved alcohol intoxication, 4,635 (37.2\%) were related to injury, and 267 (2.14\%) involved both injury and alcohol intoxication. Over the study period, there was a declining trend in the rate of ED injury visits (from $42 \%$ to $30 \%$ ) and in the rate of ED injury visits with alcohol intoxication 
(from $6.0 \%$ to $3.8 \%$ ). The percentage of alcohol intoxication among ED visits with injury also declined from $44 \%$ to $16 \%$. There were declining trends across student demographic groups, although the rate was higher among male, younger, white, fraternities/sorority association, and undergraduate students. Few students on an athletic scholarship presented to ED because of injury following alcohol intoxication. There was a substantial variation in the rates of injury ED visits with alcohol intoxication by specific university schools (e.g. engineering, architecture, medicine) or academic programs.

Conclusion: The rate of ED injury visits following alcohol intoxication varied by student demographic characteristics and campus-related factors with a declining trend over the study period. This trend suggests effectiveness of intervention efforts, such as the Brief Alcohol Screening and Intervention (BASICS) program, the general medicine clinic screening survey for alcohol use disorders, and the dean's enhanced referral system of students to appropriate services when alcohol infractions occur, in this particular university campus in mitigating the burden of injury associated with alcohol intoxication among the student population.

\section{Antidote use in Hungary: use of second-line antidotes}

\section{Csaba Pap and István Elek}

Department of Emergency Medicine and Clinical Toxicology, Péterfy Hospital, Budapest, Hungary

Objective: An antidote, as defined by the World Health Organization (WHO), is a therapeutic substance used to counteract the toxic action(s) of a specified xenobiotic. According to an expert consensus guideline (ECOG) based on a standardized and comprehensive summary of medical literature updated in 2018 [1], 45 antidotes are recommended for stocking, and one $100-\mathrm{kg}$ patient is considered as the basis for calculating the amount of antidote to stock. We determined the availability and the need of antidotes not listed in the above-mentioned ECoG, but used at our department as the only poison centre in Hungary, in order to optimize the costeffectiveness and efficiency of stocking and administering antidotes.

Methods: A retrospective record of the weekly orders of medicaments and a retrospective analysis of cases treated at our department involving the administration of selected antidotes were performed over an almost 7 year period (January 2012September 2018).

Results: During this period we used 19 antidotes/key emergency drugs/therapeutic methods (Table 1). All these antidotes are immediately available except hyperbaric oxygen therapy. As far as the stored amount of antidotes is concerned, currently we have amounts of atropine, calcium gluconate, norepinephrine, insulin, lipid emulsion, magnesium sulphate, morphine, neuromuscular blockers and sodium bicarbonate for more than 5 patients, adenosine, folic acid, lidocaine, penicillin and silibinin for 2-5 patients and propranolol, botulin antitoxin and esmolol for one patient. Folic acid, magnesium, normobaric oxygen therapy, penicillin and silibinin were routinely used, whilst the other substances were administered only in moderate to severe poisonings.

Conclusion: Several antidotes not recommended by the ECoG but used successfully both in our department and worldwide may be of great importance to treat severe poisonings.

\section{Reference}

[1] Dart RC, Goldfrank LR, Erstad BL, et al. Expert Consensus Guidelines for stocking of antidotes in hospitals that provide emergency care. Ann Emerg Med. 2018;71:314-325.

\section{Poisoned patients admitted to the intensive care unit: experience of treatments in a case series of 184 patients}

Roberto Zoppellari ${ }^{\mathrm{a}}$, Anna L Pinamonti ${ }^{\mathrm{a}}$, Filippo Tartari ${ }^{a}$, Alessandra Mallozzi Menegatti ${ }^{a}$ Luca Bagnoli ${ }^{a}$, Chiara De Fazio ${ }^{a}$, Alessandra Quaranta ${ }^{a}$, Angela Ricci Frabattista ${ }^{b}$, Anna Talarico ${ }^{c}$ and Rosa M Gaudio ${ }^{c}$

Table 1. Antidotes not listed in the Expert Consensus Guidelines but used at our department, Hungary, 2012-18.

\begin{tabular}{|c|c|c|c|c|c|}
\hline \multicolumn{2}{|c|}{ Number of poisoned patients } & \multirow[b]{2}{*}{ Type of poisoning } & \multirow[b]{2}{*}{ Antidote } & \multicolumn{2}{|c|}{$\begin{array}{l}\text { Number of patients that } \\
\text { received antidote }\end{array}$} \\
\hline 2012-15 & 2016-18 & & & 2012-15 & 2016-18 \\
\hline 27 & 13 & Theophylline & Adenosine & 1 & 0 \\
\hline 27 & 13 & & Propranolol & 4 & 0 \\
\hline 27 & 13 & Theophylline & Esmolol & 2 & 0 \\
\hline 20 & 9 & Digoxin & & 2 & 0 \\
\hline 20 & 9 & Digoxin & Lidocaine & 3 & 0 \\
\hline 20 & 9 & & Magnesium sulphate & 16 & 7 \\
\hline 14 & 8 & Mushroom, muscarine-type & Atropine & 10 & 5 \\
\hline 7 & 4 & Botulism & Botulin antitoxin & 5 & 3 \\
\hline 131 & 76 & Beta-blockers & Calcium gluconate & 44 & 39 \\
\hline 23 & 14 & Alpha-blockers & Norepinephrine & 5 & 6 \\
\hline 100 & 91 & Ethylene glycol & Folic acid & 93 & 81 \\
\hline 0 & 2 & Methanol & & 0 & 2 \\
\hline 5 & 2 & Psychiatric drugs (seizures) & Lipid emulsion & 5 & 2 \\
\hline 82 & 47 & Cardiovascular drugs (shock) & & 37 & 16 \\
\hline 3 & 2 & Drug-induced hyperthermia & Neuromuscular blockers & 3 & 2 \\
\hline 684 & 373 & Carbon monoxide & Normobaric oxygen therapy & 644 & 346 \\
\hline 684 & 373 & & Hyperbaric oxygen therapy & 3 & 2 \\
\hline 26 & 3 & Mushroom, amatoxin-type & Penicillin & 26 & 3 \\
\hline 26 & 3 & & Silibinin & 26 & 3 \\
\hline 13 & 8 & Sodium channel blockers & Sodium bicarbonate & 11 & 7 \\
\hline 13 & 8 & Sodium channel blockers & Insulin & 7 & 6 \\
\hline 89 & 68 & Calcium channel blockers & & 27 & 19 \\
\hline 7 & 4 & Lead, mercury, arsenic & Unithiol (DMPS) & 7 & 0 \\
\hline
\end{tabular}


${ }^{a}$ Anaesthesia and Intensive Care, S. Anna Hospital, Ferrara, Italy;

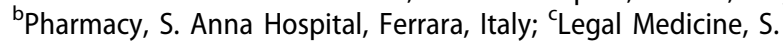
Anna Hospital, Ferrara, Italy

Objective: The correct management of some poisoned patients may require admission to the Intensive Care Unit (ICU). We studied the ICU usage in poisoned patients and the related treatments: mechanical ventilation and cardiovascular support, antidotal administration, gastrointestinal decontamination, and extracorporeal removal.

Methods: A 17-year (1 July 2001 to 30 June 2018) prospective study including all patients admitted to our adult ICU with a main diagnosis of acute poisoning. We defined three criteria for ICU admission: the presence of vital function impairment (group 1), the perception that significant organ dysfunction could appear in asymptomatic patients on the basis of toxicokinetics or toxicodynamic (group 2), and a clinical judgment for intensive observation in mildly symptomatic patients (group 3 ).

Results: In total 184 poisoned patients $(2.7 \%$ of admitted patients) were included. All toxic agents were confirmed by toxicological laboratory analysis. The number of patients was 142 for group 1, 17 for group 2 and 25 for the third. For group 1 the main causes of vital function impairment was: respiratory failure requiring ventilatory support $(n=114)$, severe cardiovascular toxicity $(n=11)$, neurological dysfunction with a Glasgow Coma Score $<11(n=14)$, and metabolic disturbance $(n=3)$. For this group antidotal administration, gastrointestinal decontamination and extracorporeal removal (dialysis) was used in 66, 99 and 6 patients, respectively. For group 2 mechanical ventilation and/or cardiovascular support was used in 7 patients, antidotal administration in 9 patients, gastrointestinal decontamination in 10 and extracorporeal removal in 4 ( 2 treated with hemoperfusion and 2 with dialysis). Group 3 required treatment with mechanical ventilation and cardiovascular support in 5 patients, antidotal administration in 4 and gastrointestinal decontamination in 15; no enhanced elimination was applied in this group.

Conclusion: A rational approach to ICU use and treatment is described. For patients who were asymptomatic (group 2) or minimally symptomatic at admission (group 3) mechanical ventilation and/or cardiovascular support was used in some cases due to an unpredictable clinical course.

\section{A tube with a view - video- assisted orogastric lavage (VAOL)}

Arie P Francis ${ }^{a}$, Elie Harmouche ${ }^{a}$, Emily S Taub ${ }^{a}$, Ben Suwing ${ }^{\mathrm{b}}$, Matthew McCarty ${ }^{\mathrm{b}}$, Rana Biary ${ }^{\mathrm{a}}$, Robert S Hoffman ${ }^{\mathrm{a}}$ and Silas W Smith ${ }^{\mathrm{a}}$

${ }^{a}$ Division of Medical Toxicology, Ronald O. Perelman Department of Emergency Medicine, NYU School of Medicine, New York, United States; ${ }^{\text {b }}$ Ronald O. Perelman Department of Emergency Medicine, NYU School of Medicine, New York, United States

Objective: Aggressive gastric emptying is often withheld in poisoning due to concerns over safety and efficacy. Despite this, endoscopy performed by emergency medicine physicians demonstrates significant retention of residual drug products many hours after ingestion [1]. Decedents may also demonstrate significant drug retention [2]. We developed a novel video technique in an attempt to improve the safety, efficacy, and completeness of orogastric lavage.

Methods: Using SolidWorks ${ }^{\mathrm{TM}}$ (Dassault Systems, Waltham, MA, 2018), we designed and produced a special Y-adapter with a polylactic acid medium using a 3D Printer (Monoprice, Rancho Cucamonga, CA, 2018) to permit side hole insertion of a disposable $\mathrm{Ambu}^{\oplus}$ aScope $^{\mathrm{TM}}$ three intubating bronchoscope (Columbia, $M D, 2018$ ) into a standard $40 \mathrm{Fr}$ orogastric lavage tube (OGT).
This allowed bronchoscope placement into the orogastric tube (OGT) with an air-tight seal and the ability to visualize through a distal side port. To simulate overdose, 50 pills (acetaminophen $500 \mathrm{mg} /$ diphenhydramine $25 \mathrm{mg}$ tablet) were placed into the mannequin stomach with $200 \mathrm{~mL}$ of tap water. The mannequin was positioned in the left lateral decubitus position; the apparatus was assembled; and gastric lavage was accomplished with 2 $L$ of tap water.

Results: We were able to easily visualize tube passage and placement into the stomach to an appropriate depth, appreciating an initially cloudy solution with numerous pill fragments. The adapted system then permitted lavage under constant bronchoscope visualization. Gastric contents were easily ascertained through the distal ports, and the stomach could be evaluated to the pylorus to exclude bezoars or remaining pill fragments. After lavage, a clear solution with no remaining evidence of pill slurry was evident. After extensive lavage, using the bronchoscope, only a small amount of pill fragments were visualized in the fundus.

Conclusion: This study demonstrates a proof of concept, linking visualization with OGT placement and active lavage. If these findings can be confirmed in vivo, our novel device may help clarify the indications, improve the safety and efficacy, and define the endpoints of orogastric lavage.

\section{References}

[1] Miyauchi M, Hayashida M, Yokota $H$. Evaluation of residual toxic substances in the stomach using upper gastrointestinal endoscopy for management of patients with oral drug overdose on admission: A prospective, observational study. Medicine. 2015;94:e463.

[2] Livshits Z, Sampson BA, Howland MA, et al. Retained drugs in the gastrointestinal tracts of deceased victims of oral drug overdose. Clin Toxicol (Phila). 2015;53:113-118.

\section{Young versus elderly patients in chronic lithium toxicity}

Sonia Cheng ${ }^{a}$, William Siu ${ }^{b}$, Angela L Chiew', Elia Vecellio ${ }^{d}$, Nicholas A Buckley ${ }^{e}$ and Betty S Chan ${ }^{c}$

${ }^{\mathrm{a}}$ Faculty of Medicine, University of New South Wales, Sydney, Australia; ${ }^{\mathrm{b}}$ Department of Emergency Medicine, Sutherland Hospital, Sydney, Australia; 'Department of Emergency Medicine \& Clinical Toxicology, Prince of Wales Hospital, Sydney, Australia; ${ }^{d}$ NSW Health Pathology, Prince of Wales Hospital, Sydney, Australia; ${ }^{e}$ Department of Clinical Pharmacology, University of Sydney, Sydney, Australia

Objective: We aimed to determine whether the predictors for severe lithium toxicity varied between an elderly and young cohort of patients with chronic lithium toxicity.

Methods: We conducted a retrospective comparison of young (15-64 years old) with elderly patients ( $\geq 65$ years old) from two hospitals with lithium concentrations $\geq 1.3 \mathrm{mmol} / \mathrm{L}$. Plasma lithium concentrations, lithium dose, medications, symptoms, treatment and outcomes were recorded. Known predictive factors of lithium toxicity were analysed in a multinomial regression analysis to quantify the risk of developing severe toxicity. The two groups were compared using either chi-square for categorical data or Mann-Whitney $U$ test for continuous data.

Results: From 2008-2018, 216 patients with elevated lithium concentrations were studied to determine if there were differences between the young and elderly cohorts (Table 1). A multinomial logistic regression revealed, that when adjusted for age and sex, mild to severe renal impairment increased the risk of moderate toxicity by 6.3 times $(95 \% \mathrm{Cl}: 1.5-25.7, \mathrm{P}=0.01)$ and increased 
Table 1. Comparison of young and elderly patients with chronic lithium toxicity. Data are medians (range) or count (proportion of relevant group).

\begin{tabular}{|c|c|c|c|}
\hline & $\begin{array}{l}\text { Young: } 15-64 \text { years } \\
(n=143)\end{array}$ & $\begin{aligned} \text { Elderly: } & \geq 65 \text { years } \\
(\mathrm{n} & =73)\end{aligned}$ & P-value \\
\hline Females (\%) & $91(63.6 \%)$ & $49(67.1 \%)$ & 0.61 \\
\hline Lithium concentration (mmol/L) & $1.400(1.3-4.1)$ & $1.500(1.3-3.2)$ & 0.31 \\
\hline Lithium dose (mg) & $1000(250-2500)$ & $500(125-1350)$ & $<0.001$ \\
\hline Estimated glomerular filtration rate (eGFR) $\left(\mathrm{mL} / \mathrm{min} / 1.73 \mathrm{~m}^{2}\right)$ & $75.5(6-90)$ & $42.0(19-90)$ & $<0.001$ \\
\hline Lithium drug interactions & $24(16.8 \%)$ & $26(35.6 \%)$ & 0.002 \\
\hline \multicolumn{4}{|l|}{ Renal impairment } \\
\hline Normal-mild (eGFR $\geq 60 \mathrm{~mL} / \mathrm{min} / 1.73 \mathrm{~m}^{2}$ ) & $102(73.9 \%)$ & $18(24.7 \%)$ & $<0.001$ \\
\hline Mild-moderate (eGFR $30-59 \mathrm{~mL} / \mathrm{min} / 1.73 \mathrm{~m}^{2}$ ) & $29(21.0 \%)$ & $43(58.9 \%)$ & $<0.001$ \\
\hline Moderate-severe $\left(\mathrm{eGFR}<30 \mathrm{~mL} / \mathrm{min} / 1.73 \mathrm{~m}^{2}\right)$ & $7(5.1 \%)$ & $12(16.4 \%)$ & 0.006 \\
\hline Infection risk & $27(18.9 \%)$ & $27(37.0 \%)$ & 0.004 \\
\hline \multicolumn{4}{|l|}{ Amdisen Score of severity of lithium toxicity [1] } \\
\hline 0 - None & $52(36.4 \%)$ & $17(23.3 \%)$ & 0.05 \\
\hline 1 - Mild & $63(44.1 \%)$ & $26(35.6 \%)$ & 0.23 \\
\hline 2 - Moderate & $9(6.3 \%)$ & $9(12.3 \%)$ & 0.12 \\
\hline 3 - Severe & $19(13.3 \%)$ & $21(28.8 \%)$ & 0.006 \\
\hline
\end{tabular}

the risk of severe toxicity by 3.9 times $(95 \% \mathrm{Cl}: 1.3-11.6, \mathrm{P}=$ 0.02). There were no fatalities.

Conclusion: Despite being on half the lithium dose, the elderly group still presented with higher rates of severe toxicity. Our results imply prescription patterns still inadequately adjust for poorer renal function in the elderly.

\section{Reference}

[1] Hansen $\mathrm{H}$, Amdisen A. Lithium intoxication (Report of 23 cases and review of 100 cases from the literature). Q J Med. 1978;47:123-144.

\section{Severe metformin poisoning in the French West Indies: incidence, early clinical and biological features and prognostic factors}

Dabor Resiere ${ }^{\mathrm{a}}$, Annuela Populo ${ }^{\mathrm{a}}$, Cyrille Charbatier ${ }^{\mathrm{a}}$, Jonathan Florentin ${ }^{\mathrm{a}}$, Didier Martin ${ }^{\mathrm{b}}$, Julien Fabre ${ }^{\mathrm{c}}$, Francois Roques ${ }^{\mathrm{b}}$, Hossein Mehdaoui ${ }^{\mathrm{a}}$ and Bruno Mégarbane ${ }^{d}$

${ }^{a}$ Critical Care Unit, University Hospital of Martinique, Martinique, France; ${ }^{b}$ Department of Cardiovascular Surgery, University Hospital of Martinique, Martinique, France; 'Department of Cardiology, University Hospital of Martinique, Martinique, France; ${ }^{d}$ Department of Medical and Toxicological Critical Care, Lariboisière Hospital, Paris-Diderot University, INSERM UMRS-1144, Paris, France

Objective: Metformin poisoning is rare but potentially life-threatening. Metformin-associated lactic acidosis (MALA) represents the main complication, combining high anion gap metabolic acidosis and hyperlactacidemia [1]. In most cases, poisoning results from metformin overdose in relation to non-compliance with contraindications in the diabetic patient. The main risk factor is the prior existence of renal failure; however, recurrent diseases leading to dehydration or tissue hypoxia have been described as precipitating factors. In the French West Indies (Martinique and Guadeloupe), metformin poisoning is likely to be accidental in the majority of cases. Poisonings due to suicide attempts are rarely reported. The incidence of metformin poisoning remains unknown as no national register exists. Thus, the aim of our study was to describe the incidence, early clinical and biological features, and prognostic factors of severe metformin poisoning in the West Indies.
Methods: Observational, prospective, multi-centric study over a period of three years between 2015 and 2018, including all successive metformin-poisoned patients admitted to the emergency medicine departments and the medical and surgical critical care units of the University Hospitals of Martinique and Guadeloupe. The clinical and biological data were collected and analyzed using univariate analysis based on chi-squared or Mann-Whitney tests as required.

Results: Forty-six patients were included [age: $67 \pm 12$ years; 23 males and 23 females; hypertension [ $n=43]$, type-2 diabetes $[n=42]$ and dyslipidemia $[n=27])$. In the majority of the cases, poisoning was due to accidental overdose. Forty-one patients presented MALA, resulting in an incidence of 35 and 39 cases per 100,000 patient-years during the last 2 years. About $88 \%$ of the patients admitted for accidental overdose presented an acute event in the recent past days. Patients most often exhibited gastrointestinal disorders with vomiting (67\%), abdominal pain (50\%) and diarrhea (39\%); however, vital signs were normal on admission in more than half of the cases. The most frequent abnormalities were oliguria (70\%), tachycardia (33\%), hypothermia $(28 \%)$ and increased respiratory rate $(80 \%)$ resulting from dehydration and lactic acidosis. Mortality rate was high (22\%). Risk factors for death were age, previous atherosclerosis disease, organ failure including shock, liver failure and coagulation disorders.

Conclusion: Incidence of metformin poisoning in the West Indies is elevated. Identification of early risk factors may improve patient management. It is mandatory to encourage general practitioners, endocrinologists, anesthesiologists, and emergency physicians to optimize therapeutic education of the diabetic patient.

\section{Reference}

[1] Seidowsky A, Nseir S, Houdret N, et al. Metformin-associated lactic acidosis: a prognostic and therapeutic study. Crit Care Med. 2009;37:2191-2196.

\section{Poisoning-associated} rhabdomyolysis: Risk factors for acute kidney injury and outcome in the intensive care unit

\section{Pierre-Franois Rogliano ${ }^{a}$, Nicolas Péron ${ }^{a}$,} Jean-Louis Laplanche ${ }^{b}$ and Bruno Mégarbane ${ }^{a}$ ${ }^{a}$ Department of Medical and Toxicological Critical Care, Lariboisière Hospital, Paris-Diderot University, INSERM UMRS-1144, Paris, 
France; ${ }^{b}$ Laboratory of Biochemistry, Lariboisière Hospital, ParisDiderot University, INSERM UMRS-1144, Paris, France

Objective: Acute kidney injury (AKI) is a major complication of rhabdomyolysis. The risk factors in cases of poisoning-related rhabdomyolysis are unknown. Our objectives were to describe the toxicants involved in poisoning-associated rhabdomyolysis and the resulting complications and to identify the predictive factors of AKI in this setting.

Methods: We conducted a single-center retrospective observational cohort study including all poisoned patients admitted in the intensive care unit (ICU) from 1 January 2012 to 30 June 2018 with rhabdomyolysis defined by elevated creatine kinase $(\mathrm{CK})>1000 \mathrm{IU} / \mathrm{L}$. AKI was defined using the Kidney Disease: Improving Global Outcomes (KDIGO) criteria. Factors associated with AKI onset were identified using a multivariate analysis based on a step-by-step logistic regression and their characteristics determined using the receiver operating characteristic (ROC) curves.

Results: Two hundred and thirty-seven patients (138 males/99 females; age $43 \pm 15$ years) were included. The main toxicants involved were benzodiazepines (14\%), antipsychotics (10\%), opioids (9\%) and serotonin reuptake inhibitors (8\%). AKI occurred in 88 patients (37\%), requiring hemodialysis in 43 patients $(49 \%$ of them). The associated complications included sepsis (51\%), cardiovascular failure (14\%), cardiac arrest $(3 \%)$, disseminated intravascular coagulation (2\%) and compartment syndrome (1\%). The overall mortality rate $(13 \%)$ was higher in the presence of $\mathrm{AKI}$ (32\% versus $2 \%, p<0.001)$. Peak serum creatinine and peak serum CK were significantly correlated $\left(r^{2}=0.3, p<0.0001\right.$; linear regression) Based on a multivariate analysis, age $(p<0.01)$, female gender $(p<0.01)$, lithium intoxication ( $<<0.001)$, hypocalcemia ( $p<0.05)$, hyperphosphotemia $(p<0.05)$, elevated serum lactate $(p<0.01)$ and creatinine concentrations $(p<$ 0.0001) were the independent predictors of AKI on ICU admission. The area under the ROC curve of our predictive model was 0.951 . Elevation in serum creatinine concentration $>125 \mu \mathrm{mol} / \mathrm{L}$ had the best accuracy (area under the curve: 0.871), sensitivity (0.71) and specificity (0.92).

Conclusion: We identified the predictive factors of AKI in the poisoned patient admitted to the ICU with rhabdomyolysis. They may help improve patient prognosis by intensifying prevention in the selected patient subgroup at risk of AKI.

\section{Massive eucalyptus oil overdose leading to prolonged coma state and associated NT-proBNP concentrations}

\author{
Joe A Rotella ${ }^{a}$, Simon Hume ${ }^{b}$ and Anselm Wong ${ }^{c}$ \\ ${ }^{a}$ Austin Toxicology Service, Austin Health and Melbourne Medical \\ School, University of Melbourne, Victoria, Australia; ${ }^{b}$ Department \\ of Medicine, St Vincent's Hospital, Victoria, Australia; 'Austin \\ Toxicology Service, Austin Health and Department of Medicine, \\ School of Clinical Sciences, Monash University, Victoria, Australia
}

Objective: Overdose of eucalyptus oil leading to a prolonged coma state is rare. We describe a case of massive eucalyptus oil ingestion leading to prolonged coma and associated $\mathrm{N}$-terminal pro-brain natriuretic peptide (NT-proBNP) concentrations. Previous studies have shown the potential of NT-proBNP to be used as a biomarker of brain injury in trauma. This has the potential as a monitor for drug-induced coma states and may prevent premature extubation of patients and multiple imaging tests.

Case report: A 53-year-old gentleman was brought to hospital by ambulance following intentional ingestion of approximately $500 \mathrm{~mL}$ of $100 \%$ eucalyptus oil on a background of schizophrenia. His medications were lithium carbonate, clozapine and benztropine. His past history included ingestion of $200 \mathrm{~mL}$ of eucalyptus oil which required four days of mechanical ventilation. The ingestion occurred within 12 hours prior to being found unconscious by his brother. He was witnessed to have two generalised tonic-clonic seizures by ambulance officers, which were aborted with intravenous midazolam. Upon arrival to the emergency department, his Glasgow Coma Score was 3 and he was intubated. He was haemodynamically stable with a blood pressure of $103 / 66 \mathrm{mmHg}$ and heart rate of 56 beats per minute. There was no evidence of other poisoning or overdose, with a sub-therapeutic lithium concentration, an unrecordable blood alcohol concentration, negative urine drug screen and undetectable serum paracetamol concentration. Activated charcoal was not administered. Neuroimaging with computerised tomography (CT) scan on days 0 and 2 post overdose as well as magnetic resonance imaging (MRI) on day 4 did not show any evidence of brain injury. Brainstem reflexes were intact. Initially there was no spontaneous breaths and no response to painful stimuli. He then slowly developed motor responses to deep painful stimulus and by day 8 he was able to follow commands and was extubated. NT-proBNP concentrations were normal on admission $(31.3 \mathrm{ng} / \mathrm{L})$, $357.1 \mathrm{ng} / \mathrm{L}$ (normal $<200 \mathrm{ng} / \mathrm{L}$ ) on day 5 and normal again prior to extubation $(31.1 \mathrm{ng} / \mathrm{L})$. There were no clinical or radiological signs of heart failure. He required a total of 15 days of mechanical ventilation and demonstrated gradual neurological recovery over this time. He made a complete neurological recovery and confirmed the ingestion.

Conclusion: Massive overdose of eucalyptus oil can lead to a prolonged coma state. Further research is needed into the use of NT-proBNP as a biomarker of drug-induced coma progression and recovery.

\section{Levetiracetam toxicity: a first step to identify the hospitalization threshold}

\author{
Jutta Trompelt ${ }^{\mathrm{a}}$, Wolfgang Klumb ${ }^{\mathrm{b}}$, Dagmar Prasa ${ }^{\mathrm{c}}$, \\ Cornelia Reichert ${ }^{\mathrm{d}}$, Elke Faerber ${ }^{\mathrm{e}}$, \\ Elisabeth Holder-Koob ${ }^{\mathrm{f}}$, Claudia Zatloukal ${ }^{\mathrm{g}}$, \\ Oliver Sauer ${ }^{\mathrm{a}}$ and Andreas Stürer ${ }^{\mathrm{a}}$ \\ ${ }^{\text {a }}$ Poisons Information Centre, Mainz, Germany; ${ }^{\text {b Poisons }}$ \\ Information Centre, Bonn, Germany; 'Poisons Information Centre, \\ Erfurt, Germany; ${ }^{\mathrm{d}}$ National Poisons Centre, Tox Info Suisse, Zurich, \\ Switzerland; ${ }^{\mathrm{e}} \mathrm{GIZ}$-Nord Poisons Centre, University Medical Centre

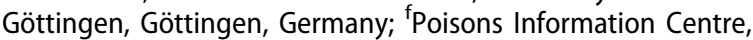 \\ Berlin, Germany; ${ }^{9}$ Poison Information Centre, Vienna, Austria
}

Objective: Levetiracetam is an anticonvulsant drug used alone or in combination. It has a wide therapeutic index but overdose data are limited. This investigation aims to determine toxic doses and a threshold for hospitalisation in overdose.

Methods: Retrospective review of acute and subacute single-substance exposures to levetiracetam reported to seven poison centres with follow-up for at least 5 hours and probable causality. Symptoms were classified according to Poisoning Severity Score (PSS).

Results: There were 91 patients $(56$ females, 31 males, 4 unknown); mean age 24.3 years (7 days-95 years), See Table 1 . Most cases involved oral overdose $(n=90)$ and one was intravenous (aged 7 days). Circumstances were suicidal $(n=48)$, accidental $(n=19)$, therapeutic error $(n=15)$, unknown $(n=8)$, and abuse $(n=1)$. In most cases (78 cases, $86 \%)$ the dose was above the recommended maximum daily dose (MDD). The predominant symptoms concerned consciousness $(n=33,36 \%)$. Severity: PSS3 $(n=1,1 \%)$ : coma. PSS2 $(n=10,11 \%)$ : seizures without documented epilepsy $(n=4)$, stupor $(n=2)$, agitation $(n=2)$, confusion $(n=2)$, QT prolongation $(n=2)$, tachycardia $158 /$ min $(n=1$, 
Table 1. Levetiracetam overdose. Age group, number of cases, Poisoning Severity Score (PSS) and range of ingested doses.

\begin{tabular}{|c|c|c|c|c|c|c|c|c|c|}
\hline \multirow[b]{2}{*}{ Patient group } & \multirow{2}{*}{$\begin{array}{c}\text { Total cases } \\
(\mathrm{n}=91) / \text { dose }>\mathrm{MDD}^{*} \\
(\mathrm{n}=78) \\
\text { (mean dose }>\text { MDD) }\end{array}$} & \multicolumn{2}{|c|}{ PSS $0(n=37)$} & \multicolumn{2}{|c|}{ PSS $1(n=43)$} & \multicolumn{2}{|c|}{ PSS $2(n=10)$} & \multicolumn{2}{|c|}{ PSS $3(n=2)$} \\
\hline & & $\mathrm{mg}$ & $\mathrm{mg} / \mathrm{kg}$ & $\mathrm{mg}$ & $\mathrm{mg} / \mathrm{kg}$ & $\mathrm{mg}$ & $\mathrm{mg} / \mathrm{kg}$ & $\mathrm{mg}$ & $\mathrm{mg} / \mathrm{kg}$ \\
\hline $\begin{array}{l}7 \text { days }-5 \text { years } \\
(n=23)\end{array}$ & $\begin{array}{c}23 / 19 \\
(163 \mathrm{mg} / \mathrm{kg})\end{array}$ & $\begin{array}{c}85-1500 \\
n=14\end{array}$ & $\begin{array}{c}21-317 \\
n=11 \\
+300 \\
n=1 \text { (iv) }\end{array}$ & $\begin{array}{c}500-2750 \\
n=7\end{array}$ & $\begin{array}{c}33-317 \\
n=7\end{array}$ & $\begin{array}{c}1500+2900 \\
n=2^{* * *}\end{array}$ & $\begin{array}{c}290+306 \\
n=2\end{array}$ & - & - \\
\hline $\begin{array}{l}\geq 6-17 \text { years } \\
\quad(n=15)\end{array}$ & $\begin{array}{c}15 / 9 \\
(240 \mathrm{mg} / \mathrm{kg})\end{array}$ & $\begin{array}{c}1000-5000 \\
n=6\end{array}$ & $\begin{array}{l}25-98 \\
n=4\end{array}$ & $\begin{array}{c}1000-100000 \\
n=9\end{array}$ & $21-385 n=5$ & - & - & - & - \\
\hline $\begin{array}{l}\text { Adults } \\
\qquad \begin{array}{l}\geq 18 \text { years } \\
\left(\mathrm{n}=53^{* *}\right)\end{array}\end{array}$ & $\begin{array}{c}53 / 50 \\
(17354 \mathrm{mg} \\
\text { or } 283 \mathrm{mg} / \mathrm{kg})\end{array}$ & $\begin{array}{c}1100-40000 \\
n=17\end{array}$ & $\begin{array}{l}23-533 \\
n=14\end{array}$ & $\begin{array}{c}1250-60000 \\
n=27\end{array}$ & $\begin{array}{c}44-1000 \\
n=17\end{array}$ & $\begin{array}{c}9000-100000 \\
n=8 \\
\left(2 \text { cases }^{* * *}\right)\end{array}$ & $\begin{array}{c}182-1250 \\
n=7\end{array}$ & $\begin{array}{l}20000 \\
n=1\end{array}$ & $\begin{array}{c}375 \\
n=1\end{array}$ \\
\hline
\end{tabular}

*MDD, maximum daily dose: $42 \mathrm{mg} / \mathrm{kg}$ ( $\leq 6$ months of age), $60 \mathrm{mg} / \mathrm{kg}$ ( $>6$ months to $<16$ years or $<50 \mathrm{~kg}$ ), $3000 \mathrm{mg}$ (adults/>50 kg).

**Including 1 case of unknown age $(80 \mathrm{~kg})$.

***Seizures, no epilepsy documented.

adult). PSS1 ( $n=43,47 \%)$ : tiredness/somnolence $(n=26)$, vertigo $(n=7)$, nausea/vomiting $(n=9)$, headache $(n=4)$, tremor $(n=3)$, restlessness/aggression $(n=3)$, visual disturbances/miosis $(n=3)$, mild hypotension $(n=2)$, sinus arrhythmia $(n=2)$. PSS-0: $n=37$, $41 \%$. There were no fatalities.

Conclusion: No clear correlation between dose and severity was found. Most overdoses $(n=80)$, were tolerated without $(n=37)$ or with minor symptoms $(n=43)$. Nevertheless, moderate symptoms (some also described as adverse effects) were seen with $182 \mathrm{mg} / \mathrm{kg}$ and severe symptoms in an adult after $20000 \mathrm{mg}$ $(375 \mathrm{mg} / \mathrm{kg})$. Only minor symptoms were found up to about threefold the MDD. Despite the limited case numbers, observation at home may be safe for small exceedances of MDD. Patients with ingestion of higher doses should be hospitalized, particularly with doses $>180 \mathrm{mg} / \mathrm{kg}$. Additional research is needed to better define a reliable toxic dose and a safe hospitalization threshold.

\section{Identification of risk factors for repetition and poor prognosis among patients presenting with acute poisoning in a one-year observational study}

\section{Kai Knudsen and Mirella Wictoran}

Anaesthesia and Intensive Care, Sahlgrenska University Hospital, Gothenburg, Sweden

Objective: Our objective was to identify possible risk factors for repetition or poor prognosis after acute poisoning, to allow identification of those patients in need of targeted intervention and better follow-up [1].

Methods: All cases of acute poisoning admitted during one year were recorded in a retrospective observational cohort study, with a subsequent one-year follow-up. Data was collected from our hospital's electronic medical records database. Main outcome measures were toxic agents, sex, age, psychiatric comorbidity, referral, suicidal intention and repetition of poisoning.

Results: A total of 668 patients were treated for 784 episodes of acute poisoning, mainly pharmaceutical agents. In total 85 patients (13\%) presented more than once with 2-10 presentations. Twenty-five different toxic substances were registered as main agents or co-agents. Most common agents taken were ethanol, benzodiazepines, opioids and paracetamol. Eighty-five percent of all patients presented with overdose of substances of abuse (SoA). We recorded 189 (24\%) episodes with suicidal intention, 336 patients $(50 \%)$ received a referral and 19 died $(2.8 \%)$ during a one-year follow-up. Risk factors for repetition were identified as psychiatric comorbidity and intake of benzodiazepines, whereas intake of ethanol or cannabis was associated with lower risk for repetition. Seventy-two percent of patients presenting with intake of SoA had a history of psychiatric comorbidity and $62 \%$ received a referral. Of those patients with intake of substances other than SoA (non-SoA), $82 \%$ had psychiatric comorbidity and $84 \%$ received a referral. Women were more likely to receive a referral than men. Intake of benzodiazepines or opioids as well as presence of psychiatric comorbidity was significantly more common among those who died within a year. There was no difference between intake of non-SoA or SoA in repeated poisoning after one year. However, within the first month after the index case, repetition was more common among patients presenting with non-SoA than SoA.

Conclusion: The risk factors for repetition of poisoning were psychiatric comorbidity and overdose with benzodiazepines. Intake of non-SoA indicates an increased risk for repetition during the first month but not after a year. A referral for follow-up was more common in patients taking non-SoA despite the same degree of psychiatric comorbidity. The high incidence of repeat overdose calls for better initial identification of risk factors for reintoxication and poor prognosis.

\section{Reference}

[1] Heyerdahl F, Bjornaas MA, Dahl R, et al. Repetition of acute poisoning in Oslo: 1-year prospective study. Br J Psychiatry. 2009;194:73-79.

\section{Intravenous immunoglobulin} (IVIG) use in subacute combined degeneration secondary to vitamin B12 deficiency from nitrous oxide

\section{abuse}

\section{Alexandra Simpson ${ }^{\mathrm{a}}$, John Rinker $^{\mathrm{b}}$ and \\ William F Rushton ${ }^{c}$}

a University of Alabama at Birmingham School of Medicine, University of Alabama at Birmingham, Birmingham, United States; ${ }^{b}$ Department of Neurology, University of Alabama at Birmingham, Birmingham, United States; ' Office of Medical Toxicology, Department of Emergency Medicine, University of Alabama at Birmingham, Birmingham, United States

Objective: Nitrous oxide $\left(\mathrm{N}_{2} \mathrm{O}\right)$ or "laughing gas," is an intoxicant that is used to obtain recreational highs. It permanently reduces vitamin $B_{12}$ to an oxidized form, inhibiting methionine synthase 
activity, thus impairing synthesis of both tetrahydrofolate and methionine. Standard treatment includes high dose vitamin $B_{12}$ and methionine, if available. Recovery from subacute combined degeneration may be slow and incomplete even after treatment with vitamin $B_{12}$. The goal of this report is to demonstrate a severe case successfully treated with IVIG.

Case report: A 28-year-old male presented with inability to ambulate, paresthesiae in his bilateral lower extremities, and a low vitamin $B_{12}$ concentration $(125 \mathrm{pg} / \mathrm{mL}$, reference range 180 $914 \mathrm{pg} / \mathrm{mL}$ ). He reported abusing $\mathrm{N}_{2} \mathrm{O}$ for one month, sometimes inhaling 200 commercial grade canisters daily. He was initially treated with a single vitamin $B_{12}$ injection. After discharge, he continued to inhale $\mathrm{N}_{2} \mathrm{O}$ and was subsequently admitted for severe proprioceptive and vibratory loss in all extremities. Initial laboratory findings were notable for hematocrit 36\%, negative infectious studies, and a vitamin $B_{12}$ concentration below the range of detection. He was treated with intramuscular vitamin $\mathrm{B}_{12}$ for two weeks without clinical improvement and then transferred to a tertiary care center where his vitamin $B_{12}$ was $270 \mathrm{pg} /$ $\mathrm{mL}$. Cerebral spinal fluid (CSF) studies demonstrated leukocytes $30 / \mathrm{mm}^{3}$ and protein $72 \mathrm{mg} / \mathrm{dL}$. Magnetic resonance imaging (MRI) of the cervical spine showed diffuse signal abnormality involving the dorsal spinal cord and associated enhancement. Methionine was unavailable for adjunctive treatment. Due to lack of improvement, he received 5 doses of IVIG, along with daily intramuscular vitamin $B_{12}$ and occupational therapy. He symptomatically improved, and after one week, his repeat vitamin $B_{12}$ concentration was $>1500 \mathrm{pg} / \mathrm{mL}$. He was transferred to acute inpatient rehabilitation for 10 days, after which he could ambulate with minimal assistance.

Conclusion: This case is noteworthy given the patient's prolonged abuse of $\mathrm{N}_{2} \mathrm{O}$, the severity of his dorsal spinal column loss, inflammatory CSF studies, and minimal improvement with vitamin $B_{12}$ alone. IVIG has effectively been employed in other forms of severe peripheral neuropathy such as acute inflammatory demyelinating polyradiculoneuropathy and multifocal motor neuropathy due to its favorable side effect profile. While its use in $\mathrm{N}_{2} \mathrm{O}$ toxicity has not been well described in the literature, it was associated with a rapid temporal improvement in the patient's symptoms. The combination of IVIG, along with vitamin $\mathrm{B}_{12}$ supplementation, could be considered for severe $\mathrm{N}_{2} \mathrm{O}$ induced vitamin $B_{12}$ deficiency in patients, particularly in those with CSF studies consistent with inflammation.

\section{Characteristics and costs of adults with acute poisoning admitted to the emergency department of a university hospital in Belgium}

\author{
Anne-Marie K Descamps ${ }^{\mathrm{a}}$, Dominique Vandijck ${ }^{\mathrm{a}}$, \\ Walter Buylaert ${ }^{b}$, Martine Mostin ${ }^{c}$ and \\ Peter De Paepe ${ }^{b}$ \\ ${ }^{\mathrm{a} P u b l i c}$ Health, Ghent University, Ghent, Belgium; ${ }^{\mathrm{b}}$ Emergency \\ Medicine, Ghent University Hospital, Ghent, Belgium; 'Poison \\ Centre, Brussels, Belgium
}

Objective: The aim of this study was to assess the characteristics of all acute poisoning admissions among adult emergency department (ED) patients, to identify factors associated with admission and to calculate direct medical costs.

Methods: Data from one year (1 January to 31 December 2017) were collected and analyzed retrospectively using patients' medical records and hospital invoices. Factors associated with type of hospitalization were identified using appropriate statistics.

Results: A total of 1,214 hospital admissions were included, accounting for $3.6 \%$ of all ED admissions. Men (62.2\%) and the age group 21-40 years (43.0\%) accounted for the largest proportion. Substances most commonly involved were ethanol (74.0\%), benzodiazepines $(11.7 \%)$, cocaine $(6.8 \%)$, cannabis $(6.5 \%)$, antidepressants (5.7\%) and amphetamines (5.8\%). A total of 4,561 treatment acts were recorded, most commonly monitoring of vital signs (63.6\%) and administration of intravenous fluids or medication (62.9\%). Patients were discharged home after having received care in the emergency department (ED-ambulatory care) in $54.5 \%$ of admissions, were admitted to the emergency-department-24-hours-observation unit or were hospitalized in $24.6 \%$ and $20.9 \%$ of admissions, respectively. Factors found to be associated with hospitalization type were age, hour of admission, location before admission, Manchester triage score, use of antidotes and need for medical imaging (all $p<0.05$ ). Total cost was $€ 1,386,317.06$, with an average of $€ 1,179.85$ per admission.

Conclusion: Poisonings entail a considerable percentage of patients admitted to an ED and financial burden. In particular, ethanol poisonings account for the largest proportion of all ED admissions. Comparison of our figures with other data is hampered by the heterogeneity in inclusion criteria. Availability of a uniform template would facilitate comparison and allow better monitoring policies for prevention and cost reduction.

\section{Should endoscopic removal of tricyclic antidepressants be performed in patients with signs of severe poisoning?}

\author{
Elena Kishinevsky, Galia Karp, Gennady Bregman and \\ Konstantin Brusin \\ Intensive Care Unit, Kaplan Medical Center, Rehovot, Israel
}

Objective: Acute gastroscopy is seldom advocated in cases of drug overdose. Tricyclic antidepressants (TCA) are discussed as potentially gastroscopic removable, as their overdose is accompanied by gastric hypomotility, which results in delayed gastric emptying. Some TCAs such as clomipramine are radiopaque $[1,2]$ and we can use X-ray to follow gut decontamination. Overdose of clomipramine can cause severe cardiovascular adverse effects, rhabdomyolysis and liver injury. A literature review found controversial opinions regarding the efficacy and safety of endoscopic removal of clomipramine bezoars [1,2], mostly due to endoscopic complications, such as gastric bleeding [1].

Case report: A 36-year-old female, treated with clomipramine due to obsessive compulsive disorder, was brought to the emergency room in deep somnolence (Glasgow Coma Score [GCS] 9), and mild hypotension (blood pressure $95 / 76 \mathrm{mmHg}$ ). Her heart rhythm was normal, 76 beats per minute, with QTc $550 \mathrm{~ms}$. Laboratory examination revealed both myoglobin and creatine kinase elevation $(42900 \mathrm{mmol} / \mathrm{L}$ and $11191 \mathrm{U} / \mathrm{L}$, respectively). Preliminary therapy included 2 liters of normal saline IV, after which the patient was transferred to the intensive care unit. Thirteen hours after hospital admission, the patient developed coma, and multiorgan failure. She was intubated, mechanically ventilated and treated with increasing doses of pressors (noradrenaline, adrenaline and vasopressin). Chemistry laboratory results showed acute renal and liver failure. A chest radiograph revealed right pneumonia and the presence of a rounded opacity in her stomach, which was later showed to be a conglomeration of tablets. Toxicological screening revealed TCA in a urine sample. At this stage bedside echocardiographic study exhibited global left ventricular dysfunction with ejection fraction $25 \%$, after which emergency insertion of intraaortic balloon pump (IABP) was performed. IABP operated for 46 hours, and pressors were used for 72 hours. Endoscopic examination performed on day 4, still found tablets in the stomach which were removed 
endoscopically. The patient gradually improved, but needed prolonged mechanical ventilation for 15 days.

Conclusion: TCA overdose causes serious life-threatening side effects. Gastric hypomotility may further complicate the patient's condition. Therefore we recommend considering early gastroscopic bezoar evacuation, to decrease the drug's long-term effects.

\section{References}

[1] Lapostolle F, Finot MA, Adnet F, et al. Radiopacity of clomipramine conglomerations and unsuccessful endoscopy: report of 4 cases. Clin Toxicol (Phila). 2000;38:477-482.

[2] Hojer J, Personne M. Endoscopic removal of slow release clomipramine bezoars in two cases of acute poisoning. Clin Toxicol (Phila). 2008;46:317-319.

\section{The Great Molasses Flood: volume overload and pulmonary symptoms after high dose insulin therapy in amlodipine poisoning}

\author{
Johanna Nordmark Grass ${ }^{\mathrm{a}}$, Johan Ahlner ${ }^{\mathrm{b}}$, \\ Fredrik C Kugelberg ${ }^{\mathrm{b}}$ and Erik Lindeman ${ }^{\mathrm{a}}$ \\ ${ }^{a}$ Swedish Poisons Information Center, Stockholm, Sweden; \\ ${ }^{\mathrm{b}}$ Department of Forensic Genetics and Forensic Toxicology, \\ National Board of Forensic Medicine, Linköping, Sweden
}

Objective: Therapeutic doses of the calcium channel inhibitor amlodipine mainly affect vessel tone. However, in large ingestions amlodipine can also cause myocardial depression, leading to severe hemodynamic compromise as a result of both vasoplegia and cardiac dysfunction. A long elimination half-life (30-60 hours) can lead to toxic symptoms that last for several days in overdose. High dose insulin therapy (HIT) is a recommended treatment in toxic myocardial compromise in calcium channel inhibitor overdose. To maintain euglycemia, glucose solutions usually need to be infused during this therapy. Data from five amlodipine overdoses treated with HIT are presented, with a focus on the duration and magnitude of insulin and glucose infusions, fluid balance and pulmonary symptoms.

Case series: All five patients had toxic amlodipine blood concentrations $(140-680 \mu \mathrm{g} / \mathrm{L})$. In all patients HIT was initiated after cardiac dysfunction had been determined by echocardiography. Insulin doses ranged from 1-10 U/kg/h and was administered for $50-90$ hours. Blood glucose concentrations were $5.5-11 \mathrm{mmol} / \mathrm{L}$ before HIT and glucose infusions were administered from the start of HIT in all patients. The maximal glucose dosage rate during HIT was $0.33-0.65 \mathrm{~g} / \mathrm{kg} / \mathrm{h}$ and the duration of glucose requirement after HIT was 28-96 hours and was correlated to the total insulin dose. The total amount of glucose administered was 39$73 \mathrm{~g} / \mathrm{kg}$ in solutions with volumes of $8650-21900 \mathrm{~mL}$. Net positive fluid balance was $3300-12800 \mathrm{~mL}$. All patients developed pulmonary signs of fluid overload and required respiratory support. Three patients met the criteria for acute respiratory distress syndrome (ARDS) and required ventilator treatment. All patients made complete recoveries.

Conclusion: While beneficial in improving cardiac function, use of HIT in amlodipine poisoning can result in very large accumulated insulin doses due to the persistence of toxic symptoms. In the present case series no significant insulin resistance was seen and maximal glucose disposal rate was similar to that in intentional insulin overdose [1]. Large amounts of glucose thus needed to be given during treatment and in some cases continued to be needed for several days after treatment discontinuation. The resultant large positive fluid balances likely contributed to the pulmonary symptoms seen, partly undermining the usefulness of HIT in these patients. Use of more concentrated glucose solutions and early efforts at limiting a positive fluid balance may lessen this complication.

\section{Reference}

[1] Megarbane B, Deye N, Bloch V, et al. Intentional overdose with insulin: prognostic factors and toxicokinetic/toxicodynamic profiles. Crit Care. 2007;11:R115.

\section{Viper envenomation with ocular neurotoxic effects managed without antidote administration: a case report}

\author{
Michele S Milella, Susanna M Signoretti, \\ Emanuela Lanni and Maria Caterina Grassi \\ Clinical Toxicology - Poison Control Centre and Drug Dependence \\ Unit, Policlinico Umberto I - "Sapienza" University of Rome, Rome, \\ Italy
}

Objective: Along with the more common clinical features, envenomation with some European vipers (Vipera species) may rarely present with neurotoxic manifestations, including muscle weakness, drowsiness, paresthesia, dyspnea and ocular complaints. Ocular neurotoxicity as an indication for antidote administration is still debated [1,2]. In this case the management with supportive/symptomatic treatment was sufficient.

Case report: In May 2018, a 19-year old man was admitted to an external emergency unit 20 minutes after a viper bite (probably Vipera aspis) on the proximal phalanges of the right hand. The Poison Control Centre (PCC) of Policlinico Umberto I of Rome was contacted immediately. Local signs of envenomation with fang marks, and swelling of the hand were present. In the following 2 hours edema extended to the middle forearm, and one episode of vomiting was registered. The patient was hydrated and analgesics administered. Four hours post-bite, edema did not progress proximally, but weakness, vertigo and mild abdominal pain were reported. Leucocytosis $\left(17.0 \times 10^{9} / \mathrm{L}, 90 \%\right.$ neutrophils) and increased creatine kinase $(245 \mathrm{U} / \mathrm{L})$ were present. The PCC provided two vials of viper venom antitoxin (Biomed) with no indication to administer at this time, but to closely monitor for any systemic and neurological manifestations. Seven hours postbite, the swelling was stable, abdominal pain and previous neurological symptoms regressed, and left ptosis appeared. Laboratory exams showed persistence of leucocytosis, glucose $123 \mathrm{mg} / \mathrm{dL}$ and creatine kinase $216 \mathrm{U} / \mathrm{L}$. The PCC recommended further monitoring. Eighteen hours post-bite ptosis ameliorated, and no systemic signs had developed. The patient was discharged 40 hours post-bite with no ptosis, edema in regression and leucocytes towards normalization.

Conclusion: This case report suggests the following observations: i) as previously reported [1], neurological manifestations due to neurotoxins in some viper species venom may be characterized by delayed onset (4-7 hours in this case, but up to 24 hours), association with mild local effects and reversibility; ii) weakness and vertigo preceded the ocular signs, resolving in a few hours; and iii) symptomatic management and close monitoring might be the best approach in such cases, with antitoxin recommended 
when rapid progression of edema and/or severe systemic/neurological symptoms appear [1].

\section{References}

[1] Lonati D, Giampreti A, Rossetto O, et al. Neurotoxicity of European viperids in Italy: Pavia Poison Control Centre case series 2001-2011. Clin Toxicol (Phila). 2014;52:269-276.

[2] de Haro L, Glaizal M, Tichadou L, et al. Asp Viper (Vipera aspis) envenomation: experience of the Marseille Poison Centre from 1996 to 2008. Toxins (Basel). 2009;1:100-112.

\section{Implementation of a protocol driven Crotalinae Envenomation Unit}

\author{
William F Rushton ${ }^{a}$, Justin Arnold ${ }^{a}$ and \\ Jessica V Riverab \\ a Office of Medical Toxicology, Department of Emergency Medicine, \\ University of Alabama at Birmingham, Birmingham, United States; \\ ${ }^{\mathrm{b}}$ Department of Pharmacy, University of Alabama at Birmingham, \\ Birmingham, United States
}

Objective: From April 2016 to September 2017, 20 patients were treated for crotalinae envenomation at our tertiary care hospital. Historical data from chart reviews reported the median length of stay (LOS) at 36 hours and the median number of crotalidae polyvalent immune $F A B$ used in treatment to be 12 vials per patient. In April 2018, a protocol was implemented for mild to moderate crotalinae envenomation. The protocol included nursing education and standardization of extremity measurements, aggressive pain control, early physical therapy consultation, timing of laboratory testing, and automatic activation of medical toxicology consultation. All patients were followed up one week post-discharge. We present a quality improvement initiative on use of the protocolized therapy that is suggestive of decreased LOS and total antivenin use.

Methods: All patients with mild to moderate envenomation as determined by medical toxicology consultation from April 2018 to September 2018 were admitted to the protocoled envenomation unit. Age, gender, number of vials of antivenin, and length of stay were recorded. Mild envenomation was defined as swelling crossing less than 2 major joints and moderate envenomation as swelling crossing two major joints.

Results: Nine patients (8 males) were admitted to the unit. Median age was 47 years [25-75\% IQR 36-52]. The median number of antivenin vials given was 8 [25-75\% IQR 4-11]. Median LOS in hospital was 25.8 hours [25-75\% IQR 13-51]. Two of the nine patients had moderate envenomation. One patient developed swelling of her entire upper extremity requiring a re-bolus of antivenin. The other patient developed severe upper extremity swelling and digital ischemia after use of tourniquet and ice therapy prior to presentation, resulting in wound management consultation. The two moderate envenomation patients had LOS of 63 and 60 hours. No other patients had LOS greater than 45 hours.

Conclusion: The median number of antivenin vials in this prospective observation was less than recommended by the manufacturer (12-18) with good outcomes in all patients. The median length of stay was also improved from the two years prior. While not explicitly recorded in this project, nursing leadership also reported increased comfort with treating envenomation. There were several limitations. Historical data was not prospectively recorded and was estimated from patients' charts making direct comparison from our prospective data unreliable and the number of patients treated remained low. A protocoled approach, directed by medical toxicologists, for treating crotalinae envenomation may decrease length of stay and the amount of antivenin given.

\section{Pharmacokinetic evaluation of Vipera ammodytes snakebites treated with currently available antivenoms}

\author{
Tihana Kurtovića, Miran Brvar ${ }^{\mathrm{b}}$, Svjetlana Karabuva ${ }^{\mathrm{c}}$, \\ Maja Lang Balijaa ${ }^{a}$, Damjan Grenc ${ }^{b}$, Igor Križaj ${ }^{d}$, \\ Boris Lukšićc and Beata Halassy ${ }^{a}$ \\ aniversity of Zagreb, Centre for Research and Knowledge Transfer \\ in Biotechnology, Zagreb, Croatia; ${ }^{b}$ Centre for Clinical Toxicology \\ and Pharmacology, University Medical Centre Ljubljana, Ljubljana, \\ Slovenia; 'Clinical Department of Infectious Diseases, University \\ Hospital of Split, Split, Croatia; ${ }^{\mathrm{d} D e p a r t m e n t}$ of Molecular and \\ Biomedical Sciences, Jožef Stefan Institute, Ljubljana, Slovenia
}

Objective: Due to shortage of Zagreb antivenom availability, Vipera ammodytes bites in Slovenia have been treated with French and UK antivenoms in recent years. In Croatia, for therapeutic purposes the remaining doses of Zagreb antivenom have been used. Composition differences between these three antivenoms exist. First, they differ in specificity. Zagreb antivenom (Croatia) is raised against $V$. ammodytes venom, but is clinically effective and used against all European venomous snakes. Viperfav (France) is raised against $V$. aspis, $V$. berus and $V$. ammodytes venoms, but was mostly used against $V$. aspis and $V$. berus. ViperaTAb (UK) is raised against $V$. berus venom solely, and used only to treat envenomations caused by $V$. berus until recently. Second, they differ in the type of active component, since Zagreb antivenom and Viperfav are $F\left(a b^{\prime}\right)_{2}$-based, while ViperaTAb is a Fab-based preparation. And third, investigated antivenoms have differently prescribed administration routes, intravenous for ViperaTAb and Viperfav and intramuscular for Zagreb antivenom. The aim was to reveal pharmacokinetic profiles of currently available antivenoms in $V$. ammodytes envenomation and their influence on venom level decrement.

Methods: A prospective case series of consecutive patients envenomed by $V$. ammodytes and treated with Zagreb antivenom or ViperaTAb at the University Hospitals of Split or Ljubljana since 2015. In serum samples concentrations of $F\left(a b^{\prime}\right)_{2}$ or Fab fragments, venom and neurotoxic ammodytoxins were determined. Pharmacokinetic parameters of antivenoms were evaluated whenever possible.

Results: Nine patients were treated with Zagreb antivenom and seven with ViperaTAb, one of which also received Viperfav. Pharmacokinetic profile differences were revealed. Systemic clearance of ViperaTAb was between 4.3 and $23.2 \mathrm{~mL} / \mathrm{h} / \mathrm{kg}$. Its distribution and elimination half-lives were in the range $0.4-3.2 \mathrm{~h}$ and 12.9-55.9 h, respectively. In the case of Zagreb antivenom, due to prolonged residence time, even the sampling period of 96 hours post-treatment was not sufficient for estimation of pharmacokinetic parameters. The exception was a patient hospitalized for 8 days, revealing systemic clearance of $0.42 \mathrm{~mL} / \mathrm{h} / \mathrm{kg}$ and a half-life of $317.2 \mathrm{~h}$. Venom was detected in the serum of all patients, in contrary to ammodytoxins. Measured concentrations correlated with the $\mathrm{F}\left(\mathrm{ab} \mathrm{b}_{2}\right)_{2} / \mathrm{Fab}$ concentration, revealing an inverse trend in venom-antivenom circulation behavior.

Conclusion: The antivenom concentration was highly dependent on the fabotherapics type and administration route, significantly affecting reappearance and systemic clearance of $V$. ammodytes venom and its neurotoxic component. 


\section{Turnaround time of antidote distribution in Canada}

\author{
Camille Tétreault ${ }^{a}$, Hugo Langlois ${ }^{b}$, Éric Villeneuve ${ }^{b}$, \\ Monique Cormier ${ }^{c}$, Juliana Lombardi ${ }^{d}$ and \\ Sophie Gosselin ${ }^{\mathrm{e}}$ \\ ${ }^{a}$ Faculty of Medicine, Sherbrooke University, Sherbrooke, Canada; \\ ${ }^{b}$ Pharmacy Department, McGill University Health Centre, Montréal, \\ Canada; ' Montreal Medical Toxicology Initiative, Montréal, Canada; \\ dPharmacy Department, Jewish General Hospital, Montréal, \\ Canada; ${ }^{E}$ Emergency Department, McGill University Health Centre, \\ Montréal, Canada
}

Objective: To evaluate the average time for Canadian hospital pharmacies to obtain antidotes from local wholesalers or directly from manufacturers on weekdays, weekday evenings (afterhours) and weekends (holidays) for urgent use. Health Canada's approval time for special access program (SAP) antidotes was also evaluated.

Methods: We elaborated three online questionnaires depending on which institution (Health Canada, manufacturers or wholesalers) controlled the approval or the distribution of approved antidotes in Canada. Google Maps was used to assess the minimum delivery time to major cities in Canada (Toronto, Montreal and Vancouver). Warehouse coordinates and city centres were used as departure and arrival points respectively. Ethics approval for the study was granted.

Results: No company, including Health Canada, completed the online questionnaire. Follow-ups were then made by telephone at least twice. Health Canada verbally replied they approve 24/7 any request within 20 minutes. Two customer service representatives were able to answer the questionnaire over the telephone. Most distributor representatives (88\%) were unable to estimate a delivery time or provide information about any transportation method. Estimated turnaround times are summarized in Table 1.

Conclusion: Relying on one distributor for most of the antidotes (86\%) implies that if a necessary antidote becomes unavailable, the user and the distributor become dependent on the manufacturer's available schedule, thus increasing delays to a patient's bedside, especially in rural areas. It was challenging to retrieve any information due to either reluctance from the distributor or simply their lack of awareness. Our results are crucial as they highlight a deficiency in antidote distribution across Canada and even more so outside regular business hours which represents $70.2 \%$ of the time emergency departments may need such antidotes. We hope those results will foster a coordinated efficient response system to make marketed or SAP antidotes available at all times.

\section{Failure of computerized tomography to detect a large bezoar after massive aspirin overdose}

\author{
Jennifer L Parker Cote ${ }^{\mathrm{a}}$, Bryan T Gerber ${ }^{\mathrm{a}}$, \\ Hyunjeong Lee ${ }^{a}$, Susan N Miller ${ }^{a}$, Robert Dietrich $^{b}$ \\ and William J Meggs ${ }^{a}$ \\ ${ }^{a}$ Emergency Medicine, East Carolina University-Brody School of \\ Medicine, Greenville, United States; ${ }^{b}$ Pulmonary and Critical Care, \\ East Carolina University-Brody School of Medicine, Greenville, \\ United States
}

Objective: To report a case of severe salicylate toxicity with a salicylate bezoar that was not evident on computerised tomography $(\mathrm{CT})$ scan.

Case report: A 64-year-old woman with a history of schizoaffective disorder called the police and stated she had ingested a bottle of aspirin to harm herself. Police and emergency medical services (EMS) found her complaining of dyspnea but no empty pill bottles. On arrival at hospital, she denied ingestion. Respiratory rate was 22/minute, Glasgow Coma Score (GCS) was 15. Initial evaluation found a salicylate concentration of $1,143 \mu \mathrm{g} /$ $\mathrm{mL}$, respiratory alkalosis, bicarbonate level $9 \mathrm{mEq} / \mathrm{L}$, anion gap 23, and normal renal function. Treatment was started with infusions of glucose and bicarbonate, multiple doses of activated charcoal, and emergent hemodialysis. She was intubated for deteriorating mental status, and admitted to the intensive care unit. After hemodialysis, salicylate concentration rebounded, so a gastroenterology $(\mathrm{Gl})$ consultation was requested to rule out bezoar formation. On hospital day 2, Gl deferred endoscopy in favor of abdominal CT scan with gastrografin due to hemodynamic instability with multiple vasopressor requirements. CT scan was negative for bezoar. After the sixth hemodialysis session and whole bowel irrigation with rebounding salicylate concentrations, the treating team requested $\mathrm{Gl}$ for reevaluation for endoscopy. On day 5, endoscopy was performed, revealing an 8 to 10 $\mathrm{cm}$ mass of foreign material containing pill fragments, blood in the stomach, gastritis, and black liquid. As much of the fragments as possible were removed with a Roth net. Another endoscopy performed on day 7 removed further fragments. Salicylate concentration began to consistently decline. Dialysis and bicarbonate infusion were discontinued on day 8. Encephalopathy did not

Table 1. The estimate turnaround times for antidote distribution in Canada.

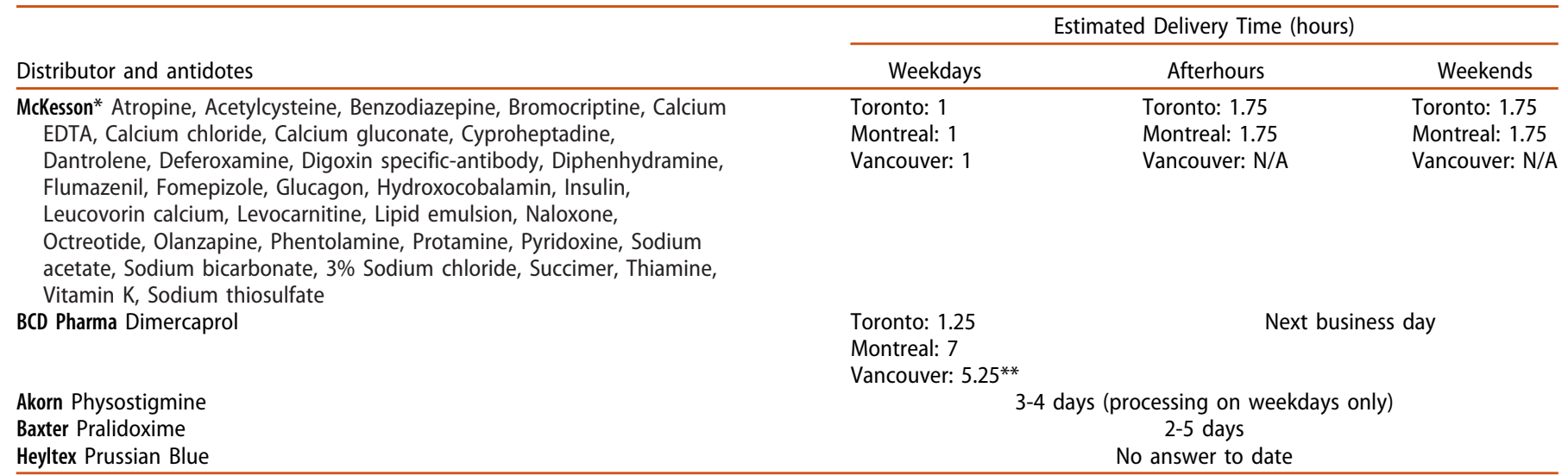

*Supervisor available on-call after business hours 24/7.

**Commercial flight. 
resolve. Brain magnetic resonance imaging (MRI) was negative for cerebral edema. Electroencephalogram was consistent with severe encephalopathy. She developed acute kidney injury requiring hemodialysis for oliguria and volume overload. Her neurological status improved minimally over her hospital course. Repeat head CT was negative for acute intracranial abnormality. After family discussion on day 11 , it was decided to provide comfort care. She died on day 11.

Conclusion: Endoscopy is diagnostic and therapeutic in the setting of rebounding salicylate concentrations. In this case, computerized tomography did not detect a pharmacobezoar. It has been used to identify phytobezoars and trichobezoars [1]. The absence of pharmacobezoar on imaging should not delay endoscopy in the setting of persistently elevated salicylate concentrations despite maximized extracorporeal therapy.

\section{Reference}

[1] Simpson SE. Pharmacobezoars described and demystified. Clin Toxicol (Phila). 2011;49:72-89.

\section{Two cases of intentional cantharidin poisoning in Hong Kong}

\author{
Chi-Keung Chan, Tin-Yat Chow and Man-Li Tse \\ Hong Kong Poison Information Centre, Hong Kong, China
}

Objective: Banmao is a traditional Chinese medicine $(\mathrm{CM})$ containing dried blister beetle from the genus Mylabris. It is highly toxic and used topically for warts or as a component in CM treatment of various malignancies. The active ingredient is cantharidin, also known popularly as Spanish fly. Clinically it is a blistering agent causing diffuse injury to the vascular endothelium, leading to mucosal inflammation, haemorrhage and multiorgan toxicity. Gastrointestinal tract and urinary tract mucosa were typically involved. The reported lethal dose is $0.5 \mathrm{mg} / \mathrm{kg}$ in human. We report two cases.

Case reports: Case 1. A 35-year-old woman attended the emergency department (ED) with haematemesis and abdominal pain. She claimed that she has intentionally ingested $250 \mathrm{~g}$ of dried insect 2 hours earlier. Physical examination revealed mild epigastric tenderness. Initial investigations were unremarkable. Due to language barrier, she was misinterpreted as ingesting a relatively non-toxic CM containing dried bees. She was given intravenous fluid, an antiemetic and was admitted to a medical ward for observation. She developed ventricular fibrillation at 6 hours post-ingestion and succumbed. Serum toxicological screening detected cantharidin and confirmed the diagnosis. Case 2. A 37year-old man presented to ED with severe haematemesis, dysuria and haematuria. He claimed that he has intentionally ingested $15 \mathrm{~g}$ of Banmao powder 2 hours earlier. He was fully conscious with stable vital signs. Venous blood gases showed compensated metabolic acidosis and hyperkalaemia. He was given an intravenous antiemetic and dextrose-insulin drip for hyperkalaemia. A nasogastric tube was inserted to empty the stomach because of repeated haematemesis. Gastric irrigation with $1 \mathrm{~L}$ of normal saline was performed to remove any residual Banmao. The patient was subsequently intubated and endoscopy was performed. Grade IIA and grade I lesions were noted in oesophagus and stomach, respectively. He developed multi-organ toxicity with shock, metabolic acidosis, fever and leucocytosis, deranged liver and renal function, and rhabdomyolysis. A computerised tomography (CT) scan on day 3 showed diffuse mucosal inflammatory changes in the stomach, terminal ileum, caecum, ascending and transverse colon, and urinary bladder. There was no visceral perforation. The patient gradually recovered with supportive treatment. Follow-up endoscopy performed at 1 month was completely normal. Cantharidin was detected in urine, gastric aspirate, and the Banmao powder.

Conclusion: We found the damaging effect of cantharidin on gastrointestinal mucosa is different from that of a typical corrosive. Despite the severe haematemesis upon initial presentation, our patient did not develop clinically significant corrosive oesophagitis or gastritis.

\section{Measurement of QRS intervals in tricyclic antidepressant poisoning}

\author{
Therese Becker ${ }^{a}$, Nancy Briggs ${ }^{b}$, Angela L Chiew ${ }^{a}$, \\ Ben $\mathrm{Ng}^{\mathrm{c}}$, Corey Smith ${ }^{\mathrm{c}}$, Jenny $\mathrm{Yu}^{\mathrm{c}}$ and Betty S Chan ${ }^{\mathrm{a}}$ \\ ${ }^{a}$ Toxicology, Prince of Wales Hospital, Randwick, Australia; \\ bStatistics, New South Wales University, Randwick, Australia; \\ 'Cardiology, Prince of Wales Hospital, Randwick, Australia
}

Objective: Traditionally the QRS interval on the electrocardiogram (ECG) has been used to determine management in tricyclic antidepressant (TCA) overdose. Inter-rater variation in measurement of the QRS intervals has raised concern about the validity of this approach to guide management [1]. The primary aim was to assess the inter- and intra-rater variability in the measurement of QRS intervals following a TCA overdose. Secondary outcomes included determining which ECG lead/s had the best inter-rater reliability and how this compared to the automatic QRS reading.

Methods: We performed a study of 50 ECGs from patients with TCA poisoning. These were reviewed by five physicians, 3 emergency consultants and toxicologists, 1 cardiology trainee and 1 consultant cardiologist and electrophysiology fellow. The ECGs were distributed to each reader and the QRS interval was measured manually and recorded for all 12 leads. This was repeated 6 weeks later. All readers were blinded to patient outcomes. The variation between each reader and themselves (intra-) and each other (inter-) was analysed. Our main outcome measure was agreement among raters and themselves. For each ECG the mean and median QRS for each reader was calculated and then compared to the automatic reading.

Results: Intra-reader variability was good with the majority of intraclass correlation coefficient (ICC) values between 0.70-0.98 on individual leads. Agreement on the measurement of QRS intervals for each ECG was also good (inter-reader) with ICC values ranging from 0.91-0.97 over individual leads. There was the least variation when looking at lead V5 (ICC 0.81, 95\% Cl: $0.69-$ 0.89) followed by lead II (ICC 0.78, 95\% Cl:0.64-0.87). The automatic reading compared very well to the mean and median QRS for each ECG (ICC 0.97).

Conclusion: We had good inter- and intra-reader reliability measuring the QRS interval manually. Comparison of the mean QRS of all leads in each ECG was reliable when compared to the automatic reading, suggesting the automatic reading alone of the ECG can be used by treating physicians. It must be noted however that QRS intervals are only weakly associated with the development of arrhythmias and should be interpreted as part of a total toxicological assessment [2].

\section{References}

[1] Buckley NA, Whyte IM, Dawson AH. Interrater agreement in the measurement if QRS interval in tricyclic antidepressant overdose: Implications for monitoring and research. Ann Emerg Med. 1996:28:515-519.

[2] Buckley NA, Chevalier S, Leditschke A, et al. The limited utility of electrocardiography variables used to predict arrhythmia in psychotropic drug overdose. Crit Care. 2003;7:R101-R107. 


\section{A comparison between acute-on- chronic versus chronic lithium toxicity}

\author{
Sonia Cheng ${ }^{a}$, Will Siu ${ }^{b}$, Angela L Chiew ${ }^{c}$, \\ Elia Vecellio ${ }^{d}$, Nicholas A Buckley ${ }^{e}$ and Betty S Chan ${ }^{c}$ \\ ${ }^{a}$ Faculty of Medicine, University of New South Wales, Sydney, \\ Australia; ${ }^{b}$ Emergency Department, Sutherland Hospital, Sydney, \\ Australia; 'Department of Emergency Medicine \& Clinical \\ Toxicology, Prince of Wales Hospital, Sydney, Australia; ${ }^{\mathrm{N}}$ NSW \\ Health Pathology, Prince of Wales Hospital, Sydney, Australia; \\ eDepartment of Clinical Pharmacology, Prince of Wales Hospital, \\ Sydney, Australia
}

Objective: We aimed to investigate and quantify the predictive factors for severe lithium toxicity in patients presenting with acute-on-chronic and chronic lithium toxicity.

Methods: We conducted a retrospective comparison of patients with acute-on-chronic with chronic lithium toxicity from two hospitals. Plasma lithium concentrations, lithium dose, medications, symptoms, treatment and outcomes were recorded. Known predictive factors of lithium toxicity were analysed alone and then in a multinomial regression analysis to quantify the risk of developing severe toxicity. The two groups were compared using either chi-square for categorical data or Mann-Whitney $U$ test for continuous data.

Results: From 2008-2018, there were 269 presentations from 239 patients. There were 53 acute-on-chronic and 216 chronic lithium toxicity presentations (Table 1). A multinomial regression analysis showed that with adjustment for age and sex, an increase in the plasma lithium concentration by $0.1 \mathrm{mmol} / \mathrm{L}$ results in an increase in the risk of severe toxicity compared to no symptoms by 1.25 times (95\% Cl: 1.1-1.34, $\mathrm{P}<0.001)$. Similarly, patients with mild to moderate renal impairment have 3.4 times the risk of severe toxicity when compared to patients with normal to mild renal impairment (95\% Cl:1.19-9.77, $\mathrm{P}=0.02$ ). Patients with chronic toxicity were 3.4 times more likely to have severe poisoning compared to patients with acute-on-chronic toxicity $(P=0.1)$. There were no fatalities.

Conclusion: Our results agree with previous publications which show chronic toxicity is strongly associated with severe toxicity. Plasma lithium concentration and renal impairment are also important predictive factors for severe lithium toxicity.

\section{Reference}

[1] Hansen $\mathrm{H}$, Amdisen A. Lithium intoxication (Report of 23 cases and review of 100 cases from the literature). Q J Med. 1978;47:123-144.

\section{An indisciplinary national antidote preparedness workgroup optimizes antidote availability}

\author{
Lotte CG Hoegberg \\ Department of Anesthesiology and Intensive Care \& The Danish \\ Poisons Information Centre, Copenhagen University Hospital \\ Bispebjerg, Copenhagen, Denmark
}

Objective: For every poison there is an anti-poison (or antidote) - this idea is attractive, but it is far from reality. Initial management, including decontamination and supportive care, in most cases is sufficient to restore body functions in poisoned patients. A specific antidote might counteract toxic actions of a poison if appropriately used. Prior to antidote administration prescribers need to consider factors such as antidote action and efficacy, balance benefit versus adverse effect, timing, availability, and price. National synchronization might ensure a uniform national supply of relevant antidotes, with a concomitant sound economy. Furthermore, healthcare staff could benefit from poisons information centre (PIC) collaboration for educational purposes.

Methods: Denmark is divided into five regions each responsible for all levels of healthcare including pre-hospital, emergency, and hospital healthcare. An antidote preparedness workgroup was formed, with the purpose of creating a robust model where a relevant selection of antidotes were available in time for all citizens, and with the lowest possible costs. The workgroup included two representatives from each region (emergency doctor; pharmacist) and three representatives (2 doctors, 1 pharmacist) from the Danish PIC.

Results: Relevant antidotes were selected based on local conditions including weighing of frequency and possible poisonings (medical, chemical, and native fauna), and were divided into three stock levels. Level 1: Located in two major university hospitals geographically separated as much possible but able to reach all parts of the country within 4 hours - Holds rarely used and/or expensive antidotes. Level 2: Located in all emergency departments receiving and providing treatment for poisoned patients holds antidotes commonly used for incidents where administration needs to be immediate or within very short time. Level 3: Supply stocks - one located in each region holding an antidote for mass casualty purposes. The antidote stocks are committed to maintain the predefined minimum selection and volume of antidotes. In emergency situations with an increased need in one hospital, other hospitals are obliged to share their antidotes intraand inter-regionally. A free guideline E-book [1] was made available to overcome challenges in prescribers' knowledge, and most

Table 1. Comparison of characteristics of patients with acute-on-chronic and chronic toxicity. Data are medians (range) or counts (proportion of relevant group).

\begin{tabular}{|c|c|c|c|}
\hline Patient characteristics & Acute-on-chronic $(\mathrm{n}=53)$ & Chronic $(n=216)$ & P-value \\
\hline Female & $42(79.2 \%)$ & $140(64.8 \%)$ & 0.04 \\
\hline Lithium concentration $(\mathrm{mmol} / \mathrm{L})$ & $1.8(1.3-5.6)$ & $1.5(1.3-4.1)$ & $<0.001$ \\
\hline Nephrotoxic drugs & $0(0.0 \%)$ & $6(27.8 \%)$ & 0.22 \\
\hline Lithium drug interactions & $9(17.0 \%)$ & $50(23.1 \%)$ & 0.33 \\
\hline Infection risk & $1(1.9 \%)$ & $54(25.0 \%)$ & $<0.001$ \\
\hline 1 - Mild & $32(60.4 \%)$ & $89(41.2 \%)$ & 0.01 \\
\hline 2 - Moderate & $5(9.4 \%)$ & $18(8.3 \%)$ & 0.80 \\
\hline 3 - Severe & 5 (9.4\%) & 40 (18.5\%) & 0.11 \\
\hline Treatment with intravenous fluids & $38(71.7 \%)$ & $109(50.5 \%)$ & 0.005 \\
\hline
\end{tabular}


antidotes are used after consultation with the PIC providing risk assessment, and treatment, including indications for the antidote. Conclusion: Establishing an interdisciplinary national antidote preparedness workgroup optimized the selection and volume of stocked antidotes, reduced costs by collecting expensive and/or rarely used antidotes at two stocks only, and provided uniform user guidelines.

\section{Reference}

[1] [Antidote preparedness in Denmark 2009 - 13th edition. Updated March 2017 [cited 2018 Oct 11]. Available from: http://sygehusberedskabet.dk/antidothaandbogen/main.html? Danish

\section{Unintentional ingestion of brimonidine: severe effects in an adult patient}

\author{
Mariapina Gallo ${ }^{a}$, Andrea Giampreti ${ }^{a}$, Raffaella Butera ${ }^{a}$, \\ Lorella Faraoni ${ }^{a}$, Georgios Eleftheriou ${ }^{\mathrm{a}}$ and \\ Giuseppe Bacis ${ }^{a}$ \\ aUOSD Poison Control Center and Toxicology, ASST Ospedale Papa \\ Giovanni XXIII, Bergamo, Italy
}

Objective: Brimonidine is a potent and highly a2-selective adrenoceptor agonist used topically to treat open-angle glaucoma or ocular hypertension. Although brimonidine is distinguished from clonidine by a chemical modification that reduces its ability to cross the blood-brain barrier some neurologic adverse effects have been reported after ocular administration. Currently, case reports and small retrospective series have revealed potentially fatal systemic side effects in children after topical use or unintentional ingestion. We report a case of severe symptoms after unintentional ingestion of a topical brimonidine preparation in an adult patient.

Case report: A 61-year-old man, $90 \mathrm{~kg}$, was brought to the emergency department (ED) because of confusion, dizziness and hypotension after ingestion, one hour before, of $1 \mathrm{~mL}$ of brimonidine $0.2 \%$ topical formulation instead of $1 \mathrm{~mL}$ of vitamin D. On examination he presented drowsiness. Arterial pressure was $80 / 60 \mathrm{mmHg}$, pulse rate $45 \mathrm{bpm}$, oxygen saturation 93\% (room air). Electrocardiogram and laboratory findings were normal. Neurologic, metabolic and traumatic etiologies were ruled out. Infusion with saline solution was started and because of the similarity to opioid toxic effects, naloxone was recommended in case of respiratory and neurological depression. The patient was monitored until complete recovery and discharged asymptomatic the following day.

Conclusion: The toxicity of brimonidine is similar to clonidine overdose, which can cause miosis, hypothermia, dizziness, agitation, ataxia, drowsiness, coma, apnea, hypotension, hypertension, bradycardia, respiratory depression, respiratory failure, and death. Central nervous system involvement in brimonidine toxicity has been reported to occur much more frequently and severely in children compared to adults probably due to blood brain barrier immaturity. To our knowledge, accidental and voluntary oral ingestion in adult patients are not well described. Human toxicity with hypotension, bradycardia, and respiratory depression has been described in infants after ocular application of $0.15-0.2 \%$ solution. Somnolence occurred in a 2-year-old patient after ingestion of $2 \mathrm{~mL}$ of $0.2 \%$ eye drops [1]. In our case, although we could not evaluate the blood concentration of brimonidine as it is not routinely available, ingestion of $1 \mathrm{~mL}$ of $0.2 \%$ solution resulted in bradycardia, mild hypotension and CNS depression in an adult that were managed with supportive therapy and rapidly resolved within 24 hours.

\section{Reference}

[1] Hoffmann U, Kuno S, Franke G, et al. Adrenoceptor agonist poisoning after accidental oral ingestion of brimonidine eye drops. Pediatr Crit Care Med. 2004;5:282-285.

\section{Intermittent sodium bicarbonate bolus with permissive hypernatremia and alkalemia in severe desipramine cardiotoxicity}

\author{
Robert Goodnough ${ }^{a}$, Ben Tsutaoka ${ }^{b}$, Kai Li $^{\mathrm{c}}$, \\ Daniel Repplinger ${ }^{\mathrm{a}}$, Adina Badea ${ }^{\mathrm{d}}$, Kara L Lynch ${ }^{\mathrm{d}}$, \\ Craig G Smollin a and Paul D Blanc ${ }^{\mathrm{e}}$ \\ ${ }^{a}$ Department of Emergency Medicine, University of California San \\ Francisco, San Francisco, United States; ${ }^{\mathrm{b}}$ California Poison Control \\ System - San Francisco Division, University of California San \\ Francisco, San Francisco, United States; 'Department of Emergency \\ Medicine, Kaiser San Leandro/Fremont, San Leandro, United

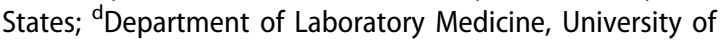 \\ California San Francisco, San Francisco, United States; ${ }^{\text {e Department }}$ \\ of Medicine, University of California San Francisco, San Francisco, \\ United States
}

Objective: Severe tricyclic antidepressant (TCA) cardiotoxicity manifests as hypotension, conduction delays, and ventricular dysrhythmias. Although intravenous boluses of sodium bicarbonate (NaBicarb) are a treatment mainstay, alkalemia $(\mathrm{pH}>7.55)$ and hypernatremia (sodium $>155 \mathrm{mmol} / \mathrm{L}$ ) may cause clinicians to curtail administration. We present a patient surviving profound desipramine cardiotoxicity in which alternate modalities (including intravenous lipid emulsion therapy [ILE]) lacked efficacy, while aggressive bolus NaBicarb, with permissive alkalemia and hypernatremia, proved the cornerstone of management.

Case report: A 30-year-old woman ingested an unknown quantity of desipramine and zolpidem. On Emergency Department presentation, her triage vital signs were blood pressure (BP) 113/ $71 \mathrm{mmHg}$, heart rate $133 /$ minute, respiratory rate 20 /minute, oxygen saturation $99 \%$ (room air) and temperature $35.4{ }^{\circ} \mathrm{C}$. She was somnolent with dilated pupils $(5 \mathrm{~mm})$. The initial electrocardiogram (ECG) displayed a widened QRS (156 ms) with a terminal $r$ wave in aVR. She received seven $50 \mathrm{mEq}$ doses of NaBicarb over one hour. The QRS narrowed (125 ms); she was not frankly alkalemic (arterial pH 7.52) before admission. After progressive QRS widening and hypotension, a lidocaine infusion at $2-4 \mathrm{mg} / \mathrm{min}$ was initiated without improvement. A NaBicarb infusion and a $3 \%$ hypertonic saline infusion were initiated. By ten hours, she manifested hypotension (systolic BP $80 \mathrm{mmHg}$ ) and profound QRS widening (244 ms). NaBicarb and hypertonic saline continuous infusions were discontinued, and bolus NaBicarb $(50 \mathrm{mEq})$ therapy was reinstituted ( 12 boluses over 1.5 hours). Each bolus of NaBicarb temporarily narrowed the QRS complex, which then re-widened. Administration of $100 \mathrm{~mL}$ ILE lacked clinical effect or substantive change in her serum desipramine concentration (pre ILE $606 \mathrm{ng} / \mathrm{mL}$; post ILE $543 \mathrm{ng} / \mathrm{mL}$; peak concentration $909 \mathrm{ng} /$ $\mathrm{mL}$ ). By 12 hours, the QRS stabilized between $190-215 \mathrm{~ms}$ with a concomitant serum sodium of $159-161 \mathrm{mmol} / \mathrm{L}$ and arterial blood $\mathrm{pH}$ 7.51-7.60. Seventeen hours after presentation, the QRS was $168 \mathrm{~ms}$. The patient recovered completely.

Conclusion: This highlights bolus NaBicarb-based treatment as the mainstay of management for TCA toxicity, allowing for permissive hypernatremia and alkalemia. Other interventions, including ILE, had little impact on the clinical course in this patient. Evidence-based serum sodium and $\mathrm{pH}$ targets for therapy are 
lacking; this patient tolerated serum sodium of $161 \mathrm{mmol} / \mathrm{L}$ and alkalemia to 7.60 .

\section{Successful prolonged cardiopulmonary resuscitation (CPR) followed by extracorporeal membrane oxygenation (ECMO) after venlafaxine- induced takotsubo cardiomyopathy}

\author{
Sune Forsberg ${ }^{\mathrm{a}}$ and Lis Abazi ${ }^{\mathrm{b}}$ \\ ${ }^{a}$ Swedish Poisons Information Centre, Stockholm, Sweden; \\ bIntensive Care, Tiohundra, Norrtälje, Sweden
}

Objective: The antidepressant venlafaxine is a serotonin and norepinephrine, and to some extent, dopamine reuptake inhibitor. Severe overdose is associated with seizures, cardiac dysfunction and fatalities. The cardiotoxic complications are conduction abnormalities, malignant arrhythmias as well as severe cardiac failure and sometimes takotsubo cardiomyopathy [1].

Case report: A 48-year-old male was admitted to the intensive care unit two hours following intoxication with $20 \mathrm{~g}$ venlafaxine. He was awake but drowsy, with stable respiratory and circulatory function, blood pressure $150 / 90 \mathrm{mmHg}$, heart rate $60 \mathrm{bpm}$ and pulse oximetry $99 \%$ (room air). Electrocardiogram (ECG) and blood samples were normal. Activated charcoal $(50 \mathrm{~g})$ was given. Seven hours later, tonic-clonic seizures developed which were treated with diazepam. At that time the patient was intubated and thereafter continuously sedated with propofol and remifentanil and required norepinephrine to gain a mean arterial blood pressure above $65 \mathrm{mmHg}$. By 15 hours post-ingestion there was an increased requirement of norepinephrine, which probably explained the subsequently worsened lactic acidosis. Echocardiography revealed prominent hypokinesia and takotsubo cardiomyopathy, with an ejection fraction of less than $20 \%$. At 18 hours post-ingestion circulation deteriorated and he suffered refractory cardiac arrest with an initial rhythm of asystole. Standard CPR continued for two hours and during transportation to a tertiary hospital with ECMO facilities. ECMO was initiated and continued for 32 hours. He was returned to the primary hospital and 3 days after the cardiac arrest cardiac function was echodynamically restored with an ejection fraction (EF) of approximately $55 \%$. The serum venlafaxine concentration 24 hours after ingestion was $12.6 \mathrm{mg} / \mathrm{L}$. The peak $\mathrm{N}$-terminal probrain natriuretic peptide (NT-proBNP) was $8360 \mathrm{ng} / \mathrm{L}$. The patient was on a ventilator for 8 days and on dialysis for 3 weeks. Two days after extubation he gradually regained consciousness and 30 days after the intoxication the patient was back to normal and could leave the hospital for psychiatric treatment from where he was discharged in good health without his former psychiatric medications.

Conclusion: A high dose of the norepinephrine reuptake inhibitor venlafaxine in combination with intravenously administrated norepinephrine probably contributed to takotsubo cardiomyopathy and cardiac arrest, but in this case, two hours of CPR followed by 32 hours of ECMO saved the patient.

\section{Reference}

[1] Batista $M$, Dugernier $T$, Simon $M$, et al. The spectrum of acute heart failure after venlafaxine overdose. Clin Toxicol (Phila). 2013;51:92-95.
188. The octreotide endgame: reducing glucose infusion after highdose insulin therapy

\author{
Erik Lindeman ${ }^{\mathrm{a}}$, Marzena Wandas ${ }^{\mathrm{b}}$, Carsten Metzsch ${ }^{\mathrm{c}}$, \\ Ala Ghazi ${ }^{d}$, Elisabeth Aardal ${ }^{\mathrm{e}}$, Johan Ahlner ${ }^{f}$, \\ Fredrik C Kugelberg ${ }^{f}$ and Johanna Nordmark Grass ${ }^{a}$ \\ ${ }^{a}$ Swedish Poisons Information Center, Stockholm, Sweden; \\ ${ }^{b}$ Department of Anesthesiology and Intensive Care, Skåne \\ University Hospital, Malmö, Sweden; 'Department of \\ Cardiothoracic Anesthesia and Intensive Care, Skåne University \\ Hospital, Lund, Sweden; ${ }^{d}$ Department of Anesthesiology and \\ Intensive Care, Vrinnevi Hospital, Norrköping, Sweden; \\ eDepartment of Clinical Chemistry, Linköping University Hospital, \\ Linköping, Sweden; 'Department of Forensic Genetics and Forensic \\ Toxicology, National Board of Forensic Medicine, Linköping, \\ Sweden
}

Objective: High-dose insulin therapy (HIT) is used for hemodynamic compromise caused by toxic myocardial depression in calcium channel blocker poisoning. To avoid hypoglycemia intravenous glucose is usually necessary during treatment and often needs to be continued for prolonged periods after insulin is stopped. Two cases of amlodipine-poisoning receiving HIT are presented; octreotide was used to shorten the requirement for post-insulin glucose infusion (PIGl) in case 2 but not in case 1 .

Case reports: Case 1 was a 26-year-old woman, case 2 an 81year-old man. Both developed severe hemodynamic compromise after ingesting $0.5-1 \mathrm{~g}$ of amlodipine. Case 1 had a blood amlodipine concentration of $510 \mu \mathrm{g} / \mathrm{L}$ at 12 hours; case 2 had $680 \mu \mathrm{g} / \mathrm{L}$ at 18 hours post-ingestion. Both patients received multiple therapies in addition to HIT and both recovered. HIT was similar in both cases with insulin infusions up to $10 \mathrm{U} / \mathrm{kg} / \mathrm{h}$ and a total of $30,000 \mathrm{U}$ administered over 3.5 days. Goals upon termination of HIT were to taper PIGI while maintaining blood glucose within a target range of $6-10 \mathrm{mmol} / \mathrm{L}$. Case 1 . PIGl remained above $0.40 \mathrm{~g} /$ $\mathrm{kg} / \mathrm{h}$ for 50 hours and was discontinued at 96 hours. During glucose-tapering C-peptide concentrations were $1 \mathrm{nmol} / \mathrm{L}$ (0 hours), $3 \mathrm{nmol} / \mathrm{L}$ (24 and 36 hours), and $0.8 \mathrm{nmol} / \mathrm{L}$ (96 hours). Glucose was $5-8 \mathrm{mmol} / \mathrm{L}$ during the last 80 hours of PIGl. Case 2: He was given octreotide $100 \mu \mathrm{g} 6$ hourly at termination of HIT with the last dose at 36 hours. PIGl started at $0.40 \mathrm{~g} / \mathrm{kg} / \mathrm{h}$ with several glucose values at $4-5 \mathrm{mmol} / \mathrm{L}$ during the first 24 hours. At 28 hours glucose rose to $8-10 \mathrm{mmol} / \mathrm{L}$ and $\mathrm{PIGl}$ was rapidly tapered to $0.10 \mathrm{~g} / \mathrm{kg} / \mathrm{h}$, and maintained until 48 hours. C-peptide concentrations were checked 4 hourly: $0.36 \mathrm{nmol} / \mathrm{L}$ ( 0 hours), $<0.15 \mathrm{nmol} / \mathrm{L}$ (6-28 hours), and $1 \mathrm{nmol} / \mathrm{L}$ (48 hours). During this time of suppressed endogenous insulin, glucose did not rise above target, suggesting lingering exogenous insulin at physiologic levels.

Conclusion: Maintaining blood glucose at $6-10 \mathrm{mmol} / \mathrm{L}$ during and after HIT is safe and avoids glycosuria, however, in non-diabetics concentrations within this range will stimulate endogenous insulin secretion as seen indirectly in case 1 where C-peptide rose to postprandial concentrations during the prolonged PIGItaper. The absence of this response in case 2, with C-peptide concentrations well below the upper fasting limit $(1 \mathrm{mmol} / \mathrm{L})$ during octreotide treatment, made a more aggressive PIGI-taper possible in this case. Thus octreotide may be a valuable agent in limiting glucose and fluid overload after HIT. 


\section{Ecstasy-associated hyponatremia: treat them like marathon runners}

\author{
Erik Lindeman ${ }^{a}$ and Inga Fredriksson ${ }^{b}$ \\ ${ }^{\mathrm{a}}$ Swedish Poisons Information Center, Stockholm, Sweden; \\ ${ }^{\mathrm{b}}$ Department of Anesthesiology and Intensive Care, Danderyd \\ Hospital, Stockholm, Sweden
}

Objective: 3,4-Methylenedioxymethamphetamine (MDMA, ecstasy) can cause impaired ability to suppress antidiuretic hormone secretion (ADH) secretion, making users less able to lose free water in the urine. The popular notion that people attending raves need to drink large amounts of water in order to stave off heat stroke puts the MDMA-user at risk of developing severe water intoxication and hyponatremic encephalopathy. This condition is identical to the encephalopathy that can develop in exercise-associated hyponatremia (EAH), an increasingly well recognized clinical entity. An expert consensus in sports medicine has established a simple goal-directed treatment protocol in symptomatic EAH [1]. We present a case of symptomatic ecstasyassociated hyponatremia treated with the EAH-protocol.

Case report: A 30-year-old male presented to the emergency department (ED) with headache and nausea a few hours after taking a dose of ecstasy $50 \%$ larger than his usual amount. After this he drank a large, but unspecified, amount of water with the intention of preventing overdose symptoms. On examination he was somnolent with slightly slurred speech, a normal body temperature, blood pressure $190 / 100 \mathrm{mmHg}$ and heart rate $70 \mathrm{bpm}$. Lungs were clear. His sodium was $116 \mathrm{mmol} / \mathrm{L}$ and ethanol not detected. He received $40 \mathrm{mg}$ of furosemide in the ED and, after consulting the poison center, two $100 \mathrm{~mL}$ boluses of $\mathrm{NaCl} 3 \%$ after transfer to the intensive care unit (ICU). Sodium was 119 and $122 \mathrm{mmol} / \mathrm{L}$ after the first and second bolus, respectively, representing a rise of $6 \mathrm{mmol} / \mathrm{L}$ in 40 minutes. The patient received no further active treatment and was discharged from the ICU in good health 10 hours after hospital arrival. His sodium had then risen to $135 \mathrm{mmol} / \mathrm{L}$ and he had produced 7.5 liters of dilute urine. Blood drawn at 2 hours after hospital arrival tested positive for MDMA ( $240 \mu \mathrm{g} / \mathrm{L})$ and tetrahydrocannabinol (THC) (4 $\mu \mathrm{g} / \mathrm{L})$.

Conclusion: Symptomatic hyponatremia that develops over the course of hours is a life-threatening condition and early symptoms such as headache and nausea can rapidly progress to loss of consciousness, seizures and death. Treatment consists of acutely raising sodium concentration by $4-6 \mathrm{mmol} / \mathrm{L}$, which is sufficient to reverse a possible impending herniation. This goal is usually achieved by $2-4$ divided $100 \mathrm{~mL}$ doses of $\mathrm{NaCl} 3 \%$ given as rapidly as symptoms dictate. This regimen is recommended for EAH and our case illustrates its implementation in ecstasyassociated hyponatremia.

\section{Reference}

[1] Moritz M, Kalantar-Zadeh K, Ayus JC. Ecstasy-associated hyponatremia: why are women at risk? Nephrol Dial Transplant. 2013;28:2206-2209.

\section{Clinical signs in a non- Parkinson's patient from therapeutic doses of pramipexole given in error - and antidotal reversal}

\author{
Soeren Bøgevig ${ }^{a}$, Sys S Johansen ${ }^{b}$, Heidi Kruse ${ }^{c}$ and \\ Lotte CG Hoegberg ${ }^{\mathrm{d}}$
}

aDanish Poisons Information Centre and Department of Clinical Pharmacology, Copenhagen University Hospital Bispebjerg, Copenhagen, Denmark; ${ }^{\mathrm{b}}$ Section of Forensic Chemistry, Department of Forensic Medicine, Faculty of Health and Medical Sciences, University of Copenhagen, Copenhagen, Denmark; 'Department of Anesthesiology, Copenhagen University Hospital Herlev, Herlev, Denmark; ${ }^{d}$ Danish Poisons Information Centre and Department of Anesthesiology and Intensive Care, Copenhagen University Hospital Bispebjerg, Copenhagen, Denmark

Objective: Pramipexole is a dopamine-D2 receptor agonist used to treat idiopathic Parkinson's disease and primary restless legs syndrome. Reports of overdoses are rare and symptoms from therapeutic doses and the treatment of pramipexole toxicity are, to our knowledge, not reported. We report symptoms in a nonParkinson's disease patient due to a medication error, and symptom control by receptor specific antidotal reversal.

Case report: A 75-year-old woman (120 kg BMI 52.76) was admitted to hospital due to acute renal failure and respiratory insufficiency. Her medical history included chronic kidney insufficiency (creatinine $150 \mu \mathrm{mol} / \mathrm{L}$ ), chronic obstructive lung disease (COLD), and insulin-dependent diabetes mellitus. On arrival to the emergency care unit she was awake and alert (Glasgow Coma Score 15). It was estimated that her weight was $17 \mathrm{~kg}$ over normal status due to overhydration. After 48 hours she was transferred to the intensive care unit (ICU) due to increased respiratory distress and insufficient reversal of the overhydrated state. Within 24 hours she deteriorated due to respiratory workload and distress, and mechanical ventilation was started along with forced diuresis. On day 9 tracheostomy was performed and propofol discontinued. She became more awake and responsive, but over the following two days developed involuntary stereotypic, choreiform movements of the face and upper extremities. Neurologic examination found no signs of convulsions, focal epilepsia, myoclonia or dystonia. A cranial computerised tomography (CT) scan showed no pathology, and an electroencephalogram (EEG) no signs of focal or paroxysmal activity. Creatinine slowly increased during ICU admission, peaking on day 12-13 (279 $\mu \mathrm{mol} / \mathrm{L})$. A medication error was discovered on day 12 . She had received pramipexole $0.7 \mathrm{mg}$ three times daily for 10 days during ICU admission. It was suspected that her involuntary movements were due to pramipexole overdose. Pramipexole cessation and treatment with aripiprazole, $5 \mathrm{mg}$ three times daily, were initiated after consultation with the Danish Poisons Information Centre. The involuntary stereotypic, choreiform movements were clearly reduced within 48 hours. Whole blood and urine sampling on days 12-14 showed elevated pramipexole concentrations: day 11 blood $0.017 \mathrm{mg} / \mathrm{L}$, urine $1.2 \mathrm{mg} / \mathrm{L}$; day 12 blood $0.015 \mathrm{mg} / \mathrm{L}$, urine $0.48 \mathrm{mg} / \mathrm{L}$; and day 13 blood $0.014 \mathrm{mg} / \mathrm{L}$, urine $0.40 \mathrm{mg} / \mathrm{L}$ (therapeutic blood concentrations in Parkinson's disease patients 0.009 $\mathrm{mg} / \mathrm{L})$.

Conclusion: This case represents the clinical presentation of a pramipexole overdose in a non-Parkinson's disease patient. The clinical effect of aripiprazole after 48 hours treatment was pronounced and improvement was caused by aripiprazole rather than the very limited observed decrease in pramipexole concentrations

\section{Delays, interruptions, and adverse reactions with IV acetylcysteine administration using traditional, 3-bag dosing}

Susan C Smolinske, Brandon J Warrick and Steven A Seifert 
New Mexico Poison and Drug Information Center, University of New Mexico College of Pharmacy, Albuquerque, United States

Objective: The current 3-bag method of intravenous $\mathrm{N}$-acetylcysteine (NAC) administration for treatment of acetaminophen overdose is complex. Each infusion has a different dose, diluent volume, and IV bag size. Retrospective reviews found medication errors in up to $33 \%$ of patients, the most frequent being interruption of therapy by $>1$ hour. Alteration of the regimen to a 2-bag method at a slower initial infusion rate has decreased errors and adverse reactions. Our objective was to quantify the rate of delays, interruptions, and adverse reactions with the 3-bag method in cases where the poison center was consulted.

Methods: Patient charts were reviewed between January 1 and December 20, 2017, of poison center-consulted patients with acetaminophen overdose treated with the 3-bag NAC regimen. Inclusion criteria were completion of at least one bag, time delay between any of the doses of $\geq 30$ minutes, and complete record of administration timing and outcomes. Measures included delay in therapy between each bag, reason for delay, adverse reactions to NAC, and presence of liver injury (defined as $\geq 3$-fold increase in AST or ALT).

Results: In total 279 records were reviewed; 27 were excluded due to time delay $<30$ minutes and 105 due to lack of complete timing information, leaving 147 records for analysis. Delay in therapy occurred in 65 (44\%), with an average total administration delay of 157 minutes (range 30-883 minutes, median 107). Of those, 11 (17\%) had a delay between bags 1 and 2, 24 (37\%) had a delay between bag 2 and 3, $40(21.8 \%)$ had a delay between both bags $1-2$ and $2-3$, and $82(56 \%)$ had no delay. Adverse reactions occurred in 40 cases (14.3\%). Delay in therapy was related to an adverse event in 10 patients (3.6\%) (including flushing, itching, swelling, and infiltration). Subgroup analysis of 71 patients (25\%) from a hospital where there was online access to medical records showed that 12 of 35 patients (34\%) with time delay developed liver injury. In this subgroup, 4 were late presenters, 2 were chronic overdoses, and 1 had a therapy delay related to an adverse event to NAC.

Conclusion: This quality assurance study documented a $44 \%$ therapeutic delay rate of the widely used 3-bag $\mathrm{N}$-acetylcysteine regimen. Time delay between bags was associated with development of liver injury in some early presenters, and provided impetus to institute a simpler 2-bag method. Repeat of this study can be used to validate effectiveness of this change.

\section{Carbon monoxide poisoning unresponsive to hyperbaric oxygen therapy: synergy between clinicians and laboratory}

\author{
Azzurra Schicchi ${ }^{\mathrm{a}}$, Anna Celentano ${ }^{\mathrm{b}}$, \\ Roberto Costagliola ${ }^{c}$, Davide Lonati ${ }^{a}$, \\ Francesca Chiara a , Stefano Paglia ${ }^{\text {, }}$ \\ Rossella Giacomello , Chiara Guerrinif \\ Damiano Vignaroli ${ }^{9}$, Pietro Papa ${ }^{\mathrm{h}}$, Fabio Garuti ${ }^{\mathrm{e}}$ \\ Carlo A Locatelli ${ }^{a}$ and Franca Davanzo ${ }^{b}$ \\ apavia Poison Control Centre - National Toxicology Information \\ Centre - Clinical and Experimental Lab, Toxicology Unit, Maugeri \\ Clinical and Scientific Institutes IRCCS and University of Pavia,

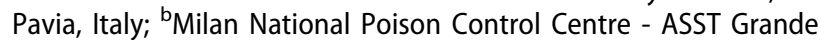 \\ Ospedale Metropolitano Niguarda, Milan, Italy; 'S.C. Anestesia e \\ Rianimazione 2, ASST Grande Ospedale Metropolitano Niguarda,

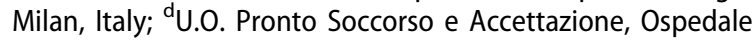 \\ Maggiore di Lodi, Lodi, Italy; ${ }^{\mathrm{e}}$ Hyperbaric Centre, ASST Grande \\ Ospedale Metropolitano Niguarda, Milan, Italy; ${ }^{f}$ Internal Medicine
}

Department, Ospedale Maggiore, Lodi, Italy; ${ }^{9}$ Emergency Medicine Fellowship Program, University of Pavia, Pavia, Italy; 'haboratory of Analytical Toxicology, Fondazione IRCCS Policlinico San Matteo, Pavia, Italy

Objective: Dichloromethane (DCM, methylene chloride) can be a source of carbon monoxide (CO) poisoning as it is metabolized by cytochrome-P450 to $\mathrm{CO}(25-34 \%)$ and to carbon dioxide (70\%). We describe a case of CO-poisoning in a patient with occupational exposure to DCM.

Case report: A 59-year-old man (non-smoker) was admitted to the emergency department (ED) due to repeated seizure at his workplace (painter in industry). Medical history was positive for arterial hypertension. At ED admission the patient was awake with normal vital parameters and neurological evaluation. Arterial blood gas (ABG) analysis showed a carboxyhaemoglobin $(\mathrm{COHb})$ concentration of $11.5 \%$ (haemoglobin $14.8 \mathrm{~g} / \mathrm{dL}$ ). Considering prehospital clinical manifestations and the $\mathrm{COHb}$ concentration, hyperbaric oxygen therapy (HBOT) was indicated (2.8 ATA for 20 minutes, followed by 60 minutes at 2.5). After HBOT, the $\mathrm{COHb}$ was $6.5 \%$ (7 hours after ED admission) and he complained of severe headache. A repeat $A B G$ was performed due to persistent signs and showed a $\mathrm{COHb} 10 \%$ ( 14 hours after ED admission). Considering the increase of $\mathrm{COHb}$ and the persistence of neurologic symptoms, a second HBOT session was performed. After this session, the $\mathrm{COHb}$ was $5.5 \%$ and the patient was asymptomatic (21 hours after admission). Repeat ABG measurements showed a decreased $\mathrm{COHb}$ of $2.7 \%$ (48 hours after ED admission). The first hypothesis was glucose-6-phosphate dehydrogenase (G6PD) deficiency (not confirmed). Sources of $\mathrm{CO}$ at home were excluded. At this stage, occupational exposure to solvents was suspected. A blood sample taken approximately 40 hours after cessation of exposure, showed a DCM concentration of $0.5 \mu \mathrm{g} /$ $\mathrm{mL}$ (cut off at the lower limit). This compound has high lipid solubility, and with slow release from tissues and the short halflife of DCM (40 minutes) this result was considered positive for toxic exposure. Echocardiography, electroencephalogram and brain magnetic resonance imaging (MRI) performed during hospitalization were normal, except for modest cortical atrophy at the vertex and bilateral mastoiditis. The patient was discharged asymptomatic on day 4.

Conclusion: DCM is highly volatile, and this property leads to rapid accumulation following inhalation exposure. DCM exposure can be responsible for hidden and chronic $\mathrm{CO}$ poisoning due to persistent metabolic production. In our experience, prolonged DCM inhalation in an occupational setting may lead to severe poisoning requiring HBOT. Personal protective equipment is essential to reduce risks. Laboratory support is the unique way to confirm exposure. A high level of $\mathrm{COHb}$ is normally related to exogenous CO intoxication. This case emphasizes the importance of carrying out different causal hypotheses when there are unsatisfactory results from HBOT sessions such as the persistence of high concentrations of $\mathrm{COHb}$.

\section{Anticholinergic syndrome treated with oral rivastigmine: an inexpensive therapy}

Fannie Péloquin ${ }^{a}$, Pierre-André Dubéb, Maude St-Onge ${ }^{c}$, Vincent Pichette ${ }^{d}$ and Sophie Gosselin ${ }^{c}$

${ }^{a}$ Emergency Medicine Residency Program, Université Laval,

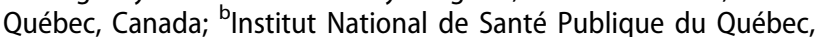
Québec, Canada; 'Centre Antipoison du Québec, Québec, Canada; ${ }^{d}$ Nephrology Department, Hôpital Maisonneuve-Rosemont, Montréal, Canada 
Objective: Physostigmine is not marketed in Canada, but some hospitals use Health Canada's Special Access Program to stock a small quantity. Clinicians are thus inexperienced in using it and are concerned about inducing adverse effects. Rivastigmine is available orally, possesses the same mechanism of action and crosses the blood-brain barrier. It has a bioavailability of $40 \%$, a half-life of 2 hours allowing prolonged control of symptoms and is not metabolized by the liver, reducing the risk of drug interaction. In addition, it is readily available in hospitals at a lower cost (Can\$ $0.15-0.55$ for $1.5 \mathrm{mg}$ tablet versus Can $\$ 15-40$ for a 2 $\mathrm{mg}$ vial of physostigmine). We report success in treating anticholinergic symptoms with oral rivastigmine.

Case report: A 21-year-old man presented to the emergency room 4 hours after ingestion of $20 \mathrm{mg} / \mathrm{kg}$ of diphenhydramine. He exhibited mydriasis, severe delirium with Lilliputian hallucinations and urinary retention with preservation of peristalsis. The initial vital signs were: blood pressure $130 / 81 \mathrm{mmHg}$, pulse 93 bpm, oxygen saturation $99 \%$, temperature $36.4{ }^{\circ} \mathrm{C}$, and an electrocardiogram (ECG) showed QRS $86 \mathrm{~ms}$ and QTc $400 \mathrm{~ms}$. Following recommendations of the Canadian Antidote Guide in Acute Care Toxicology, he received boluses of lorazepam in 1-2 $\mathrm{mg}$ IV doses every 10 minutes to control his agitation. After a total dose of $14 \mathrm{mg}$ IV, there was no improvement in his status. Consequently, our Poison Control Center (PCC) suggested the administration of $0.5 \mathrm{mg}$ of physostigmine IV, which produced a brief but complete resolution of the patient's delirium. Unfortunately, the hospital did not have enough physostigmine for an infusion. As an alternative strategy, our PCC suggested to give another $0.5 \mathrm{mg}$ of IV physostigmine concomitantly with 1.5 $\mathrm{mg}$ of oral rivastigmine. The delirium improved and patient's status was maintained for the following 24 hours with no other medication requirement and the patient was discharged home.

Conclusion: Oral anticholinesterase molecules, like rivastigmine, represent an interesting therapeutic avenue to treat anticholinergic syndrome. Indeed, this drug has several advantages over physostigmine: longer duration of action allowing prolonged symptom control without the need of an infusion, wide availability and affordability. Its use might reduce length of stay and facilitate monitoring of patients when physostigmine is unavailable. While inadvisable with concomitant gastrointestinal decontamination, it warrants further studies to consider its official inclusion in the Canadian Antidote Guide.

\section{Buprenorphine exposures reported to a regional poison center, 2011-2016}

\author{
Saumitra Rege ${ }^{a}$, Duc Anh Ngo ${ }^{b}$, Nassima Ait-Daoud ${ }^{c}$ \\ and Christopher P Holstege $\mathrm{a}^{\mathrm{a}}$ \\ aDivision of Medical Toxicology, Department of Emergency \\ Medicine, University of Virginia, Charlottesville, United States; \\ ${ }^{\mathrm{b}}$ Division of Student Affairs, Department of Student Health, \\ University of Virginia, Charlottesville, United States; ${ }^{\text {P Psychiatry and }}$ \\ Neurobehavioral Sciences, University of Virginia, Charlottesville, \\ United States
}

Objective: Ambulatory treatment visits for buprenorphine increased from 0.2 million visits in 2003 to 2.1 million visits in 2013 [1]. The extent of buprenorphine overdoses has not been clearly delineated. We investigated the trends and characteristics of buprenorphine exposures reported to a regional poison center (PC).

Methods: Toxicall ${ }^{\mathrm{TM}}$, a comprehensive case management system used by $75 \%$ of US PCs, was queried at a single regional poison center for all closed, human records where buprenorphine was reported as an exposure from 1 January 2011 through 31 December 2016. Key exposure characteristics were descriptively assessed. Annual trends in buprenorphine exposure reports during the study period were evaluated using linear regression.

Results: During the study period, there were 353 calls to the regional PC reporting buprenorphine exposures which amount to a $42 \%$ increase (50 to 71 ), despite an overall decrease in PC call volume. Of these, $45.6 \%$ were multiple substance exposures. The intentional exposures to buprenorphine significantly increased by $104 \%$ ( 22 to 45 ) with reports of abuse (10 to 18 reports) as well as suicide (10 to 20 reports) demonstrating an increase from 2011 to 2016. Females (52.1\%) and adults aged 20-29 years $(22.7 \%)$ were the most frequent demographic groups. The most frequent route of exposure was ingestion (80.9\%) and the highest proportion of cases were treated in a healthcare facility (82.1\%). Minor (24.9\%) and moderate medical effects (35.7\%) were more commonly seen. Moreover, in comparison to unintentional exposures, major effects were more common (69\% versus $24.1 \%$ ) in intentional exposures. Neurological effects (90\%) and cardiovascular effects $(44.5 \%)$ were most commonly exhibited. Intubation was reported in $9.9 \%$ of the cases while naloxone therapy was reported in $19.8 \%$. The number of buprenorphine exposure calls to the PC increased at a rate of 4 more calls per year $(95 \% \mathrm{Cl}$ : 3.10, 5.15; $\mathrm{p}<0.001$ ). Specifically, intentional buprenorphine exposures demonstrated a rate of 3 more calls per year $(95 \% \mathrm{Cl}$ : 2.17, 5.25; $\mathrm{p}<0.001)$.

Conclusion: Buprenorphine exposures reported to a regional PC increased. Our analysis highlights the severe health consequences due to intentional buprenorphine exposures, despite being considered safe. More guidelines and policies need to be developed to ensure optimal use and better outcomes with buprenorphine. PCs can be vital in managing such cases as demonstrated in the current study.

\section{Reference}

[1] Turner L, Kruszewski SP, Alexander GC. Trends in the use of buprenorphine by office-based physicians in the United States, 20032013. Am J Addict. 2015;24:24-29.

\section{Veno-arterial extracorporeal membrane oxygenation (VA-ECMO) and hydroxocobalamin in diphenhydramine-induced cardiac arrest}

\author{
Jonathan De Olano ${ }^{\mathrm{a}}$, Nicos Hadjiangelis ${ }^{\mathrm{b}}$ and \\ Rana Biary ${ }^{\mathrm{a}}$ \\ ${ }^{a}$ Division of Medical Toxicology, Ronald O. Perelman Department \\ of Emergency Medicine, NYU School of Medicine, New York, \\ United States; ${ }^{\mathrm{b}}$ Critical Care, New York Presbyterian Brooklyn \\ Methodist Hospital, Brooklyn, United States
}

Objective: Diphenhydramine is a common over-the-counter antihistamine which rarely leads to severe toxicity. We report a patient with a diphenhydramine overdose manifested with seizure and cardiac arrest. Though her hemodynamics improved with VA-ECMO and hydroxocobalamin, the patient was ultimately declared brain dead and underwent organ procurement.

Case report: A 34-year-old woman with a history of depression was found by emergency medical services with seven bottles of diphenhydramine, two of which were empty. The patient seized and moments afterwards went into cardiac arrest. Advanced cardiac life support (ACLS) was initiated, with chest compressions performed for 35 minutes, $2 \mathrm{mg}$ of epinephrine given intravenously, an epinephrine infusion and intubation. Additionally, the 
patient received intermittent boluses of sodium bicarbonate totaling $150 \mathrm{mEq}, 25 \mathrm{~g}$ dextrose and $1 \mathrm{~g}$ calcium gluconate. Toxicology was consulted and recommended an additional 150 $\mathrm{mEq}$ IV bolus of sodium bicarbonate and, within 5 minutes, the patient regained a pulse. On physical exam, she had minimally reactive pupils and was unresponsive to painful stimuli. Initial laboratory findings were remarkable for a lactate of $9 \mathrm{mmol} / \mathrm{L}$ and a potassium of $2.5 \mathrm{mmol} / \mathrm{L}$. The electrocardiogram (ECG) demonstrated a QRS of $154 \mathrm{~ms}$ and a QTc of $463 \mathrm{~ms}$ and she was placed on a bicarbonate infusion. Decontamination was performed using gastric lavage and $50 \mathrm{~g}$ of activated charcoal. Four hours following presentation, her vasopressor requirements included: $20 \mu \mathrm{g} / \mathrm{min}$ epinephrine, $60 \mu \mathrm{g} / \mathrm{min}$ norepinephrine, 200 $\mu \mathrm{g} / \mathrm{min}$ phenylephrine, and $0.04 \mathrm{U} / \mathrm{min}$ vasopressin. She developed acute respiratory distress syndrome (ARDS) so was placed on VA-ECMO. Given profound vasoplegic shock, she received $5 \mathrm{~g}$ of hydroxocobalamin and was quickly weaned off her vasopressor requirements to $5 \mu \mathrm{g} / \mathrm{min}$ epinephrine and $15 \mu \mathrm{g} / \mathrm{min}$ norepinephrine. Repeat ECG showed a QRS of $160 \mathrm{~ms}$ and QTc 565 ms. Her ECG normalized within 24 hours of arrival and bicarbonate infusion was discontinued. She was on VA-ECMO for seven days with stable hemodynamics, and never developed acute kidney or liver injury. She was declared brain dead on hospital day 7 and underwent organ procurement. The initial serum diphenhydramine concentration was $1,400 \mathrm{ng} / \mathrm{mL}$ (normal range $90-120$ $\mathrm{ng} / \mathrm{mL}$ ).

Conclusion: We present a unique case of diphenhydramine overdose who obtained return of spontaneous circulation after ACLS and $150 \mathrm{mEq}$ IV push of sodium bicarbonate, placed on VAECMO, and treated for vasoplegic shock with hydroxocobalamin. While there is increasing use of ECMO in poisoned patients, its use as a bridge to organ donation requires further ethical consideration.

\section{Complications of transport in corpore of illicit drug requiring admission to the intensive care unit}

\author{
Lise Aubry ${ }^{a}$, Karim Jaffal ${ }^{a}$, Marion Soichot ${ }^{b}$, \\ Laurence Labat ${ }^{b}$ and Bruno Mégarbane ${ }^{a}$ \\ ${ }^{a}$ Department of Medical and Toxicological Critical Care, Lariboisière \\ Hospital, Paris-Diderot University, INSERM UMRS-1144, Paris, \\ France; ${ }^{b}$ Laboratory of Toxicology, Lariboisière Hospital, Paris- \\ Diderot University, INSERM UMRS-1144, Paris, France
}

Objective: Several methods of illicit drug smuggling including their concealment in the body are used to allow cross-border transportation (body packing), avoiding a sudden police control (body stuffing) or reaching the sale area (body pushing). Drug transport in corpore may result in life-threatening complications due to the bioavailability of a large drug amount in case of pack rupture. Our objective was to describe the complications in a series of drug transporters admitted to the intensive care unit (ICU) and investigate the contribution of the measurement of the drug concentration in the plasma.

Methods: We conducted an observational retrospective singlecenter study during an 8-year period (2010-2018) including all symptomatic patients admitted to the intensive care unit for suspicion of complications in relation to illicit drug smuggled in their body. Patients arrested in the Paris area or at the two Paris international airports and suspected of drug body-transport are usually transferred in legal custody in the Medico-Judiciary Emergency Unit of a neighboring hospital and if symptomatic, immediately referred to our ICU. The plasma concentration of the various transported drugs were measured on patient admission and the drug found in the hidden bags determined using liquid chromatography coupled to mass spectrometry assay.
Results: Forty-two patients (88\% males, age: 31 years [22-43] (median [percentiles 25-75]) were included. The patients were body packers $(86 \%)$, body stuffers $(12 \%)$ and body pushers $(2 \%)$, transporting cocaine (90\%), heroin (5\%) and hashish (5\%). They had arrived from Latin America (45\%), Guyana (22\%), Metropolitan France (17\%) or French West Indies (5\%). The packs were professionally made $(83 \%)$, containing solid versus liquid drugs (83\% versus 17\%). Diagnosis was assessed based on abdominal computerised tomography (CT) scan (85\%) versus radiography (25\%). Symptoms/signs requiring ICU admission included intense abdominal pain (33\%), sustained vomiting (26\%), mydriasis $(22 \%)$, absence of abdominal noises $(8 \%)$, hyperthermia (7\%), cardiovascular failure (5\%) and cardiac arrest (2\%). The observed complications during the ICU stay included intestinal occlusion (12\%), drug overdose by pack rupture (7\%) and death (2\%). Laxative medications including paraffin oil (52\%) and polyethylene glycol (57\%) were administered to all patients. Surgery $(14 \%$ including gastrotomy [33\%], enterotomy [17\%] and colostomy [17\%]) and catecholamine infusion (9\%) were required. The plasma concentration of the drug on ICU admission was not significantly correlated to the risk of abdominal complications.

Conclusion: Life-threatening and even fatal complications may be observed in symptomatic in corpore drug transporters. Careful monitoring is required in the ICU to allow early detection and treatment of complications. Surgical removal of the packs when complications occur is warranted.

\section{Metformin intoxication requiring dialysis: a case report of extracorporeal removal and renal elimination}

\author{
Roberto Zoppellari ${ }^{a}$, Milo Vason ${ }^{a}$, Alda Storari ${ }^{b}$, \\ Anna Talarico ${ }^{c}$, Stefano Petrini ${ }^{a}$ and Margherita Neri ${ }^{d}$ \\ ${ }^{a}$ Anaesthesia and Intensive Care, S. Anna Hospital, Ferrara, Italy; \\ ${ }^{b}$ Nephrology, S. Anna Hospital, Ferrara, Italy; 'Legal Medicine, S.

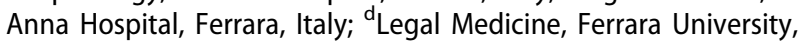 \\ Ferrara, Italy
}

Objective: Extrarenal removal in metformin intoxication has been suggested [1]. Metformin can cause severe complications such as severe lactic acidosis, especially in the setting of acute renal failure. We report a case of metformin intoxication treated with dialysis where the metformin concentrations in serum, urine and ultrafiltrate were measured.

Case report: An 80-year-old woman was admitted to the intensive care unit anuric, with abdominal pain, vomiting and diarrhoea. She had a history of hypertension and diabetes treated with metformin ( $3 \mathrm{~g} /$ day). Arterial blood gas (ABG) analysis showed severe metabolic acidosis $(\mathrm{pH} 7.07$, bicarbonate 6.4 $\mathrm{mmol} / \mathrm{L}$ ). Lactate was $5.5 \mathrm{mmol} / \mathrm{L}$. Glycaemia and abdominal ultrasound were normal. Blood urea and serum creatinine were 194 (reference $17-43 \mathrm{mg} / \mathrm{dL}$ ) and 9.04 (reference $0.5-1.2 \mathrm{mg} / \mathrm{dL}$ ), respectively. Serum metformin was $18.5 \mu \mathrm{g} / \mathrm{mL}$ (upper limit <4 $\mu \mathrm{g} / \mathrm{mL}$ ). Dialysis was started. Metformin concentration in serum, ultrafiltrate and urine was measured by ultra-performance liquid chromatography-mass spectrometry. The amount of metformin removed by dialysis was $1460 \mathrm{mg}$, whereas metformin elimination by urine during the same time was $895 \mathrm{mg}$. Serum metformin at the end of dialysis was $12.4 \mu \mathrm{g} / \mathrm{mL}$ and ABG improved $(\mathrm{pH} \mathrm{7.32,}$ bicarbonate $15.5 \mathrm{mmol} / \mathrm{L}$, lactate $0.9 \mathrm{mmol} / \mathrm{L}$ ). During the following 12 hours metformin elimination via urine was $1731 \mathrm{mg}$. At the end of this time the metformin serum concentration was 8.8 $\mu \mathrm{g} / \mathrm{mL}$. On day 3 the patient became polyuric, ABG normalized and the metformin serum concentration was $3.7 \mu \mathrm{g} / \mathrm{mL}$.

Conclusion: A concurrent disease may induce acute renal failure leading to metformin accumulation. Clinical benefit of extrarenal 
support is to restore an acceptable acid-base status and to remove the drug. In our case the benefit of dialysis was due to direct elimination of the drug itself (small removal) and particularly the correction of the metformin-induced severe lactic acidosis [2]. Metformin elimination by diuresis improved with the recovery of renal function and resulted in a lowering of the serum concentration.

\section{References}

[1] Seidowsky A, Nseir S, Houdret N, et al. Metformin-associated lactic acidosis: a prognostic and therapeutic study. Crit Care Med. 2009;37:2191-196.

[2] Zoppellari R, Felisatti G, Dallocchio G, et al. Metformin removal by extracorporeal elimination techniques in cases of overdose: a literature review. Clin Toxicol (Phila). 2014;52:404-405.

\section{Prolonged clinical course from suspected verapamil bezoar formation in an intentional massive overdose of verapamil and enalapril}

\author{
Lotte CG Hoegberg ${ }^{\mathrm{a}}$, Sys S Johansen ${ }^{\mathrm{b}}$, \\ Tonny S Petersen ${ }^{c}$, Dorte F Palmqvist ${ }^{\mathrm{a}}$ and \\ Soeren Bøgevig ${ }^{c}$ \\ ${ }^{\mathrm{a}}$ Department of Anesthesiology and Intensive Care and Danish \\ Poisons Information Centre, Copenhagen University Hospital \\ Bispebjerg, Copenhagen, Denmark; 'bection of Forensic Chemistry, \\ Department of Forensic Medicine, Faculty of Health and Medical \\ Sciences, University of Copenhagen, Copenhagen, Denmark; \\ 'Department of Clinical Pharmacology and Danish Poisons \\ Information Centre, Copenhagen University Hospital Bispebjerg, \\ Copenhagen, Denmark
}

Objective: We present a case of overdose with prolonged severe cardiovascular symptoms following ingestion of verapamil retard and enalapril tablets. The specific verapamil retard formulation ingested is prone to pharmacobezoar formation and prolonged release in vitro [1].

Case report: A 42-year old man $(90 \mathrm{~kg})$ was admitted to the emergency room (ER) after apparent suicidal ingestion of $12 \mathrm{~g}$ verapamil retard (100 tablets) and $1 \mathrm{~g}$ enalapril (100 tablets). The time of ingestion was unknown. The doses were initially questioned due to absent symptoms and normal vital parameters. Gastrointestinal decontamination was not performed. Twelve hours after admission he deteriorated; at 16 hours vital parameters were BP $63 / 47 \mathrm{mmHg}, \mathrm{HR} 105 / \mathrm{min}$, temperature $35.6{ }^{\circ} \mathrm{C}$, respirations $26 / \mathrm{min}$, oxygen saturation $86 \%$, mental status fluctuations, and diaphoresis. Electrocardiogram (ECG) showed broadened complexes. BP rapidly decreased to $45 / 30 \mathrm{mmHg}$ and HR $50 / \mathrm{min}$, with lactate $6 \mathrm{mmol} / \mathrm{L}$. He was transferred to intensive care with suspicion of continued absorption of verapamil. The extracorporeal membrane oxygenation (ECMO) team was unable to provide immediate treatment. Bedside echocardiography showed near-normal ejection fraction (45\%) and stasis. Vasopressor support, hyperinsulinemic-euglycemic therapy (HIET), bolus $90 \mathrm{U}$ fast-acting insulin followed by infusion $5 \mathrm{U} / \mathrm{kg} / \mathrm{h}$ at first, then increased to $10 \mathrm{U} / \mathrm{kg} / \mathrm{h}$, and glucose $20 \% 1 \mathrm{~L}$ was initiated, with calcium chloride $5 \mathrm{mmol}$ IV in repeated doses and crystalloids. He had respiratory insufficiency from pulmonary edema and was almost anuric at 30 hours. He was intubated and continuous renal replacement therapy (CRRT) initiated to control hypervolaemia after volume resuscitation. In the following 48 hours he received high dose epinephrine/norepinephrine.
Persistent third degree AV-block with idioventricular rhythm were present and a temporary pacemaker was planned. Slow stabilization and diminishing vasopressor support were observed from day 4 after ingestion. Whole blood concentrations at 20, 25, 28, 32,40 and 91 hours were: verapamil 3.09, 1.79, 1.98, 1.60, 2.11 and $1.20 \mathrm{mg} / \mathrm{kg}$; norverapamil 1.12, 0.90,1.34, 1.05, 1.07 and 0.42 $\mathrm{mg} / \mathrm{kg}$; enalapril $0.26,0.069,0.036,0.014,0.014$ and $0.005 \mathrm{mg} / \mathrm{kg}$; enalaprilate $1.34,0.94,1.16,0.55,0.51$ and $0.18 \mathrm{mg} / \mathrm{kg}$.

Conclusion: Verapamil/norverapamil were in toxic and possibly lethal concentrations. Late increase in blood concentrations confirmed ongoing late absorption and the suspicion of possible pharmacobezoar(s) from the specific verapamil extended release formulation ingested. Enalapril/enalaprilate concentrations were elevated by a factor of 10 compared to therapeutic concentrations.

\section{Reference}

[1] Hoegberg LCG, Refsgaard F, Pedersen SH, et al. Potential pharmacobezoar formation of large size extended-release tablets and their dissolution - an in vitro study. Clin Toxicol (Phila). 2018 [Epub ahead of print].

\section{Physostigmine for atypical antipsychotic agents (quetiapine, olanzapine and clozapine): a retrospective review}

\author{
Timothy J Wiegand ${ }^{a}$, Rachel Gorodetsky ${ }^{b}$, \\ Nicole Acquisto ${ }^{c}$, Rachel Schult ${ }^{c}$, Kimberly Kaukeinen ${ }^{a}$ \\ and Kenneth Conner ${ }^{a}$ \\ aEmergency Medicine, University of Rochester Medical Center, \\ Rochester, United States; ${ }^{\mathrm{b}} \mathrm{D}^{\prime}$ Youville College, School of Pharmacy, \\ Buffalo, United States; 'Department of Pharmacy, University of \\ Rochester Medical Center, Rochester, United States
}

Objective: Physostigmine is a carbamate acetylcholinesterase inhibitor used to treat antimuscarinic drug toxicity. Once widely accepted as a standard antidote for antimuscarinic drug intoxication, a few sentinel case reports describing adverse events including asystole and death caused a shift away from use. In recent years more critical interpretation of the data, along with a growing number of publications describing safety and efficacy, have led to a recurrence of use. We recently reported our experience of over 5 years of physostigmine administration and found it to be efficacious with low incidence of side effects [1]. Our practice is to administer $1-2 \mathrm{mg}$ over $5-10$ minutes in adults (pediatric weight-based). Here we detail a subset of this data for quetiapine, olanzapine and clozapine cases; atypical antipsychotic agents with significant antimuscarinic effects. Physostigmine use for these agents has only rarely been reported.

Methods: Retrospective chart review of all patients administered physostigmine at a single institution from 5 March 2011 to 31 July 2016. Electronic health records were reviewed and data entered into REDCap database. Cases with quetiapine, olanzapine or clozapine as primary agent were extracted and reviewed. Demographics, exposure history, symptoms, and details of physostigmine administration (dose/response/adverse events) were recorded and descriptive analysis performed.

Results: Overall 231 patients received physostigmine during the study period (15 distinct agents identified). Of these, 54 (23\%) involved atypical antipsychotics. Quetiapine was reported most frequently in 43 ( $19 \%$ total entries). Olanzapine was identified in 7, clozapine 4. Coma occurred in 29/43 (67\%) quetiapine entries, 
4/7 (57\%) olanzapine and 2/4 (50\%) clozapine. Agitation or delirium was present in 21 (49\%) and 31 (72\%) quetiapine cases, both symptoms reported in 5 (71\%) of olanzapine and 1 (25\%) and 3 (75\%) of clozapine. Symptoms recurred in 18 (range 0.2-6 hours) although only 10 received additional physostigmine (1-6 doses with one continuous infusion $2 \mathrm{mg} / \mathrm{h} / 14$ hours). Doses ranged from $0.4-2 \mathrm{mg}$ with most $(n=40)$ receiving $2 \mathrm{mg} ; 10 \quad(19 \%)$ received benzodiazepines. Adverse effects occurred in 3/54 (5\%) with one seizure (patient with pre-existing seizure disorder and additional physostigmine administered without recurrence). In two entries vomiting occurred after physostigmine. No bradycardia or other arrhythmias were reported.

Conclusion: Physostigmine is safe and effective in reversing atypical antipsychotic associated anticholinergic signs and symptoms for quetiapine, olanzapine and clozapine ingestions.

\section{Reference}

[1] Gorodetsky R, Acquisto N, Schult R, et al. Five years of physo: a retrospective review of the safety and efficacy of physostigmine. Clin Toxicol (Phila) 2018;56:73.

\section{Early diagnosis associated with pharmacological and hyperbaric oxygen therapy in a Loxosceles bite in Italy}

\author{
Franca Davanzo ${ }^{a}$, Anna Celentano ${ }^{a}$, Valeria Dimasi ${ }^{a}$, \\ Fabio Garuti ${ }^{\mathrm{b}}$, Giovanni Sesanab ${ }^{\mathrm{b}}$, Rossella Giacomello ${ }^{\mathrm{b}}$, \\ Gianluca Basso ${ }^{c}$ and Paolo Pantini ${ }^{d}$ \\ ${ }^{a}$ Milan Poison Control Center, Niguarda Great Metropolitan \\ Hospital, Milan, Italy; ${ }^{\mathrm{b}}$ Hyperbaric Medicine Center, Niguarda Great \\ Metropolitan Hospital, Milan, Italy; 'Plastic Surgery, Niguarda Great \\ Metropolitan Hospital, Milan, Italy; ${ }^{\mathrm{d}}$ Museo Civico di Scienze \\ Naturali "E.Caffi" Bergamo, Bergamo, Italy
}

Objective: The spider Loxosceles rufescens (LR) is indigenous to Mediterranean Europe. We described a patient with severe skin necrosis, caused by a Loxosceles rufescens bite, which was treated with hyperbaric oxygen therapy (HBOT).

Case report: A 38-year-old male, arrived at the emergency department 4 days after the spider's bite, which happened at home during the night. The spider was killed by the patient and was identified by the entomologist as LR. The bite was not immediately noticed by the patient and affected the distal part of the right arm. After a few hours a small lesion appeared, which progressively enlarged, complicated by pain, itching, and edema that spread both proximally and distally of the bite with functional impairment. Fever $\left(>39^{\circ} \mathrm{C}\right)$ and erythema appeared. He was treated with oral antibiotic, antihistamine and topical cortisone. The local lesion was approximately $11 \times 5 \mathrm{~cm}$, but worsened rapidly with ecchymosis, edema, hemorrhage and dermonecrosis at the bite site. Laboratory tests showed increased white blood cells $\left(11,900 \mathrm{~mm}^{3}\right)$, elevated aminotransferase $(91 \mathrm{U} / \mathrm{L})$ and hyperglycemia $(107 \mathrm{mg} / \mathrm{dL})$. Due to worsening and rapid local evolution of the lesion with increasing pain, it was decided, in agreement with the hyperbaric medicine center, to perform two sessions of HBOT daily at 2.8 ATA for seven days and then once a day for twenty days, and, after an interruption, a new cycle of 15 days. HBOT was started 4 days after the bite, and during treatment the lesion and edema decreased with injury delimitation. The eschar in some areas measured more than $3 \mathrm{~mm}$ thick, and on the 80th day after the bite, a surgical toilette was performed and the dimensions reduced to about $5 \times 2 \mathrm{~cm}$. The patient was declared healed after about another 20 days. There was no residual neurological or functional impairment.

Conclusion: Regardless of score, HBOT results in a decrease in pain and in a faster demarcation of the eschar. The timely antibiotic and antihistamine therapy, in addition to HBOT, enabled rapid healing without any local damage in this patient. Symptoms of loxoscelism tend not to appear immediately and diagnosis is frequently delayed. The venom contains anaerobic bacteria so antibiotic therapy is necessary. Even if the correct diagnosis is very delayed, HBOT may still contribute to recovery and can prevent the loss of an affected limb following a Loxosceles bite.

\section{Biological monitoring of occupational exposure to 5- fluorouracil by urinary a-fluoro- $\beta$ - alanine (FBAL) assay with a high- sensitivity analytical method}

\author{
Mireille Canal Raffin ${ }^{\mathrm{a}}$ and Antoine Villa ${ }^{\mathrm{b}}$ \\ ${ }^{a}$ Equipe Epicene - Laboratoire de Pharmacologie et de Toxicologie, \\ Inserm U1219, Université de Bordeaux, CHU de Bordeaux, \\ Bordeaux, France; 'bervice de Pathologies Professionnelles et \\ Environnement, Hôpital la Timone, AP-HM, Marseille, France
}

Objective: 5-Fluorouracil (5FU), an antineoplastic drug commonly used by health professionals, is considered as "dangerous to handle". Biological monitoring of occupational exposure is the most reliable process to identify insufficiently controlled exposures. To evaluate the potential contamination of workers exposed to 5FU, the aim of this work was to develop a high-sensitivity analytical tool allowing the assay of a-fluoro- $\beta$-alanine (FBAL), the urinary metabolite of 5FU. This method is currently applied routinely within our laboratory for prescribed biological assays as part of biological monitoring of occupational exposure to 5FU in care establishments. The main data collected to date by occupational health monitoring are presented.

Methods: After a derivatization step, urinary FBAL was extracted by a solid/liquid phase extraction and analyzed by ultra-high-performance-liquid chromatography coupled with tandem mass spectrometry. Occupational health interventions were conducted between 2015 and 2017 in eight care establishments including nurses preparing and/or infusing 5FU, caregivers and pharmacy technicians. Each subject provided a urine sample 0-10 hours after the end of their work-shift. A subject was considered as "contaminated" when FBAL was detected as trace or with urine concentration equal to or higher than the method's limit of quantification (LOQ).

Results: The validated assay method has a good linearity $(r<$ 0.996), repeatability and precision and is specific and sensitive with a lower limit of quantification (LOQ) of $20 \mathrm{ng} / \mathrm{L}$. Seventythree health professionals exposed to 5FU were followed-up. The contamination percentage was $9.6 \%$ for all participants $(n=7 /$ $73), 33.3 \%$ in Mauritanians and $4.9 \%$ in French subjects. Overall $14.3 \%$ of caregivers were contaminated, $10.4 \%$ of nurses, $6.7 \%$ of hospital workers and none of the pharmacy technicians. Urinary FBAL concentrations ranged from 25 to $301 \mathrm{ng} / \mathrm{L}$ (median $44 \mathrm{ng} /$ L). The three French cases of contamination had urinary FBAL concentrations between $25-35 \mathrm{ng} / \mathrm{L}$, which is very close to the LOQ of this method.

Conclusion: An effective analytical tool is essential for the reliable detection and routine biological monitoring of healthcare professionals occupationally exposed to 5-FU. Contaminations of French health workers would not have been detected with currently published methods (LOQ $40 \mathrm{ng} / \mathrm{L}$ by gas chromatographymass spectrometry, $1000 \mathrm{ng} / \mathrm{L}$ by liquid chromatography-tandem 
mass spectrometry). These results show the importance of the LOQ value of the analytical method; the LOQ must be as low as possible in order to reduce the number of false negatives, given that currently no biological exposure indices for interpretation of antineoplastic drug contamination exist.

\section{Respiratory impairment in workers exposed to nanoparticles}

\author{
Daniela Pelclova ${ }^{\mathrm{a}}$, Vladimir Zdimal ${ }^{\mathrm{b}}$, \\ Stepanka Vlckova ${ }^{a}$, Zdenka Fenclova $^{\mathrm{a}}$ and \\ Lucie Lischkova ${ }^{a}$ \\ aDepartment of Occupational Medicine, Charles University in \\ Prague and General University Hospital, First Faculty of Medicine, \\ Prague, Czech Republic; ${ }^{b}$ Institute of Chemical Process \\ Fundamentals of the CAS, Prague, Czech Republic
}

Objective: At present, little is known about the health effects in thousands of workers processing nanocomposites, and studies are extremely rare.

Methods: Twenty researchers ( $42 \pm 11$ years), handling nanocomposites for $18 \pm 10$ years were examined pre-shift and post-shift, together with 21 controls ( $43 \pm 12$ years). Biomarkers of oxidative stress derived from lipids, nucleic acids, proteins and markers of inflammation were analyzed in exhaled breath condensate (EBC). Aerosol exposure was monitored during nanoparticle generation operations: smelting, welding and nanocomposite machining.

Results: Mass concentrations ranged from 0.120 to $1.840 \mathrm{mg} / \mathrm{m}^{3}$, and median particle number concentrations from $4.8 \times 10^{4}$ to 5.4 x $10^{5}$ particles $/ \mathrm{cm}^{3}$. Nanoparticles accounted for 40 to $95 \%$ of particles, with iron and manganese prevailing. Significant elevations were already seen in most oxidative stress markers and in several inflammation markers in the pre-shift samples relative to the controls. Significant associations were found between working in nanocomposite synthesis and the majority of EBC biomarkers. Chronic bronchitis was more frequent in researchers. A minor, but significant post-shift decrease of lung function parameters was found.

Conclusion: Workers in nanocomposite synthesis may be at risk of developing airway disorders with time. These initial changes may not be detected by regular spirometry. The analysis of oxidative stress and inflammation markers appears more sensitive [1]. The findings were compared with previous studies of workers exposed to nano- $\mathrm{TiO}_{2}$ and nano-Fe oxides. Among the markers analyzed in $\mathrm{EBC}$, the following were most robust and could be recommended for preventive examination: 8-hydroxy-2-deoxyguanosine $(8-\mathrm{OHdG})$ and 5 -hydroxymethyl uracil $(5-\mathrm{OHMeU})$ from nucleic acids; o-tyrosine (o-Tyr) and 3-nitrotyrosine (3-NOTyr) from proteins; and malondialdehyde and aldehydes $C_{6}-C_{13}$ from lipids. Among all markers of inflammation, tumor necrosis factor and leukotriene B146 appeared the most useful [2-4].

\section{Acknowledgements}

Progres Q25/1LF, Q29/1LF.

\section{References}

[1] Pelclova D, Zdimal V, Schwarz J, et al. Markers of oxidative stress in the exhaled breath condensate of workers handling nanocomposites. Nanomaterials. 2018;8:pii:E611.

[2] Pelclova D, Zdimal V, Kacer $P$, et al. Markers of lipid oxidative damage in the exhaled breath condensate of nano $\mathrm{TiO}_{2}$ production workers. Nanotoxicology. 2017;11:52-63.
[3] Pelclova D, Zdimal V, Kacer P, et al. Markers of lipid oxidative damage among office workers exposed intermittently to air pollutants including nanoTiO 2 particles. Rev Environ Health. 2017;32:193-200.

[4] Pelclova D, Zdimal V, Kacer $P$, et al. Oxidative stress markers are elevated in exhaled breath condensate of workers exposed to nanoparticles during iron oxide pigment production. J Breath Res. 2016;10:016004.

\section{Acute transdermal chromium oxide intoxication after an industrial accident in two tanning workers}

\author{
Hyun Ho Jung, Jung Taek Park and Kyoung Ho Choi \\ Emergency Medicine, The Catholic Medical University of Korea, \\ Uijeonbu, Republic of South Korea
}

Objective: We report two workers with transdermal chromium oxide intoxication after an industrial accident.

Case series: A 58-year-old man (case 1) presented to our Emergency Department (ED) after a factory guard found him and his co-worker unconscious lying in a spill of chromium (III) oxide crystals $\left(\mathrm{Cr}_{2} \mathrm{O}_{3}, 25.5 \%\right)$ for six hours. On arrival, he was agitation with hypertension $(153 / 110 \mathrm{mmHg})$. Physical examination revealed chemical burns on $11 \%$ of total body surface area (TBSA). Blood gas analysis on oxygen ( $5 \mathrm{~L} /$ minute) revealed the following: $\mathrm{pH} 7.268, \mathrm{PCO}_{2} 42.4 \mathrm{mmHg}$, and $\mathrm{PaO}_{2} 205.9 \mathrm{mmHg}$. Radiologic examination showed orbital wall and L2 compression fracture. He had leukocytosis $\left(16,630 \mathrm{cells} / \mathrm{mm}^{3}\right)$, and elevated lactic acid $(70.4 \mathrm{mg} / \mathrm{dL})$, glucose $(178 \mathrm{mg} / \mathrm{dL})$, BUN $(25.1 \mathrm{mg} / \mathrm{dL})$, creatinine $(1.33 \mathrm{mg} / \mathrm{dL})$, AST $(416 \mathrm{U} / \mathrm{L})$, ALT $(322 \mathrm{U} / \mathrm{L})$, and CPK $(474 \mathrm{U} / \mathrm{L})$. He received supportive management. He became alert on day three and was discharged on day 27. The second worker (case 2), a 41-year-old man, was agitated on arrival with chemical burns on $3 \%$ of TBSA; radiologic examination revealed traumatic subdural hematoma, subarachnoid hemorrhage, and scapular fracture. He had leukocytosis $\left(19,950 \mathrm{cells} / \mathrm{mm}^{3}\right)$, and elevated lactic acid $(24.3 \mathrm{mg} / \mathrm{dL})$, glucose $(193 \mathrm{mg} / \mathrm{dL})$, BUN $(25.9 \mathrm{mg} / \mathrm{dL})$, creatinine $(1.52 \mathrm{mg} / \mathrm{dL})$, AST $(141 \mathrm{U} / \mathrm{L})$, ALT $(118 \mathrm{U} / \mathrm{L})$, and CPK $(566 \mathrm{U} /) \mathrm{L}$. He was treated supportively and required surgery for external ventricular drainage to control increased intracranial pressure. On day six, he became alert and was discharged on day 27 . We serially measured serum and urinary chromium concentrations and calculated serum half-lives were 35.7 hours and 25.1 hours, and urinary half-lives were 2.3 hours and 2.5 hours in case 1 and case 2, respectively. The precise cause of the accident was not determined; a drug screen was negative.

Conclusion: Following transdermal exposure to chromium (III) oxide for six hours, our patients developed agitation, hyperglycemia, leukocytosis, and elevated lactic acid, BUN, creatinine, liver enzymes, and CPK, but did not develop severe signs of chromium poisoning [1]. One patient, however, developed significant respiratory depression, which did not progress to respiratory failure. Serial chromium blood and urine concentrations in our patients differed from those reported following oral hexavalent chromium intoxication [2]. We suggest that transdermal exposure of trivalent chromium, especially to injured skin, may result in significant toxicity and morbidity.

\section{References}

[1] Barceloux DG. Chromium. J Toxicol-ClinToxicol. 1999;37:173-194.

[2] Kołaciński Z, Kostrzewski P, Kruszewska S, et al. Acute potassium dichromate poisoning: a toxicokinetic case study. J Toxicol Clin Toxicol. 1999;37:785-791. 


\section{Four incidents of mass occupational herbicide poisoning in Azerbaijan}

\author{
Ismayil Afandiyev \\ Azerbaijan Medical University, Baku, Azerbaijan
}

Objective: Herbicides are commonly ingested intentionally in suicide attempts, but relatively little has been published on occupational poisoning with herbicides. We report a case series of patients with mass acute occupational exposure to quizalofop-Pethyl herbicide in Azerbaijan.

Case series: Over a 10-day period in June 2018 we observed four incidents of mass pesticide poisoning in cotton field workers, involving 111 patients. The four incidents occurred on 11 June in Sabirabad and Saatly districts $(n=21), 17$ June in Terter district $(n=14), 19$ June in Imishli district $(n=52)$ and 20 June in Yevlakh district $(n=24)$. The main product involved in these incidents was "Formula Super 5 EC" (Turkey), containing quizalofopP-ethyl $50 \mathrm{~g} / \mathrm{L}$. Exposure to the herbicide occurred through inhalation and dermal contact. Just a day after the cotton fields had been treated with the herbicide the workers resumed their work in strong windy weather without individual protective equipment. The symptoms of exposure included nausea, vomiting, an epigastric burning sensation and dizziness. Quizalofop-p-ethyl was detected in urine samples of 24 patients by qualitative chemical toxicological analysis. Eight patients with moderate poisoning and reduced level of consciousness on admission (Glasgow Coma Score 9-13) were transferred to the Poison Center in the capital, Baku. All other patients had only minor symptoms of intoxication and received inpatient and outpatient care in regional hospitals. Only symptomatic and supportive care was required. Patients who vomited received a gastric lavage, antiemetics and intravenous saline. The median hospital stay was 1 day. No serious complications or fatal outcomes were recorded. All patients were discharged home in a normal condition.

Conclusion: Despite the relatively low toxicity of quizalofop-pethyl and the expectation that it will not volatilize from dry soil, it may cause clinical features of poisoning if workers are exposed soon after application in certain weather conditions.

\section{Workplace exposures in New Zealand: Enquiries to the poison centre over a five year period}

\section{Aleisha Jane King \\ New Zealand National Poisons Centre, University of Otago, Dunedin, New Zealand}

Objective: In New Zealand during 2017 there were 201 workplace injury claims per 1,000 equivalent full-time employees [1] A portion were related to exposures to various substances, which can lead to morbidity and productivity loss in workplaces. In April 2015 the New Zealand Government introduced the Health and Safety at Work Act (HSWA) to improve worker safety. This study investigated whether passage of the Act has been associated with a reduction of workplace exposure calls to the New Zealand National Poisons Centre (NZNPC), and the demographics surrounding these calls.

Methods: A retrospective analysis of unintentional human exposures in the workplace between 1 June 2013 and 31 May 2018. Data on type of exposure, gender, exposure route, caller's relationship to the patient, and disposition advice were extracted from the NZNPC database. An unpaired $t$-test was used to evaluate any differences in numbers of workplace exposure calls prior (June 2013-June 2015) and post (June 2016-June 2018) introduction of the HSWA.

Results: The NZNPC received 3811 calls regarding workplace exposures over the study period. Call numbers declined over the study period with 802 calls received in the first year of the study (June 2013-June 2014) and 626 calls received in the last year (June 2017-June 2018). Statistical analysis revealed there was a significant decrease in workplace exposures reported to the NZNPC following the legislation $(p=0.022)$, while there was no evidence of a statistically significant decrease in total human exposure poisoning calls over the study period. Most calls related to acute exposures (94.2\% acute, 5.8\% chronic). Males accounted for $67 \%$ of patients. The most common routes of exposure were inhalation (32.5\%), eye (24.9\%), skin (20.4\%), ingestion $(13.1 \%)$ and injection (7.12\%). A high proportion of calls were received from healthcare professionals (39.6\%) and the patient (36.4\%). Common treatment recommendations were medical referral $(59.8 \%)$, self-treatment $(20.9 \%)$ and no treatment required $(12 \%)$.

Conclusion: The introduction of the HSWA was associated with a statistically significant reduction in workplace exposure calls. Fewer calls were received annually in the last year of the study compared to the initial year. Eye and inhalational exposures were the most common routes of exposure, emphasising the importance of using adequate personal protective equipment on the job. Calls to NZNPC about workplace chemical exposures were more commonly made by healthcare professionals rather than the exposed workers.

\section{Reference}

[1] Statistics New Zealand. Injury Statistics - work related claims: 2017 [cited 2018 Oct 1]. Available from: https://www.stats.govt.nz/information-releases/injury-statistics-work-related-claims-2017

\section{Occupational exposure and contamination to antineoplastic drugs of healthcare professionals in Mauritania}

\author{
Antoine Villa ${ }^{a}$, Moustapha Mohamedou ${ }^{b}$, \\ Mathieu Molimardc, \\ Mohamed Sidatt Cheikh El Moustaph ${ }^{d}$ and \\ Mireille Canal Raffin ${ }^{\mathrm{e}}$ \\ ${ }^{a}$ Service de Santé au Travail, Hôpital la Timone, AP-HM, Marseille, \\ France; ${ }^{b}$ Centre National d'Oncologie, Nouakchott, Mauritania; \\ 'Laboratoire de Pharmacologie et de Toxicologie, Inserm U1219, \\ Université de Bordeaux, CHU de Bordeaux, Bordeaux, France; \\ ${ }^{d}$ Institut National pour la Recherche en Santé Publique,

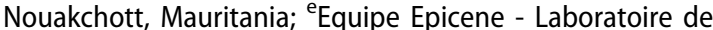 \\ Pharmacologie et de Toxicologie, Inserm U1219, Université de \\ Bordeaux, CHU de Bordeaux, Bordeaux, France
}

Objective: In Mauritania, the activity of the National Center of Oncology has steadily risen, leading to an increase in the handling of antineoplastic drugs (AD) by healthcare professionals. In this context, $A D$ contamination of those professionals is a major concern for occupational physicians and has been evaluated using biological monitoring of occupational exposure (BMOE).

Methods: The intervention took place in 2015, in two care units, and evaluated nurses preparing and/or infusing $A D$ and staff in charge of hygiene. Participants provided a single urine sample, at the end of the week, at the end of their shift. Five compounds were measured using specific high sensitivity methods (ultra-high 
performance liquid chromatography tandem mass spectrometry) with very low limits of quantification (LOQ) (cyclophosphamide, ifosfamide, methotrexate $2.5 \mathrm{ng} / \mathrm{L}$; doxorubicin $10 \mathrm{ng} / \mathrm{L}$ and a-fluoro- $\beta$-alanine [FBAL], an 5-FU metabolite) $20 \mathrm{ng} / \mathrm{L}$ ). A healthcare worker was considered "contaminated" when an AD was detected at a urine concentration equal or greater than the LOQ of the analytical method or at trace concentration.

Results: Twelve persons participated and 12 urine samples were collected and analyzed. The percentage of contamination was $66.6 \%$ for all participants $(n=8 / 12), 100 \%$ for nurses $(6 / 6)$ and $33 \%$ for agents in charge of hygiene $(2 / 6)$. In $62.5 \%(n=5 / 8)$ of the contaminated workers, two to four of the $A D$ were detected in the urine. Cyclophosphamide was found in the urine of all contaminated workers. FBAL was found in four, methotrexate in three and doxorubicin in one. Only ifosfamide was not detected. Urinary concentrations (all drugs combined) ranged from 3 to $844 \mathrm{ng} / \mathrm{L}$ for nurses and from 3 to $44 \mathrm{ng} / \mathrm{L}$ for staff in charge of hygiene. The median urinary concentrations were $87 \mathrm{ng} / \mathrm{L}, 15.1$ $\mathrm{ng} / \mathrm{L}$ and $4.4 \mathrm{ng} / \mathrm{L}$ for FBAL, cyclophosphamide and methotrexate, respectively. The doxorubicin urinary concentration found was $218 \mathrm{ng} / \mathrm{L}$.

Conclusion: There is no current biological exposure index for the interpretation of $A D$ contamination. The contamination of these healthcare professionals is therefore established by the detection of one or more $A D$ in urine. These urinary concentrations are higher than the LOQ of the analytical methods, which must be as low as possible. Given the danger of $A D$, the implementation of corrective measures is essential to protect staff. Biological monitoring of occupational exposure is the most reliable method to identify groups at risk, tracing insufficiently controlled exposures and as an alarm signal. These results show the necessity of educating professionals about the risks of handling $A D$ and how to reduce contamination when handling the drugs and caring for treated patients.

\section{Chronic methyl bromide toxicity treated with intermittent haemodialysis}

\section{Therese Becker, Betty S Chan and Angela L Chiew \\ Toxicology, Prince of Wales, Randwick, Australia}

Objective: We report a case of chronic methyl bromide toxicity treated with intermittent haemodialysis (IHD).

Case report: A 20-year-old male presented with subacute neurological symptoms. Two months prior he developed bilateral paresthesia of the lower limbs, multiple falls and erectile dysfunction. Two weeks prior to presentation his vision deteriorated. On examination he had reduced visual acuity with 6/36 left eye and 6/24 right eye. There was no disc swelling or pallor but there was extensive central visual field loss. He had normal tone, power and reflexes of the lower limbs with significant ataxia, no proprioception and symmetrical bilateral sensory loss below the knees with absent pain, vibration and temperature sensation. Lumbar puncture showed no organisms, oligoclonal bands, syphilis or viruses. Electroencephalogram and nerve conduction tests were normal. Visual evoked potentials showed significant delay bilaterally and somatosensory evoked potentials were absent on right lower limb and delayed on the left. Brain and spine magnetic resonance imaging (MRI) showed non-specific flair and T2 hyper intensities in the thalami medulla and periaqueductal grey matter suggestive of toxic encephalopathy. His history was revisited; he worked, often without protective equipment, as a fumigator using methyl bromide. Bromide concentrations were assessed in initial samples; plasma bromide concentration was $518.2 \mu \mathrm{mol} / \mathrm{L}$ (reference 37.5-150.2) and cerebrospinal fluid (CSF) $120.8 \mu \mathrm{mol} / \mathrm{L}$ (no defined reference range). His symptoms were attributed to chronic methyl bromide toxicity. IHD was trialed 9 days post-presentation. Prior to dialysis the half-life of bromide was 15.3 days. During the first session of dialysis it was 1.5 hours and in a second session 2.1 hours. He had 8 sessions of IHD at which time his plasma bromide concentration was $17.5 \mu \mathrm{mol} / \mathrm{L}$. The patient reported a subjective improvement in his visual symptoms after the first session. Ten weeks post-presentation his vision had improved enough for him to drive. Ataxia and sensory deficits in the lower limbs remained but were much improved.

Conclusion: Chronic methyl bromide exposure causes serious toxicity. There are case reports of IHD being utilised in acute methyl bromide toxicity $[1,2]$ and these demonstrated methyl bromide is dialyzable, however the impact on neurological recovery is not clear. There are no case reports of IHD in chronic exposure. In this case intermittent haemodialysis did remove methyl bromide with subjective improvement in neurological symptoms.

\section{References}

[1] Moosa MR, Jansen J, Edelstein CL. Treatment of methyl bromide poisoning with haemodialysis. Postgrad Med J. 1994;70:733-735.

[2] Yamano Y, Kagawa J, Ishizu S, et al. Three cases of acute methyl bromide poisoning in a seedling farm family. Indust Health 2001;39:353-353.

\section{Guttate psoriasis: a case of unusual evolution of dermal chemical burns}

\author{
Azzurra Schicchi ${ }^{\mathrm{a}}$, Marco Tresoldi ${ }^{\mathrm{b}}$, \\ Valeria M Petrolini ${ }^{\mathrm{a}}$, Marta Crevani $^{\mathrm{a}}$, Maria Perrone ${ }^{\mathrm{c}}$ \\ and Carlo A Locatelli ${ }^{\mathrm{a}}$ \\ ${ }^{\text {a }}$ avia Poison Control Centre - National Toxicology Information \\ Centre - Clinical and Experimental Lab, Toxicology Unit, Maugeri \\ Clinical and Scientific Institutes IRCCS and University of Pavia,

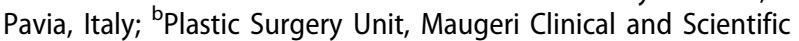 \\ Institutes IRCCS and University of Pavia, Pavia, Italy; ${ }^{\text {EEmergency }}$ \\ Department, IRCCS Policlinico San Matteo, Pavia, Italy
}

Objective: Chemical burns are a frequent problem in workplace accidents. Local lesions can be severe, and the risk of systemic effects depends on (i) toxic and kinetic characteristics of the substance involved, and (ii) the size of the burned area. We describe a case of chemical burns with unusual evolution.

Case report: A 36-year-old man was admitted to the emergency department (ED) following a workplace accident due to failure of a hydraulic system. A jet of caustic substances (hydrochloric acid and hydrofluoric acid 10-20\%) injured the patient. At the workplace, dermal decontamination was performed, and calcium topical preparation was applied. At ED admission, he presented multiple first and second-degree skin burns on his frontal region, scalp, right forearm, and earlobe. Cardiac monitoring and measurement of ionized calcium blood concentration remained normal. Calcium gel was applied in the affected area at ED, and then continued for a week. Successive plastic surgery management consisted of topical dressings with silver sulfadiazine and paraffin gauze dressing twice a week for one month. When the skin lesions were healed, a topical silastic gel and sunscreen (SPF $50+$ ) were applied daily to improve the aesthetic outcome. Two days after the start of this treatment, in the same location of the scars from the previous burns, the patient developed an unusual generalized skin reaction with itchy erythema. Topical product application was immediately suspended without any change. 
Antihistamines were used as symptomatic treatment for five days. The allergic reaction to the topical products was excluded and the personal and family history was negative for autoimmune and genetic skin pathologies. In a few days, the skin reaction with itchy erythema had evolved to a generalized guttate psoriasis. The patient was admitted to the Dermatologic Unit for appropriate treatment. His clinical condition improved in about a month, but the lesions did not disappear completely.

Conclusion: Guttate psoriasis is not described as a possible evolution of chemical burns. No direct correlation with a specific product is hypothesized. In the absence of family history positive for autoimmune and genetic skin pathologies, the local inflammatory reaction and immune activation due to chemical burns may have been the trigger factor for the development of guttate psoriasis.

\section{Poisoning by oral exposure in the work environment}

\author{
Nina Glaser ${ }^{a}$, Kathrin Begemann ${ }^{b}$, Esther Feistkorn ${ }^{b}$ \\ and Herbert Desel ${ }^{b}$ \\ ${ }^{a}$ Scientific Service, German Federal Institute for Risk Assessment, \\ Berlin, Germany; ${ }^{\text {b}}$ German Federal Institute for Risk Assessment, \\ Berlin, Germany
}

Objective: In general, it is believed that oral intoxications do not play a major role in the work environment and therefore no detailed risk assessment is necessary. However, in the past 12 years, the Poisoning Documentation Centre of the German Federal Institute for Risk Assessment (BfR) has received cases of oral poisoning at work, some of them with severe symptoms. In this analysis the circumstances of oral exposure at work were examined.

Methods: In Germany physicians report cases of poisoning to the BfR on a legal basis ( $\S 16$ e Chemicals Law). For intoxications in the work environment the statutory accident insurance institutions are obliged to notify these cases to BfR. All cases with oral exposure received by the statutory accident insurance institutions between 2005 and 2017 were analysed for the circumstances of exposure and the clinical course. Frequently involved product groups were identified.

Results: Overall 498 cases with oral route of exposure were identified. In 310 of them, oral exposure was the single route, whereas in 188 cases more than one route was registered. In cases with more than one route, the scenario was usually a spillage/splash in the face, whereas the cases with oral exposure only showed a broad spectrum of assignable causes. The most frequent circumstances were e.g. pipetting/suctioning with the mouth, mistaking bottles, transferring chemicals to beverage bottles or forgetting the presence of a descaling agent in kettles. Product categories frequently involved in oral ingestion were special purpose cleaners (e.g. descaling agent) and vehicle and machine maintenance products (e.g. petrol). The percentage of cases with moderate or severe poisoning was $4.2 \%$. Most of them showed local irritation/chemical burns of gastrointestinal tract (61\%). Nausea/vomiting were reported in $43 \%$ of cases with moderate/severe oral poisoning and breathing difficulties/dyspnoea (e.g. due to aspiration) were present in $14 \%$ of cases. Over a fifth of patients (23\%) showed signs of systemic toxicity.

Conclusion: The documentation of poisonings in the BfR database allows the identification of critical exposure scenarios. Regarding oral ingestion of chemical products at work, mistaking bottles, malpractice and accidental scenarios could be differentiated. The analysis of assignable causes facilitates the implementation of improved safety measures.

\section{Tool development for assessing antineoplastic drugs surface contamination in healthcare services and other workplaces}

\author{
Benoît Atgéa ${ }^{a}$ Oscar Da Silva Caao ${ }^{b}$, \\ Dominique Ducint ${ }^{c}$, Catherine Verdun-Esquer ${ }^{d}$, \\ Isabelle Baldi ${ }^{e}$, Mathieu Molimard ${ }^{f}$, Antoine Villa ${ }^{g}$ and \\ Mireille Canal Raffin ${ }^{\mathrm{b}}$ \\ ${ }^{a} A H I 33$, Association d'Hygiène Industrielle, Service de Santé au \\ Travail, Bordeaux, France; ${ }^{b}$ Equipe Epicene - Laboratoire de \\ Pharmacologie et de Toxicologie, Inserm U1219, Université de \\ Bordeaux, CHU de Bordeaux, Bordeaux, France; 'Laboratoire de \\ Pharmacologie et de Toxicologie, CHU de Bordeaux, Bordeaux, \\ France; ${ }^{\text {d}}$ Service de Santé au Travail, CHU de Bordeaux, Bordeaux, \\ France; ${ }^{e}$ Equipe Epicene - Service de Santé au Travail, Inserm \\ U1219, Université de Bordeaux, CHU de Bordeaux, Bordeaux, \\ France; ${ }^{f}$ Laboratoire de Pharmacologie et de Toxicologie, Inserm \\ U1219, Université de Bordeaux, CHU de Bordeaux, Bordeaux, \\ France; ${ }^{9}$ Service de Santé au Travail, Hôpital la Timone, AP-HM, \\ Marseille, France
}

Objective: Healthcare worker exposure to antineoplastic drugs is a burning issue for occupational medicine practitioners. Biological monitoring of occupational exposure (BMOE) is an essential tool for assessing antineoplastic drug contamination of healthcare workers. In addition to BMOE, surface sampling is a useful tool in order to understand how workers become contaminated, identify sources of environmental contamination, verify the effectiveness of surface decontamination and to ensure monitoring of these surfaces. The objective of this work was to develop a complete tool including a kit for surface sampling and a quantification analytical method for antineoplastic drug trace detection. The development was realized with the three following criteria: the kit capacity to sample in every professional environment (healthcare services, veterinary practices, etc), the detection of very low antineoplastic drug traces with a validated analytical method and the ease of the sampling kit use regardless of the person performing the sampling.

Methods: Antineoplastic drugs used most, in terms of quantity and frequency, were identified by analysis of the literature and consumption in different hospitals, veterinary services and home care settings. The kind of adsorbent device, surface moistening solution and mix of solvents for the extraction of antineoplastic drugs from the adsorbent device have been tested for maximal yield. Antineoplastic drug quantification was achieved by an ultra-high performance liquid chromatography method coupled with tandem mass spectrometry (UHPLC-MS/MS).

Results: With their high frequency of use across healthcare systems, 15 antineoplastic drugs (cyclophosphamide, ifosfamide, doxorubicin, daunorubicin, epirubicin, 5-fluorouracil, dacarbazin, etoposide, pemetrexed, vincristine, cytarabine, methothrexate, paclitaxel, gemcitabine, mitomycin C) were selected. The analytical method was optimized and adapted to obtain high sensitivity with very low limits of quantification ( 25 to $5000 \mathrm{ng} / \mathrm{L}$ ), equivalent or lower that those previously published (for 13/15 antineoplastic drugs). The sampling kit is easy to use, provided with support material (online video and paper protocol). There was no inter-individual variation $(n=5 /$ person; $n=5$ persons; $p=0.85$; ANOVA) regardless of the person performing the sampling.

Conclusion: This validated tool (sampling kit + analytical method) is very sensitive, easy to use and very didactic in order to control the risk of exposure to antineoplastic drugs. Moreover BMOE 
allows a focal prevention. Used routinely, this tool is available for every intervention of occupational health.

\section{Detection of occupational exposure to nanoparticles in human biological fluids by transmission electron microscopy for the assessment of chronic toxic effects}

\author{
Lucie Lischkova ${ }^{a}$, Daniela Pelclova ${ }^{a}$, Jiri Hlusicka ${ }^{a}$, \\ Marian Regenda ${ }^{b}$, Tomas Navratil $^{a}$, Alena Michalcova $^{c}$ \\ and Sergey Zakharov ${ }^{\mathrm{a}}$ \\ ${ }^{a}$ Department of Occupational Medicine and Toxicological \\ Information Centre, 1st Faculty of Medicine, Charles University, \\ General University Hospital, Prague, Czech Republic; ${ }^{\mathrm{b}}$ Department \\ of Occupational Medicine and Toxicological Information Centre, \\ General University Hospital, Prague, Czech Republic; 'Department \\ of Metals and Corrosion Engineering, University of Chemistry and \\ Technology in Prague, Prague, Czech Republic
}

Objective: The application of new nanomaterials in industry has been growing vastly during recent decades, but the health effects of nanoparticles on exposed employees are still unclear. No standard methods for the routine monitoring of exposure of workers to engineered nanoparticles are available. The intention of occupational physicians is directed to the early detection and evaluation of potential health risks and hazards, as well as the detection of suitable biomarkers of exposure in biological fluids for timely diagnostics and prevention of respiratory and/or systematic diseases. The aim of the study was to detect and evaluate the amount of nanoparticles in biological samples of the subjects occupationally exposed during the processing of different nanomaterials and to determine its association with the results of external workplace aerosol measurements.

Methods: The cohort of the workers occupationally exposed to nanoparticles consisted of two groups: the first group was exposed mainly to nanoparticles containing iron and manganese, the second group was exposed to nanoparticles containing copper oxide. The patients were interviewed by trained physicians and physically examined. The spirometry and fractional exhaled nitric oxide (FeNO) were measured and post-shift samples of urine, blood serum, and exhaled breath condensate (EBC) were collected and analyzed. Transmission electron microscope (TEM) analysis of biological samples was performed using Jeol 2200FS microscope. The images were taken by zero loss electrons with slit with $20 \mathrm{eV}$. Chemical composition analysis was obtained in scanning transmission electron microscopy (STEM) mode by energy dispersive (EDS) detector with a $1 \mathrm{~nm}$ electron beam. The TEM samples were prepared by dropping $7 \mu \mathrm{L}$ of study solution on holey carbon 300 mesh grid made from $\mathrm{Cu}$ or $\mathrm{Ni}$ (for $\mathrm{Cu}$-containing samples).

Results: Altogether 15 samples (urine, serum, and exhaled breath condensate) were collected from 5 occupationally exposed subjects. The size of detected particles in biological fluids was in the range from below $100 \mathrm{~nm}$ to $2500 \mathrm{~nm}$. Most detected particles were bigger than $100 \mathrm{~nm}$. The most common chemical elements in the spectrum of the particles from EBC were carbon, copper, calcium, chlorine, and potassium. In the urine and serum, the spectrum of the nanoparticles contained mainly carbon and copper.

Conclusion: Our results demonstrate a possibility of detection of occupational exposure to the particles in the biological fluids, blood serum, urine, and exhaled breath condensate.

\section{Jimsonweed meets Hungarian goulash - case report of a mass intoxication}

\author{
Dóra O Jakab and István Elek \\ Emergency Department and Clinical Toxicology, Péterfy Hospital \\ and National Traumatology Centre, Budapest, Hungary
}

Objective: Jimsonweed (Datura stramonium, aka Devil's snare) is a plant in the nightshade family. It contains tropane alkaloids, atropine, scopolamine and hyoscyamine. It is highly toxic and can cause anticholinergic symptoms when ingested. This case study presents a mass intoxication which happened in our hospital in April 2018.

Case report: On two consecutive nights, a total of 18 patients were admitted with similar anticholinergic symptoms. They all had an identical history: all of them had traditional Hungarian goulash for dinner at the same downtown restaurant and 30 minutes after supper they started feeling strange. Fourteen patients arrived in hospital almost at the same time. Seven of them presented severe symptoms: pronounced hallucinations, agitation, tachycardia, mydriasis, and dry, warm skin (Poison Severity Score [PSS] 2). They required multiple doses of sedatives and physostigmine, along with parenteral fluid and supportive therapy. Their laboratory results were normal. The other seven patients showed minor symptoms; they were oriented, not agitated (PSS 1) and reported dry mouth, dizziness and blurred vision. Their intoxication did not require sedatives or antidote. With only supportive therapy their intoxication subsided. Four additional patients arrived the next day; their symptoms were minor and in remission, and they did not need hospitalization. All of the patients, who were admitted and treated were discharged on the following day completely recovered, there were no complications. Urine samples were obtained from 12 patients. Our toxicology laboratory identified atropine and scopolamine with high performance liquid chromatography (HPLC) from 10 samples. Based on the clinical symptoms and the toxicology laboratory results there was a high possibility of contamination with an atropine-scopolamine containing plant in the restaurant. Later the National Food Chain Safety Office identified jimsonweed mixed in the ground cumin used in the goulash.

Conclusion: We rarely see severe mass plant intoxications in our ward. Based on the history and the anticholinergic symptoms, it is important to think about the possibility of food contamination. The indication of the antidote depends on the patient's overall clinical condition and the severity of their delirium. If the intoxication is recognized and treated in time, the prognosis is good with low risk of complications.

\section{No, this is not wild garlic... and it can kill you! A case of Colchicum autumnale poisoning involving the Danish Poison Information Center and Authorities}

\author{
Paula EC Hammer, Ellen B Pedersen and \\ Niels E Ebbehoej \\ Danish Poison Information Center, Bispebjerg and Frederiksberg \\ University Hospital, Copenhagen, Denmark
}

Objective: We report a case of severe poisoning with autumn crocus (Colchicum autumnale) illustrating an effective collaboration between the Danish Poison Information Center (DPIC), a 
Police Department and the Danish Emergency Management Agency.

Case report: A 53-year-old woman was admitted at a local hospital 16 hours after ingestion of around 10 leaves of what she believed was wild garlic (Allium ursinum) harvested from her own garden. She had abdominal and chest pain, and severe vomiting and diarrhea. At the first contact with the DPIC ingestion of lily of the valley (Convallaria majalis) was suspected. Within 24 hours after ingestion her clinical condition worsened with deterioration of hepatic and renal function. On the second discusson with DPIC, poisoning with Colchicum autumnale and/or Amanita species mushroom was suspected. The patient was confused and could not give precise information on whether she ingested mushrooms, nor whether others had ingested the same meal. However, it was known that she had leftovers from the ingested meal at home. Besides advising on risk assessment and treatment of poisoning, the DPIC offers assistance on identifying plants and mushrooms when this is relevant. Considering the potential risk of fatal poisoning of other individuals and to allow for adequate treatment the DPIC contacted the local police department requesting an investigation of the patient's house. The police found a bowl with the patient's vomit and several plants and mushrooms in her kitchen. Pictures of the mushrooms sent to a mycologist could not rule out the presence of Amanita species. Within three hours, the Chemical Division of the Danish Emergency Management Agency confirmed the presence of colchicine both in the leftovers and in the patient's stomach's content. She developed bone marrow suppression peaking on the seventh day, and had paralytic ileus for 10 days. Treatment was supportive intensive care and she was discharged 16 days after ingestion.

Conclusion: The recent increase in immigration across Europe poses higher risks of poisoning due to diverse culinary and medicinal use of plants and mushrooms. Often local poisonous species are mistaken with domestic edible species. Colchicum autumnale poisoning causes high mortality due to multi-organ failure. Adequate risk assessment and effective treatment guidance is challenged when ingestion is uncertain. Timely collaboration between PIC and authorities in such cases might be relevant and efficient, and can contribute to preventive measures, such as disseminating information on local poisonous plants and mushrooms.

\section{Anticholinergic toxicity associated with lupini beans in Europe: two case reports}

\author{
Corinne Schmitt ${ }^{a}$, Romain Torrents ${ }^{a, b}$, \\ Bastien Domangéa ${ }^{a}$ Luc De Haro ${ }^{a}$ and \\ Nicolas Simon ${ }^{a, b}$ \\ ${ }^{a}$ Clinical Pharmacology, Poison Control Center, St Marguerite

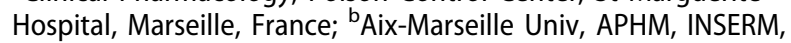 \\ IRD, SESSTIM, Marseille, France
}

Objective: All lupin species contain highly variable concentrations of quinolizidine alkaloids such as sparteine and lupanine, responsible for the bitter taste of the plant and the anticholinergic properties. Usually, the different species are divided into two groups "bitter lupins" and "sweet lupins". Bitter lupini (lupin) beans are commonly eaten as an appetizer in Southern Europe but poisoning cases are reported mainly in the US and Australia. In order to be safely consumed, bitter lupini beans need to undergo an elaborate series of steps to remove the alkaloids they contain. All these steps last for hours and it takes about 4 days before the beans are ready for consumption [1]. We describe two cases of poisoning reported to the Poison Control Center in Marseille.

Case reports: Case 1. A previously healthy 56-year-old woman of Italian origin, ate two handfuls of lupini beans she bought on the market in Italy early in the morning. One hour later she suffered from nausea, vomiting, dizziness, blurred vision and dry mouth. About 7 hours after ingestion, she consulted the nearest emergency department because her symptoms persisted. On admission she was alert, heart rate was $71 \mathrm{bpm}$, and cardiovascular and pulmonary examination was unremarkable. She admited the beans had been insufficiently prepared. She was observed and treated with oral hydration and returned home that evening. Case 2. A 38-year-old man, with no particular medical history, inadvertently ingested the cooking water of lupini beans at midday. About three hours later, he suffered from vomiting, dizziness, severe dry mouth, blurred vision and urinary retention. As the symptoms persisted at the end of the day, he presented to the emergency department. Heart rate and blood pressure were unremarkable. Cardiovascular, respiratory and neurological examinations were normal, and laboratory tests were all within the normal range, however he was anuretic. He was treated with intravenous fluids and a urinary catheter was placed to drain urine. He was monitored overnight, all the symptoms settled by the morning, the catheter was removed and he was discharged.

Conclusion: Hopefully, human poisoning is infrequent but these two cases show that, even with accidental poisoning, the patient's clinical effects may require medical care in an emergency department.

\section{Reference}

[1] Li K, van Wijk XMR, Hayashi S, et al. Anticholinergic toxicity associated with ingestion of water containing lupini bean extract. Clin Toxicol (Phila). 2017;55:687-688.

\section{Ingestion of raw Aleurites moluccana seeds: experience of the Austrian Poisons Information Centre}

\author{
Kinga Bartecka-Mino, Helmut Schiel and Tara Arif \\ Poisons Information Centre Austria, Vienna, Austria
}

Objective: The plant Aleurites moluccana is a member of the family Euphorbiaceae and is native to Southeast Asia. All parts of the plant are toxic, especially the raw seeds as they contain phorbols and saponins as toxins. The seeds are used cooked in Asian cuisine as well as in the traditional herbal medicine for various medical issues including obstipation and dermatologic conditions. In Austria the seeds can be bought in Asian shops or via the Internet.

Methods: A retrospective and descriptive review of enquiries to the Austrian Poisons Information Centre (PIC) concerning ingestion of Aleurites moluccana from 2014 to July 2018 was conducted. The extracted data include age cohort, ingested amount and symptoms.

Results: In total 13 adult patients called the PIC after eating 0.5 to 20 raw seeds. All of them were unaware of the correct way to use the seeds. Those who called within 15 minutes to 1 hour after ingestion $(n=5)$ had no symptoms at the time of the PIC consultation. Eight patients, who called after 1.5 hours, had the following symptoms: nausea $(n=8)$, vomiting $(n=4)$, diarrhoea $(n=5)$, abdominal cramps (2), sore throat $(n=1)$, vertigo $(n=3)$, and headache $(n=2)$. The duration of symptoms was up to 24 
hours. One of the patients was a 61-year-old male who developed moderate symptoms after eating 20 raw seeds. He became ill one hour after consumption with nausea and he vomited twice. He called the PIC after 13 hours complaining of diarrhoea every 20 to 60 minutes. He also developed headache, vertigo and painful abdominal cramps. He did not follow our advice to visit the hospital, but he recovered after 24 hours.

Conclusion: All of the 13 patients had eaten the seeds raw, despite the fact that they should be cooked before eating. Even the ingestion of only half a raw seed can cause symptoms. The severity of gastrointestinal symptoms depends on the amount of seeds ingested and signs can persist for up to 24 hours. Hospitalisation may be necessary.

\section{Evaluation of enquiries regarding Atropa belladonna ingestion to the Austrian Poisons Information Centre}

\author{
Tara Arif, Helmut Schiel and Kinga Bartecka-Mino \\ Poisons Information Centre Austria, Vienna, Austria
}

Objective: All parts of the plant Atropa belladonna (deadly nightshade) contain tropane alkaloids mainly atropine, hyoscyamine, and scopolamine. The highest concentration can be found in the leaves (up to $1.5 \%$ ) and the roots (0.85\%). The aim of this study was to determine what quantity of Atropa belladonna that could be expected to lead to symptoms.

Methods: The Poisons Information Centre (PIC) in Austria retrospectively evaluated enquiries regarding ingestion of Atropa belladonna from 2002 to 2017. The extracted data included age cohort, ingested amount and symptoms.

Results: Overall 176 cases of oral mono-intoxications with Atropa belladonna were extracted from the database. There were 82 children aged 13 months to 14 years. Ingestion of the berries was accidental in most cases $(n=79)$ and most commonly occurred outdoors. At the time of PIC consultation 71 out of 82 children were asymptomatic, 11 had anticholinergic symptoms. After ingestion of only one berry, 4 children developed minor anticholinergic symptoms (flushing, hoarseness, mydriasis, vomiting, and sinus tachycardia). Three patients ingested 2 to 4 berries and developed minor anticholinergic symptoms (mild visual disturbances, mydriasis, and sinus tachycardia). A 10-year-old child ingested up to 30 berries and had moderate symptoms (agitation, confusion, hallucinations, and sinus tachycardia). Three children ate an unknown amount of berries; one developed only mydriasis and two moderate anticholinergic symptoms with hallucination, confusion and agitation. Adults ( $>15$ years old) were involved in 94 cases. Three ingested less than one berry and were asymptomatic. Thirty-one adults developed minor symptoms after ingestion of 1-4 berries with mydriasis, mild visual disturbances, vertigo, headache, nausea, vomiting, xerostomia, dry skin, and mild sinus tachycardia. Forty-two adults ingested 5-30 berries and developed minor effects and also hallucination, agitation, convulsions, ataxia, delirium, somnolence, hyperthermia, hypertonia, and urinary retention. Sixteen adults ingested an unknown amount and developed minor to moderate anticholinergic symptoms. Two patients ate the root of Atropa belladonna in mistake for horseradish. They developed symptoms two hours later with flushing, confusion, vertigo, agitation, hallucination, somnolence, sinus tachycardia, and hypertonia. They required admission to an intensive care unit and received activated charcoal, benzodiazepines, and physostigmine, with recovery after 30 hours. There were no cases with severe symptoms.
Conclusion: In paediatric patients only mild symptoms can be expected after the ingestion of one berry of Atropa belladonna and these children can be observed at home. Adult patients should be hospitalised after the ingestion of more than 4 berries.

\section{Evaluation of enquiries regarding Brugmansia suaveolens exposures to the Austrian Poisons Information} Centre

\author{
Tara Arif, Dieter Genser and Kinga Bartecka-Mino \\ Poisons Information Centre Austria, Vienna, Austria
}

Objective: All parts of Brugmansia suaveolens (previously Datura suaveolens) contain tropane alkaloids $(0.25 \%$ to $0.7 \%)$, especially scopolamine (up to $75 \%$ ), L-hyoscyamine, and atropine. The plant is sometimes used for its hallucinogenic effects. The aim of this study was to evaluate enquiries regarding Brugmansia suaveolens exposures.

Methods: A retrospective review of enquiries to the Austrian Poisons Information Centre concerning Brugmansia suaveolens from 2002 to 2017 was conducted. Data extracted included age cohort, route of exposure, symptoms and poisoning severity score (PSS).

Results: Overall 245 cases were extracted from the database. Of these 110 enquiries involved children ( 6 months- 14 years) and most were oral exposures $(n=84)$. Symptoms occurred in 12 children. Minor symptoms occurred in 9 cases (nausea, vomiting, diarrhoea, hyperthermia, mydriasis, drowsiness) and moderate symptoms (confusion, hallucinations) in three patients. All 9 cases of ocular exposure developed mydriasis (PSS 1). In 2 of 17 cases of dermal exposures mild symptoms (hyperthermia, nausea) occurred, however, the causality in these cases was unclear. There were 135 enquiries regarding adults (>15 years). After excluding nine patients due to the additional intake of other substances, 126 cases were evaluated. Of 85 cases of oral exposures, symptoms occurred in 75 cases, of which 66 patients ingested Brugmansia suaveolens for its hallucinogenic effects. In 12 cases symptoms were mild with mydriasis, nausea, xerostomia, gastralgia, drowsiness, somnolence, vertigo, dyspnoea, and muscle fasciculation. In 60 patients moderate symptoms occurred with mydriasis, xerostomia, nausea, vomiting, sinus tachycardia, hypertension, facial paresis, confusion, agitation, hallucination, drowsiness, somnolence, coma responsive to pain, muscle fasciculation, infrequent seizures and delirium. Three patients had severe symptoms with mydriasis, fixed pupils, sinus tachycardia, hypertension, confusion, deep coma unresponsive to pain and frequent generalized seizures. All adult patients with ocular exposure $(n=23)$ developed mydriasis (PSS 1). In 5 of 12 patients with dermal exposure minor symptoms occurred with hyperthermia, vomiting, diarrhoea and skin lesions; the causality in these cases was unclear. In two cases parts of Brugmansia suaveolens were inhaled causing hallucinations (PSS 2). In four patients the route of exposure was unclear; two had moderate symptoms (mydriasis, confusion, hallucinations).

Conclusion: Overall there were 236 single-agent exposures to Brugmansia suaveolens. Symptoms occurred in 23 (21\%) of 110 paediatric and 107 (85\%) of 126 adult patients. Symptoms were minor in 60 patients (46\%), moderate in 67 patients (52\%) and severe in 3 cases (2\%). In adults exposure to Brugmansia suaveolens was most commonly intentional for its hallucinogenic effects. 


\section{A 16-year review of enquiries regarding Myristica fragrans ingestion to the Austrian Poisons Information Centre (PIC)}

\author{
Kinga Bartecka-Mino, Dieter Genser and Tara Arif \\ Poisons Information Centre Austria, Vienna, Austria
}

Objective: The dried seed of Myristica fragrans is used as a spice (nutmeg), as a folk treatment for dyspepsia, musculoskeletal disorders, and arthritis, and as a substance of abuse for its hallucinogenic effects. Some organic shops in Austria sell so-called "nervecookies" with high amounts of nutmeg, made according to a traditional recipe from a German Benedictine abbess.

Methods: A retrospective review of enquiries to the Austrian PIC concerning oral Myristica fragrans exposures from 2002 to 2017 was conducted. Data extracted included age cohort, circumstance and poisoning severity score (PSS).

Results: Overall there were 105 enquiries regarding Myristica fragrans ingestion. In the paediatric group there were 42 children ( 9 months to 14 years); nine (21\%) children developed symptoms. The nutmeg was ingested mainly accidentally $(n=36)$; five children had minor symptoms (nausea, vomiting, restlessness, drowsiness). It was used intentionally in 4 cases (12 to 13 years); one had minor (nausea, vertigo) and two had moderate symptoms (drowsiness, confusion, agitation). In two cases the reason for intake was unclear; one child had nausea and headache (PSS 1). There were 63 enquiries regarding adults (age $>15$ years). After excluding 11 cases due to the additional intake of other substances, 52 cases were evaluated, of which 28 patients (54\%) developed symptoms. In 14 cases nutmeg was ingested for its hallucinogenic effects; four had minor symptoms (nausea, vomiting, diarrhoea, restlessness, vertigo, somnolence, sinus tachycardia), three were also agitated (PSS 2). After accidental intake 5 out of 14 patients had nausea, headache, and mild hypertension (PSS 1). Ten patients developed adverse effects after taking nutmeg as a folk treatment, as "nerve-cookies" or in mistake for another spice. All patients had symptoms. Six had minor effects (xerostomia, nausea, vomiting, hyperhidrosis, vertigo, and headache) and four had moderate symptoms (the minor effects and confusion, agitation, paraesthesia, hallucination). In 8 patients nutmeg was ingested in a suicide attempt; one patient had mild sinus tachycardia (PSS 1), and three had moderate symptoms with nausea, vomiting, mydriasis, hypertonia, sinus tachycardia, headache, muscle fasciculation, drowsiness, and confusion. Two of 6 patients with an unknown reason for ingestion developed mild symptoms (gastralgia, headache, visual disturbances and somnolence).

Conclusion: There were 94 enquiries regarding ingestion of nutmeg only. Symptoms occurred in 9 (21\%) of 42 children and 28 (54\%) of 52 adults. Minor symptoms were seen in 25 patients, mainly following accidental ingestion, and moderate symptoms in 12 patients. There were no severe cases.

\section{Ingestion of Veratrum album due to confusion with gentian}

\section{Tara Arif, Ursula Schreiner and Kinga Bartecka-Mino Poisons Information Centre Austria, Vienna, Austria}

Objective: In Austria gentian (Gentiana lutea) is protected under the Nature Conservation Act. Nevertheless, people collect the roots to make schnapps and use as medicinal herbs. In the nonflowering condition Veratrum album can be mistaken for yellow gentian. All parts of Veratrum album contain toxic alkaloids (protoveratrin A, B, and jervin). We evaluated enquiries to the Austrian Poisons Information Centre (PIC) regarding ingestion of Veratrum album.

Methods: We retrospectively evaluated enquiries regarding ingestion of Veratrum album from 2013 to July 2018. The data collected included age cohort, ingested amount, symptoms and treatment.

Results: Overall there were 10 cases. Two children ingested a very small amount of the plant. Only one child developed hypersalivation and had an "odd feeling" in the mouth. Five of the eight adults ate roots, two drank schnapps and one ate leaves. Three adults ingested a thumbnail-sized piece of raw root and immediately developed nausea and prolonged vomiting. A 73year-old female also developed vertigo, blurred vision, diplopia, hypotension, bradycardia, and cold sensation for 3 hours. A 69year-old male additionally developed diaphoresis, diarrhoea, retrosternal pain, paraesthesia in fingers up to the thoracic area, hypotension, bradycardia $(40 / \mathrm{min})$ and repolarisation abnormalities. Both patients were treated supportively and received atropine. Duration of symptoms was 20 hours. The third patient also experienced dizziness and light-headedness, but the further course is not known. Two patients ate an unknown amount of the root. One was a 56-year-old psychiatric patient who developed pronounced vomiting, weakness, bradycardia (40/min), diaphoresis, salivation, agitation and tremor for 8 hours. Therapy included atropine, a benzodiazepine, and ondansetron. The second patient, a 57-year-old female, developed nausea and vomiting, but the further course is unknown. Two patients consumed 1-2 centilitres of homemade alcoholic beverages containing Veratrum album. Both of them developed nausea and pronounced and prolonged vomiting. The 56-year-old woman also developed dyspnoea, bradycardia $(42 / \mathrm{min})$ and hypotension for 10 hours. The 74-year-old man also suffered from mydriasis, blurred vision, hypotension and various electrocardiogram changes (fluctuating AV conduction disturbances). Both patients recovered after supportive treatment. A 41-year-old woman ate leaves and developed vomiting, hypotension and bradycardia (45/min). She was treated symptomatically and recovered after 12 hours.

Conclusion: Veratrum album ingestion has to be taken seriously. Even a thumbnail-sized piece of root or one centilitre of homemade alcoholic beverage can lead to moderate symptoms. Observation in an intensive care unit is always necessary. Symptoms can occur after 30 minutes and persist for up to 20 hours.

\section{A one-year survey of self- collected Allium ursinum ingestion of the Austrian Poisons Information Centre}

\author{
Susanna Dorner-Schulmeister, Sandra Hruby, \\ Kinga Bartecka-Mino, Dieter Genser, Helmut Schiel \\ and Tara Arif \\ Poisons Information Centre Austria, Vienna, Austria
}

Objective: Allium ursinum (wild garlic) is often used in Austrian cuisine and many people collect it in the springtime, however, it can be mistaken with plants such as Convallaria majalis, Colchicum autumnale and Arum maculatum. The aim of the study was to determine the severity of symptoms after the ingestion of self-collected wild garlic with the help of telephone follow-up.

Methods: The Poisons Information Centre (PIC) Austria conducted a prospective study of self-collected Allium ursinum in 2015. Telephone follow-up was conducted at least 24 hours after 
the ingestion. The extracted data include age cohort, ingested amount, symptoms, hospitalisation and therapy.

Results: Overall, there were 165 enquiries ( 25 children, 140 adults) regarding Allium ursinum ingestions. Telephone follow-up was performed in 100 cases, in which self-collected wild garlic was ingested. Twenty-six persons were asymptomatic and in 74 cases symptoms occurred. Sixty-four persons developed mild symptoms with nausea $(n=37)$, vomiting $(n=29)$, diarrhoea $(n=23)$, burning sensation in the mouth $(n=16)$, abdominal pain $(n=12)$, vertigo $(n=7)$, and blisters in the mouth $(n=1)$. In 9 cases the symptoms were moderate including nausea, pronounced and prolonged vomiting and diarrhoea up to 12 times. Seventeen patients were hospitalised. They were treated symptomatically and in 6 cases activated charcoal was administered. There was only one case with severe symptoms. A couple had collected wild garlic in the wood. One hour after eating the raw leaves both of them developed pronounced vomiting. The PIC recommended hospital admission. The 59-year-old female patient had no further symptoms but the 61-year-old male presented vertigo, somnolence, bradycardia and he collapsed. Atropine administration increased his heart rate from 30 to 40 beats per minute. When the PIC was contacted again intoxication with lily of the valley (Convallaria majalis) was suspected. Digoxin specific antibody fragments were recommended and given for eight hours (total dose $400 \mathrm{mg}$ ). The duration of symptoms was 12 hours. The couple were discharged in good clinical condition after three days.

Conclusion: In this one-year survey, 64 out of 100 persons had mild and nine had moderate gastrointestinal symptoms after eating self-collected Allium ursinum. Two persons mistook wild garlic for lily of the valley, leading to severe symptoms in one person, who was treated with digoxin specific antibody fragments. Hospitalisation has to be recommended for people with moderate symptoms, since wild garlic can be mistaken for poisonous plants.

\section{Yew needles poisoning: a case report}

\author{
Mojca Skafar ${ }^{\mathrm{a}}$, Tomaž Goslar ${ }^{\mathrm{a}}$, Damjan Grenc ${ }^{\mathrm{b}}$, \\ Gordana Koželj ${ }^{\mathrm{c}}$ and Miran Brvar ${ }^{\mathrm{b}}$ \\ ${ }^{a}$ Centre for Intensive Internal Medicine, University Medical Centre \\ Ljubljana, Ljubljana, Slovenia; ${ }^{b}$ Centre for Clinical Toxicology and \\ Pharmacology, University Medical Centre Ljubljana, Ljubljana, \\ Slovenia; ' Institute of Forensic Medicine, Medical Faculty, \\ University of Ljubljana, Ljubljana, Slovenia
}

Objective: Taxines are poisonous constituents in yew (Taxus spp.). Their main action is on cardiac myocytes, affecting sodium/ calcium permeability in cells, resulting in heart failure and death in animal and human poisonings [1]. We report an intentional ingestion of yew needles with life-threatening arrhythmias.

Case report: A 17-year-old male intentionally ingested two handfuls of yew needles. Three hours later, he started complaining of weakness, nausea and chest pain. Upon arrival of the emergency team he was tachycardic and hypotensive. A sustained ventricular tachycardia 200 beats/minute shifted to ventricular fibrillation and successful defibrillation with $200 \mathrm{~J}$ was performed. At the Emergency Department, he was conscious and normotensive with wide QRS complex tachycardia. Sodium bicarbonate $(100 \mathrm{~mL}$ of $1 \mathrm{M})$ had no effect on QRS width. On arrival at the intensive care unit he developed a sustained ventricular tachycardia that changed to bradycardia with wide QRS complexes without pulse and 1 minute of cardiopulmonary resuscitation was performed successfully. Afterwards, he was sedated, intubated and mechanical ventilation was started. Hypotension was treated with a continuous infusion of noradrenaline. Gastric lavage was performed and activated charcoal was given. His laboratory results, including electrolytes, were normal. Electrocardiogram (ECG) revealed a sinus rhythm with a wide QRS complex $(200 \mathrm{~ms})$ and prolonged QTc interval $(553 \mathrm{~ms})$. Treatment with $100 \mathrm{~mL}$ of lipid emulsion had no effect on QRS complex wideness and QTc interval duration. Cannulation for the potential need of veno-arterial extracorporeal membrane oxygenation (VA ECMO) was performed. In the next 8 hours wide QRS complexes and QTc interval shortened to $120 \mathrm{~ms}$ and $454 \mathrm{~ms}$, respectively, without any further intervention. Cannulas were removed and he was successfully extubated. On the second day, he developed paralytic ileus and aspiration pneumonia that were treated with neostigmine, metoclopramide, erythromycin and amoxicillin with clavulanic acid. He was discharged after psychiatric evaluation 2 weeks later. Subsequent toxicology analysis by liquid chromatography coupled to mass spectrometry revealed taxine in his serum samples taken on admission.

Conclusion: Yew ingestion can result in prolonged QT interval and sustained ventricular tachycardia and bradycardia with wide QRS without pulse. Sodium bicarbonate $(1.5 \mathrm{mmol} / \mathrm{kg})$ and lipid emulsion $(1.5 \mathrm{~mL} / \mathrm{kg})$ had no effect on QT interval prolongation and QRS widening in this case.

\section{Reference}

[1] Wilson CR, Sauer J, Hooser SB. Taxines: a review of the mechanism and toxicity of yew (Taxus spp.) alkaloids. Toxicon. 2001;39:175-85.

\section{Hepatotoxic mushroom poisoning in Northern Italy}

\author{
Giuseppe Musella ${ }^{a}$, Paolo Severgnini ${ }^{b}$ and \\ Francesca Assisi ${ }^{\mathrm{c}}$ \\ ${ }^{a}$ Anesthesia and Intensive Care, ASST Sette Laghi, Varese, Italy; \\ ${ }^{b}$ Department of Biotechnologies and Life Science, University of \\ Insubria, Varese, Italy; 'Poison Control Center, Ospedale Niguarda \\ Cà Granda, Milan, Italy
}

Objective: Analysis of prognostic factors after hepatotoxic mushroom poisoning

Methods: We retrospectively analyzed clinical records from 45 patients with confirmed amatoxin poisoning, followed by the Poison Control Center of Milan, Italy, from June to December 2010.

Results: All patients developed gastrointestinal symptoms, 40 developed acute hepatitis, while 5 had no evidence of hepatotoxicity. Severe hepatitis occurred in 28 cases, with one successful liver transplant and 5 deaths. These 6 patients with an adverse outcome, compared with the survivors, were older (mean 71 versus 59 years, $p=0,045$ ), had delayed presentation (36 versus 17 hours, $p=0,025)$, and a higher peak in serum creatinine, bilirubin and INR (3.6 versus $1.1 \mathrm{mg} / \mathrm{dL}, \mathrm{p}=0.02 ; 14.7$ versus $1.7 \mathrm{mg} / \mathrm{dL}$, $\mathrm{p}=0.002 ; 5.7$ versus $1.7, \mathrm{p}=0,001)$. Urinary amanitin was not significantly higher (44 versus $28.5 \mathrm{ng} / \mathrm{mL}, \mathrm{p}=0.89$ ) as the transaminase peak (5570 versus $3790 \mathrm{UI} / \mathrm{mL}, \mathrm{p}=0.34$ ), but the time from ingestion to the transaminase peak was longer (78 versus 59 hours, $p=0.043$ ). We retrospectively tested sensitivity and specificity of transplant criteria proposed by O'Grady [1] for paracetamol-induced hepatitis ( $83 \%$ sensitivity and $100 \%$ specificity) and for other aetiologies (50\% and 95\%), by Ganzert [2] (83\% and $100 \%$ ) and a blood lactate concentration $>3 \mathrm{mmol} / \mathrm{L}$ as proposed by Bernal [3] (100\% and 95\%). We constructed the receiver operating characteristic (ROC) curve for the Model for End-stage Liver 
Disease (MELD) score [4]: a score $>27$ showed the best diagnostic performance in our population (83\% and $96 \%$ ).

Conclusion: Amatoxin poisoning is still associated with a high mortality; no prognostic criteria were showed to be superior to the others; the blood lactate concentration and the MELD score seem promising, but a cut-off value is still needed for the latter.

\section{References}

[1] O'Grady JG, Alexander GJ, Hayllar KM, et al. Early indicators of prognosis in fulminant hepatic failure. Gastroenterology. 1989;97:439-445.

[2] Ganzert M, Felgenhauer N, Zilker T. Indication of liver transplantation following amatoxin intoxication. J Hepat. 2005;42:202-209.

[3] Bernal W, Donaldson N, Wyncoll D, et al. Blood lactate as an early predictor of outcome in paracetamol-induced acute liver failure: a cohort study. Lancet. 2002;359:558-563.

[4] Katoonizadeh A, Decaestecker J, Wilmer A, et al. MELD score to predict outcome in adult patients with non-acetaminopheninduced acute liver failure. Liver Int. 2007;27:329-334.

\section{Mushroom poisoning outbreak in Iran: lessons learnt for preparedness of future mycetism}

\author{
Hossein Hassanian-Moghaddam ${ }^{a}$, Nafiseh Mirkatouli ${ }^{b}$, \\ Pirhossein Kolivand $^{\mathrm{b}}$, Nasim Zamani ${ }^{\mathrm{c}}$, Behjat Barari ${ }^{\mathrm{d}}$ \\ and Zahra Habibi-Nodeh ${ }^{\mathrm{b}}$ \\ ${ }^{a}$ Social Determinants of Health Research Center, Shahid Beheshti \\ University of Medical Sciences, Tehran, Iran; ${ }^{\mathrm{b} C i v i l ~ D e f e n s e}$ \\ Secretariat of Ministry of Health, Tehran, Iran; ${ }^{C}$ Clinical Toxicology, \\ Loghman Hakim Hospital, School of Medicine, Shahid Beheshti \\ University of Medical Sciences, Tehran, Iran; ${ }^{\mathrm{d}}$ Toxicological \\ Research Center, Shahid Beheshti University of Medical Sciences, \\ Tehran, Iran
}

Objective: From April to June 2018, more than 1200 patients were admitted to emergency departments (ED) of north-west, west, and central parts of Iran due to mushroom poisoning (MP) [1]. Lepiota brunneoincarnata, Hypholoma fasciculare, and Coprinopsis atramentaria were identified as the causes of the outbreak by the Iranian Plant Pathology Research Institute. A new protocol on mushroom poisoning management was released by the Ministry of Health $(\mathrm{MOH})$. We aimed to evaluate possible onpresentation variables that predicted mortality and to increase public health knowledge regarding this poisoning.
Methods: Iranian $\mathrm{MOH}$ collected the data by trained locals in affected areas using a specific questionnaire.

Results: Of 872 cases with available data, $53.6 \%$ were male and patients were aged 2-86 years. The hospitalization rate was $64.1 \%$ with median [IQR] duration of 1 [>1,3] (range of $<1-23$ ) days. Mushrooms were eaten by a group of victims in $32.6 \%$ of cases. Common on-arrival symptoms/signs were vomiting (70.9\%), nausea $(70.4 \%)$, abdominal pain $(56.1 \%)$, diarrhea (22.4\%), weakness $(21.8 \%)$, sweating (21.5\%) and drooling (8.5\%). The first visit by healthcare providers was available within 5.3 [3, 12] hours post-ingestion. There were 64 cases (7.4\%) who were discharged at first visit, but readmitted due to progression of symptoms/signs, of whom 5 died $(p<0.001$ ) [OR $=6.1495 \% \mathrm{Cl}$ $2.06,18.26]$. Table 1 shows significant variables predicting mortality in univariate analysis. Conservative treatments including fluid therapy $(90.5 \%)$, antidiarrheals $(6.6 \%)$, activated charcoal $(16.3 \%)$, antiemetic (61.2\%), $\mathrm{H}_{2}$ blockers (25.2\%), proton pump inhibitors (39.1\%), non-steroidal anti-inflammatory drugs (NSAIDs) (19.9\%), $\mathrm{N}$-acetylcysteine $(19.4 \%)$, penicillin $(17.8 \%)$, hemodialysis $(0.6 \%)$, hemoperfusion $(0.2 \%)$, corticosteroids $(9.9 \%)$, silimarin $(23.8 \%)$ and 3 cases of liver transplantation were the main therapies.

Conclusion: Raising public awareness about risky behaviors of collecting and consuming wild self-picked mushrooms and increasing knowledge of physicians is of paramount importance.

\section{Reference}

[1] Soltaninejad K. Outbreak of mushroom poisoning in Iran: AprilMay, 2018. Int J Occup Environ Med. 2018;9:152-156.

\section{Calcium oxalate crystalluria and hypocalcemia induced by ingestion of Phytolacca americana root}

\author{
Samuel C Holstege ${ }^{a}$, Angela Venuto-Ashton ${ }^{b}$ and \\ Christopher P Holstege ${ }^{a}$ \\ ${ }^{a}$ Emergency Medicine/Medical Toxicology, University of Virginia, \\ Charlottesville, United States; ${ }^{\mathrm{b}}$ Department of Hospitalist Medicine, \\ Roanoke Memorial Hospital, Roanoke, United States
}

Objective: Phytolacca americana (pokeweed) is a common plant in the Americas. Acute toxicity is often reported following ingestion of the leaves or berries, however, reports of ingestion of the root are uncommon. We report a unique case of Phytolacca americana toxicity associated with ingestion of root that resulted in oxalate crystalluria and hypocalcemia.

Table 1. Significant variables predicting mortality in patients in an outbreak of mushroom poisoning in Iran in 2018

\begin{tabular}{|c|c|c|c|c|}
\hline & $\begin{array}{l}\text { Survived } \\
(\mathrm{n}=856)\end{array}$ & $\begin{array}{c}\text { Fatal } \\
(n=16)\end{array}$ & $\mathrm{P}$ & OR $(95 \% \mathrm{Cl})$ \\
\hline Age (years) & $37[27,51](2,86]$ & $54[29,66](2,68]$ & 0.032 & $1.03(1,1.06)$ \\
\hline Time of first signs after ingestion (hours) & $4[2,8](0.1,772)$ & $10[5,11](3,14)$ & 0.029 & - \\
\hline On arrival aspartate transaminase (AST) (IU/L) & $22[17,31](1,5023)$ & $890[36,1850](36,3136)$ & 0.000 & $1.01(1.01,1.01)$ \\
\hline On arrival alanine aminotransferase (ALT) (IU/L) & $20[14,35](3,6630)$ & $565[51,1950](20,7030)$ & 0.000 & $1.01(1,1.01)$ \\
\hline On arrival prothromin time (PT) (s) & $13[12,13](10,120)$ & $14[13,38](12,41)$ & 0.001 & $1.05(1.01,1.09)$ \\
\hline On arrival partial thromboplastin time (PTT) (s) & $31[28,35](3,236)$ & $35[30,49](25,73)$ & 0.038 & - \\
\hline On arrival INR & $1[1,1](0.1,9.5)$ & $1.2[1,5.8](1,6)$ & 0.000 & $1.93(1.41,2.64)$ \\
\hline On arrival total bilirubin (mg/dL) & $0.7[0.5,1.1](0.1,46)$ & $4.2[2.2,16.9](1.6,21)$ & 0.002 & $1.10(1.01,1.20)$ \\
\hline On arrival urea (mg/dL) & $29[23,36](6,133)$ & $39[31,121](20,172)$ & 0.003 & $1.05(1.03,1.07)$ \\
\hline On arrival creatinine $(\mathrm{mg} / \mathrm{dL})$ & $1[0.8,1.1](0.1,13)$ & $1.2[1,1.9](0.5,4.2)$ & 0.021 & - \\
\hline On arrival bicarbonate $(\mathrm{mEq} / \mathrm{L})$ & $23.7[21.2,26](3.2,79)$ & $19[11.4,22.7](6.7,22.8)$ & 0.000 & $0.77(0.68,0.88)$ \\
\hline Watery diarrhea & $188(22 \%)$ & $7(43.8 \%)$ & 0.038 & $2.77(1.02,7.16)$ \\
\hline Abdominal pain & $476(55.6 \%)$ & $13(81.3 \%)$ & 0.044 & $3.39(1,11.82)$ \\
\hline Edema & $4(0.5 \%)$ & $2(12.5 \%)$ & 0.005 & $20.62(5.95,71.62)$ \\
\hline Deep coma & $5(0.6 \%)$ & $5(31.3 \%)$ & 0.000 & $39.18(16.68,92.02)$ \\
\hline
\end{tabular}


Case report: A 55-year-old farmer while working in his fields pulled a plant he misidentified as edible potato. He skinned the root $(7 \mathrm{~cm}$ long by $4 \mathrm{~cm}$ in diameter), chewed on it 6 times with the intention of eating it, and then spat it out because of the bitter taste. Approximately 90 minutes later he began to experience intractable vomiting (approximately 25 times) over the course of 4 hours. Vomiting was associated with flushing, diaphoresis and one episode of syncope prompting him to seek medical care. He also complained of diarrhea with crampy abdominal pain. On examination he had tachycardia, pallor, and epigastric abdominal pain. His initial laboratory values were significant for a lactate of $3.8 \mathrm{mmol} / \mathrm{L}$, white blood cell count of 38.7 per $\mathrm{mm}^{3}$ with $57 \%$ bandemia, and creatinine $1.7 \mathrm{mg} / \mathrm{dL}$. He was initially anuric and a catheter was placed with return of scant urine. His ionized calcium concentration was $1.05 \mathrm{mg} / \mathrm{dL}$. He was managed supportively with intravenous fluids; renal function slowly improved after a peak creatinine of $2.76 \mathrm{mg} / \mathrm{dL}$ on day 2 . He was given intravenous calcium. Urinalysis demonstrated the presence of protein, blood, and numerous calcium oxalate crystals. The plant ingested was photographed and the Master Naturalists Society confirmed it as Phytolacca americana.

Conclusion: Phytolacca americana toxins are concentrated most heavily in the root system and increase with the age of the plant. The primary active components are triterpene saponins and proteinaceous mitogens, which accounted for his gastrointestinal symptoms and his marked leukocytosis, respectively. Though reported in animals, this is the first human report of the additional toxicity due to soluble oxalate within the root system which accounted for his hematuria, hypocalcemia, and urine calcium oxalate crystals. Though every part of the Phytolacca americana is toxic, the highest concentration of toxins reside in the roots and includes soluble oxalate.

\section{Severe liver injury caused by recommended doses of the food supplement kratom}

\author{
Soeren Bøgevig ${ }^{a}$, Torben Breindal ${ }^{b}$, \\ Mikkel B Christensen ${ }^{a}$, Trine Nielsen ${ }^{c}$ and \\ Lotte CG Hoegberg ${ }^{\mathrm{d}}$ \\ ${ }^{a}$ Danish Poisons Information Centre and Department of Clinical \\ Pharmacology, Copenhagen University Hospital Bispebjerg,

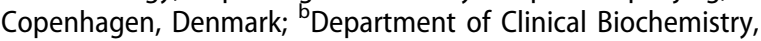 \\ North Denmark Regional Hospital (Aalborg University), Hjørring, \\ Denmark; 'Department of Internal Medicine, Zealand University \\ Hospital, Køge, Denmark; ${ }^{d}$ Danish Poisons Information Centre and \\ Department of Anesthesiology and Intensive Care, Copenhagen \\ University Hospital Bispebjerg, Copenhagen, Denmark
}

Objective: Powder from Mitragyna speciosa, a tropical tree, also named kratom, can be bought as a food supplement over the Internet. The active components of kratom are mitragynine and 7-hydroxymitragynine, both alkaloids that possess central nervous system activity with an opioid-like effect. We present a case of severe liver injury after kratom ingestion in the dose recommended on the package.

Case report: A 56-year old man was admitted to the hospital by his general practitioner (GP) with symptoms of obstipation and jaundice. The patient described 10 days of obstipation and around five days with yellow coloring of the skin, mild stomach pain, but no other complaints. Medical history included chronic pain due to spinal stenosis including spinal surgery. There was no current or past use or substance abuse including alcohol, but he had a prior history of mild elevation of the liver enzymes, but normal values 6 month before admission. He explained he had begun to use a food supplement for his back pain as recommended by his son. The powder, named Green Malay Kratom, was purchased from the Internet and used in the dose indicated, one teaspoon of powder daily starting 14 days before admission. He received no other prescription medication, but took vitamin $C$ and probiotics on a daily basis. Initial laboratory values showed liver injury; alanine aminotransferase (ALT) $887 \mathrm{U} / \mathrm{L}$; alkaline phosphatase $392 \mathrm{U} / \mathrm{L}$; bilirubin $296 \mu \mathrm{mol} / \mathrm{L}$; lactate dehydrogenase $259 \mathrm{U} / \mathrm{L}$. Blood coagulation parameters and pancreatic amylase were normal. Further tests during admission were without signs of infection including cytomegalovirus and hepatitis. Kratom powder ingestion was stopped, and symptoms decreased over the following days. Bilirubin and ALT normalized after 3 weeks. The purchased kratom powder was analyzed using high-performance liquid chromatography/tandem mass spectrometry (LC-MS/ MS) and electron impact ionization gas chromatography/mass spectrometry (El-GC-MS) using deuterium-labelled internal standards and certified reference standards. Quantification of mitragynine showed average contents of $0.590 \mathrm{mg} / \mathrm{g}(0.059 \% \mathrm{w} / \mathrm{w})$. Mitragynine contents in the range $1.6-19 \mathrm{mg} / \mathrm{g}$ have been reported for kratom products sold online.

Conclusion: This case presents severe liver injury after a food supplement kratom powder was ingested at the recommended dose for only 2 weeks. The active component mitragynine was found in relatively low concentration in the powder by LC-MS/ MS, however kratom was suspected to be the cause of liver injury in this case.

\section{Humulus lupulus (hops) ingestion in 13 domestic dogs}

\section{Tiffany Blackett and Nicola Bates \\ Veterinary Poisons Information Service, London, United Kingdom}

Objective: To examine the typical clinical signs and outcome of Humulus lupulus (hops) ingestion in dogs. The plant is known to cause severe pyrexia in dogs, but the cause is unknown.

Methods: A retrospective study of 13 cases of hops ingestion in dogs reported to the VPIS between July 2012 and July 2018.

Results: Of 13 dogs, $76.9 \%(\mathrm{n}=10)$ became symptomatic. Overall, 5 fully recovered (38.5\%), 2 died (15.4\%), one was euthanized $(7.7 \%)$ and 2 were still receiving care when follow-up was obtained (15.4\%). The sources included spent brewing hops $(n=7)$, plant material $(n=5)$ and pelleted brewing hops $(n=1)$. The earliest reported onset of effects was within 15 minutes of exposure. Common signs were panting $(n=8 / 10)$, pyrexia $(n=8 /$ $10)$, vomiting $(n=4 / 10)$, convulsions $(n=3 / 10)$ and agitation $(n=3 / 10)$. Where documented, pyrexia ranged from $39.6-41.8^{\circ} \mathrm{C}$ (average $41.0{ }^{\circ} \mathrm{C}, \mathrm{n}=7$ ), had a mean onset of 3.8 hours (range 0.5-7 hours) and on average lasted 16.3 hours (range 8-24 hours). Rapid increases in body temperature were recorded in one dog, from 39.2 to $41.7^{\circ} \mathrm{C}$ in 2 hours, along with raised alanine aminotransferase and alkaline phosphatase. In 2 symptomatic dogs, pyrexia was not recorded. One did not present for veterinary care, was tachypnoeic and observed at home, but recovered. The second dog, a greyhound, vomited within 15 minutes of eating pelleted brewing hops and was panting, but recovered following activated charcoal (AC) administration. In the two dogs that died, both ingested spent brewing hops and had convulsions prior to death; one dog died within 7 hours of exposure. A third dog with convulsions ingested hop plant material from the garden the day before presentation and on arrival was pyrexic $\left(41.3^{\circ} \mathrm{C}\right)$, lethargic, recumbent, icteric, had vomited and had forelimb rigidity and dysrhythmias; however, it also had an abdominal mass and was euthanised. The 3 asymptomatic dogs presented within 40 minutes following suspected minimal exposures (possible ingestion of spent brewing hops, one dried flower cone and licking of spent hops). Gut decontamination was undertaken in all three dogs (emetic and $A C n=2, A C$ only $n=1$ ). Treatments in this case series involved aggressive cooling measures including cool 
intravenous fluid therapy, fans, cool water sprays or baths, or placement of ice packs around large superficial blood vessels.

Conclusion: Treatment of hops toxicosis is largely supportive necessitating rapid pyrexia control. In this case series the maximum recorded pyrexia was $41.8^{\circ} \mathrm{C}$ (this dog died) and the fatality rate was $23.1 \%$.

\section{Mirabegron toxicosis in dogs: not as bad as we feared}

\author{
Nicola Bates and Nick Edwards \\ Veterinary Poisons Information Service, London, United Kingdom
}

Objective: Mirabegron, a potent, selective beta 3-adrenoceptor agonist, is used in humans with overactive bladder. In single dose toxicity studies in dogs, tachycardia occurred at all doses $(0.3-30 \mathrm{mg} / \mathrm{kg})$ with extreme skin reddening. One dog died 50 minutes after $30 \mathrm{mg} / \mathrm{kg}$ [1]. Mirabegron is not used in veterinary medicine and data on canine cases of poisoning is limited.

Methods: Retrospective analysis of cases of mirabegron ingestion in dogs reported to the VPIS between 2013 and August 2018. All cases with exposure to mirabegron as a single agent and known outcome with returned veterinary surgeon follow up were included.

Results: Follow up information was available for 12 dogs. In four, the dog was given a $50 \mathrm{mg}$ tablet in error by the owner; one ingested a dropped tablet and one stole the tablet from a handbag. Four dogs remained asymptomatic after 1.8, 2.3, 2.8 and 3.3 $\mathrm{mg} / \mathrm{kg}$. The remaining 8 dogs (Table 1) developed erythema/ hyperaemia $(n=7)$, tachycardia $(n=6)$, quiet/lethargy $(n=2)$, hypertension $(n=2)$ and pyrexia $(n=1)$. Onset of signs was $0.5-$ 4 hours and duration 1-24hours. No dog had elevated liver enzymes, but it was reportedly only measured in one case. Two dogs received atenolol for tachycardia. The duration of the erythema/hyperaemia ranged from 1-24 hours (generally 1-12 hours). All the dogs recovered fully.

Conclusion: As in a recent report [2] these clinical cases confirm that mirabegron causes rapid onset erythema and tachycardia in dogs, suggests that mirabegron-induced tachycardia rarely warrants pharmacological intervention and that recovery is expected within 24 hours.

\section{References}

[1] FDA. 2011 Mirabegron. Pharmacology/toxicology NDA/BLA review and evaluation. NDA 202-611 [cited 2018 Jul 8]. Available from: https://www.accessdata.fda.gov/drugsatfda_docs/nda/2012/ 2026110rig1s000PharmR.pdf

[2] Schmid RD, Hovda LR. Mirabegron toxicosis in dogs: a retrospective study. J Med Toxicol. 2018;14:160-167.

Table 1. Clinical signs in dogs with known or estimated dose of mirabegron.

\begin{tabular}{ll}
\hline Dosage of mirabegron ingested & \multicolumn{1}{c}{ Clinical signs } \\
\hline $1.1 \mathrm{mg} / \mathrm{kg}$ (known) & $\begin{array}{c}\text { Hyperaemia and tachycardia within } 1 \text { hour, } \\
\text { lasting } 24 \text { hours. } \\
\text { Tachycardia at } 1.5 \text { hours with hyperaemia } \\
\text { and hypertension. } \\
1.9 \mathrm{mg} / \mathrm{kg} \text { (known) }\end{array}$ \\
$\begin{array}{l}\text { Erythema at } 1 \text { hour, lasting } 8 \text { hours. } \\
\text { Severe tachycardia, hyperaemia and hyper- } \\
\text { tension within 2-3 hours; recovery by } 24 \\
\text { hours. }\end{array}$ \\
$5.6 \mathrm{mg} / \mathrm{kg}$ (known) \\
$\begin{array}{c}\text { Tachycardia, pyrexia and hyperaemia within } \\
4 \text { hours lasting } 24 \text { hours. }\end{array}$ \\
$10 \mathrm{mg} / \mathrm{kg}$ (known) & $\begin{array}{c}\text { Hyperaemia and tachycardia at } 3 \text { hours, } \\
\text { recovery by } 12 \text { hours. }\end{array}$ \\
$10-30 \mathrm{mg} / \mathrm{kg}$ (estimated) & $\begin{array}{c}\text { Erythema of the pinnae which occurred at } \\
30 \text { minutes and lasted } 1 \text { hour. }\end{array}$ \\
$28.2-35.2 \mathrm{mg} / \mathrm{kg}$ (estimated) & $\begin{array}{c}\text { Tachycardia and lethargy at } 1 \text { hour with } \\
\text { recovery by } 5 \text { hours. }\end{array}$ \\
\hline
\end{tabular}

\section{Xylitol-sweetened gingerbread: an unrecognised danger for dogs}

\author{
Dave Huiskens ${ }^{a}$, Anne A Kan ${ }^{a}$, Joris H Robben ${ }^{b}$, \\ Dylan W De Lange ${ }^{a}$ and Marieke A Dijkman ${ }^{a}$ \\ ${ }^{a}$ Dutch Poisons Information Center, University Medical Center \\ Utrecht, Utrecht University, Utrecht, Netherlands; ${ }^{\mathrm{b}}$ Clinical Sciences \\ of Companion Animals, Faculty of Veterinary Medicine, Utrecht \\ University, Utrecht, Netherlands
}

Objective: In 2015 a xylitol-sweetened gingerbread was introduced to the Dutch market. Gingerbread is very palatable for dogs and is often advised as a "remedy" for ingestion of sharp objects. Here we present a fatal case of a dog exposed to xylitolsweetened gingerbread. Additionally a retrospective analysis is presented of all veterinary gingerbread exposures to the Dutch Poisons Information Centre (DPIC), describing the circumstances of exposure and the ingested dose.

Case report: A 4.5-year-old Dachshund $(8.5 \mathrm{~kg})$ was presented to his local veterinarian with drowsiness, painful abdomen, anorexia and intermittent vomiting (possibly haematemesis) over the previous 7 hours. Severe diarrhoea occurred the previous evening but had resolved. The presumptive diagnosis was acute gastroenteritis and maropitant $(1 \mathrm{mg} / \mathrm{kg})$ was given. Symptoms worsened during the night and the next morning the dog was lethargic and hypothermic $\left(36.3^{\circ} \mathrm{C}\right)$, with a weak pulse. Laboratory results were blood glucose $4.4 \mathrm{mmol} / \mathrm{L}$, creatinine 254 $\mu \mathrm{mol} / \mathrm{L}, \mathrm{ALT}>1000 \mathrm{U} / \mathrm{L}$, BUN $15.9 \mathrm{mmol} / \mathrm{L}$ and ALP $196 \mathrm{U} / \mathrm{L}$. The dog was transferred to the Emergency Service of the Faculty of Veterinary Medicine and arrived in shock. Mucosal membranes were slightly icteric, a haematoma was present at a vena jugularis puncture site and petechiae on the abdomen were noted. Hypoglycaemia (1.6 mmol/L), hypoproteinaemia / hypoalbuminaemia, anemia and increased clotting times (PT >35 s, aPTT >200 s) were present. A presumptive diagnosis of disseminated intravascular coagulation (DIC) secondary to liver necrosis was made and owners decided on euthanasia. The history revealed that approximately 6 hours before onset of signs the dog had eaten half a xylitol-sweetened gingerbread, with an estimated xylitol dose of $4.2 \mathrm{~g} / \mathrm{kg}$. Retrospective analysis of the DPIC database from January 2015 to July 2018 revealed 26 enquiries concerning 29 dogs exposed to xylitol-sweetened gingerbread. The amount ingested ranged from less than a slice, to a whole gingerbread (475 g containing $10-15 \%$ xylitol). The estimated xylitol dose ranged from 0.03 to $4.45 \mathrm{~g} / \mathrm{kg}$. In at least 6 cases, gingerbread was given as a remedy for ingestion of sharp objects or to resolve gastrointestinal symptoms.

Conclusion: The case report illustrates the lack of awareness of dog owners and veterinarians of the risks involved in the ingestion of this new variety of gingerbread. The DPIC received an increasing number of veterinary information requests concerning dogs exposed to xylitol-sweetened gingerbread. Due to the high percentage of xylitol and palatability of gingerbread, dogs are easily exposed to a toxic dose.

\section{How "diet" gingerbread increased the awareness of xylitol toxicity in dogs}

\author{
Dave Huiskens ${ }^{\mathrm{a}}$, Anne A Kan ${ }^{\mathrm{a}}$, Joris H Robben ${ }^{\mathrm{b}}$, \\ Duc Anh Ngo ${ }^{c}$, Dylan W De Lange ${ }^{a}$ and \\ Marieke A Dijkman ${ }^{\mathrm{a}}$
}

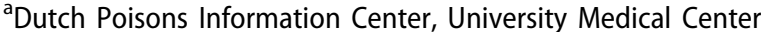
Utrecht, Utrecht University, Utrecht, Netherlands; ${ }^{\text {b}}$ Clinical Sciences of Companion Animals, Faculty of Veterinary Medicine, Utrecht 
Table 1. Number of animals exposed to xylitol-containing products reported to the Dutch Poisons Information Centre (DPIC).

\begin{tabular}{|c|c|c|c|c|c|c|c|c|c|}
\hline Year & Total & Chewing gum & $\%$ & Sweets & $\%$ & Gingerbread & $\%$ & Vitamins / medicines & $\%$ \\
\hline 2013 & 5 & 3 & 60.0 & 2 & 40.0 & 0 & 0.0 & 0 & 0.0 \\
\hline 2014 & 8 & 5 & 62.5 & 3 & 37.5 & 0 & 0.0 & 0 & 0.0 \\
\hline 2015 & 14 & 11 & 78.6 & 2 & 14.3 & 0 & 0.0 & 1 & 7.1 \\
\hline 2016 & 32 & 26 & 81.3 & 4 & 12.5 & 2 & 6.3 & 0 & 0.0 \\
\hline 2017 & 55 & 34 & 61.8 & 4 & 7.3 & 15 & 27.3 & 2 & 3.6 \\
\hline 2018 (to July) & 35 & 22 & 62.9 & 0 & 0.0 & 11 & 31.4 & 2 & 5.7 \\
\hline
\end{tabular}

University, Utrecht, Netherlands; 'Division of Student Affairs, Department of Student Health, University of Virginia, Charlottesville, United States

Objective: In 2015 gingerbread sweetened with xylitol was introduced to the Dutch market. Xylitol is toxic to dogs; it causes hypoglycaemia and in some cases severe liver toxicity. Our aim was to investigate whether the introduction of this potentially harmful food affected the number of reported xylitol poisonings to the Dutch Poisons Information Centre (DPIC), and to determine which products were involved.

Methods: Retrospective analysis of the DPIC database from January 2013 to July 2018. Only veterinary enquiries concerning small animals were included.

Results: A total of 135 enquiries were identified concerning 143 dogs, 2 cats and 1 rabbit (Table 1). At the time of the enquiry, 41 animals were experiencing signs after ingestion of chewing gum ( $n=24,23$ dogs and 1 cat), gingerbread (13 dogs), and sweets (6 dogs). Gastrointestinal signs were noted in 38 animals. Most dogs were vomiting $(n=27)$. Neuromuscular and/or central nervous system signs were reported $(n=29)$, including ataxia $(n=8)$, coma $(n=4)$ and convulsions $(n=4)$. Liver toxicity was reported in 6 animals. Three deaths were reported; two dogs after consumption of gingerbread and one dog after eating chewing gum. Conclusion: An increase in veterinary xylitol poisonings has been noted. This was not solely attributable to the amount of gingerbread exposures, but also due to the increase in chewing gumrelated cases. This may indicate that Dutch veterinarians are becoming more aware of the toxicity of xylitol in dogs. Negative (social) media attention after the death of several dogs could have increased this awareness of both veterinarians and pet owners. Additionally, due to the start of a public warning/information campaign in July 2018 by a gingerbread manufacturer, a further increase in awareness is expected.

\section{5-Hydroxytryptophan (5-HTP) exposure in 65 dogs}

\section{Emilija Kekla, Philip Rawson-Harris and Nicola Bates \\ Veterinary Poisons Information Service, London, United Kingdom}

Objective: 5-Hydroxytryptophan (5-HTP) is a readily available over-the-counter supplement for depression; it is the intermediate metabolite in the biosynthesis of the neurotransmitter serotonin. Exposure in dogs causes serotonin syndrome with neurological, gastrointestinal and neuromuscular effects. Cyproheptadine, a $5 \mathrm{HT}_{2 \mathrm{~A}}$ antagonist, appears to produce clinical improvement. We reviewed canine cases of 5-HTP exposure reported to the Veterinary Poisons Information Service (VPIS).

Methods: A retrospective study of canine cases of 5-HTP exposure reported between March 2000 and July 2018. All cases with known outcome with returned veterinary surgeon postal followup were included.

Results: There were 65 cases of 5-HTP ingestion in dogs included. Five dogs remained asymptomatic; the dose was only known in one of these cases $(7.1 \mathrm{mg} / \mathrm{kg})$. The most common signs in symptomatic dogs (92.3\%) were diarrhoea (33.8\%), hypersalivation (32.3\%), tremor (21.5\%), convulsions $(21.5 \%)$, vocalising
(20\%), dilated pupils (20\%), pyrexia (18.5\%) and ataxia (16.9\%). Most dogs recovered $(91.6 \%, n=55 / 60)$ but there were 5 fatalities (3 euthanised, 2 died); none of these dogs received cyproheptadine but one was given mirtazapine (another serotonin agonist) without effect, when cyproheptadine was unavailable. Signs in fatal and euthanised cases were convulsions $(n=3)$, pyrexia $(n=1)$, coma $(n=1)$ and ventricular fibrillation $(n=1)$. In one dog no signs were observed, and it was found dead within 4 hours of ingestion. Time to death was 4 hours in the other dog that died. The dose was known in two fatal cases, $8.3 \mathrm{mg} / \mathrm{kg}$ (euthanised) and $128.6 \mathrm{mg} / \mathrm{kg}$ (died). Cyproheptadine was used in 28 dogs (43.1\%); all these dogs recovered. In symptomatic cases time to recovery was 4-96 hours (median 19 hours, $\mathrm{n}=36$ ) and the doses ingested were 8.3-1250 $\mathrm{mg} / \mathrm{kg}$ (median $58.5 \mathrm{mg} / \mathrm{kg}, \mathrm{n}=29$ ). Recovery was longer in dogs given cyproheptadine (median 24 hours, IQR 19-30, $\mathrm{n}=19$ ) compared to dogs without cyproheptadine (median 12 hours, IQR 10$16, \mathrm{n}=17$ ), but the dose of 5-HTP was higher in the former (median $90 \mathrm{mg} / \mathrm{kg}, \mathrm{IQR} 53-208, \mathrm{n}=12$ ) compared to dogs not given cyproheptadine (median $33 \mathrm{mg} / \mathrm{kg}$, IQR 24-49, $\mathrm{n}=7$ ) and the number of cases overall was small.

Conclusion: Most dogs that ingest 5-HTP develop clinical signs and the fatality rate in VPIS cases is quite high (7.7\%). 5-HTP exposure causes gastrointestinal and neurological signs and there is no correlation between the dose ingested and severity or duration of clinical signs. The mainstay of treatment is supportive with cyproheptadine. All dogs treated with cyproheptadine recovered but there is currently insufficient information to determine if administration is beneficial.

\section{Gorilla Glue ingestion in dogs: an expanding issue}

\section{Zoe Tizzard and Nicola Bates \\ Veterinary Poisons Information Service, London, United Kingdom}

Objective: Gorilla Glue is a polyurethane expanding adhesive that it is claimed expands up to 3 times its volume as it hardens. Systemic toxicity is not expected following ingestion; the risk is from expansion of the product in the stomach. We reviewed canine cases of Gorilla Glue exposure to evaluate clinical signs and outcome.

Methods: A retrospective study of cases of Gorilla Glue exposure in dogs reported to the VPIS between 1996 and August 2018. All cases with exposure to Gorilla Glue as a single agent and known outcome with returned veterinary surgeon follow up (via postal questionnaire) were included.

Results: There were 17 cases of Gorilla Glue exposure in dogs; 16 of these occurred within the last 3 years, with 1 case in 2012. Of these cases 3 had skin and buccal exposure only and 14 involved ingestion. In one case of ingestion, the ingested product was cured and therefore not a concern, but in the remaining 13 cases, the "wet" product was consumed, mostly through piercing or chewing the container. Of these 13 dogs, only 2 remained asymptomatic. In the other 11 dogs, the most common signs were vomiting $(n=10)$, a gastric mass $(n=7)$, inappetence $(n=$ 3) and lethargy $(n=3)$. Time to consultation with VPIS was prompt in 6 cases, however, in 7 cases the time to consultation was 12 hours to 2 weeks post-ingestion. Seven dogs required 
surgery to remove a gastric mass; 6 of these were the later presenting cases. Masses were described as "hard spongy" or "an anatomical cast". Size ranged from $5 \times 4 \mathrm{~cm}$ to $20 \mathrm{~cm}$ in diameter and in no case was any glue found beyond the pylorus. In several cases the mass was described as resembling or being mistaken for ingesta on X-ray; in one case the dog had been anorexic so in hindsight the vet commented that the presence of ingesta on X-ray was unlikely. In only one case was damage to the gastric mucosa reported as "some areas of ulceration". All symptomatic dogs made a full recovery.

Conclusion: Gorilla Glue ingestion presents a risk of gastric foreign body to dogs. Dogs may present with persistent non-specific gastrointestinal signs and the gastric mass may be mistaken for food in the stomach. Prognosis following ingestion of Gorilla Glue is good provided the gastric mass is removed.

\section{Confirmed ingestion of paralytic shellfish toxins (PST) in a dog}

\author{
Andrew Turner ${ }^{\mathrm{a}}$, Nicola Bates ${ }^{\mathrm{b}}$ and Nick Edwards ${ }^{\mathrm{b}}$ \\ ${ }^{a}$ Centre for Environment, Fisheries and Aquaculture Science \\ (Cefas), Weymouth, United Kingdom; ' ${ }^{\text {Veterinary Poisons }}$ \\ Information Service, London, United Kingdom
}

Objective: Over a two-week period (31 December 2017 to 13 January 2018), nine cases including two deaths were recorded in dogs after walking on beaches on the English East Coast. Signs included vomiting, loss of motor control and muscle paralysis. Paralytic shellfish toxins (PST) including saxitoxin, decarbamoyl saxitoxin and gonyautoxin 5 , were detected in flatfish, starfish and crab samples from these beaches [1]. We report an additional case of PST ingestion in a dog that occurred in March 2018.

Case report: A 4-month-old $16 \mathrm{~kg}$ Golden Retriever ingested crab on a beach on the East Coast of England and vomited profusely 15 minutes later. She was immediately taken to a veterinary practice where she was given activated charcoal and IV fluids. She developed no neurological signs. Samples of crab claw, vomitus (including crab) and urine were taken. Liquid chromatography with fluorescence detection (LC-FLD) revealed paralytic shellfish toxin (PST) analogues in all samples (Table 1). Confirmation using liquid chromatography-tandem mass spectrometry (LC-MS/MS) was attempted, but quality control failures resulted in qualitative assessment only. Low concentrations were detected in crab claw, with higher concentrations in the vomitus. Analysis showed the near-exclusive detection of decarbamoyl saxitoxin, and rapid decline in urinary concentrations within the first few hours.

Conclusion: The European Union regulatory limit for total PST is $800 \mu \mathrm{g} \mathrm{STX} \mathrm{eq} / \mathrm{kg}$ shellfish tissue, however, there is evidence that toxic effects can occur in humans at concentrations below this. In this case PST were confirmed in crab, vomitus and urine. The absence of neurological signs is consistent with the low

Table 1. Paralytic shellfish toxins (PST): total concentrations in urine, vomit and crab claw samples from a dog.

\begin{tabular}{lcll}
\hline & $\begin{array}{c}\text { Time sample } \\
\text { taken (ingestion } \\
\text { occurred at } \\
\text { approximately 10:45) }\end{array}$ & LC-MS/MS & \multicolumn{1}{c}{ LC-FLD } \\
Sample & $13: 20$ & Detected & $47.7 \mu \mathrm{g} \mathrm{STX} \mathrm{eq/L}$ \\
Urine 1 & $14: 50$ & Detected & $10.2 \mu \mathrm{g} \mathrm{STX} \mathrm{eq/L}$ \\
Urine 2 & $16: 40$ & Detected & $5.0 \mu \mathrm{g} \mathrm{STX} \mathrm{eq/L}$ \\
Urine 3 & $19: 50$ & Detected & $6.5 \mu \mathrm{g} \mathrm{STX} \mathrm{eq/L}$ \\
Urine 4 & $22: 00$ & Not detected & $4.0 \mu \mathrm{g} \mathrm{STX} \mathrm{eq/L}$ \\
Urine 5 & $06: 20$ & Not detected & $2.9 \mu \mathrm{g} \mathrm{STX} \mathrm{eq/L}$ \\
Urine 6 & - & Detected & $88.4 \mu \mathrm{g} \mathrm{STX} \mathrm{eq/kg}$ \\
Vomitus & - & Detected & $16.6 \mu \mathrm{g} \mathrm{STX} \mathrm{eq/kg}$ \\
Crab claw & & &
\end{tabular}

concentrations of toxins detected but highlights the potential risk to animals eating washed up marine organisms.

\section{Reference}

[1] Turner AD, Dhanji-Rapkova M, Dean K, et al. Fatal canine intoxications linked to the presence of saxitoxins in stranded marine organisms following winter storm activity. Toxins (Basel). 2018;10:pii:E94.

\section{Wildlife poisoning in Emilia Romagna region, Italy: a 10 year} survey

Silva Rubini ${ }^{\mathrm{a}}$, Elena Faggionato ${ }^{\mathrm{b}}$, Alberto Biancardi ${ }^{\mathrm{b}}$, Giuseppe Merialdi ${ }^{\mathrm{b}}$, Marianna Merenda ${ }^{\mathrm{b}}$, Paola Massi ${ }^{\mathrm{b}}$, Matteo Frasnelli ${ }^{b}$, Chiara A Garbarino ${ }^{b}$, Luca Gelmini ${ }^{b}$, Andrea Luppi ${ }^{b}$, Claudia Cotti ${ }^{c}$, Carmela Musto ${ }^{c}$, Mauro Delogu ${ }^{c}$, Anna Talarico ${ }^{d}$, Rosa M Gaudio ${ }^{d}$, Paolo Frisoni ${ }^{d}$, Sabina Strano Rossi ${ }^{e}$, Carlo A Locatelli ${ }^{f}$ and Sandro Mazzariol ${ }^{\mathrm{g}}$

${ }^{a}$ Sezione di Ferrara, Experimental Zooprophylactic Institute of Lombardy and Emilia Romagna, Brescia, Italy; ${ }^{b}$ Experimental Zooprophylactic Institute of Lombardy and Emilia Romagna, Brescia, Italy; ${ }^{C}$ Wildlife and Exotics Animal Service (SeFSE), Department of Veterinary Medical Sciences, University of Bologna,

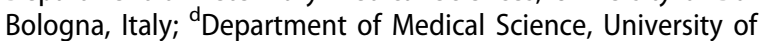

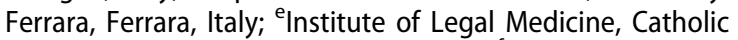
University of Sacred Heart, Rome, Italy; ${ }^{\text {PPavia Poison Control }}$ Centre - National Toxicology Information Centre, Pavia, Italy; ${ }^{9}$ Department of Comparative Biomedicine and Food Science BCA, University of Padua, Legnaro (PD), Italy

Objective: Poisoning of wild animals can be accidental, usually due to a misuse of pesticides or other products intended for agricultural use or secondary, mainly in predators that ingest poisoned prey but, in many cases, it is malicious. Many toxic substances are easy to find on the market (e.g. anticoagulant rodenticides and metaldehyde) while Italian legislation regulates sale, supply and storage of other products (i.e. pesticides) or completely prohibits them (i.e. strychnine and cyanide). In Italy, this issue has become a relevant problem for animals' health in recent years, after the application of the Ministerial decree of 18 of December 2008 and successive modifications on the prohibition of the use of poisoned baits. We present data from a 10 year survey of wildlife poisoning in Emilia Romagna region (2009 - August 2018).

Methods: The 8 Emilia Romagna laboratories of Experimental Zooprophylactic Institute of Lombardy and Emilia Romagna (IZSLER) received 603 samples belonging to 58 different species of wild animals, for toxicological investigation. Postmortem analyses were carried out on the carcasses and, on the basis of pathological findings, tissues and biological fluids were sampled for toxicological analysis.

Results: In total 220/603 animals (36.5\%) were poisoned. Among predators, $67.3 \%$ of foxes, $26.1 \%$ of wolves and $37.1 \%$ of common buzzards were poisoned. Among synanthropic animals, $57.4 \%$ of pigeons were poisoned. Carbamates and anticoagulant rodenticides were the most frequently reported substances found in poisoned animals.

Conclusion: Poisons are often used illegally for animal killing and represent a serious problem for public health. In order to face this serious problem, in addition to the good diagnostic capacities herein described, it is very important to adopt a multidisciplinary approach and to enhance collaboration among different specialists such as pathologists, practitioners, toxicologists, and biologists as well as areas of human medicine. 


\section{Pharmacokinetics of an oral lysergic acid diethylamide (LSD) solution in healthy subjects}

\author{
Friederike Holze, Urs Duthaler, Patrick Vizeli and \\ Matthias E Liechti \\ Clinical Pharmacology and Toxicology, University Hospital Basel, \\ University Basel, Basel, Switzerland
}

Objective: Lysergic acid diethylamide (LSD) is usually consumed orally on filter paper or as oral solution allowing for partial oral absorption. Controlled studies on the pharmacokinetics of LSD only used capsules with enteral absorption. The aim of the present study was to characterize the pharmacokinetics of an oral LSD solution in healthy subjects.

Methods: A target dose of $100 \mu \mathrm{g}$ (effectively $96 \mu \mathrm{g}$ LSD base in $1 \mathrm{~mL}$ ethanol corresponding to a dose of $118 \mu \mathrm{g}$ LSD tartrate) was administered in 27 (13 male, 14 female) healthy subjects using a placebo-controlled, double-blind, cross-over study design. Plasma concentration of LSD and its major metabolite 2-oxo-3hydroxy-LSD (O-H-LSD) were repeatedly assessed for up to 11.5 hours using liquid chromatography-tandem mass spectrometry (LC-MS/MS). Pharmacokinetic parameters were determined using compartmental modeling.

Results: First-order elimination kinetics were observed for LSD. Geometric mean (range) maximum concentration $\left(C_{\max }\right)$ values of $1.7(1.0-2.9) \mathrm{ng} / \mathrm{mL}$ were reached on average $\left(T_{\max }\right) 1.7(1.0-3.4)$ hours after administration. The mean plasma half-life $\left(\mathrm{T}_{1 / 2}\right)$ was 3.6 (2.4-7.3) hours. The area under the plasma concentration-time curve from time zero to infinity $\left(A \cup C_{\infty}\right)$ was $13(7.1-28) \mathrm{ng} \times \mathrm{h} /$ $\mathrm{mL}$. There were no differences in these pharmacokinetic parameters between male and female subjects [mean ( $95 \%$ confidence intervals, $\mathrm{Cl}$ ) values were $C_{\max } 1.7$ (1.4-2.1) and 1.8 (1.6-2.1) ng/ $\mathrm{mL}, \mathrm{T}_{\max } 1.8(1.5-2.2)$ and 1.7 (1.4-2.1) hours, $\mathrm{T}_{1 / 2} 3.9$ (3.2-5.0) and 3.4 (3.0-4.1) hours, AUC $_{\infty} 13$ (11-18) and 13 (12-16) $\mathrm{ng} \times \mathrm{h} / \mathrm{mL}$ in men and women, respectively. O-H-LSD plasma concentrations could be quantified in all subjects. Geometric mean (range) $\mathrm{O}-\mathrm{H}$ LSD $C_{\max }$ concentrations of $0.11(0.07-0.19) \mathrm{ng} / \mathrm{mL}$ were reached after 5 (3.2-8) hours. $\mathrm{T}_{1 / 2}$ and $\mathrm{AUC}_{\infty}$ values of $\mathrm{O}-\mathrm{H}$-LSD were 5.2 (2.6-21) hours and $1.7(0.85-4.3) \mathrm{ng} \times \mathrm{h} / \mathrm{mL}$, respectively. The mean $(95 \% \mathrm{Cl})$ first-order absorption coefficient for the present oral LSD solution was $1.2(1.1-1.7) /$ hour and not different from that previously calculated for LSD capsules [1.4 (1.2-4.1)/hour].

Conclusion: The present study provides a characterization of the pharmacokinetics of LSD and of its main metabolite O-H-LSD with administration of LSD at a known dose. Pharmacokinetic parameters of LSD were similar in men and women although dosing was not adjusted for differences in body weight. Overall the pharmacokinetic parameters including the absorption coefficient were comparable to those derived from previous studies using LSD capsules. The data may serve as reference for clinical and forensic cases of LSD intoxication.

\section{Derivation of a clinical decision rule allowing earlier discharge of patients with paracetamol poisoning after a shorter 12-hour acetylcysteine (SNAP) protocol}

Amani S Alrossies ${ }^{a}$, Ruben HK Thanacoody ${ }^{a}$, James Harnett ${ }^{\mathrm{b}}$, Kerry Layne ${ }^{\mathrm{b}}$, John RH Archer ${ }^{\mathrm{b}}$,
Simon L Hill ${ }^{\mathrm{a}}$, David M Wood ${ }^{\mathrm{b}}$, Paul I Dargan ${ }^{\mathrm{b}}$ and Simon HL Thomas ${ }^{\mathrm{a}}$

${ }^{a}$ Newcastle University, Newcastle upon Tyne, United Kingdom; ${ }^{b}$ Clinical Toxicology, Guy's and St Thomas' NHS Foundation Trust and Faculty of Life Sciences and Medicine, King's College London, London, United Kingdom

Objective: A modified 2-bag $12 \mathrm{~h}$ acetylcysteine (NAC) regimen ("SNAP") causes fewer adverse reactions, is simpler and quicker to administer than the current 3-bag 21h NAC regimen used to treat paracetamol poisoning. This research was performed to develop criteria to allow safe early discharge of selected patients soon after use of the SNAP regimen.

Methods: The modified $12 \mathrm{~h}$ SNAP regimen, comprising intravenous NAC $100 \mathrm{mg} / \mathrm{kg}$ over $2 \mathrm{~h}$ then $200 \mathrm{mg} / \mathrm{kg}$ over $10 \mathrm{~h}$, was introduced in 2016 in two UK hospitals to treat all patients requiring NAC for paracetamol overdose. NAC treatment was discontinued if all of the following criteria were met at the end of infusion ("early post-SNAP"): INR $<1.4$ and ALT $<40$ (upper limit of normal); ALT $<80$ and not more than doubled from admission; paracetamol concentration $<10 \mathrm{mg} / \mathrm{L}$. When this early post-SNAP sample occurred earlier than $24 \mathrm{~h}$ post-ingestion, an additional blood sample was taken at least $24 \mathrm{~h}$ post-ingestion ("late post-SNAP"). NAC was continued at $200 \mathrm{mg} / \mathrm{kg}$ over $10 \mathrm{~h}$ in patients who did not meet these criteria on either the early or late blood sample. Data was collected prospectively. In patients in whom there were paired "early" ( $12 \mathrm{~h}$ post-infusion) and "late" ( $24 \mathrm{~h}$ post-overdose) samples, we modelled clinical rules using different cut-offs of $A L T$, INR and paracetamol concentration iteratively to derive criteria that accurately identified patients who did or did not require further treatment after the 12h SNAP NAC infusion and those who require additional blood testing before discharge.

Results: Of 989 patients treated with the SNAP regimen, paired $12 \mathrm{~h}$ and $24 \mathrm{~h}$ blood results were available in 390. Of these 217 patients met all of the following criteria on the "early" ( $12 \mathrm{~h}$ postinfusion) blood samples: ALT $\leq 40$; paracetamol concentration $<10 \mathrm{mg} / \mathrm{L}$; ALT not doubled from admission; INR $<1.5$. None of these patients required re-institution of NAC treatment following "late" (24h post-overdose) blood samples. There were 569/989 (57.5\%) patients that met all of the above criteria for discharge at $12 \mathrm{~h}$ and $134 / 989(13.5 \%)$ met one or more of the following criteria for NAC continuation: paracetamol concentration $>20 \mathrm{mg} / \mathrm{L}_{i}$ ALT 41-80 and doubled or more from admission; ALT $>80$. Of $286 / 989$ (29\%) patients that did not meet either set of criteria, 10 developed liver injury (ALT $>100$ ) on a subsequent blood sample but none developed severe hepatotoxicity (ALT >1000 or INR $>2$ ).

Conclusion: A simple clinical decision rule may accurately identify low-risk patients who can be discharged safely after the shorter $12 \mathrm{~h}$ SNAP regimen but requires validation in larger cohorts of patients before implementation into clinical practice.

\section{Phase I and II metabolites of methylenedioxy-substituted stimulants interact with human monoamine transporters}

\author{
Karolina E Kolaczynska ${ }^{\mathrm{a}}$, Dino Luethi ${ }^{\mathrm{a}}$, Kenner C Rice ${ }^{\mathrm{b}}$, \\ Bruce E Blough ${ }^{c}$, Michael H Baumann ${ }^{\mathrm{d}}$ and \\ Matthias E Liechti ${ }^{\mathrm{a}}$ \\ aDivision of Clinical Pharmacology and Toxicology, Department of \\ Biomedicine, University Hospital Basel, University of Basel, Basel, \\ Switzerland; ${ }^{b}$ Drug Design and Synthesis Section, Intramural \\ Research Program, National Institute on Drug Abuse, National
}


Institutes of Health, Bethesda, United States; ${ }^{\circ}$ Center for Drug Discovery, Research Triangle Institute, Research Triangle Park, United States; ${ }^{d}$ Designer Drug Research Unit, Intramural Research Program, National Institute on Drug Abuse, National Institutes of Health, Baltimore, United States

Objective: To determine whether there are psychoactive metabolites potentially contributing to the effects of the parent drugs, we investigated the interactions of 10 different $\mathrm{N}$-demethylated and O-demethylenated metabolites of 3,4-methylenedioxymethamphetamine (MDMA), 3,4-methylenedioxymethcathinone (methylone) and 3,4-methylenedioxypyrovalerone (MDPV) with human monoamine transporters.

Methods: HEK 293 cells transfected with human transporters were treated with several concentrations of parent compounds and metabolites to examine inhibition of radiolabeled monoamine uptake by the dopamine (DAT), serotonin (SERT) and norepinephrine transporters (NET).

Results: MDMA metabolites inhibited the NET, DAT, and SERT with $\mathrm{IC}_{50}$ values as low as $0.18,0.13$, and $4.3 \mu \mathrm{M}$, respectively. Methylone metabolites inhibited the NET, DAT, and SERT with lowest values of $0.78,0.34$, and $13 \mu \mathrm{M}$, respectively. MDPV metabolites potently inhibited the NET and DAT with lowest values of 0.024 and $0.092 \mu \mathrm{M}$, respectively, with no effects on SERT. Ndemethylation decreased NET and DAT inhibition potency of methylone but not of MDMA. For all substances, O-demethylenation resulted in catechol metabolites and these resembled monoamine uptake inhibition profiles of the parent compounds. However, further metabolization by O-methylation of the catecholamine metabolites markedly reduced their NET inhibition potency and expected stimulant psychoactivity.

Conclusion: MDMA, methylone, and MDPV metabolites interacted with human transporters. N-dealkylated metabolites may contribute to the psychostimulation induced by these substances because these metabolites are detected in human plasma in unconjugated form and at relevant concentrations. In contrast, although the catecholamines formed by $\mathrm{O}$-demethylenation were similarly active to the parent substances, they are unlikely to contribute to their effects due to low plasma concentrations in humans and rapid $\mathrm{O}$-methylation and further conjugation to less active metabolites [1].

\section{Reference}

[1] Schmid Y, Vizeli P, Hysek CM, et al. CYP2D6 function moderates the pharmacokinetics and pharmacodynamics of 3,4-methylenedioxymethamphetamine in a controlled study in healthy individuals. Pharmacogenet Genomics. 2016;26:397-401.

\section{Mapping snake envenomation: a pilot meteorological and geospatial model based on retrospective review of case records at a single tertiary care snake bite treatment centre}

\author{
Siju V Abraham ${ }^{\mathrm{a}}$ and Ronald Jaison Melit ${ }^{\mathrm{b}}$ \\ ${ }^{a}$ Emergency Medicine, Jubilee Mission Medical College and \\ Research Institute, Thrissur, India; ${ }^{b}$ Jubilee Mission Medical College \\ and Research Institute, Thrissur, India
}

Objective: Mapping snake envenomation risk in a district might help in developing preventive strategies in the region. In a resource limited setting we intended to study the correlation between incidence of venomous snake bites and meteorological parameters and the geospatial distribution of snake bite in the region.

Methods: Case records of patients that presented to a single tertiary care snake envenomation treatment centre in central Kerala, south India, over a period of 5 years was accessed after approval from the Institutional Ethic Committee. The place where the patient was bitten was plotted on Google maps ${ }^{\circledR}$ and the shortest distance by road to the institute was recorded. Retrospective regional weather data, namely rainfall, temperature, and relative humidity over the region during the day of bite, were procured from India Meteorological Department (IMD). Kendall's Tau-b (tb) correlation coefficient was used to measure the strength of association of meteorological parameters with incidence of bite.

Results: Overall 3229 individuals were registered at the institute with alleged history of snakebite, of which 451 cases had information recorded on where the bite had occurred. Of these 248 victims (55\%) had been bitten between May and September. There was statistically significant correlation between incidence of bite with monthly rainfall ( $\mathrm{tb}=0.969, \mathrm{p}<0.001$ ) and humidity $(\tau b=0.946, p<0.001)$. Plotting on Google maps $^{\circledR}$ showed higher incidence of bites in human settlements outside forest areas and particularly around paddy fields and local plantations. The average time taken to reach the hospital was 170 minutes and $60 \%$ of victims had to travel more than $20 \mathrm{~km}$ to reach a healthcare facility.

Conclusion: Snake envenomation is more common in this region of India when relative humidity and precipitation is high. Future region specific, prospective mapping is a cost-effective strategy that could help in vulnerability analysis of the region, development of meteorological prediction models, efficient antivenom storage and dispersal and improved ambulance dispatch [1].

\section{Reference}

[1] Eiriweera DS, Diggle PJ, Kasturiratne A, et al. Evaluating temporal patterns of snakebite in Sri Lanka: the potential for higher snakebite burdens with climate change. Int J Epidemiol. 2018;47:2049-2058.

\section{Management of severe calcium channel blocker poisonings using albumin dialysis by MARS ${ }^{\mathrm{TM}}$ : benefits and limits}

Laetitia Sutterlin ${ }^{a}$, Isabelle Malissin ${ }^{a}$, Sebastian Voicu ${ }^{a}$, Nicolas Deye ${ }^{a}$, Brigitte Delhotal Landes ${ }^{a}$, Marion Soichot ${ }^{b}$, Eya Hamdi ${ }^{c}$, Jean-Louis Laplanche ${ }^{c}$, Laurence Labat ${ }^{b}$ and Bruno Mégarbane ${ }^{a}$

${ }^{a}$ Department of Medical and Toxicological Critical Care, Lariboisière Hospital, Paris-Diderot University, INSERM UMRS-1144, Paris, France; 'baboratory of Toxicology, Lariboisière Hospital, ParisDiderot University, INSERM UMRS-1144, Paris, France; 'Laboratory of Biochemistry, Lariboisière Hospital, Paris-Diderot University, INSERM UMRS-1144, Paris, France

Objective: Calcium channel blocker poisonings are rare but lifethreatening. Based on limited case reports published in the literature, possible benefit of albumin dialysis by the molecular adsorbent recirculating system $\left(\right.$ MARS $\left.^{\text {TM }}\right)$ was suggested. We aimed to determine the toxicant extraction rates by albumin dialysis using the MARS $^{\mathrm{TM}}$ system in severely calcium channel blocker-poisoned patients managed in the intensive care unit. Methods: We conducted a prospective single-center study during four years. Severely calcium channel blocker-poisoned patients admitted to the intensive care unit (ICU) and treated by albumin 
dialysis using MARS ${ }^{\mathrm{TM}}$ were included. Quantification of the serum concentrations of the involved toxicants and albumin (to compensate any hemoconcentration induced by the device) was performed. The different components of the MARS $^{\mathrm{TM}}$ circuit were sampled. The extraction ratios were calculated for the whole system and the different circuit components.

Results: Seventeen poisoned patients (35\% males, aged 51 years [50-54] (median [percentiles 25-75]) were included, corresponding to five amlodipine, three diltiazem (of which one with verapamil co-ingestion), three nicardipine (of which one with verapamil coingestion) and eight verapamil poisonings. All patients were managed with at least one albumin dialysis session using $M$ ARS $^{\text {TM }}$, in addition to intravenous calcium $(4.5 \mathrm{~g}$ [3.8-6.3], norepinephrine (maximal infusion rate, $43 \mathrm{mg} / \mathrm{h}$ [26-80]), epinephrine (maximal infusion rate, $10 \mathrm{mg} / \mathrm{h}$ [5-25]), high-dose insulin $(1.0 \mathrm{lU} / \mathrm{kg} / \mathrm{h}[0.5-$ $10.0]), 8.4 \%$ sodium bicarbonate $(71 \%)$, atropine $(41 \%)$, terlipressin $(31 \%)$, isoprenaline (29\%), methylene blue (29\%), lipid emulsion (24\%) and glucagon (18\%). Six patients died in the intensive care unit (ICU). The overall extraction ratios were relatively low (in the $0-12 \%$ range), mainly due to the charcoal column of the system $(40-70 \%)$.

Conclusion: Calcium channel blocker poisoning may be severe and refractory to the usual treatments. Based on our findings, MARS $^{\text {TM }}$ contribution to drug elimination seems limited although beneficial clinical effects were observed, however, the exact mechanism of such benefit remains to be clarified.

\section{Using EXTRIP criteria as indications for extracorporeal treatment in lithium poisoning}

\author{
Betty S Chan ${ }^{a}$, Sonia Cheng ${ }^{b}$, Angela L Chiew ${ }^{\mathrm{a}}$ and \\ Nicholas A Buckley \\ aDepartment of Emergency Medicine \& Clinical Toxicology, Prince \\ of Wales Hospital, Sydney, Australia; ${ }^{b}$ Faculty of Medicine, \\ University of New South Wales, Sydney, Australia; ${ }^{\mathrm{C} D e p a r t m e n t}$ of \\ Clinical Pharmacology, University of Sydney, Sydney, Australia
}

Objective: The Extracorporeal Treatments in Poisoning (EXTRIP) Workgroup provided recommendations on when to perform haemodialysis in lithium toxicity [1]. This study aimed to determine the impact on current practices of applying the EXTRIP criteria to determine which patients are indicated for extracorporeal treatment in lithium poisoning.

Methods: This is a retrospective review of patients presenting with acute-on-chronic or chronic lithium poisoning with an elevated lithium concentration $\geq 1.3 \mathrm{mmol} / \mathrm{L}$. EXTRIP criteria was applied to patients and compared with the number of patients that actually received extracorporeal treatment. Patient

Table 1. Applying EXTRIP criteria for extracorporeal treatment in lithium poisoning in a retrospective review of 266 patients.

\begin{tabular}{|c|c|c|c|c|}
\hline EXTRIP criteria [1] & $\begin{array}{l}\text { No. of } \\
\text { patients }\end{array}$ & $\begin{array}{l}\text { Median age } \\
\text { (range) years }\end{array}$ & $\begin{array}{l}\text { No. of patients } \\
\text { treated with } \\
\text { extracorporeal } \\
\text { treatment }\end{array}$ & Outcome of patients \\
\hline $\begin{array}{l}\text { Severe neurotoxicity (Amdisen } \\
\text { criteria) (1D) }\end{array}$ & 45 & $64(26-92)$ & 3 & $\begin{array}{l}3 \text { neuro sequelae*- no dialysis } \\
\text { - } 81 \mathrm{~F} \text { went to N/H (Li; 1.9, eGFR 36) } \\
\text { - } 84 \mathrm{~F} \text { went to N/H (Li 1.9, eGFR 37) } \\
\text { - } 65 \mathrm{~F} \text { returned to N/H (Li: 3.2, eGFR 40) } \\
32 \text { discharged to previous residential address } \\
10 \text { admitted to } \mathrm{MH} \text {. }\end{array}$ \\
\hline $\begin{array}{l}\text { Lithium }>4 \mathrm{mmol} / \mathrm{L} \text { and eGFR } \\
<90 \mathrm{~mL} / \mathrm{min} / 1.73 \mathrm{~m}^{2} \text { (1D) }\end{array}$ & 4 & $37.5(23-58)$ & $\begin{array}{l}2 \text { patients } \\
\text { - Li } 4.1 \text { \& eGFR } 13 \\
\text { - Li } 5.3 \text { \& eGFR } 75\end{array}$ & $\begin{array}{l}0 \text { neuro sequelae } \\
1 \text { discharge home } \\
1 \text { admitted to a nursing home } \\
2 \text { admitted to } \mathrm{MH}\end{array}$ \\
\hline $\begin{array}{l}\text { Decreased level of conscious- } \\
\text { ness (Glasgow Coma Score } \\
(G C S)<15)(1 D)\end{array}$ & 48 & $64.5(23-89)$ & $\begin{array}{l}3 \text { patients } \\
\text { - } \text { GCS } 13 \\
\text { - } \text { GCS } 14 \\
\text { - } \text { GCS } 13\end{array}$ & $\begin{array}{l}3 \text { neuro sequelae* } \\
30 \text { discharged to previous residential address } \\
6 \text { discharged to } \mathrm{N} / \mathrm{H} / \text { supervised home } \\
11 \text { admitted to } \mathrm{MH} \\
\text { - } 79 \mathrm{~F} \text { died from severe right heart failure and } \\
\text { pneumonia, } \mathrm{Li} 1.3 \mathrm{mmol} / \mathrm{L} \text {, no focal neurological } \\
\text { symptoms }\end{array}$ \\
\hline Seizures (1D) & 2 & $60.5(70-51)$ & 0 & $\begin{array}{l}0 \text { neuro sequelae } \\
2 \text { discharged home }\end{array}$ \\
\hline $\begin{array}{l}\text { Life threatening dysrhythmias } \\
\text { (1D) }\end{array}$ & 0 & - & 0 & 0 \\
\hline Lithium $>5 \mathrm{mmol} / \mathrm{L}(2 \mathrm{D})$ & 2 & $35.5(23-48)$ & $\begin{array}{l}1 \text { patient } \\
\text { - } \quad \text { Li } 5.3 \text { eGFR } 75\end{array}$ & $\begin{array}{l}0 \text { neuro sequelae } \\
2 \text { admitted to } \mathrm{MH}\end{array}$ \\
\hline Confusion (2D) & 38 & $65(26-92)$ & 2 & $\begin{array}{l}3 \text { neuro sequelae* } \\
26 \text { discharged to previous residential address } \\
3 \text { discharged to } \mathrm{N} / \mathrm{H} / \text { supervised home. } \\
9 \text { admitted to } \mathrm{MH} \text {. }\end{array}$ \\
\hline $\begin{array}{l}\text { If the expected time to obtain } \\
\text { a lithium }<1 \mathrm{mmol} / \mathrm{L} \text {, with } \\
\text { optimal management }>36 \mathrm{~h} \\
\text { (2D) }\end{array}$ & 141 & $55(15-92)$ & 4 & $\begin{array}{l}3 \text { neuro sequelae* } \\
87 \text { discharged to previous residential address } \\
8 \text { discharged to } \mathrm{N} / \mathrm{H} / \text { supervised home. } \\
46 \text { admitted to } \mathrm{MH}\end{array}$ \\
\hline
\end{tabular}

MH: Mental health, admissions to $\mathrm{MH}$ are suitable for patients who are mobile and independent.

Neuro: neurological.

*Same 3 patients as in previous row.

$\mathrm{N} / \mathrm{H}$ : nursing home.

$\mathrm{Li}$ concentration in $\mathrm{mmol} / \mathrm{L}$.

eGFR: estimated glomerular filtration rate. 
demographics, clinical symptoms, treatment and outcome were recorded.

Results: From 2008-2018, there were 53 acute-on-chronic and 216 chronic lithium toxicity patients. Four patients $(1.5 \%)$ were treated with extracorporeal methods (continuous veno-venous haemodialysis (CVVHD)). Two had acute-on-chronic and two with chronic toxicity. The Amdisen score was 3 in 3 patients, 1 in 1 patient; none developed persisting neurological sequelae. Ten patients were discharged to nursing home or supervised home. There were no fatalities. Time taken until lithium concentration returned to normal $(<1 \mathrm{mmol} / \mathrm{L})$ was poorly documented in 61 patients. Of the remaining 208 patients, 141 patients took $\geq 48$ hours for normalization. Various criteria were met by a variable number of patients ( 0 to 141). The 3 concentration-related criteria show remarkably little overlap. Three patients with documented neurological sequelae presented with severe neurotoxicity, did not meet the two concentration-related criteria and had no dialysis.

Conclusion: Our study had only 4 patients who received extracorporeal treatment even though at least 137 other patients fulfilled one or more of the EXTRIP criteria. Most patients responded to conservative treatment.

\section{Reference}

[1] Decker BS, Goldfarb DS, Dargan PI, et al. Extracorporeal treatment for lithium poisoning: systematic review and recommendations from the EXTRIP Workgroup. Clin J Am Soc Nephrol. 2015;10:875-87.

\section{Pediatric toxicity of clonidine: can a single pill kill?}

\author{
Asaad I Alsufyani ${ }^{\mathrm{a}}$, Daniel P Gorman ${ }^{\mathrm{b}}$ and \\ Nathan P Charlton ${ }^{\mathrm{a}}$ \\ ${ }^{a}$ Medical Toxicology, University of Virginia, Charlottesville, United \\ States; ${ }^{b}$ Emergency Medicine, Virginia Tech Carilion, Roanoke, \\ United States
}

Objective: Clonidine overdose can cause sedation, bradycardia, hypotension, and hypoventilation. It is often included on the pediatric list of "single pills that can kill." The negative stigma surrounding clonidine may influence practice patterns based on the assumed danger in toxicity. In the US clonidine is available in 100,200 and $300 \mu \mathrm{g}$ tablets. We evaluated the severity of clonidine toxicity among patients less than 6 years old.

Methods: The National Poison Data System (NPDS) was queried for all single-agent clonidine exposures using the American Association of Poison Control Centers (AAPCC) generic code from 2010 to 2017 in patients less than 6 years old. The results were reported by medical outcome and level of care. For the same time period and age group, a retrospective chart review was conducted for single-agent clonidine exposures admitted to the University of Virginia (UVA) Health System to analyze more specific outcomes and interventions.

Results: During the study period, NPDS cases for patients less than 6 years old for single-agent exposures to clonidine averaged 2,051 per year (range 1,851-2,299). Of these $24.5 \%$ of patients were admitted to the ICU, $12.4 \%$ to a non-ICU setting, and $36.1 \%$ were discharged; no deaths occurred. Of those admitted, $3.6 \%$ were determined to have a major clinical effect, $25.4 \%$ moderate effect, $30.0 \%$ minor effect, and $23.1 \%$ had no effect. No follow up was available for $6.6 \%$ of cases. There were no deaths recorded during this time period. During the same period there were 28 cases identified in the UVA Health System with mean dose ingested of $0.03 \mathrm{mg} / \mathrm{kg}$ of clonidine. Of those cases, 2 patients were intubated prior to transfer to our tertiary care center, $71.4 \%$ of patients demonstrated central nervous system depression, $25.5 \%$ bradycardia, and $17.9 \%$ had hypotension. However, none required vasoactive medication, and no deaths occurred. The average length of hospital stay was less than 1 day with only 1 child requiring a 2-day admission.

Conclusion: Single-agent clonidine exposures reported to the NPDS among patients younger than 6 years from 2010 to 2017 generally resulted in good outcomes. Only $3.6 \%$ of all cases nationally were noted to have major clinical effects, and no deaths occurred. Additionally, very few patients admitted to the local hospital system required significant interventions. These results imply that clonidine is not as dangerous in toxicity as traditionally believed. Further research is needed to determine which patients can be less aggressively triaged to reduce healthcare resources and cost.

\section{Paracetamol metabolites and paracetamol-protein adducts following repeated supratherapeutic
ingestion (RSTI) of paracetamol}

Angela L Chiew ${ }^{\mathrm{a}}$, Laura P James ${ }^{\mathrm{b}}$, Geoffrey K Isbister ${ }^{\mathrm{c}}$, Paul Stathakis ${ }^{d}$, Kirsty Ress ${ }^{d}$, Betty SH Chan ${ }^{\mathrm{e}}$ and Nicholas A Buckley ${ }^{a}$

aDepartment of Pharmacology, School of Medical Sciences,

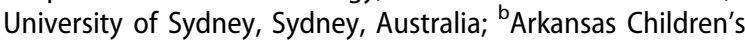
Hospital and Department of Pediatrics, University of Arkansas for Medical Sciences, Arkansas, United States; 'Department of Clinical Toxicology and Pharmacology, Calvary Mater Newcastle and School of Medical Practice, University of Newcastle, Newcastle, Australia; ${ }^{d}$ NSW Health Pathology, Prince of Wales Hospital,

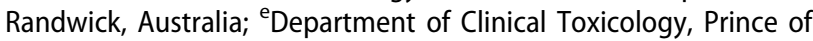
Wales Hospital, Randwick, Australia

Objective: Repeated supratherapeutic ingestion (RSTI) of paracetamol can cause acute liver injury (ALI). There has been interest in biomarkers including paracetamol protein-adducts (APAPadducts), specific biomarkers of paracetamol exposure [1], and paracetamol metabolites, particularly cytochrome P450 (CYP) conjugates as these and their ratios are sensitive for detecting ALI following acute ingestion [2]. We describe the utility of these biomarkers in RSTI.

Methods: The Australian Paracetamol Project is a multi-centre prospective observational study. Patients $>14$ years assessed for paracetamol overdose were reviewed and a subset of RSTI $(>1$ ingestion over $>8$ hours with a cumulative dose of $>4 \mathrm{~g} / 24$ hours). Serum samples were analysed for APAP-adducts, paracetamol and metabolites, including nontoxic glucuronide (APAP-Glu) and sulphate (APAP-Sul) conjugates, and NAPQI conjugatesAPAP-cysteine (APAP-Cys; unbound) and APAP-mercapturate (APAP-Mer) and CYP metabolites (APAP-Cys and APAP-Sul).

Results: Thirty patients were recruited, 21 (70\%) were female, median age 47 years (IQR: 32-51). Median ingested paracetamol dose was $10 \mathrm{~g} / 24 \mathrm{~h}$ (IQR:6-12 $\mathrm{g} / 24 \mathrm{~h}, \mathrm{n}=28$ ) with median duration of 2.5 day (IQR:1-5 d, $n=28$ ). Eighteen (60\%) had an ALT $>50 \mathrm{U} / \mathrm{L}$ on presentation, with $28(93 \%)$ receiving intravenous acetylcysteine. Nine (30\%) developed ALI, and seven hepatotoxicity. Two with ALI presented with an ALT $<50 \mathrm{U} / \mathrm{L} ; 4 / 7$ of those with hepatotoxicity, had ALT $>1000$ on presentation. Those with ALI had significantly higher APAP-adduct and ALT on presentation, but there was minimal difference in CYP metabolites and ratios (Table 1).

Conclusion: APAP-adduct concentrations were higher in those who developed ALI after RSTI of paracetamol, similar to acute ingestion [3]. However CYP metabolite concentrations and their ratios were not significantly associated with hepatotoxicity. 
Table 1. Paracetamol protein-adducts, paracetamol metabolites and ALT on presentation in patients who did and did not develop acute liver injury (ALT $>150$ $\mathrm{U} / \mathrm{L}$ ). All comparisons show median with interquartile ratio (IQR).

\begin{tabular}{|c|c|c|c|}
\hline Metabolite/biomarker on presentation & $\begin{array}{l}\text { No acute liver injury } \\
(\mathrm{n}=21)\end{array}$ & $\begin{array}{l}\text { Acute liver injury } \\
\qquad(\mathrm{n}=9)\end{array}$ & $\begin{array}{c}\text { P value } \\
\text { (Mann Whitney) }\end{array}$ \\
\hline $\mathrm{ALT}(\mathrm{U} / \mathrm{L})$ & $\begin{array}{c}52 \mathrm{U} / \mathrm{L} \\
(15-76)\end{array}$ & $\begin{array}{c}794 \mathrm{U} / \mathrm{L} \\
(241-4860)\end{array}$ & 0.0005 \\
\hline Paracetamol concentration $(\mu \mathrm{mol} / \mathrm{L})$ & $\begin{array}{c}55 \\
(34-187)\end{array}$ & $\begin{array}{c}114 \\
(58-526)\end{array}$ & 0.3435 (NS) \\
\hline APAP-adduct $(\mathrm{nmol} / \mathrm{mL})$ & $\begin{array}{c}0.32 \\
(0.21-0.53)\end{array}$ & $\begin{array}{c}2.45 \\
(0.69-12.6)\end{array}$ & $<0.0001$ \\
\hline APAP-CYS $(\mu \mathrm{mol} / \mathrm{L})$ & $\begin{array}{c}5.42 \\
(3.18-11.6)\end{array}$ & $\begin{array}{c}14.0 \\
(4.36-29.9)\end{array}$ & 0.1779 (NS) \\
\hline Sum CYP metabolites ( $\mu \mathrm{mol} / \mathrm{L})$ (APAP-CYS, APAP-Mer) & $\begin{array}{c}5.96 \\
(3.33-12.0)\end{array}$ & $\begin{array}{c}14.3 \\
(4.72-34.2)\end{array}$ & 0.2093 (NS) \\
\hline APAP-CYS/Total metabolites (\%) & $\begin{array}{c}2.6 \% \\
(1.9-4.9 \%)\end{array}$ & $\begin{array}{c}4.1 \% \\
(3.6-10 \%)\end{array}$ & 0.1041 (NS) \\
\hline APAP-CYS/APAP-Sul & $\begin{array}{c}0.18 \\
(0.10-0.42)\end{array}$ & $\begin{array}{c}0.27 \\
(0.18-0.39)\end{array}$ & 0.4224 (NS) \\
\hline
\end{tabular}

\section{References}

[1] James LP, Capparelli EV, Simpson PM, et al. Acetaminophen-associated hepatic injury: evaluation of acetaminophen protein adducts in children and adolescents with acetaminophen overdose. Clin Pharmacol Ther. 2008;84:684-90.

[2] Vliegenthart A, Kimmitt RA, Seymour JH, et al. Circulating acetaminophen metabolites are toxicokinetic biomarkers of acute liver injury. Clin Pharmacol Ther. 2017;101:531-540.

[3] Chiew AL, James LP, Letzig LG, et al. Paracetamol-protein-adducts following acute paracetamol overdose. Clin Toxicol (Phila). 2016;54:470.

\section{Efficacy of a two bag acetylcysteine regimen to treat paracetamol overdose (2NAC study)}

\author{
Anselm Wong ${ }^{\mathrm{a}}$, Geoffrey K Isbister ${ }^{\mathrm{b}}$, Richard McNulty ${ }^{\mathrm{c}}$, \\ Angela L Chiew ${ }^{\mathrm{d}}$, Colin B Page ${ }^{\mathrm{e}}$, Shaun L Greene ${ }^{\mathrm{f}}$, \\ Naren Gunja ${ }^{\mathrm{c}}$, Nicholas A Buckley ${ }^{\mathrm{g}}$ and \\ Andis Graudins ${ }^{\mathrm{h}}$ \\ ${ }^{a}$ Clinical Sciences at Monash Health, Monash University and Austin \\ Toxicology Unit and Emergency Department, Victoria, Australia; \\ ${ }^{b}$ Clinical Toxicology Research Group, University of Newcastle, \\ Newcastle, Australia; 'Department of Clinical Pharmacology \& \\ Toxicology, Western Sydney Health, New South Wales, Australia; \\ ${ }^{\mathrm{d} D e p a r t m e n t}$ of Emergency and Toxicology, Prince of Wales

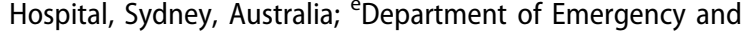 \\ Toxicology, Princess Alexandria Hospital, Brisbane, Australia; \\ ${ }^{f}$ Austin Toxicology Unit and Emergency Department, Austin \\ Health, Victoria, Australia; ${ }^{9}$ University of Sydney, New South Wales, \\ Australia; ${ }^{\mathrm{h}}$ Clinical Sciences at Monash Health, Monash University \\ and Monash Toxicology Unit and Emergency Service, Monash \\ Health, Dandenong, Australia
}

Objective: Previous studies have shown that a 2-bag 20-hour intravenous (IV) acetylcysteine regimen reduced the incidence of nonallergic anaphylactic reactions compared to the 3-bag 21-hour IV regimen for treatment of paracetamol overdose. We evaluated the efficacy and safety of the 2-bag IV acetylcysteine regimen.

Methods: This is an ongoing prospective cohort study of Australian centres using a 2-bag IV acetylcysteine regimen (200 $\mathrm{mg} / \mathrm{kg}$ over 4 hours, $100 \mathrm{mg} / \mathrm{kg}$ over 16 hours) for paracetamol overdose. Outcomes were compared with historical data of patients treated with a 3-bag IV acetylcysteine regimen $(150 \mathrm{mg} /$ $\mathrm{kg}$ over 60 minutes, $50 \mathrm{mg} / \mathrm{kg}$ over 4 hours, $100 \mathrm{mg} / \mathrm{kg}$ over 16 hours). Data was analysed from 2009 to mid-2018. Subjects all had single, acute ingestions and serum paracetamol concentration was performed 4 to 24 hours post-ingestion and was on or above the Rumack-Matthews treatment-nomogram line. The primary outcome was the development of acute liver injury (ALI), defined as peak ALT $>150 \mathrm{IU} / \mathrm{L}$ and double baseline. Secondary outcomes included adverse reactions, hepatotoxicity (ALT/AST >1000), and mortality. Categorical variables were compared using chi-squared test.

Results: Of 3996 paracetamol overdose presentations to 9 treatment centres, 1729 received acetylcysteine. Study criteria were met in 659 receiving the 2 -bag and 532 receiving the 3-bag acetylcysteine regimen. Median time to acetylcysteine was 7 hours (IQR 6,12) for 2-bag versus 7 hours $(5,10)$ for 3-bag regimens. When presentation bloods were performed $\leq 8$ hours post-overdose, 17 (3.9\%) developed ALI with the 2-bag regimen versus 11 $(2.7 \%)$ with the 3 -bag regimen $(p=0.37$ OR $1.495 \% \mathrm{Cl}: 0.66-3.07)$. When presentation bloods were performed 8-24 hours post-overdose, 39 (17.0\%) developed ALI with the 2-bag regimen versus 28 $(21.0 \%)$ with the 3 -bag regimen $(p=0.34$, OR $0.76,95 \% \mathrm{Cl} 0.45$ 1.3). The incidence of hepatotoxicity with the 2-bag regimen was: $36(5.4 \%)$ versus $23(4.3 \%)$ with the 3 -bag regimen $(p=0.36$, OR 1.3, 95\% Cl: $0.75-2.12)$ ). The incidence of cutaneous and systemic non-allergic anaphylactic reactions was: 20 (3\%) with 2-bag versus $52(9.7 \%)$ with 3-bag regimen $(\mathrm{p}=<0.0001$, OR $0.28,95 \% \mathrm{Cl}$ : $0.17-$ 0.48 ). Paracetamol associated mortality was 0 in both regimens.

Conclusion: In this multi-centre study, the 2-bag IV acetylcysteine regimen was as efficacious as the 3-bag IV regimen and resulted in significantly less non-allergic anaphylactic reactions.

\section{Paracetamol metabolites following acute overdose as a biomarker for acute liver injury}

Angela L Chiew ${ }^{a}$, Geoffrey K Isbister ${ }^{b}$, Paul Stathakis ${ }^{c}$, Kirsty Ress ${ }^{c}$, Betty SH Chan ${ }^{d}$ and Nicholas A Buckley ${ }^{\text {e }}$

${ }^{a}$ Department of Pharmacology, School of Medical Sciences, University of Sydney, Sydney, Australia; ${ }^{\mathrm{b}}$ Department of Clinical Toxicology and Pharmacology, Calvary Mater Newcastle and School of Medical Practice, University of Newcastle, Newcastle, Australia; 'NSW Health Pathology, Prince of Wales Hospital, Randwick, Australia; ${ }^{d}$ Department of Clinical Toxicology, Prince of Wales Hospital, Randwick, Australia; ${ }^{\text {eDepartment of }}$ Pharmacology, University of Sydney, Sydney, Australia

Objective: Paracetamol overdose is a common cause of acute liver injury (ALI). Paracetamol is mainly metabolized into nontoxic conjugates, a small fraction is metabolized by cytochrome P450 (CYP) into the reactive metabolite $\mathrm{N}$-acetyl-p-benzoquinoneimine (NAPQI), which reacts with glutathione to form conjugates. Studies have shown that CYP metabolites and metabolite ratios 
Table 1. Paracetamol metabolite and alanine aminotransferase (ALT) results on presentation in those patients who did and did not develop acute liver injury $(\mathrm{ALT}>150 \mathrm{U} / \mathrm{L})$.

\begin{tabular}{|c|c|c|c|c|}
\hline Metabolite/biomarker on presentation & $\begin{array}{l}\text { No acute liver injury (ALI) } \\
(\mathrm{n}=120) \\
\text { Median (IQR) }\end{array}$ & $\begin{array}{l}\text { Acute liver injury (ALI) } \\
\qquad(\mathrm{n}=18) \\
\text { Median (IQR) }\end{array}$ & $P$ value (Mann Whitney) & ROC-AUC $(95 \% \mathrm{Cl})$ \\
\hline APAP-CYS & $\begin{array}{c}8.87 \mu \mathrm{mol} / \mathrm{L} \\
(1.38-3.68)\end{array}$ & $\begin{array}{c}33.5 \mu \mathrm{mol} / \mathrm{L} \\
(25.3-57.3)\end{array}$ & $<0.0001$ & $\begin{array}{c}0.93 \\
(0.86-1.00)\end{array}$ \\
\hline Sum CYP metabolites (APAP-CYS, APAP-Mer) & $\begin{array}{c}9.85 \mu \mathrm{mol} / \mathrm{L} \\
(6.15-16.1)\end{array}$ & $\begin{array}{c}35.1 \mu \mathrm{mol} / \mathrm{L} \\
(26.8-58.4)\end{array}$ & $<0.0001$ & $\begin{array}{c}0.93 \\
(0.86-1.00)\end{array}$ \\
\hline APAP-Glu & $\begin{array}{c}436 \mu \mathrm{mol} / \mathrm{L} \\
(311-795)\end{array}$ & $\begin{array}{c}651 \mu \mathrm{mol} / \mathrm{L} \\
(466-1190)\end{array}$ & 0.0238 & $\begin{array}{c}0.66 \\
(0.54-0.79)\end{array}$ \\
\hline APAP-Sul & $\begin{array}{c}80 \mu \mathrm{mol} / \mathrm{L} \\
(57-122)\end{array}$ & $\begin{array}{c}57.7 \mu \mathrm{mol} / \mathrm{L} \\
(35.8-97.2)\end{array}$ & 0.0397 & $\begin{array}{c}0.65 \\
(0.50-0.77)\end{array}$ \\
\hline APAP-CYS/Total metabolites & $\begin{array}{c}1.4 \% \\
(1.0-2.1 \%)\end{array}$ & $\begin{array}{c}5.0 \% \\
(3.3-6.1 \%)\end{array}$ & $<0.0001$ & $\begin{array}{c}0.90 \\
(0.82-0.97)\end{array}$ \\
\hline APAP-CYS/APAP-Sul & $\begin{array}{c}0.11 \\
(0.06-0.17)\end{array}$ & $\begin{array}{c}0.85 \\
(0.33-1.08)\end{array}$ & $<0.0001$ & $\begin{array}{c}0.89 \\
(0.78-1.00)\end{array}$ \\
\hline ALT & $\begin{array}{c}18 \mathrm{U} / \mathrm{L} \\
(14-27)\end{array}$ & $\begin{array}{c}73 \mathrm{U} / \mathrm{L} \\
(23-148)\end{array}$ & $<0.0001$ & $\begin{array}{c}0.83 \\
(0.71-94)\end{array}$ \\
\hline
\end{tabular}

are sensitive for detecting ALI [1]. We investigated paracetamol metabolites as biomarkers for ALI defined as an ALT >150 U/L.

Methods: The Australian Paracetamol Project, a multi-centre prospective observational study, includes patients $>14$ years, assessed for paracetamol overdose. A subset of acute ingestions was selected in which presentation serum samples were available for analysis by liquid chromatography-tandem mass-spectrometry for paracetamol and metabolites, including non-toxic glucuronide (APAP-Glu) and sulphate (APAP-Sul) conjugates, and NAPQI conjugates, APAP-cysteine (APAP-Cys) and APAP-mercapturate (APAP-Mer). The sum of total CYP metabolites, APAP-Cys/total metabolites and APAP-Cys/APAP-Sul were calculated.

Results: Over 1.5 years, 138 patients were recruited; 100 (72\%) were females, median age 24 years (IQR:18-38 years). Median time to presentation was 2.5 hours (IQR:1.6-6.0 hours), median paracetamol dose was $20 \mathrm{~g}$ (IQR:10-40 g); 30 (22\%) ingested modifiedrelease paracetamol. Intravenous acetylcysteine was given to 126 (91\%); median time 5.3 hours (IQR: 3.4-8.5 hours) post-ingestion. Eighteen (13\%) developed ALI, of which 12 developed a peak ALT $>1000 \mathrm{U} / \mathrm{L}$. Of those with ALI, eight had ALT $<50 \mathrm{U} / \mathrm{L}$ on presentation. Those developing ALI had significantly higher paracetamol metabolites, both CYP and non-CYP (Table 1). Both CYP metabolites alone or as a fraction of APAP-Sul or total metabolites had good predictive performance for ALI (ROC-AUC >0.89).

Conclusion: Absolute concentrations of CYP pathway paracetamol metabolites were better early predictors of ALI, compared to presentation ALT. CYP metabolite measurement may allow for early recognition of high-risk patients and possibly improve treatment algorithms.

\section{Reference}

[1] Vliegenthart A, Kimmitt RA, Seymour JH, et al. Circulating acetaminophen metabolites are toxicokinetic biomarkers of acute liver injury. Clin Pharmacol Ther. 2017;101:531-540.

\section{Outcomes associated with large doses of extended and immediate release paracetamol products reported to the US National Poison Data System (NPDS)}

Kate M Reynolds, Heather Delva-Clark, Randy I Burnham, Malin Rapp-Olsson, Janetta L Iwanicki and Richard C Dart
Rocky Mountain Poison \& Drug Center - Denver Health \& Hospital Authority, Denver, United States

Objective: In February 2018, the European Medicines Agency suspended marketing of modified release paracetamol after a Swedish Poisons Information Centre report indicated that ingestions of modified release (MR) paracetamol $\geq 50 \mathrm{~g}$ involved unpredictable and persistently high serum paracetamol concentrations [1]. Similar concerns were raised about the absorption of US extended release (ER) paracetamol though the paracetamol release technology differs between $M R$ and ER paracetamol and MR paracetamol is not marketed in the US. This analysis of US poison center data aims to determine if outcomes of exposures involving large doses of ER paracetamol are associated with increased severity compared to exposures involving large doses of immediate release (IR) paracetamol.

Methods: NPDS exposures to single ingredient ER $(650 \mathrm{mg}$ formulations) and IR (325 and $500 \mathrm{mg}$ formulations) paracetamol products were identified. Exposures in adults aged $\geq 12$ years from 2007-2016 were included. Analysis compared level of healthcare facility (HCF) care, medical outcomes, and the report of AST or ALT $>1,000 \mathrm{U} / \mathrm{L}$ among $E R$ and $I R$ exposures involving $\geq 50 \mathrm{~g}$ of paracetamol. Analysis was restricted to single product acute exposures.

Results: Overall 11,868 ER and 147,621 IR paracetamol exposures were reported from 2007 to 2016, with 138 (1.2\%) ER and 1,811 (1.2\%) IR acute single product exposures involving $\geq 50 \mathrm{~g}$ of paracetamol. Among exposures involving $\geq 50 \mathrm{~g}$, median age was 27.0 years for ER and 25.0 years for IR exposures. Admission to a HCF occurred in $81.2 \%$ of ER and $79.5 \%$ of IR exposures. Death occurred in $<1 \%$ of ER and IR exposures (ER: $0.7 \%, n=1 ; I R: 0.8 \%, n=14$ ) but major effect (life-threatening medical outcome) occurred in $13.0 \%$ of ER compared to $8.7 \%$ of IR exposures. AST or ALT $>1,000$ $\mathrm{U} / \mathrm{L}$ was reported in $13.0 \%$ of ER and $10.5 \%$ of IR exposures.

Conclusion: Ingestions of $\geq 50 \mathrm{~g}$ of paracetamol occur infrequently among US poison center exposures involving ER or IR paracetamol. Most exposures of $\geq 50 \mathrm{~g}$ involved HCF admission regardless of formulation, but severe medical outcomes were slightly more common with ER compared to IR exposures. The similarities and differences of $E R$ and $I R$ exposures involving $\geq 50$ $\mathrm{g}$ should be further evaluated to determine if large doses of either product result in unpredictable absorption and increased outcome severity.

\section{Reference}

[1] Salmonson H, Sjoberg G, Brogren J. The standard treatment protocol for paracetamol poisoning may be inadequate following overdose with modified release formulation: a pharmacokinetic and clinical analysis of 53 cases. Clin Toxicol (Phila). 2018;56:63-8. 


\section{Loxoscelism: a case series from the Medical Toxicology Unit and Poison Control Centre of Florence Careggi University Hospital}

\author{
Angelo Rotulo ${ }^{\mathrm{a}}$, Alessandro Zotto ${ }^{\mathrm{a}}$, Marco Cirronis ${ }^{\mathrm{a}}$, \\ Amedeo Del Vecchio ${ }^{a}$, Francesco Gambassi ${ }^{b}$, \\ Emanuela Masini ${ }^{\mathrm{a}}$, Guido Mannaioni ${ }^{\mathrm{a}}$ and \\ Alessandra leri ${ }^{\mathrm{b}}$ \\ aDepartment of Neurofarba, Università degli Studi di Firenze, \\ Florence, Italy; ${ }^{\mathrm{b}}$ Medical Toxicology Unit and Poison Control \\ Center, Azienda Ospedaliero-Universitaria Careggi, Florence, Italy
}

Objective: There are several species of spider in Italy but spiders of medical importance include the widow spider (Latrodectus tredecimguttatus) and violin spider (Loxosceles rufescens). Epidemics of Loxosceles rufescens bites occur, especially during warmer months caused by increased human and spider activity. The venom of the violin spider contains toxins that cause necrosis and haemolysis responsible for dermo-necrotic wounds, sometimes with severe clinical presentation. Visceral-cutaneous loxoscelism is rare in Italy and results in systemic symptoms including asthenia, fever, malaise, a measles-like rash and, in severe cases, haemolysis, jaundice, rhabdomyolysis and disseminated intravascular coagulation (DIC) [1].

Methods: We studied 132 cases of suspected Loxosceles rufescens bite evaluated (by our observations) between January 2013 and June 2018 (Poison Centre telephone consultations and clinical visits). The case series was stratified by age, gender, spider identification and clinical presentation. The wound was evaluated following the typical clinical evolution: erythema, vesicles, ischemia and necrosis. The presence of systemic symptoms such as a measles-like generalized rash, fever, joint and muscular pain was carefully monitored until full recovery.

Results: Diagnosis was mainly presumptive because the bite is initially painless and identification of the spider is rare. It is based on epidemiological criteria, such as the presence of the spider in the geographical area, anamnesis, late onset of pain, clinical evolution of the wound. Out of 132 evaluated patients, diagnosis of loxoscelism was excluded in $73 \%(n=96)$, ascertained in $1 \%(n=1)$, probable in $1 \%(n=2)$ and possible in $25 \%(n=33)$ of the cases. Twenty-five percent out of ascertained/probable/possible patients had systemic symptoms, including one measles-like generalized rash. Treatment consisted of antibiotics, corticosteroids, antihistamines, low molecular weight heparin, and local care of necrotic wounds. The average healing time was 30.6 days (range 7-180 days).

Conclusion: The differential diagnosis with other clinical situations is difficult and may overrate the cases of necrotic arachnidism.

\section{Reference}

[1] Boissiere F, Masson R, Fluieraru S, et al. [Cutaneous loxoscelism, about an exceptional observation of 9 consecutive cases]. Ann Chir Plast Esthet. 2016;61:811-819. French

\section{Epidemiology and clinical aspects of mushroom-related poisonings in Italy: a 20-year retrospective study}

Francesca Assisi ${ }^{a}$, Franca Davanzo ${ }^{a}$, Maurizio Bissoli ${ }^{\mathrm{a}}$, Valeria Dimasi ${ }^{\mathrm{a}}$, Marcello Ferruzzi ${ }^{\mathrm{b}}$,
Joannhe Georgatos ${ }^{a}$, llaria Rebutti ${ }^{a}$, Angelo Travaglia ${ }^{a}$, Paolo Severgnini ${ }^{a}$, Fabrizio Sesana ${ }^{a}$, Giovanni Milanesi ${ }^{a}$, Paola Moro ${ }^{a}$ and Anna Celentano ${ }^{a}$

aPoison Control Center of Milan, ASST GOM Niguarda, Milan, Italy;

${ }^{\mathrm{b}}$ ASST GOM Niguarda, Milan, Italy

Objective: The study aims to analyze epidemiological data and clinical course of mushroom poisonings in Italy.

Methods: All cases of mushroom exposures were identified by querying the Milan Poison Control Centre (MPCC) database from 1998 to 2017. Information on age, gender, clinical effects, laboratory findings, outcomes and therapeutic measures were collected. Results: In the study period the MPCC handled 12,813 human cases of suspected poisoning due to mushroom ingestion. Of these $12 \%$ were aged $0-14,39 \%$ aged $15-50$ years and $31.2 \%$ aged $>51$ years. Age information was missing for $18.4 \%$. Most patients ( $n=7,969,62.2 \%$ ) developed at least one sign/symptom possibly related to the reported exposure; those presenting with delayed gastrointestinal symptoms (i.e. $\geq 6$ hours following ingestion) numbered 3,265 (25.5\%). Overall $75 \%$ of patients ingested unidentified mushrooms, while the species involved were identified by mycologists in 3,182 cases (24.8\%): Boletus gr. edulis $(\mathrm{n}=$ 904); Armillaria mellea $(n=658) ;$ Inocybe/Clitocybe $(n=296)$; Omphalotus olearius $(\mathrm{N}=197)$; Entoloma lividum $(\mathrm{n}=202)$; Clitocybe nebularis $(\mathrm{n}=100)$; Amanita pantherina, Amanita muscaria or Psilocybe $(n=300)$; Cortinarius orellanus $(n=33)$, Amanita proxima $(n=5)$; Amanita phalloides or amatoxin-containing species $(n=487)$. In 637 cases amatoxin poisoning was diagnosed based on mycological identification and/or detection of amatoxins in urine or the onset of hepatitis. Treatment suggested by MPCC for suspected amatoxin poisoning included: gastric lavage, repeated dose activated charcoal and forced diuresis; drugs of doubtful efficacy such as acetylcysteine, silymarin, and penicillin were not administered. The majority of cases showing delayed gastrointestinal symptoms fully recovered within a week; 19 patients required liver transplantation and 40 died. It is of note that all patients that received liver transplant or died arrived at the hospital with a delay of 2-4 days after ingestion. Five patients developed acute renal failure after the ingestion of Amanita proxima and 33 developed chronic kidney failure after Cortinarius orellanus.

Conclusion: In our case series, early gastric decontamination, administration of activated charcoal and forced diuresis seemed to improve patient outcome; late recovery of liver function is possible. The morbidity associated with $A$. phalloides is of public health concern and a nationwide advertising campaign about the risks of wild mushroom consumption could play an important role in preventing these potentially lethal intoxications. Mycological inspectors play a key role in the management of mushroom poisonings, since they provide expert assistance in rapid taxonomic identification of the suspected fungal species from leftovers of a meal. In cases of mushroom poisoning, analysis of suspect mushrooms is strongly advised.

\section{A model of big data linkage to monitor student emergency department visits with intoxication and associated risk markers in a US public university}

Duc Anh Ngo, Rege Saumitra and Christopher P Holstege

University of Virginia, Charlottesville, United States 
Objective: The objectives of this report are to describe the methodology for linking multiple student datasets with emergency department (ED) electronic medical records (EMR) to create an integrated dataset in order to monitor the burden of student alcohol intoxication and associated risk markers in a hospital ED affiliated with a US public university.

Methods: Student enrolment records were linked using student University Identification (UID) and academic term when the student enrolled with the two subsets of students: those who visited the university student health center and those who visited the university hospital ED due to alcohol intoxication identified using International Classification of Diseases (ICD) codes documented in EMR. Through this process, a comprehensive, integrated, de-identified dataset was created containing the full records of students linked to a subset of students who engaged in risky drinking that resulted in ED visits.

Results: Over 8 academic years (2009/10 to 2016/17), an integrated dataset was created that captured over 230,000 students linked to 12,450 subsequent ED visits of which $1,274(10.2 \%)$ were associated with alcohol intoxication. This dataset allows uncovering of the temporal trends in the incidence and prevalence of ED visits with alcohol intoxication in relation to a wide range of risk markers, including demographic and academic characteristics, organizational affiliation (fraternity member) and extracurricular activities (athletic scholarship), and clinical histories of those who visited the student health center clinic (e.g., depression, anxiety, injury). It also allows development of a robust risk prediction model to generate a risk score for every student at the time of enrolment in order to identify high risk students for timely referral to available outreach education and services.

Conclusion: Linking multiple student datasets provide a cost-efficient population-based data source to monitor student hazardous drinking associated with ED visits, reducing dependence on costly sample-based surveys. This initiative can serve as a data linkage model for replication in other universities, which enables a multicenter college surveillance, research, and risk screening for alcohol-related ED visits among the student population.

\section{Changes in patient demographics and poisoning severity after additional regulation of packaging and labelling for liquid laundry detergent capsules}

\author{
Patricia Casey, Feargal O'Connor and Edel Duggan \\ National Poisons Information Centre, Beaumont Hospital, Dublin, \\ Ireland
}

Objective: In an effort to protect young children, Commission Regulation (EU) No 1297/2014 introduced requirements for packaging of liquid laundry detergents contained in soluble packaging for single use. The Regulation applied from 1 June 2015 but products placed on the market before that date were not required to be relabelled and repackaged until 31 December 2015. This study compared patient age and poisoning severity during two time periods, before and after Regulation 1297 applied.

Methods: The National Poisons Information Centre prospectively collected data on enquiries about Irish patients exposed to liquid detergent capsules (LDCs) for the AISE Detergent Capsules "Accidentology" Project [1] during October 2014-April 2015 before Regulation 1297 applied (Group 1). Similar data were collected for an EU study of incidents involving LDCs [2] and the Procter \& Gamble product stewardship programme from October 2015 to September 2017, after the Regulation applied (Group 2). The hospital Research and Ethics Committee gave approval for all projects. For this paper, only patients under the age of 5 years who had ingested the product were included. Patients exposed by skin contact only, eye contact only or combined skin + eye contact were excluded from analysis. Patient ages and poisoning severity score (PSS) before and after the Regulation were compared. Chi-square test was used to compare proportions during the two periods.

Results: A larger proportion of patients in Group 2 were 2-4 years old $(p=0.01) ; 38$ of the 67 patients in Group 1 were less than 2 years old and 29 were aged 2-4 years and 151 of the 376 patients in Group 2 were less than 2 years old and 225 were 2-4 years old. Fewer patients in Group 2 were symptomatic $(p=$ 0.01): 47 patients (70\%) in Group 1 and 199 (53\%) in Group 2. The majority of symptomatic patients during both periods had a PSS of 1 (minor): $87.2 \%$ in Group 1 and $84.4 \%$ in Group 2.

Conclusion: After Regulation 1297, fewer children who ingested LDCs were less than 2 years old and fewer developed symptoms. This suggests that packaging changes made it more difficult for very young children to access these products while changes to the capsules themselves resulted in ingestion of a smaller amount of the detergent liquid.

\section{References}

[1] AISE. Detergent Capsules "Accidentology" Project Final Report [cited 2018 Oct 10]. Available from: https://www.aise.eu/documents/document/20151103103823-microsoft_word_-_detergent capsules_accidentology_-_final_report_2nov2015.pdf

[2] EU Commission. Study on hazardous detergents mixtures contained in soluble packaging for single use. Tender no 406/PP/ENT/ IMA/14/119429. Final Report [cited 2018 Oct 10]. Available from: http://ec.europa.eu/docsroom/documents/27018

\section{Documentation in the patient medical record improves adherence to poisons information centre telephone advice}

\author{
Carol J Wylie ${ }^{a}$, Genevieve H Messina ${ }^{a}$, \\ Danielle S Dean ${ }^{\mathrm{a}}$, Keith Harris ${ }^{\mathrm{b}}$, Colin B Page ${ }^{\mathrm{b}}$ and \\ Katherine Z Isoardi ${ }^{\mathrm{b}}$ \\ aPharmacy, Queensland Poisons Information Centre, Brisbane, \\ Australia; ${ }^{b}$ Emergency Department, Princess Alexandra Clinical \\ Toxicology Unit, Brisbane, Australia
}

Objective: There is little research examining adherence to specialist toxicological telephone advice. Available data suggests it is suboptimal [1]. Identified reasons include the advice being misinterpreted or deemed too complicated, compounded by the absence of documentation. This study aimed to determine if contemporaneous documentation in the medical record improves adherence to Poisons Information Centre (PIC) advice.

Methods: A prospective before and after observational study was conducted during the implementation of contemporaneous documentation of advice from Queensland Specialists in Poisons Information (SPI) into the patient's electronic record. Consecutive patient referrals to Queensland PIC from Emergency Department clinicians in hospitals using an electronic medical record were included in the study from October 2017 to September 2018. Adherence was independently assessed by two clinical toxicologists $(\mathrm{KI}$ and $\mathrm{KH}$ ) through the review of the PIC call report, PIC documentation (post-implementation), the patient's electronic record and follow-up telephone call, if performed. Adherence was designated to one of four categories; complete adherence, minor 
discrepancies not affecting patient care, major discrepancies affecting patient care and non-adherence. The primary outcome was the overall proportion of satisfactory (complete adherence, minor discrepancies) versus unsatisfactory (major discrepancies, non-adherence) adherence. Secondary outcomes include the accuracy of documented telephone advice by the receiving clinician, PIC recalls for the same patient episode and sub-optimal patient management. Sub-optimal patient management is defined as deviation from advice that results in omission of care or unnecessary treatment or observation.

Results: Overall 665 cases were included in the study, 347 in the pre-intervention phase and 318 in the post-intervention period. Documented call advice from the receiving clinician was absent in $40 / 347$ cases (12\%) and inaccurate in 40/307 (13\%). In cases where the PIC advice was documented, satisfactory adherence improved from $303 / 347(87 \%)$ to $299 / 318$ (94\%), p value 0.003 . Suboptimal management decreased from $48 / 347$ (14\%) to $14 / 318$ $(4 \%)$, p value $<0.0001$. Recalls regarding a patient's management were similar $42 / 347(12 \%)$ to $28 / 318(9 \%)$, $p$ value 0.21 . The median time to enter advice into the patient electronic record was 36 minutes (range 2-203 minutes). The most common reason for not being able to document the advice was that the SPI had a prohibitively heavy workload due to working a solo shift.

Conclusion: Contemporaneous documentation of PIC advice in the patient's electronic records improved adherence and reduced sub-optimal patient management.

\section{Reference}

[1] Becker T, Chiew A, Buckley N, et al. Poisons Information Centre Follow-up Study. Toxicology and Poisons Network Australasia (TAPNA) Scientific Meeting 2016, Brisbane.

\section{Psychological disturbances in adolescents with acute voluntary poisoning}

\section{Coriolan Ulmeanu, Dora Boghitoiu, Mihai Boscaiu, Alexandru Ulmeanu, Alexandru Baciu and Viorela Nitescu \\ Pediatric Poisoning Centre, Emergency Clinical Hospital for Children Grigore Alexandrescu, Bucharest, Romania}

Objective: To evaluate the psychological characteristics of adolescents with acute voluntary poisoning admitted to a pediatric toxicology unit.

Methods: We describe all patients with the diagnosis of acute voluntary poisoning, admitted in our unit between 1 October 2017 and 30 September 2018. The following criteria were taken into consideration: age, gender, number of hospitalizations with this diagnosis and psychological evaluation done during hospitalization.

Results: Overall 181 patients with acute voluntary poisoning were admitted in our department during the study period. Mean age was 15.4 years. With regards to gender, there was a similar percentage of girls compared to boys ( $58.1 \%$ versus $41.9 \%)$. The majority of patients $(n=137,75.7 \%)$ were from urban areas. Most adolescents involved were aged $15-17$ years (68.5\%). There were 22 patients $(12.1 \%)$ with two or more hospitalizations. In 13 cases $(7.2 \%)$ the patients were known to have a psychiatric disturbance: depression in 8 patients, conduct disorder in 2 adolescents and chronic consumption of substances of abuse in 3 patients. The main psychological disturbance was emotional disturbance and was noted in 98 patients $(54.1 \%)$ (Table 1). Suicide
Table 1. Psychological disturbances in adolescents with acute voluntary poisoning admitted to a pediatric toxicology unit over a one year period.

\begin{tabular}{lcc}
\hline Psychological disturbances & $\begin{array}{c}\text { Number of } \\
\text { patients }(\mathrm{n}=181)\end{array}$ & Percentage \\
\hline Emotional disturbance & 98 & $54.1 \%$ \\
Depression & 30 & $16.8 \%$ \\
Chronic consumer of substances of abuse & 14 & $7.7 \%$ \\
Behavioral disorder & 13 & $7.2 \%$ \\
Conduct disorder & 11 & $6.1 \%$ \\
Adaptation disorder & 4 & $2.2 \%$ \\
Borderline personality & 4 & $2.2 \%$ \\
Panic attacks & 2 & $1.1 \%$ \\
Anxiety disorder & 2 & $1.1 \%$ \\
Mood disorder & 2 & $1.1 \%$ \\
Conversion disorder & 1 & $0.5 \%$ \\
\hline
\end{tabular}

attempts were identified in 31 patients (17.1\%) and the recommendation for transfer to a psychiatric clinic was given for only 10 patients (18.1\%)

Conclusion: Emotional disturbance was the main psychological disturbance noted in adolescents with acute voluntary poisoning. A small number of patients with acute voluntary poisoning had a pre-existing psychiatric disease or were registered with this diagnosis twice or more. The term voluntary ingestion is not equivalent to suicidal attempt, this being a serious entity and rarely diagnosed.

\section{Death on demand: public debate leads to increasing use of "suicide powders"}

\author{
Antoinette JHP Van Riel ${ }^{a}$, Anja PG Wijnands-Kleukers ${ }^{a}$, \\ Douwe Dekker ${ }^{\mathrm{b}}$, Irma De Vries ${ }^{\mathrm{a}}$ and \\ Dylan W De Lange ${ }^{c}$ \\ a Dutch Poisons Information Center, University Medical Center, \\ Utrecht University, Utrecht, Netherlands; ${ }^{\text {b}}$ Dutch Poisons \\ Information Center, Department of Internal Medicine, University \\ Medical Center, Utrecht University, Utrecht, Netherlands; ${ }^{\mathrm{C}}$ Dutch \\ Poisons Information Center, Intensive Care Unit, University Medical \\ Center, Utrecht University, Utrecht, Netherlands
}

Objective: In the fall of 2017 there was a public debate in the Netherlands about the activities of the Cooperation Last Will (CLW). CLW is an association that aims to facilitate its members to end their life in an allegedly "humane" way, without reference to a medical professional. In September 2017 CLW issued a pressrelease stating they had found a, quote: "safe and humane means for its members that is legally available and leads to a worthy death in a small amount". CLW elaborated on its use as a preservative agent and on the merciful nature of the effects, when used to commit suicide, however the actual substance was not disclosed. This triggered speculation in the media on possible candidates. Sodium nitrite $\left(\mathrm{NaNO}_{2}\right)$ and sodium azide $\left(\mathrm{NaN}_{3}\right)$ have been mentioned most frequently since. We aimed to investigate the effect of the media attention for $\mathrm{NaNO}_{2}$ and $\mathrm{NaN}_{3}$ on the number of inquiries to the Dutch Poisons Information Center (DPIC).

Methods: We analyzed the number of intentional ingestions to $\mathrm{NaNO}_{2}$ and $\mathrm{NaN}_{3}$ reported to the DPIC from 2013 until 15 September 2018 and compared the 4.7 year period before the press release by CLW with the first year after this press release.

Results: In the 4.7 years before the press release we received no inquiries about intentional $\mathrm{NaNO}_{2}$ ingestion. In the year after the press release we received 13 such inquiries. In 6 cases with intentional $\mathrm{NaNO}_{2}$ ingestion, the amount ingested was unknown, in the other 7 cases it varied from 0.216 to $20 \mathrm{~g}$. One person died 
(amount unknown), at least 6 received hospital treatment, including methylene blue administration to treat methemoglobinemia. For $\mathrm{NaN}_{3}$ we received 2 inquiries in the 4.7 years before the press release and 3 in the year after the press release. All 5 cases of intentional $\mathrm{NaN}_{3}$ ingestion were lethal, the ingested amounts were unknown.

Conclusion: Following media speculation about $\mathrm{NaNO}_{2}$ and $\mathrm{NaN}_{3}$ as suicide powders, the number of intentional self-poisonings with these agents reported to the DPIC increased. $\mathrm{NaN}_{3}$ appears to be far more lethal than $\mathrm{NaNO}_{2}$. For preventive reasons, the DPIC did not comment on this issue in the media. With the growing social need for self-determination, including directing one's own death, poisons centers should expect an increasing number of questions about substances like $\mathrm{NaNO}_{2}$ and $\mathrm{NaN}_{3}$. Governmental prohibition of these substances, that have many common applications, is impractical and unenforceable.

\section{The role of extracorporeal membrane oxygenation (ECMO) in poisoning: a 10-year review of enquiries made to the UK National Poisons Information Service (NPIS) 2008-2018}

\author{
Pardeep S Jagpal ${ }^{\mathrm{a}}$, Hayley A Williams ${ }^{\mathrm{a}}$, \\ Muhammad EMO Elamin ${ }^{\mathrm{a}}$, Euan A Sandilands ${ }^{\mathrm{b}}$, \\ Simon HL Thomas ${ }^{c}$, John P Thompson ${ }^{d}$ and \\ Sally M Bradberry ${ }^{a}$ \\ ${ }^{a}$ National Poisons Information Service (Birmingham Unit), City \\ Hospital, Birmingham, United Kingdom; ${ }^{b}$ National Poisons \\ Information Service (Edinburgh Unit), Royal Infirmary, Edinburgh, \\ United Kingdom; 'National Poisons Information Service (Newcastle \\ Unit), Regional Drug and Therapeutics Centre, Newcastle, United \\ Kingdom; ${ }^{\mathrm{d}}$ National Poisons Information Service (Cardiff Unit), \\ University Hospital Llandough, Cardiff, United Kingdom
}

Objective: To investigate the incidence and circumstances in which extracorporeal membrane oxygenation (ECMO) was discussed during enquiries to the UK National Poisons Information Service (NPIS).

Methods: A retrospective analysis of UK NPIS enquiries between 1 January 2008 and 31 July 2018 was undertaken for enquiries containing the terms "ECMO" or "extracorporeal membrane oxygenation". Only cases where ECMO was referred to in the context of patient management were included in the subsequent review. Results: The search retrieved 227 enquiries of which 207, involving 182 patients fulfilled the inclusion criteria. There were 163 adults and 19 children ( $\leq 16$ years of age). Enquiries increased from one in 2008 to 19 in 2014 and 68 in 2017. Advice from a consultant clinical toxicologist was sought in 180 of 182 cases. The poisoning severity score (PSS) [1] prior to, or at the time of enquiry, was severe (PSS3) in 169 (93\%), moderate (PSS2) in 12 $(6.5 \%)$ and minor (PSS1) in $1(0.5 \%)$ patients. All enquiries with a PSS <3 involved ingestion of cardiotoxic agents and were referred to a consultant. Polypharmacy ingestions $(n=111)$ involved up to 14 agents; the three most common drug classes being calcium channel blockers $(n=46)$, beta-blockers $(n=39)$ and selective serotonin reuptake inhibitors $(n=36 ; 21$ were citalopram). Seventy-one exposures involved a single agent, most commonly beta-blockers $(n=12)$, recreational drugs $(n=10)$ or calcium channel blockers $(n=9)$. Of 182 patients, 20 (10 polypharmacy and 10 single agent poisonings) underwent ECMO, six at the admitting hospital. In 3 cases ECMO had already been initiated at the time of the initial enquiry while in 17 patients ECMO was commenced following discussion with the NPIS. Reasons why ECMO was not instituted were not routinely collected, though 57 patients were considered too unstable for the procedure or for transfer to an ECMO centre. Follow up was undertaken in 165 of 182 cases (90\%), with outcome data available in 141. Of the 20 ECMO patients: 9 died, 9 survived and the outcome was unknown in 2. Of the 123 non-ECMO cases with a known outcome there were 60 deaths and 63 survivors.

Conclusion: Enquiries to the NPIS involving discussion of ECMO are increasing. Further research on the role and efficacy of ECMO in poisoning is needed and poisons centres need to be aware of available ECMO facilities and the criteria for referral.

\section{Reference}

[1] Persson HE, Sjöberg GK, Haines JA, et al. Poisoning severity score. Grading of acute poisoning. J Toxicol Clin Toxicol. 1998;36:205-213.

\section{Three paediatric poisonings treated with extracorporeal membrane oxygenation (ECMO): the UK National Poisons Information Service (NPIS) experience 2008-2018}

\author{
Pardeep S Jagpal ${ }^{\text {a }}$, Muhammad EMO Elamin ${ }^{\text {a }}$ \\ Euan A Sandilands ${ }^{\mathrm{b}}$, Simon HL Thomas ${ }^{\mathrm{c}}$, \\ John P Thompson ${ }^{d}$ and Sally M Bradberry ${ }^{\mathrm{a}}$ \\ ${ }^{a}$ National Poisons Information Service (Birmingham Unit), City \\ Hospital, Birmingham, United Kingdom; ${ }^{\text {bNational Poisons }}$ \\ Information Service (Edinburgh Unit), Royal Infirmary, Edinburgh, \\ United Kingdom; ' National Poisons Information Service (Newcastle \\ Unit), Regional Drug and Therapeutics Centre, Newcastle, United

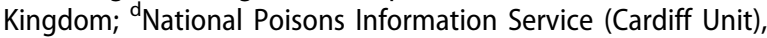 \\ University Hospital Llandough, Cardiff, United Kingdom
}

Objective: We report all enquiries of paediatric poisoning involving ECMO received by the UK NPIS over 10 years. All three patients died.

Case series: Case 1. A 15-year-old male was admitted with Glasgow Coma Score (GCS) 3, hyperpyrexia $\left(40^{\circ} \mathrm{C}\right)$, hypotension (mean arterial pressure [MAP] $30 \mathrm{mmHg}$ ) and metabolic acidosis $(\mathrm{pH} 6.96$ and lactate $18 \mathrm{mmol} / \mathrm{L})$ after collapsing in a park. He sustained a cardiac arrest. Following return of output he was commenced on ECMO and extracorporeal renal support 10 hours after presentation. NPIS were consulted seven hours later to assist with the diagnosis. Amfetamine poisoning was suggested. The patient died the following day from multi-organ failure and postmortem toxicology confirmed ecstasy use. Case 2. An 11-monthold female ingested $5 \mathrm{~mL}$ of ear wax remover (33\% camphor), and presented in respiratory distress with GCS 3 . Capillary gas analysis showed $\mathrm{pH} 6.98, \mathrm{pCO}_{2} 10.4 \mathrm{kPa}$, base excess -15 and lactate $5.8 \mathrm{mmol} / \mathrm{L}$. The child was intubated, commenced on oscillatory ventilation for pulmonary oedema and required inotropic support. The NPIS was consulted at 2.5 hours post-ingestion to advise on the suitability of ECMO, which was commenced 5 hours post-ingestion. Two days post-ingestion chest $\mathrm{X}$-ray revealed "complete white out". A head computerised tomography (CT) scan on day 5 revealed three right-sided infarcts and the patient died 9 days post-ingestion. Case 3. A 15-month-old male ingested an unknown amount of turpentine (suspected to be turpentine oil). At the time of enquiry (3 hours post-ingestion), chest $\mathrm{X}$-ray revealed pulmonary oedema with chemical pneumonitis. Arterial blood gas showed $\mathrm{pH} 7.1, \mathrm{pCO}_{2} 7.5 \mathrm{kPa}$, bicarbonate $17.2 \mathrm{mmol} /$ $\mathrm{L}$, base excess -12.6 , and lactate $3.7 \mathrm{mmol} / \mathrm{L}$. Despite improved oxygenation on oscillatory ventilation, the patient became 
hypotensive with deranged renal and clotting function. ECMO was commenced 2 days post-ingestion. Numerous infarcts were present on brain CT 4 days post-ingestion and the patient died 2 days later.

Conclusion: ECMO use in poisoning as a "bridge" to recovery or replacement is often unsuccessful. Further research is needed to establish patient subgroups most likely to benefit. Poisons centres need to be aware of the availability and indications for ECMO in paediatric poisoning.

\section{Clinical utility of extracorporeal membrane oxygenation (ECMO) in poisoned patients with cardiovascular collapse: a retrospective study of Extracorporeal Life Support
Organization's ECMO registry}

\author{
Lindsay Weiner ${ }^{a}$, Michael Mazzeffi ${ }^{b}$, Elizabeth Q Hines \\ ${ }^{c}$, Katherine Prybys ${ }^{a}$, Daniel L Herr ${ }^{d}$ and Hong K Kim ${ }^{a}$ \\ ${ }^{a}$ Emergency Medicine, University of Maryland Medical Center, \\ Baltimore, United States; ${ }^{\mathrm{b}}$ Anesthesiology, University of Maryland

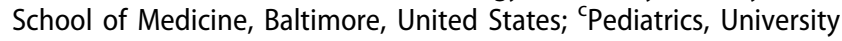 \\ of Maryland School of Medicine, Baltimore, United States; \\ ${ }^{\mathrm{d}}$ Medicine, University of Maryland School of Medicine, Baltimore, \\ United States
}

Objective: Acute overdose of cardiovascular and membrane stabilizing agents results in significant morbidity and mortality from cardiovascular failure. Extracorporeal membrane oxygenation (ECMO) support is increasingly utilized as salvage therapy in refractory cardiovascular collapse, however, there is limited evidence regarding ECMO's clinical utility in poisoned patients. Therefore, we investigated the survival benefit of ECMO support in cardiogenic shock due to acute poisoning.

Methods: We conducted a retrospective study utilizing the Extracorporeal Life Support Organization's (ELSO) ECMO registry. The ELSO registry was systematically searched using International Classification of Diseases (ICD)-9/10 codes for poisoning (960-989 and T36-T65, respectively) from 1 January 2003 to 30 July 2018. The registry contains patient demographic and clinical data (e.g. hemodynamics, acid/base status, etc.) before and 24 hours after ECMO support. Cases with cardiogenic shock, defined as systolic blood pressure $<90 \mathrm{mmHg}$ or mean arterial pressure (MAP) $<65$ $\mathrm{mm} \mathrm{Hg}$, that received veno-arterial (VA) ECMO were included in the study. The primary outcome was survival rate; secondary outcomes included changes in acid/base, oxygenation, and hemodynamics status, and complications related to ECMO

Table 1 Hemodynamic and metabolic parameters before and 24 hours after ECMO in 104 poisoned patients with cardiovascular collapse.

\begin{tabular}{llll}
\hline Variable & $\begin{array}{c}\text { Pre-ECMO } \\
\text { median, [IQR] }\end{array}$ & $\begin{array}{c}\text { Post-ECMO } \\
\text { median, [IQR] }\end{array}$ & p-value \\
\hline pH & $7.21[7.10,7.32]$ & $7.38[7.27,7.45]$ & $<0.0001$ \\
pO2 $(\mathrm{mm} \mathrm{Hg})$ & $110[62,225]$ & $168[92,247]$ & 0.004 \\
pCO2 $(\mathrm{mm} \mathrm{Hg})$ & $49[38,60]$ & $38[34,68]$ & 0.06 \\
HCO3 & $16[13,22]$ & $22[18,26]$ & $<0.0001$ \\
MAP $(\mathrm{mm} \mathrm{Hg})$ & $51[45,65]$ & $70[65,80]$ & $<0.0001$ \\
SBP $(\mathrm{mm} \mathrm{Hg})$ & $77[70,90]$ & $96[88,110]$ & $<0.0001$ \\
DBP $(\mathrm{mm} \mathrm{Hg})$ & $45[39,52]$ & $60[55,68]$ & $<0.0001$ \\
SpO2 $(\%)$ & $96[87,99]$ & $99[96,100]$ & 0.0004 \\
SvO2 $(\%)$ & $48[26,51]$ & $75[71,83]$ & 0.005 \\
\hline
\end{tabular}

Notes: p02: partial pressure of oxygen; pCO2: partial pressure of carbon diox ide; MAP: mean arterial pressure; SBP: systolic blood pressure; DBP, diastolic blood pressure; SpO2: peripheral capillary oxygen saturation; SvO2: mixed venous oxygen saturation. support. Descriptive analysis and comparison of median was performed.

Results: Overall 113 cases were identified from the ELSO registry; 9 cases were excluded, as cardiogenic shock was not due to poisoning, leaving 104 for analysis. Of these $53.5 \%$ were male with a median age of 34 years. Cardiovascular agents were responsible for cardiogenic shock in $47.1 \%(n=49) ; 34$ patients experienced pre-ECMO cardiac arrest. Norepinephrine (83.7\%), epinephrine (67.4\%), and dobutamine (44.6\%) were the most commonly used vasopressors for hemodynamic support. Median duration on VAECMO was 68 hours. Statistically significant improvements in the hemodynamic and metabolic parameters were noted due to ECMO (Table 1); $53.4 \%(n=55)$ of cases survived and were discharged home. Renal replacement therapy and arrhythmia were the most frequently reported complications.

Conclusion: VA-ECMO support can improve survival in acutely poisoned patients with cardiogenic shock.

\section{7-months-old: kind of blue}

\author{
Serena Ascione ${ }^{a}$, Margherita Rosa ${ }^{b}$, Andrea Apicella ${ }^{b}$, \\ Paolo Raimondo ${ }^{b}$, Eduardo Ponticiello ${ }^{b}$ and \\ Vincenzo Tipo ${ }^{c}$ \\ aDepartment of Medical Translational Sciences, "Federico II"

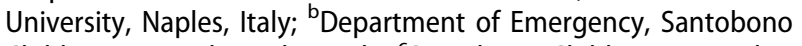 \\ Children Hospital, Naples, Italy; 'Santobono Children Hospital, \\ Naples, Italy
}

Objective: Central cyanosis results from a variety of conditions involving pulmonary, cardiac, hematological or central nervous system etiologies. It is essential to comprehend the pathophysiology of hypoxemia and a careful history taking, physical examination, and focused laboratory evaluation are helpful in diagnosis. We report a case of cyanosis in an infant.

Case report: A 7-month-old male presented with blue skin and lips and irritability. His mother reported several episodes of vomiting during feeding, one hour earlier. Vital parameters were oxygen saturation $92 \%$, heart rate $100 \mathrm{bpm}$, respiratory rate $30 \mathrm{bpm}$, capillary refill time $<2 \mathrm{~s}$, temperature $36^{\circ} \mathrm{C}$ with warm extremities and Pediatric Glasgow Coma Score 13. There were no signs of lung or heart disease, no heart murmurs, arrhythmia or preload signs. Oxygen saturation was unchanged with positive pressure ventilation $\left(\mathrm{FiO}_{2}\right.$ 60\%). Chest $\mathrm{X}$-ray and cardiologic consult excluded organ involvement. A secondary assessment revealed an otherwise healthy child with no history of drug administration. The vomiting had started after a spinach puree meal, cooked the day before and kept in the refrigerator. Arterial blood was a chocolate color and revealed $\mathrm{pH} 7.38, \mathrm{PaO}_{2} 90 \mathrm{mmHg}, \mathrm{PaO}_{2} / \mathrm{FiO} 2$ 150 and $\mathrm{PCO}_{2} 25 \mathrm{mmHg}$. Using the Masimo multi-wavelength pulse oximeter we found a methemoglobinemia (MetHb) of $25 \%$, which was also confirmed with laboratory analysis. A diagnosis of acquired methemoglobinemia was made. Methylene blue was given by slow IV injection $(1 \mathrm{mg} / \mathrm{Kg})$. In 20 seconds the MetHb fell to $<1 \% ; \mathrm{SpO}_{2}$ fell from $92 \%$ to $77 \%$ for about a minute and then became stable at $98 \%$ on room air, and the cyanosis resolved. The child was discharged after 24 hours. Methylene blue has a high peak absorption at $660 \mathrm{~nm}$ and a pulse oximeter usually measures non-oxygenated hemoglobin resulting in false desaturation.

Conclusion: Methemoglobinemia is a rare cause of cyanosis in children and must be considered when there is no cardiopulmonary involvement. The primary form has an earlier onset $(<4$ months) and is associated with low-birth weight, dehydration, acidosis and diarrhea, and was ruled out in this case. Secondary methemoglobinemia was promptly recognized as linked to spinach ingestion, due to nitrite production [1]. Supportive treatment with IV methylene blue lead to immediate reduction in MetHb and clinical improvement. The multi-wavelength pulse oximeter 
was a helpful non-invasive tool for diagnosis. The case presented two well-known phenomena, "saturation gap" (non-correspondence between $\mathrm{SpO}_{2}$ and $\mathrm{PaO}_{2}$ ) and paradoxical reduction in $\mathrm{SpO}_{2}$ when administering methylene blue [2].

\section{References}

[1] Sanchez-Echaniz J, Benito-Fernández J, Mintegui-Raso S Methemoglobinemia and consumption of vegetables in infants. Pediatrics. 2001;107:1024-1028.

[2] Cortazzo JA, Lichtman AD. Methemoglobinemia: a review and recommendations for management. J Cardiothorac Vasc Anesth. 2014;28:1043-1047.

\section{Teenagers and self-poisoning in a pediatric emergency department}

\author{
Virna Carmellino ${ }^{a}$, Claudia Bondone ${ }^{\mathrm{a}}$, \\ Andrea Vermena ${ }^{a}$, llaria Bergese ${ }^{a}$, Emanuela Garrone ${ }^{a}$, \\ Sonia Aguzzi ${ }^{\mathrm{a}}$, Francesca Bosetti ${ }^{\mathrm{b}}$ and \\ Antonio F Urbino ${ }^{\mathrm{a}}$ \\ aDepartment of Pediatric Emergency, Regina Margherita Children's \\ Hospital, AOU Città della Salute e della Scienza di Torino, Torino, \\ Italy; ${ }^{b}$ Pediatric Department, Cardinal Massaia Hospital, Asti, Italy
}

Objective: Suicide is a relatively rare event in children, however its prevalence increases significantly throughout adolescence [1]. The aim of this work is to investigate intentional poisoning with the purpose of attempted suicide in patients aged 0-18 years admitted to the Regina Margherita Children's Hospital, AOU Città della Salute e della Scienza di Torino, Turin.

Methods: We analysed data on all patients aged 0-18 years admitted in the hospital for voluntary intoxication from January 2012 to August 2018. Epidemiological and demographic information were collected.

Results: Overall there were 67 patients ( 11 males and 56 females) admitted to the Emergency Department (ED) due to voluntary intoxication. The average age of the patients was 14 years. The triage code assigned was yellow (urgent) in $14.9 \%$ cases, and in the others was red (emergency) due to the critical condition of the patient. More than half of patients (77.6\%) were receiving psychological support before the event, and for $56 \%$ of children this was not the first suicide attempt. The most common substances taken were psychotherapeutic drugs (37.3\%), non-steroidal anti-inflammatory drugs (NSAIDs) and paracetamol (23.8\%), liquid caustics (22.3\%) and other drugs (16.6\%). In $37.3 \%$ of the episodes more than one toxic agent was used. As previously reported [2] psychological problems, family and school conflicts, and eating disorders were the most important triggers for voluntary intoxication. There were no deaths in the ED, but a 14-year-old diabetic patient died in the intensive care unit after intentional under-dosage of insulin.

Conclusion: Future goals for the prevention of attempted suicide in teenagers should involve recognition and prompt handling of unstable situations to decrease the incidence of fatal events among teenagers.

\section{References}

[1] Kõlves K, de Leo D. Suicide methods in children and adolescents. Eur Child Adolesc Psychiatry. 2017;26:155-164.

[2] Nistor N, Jitareanu C, Frasinariu OE, et al. Epidemiologic profile and triggering factors of voluntary poisoning in teenagers. Medicine (Baltimore). 2017;96:e5831.

\section{Unintentional clozapine ingestion in two siblings due to a pharmacy dispensing error}

\author{
Madeline H Renny ${ }^{\mathrm{a}}$, Rana Biary ${ }^{\mathrm{a}}$, Mary Ann Howland ${ }^{\mathrm{a}}$ \\ and Mark K Su ${ }^{\mathrm{b}}$ \\ ${ }^{a}$ Ronald O. Perelman Department of Emergency Medicine, NYU \\ School of Medicine, New York, United States; ${ }^{b}$ New York City \\ Poison Control Center, Department of Health and Mental Hygiene, \\ New York, United States
}

Objective: Clozapine is a second-generation antipsychotic medication used to treat refractory schizophrenia. There are limited reports of confirmed clozapine ingestions in young children. We report a case of two siblings who ingested clozapine due to a pharmacy dispensing error; both recovered with supportive care.

Case report: A 5-year-old girl and her 19-month-old sister, both previously healthy, presented to the emergency department (ED) around $10 \mathrm{pm}$ with lethargy and confusion soon after they both took their first evening dose of what was believed to be $200 \mathrm{mg}$ cimetidine, newly prescribed to treat molluscum contagiosum. In the ED, they were both tachycardic, but otherwise had ageappropriate vital signs. On physical examination, both children were noted to be lethargic and drooling with roving eye movements. The older sibling was also confused and agitated at times with abnormal arm movements. Both children were observed for four hours and discharged home. The 19-month-old remained somnolent, but returned to baseline the following afternoon. The 5 -year-old was persistently lethargic and confused the following day, with some improvement 36 hours post-ingestion. Their mother was in close contact with their pediatrician during this time. Given the persistence of symptoms inconsistent with cimetidine, the mother examined the tablets in the prescribed bottle and through a pill identifier, identified the tablets as 200 $\mathrm{mg}$ clozapine. Both children were seen in the pediatrician's office on day two and day three post-ingestion with normal electrocardiograms and normal complete blood counts on day three. About 85 hours post-ingestion the older sibling's serum clozapine concentration was reported as $17 \mu \mathrm{g} / \mathrm{L}$ and norclozapine concentration as $55 \mu \mathrm{g} / \mathrm{L}(25-400 \mu \mathrm{g} / \mathrm{L})$. The older sibling returned to her baseline 4 days post-ingestion. When the dispensing error was reported to the pharmacy, it was discovered that the bottles of cimetidine and clozapine had been placed next to one another on the shelf in the pharmacy. Further investigation into the error was undertaken and as a preliminary safety measure, clozapine was moved to a more secure location in the pharmacy. Three months post-exposure, both children were healthy with no sequelae noted on follow-up.

Conclusion: We describe two children who ingested clozapine and developed drooling and altered mental status. It took several days for the children to return to their baseline mental status, but complete recovery occurred with supportive care. Efforts should be taken to reduce pharmacy dispensing errors that can lead to serious toxicity in children.

\section{Severe intoxication with aspiration in a toddler after ingestion of diluted benzalkonium chloride, a common quaternary ammonium compound}

\author{
Johanna Nordmark Grass and Emma Petersson
}

Swedish Poisons Information Centre, Stockholm, Sweden 
Objective: Benzalkonium chloride (BZC), a quaternary ammonium compound (QAC), is a surfactant commonly used as a disinfectant or algaecide. Solutions with concentrations of $10 \%$ or higher are known to cause local corrosive injuries. Systemic effects such as impaired consciousness, hypoxia, hypotension, muscle paralysis and metabolic acidosis have been described and appear to be dose-dependent [1]. Here a case with severe systemic effects and corrosive injuries after ingestion of a diluted solution of BZC is presented.

Case report: A previously healthy, 1.5 -year-old boy accidentally ingested an unknown amount of BZC solution. The original product had a concentration of $8 \%$ and then was diluted (end-concentration unknown). He immediately started to vomit repeatedly. One hour later he arrived at the hospital. He was hypoxic, oxygen saturation $90 \%$ (on oxygen, flow rate $10 \mathrm{~L}$ ) with stridor. Blood pressure was $80 / 50 \mathrm{mmHg}$ and heart rate 150 beats/ minute. He subsequently lost consciousness and muscle tone. Thirty minutes after admission he was intubated. Acute esophagoscopy and bronchoscopy were performed revealing corrosive mucous membrane injuries in the ventricle and esophagus. In addition the pharynx, carina and the left main bronchus had corrosive changes and there were profuse secretions in the lungs. During the first few hours after admission he needed repeated boluses of intravenous Ringer's acetate to maintain an adequate blood pressure. Laboratory tests were notable for an increased troponin- $\mathrm{T}(50.1 \mathrm{ng} / \mathrm{L})$ indicating myocardial damage. A pulmonary computerised tomography (CT) scan on day four showed bilateral consolidations. After a week the boy was extubated. His corrosive injuries eventually healed and after 22 days he was discharged home, completely recovered.

Conclusion: This exposure resulted in both severe local injuries and systemic effects in spite of the low concentration of the BZC solution. The pulmonary injuries confirmed aspiration had occured. Only a few household products marketed in Sweden are dangerous in moderate doses. Products containing QACs are among these, but have been considered to be relatively safe if sufficiently diluted. However, the presented case makes that assumption questionable and calls for healthcare providers to be vigilant when handling patients after QAC ingestion, irrespective of the concentration of the solution. It is also of utmost importance that these products are kept in child-safe containers.

\section{Reference}

[1] Van Berkel $M$, de Wolff FA. Survival after acute benzalkonium chloride poisoning. Hum Toxicol. 1988;7:191-93.

\section{Does a toxic dose of clarithromycin predispose to Kounis Syndrome in children? A case report}

\author{
Marta Crevani ${ }^{\mathrm{a}}$, Mara Pisani ${ }^{\mathrm{b}}$, Elena Bellelli ${ }^{\mathrm{b}}$, \\ Carla Olita $^{\mathrm{b}}$, Tatiana Federici ${ }^{\mathrm{b}}$, Andrea Deidda ${ }^{\mathrm{b}}$, \\ Marco Maranoc, Francesco P Rossi ${ }^{d}$ and \\ Antonino Reale ${ }^{\mathrm{b}}$

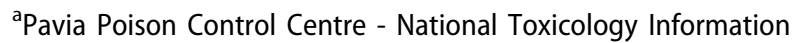 \\ Centre - Clinical and Experimental Lab, Toxicology Unit, Maugeri \\ Clinical and Scientific Institutes IRCCS and University of Pavia, \\ Pavia, Italy; ${ }^{b}$ Emergency Department, IRCCS Bambino Gesù

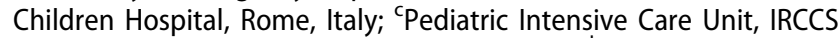 \\ Bambino Gesù Children Hospital, Rome, Italy; ${ }^{\mathrm{d} E m e r g e n c y}$ \\ Department, IRCCS Bambino Gesù Children Hospital, Rome, Italy
}

Objective: Kounis Syndrome (KS) is defined as the concurrence of angina or acute coronary syndrome following mast-cell and platelet activation in the setting of an allergic or an anaphylactic insult [1]. The most common triggers are antibiotics (particularly beta-lactams) and insect bites. Cases occurring with clarithromycin in adults are reported in literature [2]. We present a case report of $\mathrm{KS}$ in a child after an accidental overdose of clarithromycin.

Case report: A 5-year-old girl $(16 \mathrm{~kg})$ was admitted to our pediatric Emergency Department (ED) at $6 \mathrm{pm}$ because she had accidentally been given $880 \mathrm{mg}$ of clarithromycin syrup about 20 hours before. Her family medical history was unknown. She had no history of cardiac dysfunction. During the night she has complained of stomach-ache, still present at the ED evaluation. Her physical examination and vital signs were normal. An electrocardiogram (EKG) was performed because of the risk of cardiotoxicity with clarithromycin, It showed negative $T$ waves in leads $V 1$, V2, V3, V4, V5 absent on a previous electrocardiogram performed in June 2016. Both echocardiogram and high-sensitive troponins were normal. There was no apparent reason for coronary spasm (atherosclerosis, smoking, chemical exposure, energy drinks, cocaine or any other stimulant drugs). The following morning her clinical condition was stable and a second EKG was repeated. It showed a complete normalization of the T-wave inversion in V4 and V5 leads. Laboratory analyses were normal. She was then diagnosed as KS type one variant, secondary to clarithromycin ingestion. She was discharged asymptomatic the day after admission, with a clinical follow-up.

Conclusion: KS must be suspected in the presence of allergic symptoms and/or EKG abnormalities in patients with undergoing triggers (antibiotics, insect bites). This is the first case reported in a child after clarithromycin ingestion. KS may be triggered in predisposed patients, by high doses of this drug. Detecting this syndrome is crucial in patients with coronary spasm in order to prevent recurrent acute coronary syndromes and unexpected cardiac arrest.

\section{References}

[1] Kounis NG, Zavras GM. Histamine-induced coronary artery spasm: the concept of allergic angina. Br J Clin Pract. 1991;45:121-8.

[2] Bilgin $M$, Akyel $A$, Doğan $M$, et al. Acute coronary syndrome secondary to clarithromycin: the first case and review of the literature. Turk Kardiyol Dern Ars. 2014;42:461-3.

\section{Cannabinoid intoxication: experience in an Italian Pediatric Emergency Department}

\author{
Eduardo Ponticiello, Laura Ruggiero, Vincenzo Tipo, \\ Onorina Di Mita and Margherita Rosa \\ Emergency Department, AORN Santobono-Pausilipon, Naples, Italy
}

Objective: The aim of our study was to conduct an epidemiological investigation on acute cannabinoid poisoning in a highly specialized urban pediatric emergency department.

Methods: We retrospectively collected the clinical notes of patients aged 0-14 years attending our Pediatric Emergency Department from April 2016 to November 2017. The diagnosis of cannabinoid poisoning in all cases was confirmed by a urine toxicological screening kit using a rapid flow immunochromatographic technique, based on the principle of competitive binding. 
Results: Among all the poisoning cases, cannabinoid intoxication was found to have a prevalence of $1.2 \%$. We registered 10 admissions for acute cannabinoid poisoning (mean age of the patients $4.9 \pm 4.8$ years), of which 7 were due to accidental ingestion and 3 to voluntary consumption. The mean age of patients with accidental intoxication was $2.2 \pm 2.2$ years while the mean age of the 3 patients with intentional use was $11.3 \pm 1.5$ years. The diagnosis in all 10 cases was suspected on the basis of the clinical picture and on the anamnestic data. The only case studies referring to Italian pediatric population are that of the Centro Antiveleni di Pavia [1], that retrospectively evaluated (from January 2007 to November 2014) the incidence of accidental cannabinoid intoxication in patients aged 0-5 years. In this study 10 cases were also collected, but we detected the same number of cases in a significantly short period of observation (19 months versus 94 months).

Conclusion: Our data suggest that this kind of intoxication has become more frequent in the pediatric population compared to previous studies and it is crucial that healthcare workers in pediatrics consider this intoxication, especially in the presence of typical signs and symptoms.

\section{Reference}

[1] Crevani $M$, Aloise $M$, Lonati $D$, et al. [Acute poisoning from accidental ingestion of hashish in the pediatric population (20072014): case study of the Poison Control Center of Pavia]. Abstract SITOX Congress 2015 [cited 2018 Oct 11]. Available from: https:// congresso.sitox.org/archivio/2015/abs/80.pdf Italian

\section{Nalbuphine overdose in children: a case series}

\section{Colette Degrandi, Hugo Kupferschmidt and Katharina E Hofer \\ National Poisons Centre, Tox Info Suisse, Associated Institute of the University of Zurich, Zurich, Switzerland}

Objective: Nalbuphine, a synthetic opioid, acts as a k-receptor agonist and partial $\mu$-receptor antagonist. Due to its analgesic effect and ability to cause only moderate sedation with few side effects, nalbuphine is often used in pediatric patients, at a dose of $0.1-0.2$ $\mathrm{mg} / \mathrm{kg}$ intravenously. Its elimination half-life ranges from 0.9 to 1.9 hours. Nalbuphine is thought to exhibit a ceiling effect, and is considered a drug with minimal risk of inducing respiratory depression, even in overdose, but there is limited information available on this subject, especially in small children [1].
Methods: Retrospective review of acute nalbuphine overdose in children ( $<16$ years) reported to our National Poisons Centre from 2007 to 2018. Included were all cases with a follow up for at least 7 hours, and a high causality. Severity was graded according to the Poisoning Severity Score (PSS).

Results: Overall, 10 patients, 6 females and 4 males, aged from 17 days to 12 years (mean 61 months) were included. In all patients nalbuphine was administered by medical staff, with precise information on overdose. A ten-fold overdose occurred in 7 cases. Seven of the 10 patients had no symptoms. Minor symptoms occurred in two cases, and moderate symptoms in one case (Table 1).

Conclusion: Most of the nalbuphine overdoses in this case series were ten-fold medication errors, probably due to confusion of units ( $\mathrm{mL}$ instead of $\mathrm{mg}$ ). Respiratory depression was rare, and was not observed in the two newborns. Therefore, with careful surveillance including continuous measurement of oxygen saturation, a wait-and-see attitude for naloxone administration is reasonable in overdoses of up to ten-fold the therapeutic dose of nalbuphine in children.

\section{Reference}

[1] Kubica-Cielińska A, Zielińska M. The use of nalbuphine in paediatric anaesthesia. Anaesthesiol Intensive Ther. 2015:47:252-256.

\section{Morbidity and mortality of paediatric poisonings at a children's hospital in South Africa}

\section{Kate Balme and Cindy Stephen}

Department of Paediatrics, Poisons Information Centre, Red Cross War Memorial Children's Hospital and University of Cape Town, Cape Town, South Africa

Objective: According to the WHO World Report on Child Injury Prevention, poisoning comprises a significant component of injury-related disease, and poisoned children in low- and middleincome countries are four times more likely to die than those in high-income countries [1]. The few South African paediatric publications show similarities in age-group and causative substances, but reveal a paucity of data on morbidity and mortality [2-4].

Methods: Cases were identified by review of the Red Cross War Memorial Children's Hospital (RCWMCH) Poisons Information Centre hospital admission records from 2003 to 2017.

Results: There were a total of 5867 cases; the majority of children were under 5 years of age $(n=4904,84 \%)$. A single toxin was implicated in 5347 cases (91\%). Of these, kerosene was the

Table 1. Nalbuphine overdose in children. Patient characteristics, dosage and clinical effects with classification according to severity (PSS) where $0=$ no symptoms, $1=$ mild symptoms and $2=$ moderate symptoms.

\begin{tabular}{|c|c|c|c|c|c|c|c|c|c|}
\hline \multirow[b]{2}{*}{ Case } & \multirow[b]{2}{*}{ Age } & \multirow[b]{2}{*}{ Sex } & \multicolumn{2}{|c|}{ Dose } & \multirow{2}{*}{$\begin{array}{c}\text { X-fold } \\
\text { overdose }\end{array}$} & \multirow[b]{2}{*}{ Route } & \multirow[b]{2}{*}{ PSS } & \multirow[b]{2}{*}{ Clinical effects } & \multirow{2}{*}{$\begin{array}{c}\text { Naloxone } \\
\text { dosage }\end{array}$} \\
\hline & & & $\mathrm{mg}$ & $\mathrm{mg} / \mathrm{kg}$ & & & & & \\
\hline 1 & 17 days & $\mathrm{F}$ & 4 & 1.0 & 10 & IV & 0 & None & None \\
\hline 2 & 27 days & $\mathrm{F}$ & 4.6 & 1.0 & 10 & IV & 0 & None & None \\
\hline 3 & 6 weeks & $M$ & Not known & Not known & 10 & IV & 0 & None & None \\
\hline 4 & 6 weeks & $\mathrm{F}$ & 5 & 1.0 & 10 & Nasal & 0 & None & None \\
\hline 5 & 11 months & $M$ & Not known & Not known & 10 & IV & 0 & None & None \\
\hline 6 & 7 years & $\mathrm{F}$ & 30 & 1.2 & 10 & IV & 0 & None & None \\
\hline 7 & 9 years & $\mathrm{F}$ & 10 & 0.5 & 2.4 & IV & 0 & None & None \\
\hline 8 & 9 years & M & 20 & 0.7 & 6.7 & IV & 1 & Somnolence & None \\
\hline 9 & 11 years & $\mathrm{F}$ & 50 & 1.5 & 8.3 & IV & 1 & Nausea & None \\
\hline 10 & 12 years & M & 32 & 1 & 10 & IV & 2 & $\begin{array}{l}\text { Respiratory depression (no } \\
\text { information of respiratory } \\
\text { rate or oxygen satur- } \\
\text { ation), confusion }\end{array}$ & $2 \mathrm{mg}$ \\
\hline
\end{tabular}


most common specific substance $(n=1206,23 \%)$ and medications the largest group of substances $(n=1942,36 \%)$, followed by pesticides $(n=662,12 \%)$ and household products $(n=652$, $12 \%)$. Most patients ( $n=4459,83 \%)$ were asymptomatic or mildly symptomatic (Poisoning Severity Score (PSS) $\leq 1$ ). Children with moderate and severe poisonings $(n=880$; PSS $\geq 2)$ had a median hospital stay of 3 days (range 0-69 days) and included 16 deaths $(0.3 \%)$ due to pesticides $(n=8)$, medications $(n=6)$ and kerosene $(n=2)$. Further analysis of this subgroup (PSS $\geq 2)$ by substance and median hospital stay showed that pesticides $(n=270,4$ days) caused greater toxicity than medications ( $n=270,1$ day), kerosene ( $n=181,1$ day) and household products ( $n=57,1$ day).

Conclusion: The increased morbidity and mortality of paediatric pesticide poisonings exposes the need for appropriate medical training in the management of such poisonings. Furthermore, the large proportion of kerosene and pesticide cases, advocates for continued commitment towards socioeconomic uplift of the South African population at large.

\section{References}

[1] World Health Organization/UNICEF. World report on child injury prevention 2008 [cited 2018 Sep 28]. Available from: http://whqlibdoc.who.int/publications/2008/9789241563574_eng.pdf.

[2] Veale DJH, Wium CA, Muller GJ. Toxicovigilance II: A survey of the spectrum of acute poisoning and current practices in the initial management of poisoning cases admitted to South African hospitals. S Afr Med J. 2013;103:298-303.

[3] Balme K, Roberts JC, Glasstone M, et al. The changing trends of childhood poisoning at a tertiary children's hospital in South Africa. S Afr Med J. 2012;102:142-146.

[4] Marks CJ, van Hoving DJ. A 3-year survey of acute poisoning exposures in infants reported in telephone calls made to the Tygerberg Poison Information Centre, South Africa. S Afr J Child Health. 2016;10:43-46.

\section{Double dose guanfacine: harmless of not?}

\section{Jenny Westerbergh and Jonas Höjer \\ Swedish Poisons Information Centre, Stockholm, Sweden}

Objective: Guanfacine, in an extended-release formulation, was introduced on the Swedish market in 2016 for treatment of attention deficit hyperactivity disorders (ADHD). It is a non-stimulant drug which is used as monotherapy or as adjunctive treatment to stimulant medications. Guanfacine is a selective alpha-2A adrenergic receptor agonist which is structurally related to clonidine. It has previously been used as an antihypertensive. In order to assess the risk of ingestion of double dose guanfacine, a retrospective survey of telephone calls and hospital case records received by the Swedish Poisons Information Centre from January 2016 to September 2018 was carried out.

Case series: During the survey period the poison centre was consulted in 55 cases regarding ingestion of double dose guanfacine in individuals below the age of 18 years. Among these, 17 cases could be followed up. The mean age of the patients was 11.4 years (range 6-16) and $82 \%$ were boys. The most frequent symptoms were tiredness (8/17), dizziness (6/17), hypotension $(5 / 17)$ and bradycardia (4/17). Four patients were asymptomatic. The patients were all discharged within one day after an uneventful hospital course with mild or no symptoms. As an example, a 12year old girl took $10 \mathrm{mg}$ guanfacine instead of $5 \mathrm{mg}$. She became tired, developed headache and hypotension with the lowest blood pressure about 12 hours after ingestion. She was observed in hospital for 24 hours and then referred home.
Conclusion: In this material, ingestion of double dose guanfacine was benign, even though some of the children developed symptoms. Guanfacine has a narrow therapeutic interval wherefore even a small overdose can give rise to symptoms. At the Swedish Poisons Information Centre, hospital observation is recommended after ingestion of a double dose of guanfacine by mistake, if the intended dose is over $2 \mathrm{mg}$ for children up to 10 years or over 4 $\mathrm{mg}$ for children older than 10 years. After evaluation of this case series it is concluded that this recommendation is appropriate. There is only a single case report on this issue previously published and this presents an 8-year-old boy who ingested $6 \mathrm{mg}$ guanfacine instead of $3 \mathrm{mg}$ and who developed somnolence, hypotension and bradycardia [1].

\section{Reference}

[1] Walton J, Byrum M, Shumaker A, et al. Prolonged bradycardia and hypotension following guanfacine extended release overdose. J Child Adolesc Psychopharmacol. 2014;24:463-465.

\section{Out-of-hospital medication errors in children: a 6-year retrospective study}

Annalisa Troiano, Claudia Bondone, Virna Carmellino, Emanuele Castagno, Andrea Vermena and Antonio F Urbino

Department of Pediatric Emergency, Regina Margherita Children's Hospital, AOU Città della Salute e della Scienza di Torino, Torino, Italy

Objective: This is a 6-year retrospective study of 117 cases of out-of-hospital medication errors, admitted to the Emergency Department (ED) of a pediatric tertiary care hospital.

Methods: Medication errors were defined as errors during the administration of a drug by an adult, consisting of incorrect dose, incorrect route of administration, incorrect formulation and/or wrong medication given. Epidemiological data were described according to time trends, age, gender and nationality of child, type of medication error, category of pharmaceutical, number of pharmaceuticals, type of formulation, and route of exposure. Clinical and diagnostic features were analyzed by symptoms, blood and radiological tests performed, need for therapeutic interventions, specialist consultation and outcome. The severity of symptoms or signs was graded according to the Poisoning Severity Score (PSS)

Results: Overall 117 children were recruited; 62 were males (53.0\%) and 93 (79.5\%) were Italian. The median age was 2.3 years (range 0.02-14.17); most patients $(n=63,53.8 \%)$ were $1-4$ years old. The most commonly reported error was incorrect dosage. The major categories of medications involved included analgesics in 33 cases $(28.21 \%)$, drugs active on the central nervous system in 26 cases $(22.2 \%)$, antimicrobial agents in 16 cases $(13.68 \%)$, antihistamines in 10 cases $(8.6 \%)$, and vitamins in 8 cases $(6.8 \%)$. Ingestion was the most common route of exposure (105 events, 89.7\%), and liquid formulation was the most common substance status $(\mathrm{n}=81,69.2 \%)$. In particular, 44 medication errors involved drops (37.6\%), followed by syrup in 27 cases (23.1\%) and tablets/capsules in 14 patients (12.0\%). Among the four cases of parenteral exposure, two involved incorrect dosage of insulin in diabetic children, one was incorrect dosage of intramuscular antibiotic (ceftriaxone) and one was incorrect dosage of growth hormone, due to malfunction of the dosing pen. Symptoms were reported in 45 patients $(38.5 \%)$, and moderate 
to severe clinical consequences (PSS 2 or 3) occurred in only 10 patients (8.5\%). Overall, 98 patients $(83.8 \%)$ did not require any treatment.

Conclusion: Medication errors involving children represent a frequent public health problem. There are several proposed strategies for decreasing medication errors among children and increased efforts are needed for prevention. Pediatric healthcare providers should be aware of these strategies and implement them into their practices.

\section{Childhood acute poisoning: epidemiological assumptions for the elaboration of preventive strategies}

\author{
Andrea Vermena, Claudia Bondone, Virna Carmellino, \\ Emanuele Castagno, Federico Tremoloso and \\ Antonio F Urbino \\ Department of Pediatric Emergency, Regina Margherita Children's \\ Hospital, AOU Città della Salute e della Scienza di Torino, Torino, Italy
}

Objective: To identify epidemiological, socio-demographic and cultural background in childhood acute poisoning to improve prevention strategies.

Methods: In this prospective observational study we considered all patients with acute poisoning aged 0-14 years, admitted to the Emergency Department (ED) of our hospital between 1 January and 31 October 2017. Children aged 15-18 who were already followed in our hospital for chronic diseases were also included. Epidemiological and demographic information about children and caregivers, circumstances related to intoxication, characteristics of the poison, behaviors and choices of the caregiver following the episode of intoxication were collected.

Results: Overall 119 children were recruited; 108 were exposed accidentally (90.8\%), while the remaining 11 cases occurred voluntarily (9.2\%). Poisoning was significantly more frequent in males $(p=0.0131)$ and pre-school aged children $(p<0.0001)$; homecleaning products and medications were the most common substances involved $(p<0.0001)$. Thirty-one $(26.1 \%)$ children were exposed when the substance was left unattended and in 6 cases the poison was stored in unlabeled bottles. Patients who lived with other cohabitants experienced more serious episodes of poisoning $(p=0.0123)$. In 15 occasions, a previous poisoning episode had occurred within the family (12.6\%), however, in less than half of these situations a precaution was taken; 34 caregivers $(28.8 \%)$ waited before acting and caregivers who sought information on the Internet waited longer than the others (37.0\% versus $22.2 \%$, $p=0.0785$ ). The majority of caregivers gave other substances or tried to carry out procedures before reaching the ED, particularly those who had looked for information on the Internet $(66.7 \%$ versus $47.7 \%, p=0.0377)$. Only 55 caregivers claimed to be informed about childhood poisoning before the episode (46.2\%). The parents' nationality affected the sources of information usually consulted, Italian parents consult the Internet two-fold more frequently than foreigners (mother $51.6 \%$ versus $23.1 \%, p=0.0098$; father $50.0 \%$ versus $21.1 \%, p=0.0202$ ), while foreign parents referred more often to family and friends than Italians (mother $50.0 \%$ versus $22.6 \%, p=0.0062$, father $52.6 \%$ versus $24.0 \%, p=$ 0.0113). Only 63 caregivers (51.3\%) were aware of the Poison Control Center (PCC) but only 30 of them knew how to get in touch with it (25.2\%); only 15 caregivers called the PCC (12.6\%). Conclusion: Prevention strategies must be adopted taking into account the risk factors identified. It is also desirable to broaden the awareness of PCC and pay particular attention to the role of the Internet as an information source used by caregivers.

\section{Viper bites in children: experience of a tertiary care pediatric hospital in Central Italy}

\author{
Martina Di Giuseppe ${ }^{a}$, Mara Pisani ${ }^{b}$, \\ Giuseppe Pontrelli ${ }^{c}$, Barbara Scialangab ${ }^{b}$, Elena Bellelli ${ }^{b}$, \\ Gabriella Bottari ${ }^{\mathrm{d}}$, Corrado Cecchetti ${ }^{\mathrm{d}}$ and \\ Marco Marano ${ }^{d}$ \\ aPediatric University Hospital Department, Bambino Gesù Children \\ Hospital, University of Tor Vergata, Rome Italy; ${ }^{b}$ Emergency \\ Department, Bambino Gesù Children Hospital, Rome Italy; ${ }^{\mathrm{C}} \mathrm{Clinical}$ \\ Trial Centre, Bambino Gesù Children Hospital, Rome, Italy; \\ dEmergency Department PICU, Bambino Gesù Children Hospital, \\ Rome Italy
}

Objective: European viper (Vipera species) bite is relatively uncommon (about 0.4 to 1.1 people per 100,000 population per year), but can cause serious envenoming, especially in children. A total of 16 children with venomous viper bite were investigated. General characteristics of the children, localization of the bite, clinical and laboratory findings and treatment approaches were evaluated.

Case series: A retrospective investigation of 16 children admitted to the Pediatric Intensive Care Unit and Pediatric Emergency Department of Bambino Gesù Children Hospital in Rome for viper bite (Vipera species). The patients were evaluated according to the clinical grading score (CGS) of Audebert. They were aged 18 months to 15 years. Most (68\%) were male. Most bites occured in August and September, and usually (68\%) on the upper limb. The most common symptoms were local edema, ecchymosis and

Table 1. General characteristics, localization of the bite, clinical and laboratory findings in children with adder bite (Vipera species).

\begin{tabular}{lc}
\hline Parameters & Number of \\
children $(\mathrm{n}=16)$ \\
\hline Gender & \\
Male & $11(68.75 \%)$ \\
Female & $5(31.25 \%)$ \\
Month bitten & \\
March & $1(6.25 \%)$ \\
April & $1(6.25 \%)$ \\
May & $1(6.25 \%)$ \\
June & $2(12.5 \%)$ \\
July & $1(6.25 \%)$ \\
August & $5(31.25 \%)$ \\
September & $4(25 \%)$ \\
Bite site & \\
Upper limb & $11(68.75 \%)$ \\
Lower limb & $5(31.25 \%)$ \\
Clinical grading score (CGS) of Audebert & \\
O No & $3(18.75 \%)$ \\
I Minimal & $4(25.0 \%)$ \\
II Moderate & $6(37.5 \%)$ \\
III Severe & $3(18.75 \%)$ \\
Signs and symptoms & \\
Local pain & $16(100 \%)$ \\
Swelling & $14(87.5 \%)$ \\
Ecchymosis & $5(31.25 \%)$ \\
Neurological signs & $2(12.5 \%)$ \\
Anaphylaxis & $1(6.25 \%)$ \\
Laboratory findings & \\
Leucocytosis & $10(62.5 \%)$ \\
Neutrophilia & $14(87.5 \%)$ \\
Elevated creatine kinase (CK) & $4(25.0 \%)$ \\
Elevated lactic dehydrogenase (LDH) & $13(81.25 \%)$ \\
Hyperglycemia & $7(43.75 \%)$ \\
Elevated aspartate transaminase (AST) & $7(43.75 \%)$ \\
INR prolonged & $7(43.75 \%)$ \\
Activated partial thromboplastin time (aPTT) prolonged & $2(12.5 \%)$ \\
Administration of antivenom & $10(62.5 \%)$ \\
\hline & \\
\hline
\end{tabular}


pain. Among systemic symptoms, one child developed anaphylaxis and two patients had neurological involvement such as ptosis and diplopia. Blood tests were performed at admission; $62.5 \%$ of children had leukocytosis with neutrophilia. Patients with an abnormal white blood cell count (using age specific cut-off) on arrival showed a longer hospitalization time (from 3 to 14 days) than patients with normal WBC (probability $100 \%$ versus $50 \%$, $\mathrm{p}=0.038$ ) and were more often classified on the basis of clinical features as grade 2 or 3 of the CGS $(87.5 \%$ versus $25.0 \%, p=$ 0.0203). (Table 1).

Conclusion: Viper bite is a rare pediatric medical emergency in Italy, but can sometimes be severe. Leukocytosis at admission was significantly associated with a longer hospitalization and with a higher CGS. We therefore suggest the use of leukocytosis at admission as an important parameter for the severity of viper bite poisoning.

\section{Accidental repeated supratherapeutic overdose of paracetamol in a neonate with prolonged paracetamol elimination half-life}

\author{
Monica Abadier ${ }^{a}$, Anselm Wong ${ }^{b}$ and Andis Graudins ${ }^{c}$ \\ ${ }^{a}$ Monash Toxicology Unit, Monash Health, Dandenong, Australia; \\ ${ }^{\mathrm{b}}$ Clinical Sciences at Monash Health, Monash University, Clayton, \\ Australia; 'Monash Toxicology Unit and Emergency Medicine \\ Service, Monash Health and Monash University, Dandenong, \\ Australia
}

Objective: We report a case of accidental repeated supratherapeutic dosing of paracetamol in a neonate with hyperbilirubinaemia and prolonged paracetamol elimination.

Case report: A 10-day-old, $3.5 \mathrm{~kg}$, male, born at 38-weeks' gestation, was referred with an elevated paracetamol concentration. The child underwent circumcision six days after birth and was accidentally given four doses of $200 \mathrm{mg}(56 \mathrm{mg} / \mathrm{kg})$ of paracetamol over 24 hours ( $224 \mathrm{mg} / \mathrm{kg}$ total). Due to misinterpretation of syringe markings, the mother dosed $200 \mathrm{mg}(2 \mathrm{~mL})$ instead of 50 $\mathrm{mg}(0.5 \mathrm{~mL})$. Two days later, she realized the error when using a different syringe to administer $40 \mathrm{mg}$ paracetamol. Blood tests by the general practitioner 19.5 hours after this last dose revealed paracetamol concentration $381 \mu \mathrm{mol} / \mathrm{L}(57 \mathrm{mg} / \mathrm{L}), \quad A L T 18 \mathrm{IU} / \mathrm{L}$ (normal <40), with total bilirubin $262 \mu \mathrm{mol} / \mathrm{L}$ (normal <300) and gamma glutamyltransferase (GGT) $83 \mathrm{IU} / \mathrm{L}$ (normal <50). In hospital, repeat paracetamol concentration, assayed 29.5 hours postlast dose, was $236 \mu \mathrm{mol} / \mathrm{L}$. Bilirubin was $284 \mu \mathrm{mol} / \mathrm{L}(16.6 \mathrm{mg} / \mathrm{dL})$. Acetylcysteine was commenced six hours later using a 2-bag, 20hour regimen and continued for 27 hours, when serum paracetamol was undetectable and ALT was $29 \mathrm{IU} / \mathrm{L}$. He was discharged home clinically well. On follow up one week later, he remained well, however, serum ALT was $58 \mathrm{IU} / \mathrm{L}$ with total bilirubin 308 $\mu \mathrm{mol} / \mathrm{L}$. Elimination half-life calculation for paracetamol was 14.5 hours with apparent first-order elimination.
Conclusion: Elimination half-life for paracetamol in adults after therapeutic dosing is 1.5-3 hours [1] and in healthy neonates 3.5 hours [2]. After acute overdose, neonatal elimination half-life is 5.7-9 hours. In this case, hyperbilirubinemia was unlikely to interfere with the paracetamol assay, given the concentration was eventually undetectable. We hypothesize that the prolonged halflife may have been influenced by unconjugated hyperbilirubinemia which utilises glucuronidation to form conjugated bilirubin. This may prevent effective paracetamol glucuronidation. In neonates, paracetamol preferentially undergoes sulphation, however in the presence of supratherapeutic paracetamol concentration this may saturate [2]. Immaturity of neonatal cytochrome enzymes [3], particularly CYP2E1, which produces the toxic metabolite of paracetamol, may also influence the lack of hepatic injury despite the delay in treatment.

\section{References}

[1] Chiew AL, Fountain JS, Graudins A, et al. Summary statement: new guidelines for the management of paracetamol poisoning in Australia and New Zealand. Med J Aust. 2015;203(5):215-8. Erratum in: Med J Aust. 2015;203:320.

[2] Levy G, Khanna NN, Soda DM, et al. Pharmacokinetics of acetaminophen in the human neonate: formation of acetaminophen glucuronide and sulfate in relation to plasma bilirubin concentration and D-glucaric acid excretion. Pediatrics. 1975;55:818-25.

[3] Ogilvie JD, Rieder MJ, Lim R. Acetaminophen overdose in children. CMAJ. 2012;184:1492-6.

\section{Pediatric, self-harm cases comprise a large proportion of intentional exposures to methylphenidate reported to participating poison centres}

\author{
Geoff Severtson ${ }^{a}$, James J Lagrotteria ${ }^{a}$, \\ Zachary R Margolina, Samantha J Leroy ${ }^{a}$, \\ Antoine Villa ${ }^{b}$, Andreas Schaper, Martin Ebbecke ${ }^{c}$, \\ Fabrizio Sesana ${ }^{d}$, Bruno Mégarbane ${ }^{b}$, \\ Janetta L Iwanicki ${ }^{a}$ and Richard C Dart ${ }^{\mathrm{a}}$ \\ ${ }^{a}$ Rocky Mountain Poison \& Drug Center, Denver, United States; \\ ${ }^{b}$ Centre Antipoison et de Toxicovigilance de Paris, Paris, France; \\ 'GIZ-Nord Poisons Centre, University Medical Centre, Göttingen, \\ Germany; ${ }^{\mathrm{d}}$ Poison Control Centre of Milan, Milan, Italy
}

Objective: To examine characteristics of intentional exposures involving methylphenidate in Italy, Germany, and France.

Methods: Data from the Researched Abuse, Diversion and Addiction-Related Surveillance (RADARS $\left.{ }^{\circledR}\right)$ System Global Toxicosurveillance Network (GTNet) were used. The number of intentional exposures was calculated using data collected from

Table 1. Intentional exposures* in pediatric patients involving methylphenidate by country.

\begin{tabular}{|c|c|c|c|c|}
\hline Country & $\begin{array}{l}\text { Total intentional } \\
\text { exposure cases }\end{array}$ & $\begin{array}{c}\text { Pediatric } \\
\text { intentional } \\
\text { exposure } \\
\text { cases (\% of total) }\end{array}$ & $\begin{array}{l}\text { Intentional } \\
\text { exposure cases } \\
\text { involving } \\
\text { methylphenidate }\end{array}$ & $\begin{array}{c}\text { Pediatric intentional exposure } \\
\text { cases involving methylphenid- } \\
\text { ate (\% of exposures involving } \\
\text { methylphenidate) }\end{array}$ \\
\hline Germany & 7,070 & 275 (3.9\%) & 278 & 86 (30.9\%) \\
\hline Italy & 8,684 & 315 (3.6\%) & 6 & $3(50.0 \%)$ \\
\hline France & 1,612 & 252 (15.6\%) & 23 & $13(56.5 \%)$ \\
\hline
\end{tabular}

* Restricted to exposures involving select benzodiazepines (alprazolam, diazepam, etizolam, flunitrazepam, flurazepam, lorazepam, lormetazepam, nitrazepam, oxazepam), GABA analogs (gabapentin, pregabalin), opioids (buprenorphine, codeine, fentanyl, methadone, morphine, oxycodone, pethidine, meperidine, tapentadol), stimulants (methylphenidate), and Z-drugs (zaleplon, zolpidem, and zopiclone). 
participating poison centres in Italy (Milan), Germany (Göttingen), and France (Paris). Intentional exposures include exposures where the patient was attempting to gain a euphoric effect (abuse), self-harm (suicide), or intentionally improperly used a medication for reasons other than to gain a euphoric effect or for self-harm (misuse). Data collected on exposures involving methylphenidate and select benzodiazepines, opioids, and Z-drugs from the first quarter of 2012 through fourth quarter 2016 were analyzed. Pediatric cases were defined as cases ranging from age 1 to 17 years.

Results: In each country, a greater percentage of intentional exposures involving methylphenidate were pediatric exposures relative to all intentional exposures included in the analysis (Table 1). In Germany, 278 intentional exposures involved methylphenidate, 86 of which involved a pediatric patient (7 to 17 years). The intent of $70(81 \%)$ of the pediatric methylphenidate intentional exposures in Germany was self-harm. In Italy, six intentional exposures involved methylphenidate, three of which involved a pediatric patient (11 to 15 years). The intent of two $(67 \%)$ of the pediatric methylphenidate intentional exposures in Italy was self-harm. In France, 23 intentional exposures involved methylphenidate, 13 of which involved a pediatric patient (11 to 17 years). The intent of 11 of the pediatric methylphenidate intentional exposures (85\%) in France was self-harm.

Conclusion: Though methylphenidate makes up a small proportion of intentional exposures included in these analyses, a disproportionate number of these exposures are pediatric cases. Upon further investigation of these pediatric cases, self-harm was the intent for the majority.

\section{Differential diagnosis of botulism in an acutely hypotonic infant}

\author{
Elena Bellelli ${ }^{\mathrm{a}}$, Mara Pisani ${ }^{\mathrm{a}}$, Davide Lonati ${ }^{\mathrm{b}}$, \\ Francesco P Rossi ${ }^{a}$, Maia De Luca ${ }^{c}$, Daniela Perrotta ${ }^{d}$, \\ Elisabetta Pasotti ${ }^{d}$ and Marco Marano ${ }^{d}$ \\ ${ }^{\mathrm{a} E m e r g e n c y ~ D e p a r t m e n t, ~ B a m b i n o ~ G e s u ̀ ~ C h i l d r e n ~ H o s p i t a l, ~ R o m e, ~}$

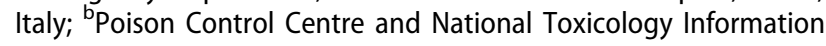 \\ Centre, Toxicology Unit, IRCCS Maugeri Foundation, Pavia, Italy; \\ ${ }^{c}$ Bambino Gesù Children Hospital, Rome, Italy; ${ }^{d}$ Emergency \\ Department PICU, Bambino Gesù Children Hospital, Rome, Italy
}

Objective: Infant botulism (IB) is a rare, life-threatening condition. We present a case of IB that is interesting because of the severe presentation, unusual source of intoxication and non-conventional treatment.

Case report: A 4-month-old male $(6.5 \mathrm{~kg})$ was admitted to hospital with a 4-day history of drowsiness, feeding difficulties and constipation. He was in poor general condition, with signs of dehydration, hyporeactivity, diffuse hypotonia and mild mydriatic pupils. Blood tests showed dehydration, acidosis and hypoglycemia ( $\mathrm{pH} 7.33$, base excess -5.9 , glucose $46 \mathrm{mg} / \mathrm{dL}$ ) without sign of infection or organ dysfunction. Treatment with fluids, glucose and electrolytes was started but his clinical condition gradually worsened and the baby was admitted to the intensive care unit where he was intubated due to respiratory failure. Medical history revealed that he has been exposed to dust from a large construction site near his home. IB was suspected. The diagnosis was confirmed by stool detection of the neurotoxin-producing clostridia. Household dust samples tested negative for botulin toxin-producing clostridia. The patient was treated with trivalent equine antitoxin $(10 \mathrm{~mL} / \mathrm{kg})$ without adverse events. After antidote treatment clostridiocidal antibiotic therapy was started and then probiotics were administered to restore intestinal flora. Supportive therapies and a rehabilitation program were started. Clinical condition progressively improved and he was discharged after 4 weeks. A 6 month-follow up showed complete recovery of motor functions and muscular tone.

Conclusion: The case described was particularly severe and required administration of the antitoxin. Antitoxin is not always readily available. In our experience, trivalent equine antitoxin can be safely administered in severe cases. Effectiveness and safety have been demonstrated in a large case series in Italy [1] and this treatment has been successfully adopted in other countries. In our case the source of spores was not identified, but the patient was exposed to a high level of dust and we suppose that this environment together with an individual predisposition of the baby is responsible for the illness. In conclusion it is important to warn clinicians to suspect IB in infants with a recent onset of hypotonia or a history suggestive of possible exposure. The excessive presence of environmental dusts should help in the diagnostic suspicion.

\section{Reference}

[1] Sabatini D, Papetti L, Lonati $D$, et al. A case of infant botulism in a 4-month-old baby. QJ Med. 2016;109:47-48.

\section{Yellow sac spider (Cheiracanthium punctorium) bite in a child: a case report}

Marcello Montibeller ${ }^{\mathrm{a}}$, Marta Crevani ${ }^{\mathrm{b}}$, Leonardo Genuini ${ }^{c}$, Mara Pisani ${ }^{d}$, Alessia Mesturino ${ }^{d}$, Roberto A Pantaleoni ${ }^{\mathrm{e}}$ and Marco Marano ${ }^{\mathrm{C}}$

${ }^{a}$ Faculty of Medicine, La Sapienza University of Rome, Rome, Italy; ${ }^{b}$ Poison Control Centre, National Toxicology Information Centre, Maugeri Clinical and Scientific Institutes IRCCS, Pavia, Italy; 'Department of Emergency PICU, Bambino Gesù Children Hospital, Rome, Italy; ${ }^{d}$ Emergency Department, Bambino Gesù Children

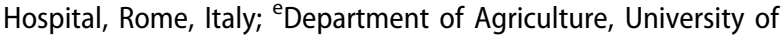
Sassari, Sassari, Italy

Objective: Although most spiders in Italy are not particularly venomous some potentially dangerous species have to be considered: Latrodectus tredecimguttatus and Loxosceles rufescens, whose venom can cause systemic effects. Steatoda paykulliana and the less dangerous Cheiracanthium punctorium (yellow sac spider) are responsible for local symptoms [1]. Cheiracanthium is an expanding species in Europe, whose venom is a unique twodomain cytotoxic polypeptide [2] and whose bite usually produces local pain, swelling and redness [3]. We present a case of Cheiracanthium punctorium bite in a child that resulted in selflimiting systemic toxicity.

Case report: A 7-year-old, otherwise healthy, child was sent to our ED by another hospital with complaint of a "possible Loxosceles rufescens bite" two days before. The patient presented bilateral non-secreting conjunctivitis with palpebral edema, lowgrade fever and rash. Netilmicin collyrium and amoxicillin clavulanate were administered. Blood tests reported several anomalies: eosinophils $8.8 \%$ (reference $0-7$ ), fibrinogen $174 \mathrm{mg} / \mathrm{dL}$ (reference 200-400) and D-dimer $740 \mathrm{ng} / \mathrm{mL}$ (reference 0-500). Physical examination showed a spider bite consisting of a tender lesion in the left knee fold, rash on the lower limb and bilateral inguinal adenitis. The spider was identified as a Cheiracanthium punctorium by an entomologist from images taken by the parents and the child was hospitalized; during the next 24 hours he became apyretic, in good clinical condition, with progressive improvement of the lesion, and resolution of the rash. Blood tests normalized and he was discharged. 
Conclusion: A yellow sac spider bite can be of medical interest in children, as signs of systemic toxicity were detected in our patient although the effects were self-limiting.

\section{References}

[1] Trotta A. [Introduction to Italian spiders (Arachnida Araneae).] Mem Soc Entomol It. 2005;83:3-178. Italian.

[2] Vassilevski AA, Fedorova IM, Maleeva EE, et al. Novel class of spider toxin: active principle from the yellow sac spider Cheiracanthium punctorium venom is a unique two-domain polypeptide. J Biol Chem. 2010;285;32293-302.

[3] Vetter RS, Isbister GK, Bush SP, et al. Verified bites by yellow sac spiders (genus Cheiracanthium) in the United States and Australia: where is the necrosis? Am J Trop Med Hyg. 2006;74:1043-1048.

\section{Steel yourself! Trends in iron packaging regulations in the US and pediatric morbidity}

\author{
James B Leonard ${ }^{\mathrm{a}}$, Elizabeth Q Hines ${ }^{\mathrm{b}}$ and \\ Wendy Klein-Schwartz ${ }^{a}$ \\ ${ }^{a}$ Maryland Poison Center, University of Maryland School of \\ Pharmacy, Baltimore, United States; ${ }^{b}$ Department of Pediatric \\ Emergency Medicine, University of Maryland School of Medicine, \\ Baltimore, United States
}

Objective: Iron poisoning was a leading cause of pediatric morbidity and mortality in the US until the Food and Drug Administration (FDA) placed strict controls on packaging in 1997. In 2003, the FDA reversed those packaging restrictions. The intent of this study is to identify if reversal of strict packaging requirements resulted in an increase in iron-related morbidity and mortality in pediatric patients.

Methods: We performed a retrospective cohort study utilizing the National Poison Data System (NPDS). Inclusion criteria were patients age 0-12 years with exposure to an iron-containing pharmaceutical product from 2000-2017. The group was split into two separate cohorts using the years 2000-2003 when packaging regulations were in place and the years 2004-2017 after its reversal. For analysis of therapies and clinical effects, only patients treated in a healthcare facility and followed to a known outcome were included. Severity of exposures was identified by comparing the rates of several clinical effects (acidosis, hypotension, liver injury, cardiac dysrhythmias) and use of life-saving therapies (deferoxamine, intubation/ventilation, and vasopressors). Descriptive analyses are presented as medians and interquartile ranges (IQR). Odds ratios (OR) are presented as changes from 2004-2017 relative to 2000-2003.

Results: There were 358,463 exposures over the 18-year period 102,197 from 2000-2003 and 256,264 cases from 2004-2017. There was a reduction in the total number of calls per year over the time period from 24,939 in 2000 to 11,247 in 2017 (R-squared $=0.8711$ ). Of these cases, 22,820 met final inclusion criteria; 6,670 $(6.5 \%)$ in the early period and $16,150(6.3 \%)$ in the late period. The median age was 2 years (IQR: $2-3$ ) and $56.7 \%$ were male. Two deaths occurred, both in the 2004-2017 group. The frequency of deferoxamine use was lower in the later time period (115/16,150 versus 109/6,670; OR: 0.4317, 95\% Cl: 0.3319-0.5627). There was no difference in the use of vasopressors or intubation/ ventilation. Additionally, we did not identify a difference in the frequency of key serious effects. There was a reduction in the use of whole bowel irrigation (OR $0.5481 ; 95 \% \mathrm{Cl}$ : 0.4623-0.6486) and gastric lavage (OR $0.1544 ; 95 \% \mathrm{Cl}: 0.1281-0.1587)$; there was an increase in the use of IV fluids (OR 1.368; 95\% Cl: 1.179-1.591).

Conclusion: Reversal of the iron packaging regulations did not result in an increase in severe iron exposures reported to the NPDS.

\section{Maternal use of acetaminophen- butalbital-caffeine product resulting in neonate butalbital exposure through breast milk}

\author{
Varun Vohra and Jeanna M Marraffa \\ SUNY Upstate Medical University, Upstate New York Poison \\ Center, Syracuse, United States
}

Objective: A medication product containing acetaminophen 325 $\mathrm{mg}$, butalbital $50 \mathrm{mg}$, and caffeine $40 \mathrm{mg}$ is indicated by the Food and Drug Administration (FDA) for the treatment of muscular and tension-type headaches. Butalbital has a low therapeutic index with toxic effects including respiratory and central nervous system (CNS) depression. We report a case of a 7-day-old breastfed neonate presenting with lethargy and poor feeding following maternal use of an acetaminophen-butalbital-caffeine product.

Case report: A 7-day-old infant presented with poor feeding, lethargy and emesis for 1-2 days prior to hospital admission. The infant's mother reported breastfeeding while taking a product containing acetaminophen $325 \mathrm{mg}$, butalbital $50 \mathrm{mg}$, and caffeine $40 \mathrm{mg}$ every 6 hours for 24 hours for a spinal headache. Her last dose occurred a day prior to the infant's hospital presentation. The patient presented somnolent, lethargic, and demonstrating diminished reflexes. An immunoassay urine drug screen was negative for acetaminophen but positive for barbiturates. Confirmatory urine test results on a sample drawn from the neonate approximately 15 hours after presentation detected a caffeine concentration of $1 \mu \mathrm{g} / \mathrm{mL}$. Quantitative butalbital concentrations were not detected at that time. The patient's symptoms and neurological status improved within 24 hours allowing for discharge within 48 hours.

Conclusion: We report a case of neonatal exposure via breast milk of butalbital with resultant CNS depression. Though there is limited data regarding butalbital elimination in breast milk, pentobarbital has been shown to be detectable in breast milk with levels of $0.17 \mu \mathrm{g} / \mathrm{mL}$ up to 19 hours post-maternal use [1]. Butalbital possesses an elimination half-life of 35 hours [2] compared to a half-life of up to 50 hours for pentobarbital [3]. Confirmatory drug testing was negative for butalbital, however was drawn on a sample 15 hours after hospital presentation. This case highlights the need for clinical awareness of butalbital excretion through breast milk in sufficient quantity to result in clinical manifestations of toxicity.

\section{References}

[1] Briggs GG, Freeman RK, Yaffe SJ. A Reference Guide to Fetal and Neonatal Risk. Drugs in Pregnancy and Lactation. 4th ed. Baltimore, MD: Williams \& Wilkins.1994. p.670.

[2] Drost ML, Walter L. Blood and plasma concentrations of butalbital following single oral doses in man. J Anal Toxicol. 1988;12:322-4.

[3] Reynolds JEF, Prasad AB. Martindale-The Extra Pharmacopoeia. 28th ed. London: The Pharmaceutical Press. 1982. p. 810. 


\section{Neurotoxicity of high doses intrathecal baclofen in a pediatric patient}

\author{
Davide Lonati ${ }^{\mathrm{a}}$, Azzurra Schicchi $^{\mathrm{a}}$, Sara Olivotto ${ }^{\mathrm{b}}$, \\ Silvia Masnadab, Rossella Gottardoc, \\ Nadia M Porpiglia ${ }^{c}$, Franco Tagliaro ${ }^{c}$ and \\ Carlo A Locatelli ${ }^{\mathrm{a}}$ \\ apavia Poison Control Centre - National Toxicology Information \\ Centre - Clinical and Experimental Lab, Toxicology Unit, Maugeri, \\ Pavia, Italy; ${ }^{\mathrm{b}}$ UOC Pediatric Neurology V. Buzzi-ASST \\ Fatebenefratelli Sacco Hospital, Milan, Italy; ' Department of \\ Diagnostics and Public Health, Unit of Forensic Medicine, \\ University of Verona, Policlinico "G.B. Rossi", Verona, Italy
}

Objective: Intrathecal baclofen therapy is an option to obtain better clinical response in children with spasticity, reducing lethargy due to oral administration (20-60\% versus $5-10 \%$ for the intrathecal route). Usually, clinical response for cerebral spasticity treatment is obtained by continuous intrathecal baclofen infusion at a rate of $70-400 \mu \mathrm{g} /$ day. We describe a child with severe spastic quadriplegia, treated with high doses of intrathecal baclofen with neurotoxicity and quantified baclofen serum concentrations. Case report: A 7-year-old child $(18 \mathrm{~kg})$ treated with benzodiazepines and intrathecal baclofen pump (implanted two months before) was admitted to the emergency department (ED) for lethargy. Baclofen infusion rate was gradually increased during the last two months up to $1700 \mu \mathrm{g} /$ day to obtain a successful clinical response. A neurotoxic effect of high baclofen doses was supposed, and the infusion rate reduced, first to 1000 , and then to $800 \mu \mathrm{g} /$ day. A gradual clinical improvement was observed during the following 24 hours. At this stage, a multichannel electroencephalogram (EEG) registered during the reduction of infusion rate (from 700 to $500 \mu \mathrm{g} /$ day) revealed the presence of a normal sleep pattern with only a mild and paroxystic excess of slow waves especially in the frontal regions and no epileptiform activity. Awakening was reached 36 hours after admission. To avoid lethargy, during the following 4 weeks the infusion rate was progressively reduced until $400 \mu \mathrm{g} /$ day (while an optimal clinical response to spasticity was not achieved); during this month, the plasma concentrations were below the limit of quantification (LOQ) $(10 \mathrm{ng} / \mathrm{mL})$ but above the limit of detection (LOD) $(3 \mathrm{ng} / \mathrm{mL})$ in all collected samples. No withdrawal symptoms were registered. Diazepam serum measurement confirmed therapeutic concentrations.

Conclusion: Intrathecal baclofen administration can cause lethargy. This effect is dose-dependent and limits clinical use of the drug, especially in pediatric patients (age $<12$ years) with severe spasticity requiring higher baclofen doses. In our experience, a maximum intrathecal infusion rate of $500 \mu \mathrm{g} /$ day should be considered in pediatric patients with spasticity non-responsive to usual baclofen dosage. When a high dose is required, close monitoring of the patient is necessary. Despite different intrathecal baclofen infusion rates at time of sample collection $(800,500,400 \mu \mathrm{g} /$ day), no difference in plasma concentrations were detected, and baclofen concentrations were below the LOQ in all samples. These data confirm that plasma concentration is not useful as a guide to choosing an effective infusion rate or to predict neurotoxicity.

\section{Safekeeping of cleaning agents reported by parents of preschool children in Croatia}

\author{
Željka Babića , Jelena Kovačićc ${ }^{b}$ Zrinka Franićc \\ Adrijana Bjelajac ${ }^{\mathrm{b}}$, Veda M Varnai ${ }^{\mathrm{b}}$, Jelena Macan ${ }^{\mathrm{b}}$ \\ and Rajka Turk ${ }^{\mathrm{b}}$
}

${ }^{a}$ Croatian Poison Control Centre, Institute for Medical Research and Occupational Health, Zagreb, Croatia; ${ }^{b}$ Institute for Medical Research and Occupational Health, Zagreb, Croatia

Objective: Cleaning agents are the leading source of poisoning in preschool children based on the experience of the Croatian Poison Control Centre (CPCC) telephone information service [1].

Methods: During February-June 2018 CPCC conducted a pilot study with parents of preschool children attending kindergartens in the capital, Zagreb, which included a questionnaire on safekeeping of common sources of poisoning. Possible answers to the question about storing cleaning products were: in child-proof compartments (i.e. locked with a key or secured by child-proof lock); out of reach of children, or in any other place. They were also asked whether or not they sometimes store cleaning agents, products for personal hygiene, cosmetics and medications out of the original packaging.

Results: There were 138 participants in total. Median age of parents was 38 years (IQR 34-40, range 24-55 years). Most of them were women $(73 \%)$, highly educated (83\% with post-secondary education) and currently employed (93\%). They most commonly had one $(42 \%)$ or two children $(44 \%)$, and rarely three or more (14\%). Storing substances out of reach of children was by far the most prevalent way of safekeeping cleaning agents at home (reported by $72 \%$ of the parents). The safest storage method (in child-proof compartments) was reported by the minority of parents $(11 \%)$, while $17 \%$ reported keeping cleaning agents in other places that may be accessible to children. Almost one third (29\%) of the parents stated they sometimes store household products including cleaning agents out of the original packaging. Women were twice as likely to men to report this habit (34 women which was $34 \%$ of all participating women versus 6 men which was $16 \%$ of all men, chi-square test, $p=0.045$ ).

Conclusion: Only $11 \%$ of parents interviewed store cleaning agents in child-proof compartments. Educational prevention action should be aimed at promoting this way of safekeeping as preferable to the currently used method of keeping products "out of reach of children" which is open to interpretation by each parent. Additionally, parents (especially women given their frequent use of cleaning products) need to be warned that storing products out of the original packaging (especially in food or beverage containers) could easily lead to poisoning.

\section{Reference}

[1] Babić Ž, Turk R. Analysis of Croatian Poison Control Centre consultations involving poisonings in preschool children (2007-2016) as a basis for poisoning prevention action. Clin Toxicol (Phila). 2018;56:557.

\section{Pediatric patients with acute neurological disorders: are they poisoned?}

\author{
Mariapina Gallo ${ }^{a}$, Maria D'Agostino ${ }^{b}$, \\ Georgios Eleftheriou ${ }^{a}$, Lorella Faraoni ${ }^{a}$, \\ Raffaella Butera ${ }^{\mathrm{a}}$, Andrea Giampreti ${ }^{\mathrm{a}}$ and \\ Giuseppe Bacis $^{a}$ \\ aUOSD Poison Control Center and Toxicology, ASST Ospedale Papa

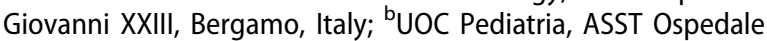 \\ Papa Giovanni XXIII, Bergamo, Italy
}

Objective: Acute neurological disorders in pediatric patients with altered level of consciousness, ranging from confusion, ataxia, acute dyskinesia, convulsions and coma, are a common cause of 
hospital admission. The etiology can be multiple and the differential diagnosis includes both neurological and non-neurological pathologies. We describe two cases of acute onset neurologic symptoms in pediatric patients with a toxicological cause.

Case reports: Case 1. A 13-month-old female, previously healthy, was brought to the emergency department after developing ataxia. On examination her vital functions were normal. She had hypotonia of the lower limbs and she could not keep upright. No relevant details emerged from the medical history and the parents denied drugs and/or other substance intake. The differential diagnosis involved acute cerebellar ataxia, initial encephalitis, or posterior cranial fossa tumor. Brain nuclear magnetic resonance spectroscopy (NMR), lumbar puncture, electroencephalogram (EEG), fundus oculi and pediatric neuropsychiatric evaluation were all negative. An exogenous cause was hypothesized. Urine toxicology screening was positive for benzodiazepines. Since the patient had been sedated with midazolam for imaging, it was necessary to perform second level screening which highlighted the presence of delorazepam. This has not been administered in hospital but was available at patient's home. The patient was discharged the following day fully recovered. Case 2. An 11-month-old male was brought by his parents to the emergency room for drowsiness. On arrival vital parameters were normal; the patient, previously healthy, was hypotonic, hyporeflexic with extreme restlessness and sedation. The father was on valproic acid. Drug abuse and/or other substances were denied by the parents. A valproic acid blood measurement was negative $(<10 \mu \mathrm{g} / \mathrm{mL})$. The differential diagnosis considered meningoencephalitis and intestinal invagination. Instrumental analyses were performed: EEG highlighted a global slowdown in brain activity; brain computerised tomography (CT) scan, lumbar puncture and abdomen ultrasound were normal. After pediatric neuropsychiatric evaluation, the patient was admitted to a Pediatric Intensive Care because of worsening of neurological status. Toxicological screening resulted positive for cannabinoids. The following day, given the clinical improvement, the baby was transferred to pediatric ward and discharged asymptomatic two days later.

Conclusion: Toxic ingestions must be evaluated when managing children with a reduced level of consciousness and when other diseases such as neurological infection or trauma are unlikely. Rapid urine toxicology may prevent unnecessary investigations and treatments.

\section{Infant botulism - a misjudged rarity?}

\author{
Maike Borchers ${ }^{a}, G$ Christoph Korenke ${ }^{b}$, \\ Marie-Madeleine Baeck ${ }^{\mathrm{a}}$, Andreas Schaper ${ }^{\mathrm{a}}$ and \\ Martin Ebbecke ${ }^{a}$ \\ aPoisons Information Centre Göttingen, Göttingen, Germany; \\ ${ }^{b}$ Pediatrics Hospital, Klinikum Oldenburg, Oldenburg, Germany
}

Objective: Infant botulism is a neuroparalytic disorder caused by toxin-producing clostridia. It is characterized by flaccid muscle paralysis and autonomic nervous system disorders [1]. While only a few cases are diagnosed annually in Germany (1-4 cases/year in 2007-2016), the frequency in the US is more than 10 times higher $[2,3]$. Honey consumption in the first year of life is a known risk factor for botulism but spore-containing dust is also a possible source. We report two cases from a rural environment diagnosed in five months (in April and September) in the same hospital in Northern Germany, and discuss a possible reason for the different incidences.

Case report: In our first case a 5-month-old breast-fed girl showed typical symptoms of infant botulism, with mydriasis, ptosis, decreased motor function and intestinal atony. She initially received cefotaxime but symptoms continued to progress. She was given physiotherapy and measures to support intestinal activity but did not require intubation. She improved over 16 days of hospitalization and was sent for rehabilitation. Five months later a 4-month-old breast and bottle-fed boy presented to the same clinic in Northern Germany with non-specific symptoms including general weakness. He was hospitalised for 13 days and received nutritional support with nasogastric feeding; he did not require intubation. In both cases mouse bioassays with faecal samples were used for detection and typing botulism. Additionally, in the first case serum was also used for the mouse bioassay and selective clostridium culture from a faecal sample as well as Clostridium botulinum-polymerase chain reaction (PCR) from faeces were also used to confirm the infection. Dust containing spores from an agricultural environment were thought to be the likely source of infection. Neither child was fed honey.

Conclusion: While the girl showed typical symptoms for infant botulism, the symptoms in the second case were more non-specific. Also because of the first case a few months earlier, the doctors were aware of the rare diagnosis "infant botulism" and tested the boy with positive results. We conclude that one of the reasons for the different incidences of infant botulism in Germany and the US may be due to underdiagnosis in Germany.

\section{References}

[1] Francisco AM, Arnon SS. Clinical mimics of infant botulism. Pediatrics. 2007;119:826-8.

[2] Robert Koch Institute: Infectious Disease Epidemiology Annual Reports 2007-2016 [cited 2018 Oct 16]. Available from: https:// www.rki.de/EN/Content/infections/epidemiology/inf_dis_Germany/ yearbook/Yearbook_inhalt.html

[3] Khouri JM, Payne JR, Arnon SS. More clinical mimics of infant botulism. J Pediatr. 2018;193:178-182.

\section{Creation of a Choosing Wisely list in the care of paediatric patients with suspected toxic exposure following a modified-Delphi approach}

Lidia Martínez-Sánchez Carlos Miguel Angelats-Romero ${ }^{\mathrm{b}}$, Alberto Barasoain-Millán ${ }^{c}$, Javier López-Ávila ${ }^{d}$ and Juan Carlos Molina-Cabañero ${ }^{\mathrm{e}}$; on behalf of the Intoxications Working Group, Spanish Society of Paediatric Emergencies

aPaediatric Emergency Department, SJD Barcelona Children's Hospital, Barcelona, Spain; ${ }^{b}$ Francesc de Borja University Hospital, Gandía, Spain; 'University Hospital Foundation of Alcorcon, Madrid, Spain; 'University Hospital of Salamanca, Salamanca, Spain; 'Niño Jesús University Children's Hospital, Madrid, Spain

Objective: Previous studies performed by the Intoxications Working Group (IWG) of the Spanish Society of Paediatric Emergencies have shown that there are aspects in the care of paediatric poisoned patients, specially related to gut decontamination, which must improve. We elaborate the process of a Choosing Wisely list in the care of paediatric patients with suspected toxic exposure, by the IWG.

Methods: The process was completed in 3 phases. Phase 1 (MayJune 2017): Proposal of Choosing Wisely. All the IWG members, from 57 Spanish Paediatric Emergency Departments, were invited to propose actions which must be avoided in the care of paediatric patients with suspected toxic exposure. Phase 2 
(July-October 2017): Selection of Choosing Wisely. All the IWG members were asked to rate the Choosing Wisely list proposed using a nine-point rating scale, ranging from "strongly disagree $=1$ " to "strongly agree $=9$ ". They could include their scoring reasons and suggestions for improving the wording. According to a predetermined decision rule, a Choosing Wisely action was included if it reached a median score $>8$ and more than two thirds of participants rated it highly agree (rating 7-8-9). The proposals with mean score 6-8 were considered indeterminate and were included in a second round [1,2]. Phase 3 (November 2017): Editing the definitive Choosing Wisely list.

Results: Thirty-two IWG members participated throughout the process. We obtained 11 proposals of Choosing Wisely. Seven were included initially and another added in the second round. They are:

1. Treat a nontoxic exposure.

2. Underestimate the toxicity (especially highly toxic substances).

3. Induce vomiting.

4. Perform a gastric lavage (with some specified exceptions).

5. Administer activated charcoal when it has no indication: a) No toxic ingestion; b) No adsorbent ability; c) $>2$ hours since ingestion (with some specified exceptions); d) Unprotected airway.

6. Administer water, milk or activated charcoal after ingestion of household or industrial products.

7. Administer flumazenil to patients with seizures or suspected tricyclic antidepressant ingestion.

8. Administer $\mathrm{N}$-acetylcysteine after a recent ( $<8$ hour) ingestion of paracetamol without confirming toxic serum concentrations (with some specified exceptions).

Conclusion: The followed process, based on Delphi methodology, has been useful to develop a Choosing Wisely list in the care of paediatric patients with suspected toxic exposure.

\section{References}

[1] Stang AS, Sharon SE, Crotts J, et al. Quality indicators for high acuity pediatric conditions. Pediatrics. 2013;132:752-762.

[2] Friedman JN, Mahant S. Making it easier to 'choose wisely'. Paediatr Child Health. 2017;22:66-67.

\section{Use of veno-arterial extracorporeal membrane oxygenation in life-threatening bupropion ingestion in a pediatric patient}

\author{
James B Leonard ${ }^{\mathrm{a}}$, Elizabeth Q Hines ${ }^{\mathrm{b}}$, \\ K Barry Deatrick ${ }^{c}$, Katherine Prybys ${ }^{d}$ and Hong K Kim ${ }^{d}$ \\ ${ }^{a}$ Maryland Poison Center, University of Maryland School of \\ Pharmacy, Baltimore, United States; ${ }^{b}$ Department of Pediatric \\ Emergency Medicine, University of Maryland School of Medicine, \\ Baltimore, United States; 'Department of Pediatric Cardiothoracic \\ Surgery, University of Maryland School of Medicine, Baltimore,

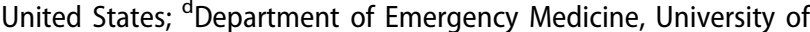 \\ Maryland School of Medicine, Baltimore, United States
}

Objective: Extracorporeal membrane oxygenation (ECMO) is increasingly utilized with acute poisoning. ECMO can provide cardiovascular support in patients with refractory hypotension, allowing time for metabolism and elimination of toxins. We present a pediatric patient with bupropion overdose that showed hemodynamic improvement with ECMO.

Case report: A 12-year-old male with a history of long QT syndrome, presented to the emergency department at an outside hospital $(\mathrm{OSH})$ after a witnessed seizure at school. Social history was significant for his hesitancy to attend school including recent absence due to "gastrointestinal issues." He was not taking any medications; but bupropion, lisdexamphetamine, and multiple over-the-counter agents were available. At the $\mathrm{OSH}$, he received lorazepam, fosphenytoin and levetiracetam, and was intubated due to continued seizures. Upon transfer to our tertiary center's pediatric intensive care unit (PICU), vital signs were: temperature $34{ }^{\circ} \mathrm{C}$, blood pressure $77 / 38 \mathrm{mmHg}$ (on dopamine and epinephrine), heart rate (HR) 119 beats/min, respiratory rate 25 breaths/ min, oxygen saturation $100 \%$ (intubated on $55 \%$ FiO2). Initial laboratory results were significant for potassium $2.5 \mathrm{mEq} / \mathrm{L}$, calcium $5.9 \mathrm{mg} / \mathrm{dL}$, lactate $5.3 \mathrm{mmol} / \mathrm{L}$, venous $\mathrm{pH} 7.30$, and bicarbonate $22 \mathrm{mEq} / \mathrm{L}$. Electrocardiogram (EKG) showed a widecomplex tachycardia (110 beats/min), QRS $130 \mathrm{~ms}$, and QT/QTc, $436 / 590 \mathrm{~ms}$. He was administered magnesium, calcium, sodium bicarbonate and norepinephrine. In the PICU, he experienced bradycardic cardiac arrest and tachydysrhythmias including torsades de pointes. Isoproterenol was initiated after return of spontaneous circulation, but EKG abnormalities worsened (QRS $160 \mathrm{~ms}$ and QTc $611 \mathrm{~ms})$. Intravenous lipid emulsion $(1.5 \mathrm{~mL} / \mathrm{kg})$ was administered without hemodynamic improvement. Due to rising lactate $(13.7 \mathrm{mmol} / \mathrm{L})$ and refractory hypotension with wide complex tachyarrhythmia, veno-arterial (VA) ECMO was initiated. Within 2 hours, hemodynamic status improved with clearance of lactate by 6 hours. He was decannulated 5 days after ingestion and was discharged home on hospital day 13. Bupropion concentration was $>400 \mathrm{ng} / \mathrm{mL}$ (therapeutic $50-100 \mathrm{ng} / \mathrm{mL}$ ). Hydroxybupropion concentration was not measured.

Conclusion: This is the youngest reported patient given VAECMO in bupropion-induced refractory cardiogenic shock. A large proportion of published cases report the use of veno-venous ECMO for acute respiratory distress syndrome (ARDS) in poisoned patients. A growing number of cases, however, show potential survival benefit of VA-ECMO in patients with refractory cardiogenic shock. Our patient's dramatic improvement in both hemodynamic and metabolic status provides additional support to consider VA-ECMO in acutely poisoned patients. VA-ECMO is a potentially life-saving intervention that should be considered in life-threatening cardiac toxicity from bupropion.

\section{Pediatric opioid exposures reported to US Poison Centers, 2011- 2017}

\author{
Saumitra Rege ${ }^{a}$, Duc Anh Ngo ${ }^{b}$, Marissa Kopatic ${ }^{a}$ and \\ Christopher P Holstege ${ }^{a}$ \\ aDivision of Medical Toxicology, Department of Emergency \\ Medicine, University of Virginia, Charlottesville, United States; \\ ${ }^{b}$ Division of Student Affairs, Department of Student Health, \\ University of Virginia, Charlottesville, United States
}

Objective: The US has the highest usage of prescription opioids in the world, with related overdose deaths increasing four-fold between 1999 and 2016 [1]. Opioid exposures can result in lifethreatening effects in children. We investigated the trends and characteristics of opioid exposures in the US pediatric population. Methods: We identified pediatric exposures ( $\leq 19$ years) to opioids reported to the National Poison Data System (NPDS) from 2011 to 2017 using the American Association of Poison Control Centers (AAPCC) generic codes. Descriptive statistics were used 
to analyze key characteristics. Calls from acute care hospitals and emergency departments $(\mathrm{ACH})$ were evaluated. Poisson regression was used to evaluate the trends in the number and rates of exposures. The percentage changes during the study period were reported.

Results: Overall there were 133,585 pediatric opioid exposures during the study period, with $41.5 \%$ of calls originating in an $\mathrm{ACH}$. Children $\leq 5$ years (58.4\%) and females (53.5\%) constituted the highest percentage of exposures in the pediatric age group. Most exposures occurred in a residence $(95.5 \%)$ and via ingestion (95.8\%). Unintentional exposures (47.1\%) were most common, followed by suspected suicides (24.0\%). Abuse (7.2\%) and misuse $(3.2 \%)$ were less frequently observed in exposures. Single substance exposures were more common in children under 6 years of age compared to teenagers ( $86.0 \%$ versus $50.3 \%$ ). Major clinical effects were demonstrated in $2.1 \%$ of exposures and 265 deaths were reported. Major effects were more common in the teenage group ( $1.1 \%$ versus $4.3 \%)$ with suspected suicides $(0.5 \%$ versus $56.2 \%)$ and abuse $(0.3 \%$ versus $16.3 \%)$ being more frequent. Among cases, $10 \%$ were admitted to a critical care unit (CCU). The proportion of cases originating from $\mathrm{ACH}$ increased during the study period (34.7\% versus $49.2 \%$ ). Hydrocodone was the most common opioid reported and was more frequent among the teenage population (24.2\% versus $32.9 \%)$. Pediatric opioid exposures decreased by $18.9 \%$ (95\% Cl: $-4.6 \%,-34.2 \%$, p < 0.001) from $2011(20,596)$ to $2017(16,699)$.

Conclusion: Pediatric opioid exposures decreased during the study. The observed trends in the exposures in our study parallels the changing prescribing practices, and growing efforts to increase patient awareness. Pediatric exposures and fatalities further highlight the need for greater attention to managing prescriptions and patient education regarding safe storage and adverse effects of these medications.

\section{Reference}

[1] Hedegaard $H$, Warner $M$, Miniño AM. Drug overdose deaths in the United States, 1999-2016. NCHS Data Brief, no 294. Hyattsville, MD: National Center for Health Statistics. 2017 [cited 2018 Oct 23]. Available from: https://www.cdc.gov/nchs/products/databriefs/db294.htm

\section{Digoxin overdose with unstable bradycardia: an uncommon poisoning in a child}

\author{
Chi Fong Miu', Sio Teng Lam ${ }^{\mathrm{b}}$ and Tam Fei Chang ${ }^{\mathrm{a}}$ \\ ${ }^{a}$ Emergency Medicine, Centro Hospitalar Conde de São Januário, \\ Macao, China; ${ }^{b}$ Oncology, Centro Hospitalar Conde de São \\ Januário, Macao, China
}

Objective: To describe the management of digoxin poisoning in a child.

Case report: A 4-year-old Filipino girl $(13.6 \mathrm{~kg})$ with atrial septal defect taking long-term digoxin $(50 \mu \mathrm{g}$ twice daily), accidentally ingested approximately $30 \mathrm{~mL}(1.5 \mathrm{mg})$ of digoxin syrup while travelling to Macao with her mother. After taking the syrup, she vomited clear liquid gastric content twice (volume unknown) within 3 hours. Approximately 10 hours later she complained of mild dizziness and presented to the emergency room (ER). On arrival she had bradycardia (heart rate around 50 beat per minute) with borderline blood pressure, the serum digoxin concentration was $3.2 \mathrm{ng} / \mathrm{mL}$ (11 hours post-exposure), and potassium $4.5 \mathrm{mmol} / \mathrm{L}$. An electrocardiogram (ECG) showed highdegree AV block. Antidote (DigiFab $40 \mathrm{mg}$ ) was indicated and given in view of her clinical pattern. Her blood pressure (BP) normalized and the ECG showed normal sinus rhythm, however, she had intermittent bradycardia with normal BP during observation on the ward. A 12-lead ECG showed first-degree AV block and second-degree AV block type I at various times. Thus, a second dose of DigiFab $40 \mathrm{mg}$ was administrated. Dopamine was given and transcutaneous pacing prepared for backup, because she had persistent bradycardia. About 72 hours after exposure, she was free from arrhythmia and serial ECG showed normal sinus rhythm without inotrope support.

Conclusion: Poisoning in children is common but fatal cases are rare, because the amount of the toxin ingested is usually limited. However, some common drugs could be fatal in one pill because they have a narrow therapeutic window (e.g. calcium channel blockers, camphor, gliclazide, digoxin, etc.) [1]. Antidotal therapy is effective in acute digoxin poisoning [2] and seems effective in patients with chronic digoxin exposure [3]. However, antidotal therapy was not as effective as expected in our case. Treating refractory AV block in digoxin poisoning with dopamine and transcutaneous pacing backup seems a reasonable adjuvant therapy in patients with poor response to antidotal therapy.

\section{References}

[1] Michael JB, Sztajnkrycer MD. Pediatric poisons: nine common agents that kill at low doses. Emerg Med Clin North Am. 2004;22:1019-50.

[2] Bauman JL, Didomenico RJ, Galanter WL. Mechanisms, manifestations, and management of digoxin toxicity in the modern era. Am J Cardiovasc Drugs. 2006;6:77-86.

[3] Chan BS, Isbister GK, O'Leary M, et al. Efficacy and effectiveness of anti-digoxin antibodies in chronic digoxin poisonings from the DORA study (ATOM-1). Clin Toxicol (Phila). 2016;54(6):488-94.

\section{Poisoning by ammonia solution: reasons, clinical effects and prevention opportunities}

\author{
Brigita Petrik $^{\mathrm{a}}$, Robertas Badaras ${ }^{\mathrm{b}}$ and Gabija Laubner \\ ${ }^{a}$ Vilnius University, Vilnius, Lithuania; ${ }^{b}$ Toxicology Centre of Vilnius \\ University, Vilnius, Lithuania; 'Vilnius University Emergency \\ Hospital, Vilnius, Lithuania
}

Objective: Poisoning by ammonia in a domestic environment is a very particular issue in Lithuania. Ammonia solution $10 \%$ is sold in pharmacies without prescription, which then results in accidental ingestion when it is mistaken for similar-looking medicines. In most cases ammonia is used for an unlabelled indication, for inhalation of ammonia gas to stimulate the trigeminal nerve when it is held under the nose to wake a person up after loss of consciousness (smelling salts). We review cases of ammonia ingestion presenting to our hospital.

Methods: Retrospective analysis of cases presenting to the Vilnius University Emergency Hospital. Collected data was processed by "SPSS 24.0 for Windows" statistical software. Data differences were evaluated as reliable and significant if $p \leq 0.05$. The statistical relation of qualitative attributes was evaluated using chi-square criteria.

Results: Overall 42 patients were included; $45.2 \%$ female and $54.8 \%$ male. Patients were aged $22-90$ years; $20-39$ years $19 \%$, $40-59$ years $40.5 \%$ and $\geq 60$ years $40.5 \%$. Where known the volume ingested ranged from 1 tablespoon (approximately $15 \mathrm{~mL}$ ) to a whole bottle $(40 \mathrm{~mL})$. There was no difference when comparing age groups (20-39, 40-59, $\geq 60$ years) and gender. The highest number (9.5\%) of accidents occured at 10:00h, 18:00h and 20:00h. The most common reason for ingestion was when the 
ammonia was mistaken for a similar-looking household medicine. The most frequent result was damage to the digestive tract, classified as $2 \mathrm{~A}$ according to the Zargar scale (hemorrhage, erosions, blisters, superficial ulcers). There was no connection between the volume of ammonia ingested and the degree of damage ( $p=$ 0.656). The most common residual symptoms were throat and pharyngeal pain, only being able to eat liquid or soft foods, and esophageal and / or gastric strictures. Statistically there was no significant correlation between the degree of damage according to the Zargar scale and the need for intubation $(p=0.524)$. Hospitalisation time ranged from 1 to 34 days. There was no direct connection between the severity of the gastrointestinal injury and hospitalization time $(p=0.373)$. There were no fatalities.

Conclusion: The substantial increase in poisoning by ammonia has been linked to thoughtless manufacturing of similar-looking packages for other medicaments commonly available in the home environment. Unsuspecting users of these products can easily mix up similar-looking packaging and accidentally drink the ammonia solution in error. There is no direct connection between age groups, the severity of the poisoning and the hospitalization time.

\section{Perspectives in the pharmacological treatment of corrosive ingestions}

\section{Daniela Pelclova}

Toxicological Information Centre, Charles University in Prague and General University Hospital, First Faculty of Medicine, Prague,

Czech Republic

Objective: Accidental ingestion of caustic substances represents a medical challenge. No pharmaceutical treatment has been approved to prevent esophageal strictures.

Methods: PubMed search for the years 2008-2018 was performed using the words: corrosive, caustic, ingestion, experimental.

Results: Of nine experimental studies found, five had aims other than pharmacological treatment, such as pathophysiological mechanisms, diagnostics or surgical treatment. Four studies used pharmaceuticals to prevent strictures in rats injured with sodium hydroxide [1-4]. Under certain conditions, all four agents were successful in preventing esophageal damage, measured by esophageal stenosis/index and histopathology score, collagen content and/or hydroxyproline (measure of collagen formation), and/or pro-fibrogenic genes expression [3]. The application was oral (p.o.), intraperitoneal (i.p.), or topical by esophageal instillation for 1-28 days (Table 1).
Conclusion: Four pharmaceutical treatments have been effective in reducing esophageal damage caused by sodium hydroxide in mice. These agents are already in use for other diagnoses, mostly fibroses. Topical garlic oil and 5-fluorouracil seem most practical. This promising data need evaluation in clinical studies.

\section{Acknowledgements}

Progres Q25/1LF, Q29/1LF.

\section{References}

[1] Ozbayoglu A, Sonmez K, Karabulut R, et al. Effect of polaprezinc on experimental corrosive esophageal burns in rats. Dis Esophagus. 2017;30:1-6.

[2] Şen Tanrıkulu C, Tanrikulu Y, Kılın F, et al. Beneficial effects of garlic (Allium sativum) oil in experimental corrosive esophageal burns effects of garlic oil in esophageal burns. Ulus Travma Acil Cerrahi Derg. 2017;23:181-187

[3] Orozco-Perez J, Aguirre-Jauregui O, Salazar-Montes AM, et al. Pirfenidone prevents rat esophageal stricture formation. J Surg Res. 2015;194:558-564.

[4] Duman L, Büyükyavuz Bl, Altuntas I, et al. The efficacy of singledose 5-fluorouracil therapy in experimental caustic esophageal burn. J Pediatr Surg. 2011;46:1893-1897.

\section{Review of features in 4,313 exposures to liquid laundry detergent capsules reported to the UK National Poisons Information Service (NPIS) over a 10-year period (2008-2017)}
Rachael Day ${ }^{a}$, Sally M Bradberry ${ }^{a}$, Gillian Jackson ${ }^{b}$, David J Lupton ${ }^{\mathrm{b}}$, Euan A Sandilands ${ }^{\mathrm{b}}$, Simon HL Thomas ${ }^{c}$, John $\mathrm{P}$ Thompson ${ }^{\mathrm{d}}$ and J Allister Vale ${ }^{a}$
${ }^{a}$ National Poisons Information Service (Birmingham Unit), City

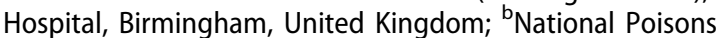 Information Service (Edinburgh Unit), Royal Infirmary, Edinburgh, United Kingdom; ' National Poisons Information Service (Newcastle Unit), Regional Drug and Therapeutics Centre, Newcastle, United

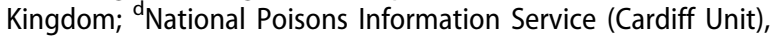 University Hospital Llandough, Cardiff, United Kingdom

Table 1. Experimental studies in mice to prevent corrosive stricture of the esophagus (2008-2018).

\begin{tabular}{|c|c|c|c|c|c|c|c|c|}
\hline \multirow[b]{2}{*}{ Reference } & \multicolumn{2}{|c|}{ Corrosive } & \multicolumn{3}{|c|}{ Treatment } & \multicolumn{2}{|c|}{ Efficiency Criteria } & \multirow{2}{*}{$\begin{array}{l}\text { Efficiency } \\
\text { conclusion }\end{array}$} \\
\hline & hydroxide & Duration & Pharmaceutical & Days & Mechanism & Stenosis index & Histopathology & \\
\hline Ozbayoglu 2017 & $10 \%$ & $3 \min$ & $\begin{array}{l}\text { Polaprezinc i.p. }+ \\
\text { p.o. }\end{array}$ & 28 & Anti-fibrogenic, antioxidant & $p=0.005$ & $p=0.01$ & yes \\
\hline Sen Tanrikulu & $37.5 \%$ & $1.5 \mathrm{~min}$ & Garlic oil topical & 1 & Anti-inflammatory, antioxi- & $p=0.016$ & $p=0.026$ & yes \\
\hline 2017 & & & $\begin{array}{l}\text { Garlic oil topical } \\
\text { + i.p. }\end{array}$ & 10 & dant, antibacterial & NS & $P=0.033$ & no \\
\hline $\begin{array}{l}\text { Orozco-Perez } \\
2014\end{array}$ & $32 \%$ & $1.5 \mathrm{~min}$ & $\begin{array}{l}\text { Pirfenidone gel } \\
\text { p.o. }\end{array}$ & 21 & $\begin{array}{l}\text { Antifibrogenic- downregula- } \\
\text { tion growth factors }\end{array}$ & $p=0.005$ & $p=0.027$ & yes \\
\hline \multirow[t]{2}{*}{ Duman 2011} & $10 \%$ & $3 \mathrm{~min}$ & $\begin{array}{l}\text { 5-Fluorouracil } \\
\text { topical }\end{array}$ & 1 & $\begin{array}{l}\text { Inhibition of fibroblast } \\
\text { proliferation }\end{array}$ & NS & NS & no \\
\hline & & & 5-Fluorouracil i.p. & 1 & & $p<0.05$ & $\mathrm{p}<0.05$ & yes \\
\hline
\end{tabular}


Objective: To provide a 10-year review of exposures to liquid laundry detergent capsules reported to the UK National Poisons Information Service (NPIS).

Methods: Telephone enquiries to the NPIS were analysed for the period January 2008 to December 2017. In addition, exposure data were collected from questionnaires on TOXBASE $^{\oplus}$, the NPIS computerized database, that were completed between July 2014 (the initiation of the project) and 31 December 2017.

Results: Data on 4,313 exposures were analysed: 4,271 were reported by telephone and 42 by questionnaire, of which $95.5 \%$ involved children aged $\leq 5$ years. Overall, 36.7\% $(n=1,562)$ of 4,260 patients remained asymptomatic (Poisoning Severity Score [PSS] 0), 60.4\% ( $n=2,575)$ developed minor (PSS 1) features, 103 suffered moderate features (PSS 2), 19 were graded as severe (PSS 3) and one (elderly patient) died. The mean annual number of paediatric exposures ( $\leq 5$ years) reported to the NPIS fell over the period of the study and was significantly lower $(p=0.004)$ when data for 2014-2017 were compared with data for 20082012. In contrast, the percentage of more serious cases (PSS $\geq 2$ ) was significantly higher $(p=0.02)$ during 2014-2017 than 20082012. Most exposures $(n=3,881,90.0 \%)$ involved ingestion and the most frequently reported features were vomiting (47.7\%), coughing (4.3\%), central nervous system (CNS) depression (3.3\%), foaming at the mouth (2.4\%), oral discomfort $(2.2 \%)$, diarrhoea $(2.0 \%)$, nausea $(1.9 \%)$, hypersalivation $(1.8 \%)$, abdominal pain $(1.0 \%)$ and stridor (1.0\%). Nine $(0.2 \%)$ children were intubated and ventilated. Ocular exposure occurred in 608 cases: 345 (59.2\%) suffered conjunctivitis or eye irritation and 20 (3.4\%) had keratitis/corneal damage, which persisted in one patient for 9 days. Dermal exposure occurred in 318 cases; 112 (35.9\%) reported minor dermal features including erythema, irritation and rash. Eye contact (4.1\%) was associated with more serious (PSS $\geq 2$ ) features, more often than other routes $(p=0.001)$. The most common features present in the 123 more serious (PSS $\geq 2$ ) cases were vomiting $(n=72)$, stridor $(n=34)$, CNS depression $(n=21)$, keratitis/corneal damage $(n=20)$, coughing $(n=18)$, conjunctivitis $(n=12)$, hypersalivation $(n=12)$, foaming at the mouth $(n=$ 11 ) and hypoxaemia $(n=10)$. However, respiratory features (stridor, hypoxaemia, bronchospasm, respiratory distress, dyspnoea, pulmonary aspiration, tachypnoea) were the reason for grading 55 of 123 cases as PSS $\geq 2$.

Conclusion: Most (97.1\%) exposures to these detergents caused no or only minor features. More serious features (PSS $\geq 2$ ), $45 \%$ of which were respiratory, were found in 123 of 4,313 cases.

\section{Unintentional paediatric ingestion of firelighters reported to the National Poisons Information Centre, Dublin}

\author{
Nicola Cassidy and Edel Duggan \\ National Poisons Information Centre, Beaumont Hospital, Dublin, \\ Ireland
}

Objective: A firelighter is a solid substance composed of wood shavings, sawdust and wax soaked in kerosene that is used to accelerate the lighting of a domestic wood or coal fire for homeheating. Pulmonary aspiration is a potential complication following firelighter ingestion due to the hydrocarbon content. The aim of the study was to characterise the epidemiology of unintentional paediatric firelighter ingestions reported to the National Poisons Information Centre (NPIC) and determine if home observation is appropriate in asymptomatic cases.

Methods: Consecutive cases of inadvertent paediatric firelighter ingestion reported to the NPIC from 1 January 2017 to 30 June
2018 were studied prospectively. Data on patient demographics, enquiry source, symptoms and treatment recommended were collated. The NPIC requested parental consent to perform a follow-up telephone enquiry to ascertain the outcome of cases reported by members of the public, general practitioners (GPs) and healthcare professionals in Emergency Departments (ED). Enquiries received from out-of-hours primary care helplines were excluded. Follow-up enquiries were performed at least 3 days post ingestion to determine whether the children developed delayed features or further medical advice/treatment after contacting the NPIC.

Results: During the 18-month study period, the NPIC received 129 enquiries relating to paediatric firelighter ingestion; 17 cases were excluded as the enquiries were received from out-of-hours primary care helplines. Consent was not requested for 7 cases, and refused in 2 cases. Overall 103 cases met the inclusion criteria; 71 cases (68.9\%) were successfully followed-up and subjected to data analysis. Enquiries were received from members of the public $(n=66), \operatorname{GPs}(n=3)$ and the ED $(n=2)$. There were 38 males and 33 females, mean age 19 months (range 7 months to 11 years). The majority of cases were asymptomatic when the NPIC was contacted ( $n=67,94.4 \%)$. Two children had coughed, one child vomited, and one child presented to the ED after 4 episodes of vomiting but had no respiratory symptoms and a normal chest X-ray and biochemistry. One child was observed in the ED for 8 hours and one was admitted to hospital overnight but neither developed further symptoms. Five children developed minor features that were considered to be unrelated to the firelighter exposure.

Conclusion: The majority of paediatric firelighter ingestions did not result in significant toxicity. Our results suggest that following accidental firelighter ingestion, asymptomatic children can be managed at home with instructions to seek medical advice if features develop.

\section{Accidental ammonia ingestion in cocaine users: a French case series}

\author{
Corinne Schmitt ${ }^{a}$, Mathieu Glaizal ${ }^{\mathrm{a}}$, \\ Romain Torrents ${ }^{a, b}$, Bastien Domangé ${ }^{a}$, Luc De Haro ${ }^{a}$ \\ and Nicolas Simon ${ }^{\mathrm{a}, \mathrm{b}}$ \\ ${ }^{a}$ Clinical Pharmacology, Poison Control Center, St Marguerite \\ Hospital, APHM, Marseille, France; ${ }^{b}$ Aix-Marseille Univ, APHM, \\ INSERM, IRD, SESSTIM, Marseille, France
}

Objective: The drug user population is at risk of many health problems. We know that cocaine drug users need to use basic products like ammonia to prepare free-base or crack cocaine [1]. Cocaine poisoning is a well described collateral hazard but the risks from use of ammonia seem to be underestimated. The aim of this study is to describe a case series of poisoning with ammonia reported to the Poison Control Center in Marseille (PCCM).

Methods: All cases of ammonia poisoning reported to the PCCM during 1 year (1 September 2017 to 31 August 2018) were retrospectively analyzed to evaluate medical history, circumstances and severity of each case.

Results: In total 71 cases of ammonia poisoning were analyzed with 39 inhaled, 30 oral, 1 ocular and 2 cutaneous exposures. There were 12 patients with drug abuse history, especially cocaine (7/12). All the 12 abusers' exposures were oral $(40 \%$ of oral exposures). Among these 12 patients, there were 2 suicidal and 10 accidental poisonings. In the accidental exposures, 9 were a consequence of a repackaging (in a water bottle or a beer can). Among the 12 cases, 5 had moderate and 5 had severe effects according to the Poisoning Severity Score (PSS). Seven patients required monitoring in a critical care unit, one patient had oesophagectomy, three had jejunostomy, and two had sequelae one month later. In the same period, the patients with no drug abuse 
history ( $n=59$ ), had mainly mild effects with $97 \%$ minor or no severity. The only severe case was a 15-month-old boy with accidental ingestion. We do not know if his parents where drug users or not.

Conclusion: When ammonia is decanted into other containers, cocaine abusers are more likely to mistake the caustic liquid for soda or beer. These accidental ingestions occur mainly during parties or early in the morning, after the party when the patients are thirsty. Our results probably underestimated the issue since drug users are suspicious and often refuse to declare their medical history or the poisoning circumstances. This small study suggests that accidental poisoning with ammonia in cocaine users is not anecdotal. A larger study would be useful to show if there is a need to raise an alert. Meanwhile, further public education may help prevent many cases of caustic ingestion.

\section{Reference}

[1] Massa N, Ludemann JP. Pediatric caustic ingestion and parental cocaine abuse. Int J Pediatr Otorhinolaryngol. 2004;68:1513-1517.

\section{Oxygen unleached: cardiac and neurologic complications following unintentional ingestion of $35 \%$ hydrogen peroxide necessitating hyperbaric therapy}

\author{
Calvin J Tan ${ }^{\mathrm{a}}$, Mark K Su${ }^{\mathrm{b}}$, Sage W Wiener ${ }^{\mathrm{c}}$, \\ Elie Harmouche ${ }^{d}$ and James Willis ${ }^{a}$ \\ ${ }^{a}$ SUNY Downstate Medical Center; NYC H + H Kings County, \\ Brooklyn, United States; ${ }^{b}$ New York City Poison Control Center, \\ New York, United States; ' $S$ UNY Downstate Medical Center; NYC H \\ + H Kings County; New York City Poison Control Center, Brooklyn, \\ United States; ${ }^{d}$ New York University School of Medicine, New York \\ City Poison Control Center, New York, United States
}

Objective: To highlight the variety of possible complications that may arise following ingestion of 35\% hydrogen peroxide.

Case report: A 40-year-old woman presented to the emergency department (ED) with blood-tinged emesis episodes following unintentional ingestion of $35 \%$ hydrogen peroxide. She complained of epigastric pain radiating to her right shoulder, headache, sore throat, and chest pain. Her triage vital signs were blood pressure $111 / 87 \mathrm{mmHg}$, heart rate $103 / \mathrm{min}$, respiration rate $19 /$ min, temperature $37.2^{\circ} \mathrm{C}$, oxygen saturation $96 \%$ (room air) with unremarkable initial physical exam aside from epigastric and diffuse abdominal tenderness. There was no obvious caustic injury to the oropharynx. Later, the patient complained of blurry vision. Initial testing was notable for elevated troponin $(0.286 \mathrm{ng} /$ $\mathrm{mL}$, reference $<0.08 \mathrm{ng} / \mathrm{mL}$ ), Non-specific $T$ wave inversions were seen on the electrocardiogram (ECG). A computerised tomography (CT) scan of chest and abdomen showed diffuse gastric pneumatosis and pneumobilia without obvious evidence of coronary air emboli. A head CT showed no acute infarct or pneumocephalus. She was transferred for hyperbaric oxygen therapy; after one session her symptoms resolved, except for continued blurred vision. Additional evaluation including echocardiography and magnetic resonance imaging (MRI) was planned, but she left against medical advice before these were completed. At ophthalmology follow-up, she reported return to normal vision; however, she had left homonymous hemianopia, likely due to the ingestion.
Conclusion: Both gastrointestinal and neurologic complications following 35\% hydrogen peroxide ingestion are reported as well as successful treatment with hyperbaric oxygen [1-2]. However, to our knowledge, this is the first case with cardiac involvement as evidenced by the elevated troponin concentration and an unusual case of homonymous hemianopia. We believe that these novel findings may be complications caused by paradoxical gas emboli that transited through a patent foramen ovale; a mechanism that has been described with iatrogenic air emboli [3], but not after hydrogen peroxide ingestion. This illustrates the variety of possible complications following ingestion of industrial strength hydrogen peroxide.

\section{References}

[1] Byrne B, Sherwin R, Courage C, et al. Hyperbaric oxygen therapy for systemic gas embolism after hydrogen peroxide ingestion. $J$ Emerg Med. 2014;46:171-175.

[2] Hendriksen SM, Menth NL, Westgard BC, et al. Hyperbaric oxygen therapy for the prevention of arterial gas embolism in food grade hydrogen peroxide ingestion. Am J Emerg Med. 2017;35:809.e5809.e8.

[3] Eichhorn V, Bender A, Reuter DA. Paradoxical air embolism from a central venous catheter. Br J Anaesth. 2009;102:718-718.

\section{Role of home-made slime in the rise of boric acid intoxications}

\author{
Marta Crevani ${ }^{a}$, Santi Di Pietro ${ }^{b}$, Francesca Chiara ${ }^{a}$, \\ Azzurra Schicchi ${ }^{\mathrm{a}}$, Valeria M Petrolini ${ }^{\mathrm{a}}$ and \\ Carlo A Locatelli ${ }^{a}$ \\ ${ }^{\text {a }}$ Pavia Poison Control Centre - National Toxicology Information \\ Centre - Clinical and Experimental Lab, Toxicology Unit, Maugeri \\ Clinical and Scientific Institutes IRCCS and University of Pavia, \\ Pavia, Italy; ${ }^{b}$ Emergency Medicine Fellowship Program, University \\ of Pavia, Pavia, Italy
}

Objective: Boric acid is a colourless, tasteless and odourless white powder used in low concentrations as an antiseptic. Toxic dose for ingestion exceeds $50 \mathrm{mg} / \mathrm{kg}$. Recently we registered an increase in cases of boric acid ingestion referred to our Poison Control Centre (PCC). One reason is the rising trend of homemade slime (skifidol); YouTube tutorials explain how to make it using simple ingredients, including boric acid. Children often put it in anonymous bottles, causing unintentional ingestion by other members of the family. We evaluated the rising trend of boric acid ingestion in the last 2 years.

Methods: Retrospective analysis of accidental boric acid ingestions referred to our PCC in 2016-2017.

Results: We registered 168 cases of accidental ingestion of boric acid during the study period. Among these, 70\% occured in 2017, with a $40 \%$ increase from the previous year. There were 95 females $(56 \%)$, median age 48 years $(0-95)$ with $30 \%$ aged under 14 years. For 150 patients (89\%) the dose ingested was assessed by the history and in 68 patients (45\%) was estimated as toxic. Among the 68 patients with an estimated toxic dose, 16 were at home at the first evaluation, and for 11 of them (69\%) emergency department (ED) admission was indicated. ED admission was indicated for one patient in a pharmacy. In addition, 51/68 patients were already at the ED. For 16 of the 34 patients with an estimated non-toxic dose who were at home at the first call, a medical evaluation was suggested because they complained of mild gastrointestinal symptoms. All the patients admitted with an estimated toxic dose underwent forced diuresis and renal function was monitored at 
admission and checked after 24 hours, 48 hours and 15 days with a specific follow-up. None of them showed impairment of renal function and they all recovered completely. Dialysis was indicated for none of them. Symptomatic patients (32\%) manifested mild gastrointestinal symptoms (nausea, vomiting, stomach ache, abdominal pain); no neurological symptoms were described.

Conclusion: Although our study did not show any case of severe intoxication, for almost $86 \%$ of all patients there was an ED admission and $43 \%$ of them required hospitalization for at least 2 days, with consequent healthcare costs and loss of working days. Diversion of dangerous substances in water bottles is a common, and potentially dangerous habit that can cause excessive ED admissions. Given this, much more concerning are the tutorials showing children how to make these mixtures.

\section{Isopropyl alcohol: a cause of anion gap metabolic acidosis with elevated lactate}

\author{
Elie Harmouche ${ }^{a}$, Mark Su ${ }^{b}$ and Rana Biary ${ }^{c}$ \\ ${ }^{a}$ Division of Medical Toxicology, Ronald O. Perelman Department \\ of Emergency Medicine, NYU School of Medicine, New York,

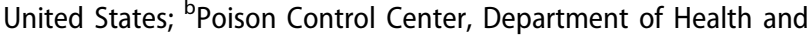 \\ Mental Hygiene, New York, United States; 'Division of Medical \\ Toxicology, Ronald O. Perelman Department of Emergency \\ Medicine, New York, United States
}

Objective: Isopropyl alcohol ingestion is reported to cause ketosis without acidosis. We report a case of confirmed isopropyl alcohol ingestion associated with an anion gap metabolic acidosis with no preceding hypotension.

Case report: A 15-year-old female with a past medical history of depression presented to the emergency department after being found somnolent at home. Thirty minutes prior to arrival, she drank $300 \mathrm{~mL}$ of $70 \%$ isopropyl alcohol. She had one episode of vomiting and was intubated for airway protection. Initial vital signs were blood pressure 115/66 $\mathrm{mmHg}$, heart rate 86 beats/minutes, respiratory rate $20 /$ minute, temperature $36.3^{\circ} \mathrm{C}$, and oxygen saturations $100 \%$ (on supplemental oxygen). Electrocardiogram (ECG) showed sinus rhythm (92 beats/minute) with normal intervals. Venous blood gas analysis showed $\mathrm{pH} 7.2, \mathrm{PCO}_{2} 50.4 \mathrm{mmHg}$, and lactate 0.34 $\mathrm{mmol} / \mathrm{L}$. Basic metabolic profile showed sodium $137 \mathrm{mmol} / \mathrm{L}$, potassium $2.9 \mathrm{mmol} / \mathrm{L}$, chloride $105 \mathrm{mmol} / \mathrm{L}$, bicarbonate $24 \mathrm{mmol} / \mathrm{L}$, blood urea nitrogen $11 \mathrm{mmol} / \mathrm{L}$, creatinine $244 \mu \mathrm{mol} / \mathrm{L}$, glucose 9 $\mathrm{mmol} / \mathrm{L}$, and anion gap $8 \mathrm{mmol} / \mathrm{L}$. Measured serum osmoles were $353 \mathrm{mOsm} / \mathrm{L}$ with a calculated osmolar gap of 65 . Ethanol, acetaminophen and salicylate were undetectable. Urine ketones were undetectable but serum acetone was $21 \mathrm{mg} / \mathrm{dL}$. Repeat laboratory tests seven hours after admission were arterial blood gases $\mathrm{pH} 7.21$, $\mathrm{PCO}_{2} 27 \mathrm{mmHg}$, lactate $1.1 \mathrm{mmol} / \mathrm{L}$, with sodium $141 \mathrm{mmol} / \mathrm{L}$, potassium $3.5 \mathrm{mmol} / \mathrm{L}$, chloride $102 \mathrm{mmol} / \mathrm{L}$, bicarbonate $15 \mathrm{mmol} /$ $\mathrm{L}$, blood urea nitrogen $10 \mathrm{mmol} / \mathrm{L}$, creatinine $244 \mu \mathrm{mol} / \mathrm{L}$, glucose 8 $\mathrm{mmol} / \mathrm{L}$ and anion gap 23 . Given the rising anion gap and elevated lactate, a loading dose of fomepizole $15 \mathrm{mg} / \mathrm{kg}$ in addition to thiamine and folate were administered. She was started on continuous veno-venous hemofiltration (CVH) 15 hours post-admission for 2 hours and was later switched to hemodialysis. Toxic alcohol concentrations sent after 2 hours of CVVH were isopropyl alcohol 69 $\mathrm{mg} / \mathrm{dL}$ and acetone $82 \mathrm{mg} / \mathrm{dL}$. Ethylene glycol and methanol were undetectable. She completed 4 hours of hemodialysis, with resolution of acidosis. Following extubation, she admitted to ingesting only isopropyl alcohol.

Conclusion: Isopropyl alcohol is either eliminated unchanged via kidneys or lungs or is metabolized by alcohol dehydrogenase to acetone, the latter eliminated by the liver or lungs. However, rodent studies show that acetone may be further metabolized to lactate, generating formate, which can contribute to an anion gap metabolic acidosis. While this pathway may explain the acidosis and hyperlactemia in our patient, it has not been demonstrated in humans. The development of acidosis in this patient is puzzling and unexpected.

\section{Neutropenia associated with metamizole: a case-control study}

\author{
Deborah Rudin ${ }^{a}$, Stephan Krähenbühl ${ }^{\mathrm{a}}$ and \\ Manuel Haschke ${ }^{b}$ \\ ${ }^{a}$ Clinical Pharmacology \& Toxicology, University Hospital Basel,

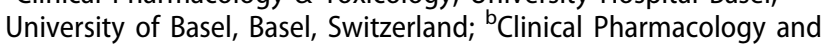 \\ Toxicology, University Hospital Bern, University of Bern, Bern, \\ Switzerland
}

Objective: Metamizole is a widely prescribed non-opioid analgesic prodrug, which rarely causes life-threatening neutropenia [1]. Besides metamizole dose and treatment duration, various factors, including comedication, comorbidities, and allergic predisposition, may influence the risk of developing neutropenia during metamizole treatment. The aim of this study was to identify possible risk factors for developing neutropenia associated with metamizole use.

Methods: An observational, multi-center case-control study was performed. Cases of metamizole-associated neutropenia attending the University Hospitals of Basel and Bern between 2005 and 2017 were characterized and compared with tolerant controls, who took metamizole for at least 28 days without developing neutropenia, and with gender- and age-matched metamizolenaive healthy controls. Medical data and blood samples of all study participants were collected.

Results: Fifty-two cases, 39 tolerant controls, and 161 metamizole-naive healthy controls were included. Median metamizole intake and duration among cases was $2000 \mathrm{mg}(350-5700 \mathrm{mg})$ for 20 days (1-382) until onset of the neutropenia compared to 1000 $\mathrm{mg}(500-4000 \mathrm{mg})$ for 177 days (30-5480) among tolerant controls without any adverse drug reaction. Concomitant medication was almost equal in cases (94\%) and tolerant controls (97\%), whereas the mean number of concomitant drugs per individual was significantly higher in the tolerant control group $(n=9 \pm 6)$ than in the case group $(n=6 \pm 4)(p=0.0035)$. Thereof $41 \%$ and $32 \%$ among tolerant controls and cases, respectively, were drugs with listed risk to induce neutropenia according to information from Swiss Drug Authorities. Accompanying infections were significantly more frequent among cases (72\%) than among tolerant controls $(31 \%)(p=0.0085)$. Conversely, tolerant control patients had autoimmune diseases (21\%) more often than neutropenia patients (15\%). Likewise, previous allergic reactions to drugs were more frequent among tolerant controls (33\%) than among cases (26\%) and even less among unexposed healthy controls (7\%).

Conclusion: Accompanying infections seem to be a possible risk factor for metamizole associated neutropenia. There was no association between concomitant medication, autoimmune diseases or drug allergy history and the development of neutropenia. A genetic association study is currently running, analyzing collected blood samples to identify potential genetic markers for metamizole-associated neutropenia.

\section{Reference}

[1] Huber M, Andersohn F, Sarganas G, et al. Metamizole-induced agranulocytosis revisited: results from the prospective Berlin CaseControl Surveillance Study. Eur J Clin Pharmacol. 2015;71:219-227. 


\section{A case of liraglutide toxicity}

\author{
Anselm Wong and Joe A Rotella \\ Austin Toxicology and Victorian Poisons Information Centre, Austin \\ Health, Victoria, Australia
}

Objective: Liraglutide is a glucagon-like peptide 1 (GLP-1) agonist, a newer class of antidiabetic medication. It stimulates glucose-dependent insulin secretion from pancreatic islets, suppresses glucagon secretion, slows gastric emptying and increases satiety. Liraglutide is slowly absorbed via subcutaneous injection with a plasma half-life of approximately 13 hours prior to metabolism in the plasma by dipeptyl-peptidase-4 and neutral endopeptidase (NEP) and excreted in the urine. There have been few cases of reported overdose, especially in non-diabetic patients.

Case report: We report a case of a 36-year-old male who presented to our Emergency Department following an accidental overdose of liraglutide. He was prescribed liraglutide for weight loss (body mass index 30.7) and did not have a history of diabetes mellitus. He was not on any other prescribed medication and denied any recreational substance use. The patient commenced liraglutide on the day of presentation and misread the instructions provided with the medication. Instead of the prescribed dose of $0.5 \mathrm{mg}$, he injected $3.0 \mathrm{mg}$ subcutaneously. Three hours later he developed profuse vomiting and epigastric pain As it was not resolving, he initially presented to the Emergency Department at 12 hours post-injection and left after receiving 4 mg ondansetron, which temporarily resolved his symptoms. He subsequently represented at 16 hours post-overdose with severe vomiting episodes followed by dry retching. He did not report any other symptoms. On examination, he had normal vital signs and some mild epigastric tenderness. He had a normal full blood count, urea, creatinine and electrolytes and was treated with metoclopramide and ondansetron (oral and IV). He was admitted overnight, symptoms had improved the following day and he was discharged 26 hours post-injection. There were no episodes of hypoglycaemia on admission.

Conclusion: From the available literature and in this case, liraglutide overdose in isolation appears relatively benign in diabetic patients with predominantly prolonged gastrointestinal features (vomiting and nausea). In addition, hypoglycaemia requiring dextrose infusion has been observed in a non-diabetic patient. This is likely due to the stimulation of insulin release and reduction in glucagon secretion facilitated by liraglutide, which would have a more marked effect in a non-diabetic patient. In this instance, regular blood glucose measurement and utilisation of intravenous dextrose when required is recommended. The long half-life likely contributed to the ongoing symptomatology in our case. There is limited information available regarding the toxicity of liraglutide. Supportive care, observation for hypoglycaemia and antiemetics are the mainstays of management.

\section{Use of rasburicase in "hidden" favism: a case report}

\author{
Georgios Eleftheriou ${ }^{a}$, Mariapina Gallo ${ }^{a}$, \\ Raffaella Butera ${ }^{a}$, Andrea Giampreti ${ }^{a}$, Lorella Faraoni ${ }^{a}$, \\ Francesca Bedussi ${ }^{\mathrm{a}}$, Giuseppe Bacis ${ }^{\mathrm{a}}$ and \\ Roberto Fiocchi ${ }^{\mathrm{b}}$ \\ aPoison Control Center, ASST Papa Giovanni XXIII Hospital, \\ Bergamo, Italy; ${ }^{\mathrm{b}}$ Cardiovascular Unit, ASST Papa Giovanni XXIII \\ Hospital, Bergamo, Italy
}

Objective: Rasburicase, a recombinant form of urate oxidase, is indicated for the management of malignancy-related hyperuricemia and off-label for severe tophaceous gout. The drug catalyzes conversion of uric acid to allantoin, which is 5-10 times more soluble than uric acid and undergoes rapid renal excretion. In patients with glucose-6-phosphate dehydrogenase (G6PD) deficiency rasburicase can cause severe oxidative hemolytic anemia. We report a case of rasburicase administration in a patient who reported not to be affected from favism as he had always eaten fava beans with no ill effects.

Case report: A 65-year-old heart transplanted male with renal failure received a single dose of rasburicase $7.5 \mathrm{mg}$ IV for hyperuricemia. Three days later he developed confusion with severe anemia (hemoglobin $45 \mathrm{~g} / \mathrm{L}$ ), hemoglobinuria and jaundice. A red blood cell (RBC) transfusion was begun and 24 hours after resolution of the hemolytic episode a hemoglobin concentration of $80 \mathrm{~g} / \mathrm{L}$ was archived. Soon after the hemolytic episode, a falsely negative qualitative G6PD deficiency test was $311 \mathrm{mU} / 10^{9} \mathrm{RBC}$ (normal value 220-570), but two months later G6PD activity decreased to $18 \mathrm{mU} / 10^{9} \mathrm{RBC}$, strongly suggestive of G6PD deficiency. Pharmacological causes of the hemolytic episode other than rasburicase were excluded: medications prior to rasburicase were pyrimethamine $75 \mathrm{mg}$ (plus levofolinic acid calcium, as perprotocol, for generalized toxoplasmosis prophylaxis), acetaminophen $500 \mathrm{mg}$, cyclosporine $160 \mathrm{mg}$, everolimus $0.50 \mathrm{mg}$, aspirin $100 \mathrm{mg}$, furosemide $12.5 \mathrm{mg}$, ursodeoxycholic acid $300 \mathrm{mg}$, lansoprazole $60 \mathrm{mg}$, levothyroxine $50 \mu \mathrm{g}$; no drug interactions exist and no link between the use of these drugs and G6PD deficiency is reported.

Conclusion: Although the incidence of hemolytic anemia in patients with G6PD deficiency is low, the severity of the potential reaction resulted in a boxed warning in the package labeling of rasburicase. The conversion of uric acid to allantoin is catalyzed by uricase, which produces hydrogen peroxide and the inability to break this down in G6PD-deficient patients can cause hemolytic anemia. This patient's history strongly suggests unrecognized G6PD deficiency and eating fava beans without any untoward effects does not mean that the patient in unaffected by G6PD deficiency. A G6PD assay is likely to be normal shortly after acute hemolysis in the $A 2$ variant because of the normal level of the enzyme in the newly produced reticulocytes. This case highlights the risk associated with rasburicase and the need for a G6PD screening before use, in order to avoid this adverse event in high-risk individuals with G6PD deficiency.

\section{Acute renal failure after the administration of the single dose of ketorolac: report of three cases}

\author{
Gabija Valauskaite ${ }^{\mathrm{a}}$, Greta Sakalauskaite ${ }^{\mathrm{a}}$ and \\ Robertas Badaras ${ }^{\mathrm{b}}$ \\ ${ }^{a}$ Vilnius University, Faculty of Medicine, Vilnius, Lithuania; ${ }^{\text {bVilnius }}$ \\ University Emergency Hospital, Centre of Toxicology, Vilnius \\ University, Faculty of Medicine, Vilnius, Lithuania
}

Objective: Ketorolac is a non-steroidal anti-inflammatory drug; it is associated with nephrotoxic adverse effects, including acute kidney injury (AKI) after prolonged use or overdose [1,2]. We present and analyse three new cases of AKI after the administration of a single dose of ketorolac.

Case series: Case 1. A 46-year-old man with a deviated nasal septum underwent conchoplasty and septoplasty. The patient received a single dose of ketorolac $60 \mathrm{mg}$ during anesthesia. Blood chemistry examination prior to administration revealed a serum creatinine of $103.3 \mu \mathrm{mol} / \mathrm{L}$. After 48 hours the serum 
creatinine started to increase and reached its peak at $891 \mu \mathrm{mol} / \mathrm{L}$. The patient was treated with hemodialysis until the creatinine decreased to $756 \mu \mathrm{mol} / \mathrm{L}$. The patient had a positive family history of chronic kidney disease. There was also a history of drinking alcohol some days before surgery and using protein supplements for 6 months. Case 2. A 57-year-old man with nasal polyps underwent maxillary antrostomy and ethmoidectomy. He received a single dose of ketorolac $60 \mathrm{mg}$ during anesthesia. Two days after the surgery he had urinary retention, and serum creatinine was $673 \mu \mathrm{mol} / \mathrm{L}$. After 24 hours this had risen to 826 $\mu \mathrm{mol} / \mathrm{L}$ and hemodialysis was given until the creatinine concentration decreased to $337 \mu \mathrm{mol} / \mathrm{L}$. The patient had predisposing risk factors for AKI including arterial hypertension and myocardial infarction. Case 3. A 29-year-old man with a deviated nasal septum underwent septoplasty and also received a single dose of ketorolac $60 \mathrm{mg}$ during anesthesia. After 10 hours the serum creatinine started to increase from $241 \mu \mathrm{mol} / \mathrm{L}$ and peaked at $391 \mu \mathrm{mol} / \mathrm{L}$ in four days. There was a history of the patient using protein supplements.

Conclusion: The present report indicates that predisposing risk factors for kidney injury and ketorolac can result in AKI. Ketorolac should be used with caution and monitoring of renal parameters may be preferable during short-term ketorolac therapy [3].

\section{References}

[1] Lewis S. Ketorolac in Europe [letter]. Lancet. 1994;343:784.

[2] Haragsim L, Dalal R, Bagga $\mathrm{H}$, et al. Ketorolac-induced renal failure and hyperkalemia: Report of three cases. Am J Kidney Dis. 1994;24:578-580.

[3] Feldman HI, Kinman JL, Berlin JA, et al. Parenteral ketorolac: the risk for acute renal failure. Ann Intern Med. 1977;126:193-9.

\section{Paliperidone-induced neuroleptic malignant syndrome: a case report}

\author{
Eddie C Garcia ${ }^{a}$, Robert Goodnough ${ }^{a}$, \\ Jeffrey Whitman ${ }^{b}$, Michael Wu ${ }^{c}$, Craig G Smollin ${ }^{a}$ and \\ Timur Durrani ${ }^{\mathrm{a}}$ \\ ${ }^{a}$ Medical Toxicology, University of California San Francisco, San \\ Francisco, United States; ${ }^{b}$ Laboratory Medicine, University of \\ California San Francisco, San Francisco, United States; 'Critical Care \\ Medicine, University of California San Francisco, San Francisco, \\ United States
}

Objective: We describe a case of neuroleptic malignant syndrome (NMS) after an intramuscular depot administration of paliperidone. The gradual increase of paliperidone to therapeutic concentrations was confirmed using liquid chromatography mass spectroscopy (LC-MS). We also describe a successful bromocriptine and dantrolene taper despite the inability to remove the offending agent.

Case report: A 43-year-old man with a past medical history of acquired immune deficiency syndrome (AIDS), polysubstance use disorder and schizoaffective disorder presented after being found in prison collapsed with "abnormal muscle movements". Further history revealed that he had received an intramuscular paliperidone injection 36 hours prior to presentation in an effort to replace his daily olanzapine. Initially, his vital signs were within normal limits but he displayed rigidity in his extremities and a Glasgow Coma Score of 8 . He was medicated with etomidate and succinylcholine and then intubated. After admission to the intensive care unit, he became hyperthermic, hypertensive, tachycardic and rigid. Given concern for both NMS and malignant hyperthermia, he was treated with bromocriptine and dantrolene. His symptoms improved dramatically during the next 12 hours but returned intermittently over the next five days, requiring repeated paralysis and dose escalation of bromocriptine and dantrolene. Blood paliperidone concentrations were quantified via LC-MS. Over the next 9 days, paliperidone concentrations increased to a peak of $37 \mathrm{ng} / \mathrm{mL}$ (therapeutic range $20-60 \mathrm{ng} / \mathrm{mL}$ ) and then plateaued. The decline in his olanzapine concentrations from $15 \mathrm{ng} / \mathrm{mL}$ (therapeutic range $20-40 \mathrm{ng} / \mathrm{mL}$ ) to undetectable was consistent with the expected half-life of 21 to 54 hours. By hospital day 10, the patient was back to his mental and physical baseline despite persistent therapeutic concentrations of paliperidone. The patient was tapered from his dantrolene over 7 days, followed by a 14-day bromocriptine taper. Follow up two months after discharge showed no return of symptoms.

Conclusion: A fundamental tenet of treating NMS is the removal of offending agents. It is logical to expect a depot neuroleptic agent to cause a prolonged course of NMS due to continued exposure to the drug. However, the duration of illness was similar to the typical reported 7-10 days, albeit the manifestation of the disease may have been more severe than usual [1]. The patient's improvement reflects either a successful dose escalation and taper of bromocriptine and dantrolene or that the relevant target organ effect cannot be predicted by serum drug concentrations alone.

\section{Reference}

[1] Strawn JR, Keck Jr PE, Caroff SN. Neuroleptic Malignant Syndrome. Am J Psychiatry. 2007;164:870-6.

\section{Prolonged anesthetic emergence after low dose methylene blue for parathyroid gland visualization}

\author{
Pierre Lavry and Martine Mostin ${ }^{\mathrm{b}}$ \\ ${ }^{a}$ Anesthesiology, André Vésale Hospital, Montigny-le-Tilleul, \\ Belgium; ${ }^{b}$ Belgian Poison Centre, Brussels, Belgium
}

Objective: The monoamine oxidase (MAO) inhibitor properties of methylene blue can lead to a serotonin syndrome in patients on serotonergic drugs. We report a case of prolonged anesthetic emergence diagnosed as a serotonin syndrome after the use of methylene blue in a patient on paroxetine.

Case report: A 52-year-old overweight female patient (BMI 42) with primary hyperparathyroidism was scheduled for right inferior parathyroidectomy and adenoma removal. The patient's medical history was significant for hypertension, hyperlipidemia, depression, vascular thrombosis-related pulmonary embolism and rheumatoid arthritis. She had several previous general anesthesia procedures without notable adverse event. Her medications included bisoprolol, atorvastatin, paroxetine, alprazolam, methylprednisolone, methotrexate, etanercept, rivaroxaban and zoledronate. Rivaroxaban was stopped 2 days before surgery without bridging. She underwent general anesthesia with administration of droperidol, fentanyl, xylocaine, propofol, suxamethonium, and sevoflurane maintenance. After induction, $150 \mathrm{mg}(1.5 \mathrm{mg} / \mathrm{kg})$ methylene blue was administered by intravenous injection over 20 minutes. After an uneventful 60 minute surgical procedure, a bilateral horizontal nystagmus was noticed on extubation. In the recovery room, she was slightly agitated, with uncoordinated limb movements and remained aphasic. The laboratory 
examination showed normal results for sodium, calcium and blood glucose. Encephalography showed a bilateral slow basic rhythm indicative of an encephalopathy with no evidence of nonconvulsive seizures. The patient developed slight hyperthermia $\left(38.2^{\circ}\right.$ C) and was transferred to the intensive care unit. She was unreactive to verbal or painful stimuli (Glasgow Coma Score 6) with motoric agitation and bilateral reactive mydriasis. Ocular clonus and bilateral Babinski sign were noted. Her body temperature remained between $38-38.5^{\circ} \mathrm{C}$. Further examinations including a head computerized tomography (CT) scan, lumbar puncture and bacterial culture of catheters, revealed no abnormalities. She gradually recovered over 24 hours. Review of patient medication lead to suspicion of a serotonin syndrome due to a drug interaction between methylene blue and the selective serotonin reuptake inhibitor paroxetine.

Conclusion: Diagnosis of serotonin syndrome is a diagnosis of exclusion. In our patient, the symptoms of agitation, ocular clonus, impaired consciousness with moderate elevation of body temperature with no evidence of infection or neurological lesion lead to the diagnosis of a serotonin syndrome due to interaction between paroxetine and methylene blue. Depression is frequent in patients with hyperparathyroidism. Tapering of serotonin reuptake inhibitor antidepressants before surgery should be considered when the use of methylene blue is planned.

\section{A heart-breaking case of loperamide toxicity}

\section{Timothy C Backus ${ }^{\mathrm{a}}$, Catherine Steger ${ }^{\mathrm{a}}$, Jonathan De Olano ${ }^{\mathrm{b}}$ and Dean Olsen ${ }^{\mathrm{a}}$ \\ ${ }^{a}$ Emergency Department, Nassau University Medical Center, East

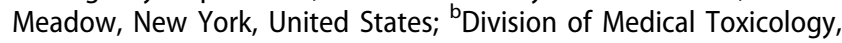 NYU School of Medicine, New York, United States}

Objective: Loperamide abuse is increasing in the US [1]. In addition to opioid effects, loperamide toxicity is associated with cardiac conduction disturbances [2]. Loperamide and its metabolite $\mathrm{N}$-desmethyl loperamide inhibit sodium and hERG potassium channels [3]. We present a case of loperamide toxicity with QRS, QTc prolongation, and delayed ventricular dysrhythmias.

Case report: A 31-year-old male with a history of opioid abuse was brought to the emergency department having been found unresponsive at home. Naloxone was given prehospital with incomplete response. The patient was unwilling to provide a history. Initial vital signs were heart rate $103 \mathrm{bpm}$, respiratory rate $24 / \mathrm{min}$, blood pressure $140 / 101 \mathrm{mmHg}$, temperature $37.1{ }^{\circ} \mathrm{C}$, oxygen saturation $95 \%$, and point-of-care blood glucose $214 \mathrm{mg} / \mathrm{dL}$. On physical examination, he was somnolent, diaphoretic, had bilaterally reactive non-miotic pupils, and tachycardic without murmurs. Laboratory tests were normal except for potassium (3.4 $\mathrm{mEq} / \mathrm{L}$ ). Paracetamol, salicylate, and ethanol concentrations were undetectable. The initial electrocardiogram revealed a QRS duration $282 \mathrm{~ms}$, QTc $675 \mathrm{~ms}$, and a terminal R wave in V1. Sodium bicarbonate was given, reducing the QRS to $148 \mathrm{~ms}$, and an infusion was started. When confronted, the patient admitted to taking fifty $2 \mathrm{mg}$ loperamide tablets shortly before presentation, as he had been doing on alternating days for at least a month. He reported that such a dose had not previously rendered him unconscious. The patient was admitted and approximately 26 hours post-ingestion had short episodes of ventricular tachycardia. An electrocardiogram obtained at that time showed QRS $132 \mathrm{~ms}$, QTc $680 \mathrm{~ms}$, and changes resembling a Brugada syndrome pattern with deeply inverted T waves; the largest in V3 at $-25 \mathrm{mV}$. On the seventh hospital day, his QRS was $110 \mathrm{~ms}$ and QTc $475 \mathrm{~ms}$ thus he was discharged on carvedilol $3.125 \mathrm{mg}$ every 12 hours and external defibrillator.

Conclusion: This case illustrates the acute and delayed cardiac effects of loperamide toxicity; recognizing and treating the lethal complications are paramount.

\section{References}

[1] Daniulaityte R, Carlson R, Falck R, et al. "I just wanted to tell you that loperamide WILL WORK": a web-based study of extra-medical use of loperamide. Drug Alcohol Depend. 2013;130:241-244.

[2] Wu PE, Juurlink DN. Clinical Review: Loperamide toxicity. Ann Emerg Med. 2017;70:245-252.

[3] Vaz RJ, Kang J, Luo Y, et al. Molecular determinants of loperamide and $\mathrm{n}$-desmethyl loperamide binding in the hERG cardiac $\mathrm{K}+$ channel. Bioorg Med Chem. 2018;28:446-451.

\section{Drug reactivation with eosinophilia and systemic symptoms (DRESS): a severe adverse drug reaction}

\author{
Ilaria Melara ${ }^{a}$, Valeria M Petrolini ${ }^{b}$, Giampiero Martini ${ }^{c}$, \\ Chiara Fulcheri ${ }^{c}$, Alessandro Raviolo ${ }^{c}$, Maria Di Primo ${ }^{b}$ \\ and Carlo A Locatelli ${ }^{\mathrm{b}}$ \\ ${ }^{a}$ Emergency Medicine Fellowship Program, University of Pavia,

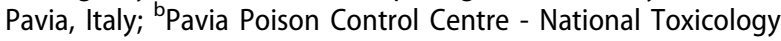 \\ Information Centre - Clinical and Experimental Lab, Toxicology \\ Unit, Maugeri Clinical and Scientific Institutes IRCCS and University \\ of Pavia, Pavia, Italy; ' Emergency Department, AO Santa Croce e \\ Carle, Cuneo, Italy
}

Objective: Drug reactivation with eosinophilia and systemic symptoms (DRESS) is a cutaneous adverse reaction with a mortality of $10-20 \%$ [1]. It develops after 3-6 weeks of exposure to the offending drug which includes aromatic anticonvulsants, allopurinol, sulphonamides, antiretroviral and antibiotics [1]. It is characterised by fever, rash, internal organ involvement (hepatitis, interstitial nephritis, pneumonitis, myocarditis) and eosinophilia or other leukocyte abnormalities [1]. Treatment involves topical and IV corticosteroids, with IV immunoglobulin for life-threatening signs. Antiviral treatment is recommended in major viral reactivation [2].

Case report: A 24-year-old man was admitted to hospital because of fever, an itchy and exfoliative rash and bronchitis. These signs started two weeks earlier, at the fourth week after starting salazopyrin (sulfasalazine) at increasing dosage because of seronegative enteritis. At admission, blood analysis revealed mild leucocytosis with eosinophils within the normal range, severe liver failure (ALT $1620 \mathrm{U} / \mathrm{L}$, AST $1152 \mathrm{U} / \mathrm{L}$, bilirubin $1.7 \mathrm{mg} / \mathrm{dL}$, INR 1.9) and a negative serology for cytomegalovirus (CMV) and Epstein-Barr virus (EBV). Serology for human herpesvirus 6 (HHV-6) and investigations for other organ damage were performed. Treatment with IV corticosteroids, immunoglobulin, valganciclovir and $\mathrm{N}$-acetylcysteine $(150 \mathrm{mg} /$ $\mathrm{kg} /$ day) were started. The patient improved over the following days, and laboratory tests normalized in a week.

Conclusion: Prompt recognition of DRESS is important to discontinue the responsible drug and start specific treatment. Biochemical indexes of organ damage and viral serology are essential for the diagnosis and the evaluation of severity. Eosinophilia is an early sign, but it can be absent in advanced phases and/or when hepatic cytolysis indices increase (as in this case). Usual treatment is based on corticosteroids and immunoglobulin administration. Nevertheless, treatment of the severe form of the syndrome is not well defined. Adjunctive treatment with $\mathrm{N}$-acetylcysteine has been tried with varying results and, in this case was employed because of the severe liver damage present [3].

\section{References}

[1] Fernando SL. Drug-reaction eosinophilia and systemic symptoms and drug-induced hypersensitivity syndrome. Australasian J Dermatol. 2014;55:15-23. 
[2] Descamps V, Ben Said B, Sassolas B, et al. [Management of drug reaction with eosinophilia and systemic symptoms (DRESS)]. Ann Dermatol Venereol. 2010;137:703-8. French

[3] Moling O, Tappeiner L, Piccin A, et al. Treatment of DIHS/DRESS syndrome with combined $\mathrm{N}$-acetylcysteine, prednisone and valganciclovir - a hypothesis. Med Sci Monit. 2012;18:CS57-62.

\section{Zidovudine overdose due to therapeutic error in a newborn}

\author{
Maria Di Primo ${ }^{a}$, Giulia Scaravaggi ${ }^{a}$, Davide Lonati ${ }^{a}$, \\ Eleonora Buscaglia ${ }^{a}$, Giuseppe Bruglieri ${ }^{\mathrm{b}}$ and \\ Carlo A Locatelli ${ }^{a}$

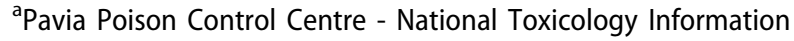 \\ Centre - Clinical and Experimental Lab, Toxicology Unit, Maugeri \\ Clinical and Scientific Institutes IRCCS and University of Pavia,

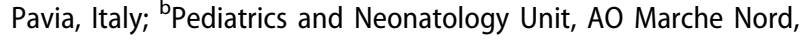 \\ Pesaro, Italy
}

Objective: Zidovudine (azidothymidine, AZT) is routinely prescribed in antiretroviral therapy and for primary human immunodeficiency virus (HIV) prophylaxis in newborns. Few cases of therapeutic error involving AZT, with various degrees of clinical manifestations (one lethal case is also described) are reported in the medical literature [1]. We describe a case involving a newborn that received an overdose of AZT as a consequence of incorrect understanding of the indication provided by health professionals.

Case report: A 5-day-old infant $(3.2 \mathrm{~kg})$ was accidentally given a 10 -fold overdose $(10.3 \mathrm{~mL}$ of AZT $100 \mathrm{mg} / 10 \mathrm{~mL}$ every 12 hours for 2 days) of AZT by the parents at home. The recommended dose in newborns is $8.0 \mathrm{mg} / \mathrm{kg} /$ day, corresponding to $1.3 \mathrm{~mL}$ every 12 hours in our patient. Close monitoring in a pediatric ward for possible appearance of neutropenia, anemia and metabolic acidosis (lactate) was scheduled. Pediatricians were alert also for late appearance of AZT-induced neutropenia. AZTprophylaxis restarted 6 days after the overdose. The patient remained asymptomatic during hospitalization and the following two weeks; none of the expected clinical manifestations were observed. The history revealed that, at discharge from nursery, the parents received from the nurses instructions to use a $1 \mathrm{~mL}$ syringe (not included in the drug box) to obtain the prescribed dose ( $1.3 \mathrm{~mL}$ every 12 hours). At home, however, the parents dispensed the drug using the $10 \mathrm{~mL}$ syringe included in the drug box and $0.3 \mathrm{~mL}$ using an insulin-syringe ( $1 \mathrm{~mL}$ capacity).

Conclusion: Accidental overdose of AZT happens and the modality of error is always the same (incorrect use of the syringe included on the drug box) in the few cases reported in the medical literature. The syringe included in the box can only be used safely with the administration of AZT in infants over $10 \mathrm{~kg}$. In cases of HIV prophylaxis in newborns (when the recommended dose is a few $\mathrm{mL}$ of solution) the instruction can confuse parents, causing overdose with potentially severe consequences. In our case the administration occurred for "only" 48 hours, probably not sufficient to cause toxicity. To avoid this preventable overdose parents should be advised correctly and manufacturers should evaluate the appropriate size of the syringe included in the drug box when this drug is used in babies weighing less than $5 \mathrm{~kg}$.

\section{Reference}

[1] Livshits Z, Hoffman RS, Nelson LS, et al. Zidovudine (AZT) overdose in a healthy newborn receiving postnatal prophylaxis. Clin Toxicol (Phila). 2011;49:747-749.

\section{Drug-induced liver injury in androgen anabolic steroid users: a case of acute cholestatic syndrome}

\author{
Azzurra Schicchi ${ }^{a}$, Valeria M Petrolini ${ }^{a}$, \\ Giulia Scaravaggi ${ }^{a}$, Damiano Cantone ${ }^{b}$, Sergio Neri ${ }^{b}$ \\ and Carlo A Locatelli ${ }^{a}$ \\ ${ }^{a}$ Pavia Poison Control Centre - National Toxicology Information \\ Centre - Clinical and Experimental Lab, Toxicology Unit, Maugeri \\ Clinical and Scientific Institutes IRCCS and University of Pavia,

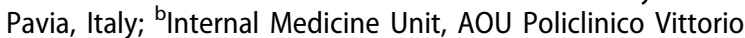 \\ Emanuele, Catania, Italy
}

Objective: Androgen anabolic steroids (AAS) are illegally used to increase muscle mass and athletic performance. Adverse reactions from their use are described, and the Drug-Induced Liver Injury (DILI) Network found an increase in cases of body-building related hepatotoxicity. We describe a case of acute AAS-related cholestatic hepatitis.

Case report: A 41-year-old man was admitted to the emergency department with jaundice lasting 6 days. His history was negative for chronic disease, medications or drug consumption. Biochemistry results showed: AST $300 \mathrm{IU} / \mathrm{L}$, ALT $200 \mathrm{IU} / \mathrm{L}$, bilirubin $12 \mathrm{mg} / \mathrm{dL}$ ( $5 \mathrm{mg} / \mathrm{dL}$ unconjugated). Abdomen ultrasound and computerised tomography (CT) scan were negative for biliary obstructive disorders. During his hospitalization, biochemistry results showed a progressive worsening of cholestasis (peak bilirubin concentration $43 \mathrm{mg} / \mathrm{dL}$, of which $25 \mathrm{mg} / \mathrm{dL}$ unconjugated) with an amelioration of transaminases (AST $45 \mathrm{IU} / \mathrm{L}$, ALT $51 \mathrm{lU} / \mathrm{L}$ ). The clinical picture was characterized by jaundice and severe itching with scratch wounds. No causes of hyperbilirubinemia were found until the patient admitted the use of methylepitiostanol (30 mg/day orally) for 6 weeks, stopped one week before the onset of jaundice). Pathological findings of liver biopsy were drug-induced chronic hepatitis with sections showing bile plugs in canaliculi and bile accumulation in hepatocytes. The Roussel Uclaf Causality Assessment Method (RUCAM) score was 9, meaning high probably of a relation between hepatotoxicity and the supposed drug (AAS in this case). He was treated with ursodeoxycholic acid and antihistamines to reduce itching. To control the elevated plasma bilirubin repeated plasmapheresis sessions (one every 3 days for a month) were performed. He recovered completely and was discharged after 3 months of hospitalization.

Conclusion: The increase of AAS-related DILI diagnosis may be due, not only to a rise in their use, but also to the better knowledge of this type of hepatotoxicity related to more signaled cases. Patterns of liver injury can vary: peliosis hepatis, nodular regeneration, transient serum enzyme elevations with many cases of acute cholestatic hepatitis. The causality of liver injury is difficult to determine if patients do not admit AAS use. The timing of onset is insidious (1-4 months from starting use), and there are no specific diagnostic tests. AAS-related DILI diagnosis is based on history of AAS-use, clinical manifestation and causality assessment methods like RUCAM (even if not completely validated for this purpose).

\section{Prolonged toxicity following self- administration of a kambô cleansing ritual}

\author{
Shireen Banerji ${ }^{\mathrm{a}}$, Krissy Melvin ${ }^{\mathrm{b}}$, Ken Rooks ${ }^{\mathrm{b}}$ and \\ Christopher O Hoyte
}

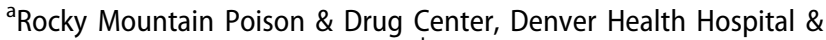
Authority, Denver, United States; ${ }^{\text {b } G o o d ~ S a m a r i t a n ~ M e d i c a l ~ C e n t e r, ~}$ US Acute Care Solutions, Lafayette, United States 
Objective: Kambô comes from the secretion of the giant monkey frog (Phyllomedusa bicolor) and is used widely amongst indigenous people in the Amazon as a medicine. Kambô cleanses are touted online as a shamanic detoxification remedy as well as treatment for a variety of different conditions, including chronic pain and drug dependence. The kambô cleansing ritual consists of burning small holes into the skin of the person taking the cleanse and then applying the secretion of the frog to the burns. The end result varies, but kambô's cleansing effects are purported to begin within minutes of administration, and the hope for users is to feel less pain in the days and weeks after treatment. We report a case of a patient with prolonged adverse effects after using a kambô cleansing ritual purchased online as a detoxification aid.

Case report: Our regional poisons center was called by an emergency department healthcare provider regarding a 29-year-old, otherwise healthy, male who attempted to detoxify himself from heavy metals using a kambô cleansing product he purchased on the Internet. He reported flu-like symptoms which persisted for 6 days. These included nausea, over 50 episodes of vomiting, and diarrhea, as well as chills, facial numbness, abdominal pain, hematemesis, and dry mucous membranes. Three round burn "holes" were seen on his upper arm. There was no clinical evidence of infection. Neurological exam was non-focal and his vital signs were normal. Blood chemistry abnormalities included hypokalemia (potassium 2.8 $\mathrm{mEq} / \mathrm{L}$ ) and hypophosphatemia. No abnormalities were seen on the electrocardiogram. Complete blood count and coagulation markers were normal. The patient was given intravenous fluids and antiemetic agents and despite plans to admit to the hospital, he chose to be discharged home due to his uninsured status.

Conclusion: This is an important case to illustrate the concerns with self-diagnosis and self-treatment of a condition with an alternative remedy. This patient suffered prolonged symptoms as a result of a detoxification remedy that he purchased online, without the advice of a healthcare provider. There are likely other alternative remedies with potential serious consequences readily available to the general public, and for this reason, healthcare providers need to include non-traditional treatments in their differentials when evaluating patients.

\section{1. latrogenic in-flight buprenorphine overdose on a large European airline caused by a dangerous emergency medical kit}

\author{
Josh J Wang ${ }^{\mathrm{a}}$, Mark K Su${ }^{\mathrm{a}}$, Rana Biary ${ }^{\mathrm{a}}$ and \\ Robert S Hoffman ${ }^{\mathrm{a}}$ \\ ${ }^{a}$ Emergency Medicine, Division of Medical Toxicology, New York \\ University School of Medicine, New York, United States
}

Objective: In-flight pain emergencies are responsible for $17 \%$ of medical diversions on commercial airlines [1]. While the Federal Aviation Authority mandates that airlines carry aspirin in the emergency medical kit (EMK), some airlines carry opioids. This case highlights the risks associated with in-flight administration of opioid analgesics from an ill-equipped EMK.

Case report: The poison center was consulted about a 38-yearold, opioid-naive woman who was brought to an emergency department directly from the airport following a transatlantic flight on a large European airliner. She had normal vital signs but was somnolent and nauseous with bilateral miosis. In her possession was a physician note attesting that she had complained of leg pain during the flight and was given buprenorphine $(400 \mu \mathrm{g}$ sublingual) and aspirin ( $300 \mathrm{mg}$ oral) from the emergency medical kit on board the flight. In the absence of hypoventilation, we recommended against naloxone administration. The patient was admitted to the intensive care unit for monitoring and discharged home 24 hours later without sequelae. We investigated the EMK contents of the patient's airline and were extremely concerned by our findings. The quantity of buprenorphine $(30 \times 200 \mu \mathrm{g}$ tablets), its convenient location within the lid compartment (next to the stethoscope and face mask), and the relative scarcity of naloxone ( $2 \times 0.4 \mathrm{mg}$ ampules) were all striking. While buprenorphine has a ceiling effect on respiratory depression in healthy volunteers, its high mu-opioid receptor affinity makes it difficult to treat with standard doses of naloxone [2]. Many physicians are also unfamiliar with analgesic buprenorphine doses which are 10-fold lower than doses used for opioid medication assisted therapy. Lastly, sublingual buprenorphine has a peak therapeutic effect 1-4 hours following administration: too late and too long for pain on a plane for shorter flights.

Conclusion: The large quantity of easily-accessible buprenorphine in an airline's EMK directly contributed to this patient's overdose. While the patient did not suffer permanent injury, she was subjected to many medical tests and hospitalized for 24 hours in a costly ICU bed. We seriously question the role of buprenorphine in the management of in-flight pain crises.

\section{References}

[1] Peterson DC, Martin-Gill C, Guyette FX, et al. Outcomes of medical emergencies on commercial airline flights. $N$ Engl J Med. 2013:368:2075-2083.

[2] Van Dorp E, Yassen A, Sarton E, et al. Naloxone reversal of buprenorphine-induced respiratory depression. Anesthesiol: J Am Soc Anesth. 2006;105:51-57.

\section{A case series of medication errors due to changes in the electronic prescription of paracetamol}

\author{
Kim P Dalhoffa, Søren Bøgevig ${ }^{b}$, Tonny S Petersen ${ }^{b}$ \\ and Lene Reuther ${ }^{\mathrm{b}}$ \\ ${ }^{a}$ Clinical Pharmacology, Bispebjerg and Frederiksberg University \\ Hospital, Copenhagen, Denmark; ${ }^{b}$ Bispebjerg and Frederiksberg \\ University Hospital, Copenhagen, Denmark
}

Objective: A description of four patients who were treated inadvertently with $8 \mathrm{~g}$ of paracetamol daily due to a change in the electronic prescription system.

Case series: Two major regions in Denmark use an electronic prescription module (Sundhedsplatformen, Epic) from which patients' medication lists at discharge are aligned with an online

Table 1. Patient parameters, treatment and outcome in four patients that were inadvertently prescribed paracetamol $8 \mathrm{~g}$ daily.

\begin{tabular}{|c|c|c|c|c|}
\hline Gender/age & Indication & Paracetamol exposure & Risk Assessment Score & Outcome \\
\hline $\mathrm{F} / 65$ & Hip fracture & $8 \mathrm{~g}$ for 9 days & 3 & 14 hours NAC, liver function tests (LFTs) normal \\
\hline$M / 32$ & Back surgery & $8 \mathrm{~g}$ for 14 days & 3 & 16 hours NAC, $\max$ ALT $138 \mathrm{U} / \mathrm{L}$ \\
\hline
\end{tabular}


drug list (FMK), used by pharmacies. Due to a change in the transfer of the prescription of paracetamol from Sundhedsplatformen to FMK, paracetamol in the dose $2 \times 500 \mathrm{mg} 4$ times daily prescribed in the hospital was automatically changed to $1 \times 1,000 \mathrm{mg} 4$ times daily and handed out to the patients when they redeemed the prescription. Neither the prescribing physicians nor the patients were aware of this change. The patients therefore misunderstood the prescribed dose and took a double dose with the risk of hepatotoxicity. The Danish Poisons Information Centre (DPIC) received enquiries from 4 patents discharged from surgery taking $8 \mathrm{~g}$ of paracetamol daily (Table 1). They were hospitalised and treated with $\mathrm{N}$-acetylcysteine (NAC). None of them were risk patients e.g. chronic alcohol abuse. One patient had a slight increase in ALT, but they were all discharged without symptoms.

Conclusion: Prescription of drugs and inappropriate changes in the transfer of drug information between electronic prescription databases may lead to potentially harmful medication errors. PICs play an important role in discovering these incidents.

\section{Fluoxetine-induced seizure in a pediatric patient}

\author{
Anthony Scoccimarro, Ryan Marino and \\ Anthony Pizon \\ University of Pittsburgh School of Medicine, Pittsburgh, United \\ States
}

Objective: Fluoxetine is a commonly prescribed selective serotonin re-uptake inhibitor (SSRI), known to be associated with serotonin toxicity, but with a relatively low risk of seizure. We present a case of fluoxetine-induced seizure in an adolescent.

Case report: A 15-year-old male with a history of depression, attention deficit hyperactivity disorder, and oppositional defiant disorder, presented to a community emergency department after intentional ingestion of $500 \mathrm{mg}$ fluoxetine $(25 \times 20 \mathrm{mg}$ tablets, 8.1 $\mathrm{mg} / \mathrm{kg}$ ) 3 hours prior to arrival. He was transferred to a tertiary care center for anticipated clinical course. Prior to transfer, the patient had 45 seconds of generalized tonic-clonic seizure activity, followed by a post-ictal period of 15 minutes. He was given lorazepam $1 \mathrm{mg}$ intravenously following the seizure activity. Upon arrival at the tertiary care facility, his mental status and vital signs had returned to baseline. He was found to have patellar hyperreflexia with normal upper extremity reflexes, and continuous bilateral ankle clonus. Examination of head and neck revealed a left lateral tongue laceration. Serum ethanol, acetaminophen, and salicylates were undetectable. Qualitative urine gas chromatography/mass spectrometry (GS/MS) identified fluoxetine, caffeine, and nicotine. Serum chemistry was notable for a potassium concentration of $4.0 \mathrm{mmol} / \mathrm{L}$ and creatinine $0.75 \mathrm{mg} / \mathrm{dL}$. A serum sample obtained approximately 12 hours after ingestion demonstrated serum fluoxetine and norfluoxetine concentrations of $434 \mu \mathrm{g} / \mathrm{L}$ (normal range $47-469 \mu \mathrm{g} / \mathrm{L}$ ) and $103 \mu \mathrm{g} / \mathrm{L}$ (normal range 52 to $446 \mu \mathrm{g} / \mathrm{L}$ ), respectively. His serotonin toxicity resolved by hospital day 2 and he was subsequently transferred to an inpatient psychiatric facility on day 4 without neurologic sequelae or recurrence of seizure activity.

Conclusion: Fluoxetine was described early in the literature as having anticonvulsant effects both in animals and humans. Of commonly prescribed SSRIs, retrospective studies have demonstrated that fluoxetine is the least likely to cause seizure, occurring in $1 \%$ of patients presenting with fluoxetine overdose. There is at least one other case reported in children, however data is lacking in the pediatric population. Although our patient had apparently therapeutic concentrations of fluoxetine and norfluoxetine, this may have been due to the delay in sampling. He had no known preexisting history of seizure disorder or predilection. Caffeine was also found in the patient's urine and although methylxanthines can cause seizure, he did not demonstrate any other signs of caffeine toxicity. Nicotine was also found, however he did not display any clinical signs of nicotine toxicity. This pediatric patient's seizure is most appropriately attributed to fluoxetine.

\section{Dose-dependent increased risk of adverse cardiovascular events and mortality in patients with acute drug overdose treated with sodium bicarbonate}

\author{
Judson Ellis ${ }^{a}$, Lynne D Richardson ${ }^{b}$, \\ Rajesh Vedanthan ${ }^{c}$ and Alex F Manini ${ }^{d}$ \\ ${ }^{a}$ Brown University, Providence, United States; ${ }^{b}$ Emergency \\ Medicine, Icahn School of Medicine at Mount Sinai, New York, \\ United States; 'Cardiology, New York University Langone School of \\ Medicine, New York, United States; ${ }^{d}$ Medical Toxicology, Icahn \\ School of Medicine at Mount Sinai, New York, United States
}

Objective: Sodium bicarbonate therapy (SBT) is provided in the emergency department (ED) for a variety of indications but its use is controversial. Some authors recommend SBT for overdoses of salicylates and sodium channel antagonists (e.g. tricyclic antidepressants [TCAs]). Due to its effect in lowering serum potassium, however, SBT administration may prolong the QTc interval and potentially cause increased rates of adverse cardiovascular events (ACVE), such as ventricular dysrhythmias. To quantify this risk, we evaluated an emergency department (ED) overdose patient population for the association between SBT and ACVE.

Methods: We prospectively analyzed consecutive ED patients with acute drug overdose who were given SBT at two urban teaching hospitals from 2015-present. Data included SBT indication, dose, duration, and QTc (initial/peak from computer generated Bazett correction) during hospital stay. We used median values to dichotomize total dose (high/low) and total duration (long/short) of SBT. Patients were prospectively followed to hospital discharge for the occurrence of the primary outcome: ACVE and/or mortality. The previously validated definition of ACVE was used for in-hospital occurrence of any of the following: ventricular dysrhythmia, myocardial infarction, shock requiring vasopressors, and cardiac arrest [1]. Severe QTC prolongation was defined as previously validated using the cut off $\geq 500 \mathrm{~ms}$. Occurrence of ventricular dysrhythmia was adjudicated by a blinded cardiologist.

Results: Indications for SBT in 30 patients analyzed were: salicylism $(n=5)$, sodium channel antagonist (6 TCA, 5 other), wide QRS in absence of known drug $(n=9)$, acidosis or cardiac arrest $(n=$ $3)$, and unknown $(n=2)$. After SBT, severe QTc prolongation occurred in $6(20 \%)$, ACVE in $17(57 \%)$, and 6 patients (20\%) died. There was a significant association between severe QTc prolongation in-hospital for both high dose and long duration groups $(p<$ 0.05 for both). There was a significant correlation between both SBT dose $(83 \%$ high, 38\% low, $\mathrm{p}<0.05)$ and SBT duration $(100 \%$ long, $25 \%$ short, $\mathrm{p}<0.05$ ) with the primary outcome.

Conclusion: ED patients with acute drug overdose receiving SBT had very high rates of mortality and ACVE, which were strongly associated with higher dose and longer duration of SBT. Severity of overdose may represent a limitation to interpretation as a potential confounder. Overall, these results are consistent with the hypothesis that SBT may cause unintended ACVE, and validates previous safety concerns regarding the administration of SBT to overdose patients.

\section{Reference}

[1] Manini AF, Nelson LS, Stimmel B, et al. Incidence of adverse cardiovascular events in adults following drug overdose. Acad Emerg Med. 2012;19:843-849. 


\section{Human exposures to irritants for treatment of common warts}

\author{
Daniela Acquarone ${ }^{a}$ and Maren Hermanns-Clausen ${ }^{b}$ \\ aPoisons Information Center, Charité Universitätsmedizin Berlin, \\ Berlin, Germany; ${ }^{\text {b}}$ Poisons Information Center, Center for \\ Pediatrics, Medical Center - University of Freiburg, Freiburg, \\ Germany
}

Objective: Warts are a very common viral skin infection which usually resolves spontaneously; however, as they may last months to years, treatment is usually sought for cosmetic reasons. There are numerous remedies used, but none is based on strong evidence [1]. Among other caustics, particularly monochloroacetic acid, trichloroacetic acid, and formic acid are used for treatment in concentrations of $40-70 \%$. The aim of our study was to analyze calls to two poison centers regarding human exposures to caustic wart remedies.

Methods: A retrospective study of calls regarding human exposures to caustic wart removers reported to two poison centers from 2009 to 2017 was conducted. All cases were anonymized before further data analysis.

Results: Overall 49 calls regarding suspected exposures to caustic wart removers were collected; 37 out of these 49 cases with verified exposure were analyzed further. Exposed patients were aged 0.7-65 years (median 10.5 years). Dermal exposures dominated $(n=27,73 \%)$, followed by oral $(n=8,22 \%)$ and ocular $(n=2$, $5 \%)$ exposures. The majority of exposures were unintentional $(n=35,94 \%)$; in six cases the wart removers were mistaken for cough drops, mouthwash, perfume or others. Severity of poisoning was minor $(n=23,62 \%)$, moderate $(n=11,30 \%)$ or severe $(\mathrm{n}=3,8 \%)$. Agents involved were monochloroacetic acid in a concentration of about $50 \%(n=21,7 \%)$, formic acid $70 \%(n=$ $12,32 \%)$, and trichloroacetic acid $40 \%(n=3,8 \%)$. One wart remover consisted of a mixture of monochloroacetic, dichloroacetic and trichloroacetic acid (3\%). Severe chemical burns were caused by monochloroacetic acid $(n=2)$ and formic acid $(n=1)$ after treating common warts on fingers $(n=1)$ or after spilling the wart remover on the skin $(n=2)$.

Conclusion: Reports about adverse effects of caustic wart remedies were infrequent. Nevertheless, moderate to severe chemical burns occurred even after application with therapeutic intent. Application of caustic wart remedies must be meticulous because spills may result in rapid damage to the epidermis of the surrounding skin. In addition, caustic wart treatment solutions may be mistaken for other medical drugs or cosmetics. Since caustic wart removers are purchased over-the-counter, these products might be perceived to be less hazardous than prescription drugs.

\section{Reference}

[1] Sterling J. Treatment of warts and molluscum: what does the evidence show? Curr Opin Pediatr. 2016:28:490-499.

\section{Adverse effects of concomitant intravenous administration of beta- adrenergic antagonists and calcium channel blockers}

\section{Emily S Taub, Chester Poon and Silas W Smith}

Division of Medical Toxicology, Ronald O. Perelman Department of Emergency Medicine, NYU School of Medicine, New York, United States
Objective: Beta-adrenergic antagonists (BAAs) and calcium channel blockers (CCBs) negatively affect chronotropy and inotropy and are administered for many indications. The frequency of adverse reactions when BAAs and CCBs are administered concomitantly is infrequently described [1]. We reviewed the incidence of hemodynamic instability in patients in whom both a $B A A$ and $C C B$ were administered within a pharmacologically relevant time period.

Methods: This was a quality improvement initiative in emergency department (ED) patients, performed at an urban, tertiary care hospital network from 1 October 2016 to 30 September 2018. Adult patients ( $\geq 18$ years) who received both an intravenous BAA and CCB (in either order) within 6 hours were included. Primary outcomes were the incidence of bradycardia (heart rate $<60$ bpm) or hypotension (systolic blood pressure $<90 \mathrm{mmHg}$ ) after administration of the second medication. Secondary outcomes were associated diastolic blood pressure changes and administratively assigned primary and secondary diagnoses.

Results: Overall 56 ED patients met inclusion criteria. The median time between medication administration was 110 minutes for the cohort. The median decrease in pulse was $42 \mathrm{bpm}$. The median decrease in systolic blood pressure was $26 \mathrm{mmHg}$, and the median diastolic blood pressure decrease was $11 \mathrm{mmHg}$. According to the prespecified endpoints, $8.9 \%$ developed bradycardia, $8.9 \%$ developed systolic hypotension, and $17.9 \%$ developed either complication. These complications occurred at median times after second medication administration of 36 minutes for bradycardia and 10 minutes for hypotension. The most common diagnosis in patients who received concomitant $B A A$ and CCB administration was atrial fibrillation $(n=39)$. All patients who developed bradycardia had atrial fibrillation $(n=4)$ or atrial flutter $(n=1)$. All patients who developed hypotension had atrial fibrillation $(n=5)$.

Conclusion: Despite a lack of published data, the administration of both BAAs and CCBs within 6 hours can cause significant hypotension and bradycardia in emergency department patients. Avoidance of concurrent administration of these medication classes or assurance of antidotal availability or pretreatment (i.e. with calcium salts) should be strongly encouraged.

\section{Reference}

[1] Oesterle SN, Alderman EL, Beier-Scott L, et al. Diltiazem and propranolol in combination: hemodynamic effects following acute intravenous administration. Am Heart J. 1986;111:489-497.

\section{Back pain and muscle stiffness: a case of valbenazine-associated neuroleptic malignant syndrome}

\author{
Monica R Noori ${ }^{\mathrm{a}}$, William J Boroughf ${ }^{\mathrm{b}}$ and \\ Benjamin W Hatten ${ }^{\mathrm{b}, \mathrm{c}}$ \\ ${ }^{a}$ Department of Emergency Medicine, Denver Health Medical \\ Center, Denver, United States; ${ }^{\mathrm{b}}$ Toxicology Associates, Rocky \\ Mountain Poison \& Drug Center, Denver, United States; ' University \\ of Colorado School of Medicine, Denver, United States
}

Objective: Tardive dyskinesia (TD) is an involuntary movement disorder involving repeated stereotyped movements of the face, extremities and/or trunk in patients with prolonged exposure to dopamine receptor-blocking agents. In April 2017, the US Food and Drug Administration approved the vesicular monoamine transporter 2 (VMAT2) inhibitor, valbenazine, as the first and only agent in the US specifically indicated for treatment of TD. Inhibition of VMAT2, a presynaptic protein that regulates 
packaging and synaptic release of monoamines, reduces dyskinetic movements via decreased dopamine stimulation in the nigrostriatal pathway. We report a patient with tardive dyskinesia treated with valbenazine who developed neuroleptic malignant syndrome (NMS).

Case report: A 21-year-old male with schizophrenia on courtordered monthly depot aripiprazole was initiated on valbenazine for TD three months prior to presentation. Three weeks prior to hospitalization, he reported back pain and generalized muscle stiffness that increased over subsequent weeks. His last injection of aripiprazole was 2 weeks prior to presentation and access to other medications was restricted. The day before presentation, he developed hyperthermia $\left(4{ }^{\circ} \mathrm{C}\right)$ and inability to ambulate due to muscle stiffness. He was transferred from his assisted living facility to the emergency department with blood pressure $115 / 82$ $\mathrm{mmHg}$, heart rate 128 beats/minutes, and temperature $38.4{ }^{\circ} \mathrm{C}$. He exhibited altered mentation, tachycardia, increased tone in proximal muscles and trunk, and flushed skin. Laboratory data was significant for white blood cell $16,920 / \mu \mathrm{L}$, creatinine $1.04 \mathrm{mg} /$ $\mathrm{dL}$, troponin $0.115 \mathrm{ng} / \mathrm{mL}$, aspartate aminotransferase $1337 \mathrm{U} / \mathrm{L}$, alanine aminotransferase $288 \mathrm{U} / \mathrm{L}$, and creatine kinase 107,464 U/ L. Spine magnetic resonance imaging (MRI) was unremarkable, and no infectious source was identified. Over his 8-day hospitalization, he improved with diphenhydramine, benztropine, benzodiazepines, intravenous fluids and valbenazine cessation.

Conclusion: To our knowledge, this is the first reported case of valbenazine-associated NMS. This syndrome is a rare, potentially fatal disorder requiring a high index of suspicion for diagnosis. We hypothesize that valbenazine-related decrease of intrasynaptic dopamine precipitated NMS. However, the aripiprazole injections cannot be excluded as a factor and likely played a role in disease severity. Notably, the patient had been receiving aripiprazole injections for at least a year without development of NMS. Furthermore, his symptoms resolved despite an ongoing antipsychotic burden, and he has continued aripiprazole injections without return to the local emergency department. The recent approval of valbenazine fulfils an unmet need for TD management. Nevertheless, development of neuroleptic malignant syndrome, especially when used in conjunction with antipsychotic medications, may limit its use.

\section{Clonidine administration error during growth hormone deficiency testing}

\author{
Katharina Von Fabeck ${ }^{\mathrm{a}}$, Soizic Beaumier ${ }^{\mathrm{b}}$, \\ Luc De Haro ${ }^{\mathrm{a}}$ and Nicolas Simon ${ }^{\mathrm{a}, \mathrm{c}}$ \\ ${ }^{a}$ Service de Pharmacologie Clinique, Centre Antipoison et de \\ Toxicovigilance, Hôpital Sainte Marguerite, APHM, Marseille,

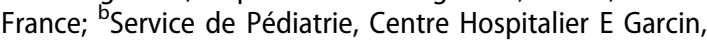 \\ Aubagne, France; ' ${ }^{\text {Aix }}$-Marseille Univ, APHM, INSERM, IRD, \\ SESSTIM, Marseille, France
}

Objective: Clonidine is a centrally-acting imidazoline alpha-2 adrenoreceptor agonist acting as an antihypertensive agent. It is also used in children with poor growth rate to investigate somatropic function by stimulating the growth hormone [1]. The aim is to emphasize the high risk of clonidine adverse effects during this medical test as previously reported [2].

Case report: A 21-month-old boy with slow growth was hospitalised in order to perform a clonidine growth hormone test. The nurse did not understand the protocol and crushed the tablet $(0.15 \mathrm{mg})$, dissolved the resulting powder in water and injected the solution intravenously. By 15 minutes drowsiness, hypertension $(136 / 98 \mathrm{mmHg})$ and difficulties in breathing were reported. The pediatrician consulted the poison control center of Marseille where the clinical toxicologist proposed to transfer the boy into the intensive care unit due to the risk of respiratory depression, pulmonary embolism, hypotension and cardiac dysrhythmia. The use of naloxone in case of respiratory depression was discussed. The same pediatrician called 10 minutes later because hypotension and miosis were observed. The naloxone protocol $0.1 \mathrm{mg} / \mathrm{kg}$ as infusion was advised. The child was then transferred in the university hospital and treated with naloxone. Drowsiness and miosis resolved, blood pressure normalized and he recovered completely the same day. He did not develop any complications.

Conclusion: Medication errors can have serious consequences and if an error occurs it is important to admit the error as soon as possible in order to take appropriate action. Adequate training is essential in performing investigative tests to avoid therapeutic and medication administration errors. In this case it was necessary to transfer the patient to an intensive care unit where close monitoring, treatment with an antidote and in consequence complete recovery occured.

\section{References}

[1] Ibba A, Guzzetti C, Casula L, et al. Reliability of clonidine testing for the diagnosis of growth hormone deficiency in children and adolescents. Clin Endocrinol (Oxf). 2018;89(6):765-770.

[2] Schmitt $C$, Kervégant $M$, Ajaltouni $Z$, et al. [Clonidine poisoning in a child: a case report]. Arch Pediatr. 2014;21:1213-1215. French.

\section{Pumping away: Use of the Impella ${ }^{\circledR}$ ventricular assist device for cardiogenic shock from clozapine- induced fulminant myocarditis}

\author{
Madeline H Renny ${ }^{a}$, Xingchen Mai ${ }^{b}$, \\ Robert S Hoffman ${ }^{a}$ and Rana Biary ${ }^{a}$ \\ ${ }^{a}$ Ronald O. Perelman Department of Emergency Medicine, NYU

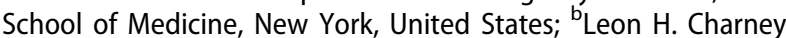 \\ Division of Cardiology, NYU School of Medicine, New York, United \\ States
}

Objective: Clozapine-induced myocarditis has a high mortality rate secondary to cardiogenic shock. The Impella ${ }^{\circledast}$ is a percutaneously inserted, microaxial flow, short-term ventricular support device. We describe a case of a patient with clozapine-induced fulminant myocarditis successfully bridged to recovery with placement of an Impella ${ }^{\oplus}$.

Case report: A 26-year-old man with a history of schizoaffective disorder, epilepsy, and polysubstance use developed fever associated with malaise, nausea, and myalgias while admitted to inpatient psychiatry. His medications included: clozapine, valproic acid, lacosamide, lithium, trazodone, benztropine, and risperidone. Clozapine was started 18 days prior to onset of symptoms. Fever to $39.4{ }^{\circ} \mathrm{C}$ persisted for 2 days. On evaluation, his vital signs were: blood pressure $102 / 62 \mathrm{mmHg}$, heart rate $120 /$ minute, respiratory rate $20 /$ minute, oxygen saturations $90 \%$ (room air) and temperature $38.3^{\circ} \mathrm{C}$. On examination, he had tachycardia with no murmur, bibasilar rales, and jugulovenous distension. Laboratory testing was notable for a troponin of $45.6 \mathrm{ng} / \mathrm{mL}$, brain natriuretic peptide (BNP) $696.8 \mathrm{pg} / \mathrm{mL}$, lactate $5.6 \mathrm{mmol} / \mathrm{L}$, white blood cell count $11.0 \times 10^{3}$ with $2.2 \%$ eosinophils, erythrocyte sedimentation rate (ESR) $6 \mathrm{~mm} / \mathrm{h}$, and C-reactive protein $194.3 \mathrm{mg} / \mathrm{L}$. His electrocardiogram showed sinus tachycardia with a right bundle branch block (RBBB), ST depressions in V1 and V2, ST elevations in III, aVF, and aVR. Echocardiogram revealed left 
ventricle ejection fraction (LVEF) $35 \%$ with infero-lateral wall motion abnormalities. Clozapine was discontinued. The patient was transferred to the Cardiac Care Unit, where he became more tachypneic and was intubated. Cardiac catheterization found evidence of right heart failure with no coronary artery disease. An Impella $\mathrm{CP}^{\oplus}$ device was placed via the right femoral artery for circulatory support. He was treated with vasopressors, inotropes, high-dose steroids and antibiotics. He developed worsening cardiogenic shock with an LVEF 10\%. A left ventricular assist device (LVAD) and extracorporeal membrane oxygenation (ECMO) therapy were discussed, but he was not a candidate. Despite minimal pulsatile flow, the patient was supported by the Impella ${ }^{\circledR}$ with flow up to $3.3 \mathrm{~L} / \mathrm{min}$. After 5 days, cardiac output improved, and the Impella ${ }^{\circledR}$ was removed seven days after placement. He was extubated after 9 days. Prior to discharge, echocardiogram was repeated, and LVEF improved to 50\%. Laboratory analysis for all rheumatologic and infectious studies were negative, and the onset of disease within 3 weeks of starting clozapine made clozapine-induced myocarditis the most likely diagnosis.

Conclusion: Circulatory support devices, such as the Impella ${ }^{\circledR}$ should be considered a therapeutic option for management of cardiogenic shock in patients with clozapine-induced myocarditis.

\section{Two consecutive massive iron intoxications in the same patient: one treated with laparotomic gastrostomy}

\section{Maria Kaista}

Poison Information Center, Department of Emergency Medicine and Services, Helsinki University and Helsinki University Hospital, Helsinki, Finland

Objective: Iron tablets are poorly soluble, too large for orogastric tubes and have an ability to form masses. Gastric lavage and whole bowel irrigation are both considered ineffective and other methods of their removal should be considered [1]. A gastrostomy may be life-saving in extracting retained tablets and preventing toxic accumulation or intestinal perforation in iron overdose [2]. For gastrointestinal bezoars a laparotomic or laparoscopic gastrostomy is a valid option if a skilled endoscopist is not available [3]. We describe two intentional iron intoxications in one adult, treated with laparotomic gastrostomy.

Case report: A 26-year-old woman with obesity $(115 \mathrm{~kg})$ and severe mental disorders took a potentially lethal dose of ferrous sulfate $100 \times 100 \mathrm{mg}$ Retafer $^{\oplus}$ depot tablets in total $(145 \mathrm{mg} / \mathrm{kg}$, ideal weight). On arrival to hospital she was vomiting but stable. Gastric lavage was performed twice unsuccessfully, with an X-ray showing numerous tablets remaining in the ventricle. Wholebowel irrigation and IV deferrioxamine were started. Five hours after the oral intake a gastrostomy from a small laparotomic incision was performed extracting the remaining 86 tablets adhered to each other. Gastric mucosa was irritated but intact. Somatic recovery was good (peak plasma iron concentration $84.2 \mathrm{mmol} / \mathrm{L}$ ) and three days later the patient was discharged home after a psychiatric evaluation. A month later she took the same amount of Retafer $^{\circledR}$ tablets $(100 \times 100 \mathrm{mg})$. Gastric lavage was again unsuccessful with an X-ray showing numerous tablets still present in the stomach. Whole-bowel irrigation and IV deferrioxamine were started. This time an endoscopy was performed 7 hours after ingestion. At this time point only 5 tablets were detected and removed from the ventricle, the rest were thought to have been vomited during the wait. She was again monitored (peak plasma iron concentration $75.7 \mathrm{mmol} / \mathrm{L}$ ) and discharged to involuntary psychiatric care after two days.

Conclusion: Gastric lavage is not effective and should not be used in massive iron intoxications. Laparotomic gastrostomy, however, remains a valid option for elimination when endoscopy is not available.

\section{References}

[1] Velez LI, Gracia R, Mills LD, et al. Iron bezoar retained in colon despite 3 days of whole bowel irrigation. J Toxicol Clin Toxicol. 2004;42:653-6.

[2] Haider F, De Carli C, Dhanani S, et al. Emergency laparoscopicassisted gastrotomy for the treatment of an iron bezoar. J Laparoendosc Adv Surg Tech A. 2009;19 Suppl 1:S141-S3.

[3] Park SE, Ahn JY, Jung HY, et al. Clinical outcomes associated with treatment modalities for gastrointestinal bezoars. Gut Liver. 2014;8:400-7.

\section{Mercury poisoning in a fisherman}

\section{Davide Lonati ${ }^{\mathrm{a}}$, Sollaku Irena ${ }^{\mathrm{b}}$, Arturo Zancan ${ }^{\mathrm{c}}$, Eleonora Buscaglia ${ }^{a}$, Anna Ronchi ${ }^{a}$, Giuseppe De Palma ${ }^{\mathrm{b}}$ and Carlo A Locatelli ${ }^{\mathrm{a}}$}

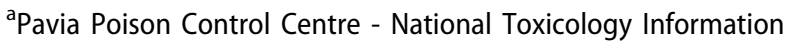
Centre - Clinical and Experimental Lab, Toxicology Unit, Maugeri Clinical and Scientific Institutes IRCCS and University of Pavia, Pavia, Italy; ${ }^{b}$ Department of Medical and Surgical Specialties, Radiological Sciences, and Public Health, Section of Public Health and Human Sciences, University of Brescia, Brescia, Italy; 'Subacute Care Unit, Istituti Clinici Scientifici Maugeri, IRCCS Maugeri Hospital, Pavia, Italy

Objective: Mercury is a chemical element diffuse in the biosphere, that can be present in either inorganic (with three different oxidation states) or organic form. There are abundant data and advisories for mercury concentrations in food and supplements containing material of marine origin. Mercury intoxication is rare in our country. We describe a case of an adult treated with chelators for severe mercury poisoning.

Case report: A 68-year-old male $(80 \mathrm{~kg})$ was evaluated at an Occupational Health Unit for neuropsychiatric symptoms (starting 18 months earlier), that progressively worsened. The patient presented with severe asthenia, hyperacusis and tinnitus, insomnia, mood changes, memory loss, inferior limb paresthesia and myalgia. Medical history revealed hypothyroidism, thalassemia trait and maculopathy. He had been retired 10 years, and in the summer fished daily in the Grado and Marano Lagoon (North East Adriatic Sea), and regularly consumed his catch (one sea bream per day) and shellfish (3-4 times per week). One year after the appearance of symptoms his blood mercury concentration was $79 \mu \mathrm{g} / \mathrm{L}$ (reference 0.35-5.2 $\mu \mathrm{g} / \mathrm{L}$ ) and urine was $2.7 \mu \mathrm{g} / \mathrm{L}$ (reference 0.1-5.0 $\mu \mathrm{g} / \mathrm{L}$ ). $\mathrm{He}$ stopped the fish intake. Blood $(\mathrm{Hg}-\mathrm{B})$, urine $(\mathrm{Hg}-\mathrm{U})$, hair, and exhaled breath condensate (EBC) have been repeatedly tested by inductively coupled plasma mass spectrometry (ICP-MS). In the EBC the concentration was $0.2 \mu \mathrm{g} / \mathrm{L}$ with the presence of methylmercury, while in hair the concentration was $5.8 \mu \mathrm{g} / \mathrm{g}$. Lymphocyte muscarinic receptors were altered (46.2; reference $6.0-13.0 \mathrm{fmol} / \mathrm{mil}-$ lion lymphocytes). He received chelation therapy with succimer (dimercaptosuccinic acid, DMSA) $30 \mathrm{mg} / \mathrm{kg} /$ day for 5 days and 20 $\mathrm{mg} / \mathrm{kg} /$ day for 14 days. One month later the $\mathrm{Hg}-\mathrm{B}$ concentration was $7 \mu \mathrm{g} / \mathrm{L}$. The patient complained of persistent symptoms for about one year, with a slight improvement in recent months. Abnormal Hg-B concentrations (not requiring chelating therapy) have also been found also in his cohabitants.

Conclusion: Based on clinical symptoms and analytical data, a diagnosis of neurotoxicity by organic mercury ingestion was made. The Grado e Marano lagoons are known for the presence of mercury pollution caused by spillage of a factory working with 
the chloralkali process and by the Isonzo river which flows into them, and drains the Idria mines containing cinnabar (mercury sulphide). Environmental exposure to $\mathrm{Hg}$ remains a possible source of intoxication. In this case a warning for people regularly fishing in these waters and consuming the fish is advisable to avoid serious poisoning.

\section{Lymphocyte muscarinic receptors: an early biomarker for clinical assessment of mercury exposure}

\author{
Marta Crevani, Davide Lonati, Teresa Coccini, \\ Elisa Roda, Anna Ronchi and Carlo A Locatelli \\ Pavia Poison Control Centre - National Toxicology Information \\ Centre - Clinical and Experimental Lab, Toxicology Unit, Maugeri \\ Clinical and Scientific Institutes IRCCS and University of Pavia, \\ Pavia, Italy
}

Objective: Exposure to neurotoxicants causes biochemical/ molecular events indicating different stages of toxic injury. Biomarkers indicating subclinical effects may be a useful support for clinicians in making an early differential diagnosis, especially in case of long-term low-dose exposure to mercury $(\mathrm{Hg})$. Lymphocyte muscarinic receptors (L-MRs) have been suggested as a biomarker in mercury intoxication.

Case report: A 40-year-old female was referred to our Unit for mercury exposure after recent removal of four dental amalgams without ad hoc procedure. The patient was asymptomatic and blood mercury concentration was $12 \mu \mathrm{g} / \mathrm{L}$ (reference range 0.2 1.5). She remained asymptomatic and no organ damage related to mercury was recorded during a 3-year follow-up program. Blood mercury $(\mathrm{Hg}-\mathrm{B})$, urinary mercury $(\mathrm{Hg}-\mathrm{U})$ and L-MRs were monitored. L-MRs normalized in line with $\mathrm{Hg}-\mathrm{B}$ concentrations (Table 1).

Conclusion: Peripheral MRs have been used clinically as predictors of pharmacological response in psychotropic drug-treated subjects, and to investigate the role of neurochemical disturbances. An evident association among mercury exposure concentration in biological specimens and blood cholinergic markers has been previously reported in a case of acute $\mathrm{Hg}$ intoxication [1]. Our results demonstrated that high/low $\mathrm{Hg}-\mathrm{B}$ were accompanied by high/low MRs in lymphocytes. Although no neurological symptoms were documented and declared by the patient, the LMRs were above the normal reference values. However, L-MRs in our asymptomatic patient are not as high as those found in a mercury-intoxicated patient (L-MRs around $200 \mathrm{fmol} / \mathrm{million}$ lymphocytes associated with $\mathrm{Hg}-\mathrm{B}$ of $25 \mu \mathrm{g} / \mathrm{L}$ ) [1]. This finding further supports the application of a specific neurochemical biomarker in clinical settings enabling clinicians to support specific early differential diagnosis (i.e., $\mathrm{Hg}$ exposure/poisoning), and to monitor the efficacy of therapy.

\section{Reference}

[1] Roda E, Giampreti A, Vecchio S, et al. Mercury vapour long-lasting exposure: lymphocyte muscarinic receptors as neurochemical markers of accidental intoxication. Case Rep Med. 2016;2016:9783876

\section{Puberty and resultant increased bone turnover as a possible etiology of an increased lead concentration in a pre-adolescent girl}

\section{Rebecca Bruccoleri and Alan D Woolf \\ Boston Children's Hospital, Boston, United States}

Objective: Lead poisoning generally affects small children, given their increased hand-to-mouth behaviors. Blood lead increases can also be seen in previously poisoned individuals who have increased bone turnover from thyroid disease or pregnancy. This case report describes an increased blood lead level (BLL) in a pre-adolescent which was likely related to increased bone turnover from puberty.

Case report: A 12-year-old girl was referred to the pediatric environmental health clinic (PEHC) after her BLL ranged from 27 to $30 \mu \mathrm{g} / \mathrm{dL}$ (reference $<5 \mu \mathrm{g} / \mathrm{dL}$ ) at her pediatrician's office. She had been seen in the PEHC 6 years previously for management of a BLL $33 \mu \mathrm{g} / \mathrm{dL}$, treated with 4 cycles of succimer (dimercaptosuccinic acid, DMSA), and discharged with a BLL 21 $\mu \mathrm{g} / \mathrm{dL}$ to have subsequent serial BLL monitored by her pediatrician. Approximately 15 months prior to her repeat PEHC referral, her BLL was $10 \mu \mathrm{g} / \mathrm{dL}$. In those intervening 15 months, she grew $10.42 \mathrm{~cm}$. Thyroid studies were normal. Initial vitamin D concentration was $16.9 \mathrm{ng} / \mathrm{mL}$ (normal $30-50 \mathrm{ng} / \mathrm{mL}$ ) and iron concentration was $49 \mu \mathrm{g} / \mathrm{dL}$ (normal 50-170 $\mathrm{gg} / \mathrm{dL}$ ), both of which were supplemented. Osteocalcin concentrations were also checked once it became apparent that external exposure was unlikely. Osteocalcin was initially $212 \mathrm{ng} / \mathrm{mL}$ (normal 9-42 $\mathrm{ng} / \mathrm{mL}$ in adults $>18$ years) when her BLL was $27 \mu \mathrm{g} / \mathrm{dL}$. Her BLL increased to $32 \mu \mathrm{g} / \mathrm{dL}$ with an osteocalcin concentration of $208 \mathrm{ng} / \mathrm{mL}$. Both BLL and osteocalcin subsequently decreased with the last osteocalcin concentration of $69 \mathrm{ng} / \mathrm{mL}$. Approximately 10.5 months later, her BLL was $16 \mu \mathrm{g} / \mathrm{dL}$ and her rate of growth had declined to $0.20 \mathrm{~cm} / 30$ days (peak growth was $1.07 \mathrm{~cm} / 30$ days). A thorough investigation was performed by the state lead inspector, including a home visit by the treating physician. No source of lead was found to explain her increased BLL. Given that the source of her exposure was unknown and she was asymptomatic, no succimer was given throughout the subsequent period of evaluation.

Conclusion: Increased bone turnover during puberty, represented by elevated amounts of osteocalcin protein being secreted by osteoblasts, may result in increased BLL in children with a body burden of lead. Additional investigations of lead

Table 1. Concentrations of lymphocyte muscarinic receptors and mercury during 3-year follow-up in an asymptomatic patient following removal of four dental amalgams.

\begin{tabular}{lccc}
\hline Year & Lymphocyte muscarinic receptors & Blood mercury concentration & Urinary mercury concentration \\
\hline April 2015 & - & 12.1 & 1 \\
October 2015 & 21.3 & 6.8 & 1.9 \\
March 2016 & 19.20 & - & - \\
January 2017 & 30.4 & 2.2 & 1.2 \\
October 2017 & 10.6 & $<0.01$ & $<0.1$ \\
Normal range & $6.0-13.0 \mathrm{fmol} /$ million lymphocytes & $0.2-1.5 \mu \mathrm{g} / \mathrm{L}$ & $0.1-4.5 \mu \mathrm{g} / \mathrm{L}$ \\
\hline
\end{tabular}


kinetics in adolescents undergoing rapid skeletal growth are needed to support this conclusion. Osteocalcin concentrations and plotting the growth curve may assist clinicians in determining if pubertal growth is playing a role in increased BLL in adolescents for whom no external source of lead exposure can be found.

\section{Successful treatment of acute arsenic poisoning with unithiol}

\section{Ildikó Urbán}

Clinical Toxicology, Péterfy Sándor Hospital, Budapest, Hungary

Objective: Acute arsenic poisoning is rare, life-threatening and there is not enough clinical experience with appropriate treatment, especially with unithiol (2,3-dimercaptopropane-1-sulphonate, DMPS) [1]. We report a case of acute self-poisoning, which was managed with unithiol without side-effect or late consequences.

Case report: A 31-year-old-man ingested 2-3 g arsenic trioxide. Gastric lavage was performed and activated charcoal was administered 1.5 hours later. Within 10 hours post-ingestion he developed vomiting, diarrhoea, dyspnoea, abdominal and chest pain. He was admitted to our hospital 25 hours after ingestion. In the intensive care unit he exhibited hypotension, tachycardia, dehydration, electrolyte and metabolic disturbances, elevated liver function tests, creatine kinase, C-reactive protein and D-dimer. He was treated with supportive care (fluid resuscitation, ulcer prophylaxis and thrombosis prophylaxis, circulation support with norepinephrine, and hepatoprotectants) and chelation therapy with unithiol. His metabolic and electrolyte disturbances improved and his circulation rapidly stabilized. Unithiol was administered intravenously for 5 days $(250 \mathrm{mg}$ every 3 hours, then every 4, 6, 12 and 24 hours) then orally $100 \mathrm{mg}$ four times daily for 13 days in our hospital. It was continued in the psychiatric department and at home. Urine arsenic concentration in 24hour specimens was measured on hospital days 4, 7, and 17 and was $2106 \mu \mathrm{g} / \mathrm{L}$ (10951.2 $\mu \mathrm{g} /$ day), $409 \mu \mathrm{g} / \mathrm{L}$ (1636 $\mu \mathrm{g} /$ day), 29.9 $\mu \mathrm{g} / \mathrm{L}(125.58 \mu \mathrm{g} / \mathrm{day})$, respectively. Abdominal ultrasound showed fatty degeneration in the liver and hepatomegaly. Transient elevations in transaminases, gamma glutamyl transferase, and low INR, thrombocytopenia, and hyperglycaemia were detected during his treatment. These symptoms ceased or started to improve in our hospital. He was transferred to the psychiatric department on hospital day 19 with proposal to continue unithiol treatment. His course after discharge was uneventful, and he recovered completely.
Conclusion: This was a potentially lethal dose of arsenic with multi-organ complications successfully treated with early decontamination, appropriate supportive care and chelation therapy (unithiol). Unithiol was given parenterally and orally with no side effects, and our patient did not develop any lasting consequences of the ingestion or treatment. Unithiol is a less toxic alternative for chelation than dimercaprol (British Anti-Lewisite, BAL) or penicillamine, and can be used effectively and safely in acute arsenic poisoning.

\section{Reference}

[1] Lu PH, Tseng JC, Chen CK, et al. Survival without peripheral neuropathy after massive acute arsenic poisoning: Treated by 2,3-dimercaptopropane-1-sulphonate. J Clin Pharm Ther. 2017;42:506-508.

\section{Non-medical use of prescription GABA analogues (gabapentin and pregabalin) in Europe in the Non- Medical Use of Prescription Drug (NMURx) National Surveys}

\author{
Caitlin F Bonney ${ }^{\mathrm{a}}$, Janetta L Iwanicki ${ }^{\mathrm{b}}$, Joshua C Black ${ }^{\mathrm{c}}$ \\ Colleen M Haynes ${ }^{c}$ and Richard C Dart ${ }^{b}$ \\ ${ }^{a}$ Medical Toxicology, Rocky Mountain Poison \& Drug Center, \\ Denver, United States; ${ }^{\text {b }}$ Rocky Mountain Poison \& Drug Center, \\ Denver, United States; 'RADARS System, Rocky Mountain Poison \& \\ Drug Center, Denver, United States
}

Objective: Non-medical use (NMU) of prescription GABA analogues in Europe has been reported. We determined the prevalence of prescription GABA analogue NMU and associated demographics in five European countries.

Methods: The Survey of Non-Medical Use of Prescription Drugs Program (NMURx) is a series of cross-sectional surveys which collects data from the general population in each participating country about NMU of prescription drugs and demographics. NMU was defined as "using a medication without a doctor's prescription or for any reason other than what was recommended by their doctor." Responses France (2Q2017, $n=10,012)$, Germany (4Q2017, $\mathrm{n}=15,051)$, Italy $(2 \mathrm{Q} 2017, \mathrm{n}=10,084)$, Spain (4Q2017, $\mathrm{n}=10,062)$, and the UK $(1 \mathrm{Q} 2017, \mathrm{n}=10,004)$ were analyzed. Adult age was defined as ages 15-110 years in Spain,

Table 1. NMURx respondents reporting non-medical use of GABA analogues in the past 12 months versus all respondents. Percentage of all responders (95\% confidence interval).

\begin{tabular}{|c|c|c|c|c|c|c|c|c|c|c|}
\hline & \multicolumn{2}{|c|}{ France } & \multicolumn{2}{|c|}{ Germany } & \multicolumn{2}{|c|}{ Italy } & \multicolumn{2}{|c|}{ Spain } & \multicolumn{2}{|c|}{ UK } \\
\hline & $\begin{array}{c}\text { GABA } \\
\text { Analogue } \\
\text { NMU }\end{array}$ & $\begin{array}{c}\text { General } \\
\text { Population }\end{array}$ & $\begin{array}{c}\text { GABA } \\
\text { Analogue } \\
\text { NMU }\end{array}$ & $\begin{array}{c}\text { General } \\
\text { Population }\end{array}$ & $\begin{array}{c}\text { GABA } \\
\text { Analogue } \\
\text { NMU }\end{array}$ & $\begin{array}{c}\text { General } \\
\text { Population }\end{array}$ & $\begin{array}{c}\text { GABA } \\
\text { Analogue } \\
\text { NMU }\end{array}$ & $\begin{array}{c}\text { General } \\
\text { Population }\end{array}$ & $\begin{array}{c}\text { GABA } \\
\text { Analogue } \\
\text { NMU }\end{array}$ & $\begin{array}{c}\text { General } \\
\text { Population }\end{array}$ \\
\hline Male & $\begin{array}{c}56.8(48.19 \\
65.36)\end{array}$ & $\begin{array}{c}47.6(46.59 \\
48.68)\end{array}$ & $\begin{array}{c}54.0 \%(48.13 \\
59.90)\end{array}$ & $\begin{array}{c}48.6 \%(47.79 \\
49.49)\end{array}$ & $\begin{array}{c}45.0 \%(33.54 \\
56.38)\end{array}$ & $\begin{array}{c}49.1 \%(47.89 \\
50.21)\end{array}$ & $\begin{array}{l}65.7 \%(53.60, \\
77.79)\end{array}$ & $\begin{array}{c}48.6 \%(47.47 \\
49.79)\end{array}$ & $\begin{array}{c}46.8 \%(39.82 \\
53.83)\end{array}$ & $\begin{array}{c}48.8 \%(47.61 \\
49.94)\end{array}$ \\
\hline $\begin{array}{l}\text { Lifetime illicit } \\
\text { drug use }\end{array}$ & $\begin{array}{l}33.9 \%(25.71, \\
42.05)\end{array}$ & $\begin{array}{c}18.2(17.39 \\
18.94)\end{array}$ & $\begin{array}{c}28.7 \%(23.42 \\
34.05)\end{array}$ & $\begin{array}{c}25.4 \%(24.70 \\
26.17)\end{array}$ & $\begin{array}{l}37.8 \%(26.47 \\
49.04)\end{array}$ & $\begin{array}{c}20.5 \%(19.64 \\
21.38)\end{array}$ & $\begin{array}{l}42.0 \%(30.86, \\
53.20)\end{array}$ & $\begin{array}{c}24.4 \%(23.45 \\
25.32)\end{array}$ & $\begin{array}{l}48.0 \%(40.95 \\
54.98)\end{array}$ & $\begin{array}{c}27.3 \%(26.22 \\
28.31)\end{array}$ \\
\hline $\begin{array}{l}\text { Previous sub- } \\
\text { stance abuse } \\
\text { treatment }\end{array}$ & $\begin{array}{l}11.5 \%(6.04 \\
16.89)\end{array}$ & $\begin{array}{c}1.7 \%(1.46 \\
1.97)\end{array}$ & $\begin{array}{l}7.7 \%(4.56 \\
10.91)\end{array}$ & $\begin{array}{c}1.8 \%(1.59 \\
2.05)\end{array}$ & $\begin{array}{c}3.8 \%(0.00 \\
8.40)\end{array}$ & $\begin{array}{c}0.6 \%(0.47 \\
0.80)\end{array}$ & $\begin{array}{l}13.3 \%(6.45 \\
20.21)\end{array}$ & $\begin{array}{c}2.2 \%(1.92 \\
2.55 \%)\end{array}$ & $\begin{array}{l}19.3 \%(13.35 \\
25.21)\end{array}$ & $\begin{array}{c}1.7 \%(1.41 \\
2.02)\end{array}$ \\
\hline \multicolumn{11}{|l|}{ DAST-10 } \\
\hline $\begin{array}{l}\text { Moderate } \\
(3-5)\end{array}$ & $\begin{array}{l}11.7 \%(6.19 \\
17.19)\end{array}$ & $\begin{array}{c}2.2 \%(1.86 \\
2.45)\end{array}$ & $\begin{array}{l}12.0 \%(8.08, \\
15.99)\end{array}$ & $\begin{array}{c}3.2 \%(2.86 \\
3.46)\end{array}$ & $\begin{array}{c}7.9 \%(2.50 \\
13.28)\end{array}$ & $\begin{array}{c}2.9 \%(2.51 \\
3.21)\end{array}$ & $\begin{array}{l}19.3 \%(11.11, \\
27.44)\end{array}$ & $\begin{array}{c}4.0 \%(3.58 \\
4.38)\end{array}$ & $\begin{array}{l}14.1 \%(9.62, \\
18.62)\end{array}$ & $\begin{array}{c}3.2 \%(2.72 \\
3.64)\end{array}$ \\
\hline $\begin{array}{l}\text { Substantial } \\
(6-8)\end{array}$ & $\begin{array}{l}8.8 \%(3.82, \\
13.87)\end{array}$ & $\begin{array}{c}0.8 \%(0.60 \\
0.94)\end{array}$ & $\begin{array}{l}3.5 \%(1.27 \\
5.77)\end{array}$ & $\begin{array}{c}0.9 \%(0.69 \\
1.01)\end{array}$ & $\begin{array}{c}1.9 \%(0.00 \\
4.13)\end{array}$ & $\begin{array}{c}0.5 \%(0.39 \\
0.68)\end{array}$ & $\begin{array}{l}6.7 \%(1.63, \\
11.70)\end{array}$ & $\begin{array}{c}1.2 \%(0.98 \\
1.42)\end{array}$ & $\begin{array}{l}11.3 \%(6.15 \\
16.46)\end{array}$ & $\begin{array}{c}1.0 \%(0.73 \\
1.23)\end{array}$ \\
\hline
\end{tabular}


16-110 years in the UK, and 18-110 years in France, Germany and Italy. Responses were collected using non-probability quota sampling and post-stratification population weighting was applied. Rates of NMU and associated demographics were reported as rate of past 90 day NMU per 100,000 adult population with $95 \%$ confidence intervals.

Results: Prevalence of past 12 month GABA analogue NMU was highest in Germany $(1,191.7$ per 100,000 population, $95 \% \mathrm{Cl}$ 1,004.3-1,379.1) and the UK $(1,067.2,95 \% \mathrm{Cl} 851.3-1,283.2)$, and lowest in Spain $(344.4,95 \% \mathrm{Cl} 204.8-484.0)$ and Italy $(366.2,95 \%$ $\mathrm{Cl}$ 207.7-524.6). NMU was evenly distributed between genders except in Spain which showed a male predominance (Table 1). Participants reporting NMU of GABA analogues reported higher incidence of lifetime chronic pain, lifetime illicit drug use, and previous substance abuse therapy, and were more likely to have moderate or substantial Drug Abuse Screening Test (DAST)-10 scores than the general population.

Conclusion: Respondents to the NMURx Survey in five European countries who reported NMU of GABA analogues were more likely to report chronic pain, use of illicit substances, and history of substance abuse treatment than the general population.

\section{Clinical outcomes of cardiac glycoside poisoning in Thailand}

\author{
Satariya Trakulsrichai ${ }^{a}$, Kritsada Chumvanichaya ${ }^{b}$, \\ Charuwan Sriapha ${ }^{c}$, Achara Tongpoo', \\ Umaporn Udomsubpayakul ${ }^{\mathrm{d}}$ and Winai Wananukul ${ }^{\mathrm{e}}$ \\ aDepartment of Emergency Medicine, Ramathibodi Poison Center, \\ Faculty of Medicine, Ramathibodi Hospital, Bangkok, Thailand; \\ ${ }^{b}$ Department of Emergency Medicine, Faculty of Medicine, \\ Ramathibodi Hospital, Bangkok, Thailand; 'Ramathibodi Poison \\ Center, Faculty of Medicine, Ramathibodi Hospital, Bangkok, \\ Thailand; ${ }^{d}$ Section for Clinical Epidemiology and Biostatistics, \\ Research Center, Faculty of Medicine, Ramathibodi Hospital, \\ Bangkok, Thailand; 'Department of Medicine, Faculty of Medicine, \\ Ramathibodi Hospital, Bangkok, Thailand
}

Objective: To describe clinical characteristics and outcomes of patients with cardiac glycoside poisoning in Thailand including differences of clinical features between pharmaceutical and nonpharmaceutical poisonings.

Methods: We performed a retrospective study of patients with cardiac glycoside (including toad as bufotoxin, plants and digoxin) poisoning from Ramathibodi Poison Center Toxic Exposure Surveillance System, during a 5-year period (2012-2016). Results: There were 108 patients included that been exposed to bufotoxin (33.3\%), plants (14.8\%) and digoxin (51.9\%). Plants included Strophanthus caudatus, Adenium obesum, Nerium oleander and yellow oleander (Cascabela thevetia). The median age was 43 years ( 9 days to 95 years). Most were male and presented with gastrointestinal $(\mathrm{Gl})$ symptoms. At presentation, 35 patients had bradyarrhythmia (no tachyarrhythmia reported). Twenty-six patients had shock. Patients with digoxin poisoning were older and had more underlying diseases, abnormal serum potassium and bradyarrhythmia at presentation than patients with nonpharmaceutical poisoning. For bufotoxin patients, three developed cardiac arrest (at presentation and in the emergency room [ER]). For patients with plant poisoning, all had Gl symptoms and survived, one developed junctional bradycardia. During admission, 17 patients developed bradyarrhythmia and three patients developed tachyarrhythmia. Ventricular tachycardia was detected in three digoxin-poisoned patients. Some had hypokalemia $(9.3 \%)$ or hyperkalemia (12.3\%) at presentation. Serum digoxin concentrations were measured by immunoassay methods in 43 patients with the median concentration of $3.4 \mathrm{ng} / \mathrm{mL}$, and also in four bufotoxin patients ( 0.43 to $>8 \mathrm{ng} / \mathrm{mL}$ ). Most patients $(75.9 \%)$ were admitted to hospital including intensive care. The median hospital stay was 72 hours. The mortality rate was $7.4 \%$. Besides supportive care, some received gastric lavage (25\%), activated charcoal (31.5\%), endotracheal intubation (10.2\%), inotropic drugs $(8.3 \%)$, transcutaneous cardiac pacing $(7.8 \%)$ and management of hyperkalemia (17\%). Two patients did not develop arrhythmia following intravenous calcium administration for hyperkalemia. Forty-two patients met criteria for treatment with digoxin immune FAB (DigiFab), however only 10 digoxin patients and one bufotoxin patient received DigiFab (1-10 vials), ten of them survived. After we excluded three patients who had cardiac arrest early in the ER, of 28 patients who should have received DigiFab, but were not treated, four of them died, while the others survived. We found that shock, coma and bradyarrhythmia at presentation were statistically significantly different between fatal and survival groups.

Conclusion: Cardiac glycoside poisoning caused deaths in Thailand. Clinical features among bufotoxin, plants and digoxin poisonings were not absolutely similar. Of the patients that should have been treated with DigiFab, but did not receive it, most survived with supportive measures.

\section{Comparison of adverse reactions to a two-bag versus three-bag intravenous acetylcysteine regimen during treatment of paracetamol overdose}

Anselm Wong ${ }^{\mathrm{a}}$, Mark Yarema ${ }^{\mathrm{b}}$, Geoffrey K Isbister ${ }^{\mathrm{c}}$, Marco LA Sivilotti ${ }^{\mathrm{d}}$, Richard McNulty ${ }^{\mathrm{e}}$, Angela L Chiew ${ }^{f}$, Colin B Page ${ }^{g}$, Shaun L Greene ${ }^{\text {, }}$, Naren Gunja ${ }^{e}$, Nicholas A Buckley ${ }^{i}$ and

Andis Graudins ${ }^{j}$

${ }^{a}$ Clinical Sciences at Monash Health, Monash University, and Austin Toxicology Unit and Emergency Department, Victoria,

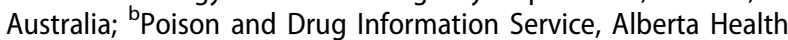
Services, Calgary, Canada; ' Clinical Toxicology Research Group, University of Newcastle, Newcastle, Australia; ${ }^{d}$ Departments of Emergency and of Biomedical and Molecular Sciences, Queen's University, Kingston, Canada; ${ }^{e}$ Department of Clinical Pharmacology \& Toxicology, Western Sydney Health, New South Wales, Australia; fDepartment of Emergency and Toxicology, Prince of Wales Hospital, Sydney, Australia; ${ }^{9}$ Department of Emergency and Toxicology, Princess Alexandria Hospital, Brisbane, Australia; ${ }^{\mathrm{h}}$ Austin Toxicology Unit and Emergency Department, Austin Health, Victoria, Australia; 'University of Sydney, New South Wales, Australia; ${ }^{j}$ Clinical Sciences at Monash Health, Monash University, and Monash Toxicology Unit and Emergency Service, Monash Health, Dandenong, Australia

Objective: Previous studies have shown that a 2-bag 20-hour intravenous (IV) acetylcysteine regimen reduced the incidence of non-allergic anaphylactic (or "anaphylactoid") reactions compared to the 3-bag 21-hour IV regimen for treatment of paracetamol overdose in smaller cohort studies. We evaluated these reactions from the 2-bag IV acetylcysteine regimen in a larger international collaborative study.

Methods: This is an ongoing prospective cohort study of Australian centres using a 2-bag IV acetylcysteine regimen (200 $\mathrm{mg} / \mathrm{kg}$ over 4 hours, $100 \mathrm{mg} / \mathrm{kg}$ over 16 hours) for paracetamol overdose, with data analysed from early 2014 to mid-2018. The comparison group consisted of patients from the Canadian Acetaminophen Overdose Study (CAOS) treated with a 3-bag IV 
acetylcysteine regimen $(150 \mathrm{mg} / \mathrm{kg}$ over $15-60$ minutes, $50 \mathrm{mg} / \mathrm{kg}$ over 4 hours, $100 \mathrm{mg} / \mathrm{kg}$ over 16 hours) from 1980 to 2005 . Subjects all had single, acute ingestions and serum paracetamol concentration 4 to 24 hours post-ingestion that was on or above the Rumack-Matthew treatment-nomogram line. Outcomes including anaphylactoid reactions, and mortality attributed to acetylcysteine are reported. Anaphylactoid reactions to acetylcysteine included cutaneous (oedema, facial flushing, pruritus, urticaria) and systemic reactions (hypotension, respiratory symptoms). Categorical variables were compared using chisquared test.

Results: Of 14479 paracetamol overdose presentations to 43 treatment centres, 10342 received acetylcysteine. Study criteria were met in 659 patients receiving the 2-bag and 2086 patients receiving the 3-bag acetylcysteine regimen. Median age was 21 years (IQR 17,32) versus 22 years $(7,36)$ in the 2 versus 3-bag acetylcysteine regimen, respectively. Median time to acetylcysteine was 7 hours (IQR 6,12$)$ for the 2-bag versus 8 hours $(6,8.8)$ for the 3 -bag regimen. The incidence of cutaneous and systemic anaphylactoid reactions was: 20 (3\%) with 2-bag versus $148(7.1 \%)$ with 3 -bag regimen $(p=0.002$, OR $0.41,95 \% \mathrm{Cl}$ : $0.25-0.66)$. There were no deaths related to acetylcysteine in either group.

Conclusion: In this multi-centre study of patients treated for acute paracetamol overdose, the 2-bag IV acetylcysteine regimen was as safe as the 3-bag IV regimen and resulted in significantly less non-allergic anaphylactic reactions.

\section{Delayed arrhythmia after amisulpride overdose}

\section{Mandy Gollmann and Dagmar Prasa \\ Poisons Information Centre Erfurt, Erfurt, Germany}

Objective: Amisulpride is an atypical antipsychotic indicated for acute and chronic schizophrenia. In overdose, amisulpride causes cardiotoxicity [1] and is associated with a very high risk of QT prolongation and torsade de pointes (TdP) [2]. Amisulpride overdoses are rarely reported to the Poisons Information Centre Erfurt and cardiac symptoms are uncommon in these cases. We present a patient with delayed arrhythmia after amisulpride overdose.

Case report: A 50-year-old woman presented after ingesting $10 \mathrm{~g}$ of amisulpride $(125 \mathrm{mg} / \mathrm{kg})$ in a suicide attempt. No co-ingestants were reported. She was initially unconscious with appropriate response to pain. On arrival, she was somnolent with hypotension but no arrhythmia. She was immediately admitted to the intensive care unit, where she was treated with IV fluids, diuretics, and noradrenaline. In the further course, she woke up and there were no cardiac abnormalities on the electrocardiogram (ECG) or any significant laboratory anomalies. Approximately 27 hours after ingestion, the ECG showed sinus rhythm with heart rate $89 \mathrm{bpm}$, ventricular arrhythmias with QT prolongation of $500 \mathrm{~ms}$ and TdP. She received magnesium infusion over 24 hours and no further cardiac events occurred.

Conclusion: Few cases describing delayed cardiac symptoms are reported, however, in these cases the first onset of cardiac symptoms appeared within 24 hours [1,3]. The mechanism of cardiotoxicity and the delay in onset is poorly understood. We assume that delayed arrhythmia occurred due to the high dose and the half-life of 12-17 hours. The plasma concentration may still be in the toxic range 27 hours after the ingestion of $10 \mathrm{~g}$. This case shows that amisulpride overdoses have a high risk for developing cardiac effects. Cardiac symptoms can occur late and very spontaneously, even after central nervous system depression has resolved. The current recommended observation time for cardiac monitoring and serial ECGs should be extended from 16 hours up to 36 hours in these patients [3]. Further investigation is required to evaluate the frequency and severity of delayed cardiac arrhythmia in amisulpride overdose.

\section{References}

[1] Isbister GK, Balit CR, Macleod D, et al. Amisulpride overdose is frequently associated with QT prolongation and torsade de pointes. J Clin Psychopharmacol. 2010;30:391-395.

[2] Wenzel-Seifert K, Wittmann M, Haen E. QTc prolongation by psychotropic drugs and the risk of torsade de pointes. Dtsch Arztebl Int. 2011;10:687-693.

[3] Isbister GK, Murray L, John S, et al. Amisulpride deliberate self-poisoning causing severe cardiac toxicity including QT prolongation and torsades de pointes. Med J Aust. 2006;184:354-356.

\section{Toxicity of clozapine in elderly patients}

\section{Undine Burmeister and Maren Hermanns-Clausen}

Poisons Information Center, Department of General Pediatrics, Adolescent Medicine and Neonatology, Center for Pediatrics, Medical Center - University of Freiburg, Freiburg, Germany

Objective: Clozapine is an atypical antipsychotic used for treatment of schizophrenia with a daily dosage of $25-900 \mathrm{mg} /$ day. In elderly patients the initial dose should not exceed $12.5 \mathrm{mg}$ in order to minimize potential adverse reactions. The aim of the present study was to analyze acute exposures to clozapine doses within the therapeutic range in elderly patients.

Methods: A retrospective study was conducted in patients aged $\geq 65$ years with exposures to clozapine reported by physicians to our Poison Centre between 2006 and 2015.

Results: Overall 31 mono-exposures to $25-900 \mathrm{mg}$ clozapine were registered. The ingested dose was quantifiable and reliable information about clinical signs was available in 20 patients, aged 6784 years (median 73 years). Pretreatment with clozapine was reported in three patients. Exposures were mainly unintentional (95\%). The ingested dose varied from $25-750 \mathrm{mg}$ (median 165 $\mathrm{mg})$. Severity of poisoning was asymptomatic $(n=3)$, minor $(n=$ $3)$, moderate $(n=11)$, or severe $(n=3)$. The most common symptoms were central nervous system depression $(n=14,70 \%)$, electrocardiogram (ECG) changes $(n=8,40 \%)$, tachycardia $(n=7$, $35 \%)$, as well as dysarthria, restlessness/agitation, disorientation/ hallucination and hypertonia (each in four patients, 20\%). The lowest dose associated with moderate or severe intoxication symptoms was $50 \mathrm{mg}$ and $150 \mathrm{mg}$, respectively.

Conclusion: The intake of as little as $50 \mathrm{mg}$ clozapine may elicit moderate intoxication in elderly patients. In our study the majority of exposures to therapeutic doses of clozapine were associated with moderate to severe symptoms (70\%). Elderly patients seem to be more susceptible to clozapine than younger patients, and they should be monitored following unintentional ingestion of $\geq 50 \mathrm{mg}$ clozapine, especially when they are not pretreated.

\section{Bromazepam poisoning in the intensive care unit: is measurement of the plasma bromazepam concentration useful for patient management?}

Sybille Riou ${ }^{a}$, Ahmed S Gouda ${ }^{b}$, Marion Soichot ${ }^{c}$, Hélène Migueres ${ }^{a}$, Laurence Labat ${ }^{c}$ and Bruno Mégarbane ${ }^{a}$

${ }^{a}$ Department of Medical and Toxicological Critical Care, Lariboisière Hospital, Paris-Diderot University, INSERM UMRS-1144, Paris, France; ${ }^{b}$ National Egyptian Center of Environmental and 
Toxicological Research (NECTR), Cairo University, Cairo, Egypt; 'Laboratory of Toxicology, Lariboisière Hospital, Paris-Diderot University, INSERM UMRS-1144, Paris, France

Objective: Bromazepam is the most commonly used and the most frequently involved benzodiazepine in acute drug poisonings in France. Our objectives were to report the complications and management of bromazepam-poisoned patients admitted to the intensive care unit (ICU) and to investigate bromazepam pharmacokinetics in overdose and the relationships between coma depth and the plasma bromazepam concentration on admission.

Methods: We conducted a retrospective single-centre observational study including all bromazepam-poisoned patients admitted between 2011 and 2018, evidenced by at least one plasma bromazepam concentration in the toxic range during their ICU stay. We studied the correlation between the presumed ingested dose of bromazepam, the plasma concentration of bromazepam and coma depth determined by the Glasgow Coma Score (GCS) by calculating the Pearson's coefficients and using the Bartlett's test for sphericity.

Results: One-hundred and sixty-four patients [112 females and 52 males; age 51 years (41-63) (median (percentiles 25-75))] were included. Toxicity resulted from multidrug ingestions (75\%), with a presumed bromazepam ingested dose of $180 \mathrm{mg}$ (113-180) and plasma bromazepam concentration on admission of $1.88 \mathrm{mg} / \mathrm{L}$ (0.87-2.70). Consciousness impairment was marked [GCS 9 (3-14)] with hypotonic coma (43\%) and decrease/loss in tendon reflexes (26\%)]. Complications included aspiration pneumonia (49\%), increase in liver enzymes (79\%), cardiovascular effects (21\%) and renal failure (10\%). Sino- and atrio-ventricular blocks were found in $17 \%$ of the cases. Flumazenil [bolus dose of $0.3 \mathrm{mg}(0.2-0.4)$ followed by infusion rate of $0.4 \mathrm{mg} / \mathrm{h}(0.3-0.6)$ during 24 hours (17-60)] was administered in $28 \%$ of patients while $40 \%$ of patients were intubated and mechanically ventilated. In the subgroup of mono-intoxications with bromazepam, no significant correlation between coma depth and the plasma bromazepam concentration was observed $\left(R^{2}=0.1\right.$; Bartlett's test, $\left.p=0.3\right)$. Factors associated with the requirement of tracheal intubation (versus flumazenil use) included lower GCS $(p=0.002)$ and more elevated serum lactate concentration $(p=0.03)$. No significant relationship was evidenced between the ingested dose and the plasma concentration of bromazepam on admission.

Conclusion: Acute bromazepam poisoning is frequent and may be responsible for life-threatening consequences requiring ICU admission. The ingested dose and the plasma concentration of bromazepam are not correlated with coma depth and do not predict the necessity of tracheal intubation, thus suggesting high inter-individual variability in the drug toxicity and optimal management.

\section{Acute quetiapine poisoning admitted in the intensive care unit: features, complications and usefulness of plasma concentration measurement}

\author{
Sara Chenafi ${ }^{a}$, Karim Jaffal ${ }^{a}$, Marion Soichot ${ }^{b}$, \\ Laurence Labat ${ }^{\mathrm{b}}$ and Bruno Mégarbane ${ }^{\mathrm{a}}$ \\ ${ }^{a}$ Department of Medical and Toxicological Critical Care, Lariboisière \\ Hospital, Paris-Diderot University, INSERM UMRS-1144, Paris, \\ France; ${ }^{b}$ Laboratory of Toxicology, Lariboisière Hospital, Paris- \\ Diderot University, INSERM UMRS-1144, Paris, France
}

Objective: Since its marketing in France in 2011, prescriptions of quetiapine, a second generation antipsychotic drug, has exponentially increased with the risk of increased numbers of acute poisonings. Data regarding acute quetiapine overdose are scarce.
Our objectives were to describe the features, complications and management of acute quetiapine poisoning patients admitted to the intensive care unit (ICU), to evaluate the prognostic contribution of plasma quetiapine concentration on ICU admission and model quetiapine pharmacokinetics in overdose.

Methods: We conducted a retrospective single-center observational study. All quetiapine-poisoned patients (evidenced by measurement of plasma concentration in the toxic range) admitted to the ICU during a 6-year period (2013-2018) were included. Severe complications, other than neurological signs, were defined as the onset of major electrocardiogram (ECG) abnormalities, catecholamine requirement, cardiac arrest and death. Plasma quetiapine concentration was determined using high-performance liquid chromatography coupled to tandem mass spectrometry.

Results: Thirty-eight quetiapine-poisoned patients $(11$ males and 27 females; median age of 45 years [interquartile range, 16]; presumed ingested dose of $3.0 \mathrm{~g}$ [6.9]) were included. Poisoning resulted from multidrug ingestions (97\%). The patients presented impaired consciousness (Glasgow Coma Score 12 [8] requiring mechanical ventilation in $47 \%$ of cases), seizures (8\%), hyperglycemia $(68 \%)$, hypokalemia (18\%), intra- $(17 \%)$ and atrio-ventricular block (7\%) and QTC prolongation on the ECG (28\%). Complications included cardiovascular failure (13\%), aspiration pneumonia $(39 \%)$ and cardiac arrest $(5 \%)$. One patient died. The length of ICU stay was 58 hours [57]. The plasma quetiapine concentration on admission $(685 \mathrm{ng} / \mathrm{mL}$ [1631]) did not allow prediction of the onset of severe complications in the ICU. Delayed quetiapine peak was observed at 27 hours [23] post-ingestion suggesting prolonged gastrointestinal absorption while quetiapine elimination was prolonged in comparison to the pharmacological condition.

Conclusion: Quetiapine poisoning is relatively rare but may lead to life-threatening cardiovascular and neurological features. The plasma concentration on admission is not helpful to predict the onset of complications, thus suggesting marked inter-individual variability.

\section{An analysis of fatal iatrogenic therapeutic errors reported to US poison centers}

\author{
James B Leonard and Wendy Klein-Schwartz \\ Maryland Poison Center, University of Maryland School of \\ Pharmacy, Baltimore, United States
}

Objective: This is a descriptive study evaluating fatal iatrogenic and in-hospital medication errors reported to US poison centers.

Methods: A retrospective evaluation of the National Poison Data System from 2000-2017 of all therapeutic errors with a scenario coded as iatrogenic/healthcare professional or occurring in a healthcare facility. Death abstracts were reviewed for details of the exposure and therapeutic error scenarios were recoded or added to the case as appropriate based on the text from the abstract for all cases. Cases where death was considered not related to the exposure were excluded. Additionally, we created one additional scenario (rate-related) and one additional route of administration (intrathecal) to better describe the cases. Data are presented as median and interquartile range (IQR). Comparisons of age by substance were analyzed with Kruskal-Wallis.

Results: A total of 231 cases were initially identified. Thirty cases were excluded because the drug in question was not contributory to death, leaving 201 cases of drug-related deaths. There was a similar number of cases per year $(r=0.003 ; p=0.9846)$. Median age of this group was 60.5 years (IQR: 39-77) but distribution was bimodal with peaks in $<6$ years old and centered around 70 years of age. The cohort was $54.2 \%$ female. Most 
common exposures were to cardiovascular agents $(23.4 \%)$, followed by analgesics (14.4\%), anticoagulants (8.0\%), sedative hypnotics (7.0\%), antineoplastics (6.5\%) and anesthetics (6.5\%). Routes of administration were primarily parenteral (57.2\%), ingestion (41.3\%), and intrathecal (6.0\%). Specific errors occurring with the highest frequency were other incorrect dose (22.9\%), other/ unknown error (21.4\%), wrong medication given (12.4\%), incorrect dosing route $(10.4 \%)$, and rate-related errors $(10.0 \%)$. Categorizing age into 0-18 years, $19-64$ years and 65+ years, there was no difference in age distribution across different substance classes $(p=0.088)$.

Conclusion: latrogenic and in-hospital medication errors have been studied extensively with goals to reduce the occurrence. Specific controls to prevent incorrect dosing routes, 10-fold overdoses, and incorrect intrathecal administration have been instituted. Despite interventions, all three of these therapeutic errors continued to occur in 2017, suggesting that more preventive controls should be instituted.

\section{Impaired high-dose methotrexate clearance: does glucarpidase use conform with current guidelines?}

\author{
Lucile Bourgue ${ }^{a}$, Sabrina Pierre ${ }^{a}$, Delphine Castellan ${ }^{b}$, \\ Julien Mahe ${ }^{c}$, Dominique Hilaire-Buys ${ }^{d}$, Kevin Bihan ${ }^{e}$ \\ and Thierry Vial ${ }^{\mathrm{a}}$ \\ ${ }^{a}$ Hospital University Department of Pharmacotoxicology, Hospices \\ Civils de Lyon, Lyon, France; ${ }^{b}$ Pharmacovigilance Center, \\ Assistance Publique Hôpitaux de Marseille, Marseille, France; \\ 'Pharmacovigilance Center, Hospital University of Nantes, Nantes, \\ France; ${ }^{d}$ Pharmacovigilance Center, Hospital University of

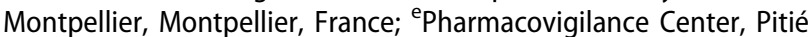 \\ Salpêtrière, Assistance Publique Hôpitaux de Paris, Paris, France
}

Objective: The antidotal management of impaired methotrexate (MTX) clearance in oncology settings is still a matter of debate. Our aim was to analyze the conformity of glucarpidase (GP) use according to current guidelines.

Methods: Any case involving high-dose MTX in the occurrence of an adverse drug reaction (ADR) coded under MedDRA terms indicative of delayed MTX clearance were extracted from the French pharmacovigilance database for two periods (January 2015-July 2016 and January 2017-July 2018). Inclusion criteria were documented delayed MTX elimination as defined by any plasma MTX concentration above $15 \mu \mathrm{mol} / \mathrm{L}$ at 24 hours, 1.5 $\mu \mathrm{mol} / \mathrm{L}$ at 48 hours or $0.75 \mu \mathrm{mol} / \mathrm{L}$ at 72 hours. Renal failure was defined by any increase of plasma creatinine of more than $50 \%$ the baseline level. To assess eligibility and relevance of GP administration, the manufacturer guideline was applied, i.e. at least one plasma MTX concentration $>50 \mu \mathrm{mol} / \mathrm{l}$ at 24 hours (only for patients exposed to more than $8 \mathrm{~g} / \mathrm{m}^{2}$ ), $>30 \mu \mathrm{mol} / \mathrm{L}$ at 36 hours, $>10 \mu \mathrm{mol} / \mathrm{L}$ at 42 hours or $>5 \mu \mathrm{mol} / \mathrm{L}$ at 48 hours.

Results: Overall 182 cases were retrieved and 122 were retained for analysis. There were 83 males/39 females with a median age of 44 years (range 2-84). Eighteen patients had previous renal insufficiency. The median dose was $3.1 \mathrm{~g} / \mathrm{m}^{2}$ (range 0.5-14.6, $\mathrm{n}=$ 83 ) and 32 patients concomitantly received a drug that may impair MTX clearance with proton pump inhibitors $(n=18)$ as the most common. Delayed MTX elimination occurred after the first cycle in 55 patients (45\%) and was associated with an increase in serum creatinine or acute renal failure in 108 patients $(89 \%)$, of whom $73(60 \%)$ had a stage $>2$. Other ADRs consisted of hematological toxicity $(n=27)$, liver injury $(n=16)$ and mucositis $(n=10)$. Fifteen patients underwent dialysis and $75(62 \%)$ received GP within 24 to 144 hours after MTX perfusion $(30<48$ hours, 30 at $48-60$ hours, $12>60$ hours, unknown in 3). Of the 103 patients retrospectively evaluable for GP eligibility, 41 of the 54 eligible patients (76\%) received at least one dose versus 22 of the 49 non-eligible patients (51\%). There was no significant difference in the rate of GP use between the two assessed periods $(60 \%$ versus $62 \%)$

Conclusion: GP was commonly used in patients who experienced delayed MTX elimination. Whereas most of the eligible patients adequately received GP, half of the patients underwent GP treatment while eligible criteria were not satisfied. Harmonization of practices in France is awaited.

\section{Epidemiology of benzodiazepine exposures reported to US Poison Centers, 2014-2017}

\author{
Saumitra Rege ${ }^{a}$, Duc Anh $\mathrm{Ngo}^{\mathrm{b}}$ and \\ Christopher P Holstege ${ }^{a}$ \\ aDivision of Medical Toxicology, Department of Emergency \\ Medicine, University of Virginia, Charlottesville, United States; \\ ${ }^{b}$ Division of Student Affairs, Department of Student Health, \\ University of Virginia, Charlottesville, United States
}

Objective: Drug overdoses are a leading cause of accidental death in the US, with more than $30 \%$ involving benzodiazepines. Benzodiazepine prescriptions increased by $67 \%$ between 1996 and 2013 [1]. The objective of this study was to outline the epidemiology of benzodiazepine exposures reported to US poison centers (PCs).

Methods: The National Poison Data System (NPDS) was queried for all closed, human exposures to benzodiazepines from 2011 to 2017 using the generic code identifiers. Key demographic and clinical characteristics were descriptively assessed. Benzodiazepine reports from acute care hospitals and emergency departments $(\mathrm{ACH})$ were analyzed. Trends in benzodiazepine frequencies and rates (per 100,000 human exposures) were analyzed using Poisson regression. Percent changes during the study period were reported.

Results: There were 530,530 benzodiazepine exposures reported to the PCs, with the calls decreasing $(82,278$ to 69,632$)$ during the study period. Among the overall benzodiazepine calls, $65.2 \%$ of calls were received from $\mathrm{ACH}$, with this proportion demonstrating an increase $(60.2 \%$ to $69.7 \%)$. Multiple substance exposures accounted for $63.2 \%$ of the overall calls and $80.6 \%$ of the calls from $\mathrm{ACH}$. Residence was the most common site of exposure (93.8\%). Demographically, $61.2 \%$ cases were females, and the most frequent age group was $20-39$ years (36.6\%). Suspected suicides (59.2\%) and unintentional misuse $(11 \%)$ were commonly observed reasons for exposure. The proportion of suspected suicides (73.7\%) was higher in cases reported by $\mathrm{ACH}$. The most frequent co-occurring substance reported was alcohol (15.4\%), with opioids reported in approximately $20 \%$ cases. Approximately $25 \%$ of the patients reporting benzodiazepine exposure were admitted to the critical care unit (CCU). Drowsiness and tachycardia were the most frequent clinical effects. Naloxone administration was reported for $7.7 \%$ of cases, with this therapy being performed prior to PC contact in most cases. Moderate (24.7\%) and major (5.4\%) effects were frequent. There were 1,553 deaths reported within $\mathrm{ACH}$ during the study period. The frequency of benzodiazepine exposures decreased by $15.7 \%$ (95\% Cl: $-16.2 \% ;-14.6 \%, p<0.001)$, and the rate decreased by $6.7 \%(95 \% \mathrm{Cl}:-10.9 \%,-2.0 \% ; \mathrm{p}=0 / 04)$.

Conclusion: PC data demonstrated a decreasing trend of benzodiazepine exposures. Despite this decreasing trend, the number of benzodiazepine exposures reported remains high, especially in combination with opioids. The proportion of cases reported from $\mathrm{ACH}$ increased. PCs can be a key early intervention of such cases. 


\section{Reference}

[1] Bachhuber MA, Hennessy S, Cunningham CO, et al. Increasing benzodiazepine prescriptions and overdose mortality in the United States, 1996-2013. Am J Public Health. 2016;106:686-688.

\section{Heart of stone: excessively elevated digoxin concentrations with no cardiac toxicity in an infant}

\author{
Elie Harmouche ${ }^{a}$, Robert S Hoffman ${ }^{a}$, \\ Mary Ann Howland ${ }^{\mathrm{b}}$ and Rana Biary ${ }^{\mathrm{a}}$ \\ ${ }^{a}$ Division of Medical Toxicology, Ronald O. Perelman Department \\ of Emergency Medicine, NYU School of Medicine, New York,

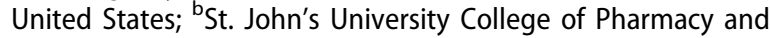 \\ Health Sciences, Jamaica (NY), United States
}

Objective: Digoxin toxicity is determined largely by physical examination and electrocardiogram (ECG) findings; concentrations serve for confirmation. Some reports suggest decreased digoxin sensitivity in younger patients. We report a case of an infant with a very elevated digoxin concentration successfully managed conservatively.

Case report: A 2-month-old boy $(3.1 \mathrm{~kg})$ with a past medical history of congestive heart failure was admitted to the hospital for vomiting and failure to thrive. The patient was started on oral digoxin, ordered as $0.015 \mathrm{mg}$ twice daily. Due to a medication error, he was instead given $0.15 \mathrm{mg}$ twice a day for four days. His only other medication was furosemide. He had no new symptoms or change in the frequency of his vomiting. During a new medication reconciliation, the error was discovered. At that time, his vital signs were blood pressure $88 / 43 \mathrm{mmHg}$, heart rate 123 beats/minute, respiratory rate 52 , temperature $36.9^{\circ} \mathrm{C}$, and oxygen saturations $100 \%$ (on supplemental oxygen). His electrocardiogram (ECG) showed a sinus rhythm at 122 beats/minute with normal intervals. There was no evidence of increased automaticity or any arrhythmias. A serum digoxin concentration drawn 5 hours after his last dose was $16 \mathrm{nmol} / \mathrm{L}$. Other laboratory findings at that time were notable for a potassium of $5.2 \mathrm{mmol} / \mathrm{L}$ with slight hemolysis and a creatinine of $3.5 \mu \mathrm{mol} / \mathrm{g}$. Repeat laboratory tests 8 hours after his last dose revealed a digoxin concentration of $15 \mathrm{nmol} / \mathrm{L}$, potassium $5.3 \mathrm{mmol} / \mathrm{L}$ (hemolyzed), creatinine 2.7 $\mu \mathrm{mol} / \mathrm{g}$ and magnesium $0.72 \mathrm{mmol} / \mathrm{L}$. He remained hemodynamically stable despite occasional episodes of vomiting. No cardiac ectopy or other events were noted. Oral digoxin was withheld and Digifab was not given. Repeat digoxin concentrations on day 1, 2, 4 and 5 were 9.6, 4.9, 2.9 and $1.92 \mathrm{nmol} / \mathrm{L}$, respectively.

Conclusion: Digoxin toxicity often manifests with cardiac and constitutional symptoms. While toxicity is usually expected with elevated digoxin concentrations, only $63 \%$ of infants with a digoxin concentration greater than $6.4 \mathrm{nmol} / \mathrm{L}$ developed toxicity [1]. In fact, some evidence suggests that infants have decreased sensitivity to digoxin [2]. In this case the significantly elevated post-distribution digoxin concentration with no clear signs or symptoms of toxicity supports this presumption.

\section{References}

[1] Koren G, Parker R. Interpretation of excessive serum concentrations of digoxin in children. Am J Cardiol. 1985;55:1210-1214.

[2] Kearin M, Kelly J, O'Malley K. Digoxin receptors in neonates: an explanation of less sensitivity to digoxin than in adults. Clin Pharmacol Ther. 1980;28:346-349.

\section{Symptoms and toxicokinetics of azilsartan medoximil in overdose: a}

\section{case report}

\author{
Katrin Faber ${ }^{\mathrm{a}}$, Daniel Müller ${ }^{\mathrm{b}}$, Stefan Weiler ${ }^{\mathrm{a}}$ and \\ Hugo Kupferschmidt ${ }^{\mathrm{a}}$

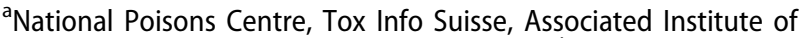 \\ the University of Zurich, Zurich, Switzerland; ${ }^{b}$ Institute for Clinical \\ Chemistry, University Hospital Zurich, Zurich, Switzerland
}

Objective: Azilsartan (AZL) medoximil is an antihypertensive prodrug that selectively blocks binding of angiotensin-II to the AT1receptor. Following an $80 \mathrm{mg}$ oral dose mean peak plasma concentrations of the active metabolite (about $5000 \mathrm{mg} / \mathrm{L}$ ) are expected within 1.5-3 hours, elimination half-life is 11 hours, and safety is generally good. There are limited data on overdose and to our knowledge no case has been published. We present a patient with a tenfold oral overdose of the maximum daily dose and provide toxicokinetics of AZL.

Case report: A 51-year-old Caucasian man with a history of hypertension was admitted to the emergency department after intentional ingestion of $800 \mathrm{mg}(10 \times 80 \mathrm{mg})$ AZL, $300 \mathrm{mg}$ mirtazapine and ethanol. At presentation 2.2 hours after ingestion blood pressure decreased to $85 / 48 \mathrm{mmHg}$ with a heart rate of 91 beats/minute. Glasgow Coma Score (GCS) was 14-15, temperature and electrocardiogram (ECG) were normal. Initial laboratory results revealed normal renal function including normal potassium. Liver function tests, full blood count $(\mathrm{FBC})$ and blood glucose $(4.6 \mathrm{mmol} / \mathrm{L})$ were also within the normal range. Soon after arrival (approximately 3 hours after ingestion) he became pale, diaphoretic and exhibited a systolic pressure of $43 \mathrm{mmHg}$ (measurement by Riva-Rocci). With a bolus of intravenous fluids systolic blood pressure increased to $80 \mathrm{mmHg}$, and treatment with vasopressors was not started. Hypotensive episodes occurred repeatedly, with decreases of blood pressure as low as $58 / 42 \mathrm{mmHg}$ (nadir at 3 hours and 7 hours after ingestion, recorded with intraarterial monitoring); these responded well to intravenous crystalloids. He fully recovered with normalization of blood pressure 12 hours after ingestion, and was discharged 6 hours later. Plasma and serum concentrations of AZL (difference plasma/ serum <5\%) measured in 4 samples by liquid-chromatography coupled with high-resolution-mass-spectrometry (LC-HRMS): 18.23 $\mathrm{mg} / \mathrm{L}$ ( 2.2 hours), $13.53 \mathrm{mg} / \mathrm{L} \quad(6.5$ hours), $7.39 \mathrm{mg} / \mathrm{L}$ ( 10.5 hours), $11.12 \mathrm{mg} / \mathrm{L}$ (11.8 hours). AZL elimination half-life was 9.5 hours, volume of distribution $37 \mathrm{~L}$ and clearance $2.7 \mathrm{~L} / \mathrm{h}$. Mirtazapine concentration was $1425 \mathrm{nmol} / \mathrm{l}$, and ethanol $1.2 \%$ o (2.2 hours). Caffeine and nicotine metabolites, but no other antihypertensive agents, were found by library-based liquid chromatography-mass spectrometry (LC-MS). Pharmacokinetic drug interactions of AZL with mirtazapine, caffeine or ethanol are not reported.

Conclusion: Marked hypotension may occur with AZL overdose and respond to intravenous fluids and crystalloids without vasopressors. The hypotensive effect might have been more pronounced by the coingestion of mirtazapine and ethanol in the present case. The clinical course corresponded to plasma concentrations and elimination half-life was not prolonged.

\section{Fatal metaxalone-induced serotonin toxicity}

\footnotetext{
Elie Harmouche ${ }^{\mathrm{a}}$, Jonathan De Olano ${ }^{\mathrm{a}}$, Anas Mashlah ${ }^{\mathrm{b}}$, Mary Ann Howland ${ }^{c}$ and William Chiang ${ }^{a}$

${ }^{a}$ Division of Medical Toxicology, Ronald O. Perelman Department of Emergency Medicine, NYU School of Medicine, New York, United States; ${ }^{b}$ Department of Forensic Medicine, NYU School of
} 
Medicine, New York, United States; 'St. John's University College of Pharmacy and Health Sciences, Jamaica (NY), United States

Objective: Metaxalone is a central nervous system depressant utilized in the treatment of acute skeletal muscle pain. The exact mechanism of action has not been established but large ingestions have been associated with serotonin toxicity. We report on a patient who expired from complications of serotonin toxicity induced by massive metaxalone ingestion.

Case report: A 20-year-old female with a prior history of depression presented to the emergency department unresponsive. History was unclear, however, her mother reported that the patient had access to her medications which included metaxalone, duloxetine, gabapentin, acetaminophen and oxycodone. She was intubated immediately and was found to be rigid with hyperreflexia and clonus. Initial vital signs were blood pressure $141 / 76 \mathrm{mmHg}$, heart rate 171 beats/minutes, respiratory rate 9/ minute, temperature $41.6^{\circ} \mathrm{C}$, oxygen saturations $98 \%$ (on supplemental oxygen). Initial venous blood gas showed $\mathrm{pH} 7.19, \mathrm{PCO}_{2}$ $23 \mathrm{mmHg}$, and lactate $1.2 \mathrm{mmol} / \mathrm{L}$. Initial blood tests revealed sodium $144 \mathrm{mmol} / \mathrm{L}$, potassium $2.6 \mathrm{mmol} / \mathrm{L}$, chloride $122 \mathrm{mmol} / \mathrm{L}$, bicarbonate $8 \mathrm{mmol} / \mathrm{L}$, blood urea nitrogen $21 \mathrm{mmol} / \mathrm{L}$, creatinine $244 \mu \mathrm{mol} / \mathrm{L}$, glucose $8.82 \mathrm{mmol} / \mathrm{L}$ and an acetaminophen concentration $662 \mu \mathrm{mol} / \mathrm{L}$ with an unknown time of ingestion. Despite supportive measures, external cooling, intravenous benzodiazepines and $\mathrm{N}$-acetylcysteine therapy she developed multiorgan failure, cerebral edema and expired on day 3 of admission. High performance liquid chromatography/tandem mass spectrometry of admission blood samples revealed a serum metaxalone of 59 $\mathrm{mg} / \mathrm{dL}$ (therapeutic less than $29.6 \mathrm{mg} / \mathrm{dL}$ ) in addition to detectable dextromethorphan, diphenhydramine and gabapentin concentrations but no detectable serum duloxetine.

Conclusion: Metaxalone is an oxazolidinone analog which is a class that was initially developed as potential antidepressant (toloxatone) and antimicrobial agents (linezolid). Based on the structure, metaxalone is expected to have monoamine oxidase inhibitor activity and may cause serotonin toxicity by itself or in conjunction with additional serotonergic medications [1]. Here we present a severe case of serotonin toxicity associated with death of a young patient with confirmed elevated metaxalone concentrations.

\section{Reference}

[1] Bosak AR, Skolnik AB. Serotonin syndrome associated with metaxalone overdose. J Med Toxicol. 2014;10:402-405.

\section{Do patients die with or from metformin-associated lactic acidosis (MALA)? Systematic review and meta- analysis of $\mathrm{pH}$ and lactate as predictors of mortality in MALA}

\author{
Sage W Wiener ${ }^{a}$, Roshanak Benabbas ${ }^{a}$, Amy Jeng ${ }^{b}$, \\ Adam Blumenberg ${ }^{c}$ and Richard Sinert ${ }^{\mathrm{a}}$ \\ a Emergency Medicine, SUNY Downstate Medical Center, Brooklyn,

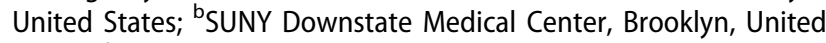 \\ States; 'Emergency Medicine, Oregon Health \& Science University, \\ Portland, United States
}

Objective: Metformin-associated lactic acidosis (MALA) may occur after acute metformin overdose, or from therapeutic use in patients with renal compromise, and mortality is high (historically $50 \%$ and more recently $25 \%$ ). Elevated lactate concentration is strongly associated with mortality in many disease states. The aim of this systematic review and meta-analysis is to investigate the utility of $\mathrm{pH}$ and lactate concentration in predicting mortality in patients with MALA.

Methods: We searched PubMed, EMBASE, and Web of Science from their inception to December 2017 for case reports, case series, prospective and retrospective studies investigating mortality in patients with MALA. Cases and studies were reviewed by all authors and included if they reported data on $\mathrm{pH}$, lactate, and outcome. Where necessary, authors of studies were contacted for patient level data. Receiver operating characteristic (ROC) curves were generated for $\mathrm{pH}$ and lactate for predicting mortality in patients with MALA.

Results: Overall 169 cases of MALA were included with the median age of 68 years old. Median pH and lactate were 7.02 and $14.5 \mathrm{mmol} / \mathrm{L}$, respectively. Overall mortality was $36.7 \%(95 \%$ $\mathrm{Cl} 29.8-44.2 \%)$. The area under the ROC curve for lactate and $\mathrm{pH}$ were $0.59(0.50-0.68)$ and $0.43(0.36-0.52)$ and therefore, neither lactate nor $\mathrm{pH}$ were good predictors of mortality among patients with MALA.

Conclusion: Mortality from MALA was higher in our review than most current series because we included patients from decades ago when mortality was higher than today. The ROC curves showed poor predictive power of either lactate or $\mathrm{pH}$ for mortality in MALA. With the exception of patients with acute metformin overdose, patients with MALA usually have coexisting precipitating illness such as sepsis or renal failure, though lactate from MALA is generally higher than would be considered survivable for those disease states on their own. It is possible that mortality is more related to that coexisting illness than MALA itself, and many patients die with MALA rather than from MALA. Additional work looking solely at MALA in healthy patients with acute metformin overdose may show a closer relationship between lactate, $\mathrm{pH}$ and mortality.

\section{Supratherapeutic digoxin concentration without cardiac manifestations}

\author{
Emily Green Vance ${ }^{a}$, Justin Arnold ${ }^{b}$ and \\ William F Rushton ${ }^{\mathrm{b}}$ \\ aDepartment of Pharmacy, UAB Health System, Birmingham, \\ United States; ${ }^{b}$ UAB Office of Medical Toxicology, Department of \\ Emergency Medicine, Birmingham, United States
}

Objective: Digoxin toxicity is not uncommon, however, there are no reports of sustained cardiac stability with a post-distribution concentration of $9.1 \mathrm{ng} / \mathrm{mL}$. We report such a case.

Case report: A 61-year-old woman with a history of atrial fibrillation on digoxin, cirrhosis due to hepatitis $C$ virus and non-alcoholic steatohepatitis, and orthotopic liver transplant, presented to the emergency department complaining of abdominal pain and swelling. Laboratory evaluation showed a total serum digoxin concentration of $9.1 \mathrm{ng} / \mathrm{mL}$ (confirmed to be post-distribution based on last dose date/time), potassium of $5.3 \mathrm{mmol} / \mathrm{L}$ (not hemolyzed), and creatinine $2.6 \mathrm{mg} / \mathrm{dL}$ (baseline $1.2 \mathrm{mg} / \mathrm{dL}$ ). She denied palpitations, chest pain, or confusion. She did complain of dyspnea, nausea, vomiting, and dizziness. Her electrocardiogram revealed normal sinus rhythm without ectopy. She denied overdose or increased dosing and had not been treated with digoxin Fab fragments. She was admitted for worsening ascites and acute kidney injury that was felt to be due to medication non-compliance. Her heart rate remained stable throughout her hospitalization. Digoxin was withheld and the drug concentration trended 
down over the next 7 days $(9.1,4.8,4.3,3.2,1.9,1.4,1.1,0.9 \mathrm{ng} /$ $\mathrm{mL}$ ). Her creatinine improved to $1 \mathrm{mg} / \mathrm{dL}$ and potassium returned to normal range. The Emit 2000 Digoxin Assay was used and accurately quantitates digoxin concentrations in serum or plasma containing $0.2-5 \mathrm{ng} / \mathrm{mL}$ digoxin. To estimate concentrations above the assay range, samples containing more than digoxin 5 $\mathrm{ng} / \mathrm{mL}$ are diluted, repeated, and multiplied by the dilution factor. Endogenous digoxin-like immunoreactive factors may also have been present due to her hepatic disease and acute kidney injury, which can result in a falsely elevated concentration. This patient also had new ascites and acute kidney injury, both of which can also cause these symptoms. Given the patient was hemodynamically stable, we postulate that the analyzer most likely had detected digoxin-like immunoreactive factors that have been reported to be present in patients with both hepatic disease or renal injury or failure.

Conclusion: We report a case of significantly elevated serum digoxin concentrations using the Emit 2000 Digoxin Assay with no cardiac manifestations. Clinicians should search for other etiologies or confounding interactions with the total serum digoxin assay and should consider not reflexively treating with digoxin Fab fragments in the setting of a minimally symptomatic individual with a reported high digoxin concentration.

\section{Paracetamol poisoning: cases treated at the National University Hospital of Iceland (NUH)}

\author{
Porbjörg Andrea Friðdriksdottir ${ }^{a}$, Curtis P Snook ${ }^{b}$, \\ Helena Lindal Baldvinsdottir ${ }^{c}$, Einar S Bjornsson ${ }^{d}$ and \\ Freyja Jonsdottir \\ ${ }^{a}$ Faculty of Pharmaceutical Sciences at University of Iceland, \\ Reykjavik, Iceland; ${ }^{b}$ Emergency Department, National University \\ Hospital, Reykjavik, Iceland; 'Poison Information Center, National \\ University Hospital, Reykjavik, Iceland; ${ }^{d}$ Gastrointestinal and Renal \\ Unit, National University Hospital, Reykjavik, Iceland; ${ }^{\mathrm{e}}$ Faculty of \\ Pharmaceutical Sciences at University of Iceland, Hospital \\ Pharmacy of National University Hospital of Iceland (NUH), \\ Reykjavik, Iceland
}

Objective: The aim was to examine the extent of paracetamol poisoning based on annual incidence 2010-2017. In addition the nature of this poisoning was explored and prognosis and risk by gender and age group were investigated.

Methods: A retrospective descriptive study was conducted. Data about all patients where paracetamol concentrations were measured from 1 January 2010 to 31 December 2017 were retrieved from the electronic medical record system at NUH. The concentrations were classified in different groups, $<66 \mu \mathrm{mol} / \mathrm{L}$, therapeutic range $66-200 \mu \mathrm{mol} / \mathrm{L}$, and concentrations $\geq 66 \mu \mathrm{mol} / \mathrm{L}$. Patients' medical records were examined and further information was gathered regarding those who met the criteria of paracetamol poisoning.

Results: Overall 542 cases were identified during 2010-2017. The mean annual incidence was 26.7/100,000 inhabitants over the study period (range 22-33) and was stable. Intentional overdose was observed most frequently among females aged 16-25 years, whereas males aged 65 years and older were more likely to overdose by accident. Accidental overdoses increased during the study period associated with a worse outcome. Overall $43 \%$ of patients received acetylcysteine treatment, but 35\% received no treatment. Paracetamol-induced liver injury occurred in 29/542 (5.4\%) of patients and six died where paracetamol poisoning contributed to the cause of death; two were intentional and four accidental.
Conclusion: Intentional paracetamol poisoning was most common among young females. Accidental overdoses were more likely in males aged $\geq 65$ years and were associated with worse outcomes. Most patients had favourable outcomes but the increase in accidental overdose associated with increased morbidity and mortality is of concern.

\section{Survival following large verapamil overdose after use of extracorporeal membrane oxygenation (ECMO) and multiple complications}

\author{
Pardeep S Jagpal ${ }^{\mathrm{a}}$, Muhammad EMO Elamin ${ }^{\mathrm{a}}$, \\ Mark Puccib ${ }^{\mathrm{b}}$, Loretta Ford ${ }^{\mathrm{c}}$, Dacia Jones ${ }^{\mathrm{d}}$, \\ Simon HL Thomas ${ }^{d}$ and Sally M Bradberry ${ }^{a}$ \\ ${ }^{a}$ National Poisons Information Service (Birmingham Unit), City \\ Hospital, Birmingham, United Kingdom; ${ }^{b}$ Department of Clinical \\ Pharmacology, Queen Elizabeth Hospital, Birmingham, United \\ Kingdom; 'Clinical Biochemistry, City Hospital, Birmingham, United \\ Kingdom; ${ }^{d}$ National Poisons Information Service (Newcastle Unit), \\ Regional Drug and Therapeutics Centre, Newcastle, United \\ Kingdom
}

Objective: We describe a patient who survived an intentional large overdose of verapamil but developed adverse effects following institution of ECMO.

Case report: A 17-year-old male presented to hospital with bradycardia (52 bpm), confusion and drowsiness (Glasgow Coma Score [GCS] 13) following an out of hospital tonic-clonic seizure. Arterial blood gas demonstrated metabolic acidosis $(\mathrm{pH} \quad 7.2$, bicarbonate $19.4 \mathrm{mmol} / \mathrm{L}$, lactate $16.3 \mathrm{mmol} / \mathrm{L})$. Electrocardiogram (ECG) revealed atrial fibrillation with slow ventricular rate, QRS widening (144 ms) and left bundle branch block. Echocardiography revealed normal left ventricular/right ventricular (LV/RV) function but abnormal septal movement. He was commenced on broad spectrum antimicrobials in view of a recent 4 day history of diarrhoea, vomiting and abdominal pain following travel to the Middle East. Lumbar puncture and blood cultures did not reveal any infective cause. Within 24 hours he was commenced on inotropes and vasopressors for haemodynamic instability and continuous veno-venous hemofiltration (CVVH) for acute kidney injury (urea $7.6 \mathrm{mmol} / \mathrm{L}$, creatinine $303 \mu \mathrm{mol} / \mathrm{L}$ ). On day two he required intubation and ventilation for worsening hypoxia despite continuous positive airway pressure (CPAP) and was transferred for veno-arterial ECMO with a working diagnosis of sepsis and secondary cardiomyopathy. While ECMO was being instituted, he developed cardiac arrest and was commenced on peripheral ECMO. Urine toxicology screening (time-of-flight exact mass analyser) identified verapamil and metabolites. His parents also found empty packets of around $10 \mathrm{~g}$ sustained release verapamil at home. He required 33 days of intensive care support involving intravenous ephedrine, calcium gluconate and high dose insulin dextrose therapy (35 units/hour) for hypotension. He also received lipid emulsion. He continued on ECMO, CVVH and treatment for coagulopathy. He developed compartment syndrome attributed to a miss-sited ECMO reperfusion line, requiring fasciotomy on day four. The femoral artery was repaired following discontinuation of ECMO. He required extensive debridement, topical negative pressure (TNP) dressings and antibiotics for vancomycin-resistant enterococci infection. One month after overdose, he required further debridement, TNP application and split skin grafting to the left leg. He was transferred to a 
specialist burns department where he continued his recovery with $100 \%$ graft take. Despite good recovery, he continued to experience nausea and pain. He was discharged on analgesia with long-term physiotherapy and psychiatry follow up.

Conclusion: ECMO use in poisoning as a bridge to "recovery" or "replacement" is not always without complications. Clinicians need to be aware both of the indications for ECMO, and its associated risks.

\section{Flecainide poisoning in the intensive care unit: predictive factors and usefulness of plasma flecainide concentration on admission}

\author{
Katia Carvalho Alves ${ }^{a}$, Brigitte Delhotal Landes ${ }^{b}$, \\ Sébastian Voicu ${ }^{a}$, Isabelle Malissin ${ }^{a}$, Nicolas Deye ${ }^{a}$ \\ and Bruno Mégarbane ${ }^{a}$ \\ ${ }^{a}$ Department of Medical and Toxicological Critical Care, Lariboisière \\ Hospital, Paris-Diderot University, INSERM UMRS-1144, Paris, \\ France; 'baboratory of Toxicology, Lariboisière Hospital, Paris- \\ Diderot University, INSERM UMRS-1144, Paris, France
}

\begin{abstract}
Objective: Acute poisoning with flecainide, a 1c anti-arrhythmic drug of the Vaughan-Williams' classification is rare but may be responsible for life-threatening consequences. Our objectives were to report the features, complications and management of flecainide poisonings admitted in the intensive care unit (ICU) and to investigate the prognostic contribution of the plasma flecainide concentration measured on admission.
\end{abstract}

Methods: We conducted a retrospective single-centre observational study including all flecainide poisoned-patients admitted to the ICU during a 20-year period (1998-2018), as evidenced by at least one plasma flecainide concentration in the toxic range. Plasma flecainide concentration was measured using liquid chromatography coupled to mass spectrometry. Identification of the predictive factors of death was performed using an univariate analysis (chi-squared and Mann-Whitney tests, as required).

Results: Forty-eight flecainide-poisoned patients (54\% males and $46 \%$ females; aged 53 years [32; 58] (median [percentiles 25; 75])) were included. The patients had ingested $3.0 \mathrm{~g}[1.7 ; 3.0]$ of flecainide (including one third with slow-release formulations) and were admitted 3.0 hours $[1.9 ; 7.6]$ post-ingestion. They developed cardiovascular failure with membrane stabilizing effect on the electrocardiogram (ECG) (58\%). Plasma flecainide concentration was $2.3 \mathrm{mg} / \mathrm{L}[1.3 ; 3.0]$ on ICU admission. Management included mechanical ventilation (77\%), catecholamines (81\%), 8.4\% sodium bicarbonate (63\%), activated charcoal (54\%), defibrillation (23\%) and veno-arterial extracorporeal membrane oxygenation (VAECMO) (3\%). Ten patients (21\%) died. Based on an univariate analysis, the non-survivors presented more marked hypotension ( $\mathrm{p}=$ $0.05)$, bradycardia $(p=0.02)$, elevation in plasma lactate concentration $(p=0.04)$, elevation in transaminases $(p=0.006)$ and decrease in serum bicarbonate concentration $(p=0.04)$ than the survivors. The presumed ingested dose $(p=0.02)$ and the plasma concentration of flecainide on admission $(p=0.0003)$ were significantly correlated to the onset of death.

Conclusion: Flecainide poisoning is responsible for acute cardiovascular failure due to membrane stabilizing effects leading to a high mortality rate. The plasma flecainide concentration on admission is highly predictive of the onset of death and its bedside availability should be strongly encouraged. The identification of the clinical prognosticators is helpful when deciding to implement ECMO in the flecainide-poisoned patient.

\section{Status epilepticus and cardiac arrest after overdose of lacosamide: a case report}

\author{
Peter Hultén, Ann-Sofi Skagius and Jonas Höjer \\ Swedish Poisons Information Centre, Stockholm, Sweden
}

Objective: The novel antiepileptic drug lacosamide is involved in the slow inactivation of voltage-gated sodium channels (VGSC). Lacosamide was approved in Sweden 2008. A few overdose cases with severe cardiac symptoms or fatal outcome have been published [1,2]. The Swedish poisons centre has been consulted in 12 lacosamide overdose cases (1.8-8.1 g). Ten cases resulted in minor or moderate symptoms, including central nervous system depression, agitation and seizures. Two cases were severe, one $(4.2 \mathrm{~g})$ led to repeated seizures and the second is presented here.

Case report: A 72-year-old woman with a suspected seizure disorder treated with lacosamide and lamotrigine, ingested $5.6 \mathrm{~g}$ lacosamide in a suicidal attempt and was found unconscious. In the ambulance convulsive status epilepticus and two episodes of ventricular tachycardia finally turning into asystole occurred. Cardiopulmonary resuscitation (CPR) including boluses of adrenaline and amiodarone was successful. In the emergency room she had dilated pupils with no response to light, ongoing status epilepticus, wide QRS complex (138 ms), and bradycardia with irregular rhythm (36 bpm). She was sedated with propofol, intubated and transferred to intensive care where an amiodarone infusion was started. She was hyperventilated and given sodium bicarbonate to maintain alkalinisation. A bolus of atropine and low dose noradrenaline was administered, but the bradycardia persisted. When propofol was later terminated, seizures returned and the drug was restarted and midazolam added. On day 2 the QRS had narrowed (105 ms), but sinus arrest for one minute occurred and was spontaneously followed by an escape nodal rhythm. More sodium bicarbonate was given as was an isoproterenol infusion. On the third day the bradycardia had disappeared. The patient started to wake up on days 45 and was successfully extubated. She was discharged on the sixth day, awake and in a stable condition.

Conclusion: It is still unclear whether lacosamide alone may give rise to severe cardiac symptoms in healthy individuals. The sparse literature indicate that a mixed overdose with other VGSC-blocking agents may increase this risk [1,3].

\section{References}

[1] Chua-Tuan JL, Cao D, Iwanicki JL, et al. Cardiac sodium channel blockade after an intentional ingestion of lacosamide, cyclobenzaprine, and levetiracetam: case report. Clin Toxicol (Phila). 2015;53:565-568.

[2] Malissin I, Baud FJ, Deveaux M, et al. Fatal lacosamide poisoning in relation to cardiac conduction impairment and cardiovascular failure. Clin Toxicol (Phila). 2013;51:381-382.

[3] Lachuer C, Corny J, Bézie Y, et al. Complete atrioventricular block in an elderly patient treated with low-dose lacosamide. Cardiovasc Toxicol. 2018;18:579-582.

\section{Monitoring and management of the 2018 Kilauea Lower East Rift Zone Eruption}

\author{
Alvin C Bronstein ${ }^{\mathrm{a}}$, G Fenix Grange ${ }^{\mathrm{b}}$, Diana J Felton ${ }^{\mathrm{b}}$ \\ and Daniel A Spyker \\ ${ }^{a}$ Emergency Medical Services Injury Prevention System Branch, \\ Hawaii Department of Health, Honolulu, United States; ${ }^{\text {b Hazard }}$
}


Table 1. Sulfur dioxide and particulate material time weighted average (TWA) and maximums by sensor location (sorted by $\mathrm{SO}_{2} 30$ minute $\mathrm{TWA}$ ) during the 2018 Kilauea Lower East Rift Zone Eruption.

\begin{tabular}{|c|c|c|c|c|c|c|c|c|c|c|c|c|}
\hline \multirow[b]{3}{*}{ Sensor Location } & \multicolumn{6}{|c|}{ Sulfur dioxide $\left(\mathrm{SO}_{2}\right)$ parts per million (ppm) } & \multicolumn{6}{|c|}{ Particulate materials $<2.5$ microns $\left(\mathrm{PM}_{2.5}, \mu \mathrm{g} / \mathrm{m}^{3}\right)$} \\
\hline & \multirow[b]{2}{*}{ Overall Max } & \multicolumn{2}{|c|}{$30 \mathrm{~min}$ TWA } & \multirow[b]{2}{*}{ Start Date } & \multirow{2}{*}{$\begin{array}{c}\text { Number } \\
\text { Days }\end{array}$} & \multirow{2}{*}{$\begin{array}{l}\text { Number } \\
\text { samples }\end{array}$} & \multirow{2}{*}{$\begin{array}{c}\text { Overall } \\
\text { Max }\end{array}$} & \multicolumn{2}{|c|}{$30 \mathrm{~min}$ TWA } & \multirow{2}{*}{$\begin{array}{l}\text { Start } \\
\text { Date }\end{array}$} & \multirow{2}{*}{$\begin{array}{l}\text { Num } \\
\text { Days }\end{array}$} & \multirow{2}{*}{$\begin{array}{l}\text { Number } \\
\text { samples }\end{array}$} \\
\hline & & Mean & Max & & & & & Mean & Max & & & \\
\hline Leilani Estates Lower & 20.00 & 8.405 & 19.975 & 21-May & 3.5 & 132,065 & & & & & & \\
\hline Kaueleau Kamaili Road & 5.90 & 0.115 & 3.191 & 25-May & 43.9 & 214,946 & & & & & & \\
\hline Leilani Estates & 16.60 & 0.113 & 5.965 & 20-May & 51.1 & 269,397 & 1710 & 20.64 & 510.0 & 20-May & 51.1 & 603,284 \\
\hline Volcano & 1.80 & 0.060 & 1.236 & 31-May & 36.1 & 603,139 & 870 & 15.32 & 244.7 & 31-May & 35.9 & $1,652,656$ \\
\hline Kalapana Seaview & 7.80 & 0.074 & 4.777 & 18-May & 57.7 & 335,525 & 265 & 4.99 & 92.2 & 19-May & 57.1 & 753,722 \\
\hline Pahoa HAAS & 5.50 & 0.022 & 2.300 & 17-May & 49.8 & 406,248 & 714 & 2.63 & 237.4 & 17-May & 49.8 & $1,509,796$ \\
\hline Pahoa Community Center & 2.40 & 0.011 & 1.214 & 28-May & 38.9 & 275,854 & 344 & 3.91 & 185.1 & 28-May & 38.9 & $1,022,210$ \\
\hline Kalapana Painted Church & 1.90 & 0.007 & 1.003 & 18-May & 57.9 & 312,680 & 163 & 7.59 & 39.0 & 18-May & 50.8 & $1,668,939$ \\
\hline Nanawale Kua O Ka La School & 20.90 & 0.007 & 11.136 & 17-May & 55.1 & 395,156 & 380 & 3.95 & 143.7 & 17-May & 54.9 & $1,222,264$ \\
\hline Leilani Estates Upper & 16.00 & 0.005 & 1.755 & 21-May & 45.1 & 121,054 & 593 & 2.41 & 92.8 & 21-May & 45.1 & $1,207,529$ \\
\hline Naalehu Fire Department & 0.80 & 0.004 & 0.582 & 27-May & 45.1 & 47,050 & 1750 & 5.16 & 46.7 & 27-May & 26.1 & 546,702 \\
\hline Black Sands Estates Aloha Road & 3.00 & 0.004 & 0.333 & 20-May & 48.9 & 255,945 & 800 & 1.49 & 24.4 & 20-May & 48.5 & 996,799 \\
\hline Hawaiian Beaches Keonepoko School & 0.50 & 0.001 & 0.100 & 17-May & 54.4 & 298,957 & 2280 & 2.56 & 56.3 & 17-May & 55.1 & $1,902,353$ \\
\hline Keaau Ke Kula School & 1.40 & 0.001 & 0.412 & 17-May & 58.9 & 351,831 & 106 & 4.78 & 60.5 & 17-May & 49.8 & $1,507,845$ \\
\hline Max & 20.9 & 8.405 & 19.98 & 31-May & 58.9 & 603,139 & 2280 & 20.64 & 510.0 & 31-May & 57.1 & $1,902,353$ \\
\hline Mean & 7.46 & 0.631 & 3.86 & 21-May & 46.2 & 287,132 & 831 & 6.29 & 144.4 & 21-May & 46.9 & $1,216,175$ \\
\hline Sum & & & & & 646 & $4,019,847$ & & & & & 563 & $14,594,099$ \\
\hline
\end{tabular}

Evaluation and Emergency Response (HEER), Hawaii Department of Health, Honolulu, United States; ' ${ }^{\mathrm{C}}$ mergency Medicine, Oregon Health \& Science University, Portland, United States

Objective: Hawaii island's Kilauea Lower East Rift Zone Eruption began 3 May 2018 producing a variety of toxicological hazards: sulfur dioxide $\left(\mathrm{SO}_{2}\right)$, sulfate aerosol particulates, and volcanic ash. Some aerosols and ash were respirable particulate matter $\left(\mathrm{PM}_{2.5}\right)$. Established guidance using air monitoring for public health decisions related emissions is sparse. Civil Defense, public, and Hawaii Department of Health $(\mathrm{HDOH})$ all identified an air monitoring need. US Environmental Protection Agency (EPA) provided a wireless real-time $\mathrm{SO}_{2}$ and $\mathrm{PM}_{2.5}$ data system from 20 sensors. $\mathrm{HDOH}$ analyzed these data to provide civil defense with public health recommendations for emergency advisories including "shelter in place" and "evacuation."

Methods: We used available $\mathrm{SO}_{2}$ and $\mathrm{PM}_{2.5}$ air concentration data from 17 May through 15 July 2018. We calculated the time weighted average (TWA) and interval TWA (1, 5, 10 and 30 minutes) by sensor and across all sensors using SAS JMP 12.0.1 (SAS, Cary, NC, US). The HDOH team utilized a 6-level model based on $\mathrm{SO}_{2}$ short-term concentrations developed as Hawaiispecific guidance during the 2008 eruption.

Results: Table 1 summarizes the maximum and $30 \mathrm{~min}$ TWA $\mathrm{SO}_{2}$ and $\mathrm{PM}_{2.5}$ from the 14 most important sensor locations. The Leilani Estates Lower sensor reported the highest $\mathrm{SO}_{2}$, but ceased reporting after 3.5 days when incinerated by lava. This sensor reported its maximum, $20 \mathrm{ppm}$ (saturated) for 17,868/132,065 samples and provided no $\mathrm{PM}_{2.5}$ data. The $\mathrm{HDOH}$ 6-level shortterm $\mathrm{SO}_{2}$-based guidance included: $\mathrm{SO}_{2}<0.10$ good, $>0.1 \mathrm{ppm}$ moderate health concern, $>0.2$ unhealthy for sensitive persons, $>1.0$ unhealthy, $>3.0$ very unhealthy, $>5.0$ hazardous. Air concentrations fluctuated widely with wind shifts making firm recommendations for shelter in place or mandatory evacuation difficult.

Conclusion: Using point-source, real-time air data to guide public health decisions and clinical health effect assessment proved difficult. Further work is needed to develop optimal air data use methods.

\section{Go-kart engine oil ingestion causing seizure}

Peter Akpunonu and Jeremiah Phelps
Emergency Medicine, University of Kentucky, Lexington, United States

Objective: Accidental ingestion of racing car engine oil is an uncommon occurrence that can result in life-threatening multisystem toxicity which may prove fatal. This case report describes severe metabolic derangement, seizure and coma in an adult male after ingestion of a go-kart engine oil.

Case report: The patient was a 32 year-old male who was an active methamphetamine abuser. Witnesses reported that he slumped over and began having seizure-like activity immediately after consumption of approximately $360 \mathrm{~mL}$ of an unknown redbrown fluid in a sports drink bottle. Upon paramedic arrival the patient required intubation and benzodiazepines for encephalopathy and seizure control. On arrival to the Emergency Department he continued to have seizure-like activity suggesting status epilepticus. Propofol and pyridoxine were started and seizures abated. Initial laboratory analyses demonstrated severe hypokalemia $(<1.5 \mathrm{mmol} / \mathrm{L})$, rhabdomyolysis (creatine kinase $3803 \mathrm{U} / \mathrm{L}$ ), acute kidney injury (creatinine $2.0 \mathrm{mg} / \mathrm{dL}$ ), hypermagnesemia $(5.1 \mathrm{mg} / \mathrm{dL})$, with a comprehensive liquid chromatography-mass spectrometry (LC-MS) urine drug screen positive for methamphetamine. After 36 hours of intubation, aggressive electrolyte replacement and fluid resuscitation he was safely extubated. He remained in intensive care for 2 more days as rhabdomyolysis resolved and he was discharged. Further investigation suggested the unknown substance was Red Line ${ }^{\circledR}$ TwoStroke Kart Oil, a commonly used go-kart racing oil in the Appalachian region of the US. This contains 2,6-di-tert-butylphenol, phenol didoecyl branched, and diphenylamine, aromatic compounds [1], which likely caused the dramatic electrolyte shifts and direct tissue toxicity resulting in life-threatening seizure, endorgan renal damage, and coma as observed in our patient.

Conclusion: Though seizures are associated with methamphetamine use, the rapid onset of seizures with electrolyte derangements suggests an alternative etiology. Two-cycle kart racing engine oils commonly contain aromatic hydrocarbons and metals (e.g. barium) which affect ion channels and electrolyte homeostasis resulting in cardiovascular and neurological side-effects. Onset of effect is rapid after ingestion and clinical manifestations may be profound and potentially lethal. Overall this case demonstrates the importance of proper chemical storage and labeling to avoid accidental ingestion as failure to comply may result in a critical toxicologic event. 


\section{Reference}

[1] Red Line ${ }^{\circledast}$ Two-Stroke Kart Oil Safety Data Sheet [cited 2019 Oct 23]. Available from: https://www.redlineoil.com/two-stroke-kart-oil

\section{The Toxicology Investigators Consortium (ToxIC): an innovative multicenter medical toxicology research network}

\author{
Paul Wax ${ }^{\mathrm{a}}$, Jeffrey Brent ${ }^{\mathrm{b}}$, Diane Colello ${ }^{\mathrm{c}}$ and \\ Sharan Campleman ${ }^{\mathrm{d}}$

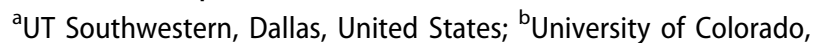 \\ Denver, United States; 'Rutgers Medical School, Newark, United \\ States; ${ }^{\mathrm{d}}$ American College of Medical Toxicology, Phoenix, United \\ States
}

Objective: In the past, few medical toxicologists in the US provided direct care for patients at the bedside. Consequently, the most common poisoning research methodology employed poison center data collection. With the growth of the medical toxicology specialty, an increasing number of medical toxicologists now provide direct care for patients at the bedside. In 2010 the American College of Medical Toxicology established the Toxic Investigators Consortium (ToxIC) to provide a national network platform for multicenter collaborative research [1]. We investigated the research output of ToxIC from inception in 2010 to September 2018 in order to assess whether this model is viable for the generation of toxicologic studies.

Methods: Research output were measured by the following variables: the number of participating investigators, research sites and hospitals; case accrual, published abstracts, published papers, and extramural funding obtained. Data was obtained from the ToxIC Annual Reports [2] and internal grant and publications tracking records.

Results: From January 2010 through September 2018, 65,000 cases were entered into the ToxIC Registry. These cases were entered from 45 sites that provide bedside care at 69 hospitals. More than 100 medical toxicologists participated in the data collection. Aside from the core registry data collection, seven subregistries were set up during this period to collect more detailed data on a variety of toxicologic research investigations. During this 8.5 year period 109 abstracts were presented at national and international meetings, and 32 manuscripts were published in the peer reviewed literature. Grant funding was obtained from the National Institutes of Health (NIH), US Food \& Drug Administration (FDA), and industry. Total grant funding to date has exceeded $\$ 1,350,000$; about half from governmental sources and half from industry.

Conclusion: Constructing a large multicenter medical toxicology research network resulted in more than 100 abstracts and 30 papers published in its first eight years indicating that it is a viable research tool. Maintaining such a network requires ongoing and continuous grant support.

\section{References}

[1] Wax PM, Kleinschmidt KC, Brent J. The Toxicology Investigators Consortium (ToxIC) Registry. J Med Toxicol. 2011;7:259-265.

[2] Farrugia LA, Rhyee SH, Campleman SL, et al. The Toxicology Investigators Consortium Case Registry - the 2017 Annual Report. J Med Toxicol. 2018;14:182-211.

\section{E-learning in toxicology: development of the new IT-based International Diploma and Continuous Professional Development Courses in Clinical Toxicology in Egypt}

\author{
Noha Fawzy Abdelkader ${ }^{a}$, Sahar El-Gowilly ${ }^{b}$, \\ Sherif Tarek Elnabarawyc, Safwat A Mangourad, \\ Yasser HRM Kazem ${ }^{e}$, Mohamed Seif Eldin ${ }^{f}$, \\ Janet Mifsud ${ }^{\mathrm{g}}$, Matthew C Wright ${ }^{\mathrm{h}}$, Mark L Zammit ${ }^{\mathrm{g}}$ \\ and Alexander Campbell \\ ${ }^{a}$ Department of Pharmacology and Toxicology, Faculty of \\ Pharmacy, Cairo University, Cairo, Egypt; ${ }^{b}$ Faculty of Pharmacy, \\ Alexandria University, Alexandria, Egypt; 'Faculty of Engineering, \\ Ain Shams University, Cairo, Egypt; ${ }^{d}$ Department of Pharmacology,

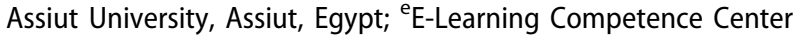 \\ Director, Ministry of Communications and Information Technology,

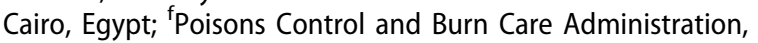 \\ Ministry of Health and Population of Egypt, Cairo, Egypt; \\ ${ }^{9}$ Department of Clinical Pharmacology and Therapeutics, Faculty of \\ Medicine and Surgery, University of Malta, Msida, Malta; ${ }^{\text {h Institute }}$ \\ of Cellular Medicine, Newcastle University, Newcastle-upon-Tyne, \\ United Kingdom; 'London, United Kingdom
}

Objective: Multi-stakeholder creation and implementation of a series of post-graduate courses in aspects of clinical, medical and occupational toxicology in Egypt, to be delivered predominantly through a new customised IT platform. Courses are aimed at professionals with or intending careers in poison centres and/or medical emergency related domains.

Methods: A comprehensive gap analysis of Egypt's toxicological exposures, their impact and a review of existing Egyptian training programs in clinical toxicology was undertaken. A funding bid to the EU-Erasmus + program, involving external partners in Malta, Sweden and the UK was successful. Academic and technological partners established the core educational content and structure, with each academic institution taking responsibility for specific modules with subsequent collective critical review from the others and the external partners through electronic file sharing and periodic face-to-face meetings. A novel feature was the development of e-labs to expand and enhance the exposure of diploma participants to practical aspects of patient management in more remote and nomadic areas in Egypt. Rules ensuring consistency of style and format on the IT platform were agreed in advance by all partners. European Credit Transfer and Accumulation System (ECTS) for educational courses was adopted to facilitate international reach and accreditation. Four levels of Quality Assurance were applied to scrutinise all aspects of the project and content for uniformity of approach and currency. Evaluation Capacity Building (ECB) check certification is being sought.

Results: The diploma course was launched on 22 September 2018 attracting over 360 registrants in 16 countries worldwide by 1 October 2018. It comprises 18 modules, each with 15 e-chapters, on an interactive IT-platform. Each e-chapter contains educational material, graphics, notes and references, self-assessment and other learning tools including 23 e-labs, online discussion seminars and forums. Final assessments are through written exams and projects. Module titles include epidemiology, toxinology, pharmaceuticals, substances of abuse, occupational and industrial toxicology, disaster preparedness, analytical toxicology, drug development, special populations and case management. In 
addition, three Continuous Professional Development courses on management of poisoning, medication errors and occupational toxicology are in their final pre-launch phase.

Conclusion: In under three years this modern educational course has been developed, launched and is enrolling its first students. Their achievements and feedback are keenly anticipated, and courses can be continuously refined to ensure a new generation of medical professionals, scientists and poisons centre staff are qualified with current knowledge and experience to handle, monitor and prevent toxicological exposures in Egypt and elsewhere.

\section{8. "Gaslighting" by atropine?}

\section{Erik Lindeman}

Swedish Poisons Information Center, Stockholm, Sweden

Objective: Anticholinergic symptoms are common non-specific features of many toxic exposures, while the pure anticholinergic toxidrome is classically associated with poisoning by belladonna alkaloids. Poisonings with these alkaloids rarely result in death, due to large differences between toxic and lethal doses. They reliably cause incapacitation however, which seems to be the purpose for their criminal use (to facilitate robbery or sexual assault). We present a case of atropine poisoning where a criminal motive may have been present, albeit with a novel purpose.

Case report: A 50-year-old male was brought to the emergency room (ER) with a delirium of acute onset. Symptoms were consistent with an anticholinergic toxidrome and administration of physostigmine led to an immediate resolution. Urine analysis revealed atropine at $5000 \mu \mathrm{g} / \mathrm{L}$ but no other belladonna alkaloids, consistent with exposure to a medicinal rather than a natural product. The patient was reluctant to believe that his symptoms had been of acute toxic origin as he had suffered bouts of distressing symptoms on several occasions and was very concerned for his physical and mental health. One of these bouts had led to an ER visit four weeks prior to the present admission. Medical records revealed that his principal complaints on this visit had been anxiety and palpitations and had also included a dry mouth and blurred vision, consistent with atropine toxicity. At follow-up the patient had himself come to suspect that his "illness" was indeed the result of poisoning. He was the owner of a small investment company. A few days after hospital discharge he had been approached by an employee who volunteered to temporarily take charge of the company to give the patient "time to recuperate". He presented documents for signing granting power of attorney over the company's financial dealings. The patient immediately terminated all dealings with the employee and reported the matter to the authorities. The police investigation was eventually suspended for lack of evidence.

Conclusion: The psychological term for manipulating a person into questioning their own sanity is "gaslighting", after the eponymous film from 1944 where the heroine (Ingrid Bergman) is nearly driven mad by her husband, whose motive is financial gain. To our knowledge, the presented case is the first description of possible "gaslighting" by poisoning. Although the suspected crime was never proven in court and important details therefore remain speculative, the unfolding of events nevertheless seem plausible from a toxicological perspective.

\section{Late, new-onset thrombocytopenia following Fab- treated rattlesnake envenomation without early thrombocytopenia or post-antivenom platelet increase}

\author{
Steven A Seifert and Damon N Cano
}

New Mexico Poison and Drug Information Center, University of New Mexico Health Sciences Center, Albuquerque, United States

Objective: To report a case of unpredicted, late thrombocytopenia following a CroFab ${ }^{\mathrm{TM}}$ (FabAV)-treated rattlesnake envenomation.

Case report: A 19 year-old male was bitten by a rattlesnake of unknown species on the dorsum of the hand. On presentation, there was a single puncture between the second and third metacarpals with swelling above the wrist and severe pain. He was treated with morphine and an initial dose of six vials of FabAV antivenom approximately one hour after envenomation with local control. Initial laboratory analyses showed normal hematological parameters. He received the standard maintenance doses of FabAV. Subsequent laboratory testing showed a positive D-dimer and a mild hypofibrinogenemia during hospitalization. The platelet count increased by $4 \%$ post-FabAV treatment and remained normal throughout hospitalization. Post-discharge monitoring showed a normal platelet count on Day 4 and a severe thrombocytopenia on Day 9, which resolved spontaneously over three days without treatment or bleeding (Table 1).

Conclusion: There was no indication of risk for late, new-onset thrombocytopenia, as there was no thrombocytopenia during hospitalization nor a $>20 \%$ increase in the platelet count following FabAV, a highly sensitive indicator [1]. His late, new-onset thrombocytopenia may have been caused by recurrent direct venom effect or increased clearance of previously venom-affected platelets, or this may have been unrelated to a venom effect, such as platelet sequestration in an area of tissue damage. Regardless, the patient developed severe late, new-onset thrombocytopenia that was unpredicted by current assessment methods. All FabAV treated rattlesnake envenomed patients should be followed post-discharge for recurrent and late, newonset hematologic abnormalities, which may not manifest during hospitalization or within 4 days post-treatment.

\section{Reference}

[1] Seifert SA, Kirschner RI, Martin N. Recurrent, persistent, or late, new-onset hematologic abnormalities in Crotaline snakebite. Clin Toxicol (Phila). 2011;49:324-329.

Table 1. Selected hematological laboratory tests in a patient with rattlesnake envenomation.

\begin{tabular}{|c|c|c|c|c|c|c|c|c|c|}
\hline Hematological parameters & Pre-FabAV & Post-FabAV & Increase in platelets $\%$ & At discharge & Day 4 & Day 9 & Day 10 & Day 11 & Day 12 \\
\hline Platelets & 239 & 249 & 4 & 219 & 217 & 50 & 55 & 64 & 131 \\
\hline D-dimer & - & $>7,650$ & - & - & - & - & - & - & - \\
\hline
\end{tabular}




\section{Beware of inhalatory methanol poisoning in illegal drug production}

\author{
Anja PG Wijnands-Kleukers ${ }^{a}$, Maaike A Sikma ${ }^{b}$, \\ Wouter Dijkman ${ }^{c}$, Jeroen Brogtrop ${ }^{d}$, \\ Marlijn JA Kamps ${ }^{\mathrm{e}}$ and Dylan W De Lange ${ }^{\mathrm{a}}$
}

${ }^{a}$ Dutch Poisons Information Center, University Medical Center Utrecht, University Utrecht, Utrecht, Netherlands; ${ }^{\mathrm{b}}$ Intensive Care Center, University Medical Center Utrecht, University Utrecht, Netherlands; ' Intensive Care Center, Máxima Medical Center, Veldhoven, Netherlands; ${ }^{\mathrm{d}}$ Department of Clinical Pharmacy, Máxima Medical Center, Veldhoven, Netherlands; ${ }^{\mathrm{e}}$ Intensive Care, Catharina Hospital, Eindhoven, Netherlands

Table 1. Laboratory results and treatment in patients with inhalational methanol toxicosis from working in an illegal drug laboratory.

\begin{tabular}{|c|c|c|c|}
\hline Patient characteristics & Patient 1 & Patient 2 & Patient 3 \\
\hline Age (years) & 28 & 26 & 20 \\
\hline Height $(\mathrm{cm})$ & 185 & 183 & - \\
\hline \multicolumn{4}{|l|}{ Heart rate $(/ \mathrm{min})$} \\
\hline Baseline (on admission) & 129 & 113 & 105 \\
\hline Range (during admission) & $103-143$ & $72-113$ & $80-130$ \\
\hline Range & $105 / 45-166 / 78$ & $100 / 56-165 / 89$ & $100 / 55-150 / 80$ \\
\hline \multicolumn{4}{|l|}{ Glasgow Coma Score } \\
\hline Baseline & 3 & 14 & 15 \\
\hline Range & $3-3$ & $13-15$ & $13-15$ \\
\hline \multicolumn{4}{|l|}{ Laboratory results } \\
\hline \multicolumn{4}{|l|}{$\mathrm{pH}$} \\
\hline Baseline & 6.3 & 7.1 & 7.2 \\
\hline \multicolumn{4}{|l|}{$\mathrm{PaCO}_{2}(\mathrm{mmHg})$} \\
\hline Baseline & 121 & 126 & 146 \\
\hline Range & $58-168$ & $76-159$ & $87-146$ \\
\hline \multicolumn{4}{|l|}{ Bicarbonate $(\mathrm{mmol} / \mathrm{L})$} \\
\hline Baseline & 4 & 4 & 5 \\
\hline Range & $4-24$ & $4-30$ & $5-26$ \\
\hline \multicolumn{4}{|l|}{ Oxygen saturation (\%) } \\
\hline Baseline & 88 & 98 & 99 \\
\hline Range & $40-100$ & $97-99$ & $96-99$ \\
\hline \multicolumn{4}{|l|}{ Osmolality (mOsm/kg) } \\
\hline Baseline & 424 & 339 & 326 \\
\hline \multicolumn{4}{|l|}{ Osmolar gap (mOsm/kg) } \\
\hline Baseline & 113 & 54 & 41 \\
\hline Range & - & $29-60$ & - \\
\hline \multicolumn{4}{|l|}{ Lactate $(\mathrm{mmol} / \mathrm{L})$} \\
\hline \multicolumn{4}{|c|}{ Aspartate aminotransferase $(\mathrm{U} / \mathrm{L})$} \\
\hline Baseline & 27 & 36 & 51 \\
\hline Range & $27-1392$ & $21-36$ & $46-65$ \\
\hline \multicolumn{4}{|l|}{ Toxicological screening } \\
\hline \multicolumn{4}{|l|}{ Serum methanol (per mille) } \\
\hline Baseline & 1.6 & 1.1 & 0.9 \\
\hline Range (min-max) & $0.1-1.6$ & $0.07-1.1$ & $0.1-0.9$ \\
\hline Blood ethanol (per mille) & & & \\
\hline Baseline & $<0.10$ & $<0.10$ & $<0.10$ \\
\hline Range (min-max) & $<0.10-1.60$ & $<0.10-2.26$ & $<0.10-1.58$ \\
\hline Therapies & & & \\
\hline $\begin{array}{l}\text { Continuous veno-venous } \\
\text { haemofiltration }\end{array}$ & During 48 hours & - & During 16 hours \\
\hline Intermittent haemodialysis & One 4 hour session & Two 4 hour sessions & One 4 hour session \\
\hline Ethanol & $\begin{array}{l}\text { Loading dose ( } 13 \text { hours after hospital- } \\
\text { ization), followed by a maintenance } \\
\text { dose for } 3 \text { days }\end{array}$ & $\begin{array}{l}\text { Loading dose ( } 2 \text { hours after hospitaliza- } \\
\text { tion), followed by a maintenance dose } \\
\text { for } 2 \text { days }\end{array}$ & $\begin{array}{l}\text { Loading dose ( } 1 \text { hour after hospitaliza- } \\
\text { tion), followed by a maintenance dose } \\
\text { for } 3 \text { days }\end{array}$ \\
\hline Folinic acid & $80 \mathrm{mg}$ every 6 hours & $\begin{array}{c}110 \mathrm{mg} \text { every } 6 \text { hours, } 100 \mathrm{mg} \text { every } 6 \\
\text { hours }\end{array}$ & $100 \mathrm{mg}$ every 4 hours \\
\hline Outcome & Died after 4 days & Survived & Survived \\
\hline
\end{tabular}


Objective: In the past methanol poisoning was predominantly reported after drinking illicit alcohol as a cheap surrogate for ethanol. Nowadays, methanol is used as a solvent in illegal drug production, therefore unexpected poisoning after inhalation may occur in people working in drug laboratories. Within $0.5-4$ hours after exposure, patients may develop symptoms such as ataxia, depressed consciousness, and disinhibition but, these initial effects may be absent, and victims fail to seek medical assistance. This is because methanol is metabolized to formic acid through alcohol dehydrogenase. Later patients may complain of gastrointestinal effects and headache. In more severe poisoning, metabolic acidosis and visual disturbances occur. Emergency treatment includes administration of an alcohol dehydrogenase inhibitor (fomepizole or ethanol) accompanied by enhancement of excretion by haemodialysis and administration of folate as a cofactor for formic acid metabolism. We present three patients with severe poisoning probably caused by methanol inhalation during work in an illicit drug laboratory.

Case reports: A 28-year-old male (patient 1) arrived at hospital unresponsive (Glasgow Coma Score (GCS) 3, fixed dilated pupils), with severe respiratory insufficiency and lactic acidosis. Two other patients arrived approximately 8 hours later. They were both feeling ill and were communicative (GCS 15). Medical history of all patients was unremarkable. Patient 2 admitted working in an ecstasy laboratory (for 40 hours) more than 24 hours earlier. Suspicion of methanol exposure arose and treatment was started (Table 1). Patient 1 died after 4 days due to severe neurological damage; the other patients recovered completely.

Conclusion: Severe methanol poisoning may occur from inhalation and requires prompt diagnosis and treatment to prevent severe morbidity and mortality. Symptoms of methanol intoxication may be delayed for one to four days after exposure, and there may be delay in seeking help and patients may refuse to cooperate because of working in an illegal drug laboratory.

\section{Vaginal button battery insertion in an adult patient}

\section{Alexander C Capleton ${ }^{a}$, Rita Arya ${ }^{b}$, Charlotte Palmer ${ }^{b}$, Alison Thomas ${ }^{\mathrm{a}}$ and John $\mathrm{P}$ Thompson ${ }^{\mathrm{a}}$ \\ ${ }^{a}$ National Poisons Information Service (Cardiff Unit), University Hospital Llandough, Cardiff, United Kingdom; ${ }^{b}$ Warrington and Halton Hospitals NHS Foundation Trust, Warrington, United Kingdom}

Objective: Button batteries are well recognised to pose a risk of serious injury following ingestion, particularly in children. However, exposure of other areas of the body to button batteries is rare. We describe an unusual presentation of button battery injury in an adult patient

Case report: A 28-year-old female presented to the Emergency Department reporting self-insertion of some batteries and pieces of a ceramic mug into her vagina. The patient requested removal of the foreign bodies as they were now causing discomfort. The presence of the batteries was confirmed by X-ray of the pelvis. An attempt was made to remove the foreign bodies, but the patient was unable to tolerate the examination. She was therefore examined under anaesthesia and a lithium button battery, two AAA batteries, and pieces of a ceramic mug were removed. The button battery had caused burns to the cervix and vagina, but the integrity of the bladder and rectum were preserved. The area was desloughed and vaginal irrigation performed. Advice was sought from the National Poisons Information Service which recommended performing arterial blood gases (ABGs), including lactate, and observation of the patient to ensure that the integrity of the tissues had not been damaged and that the patient was passing urine and opening her bowels normally. The patient was acidotic while in theatre $\left(\mathrm{pH} 7.3, \mathrm{pCO}_{2} 6.6 \mathrm{KPa}\right.$, bicarbonate $22 \mathrm{mmol} / \mathrm{L}$, lactate $1.4 \mathrm{mmol} / \mathrm{L}$ ), but repeat $A B G s$ and lactate were normal. A urinary catheter was inserted post-operatively overnight, and urine which drained was clear. The patient's observations remained stable; the following day the urinary catheter was removed and she was mobilised. The initial follow-up plan was to perform magnetic resonance imaging (MRI) to assess for fistulas. However, the patient disclosed that she had metallic foreign bodies in situ in her leg, thus precluding MRI. No further imaging or investigations were undertaken. Upon discharge the patient was advised of signs and symptoms to look out for that could indicate fistula formation.

Conclusion: We conclude that examination under anaesthetic may be necessary to visualise the areas of injury and facilitate removal of button batteries in patients presenting with a history of button battery insertion into the vagina. Expeditious identification and removal of button batteries in the vagina are essential to prevent further injury and reduce the risk of long-term complications. Patients should be followed-up at regular intervals to check for the development of long-term sequelae.

\section{Seasonal and temperature effect on plasma lithium concentration}

\author{
Sonia Cheng ${ }^{a}$, William Siu ${ }^{b}$, Angela L Chiew ${ }^{c}$, \\ Elia Vecellio ${ }^{d}$, Nicholas A Buckley and Betty S Chan ${ }^{\mathrm{c}}$ \\ ${ }^{\mathrm{a}}$ Faculty of Medicine, University of New South Wales, Sydney, \\ Australia; ${ }^{b}$ Department of Emergency Medicine, Sutherland \\ Hospital, Sydney, Australia; 'Department of Emergency Medicine \& \\ Clinical Toxicology, Prince of Wales Hospital, Sydney, Australia; \\ ${ }^{\mathrm{d}}$ NSW Health Pathology, Prince of Wales Hospital, Sydney,

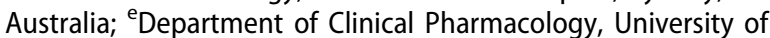 \\ Sydney, Sydney, Australia
}

Objective: We aimed to investigate the seasonal and temperature effect on plasma lithium concentrations in Australia and compare these results to those published in the Northern Hemisphere [1].

Methods: We conducted a retrospective longitudinal analysis of 27 patients from 2 hospitals with at least one plasma lithium concentration analysed per season for one year. Lithium dose, patient demographics, medications, comorbidities, symptoms of lithium toxicity and outcomes were also recorded. We also conducted a secondary study using available records of all plasma lithium concentrations recorded from the two hospitals. These concentrations were analysed to investigate correlations with the month, season and temperature at the time of collection. Temperature data, sourced from the Bureau of Meteorology, was recorded as: (1) the maximum daily temperature prior to collection and (2) the average daily maximum temperature in the 5 days prior to collection. ANOVA and Pearson correlation were used for statistical analysis.

Results: From 2008-2018, 13 males and 14 females were included in the study. They had a mean age of 44 years (SD 13.5). Patients were on average taking $1036 \mathrm{mg}$ lithium per day (SD 440, range 250-2000 mg). Lithium dose across seasons did not vary significantly $\left(R^{2}=0.05, P=0.25\right)$. The average plasma lithium concentration was $0.8 \mathrm{mmol} / \mathrm{L}$ (SD 0.46, range $0.3-3.6 \mathrm{mmol} / \mathrm{L}$ ) and also did not vary significantly across seasons amongst patients $\left(R^{2}=\right.$ $0.09, \mathrm{P}=0.06$ ). In our secondary study, there were 11977 plasma lithium concentrations that corresponded with 2887 patients. It showed a weak downhill correlation between plasma lithium concentrations and temperature $(r=-0.02, P=0.023)$. One-way ANOVA showed no variation in plasma lithium concentration across seasons. The mean plasma lithium concentration in June $(0.8 \pm 0.4 \mathrm{mmol} / \mathrm{L})$ was higher than the December mean $(0.7 \pm 0.5$ $\mathrm{mmol} / \mathrm{L}, \mathrm{P}<0.001)$. 
Conclusion: We were unable to prove any statistically significant variance in plasma lithium concentrations across seasons or months. Whilst we did find a weak correlation between plasma lithium concentration and temperature, the relationship was contrary to that established in the Northern Hemisphere, however, this is unlikely to bear any clinical significance.

\section{Reference}

[1] Medhi B, Pradhan B, Chakrabarty S, et al. Seasonal variation in plasma levels of lithium in the Indian population: is there a need to modify the dose? Singapore Med J. 2008;49:724-727.

\section{Medical toxicology as a distinct medical specialty: the US experience}

\section{Paul Wax}

UT Southwestern, Dallas, United States

Objective: Poisonings have caused havoc, life-threatening injury, and death since time immemorial. Physicians trained to provide consultation, treatment, and pursue scientific inquiry regarding poisoning challenges have traditionally trained in a variety of medical specialties such as anesthesiology, critical care (intensive care) medicine, internal medicine, pediatrics, occupational medicine, and emergency medicine. Additional subspecialty training in medical toxicology (MT) has been available in the US since the 1980 s but only received formal accreditation in 2000. A uniform acceptance of medical toxicology as a stand-alone sub-specialty with its own training program and certification examinations has not been universally adopted outside the US. Many barriers have prevented such an adoption. We report on the growth of medical toxicology as a distinct specialty in the US over the last 30 years. Methods: We reviewed the growth of medical toxicology in the US utilizing several databases including the American Board of Medical Specialties (ABMS) database of Medical Toxicology diplomates, the Accreditation Council for Graduate Medical Education (ACGME) database of MT training programs, and internal data collected by the American College of Medical Toxicology.

Results: The American Board of Medical Toxicology (ABMT), a stand-alone board associated with the American Academy of Clinical Toxicology (AACT), certified the first Medical Toxicologists (predominantly US based) from 1975-1992. Pathways to certification during this time included a 2-year fellowship training (without formal national accreditation of these programs), or a practice/mentorship pathway. Since 1994 the ABMS has overseen Board Certification in MT, and since 2000 the ACGME has overseen fellowship training accreditation. Since 2000 successful completion of a 2-year fellowship in an ACGME-approved program was required in order to quality to sit for the MT board certification exam. From 1994-2016, 541 physicians have become board certified in MT in the US. At the present time, 28 2-year MT fellowships are ACGME approved. There are 75 physicians currently training in US ACGME approved MT training programs.

Conclusion: Since 1994 more than 500 US physicians have been become ABMS board certified medical toxicologists. These physicians now have similar subspecialty credentials to other subspecialists such rheumatologists, infectious disease specialists, and pediatric cardiologists. The impact of MT as a distinct specialty is unknown. Attempts at developing performance metrics for MT are ongoing.

\section{Methanol inhalation poisoning: a life-threatening condition}

\author{
Hoiip Leong ${ }^{a}$, Puii leong ${ }^{a}$, lekhou $\mathrm{Chu}^{\mathrm{b}}$ and \\ Chumang $\mathrm{Ho}^{\mathrm{b}}$ \\ ${ }^{a}$ Emergency Department, Centro Hospitalar Conde de São \\ Januário, Macao, China; ${ }^{b}$ Centro Hospitalar Conde de São Januário, \\ Macao, China
}

Objective: To highlight that methanol inhalation is a life-threatening condition and the blood osmolality gap is very important in severe metabolic acidosis.

Case report: A previously healthy 17-year-old girl presented with general weakness and refused to talk after she had been missing for a day. The family suspected sexual assault. During observation, she progressively worsened with tachycardia, tachypnea and poor response. Arterial blood gas analysis showed $\mathrm{pH} 7.15$ and high anion gap metabolic acidosis. Her white blood cell count, hemoglobin, glucose, renal function and liver enzymes were normal. An electrocardiogram (ECG) showed tachycardia with normal QRS and QTc. The cause of clinical signs was unknown. Brain computerised tomography (CT) scan, septic workup, drug screen (including paracetamol, tricyclic antidepressants and ethanol measurement) and blood osmolality measurement were undertaken for differential diagnoses. She was intubated and fluid resuscitation, antibiotics, and bicarbonate were given, but she failed to improve and had two generalized convulsions. The ethanol concentration was normal but blood osmolality was 386 $\mathrm{mOsm} / \mathrm{kg} \mathrm{H}_{2} \mathrm{O}$ and osmolality gap was 82 . Toxic alcohol poisoning was suspected. Ethanol via nasal gastric tube infusion and urgent hemodialysis were started. She regained consciousness after 24 hours of hospitalization and was then extubation. The repeat blood osmolality showed $288 \mathrm{mOsm} / \mathrm{kg} \mathrm{H} \mathrm{H}_{2} \mathrm{O}$. The methanol concentration more than 34 hours after exposure was $105 \mathrm{mg} /$ $\mathrm{dL}$. She reported that she had inhaled $4.5 \mathrm{~L}$ of burning alcohol using an ultrasonic nebulizer with suicidal intent more than 30 hours prior to admission and that the burning alcohol contained ethanol adulterated with methanol. Ophthalmologic examination was normal. Nevertheless, the patient had a mild Parkinson-like syndrome and magnetic resonance imaging (MRI) showed bilateral putamen and caudate nucleus necrosis. She was discharged at day 20 and followed up at neurology.

Conclusion: The blood osmolality gap should be calculated in patients with metabolic acidosis of unknown cause or severe metabolic acidosis. Methanol inhalation can lead to life-threatening poisoning. Hemodialysis and fomepizole (or ethanol) are the corner stone of management.

\section{False positive acetaminophen concentration due to parenteral acetaminophen administration in a dual port intravenous line}

\author{
Anthony Scoccimarro, Ryan Marino and \\ Anthony Pizon \\ University of Pittsburgh School of Medicine, Pittsburgh, United \\ States
}

Objective: We present a case of a falsely elevated acetaminophen concentration due to residual acetaminophen in an intravenous catheter following iatrogenic administration.

Case report: A 53-year-old woman presented from an outside facility for management of a transverse process fracture of the seventh cervical spine and bilateral mandibular fracture. The 
patient admitted to using crack cocaine the night before presentation and had choreoathetosis. Toxicology screening found a serum acetaminophen concentration of $>800 \mu \mathrm{g} / \mathrm{mL}$. She was started on an intravenous (IV) $\mathrm{N}$-acetylcysteine 21-hour protocol and the Medical Toxicology service was consulted. She adamantly denied acetaminophen use and did not have any gastrointestinal symptoms. The remainder of the laboratory findings included anion gap 11, aspartate transaminase $40 \mathrm{U} / \mathrm{L}$, alanine transaminase $25 \mathrm{U} / \mathrm{L}$, and prothrombin time $12.9 \mathrm{~s}$. The case was discussed with the laboratory who stated a precise measurement of the acetaminophen concentration was not possible despite 4 serial dilutions, the maximal allowable for an accurate measurement. A repeat sample was drawn from a separate peripheral venous site, and returned with acetaminophen undetectable. $\mathrm{N}$-acetylcysteine was discontinued. She had preserved mental status and no signs or symptoms of metabolic acidosis, expected after a massive acetaminophen overdose, prompting further investigation. After discussion with nursing staff, it was discovered that the initial blood had been drawn from an existing peripheral line that had been placed at the first facility. The peripheral IV was a single lumen dual-port access and only a single port was flushed prior to obtaining the blood sample. The patient had received $1 \mathrm{~g} \mathrm{IV}$ acetaminophen 3.5 hours before arrival. Although the port that was accessed was flushed prior to blood draw, there was a second port connected to the same lumen. The typical concentration of IV acetaminophen is $1 \mathrm{~g} / 100 \mathrm{~mL}$ (i.e. $100 \mathrm{mg} / \mathrm{mL}$ ), which would explain why the concentration remained greater than a discernible level despite serial dilutions. This false positive acetaminophen prompting unnecessary administration of $\mathrm{N}$-acetylcysteine may have been avoided, e.g. reporting of medications administered at the transferring facility, adequate flushing of all access ports prior to blood draw, and drawing samples from a separate venous site.

Conclusion: This case illustrates the necessity of interpreting laboratory data in concert with a clinical assessment. Clinicians should be aware that iatrogenically-administered xenobiotics may remain in intravenous catheter ports, despite flushing the accessed port. Xenobiotics from other ports may be introduced into samples drawn from separate ports of the same intravenous catheter.

\section{6. "Rhino 7"-induced priapism leading to penile fibrosis complicated by delirium tremens}

\author{
Anthony Scoccimarro, Ryan Marino and \\ Joshua Shulman \\ University of Pittsburgh School of Medicine, Pittsburgh, United \\ States
}

Objective: "Rhino 7" is an over-the-counter (OTC) supplement marketed for male sexual enhancement, which contains several herbal ingredients as well as an unknown amount of either sildenafil or tadalafil depending on the formulation.

Case report: A 51-year-old man with alcohol use disorder presented to a community hospital with 3-4 days of priapism after using the OTC supplement, "Rhino 7." Ischemic priapism was confirmed by penile arterial blood gas analysis. Two attempts were made at bedside detumescence without avail and the patient was transferred to a tertiary care center for further management. Upon arrival, urology was consulted who after two additional attempts at detumescence deemed further efforts to be futile. During evaluation, the patient was also found to be increasingly tachycardic, hypertensive, and tremulous, at which time he stated that his last drink was 2 days prior to arrival, although his serum ethanol concentration at this point was $89 \mathrm{mg} / \mathrm{dL}$. He was treated initially with escalating doses of diazepam and phenobarbital but was transferred to the intensive care unit due to worsening delirium. He required intubation and treatment with propofol, ketamine, and dexmedetomidine infusions, and was ultimately extubated on day 8 . His clinical course was complicated by fever secondary to necrotic penile tissue. His fever improved with the administration of antibiotics, although his penile tissue eventually fibrosed. He was discharged after a 15-day stay with urological follow-up with plans for a penile prosthesis.

Conclusion: Rhino 7 is purported to have several "natural" ingredients including extracts from several plant and fungi genera including Atractylodes, Codonopsis, Cinnamomum, Cornus, Cordyceps and Panax. As per several reports from the Food and Drug Administration (FDA), phosphodiesterase (PDE)-type 5 inhibitors, including sildenafil and tadalafil, have also been identified in this product. PDE5 inhibitors prevent the degradation of cyclic guanosine monophosphate (cGMP), which induces smooth muscle relaxation via multiple mechanisms including activation of a cGMP-dependent protein kinase that activates myosin light chain phosphatase leading to dephosphorylation of myosin light chains. Clinically, PDE5 inhibitors are used in the treatment of erectile dysfunction and pulmonary hypertension. This is the first known case of this product causing prolonged priapism leading to penile fibrosis, attributable to the presence of PDE5 inhibitors in this supplement.

\section{Therapeutic errors with hearing aid battery ingestion}

\author{
Anna Celentano ${ }^{\mathrm{a}}$, Marcello Ferruzzi ${ }^{\mathrm{b}}$, \\ Angelo Travaglia ${ }^{b}$, Andrea F Stella $^{b}$, Fabrizio Sesana ${ }^{b}$, \\ Giovanni Milanesi ${ }^{b}$ and Franca Davanzo ${ }^{b}$ \\ ${ }^{a}$ Milan Poison Control Center, Great Metropolitan Niguarda

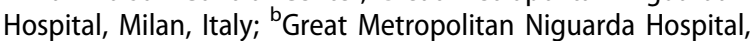 \\ Milan, Italy
}

Objective: Microbatteries (MBs) are used to power toys or other devices like hearing aids. The ingestion of these batteries can cause damage primarily to the esophagus but also to the stomach due to the electric effect alone, regardless of the release of toxic substances following the opening of battery itself. The consequences can be severe, including esophageal perforation. The aim of this study is to underline the risk in the elderly who make take $\mathrm{MB}$ in mistake for medicines because they have similar dimensions.

Methods: From January 2015 to December 2017 cases of ingestion of MB by elderly patients were investigated. Follow-up was performed confirming the ingestion and, when possible, the type of MBs involved.

Results: During the study period, a total of 21 cases of ingestion in patients $>65$ years were identified. Of these, $19.0 \%(n=4)$ had accidentally swallowed a MB and $23.1 \%(n=5)$ had swallowed them because of senile dementia. A total of 12 cases of therapeutic errors with hearing aid MB were identified (57.1\%) involving 9 females and 3 males. In one case the ingestion involved 3 $M B$, while in 11 cases it involved a single MB. Follow-up was performed in 8 cases. All of the mistakes occurred at home and all patients were hospitalized. The MBs had passed the ileocecal valve, were evacuated spontaneously and no patients presented any symptoms. It is to be noted that, in all cases, alkaline MBs were involved. The therapeutic error likely occurs because medicines are kept ready to use, near the hearing aid battery to be replaced.

Conclusion: Hospitalization is essential after ingestion of a MB even if patients do not show any symptoms. No severe cases were reported. It is imperative to recover all available information 
in order to identify the type of battery that was ingested. Therefore, it is necessary to bring the original retail packaging of the battery itself or another MB still present in the device or on the nightstand to the local emergency room. It is important that the indication warns about the risk of therapeutic errors due to ingestion and that the package itself be kept away the medicine boxes. Some elderly people may already have difficulty swallowing, so there is an increased risk of adhesion to the esophageal wall after ingestion of a MB.

\section{Acute poisoning and use of antidotes during pregnancy: the experience of the Florence Teratology Information Service and Poison Control Centre}

\author{
Sara Traversoni ${ }^{a}$, Maya Salimova ${ }^{a}$, Alessandra leri ${ }^{b}$, \\ Francesco Gambassi, Andrea Missanellib, \\ Brunella Occupati ${ }^{b}$, Cecilia Lanzi ${ }^{b}$, Cecilia Baccaro ${ }^{b}$, \\ Emanuela Masini ${ }^{\mathrm{a}}$, Guido Mannaioni ${ }^{\mathrm{a}}$ and \\ Alessandra Pistellib \\ ${ }^{a}$ Department of Neurofarba, Università degli Studi di Firenze, \\ Florence, Italy; ${ }^{\mathrm{b}}$ Toxicology Unit and Centro di Riferimento \\ Regionale Tossicologia Perinatale, Azienda Ospedaliero- \\ Universitaria Careggi, Florence, Italy
}

Objective: To evaluate acute intentional and accidental poisoning during pregnancy.

Methods: We conducted a retrospective analysis of the data obtained from the clinical records of pregnant women admitted to the Toxicology Unit of Florence University Hospital following acute intoxication between 1995 and 2017.

Results: Forty-nine women with acute intentional or accidental poisoning during pregnancy were admitted to the Toxicology Unit. Seventeen patients were accidentally exposed to carbon monoxide, 11 had high ethanol consumption, 4 had ingested mushrooms, and 17 women attempted suicide with drug overdose or bleach ingestion. The pharmacological agents employed for attempted suicide were psychoactive drugs, analgesics and hormones. One case of delayed onset of signs and three cases of rapid onset of signs following mushrooms ingestion were reported. Among drug overdose, only one patient was unconscious when admitted to the hospital following benzodiazepine overuse and received flumazenil with prompt recovery. Antidotal therapy with hyperbaric oxygen was administered to all CO intoxicated women. Obstetric ultrasound was performed in 33 of the 49 admitted women. Out of these 33, poor viability of the fetus was reported in only one case. This pregnancy ended in a miscarriage caused by carbon monoxide intoxication at week 8 , the mother showing a high carboxyhaemoglobin concentration (22\%) on emergency room admission. Among the 49 patients 3 more miscarriages were reported following opioid, ethanol and drug overdose. Twenty four healthy babies (including one twin birth) were delivered; there were three elective terminations of pregnancy (ETOP), and two therapeutic terminations in psychiatric patients were reported. No congenital anomalies were detected in the newborns. Seventeen women could not be contacted for follow-up.

Conclusion: Acute intoxication during pregnancy is a rare event in an emergency department and requires a rapid maternal and fetal assessment and medical management. The treatment of a pregnant intoxicated patient must be tailored to each single case taking into account maternal toxicity, xenobiotic involved and stage of pregnancy [1]. Our experience with antidotes, although limited, is favourable to their application during pregnancy in accordance with data about the use of most common antidotes. Acute intoxication in pregnancy may be a challenging situation because of the limited medical knowledge on this specific topic, which requires a specialist advice from a reproductive and perinatal toxicologist.

\section{Reference}

[1] Fine JS. Reproductive and perinatal principles In: Goldfrank's Toxicologic Emergencies, 10th ed. McGraw-Hill Professional, 2015:423-446.

\section{The utility of non-contrast head computed tomography in poisoned patients}

\author{
Richard McNulty ${ }^{\mathrm{a}}$, Alex Garner ${ }^{\mathrm{b}}$, Timothy Wong ${ }^{\mathrm{c}}$, \\ Lashnika Bandaranayake ${ }^{c}$, Farhan Tahmid ${ }^{c}$, \\ Emily Symes ${ }^{\mathrm{d}}$, Mohammad Mohammad ${ }^{\mathrm{b}}$, Mark Salter ${ }^{\mathrm{d}}$ \\ and Naren Gunja ${ }^{e}$ \\ ${ }^{a}$ Emergency Medicine Blacktown Mount Druitt Hospital; \\ Department of Clinical Pharmacology \& Toxicology, Western \\ Sydney Health, School of Medicine University of Western Sydney, \\ Sydney, Australia; ${ }^{b}$ Emergency Medicine Blacktown Mount Druitt \\ Hospital, Sydney, Australia; 'School of Medicine, University of \\ Western Sydney, Sydney, Australia; ${ }^{\mathrm{d} D e p a r t m e n t}$ of Clinical \\ Pharmacology \& Toxicology, Western Sydney Health, Sydney, \\ Australia; ${ }^{e}$ Department of Clinical Pharmacology \& Toxicology, \\ Western Sydney Health, Discipline of Emergency Medicine, Sydney \\ Medical School, Sydney, Australia
}

Objective: To investigate the utility of non-contrast head computed tomography (CT) in poisoned patients. Previous studies emphasised head CT as a diagnostic aid, but it is unclear whether it improves any patient-centred outcomes [1].

Methods: A retrospective cohort study of all patients presenting with intoxication at a tertiary hospital in Western Sydney between August 2010 and December 2017. Following human research ethics approval, all cases (including consultations) referred to the Western Sydney Toxicology Service were extracted from the service database. Blinded data collectors completed a study spreadsheet with demographics, characteristics of the poisoning, details of $\mathrm{CT}$ report findings and their consequences for the admission.

Results: Of 3014 patients during the 7 year study period, 523 had a head CT. Mean age was 43 (range 16-88 years). There were 213 recreational and 243 deliberate self-poisonings. Median Glasgow Coma Score (GCS) was 11, and 164 patients were intubated. Seizure occurred in 48 patients; 62 patients had signs of head injury and 28 patients had focal neurological signs. CT reports showed only chronic changes in 88 cases. There were 3 incidental findings requiring follow-up ( 1 meningioma, 1 air bubble at skull base which resolved and 1 chronic hypodensity). Seven patients had facial bone fractures, all of whom had clinical signs of injury on examination. Acute findings included 5 cases of oedema, 2 cases of hypoxic-ischaemic changes and 3 cases of intracranial haemorrhage ( 1 intra-parenchymal haemorrhage in a patient vomiting after corrosive ingestion, and 2 cases of subarachnoid haemorrhage: one in a phenelzine overdose with persistent headache, and another in methamphetamine overdose with agitation). CT head did not lead to a change in management or an interventional procedure in any patient. Fourteen adverse events occurred during head CT acquisition: 13 patients required sedation for agitation and 1 prisoner escaped. 
Conclusion: Head CT was a low yield investigation which did not change patient treatment or outcomes in this cohort. While it did add diagnostic information in a number of cases, this was at the cost of considerable resource consumption and radiation risk. We suggest no emergent head CT is needed in non-intubated poisoned patients whose presentation is consistent with the stated ingestion, and there are no signs of injury or deficits.

\section{Reference}

[1] Behnoush B, Marvash G, Fakhredin T, et al. Brain CT scan findings in patients admitted to the poisoning ICU of Baharloo Hospital, Tehran, Iran. Int J Med Toxicol Forensic Med. 2017;7:215-220.

\section{Management of patients with acute drug overdose in an emergency department observation unit}

\author{
Siri Shastry ${ }^{a}$, Lynne D Richardson ${ }^{b}$, Jonathan $Y^{c}{ }^{c}$ \\ and Alex F Manini ${ }^{\mathrm{d}}$ \\ aDepartment of Emergency Medicine, Icahn School of Medicine at \\ Mount Sinai, New York, United States; ${ }^{b}$ Department of Emergency \\ Medicine and Department of Population Health Science and \\ Policy, Icahn School of Medicine at Mount Sinai, New York, United \\ States; 'Division of Hospital Medicine, Department of Emergency \\ Medicine, Icahn School of Medicine at Mount Sinai, New York, \\ United States; ${ }^{\text {DDepartment }}$ of Emergency Medicine, Icahn School \\ of Medicine at Mount Sinai and Division of Medical Toxicology, \\ Icahn School of Medicine at Mount Sinai, Elmhurst Hospital \\ Center, New York, United States
}

Objective: Observation units (OU) are being increasingly used within the Emergency Department (ED) to optimize quality of care and resource utilization. Making greater use of dedicated hospital OUs for many short-stay patients has been shown to be cost-effective [1]. Use of OUs in the management of ED patients presenting with poisoning has been described for acetaminophen [2]; however outcomes and bounce-backs are poorly described [3]. This study aims to examine demographics, disposition, and outcomes for this population managed in an OU.

Methods: A retrospective consecutive cohort study of ED patients presenting to a single site, academic, tertiary care center with overdose and managed in an OU from March 2015 to present. Patients over 18 years with a diagnosis of overdose were included. Patients with an alternate diagnosis, non-drug ingestion, inhaled/dermal substance use, chronic toxicity or do not resuscitate status were excluded. Hemodynamic instability, new electrocardiogram abnormalities, severe electrolyte imbalance, severe altered mental status, recurrent seizures, toxic drug concentration requiring ongoing treatment and high psychiatric risk excluded patients from management in the OU. Data on patient demographics, adverse events, survival, final disposition and return visits was obtained via chart review. The primary study outcome was occurrence of adverse events and in-hospital mortality. Secondary outcomes were demographics, disposition and return visits. Patients were primarily managed under an overdose protocol which delineated required testing, management and disposition criteria.

Results: Of 947 ED overdose patients, 25 (2.4\%) were managed in the OU. The majority of patients were discharged home, with only two (8\%) requiring admission. No patients required airway management and only two patients (8\%) experienced an adverse cardiovascular event. No patients died while in hospital. Three patients (12\%) returned to the hospital within 30 days.

Conclusion: While only a small proportion of ED overdose patients were managed within an OU, our data supports the safety of an OU protocol. Further studies are needed to better examine outcomes and generalizability.

\section{References}

[1] Baugh CW, Venkatesh AK, Hilton JA, et al. Making greater use of dedicated hospital observation units for many short-stay patients could save $\$ 3.1$ billion a year. Health Aff (Millwood). 2012;31:2314-2323.

[2] Beauchamp GA, Hart KW, Lindsell CJ, et al. Performance of a multidisciplinary emergency department observation protocol for acetaminophen overdose. J Med Toxicol. 2013;9:235-241.

[3] Judge BS, Ouellette LM, VandenBerg $M$, et al. Utilization of observation units for the care of poisoned patients: Trends from the Toxicology Investigators Consortium Case Registry. J Med Toxicol. 2016;12:111-120.

\section{Keep the buprenorphine in place! Treating severe pain in a hospitalized patient with opioid dependence}

\author{
Timothy J Wiegand \\ Emergency Medicine, University of Rochester Medical Center, \\ Rochester, United States
}

Objective: Buprenorphine is a partial opioid receptor agonist used to treat opioid dependence. It is available in various formulations including buprenorphine alone and co-formulated with naloxone in a 4:1 ratio for the treatment of opioid dependence. A growing number of patients are prescribed buprenorphine as the US is in the midst of an opioid crisis with increasing overdose rates and other complications associated with opioid use disorder. Despite buprenorphine being available both for pain and dependence clinicians are often unprepared to appropriately dose it or add the right agents to maximize pain relief when it is continued. Additionally, a variety of misunderstandings and confusion exist with regard to buprenorphine, its pharmacology and the treatment of pain. Due to the ceiling effect it is typically suggested that with severe pain buprenorphine be discontinued and full opioid receptor agonists substituted until the pain dissipates. We present a case and describe an approach to treating severe pain while leaving buprenorphine in place.

Case report: A 44-year-old male with intravenous drug use was hospitalized for lower extremity cellulitis, abscess and septic arthritis of the left knee. He had been using heroin and cocaine daily prior to admission. Shortly after admission he was started on buprenorphine $(2 / 0.5 \mathrm{mg}$ sublingual $\times 1)$ which was titrated to $8 /$ $2 \mathrm{mg}$ sublingual three times daily. For the incision and drainage procedure he had full opioid receptor agonists added along with adjunctive medications acetaminophen, a tricyclic antidepressant and toradol (NSAID). After the final debridement surgery he was left with a large and complex wound requiring daily dressing changes/cleaning which caused "severe pain." Ultimately, $6 \mathrm{mg}$ hydromorphone was administered intravenously immediately prior to changes with good analgesic effect without sedation or respiratory depression. Adjunctive agents were continued along with a sedative (oral lorazepam $0.5 \mathrm{mg}$ ) one hour earlier adjunctive to the hydromorphone for the initial changes. 
Conclusion: Common teaching is that with severe pain high doses of buprenorphine are not effective for analgesia and that the buprenorphine "blocks" other opioids in this regard. In our patient buprenorphine was split ( $8 / 2 \mathrm{mg}$ three times daily) and 6 mg IV hydromorphone administered prior to the painful procedure with excellent effect. This combination was effective without requiring discontinuation of the buprenorphine. Providers should maximize doses and appropriately split buprenorphine, use adjunctive agents and consider high doses of full agonists with buprenorphine in place as an option to treat severe pain instead of discontinuing buprenorphine.

\section{Enhanced monitoring of glyphosate exposures by TOXBASE ${ }^{\circledR}$ : the National Poisons Information Service (NPIS) pesticide surveillance project 2004-2018}

\author{
Richard D Adams ${ }^{\mathrm{a}}$, David Stewart ${ }^{\mathrm{a}}$, Michal Klatka ${ }^{\mathrm{a}}$, \\ Simon HL Thomas ${ }^{b}$, Sally M Bradberry, \\ John P Thompson ${ }^{\text {, }}$, Gillian Jackson ${ }^{\mathrm{a}}$ and \\ Euan A Sandilands ${ }^{a}$ \\ ${ }^{a}$ National Poisons Information Service (Edinburgh Unit), Royal

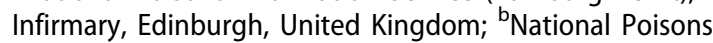 \\ Information Service (Newcastle Unit), Regional Drug and \\ Therapeutics Centre, Newcastle Hospitals NHS Foundation Trust, \\ Newcastle, United Kingdom; ${ }^{C}$ National Poisons Information Service \\ (Birmingham Unit), City Hospital, Birmingham, United Kingdom; \\ ${ }^{d}$ National Poisons Information Service (Cardiff Unit), University \\ Hospital Llandough, Cardiff, United Kingdom
}

Objective: Glyphosate is a broad spectrum herbicide widely used in agriculture, horticulture and domestic circumstances and the second most frequently reported ingredient to the UK National Poisons Information Service (NPIS) pesticide surveillance project. We analysed glyphosate exposures reported to the NPIS over a 14 year period.

Methods: The NPIS monitors pesticide exposures through analysis of TOXBASE accesses and national telephone enquiries and follow-up electronic and paper questionnaires. We retrospectively analysed data regarding glyphosate exposures between 1 April 2004 and 31 March 2018.

Results: During the study period, a total of 13,179 pesticide exposures were reported with 1,209 (9.2\%) of these involving glyphosate. The majority of cases involved accidental exposures $(1,062,87.7 \%)$, while $147(12.2 \%)$ were as a result of self-harm. Of the accidental exposures, 769 (72\%) involved adults, with 687 (64.7\%) being male. Routes of exposure reported included ingestion $(318,29.9 \%)$, skin contact $(203,19.1 \%)$, eye contact $(166$, $15.6 \%)$, inhalation $(148,13.9 \%)$ and unknown (16, 1.5\%); 211 (19.9\%) cases occurred by multiple routes of exposure. Most exposures caused only minor or no clinical features, with only 2 cases of severe toxicity (Poisoning Severity Score [PSS]) (0.2\%); moderate 45 (4.2\%); minor 531 (50\%); none 449 (42.3\%); uncertain 35 (3.3\%). Symptoms most frequently reported by single route exposure included: ingestion - vomiting $(n=34)$, abdominal pain $(n=24)$, oral irritation $(n=23)$, diarrhoea $(n=11)$, nausea $(n=10)$; skin - skin irritation $(n=67)$, burn $(n=13)$, paraesthesia $(n=10)$; inhalation - nausea $(n=31)$, oral irritation $(n=17)$, dizziness $(n=17)$, vomiting $(n=17)$, headache $(n=17)$, malaise $(n=$ 15), abdominal pain $(n=14)$, dyspnoea $(n=13)$; eye - irritation $(n=82)$, conjunctivitis $(n=27)$. In 777 cases $(73.2 \%)$, exposure occurred during non-occupational use, with only $97(9.1 \%)$ cases occurring during occupational use. In 188 (17.7\%) cases the circumstances surrounding the exposure were unknown. Intentional exposures to glyphosate $(147,12.2 \%)$ were more commonly associated with severe or fatal toxicity $(P<0.0001)$ (PSS fatal $5(3.4 \%)$; severe 11 (7.5\%); moderate 17 (11.6\%); minor 46 (31.3\%); none 55 (\%); uncertain 13 (8.8\%). The vast majority (138, 93.9\%) of these intentional exposures occurred by ingestion.

Conclusion: Overall most glyphosate exposures reported to the NPIS were accidental following domestic use. Reassuringly, the majority of these exposures were of low clinical severity with more severe cases associated with deliberate self-harm. It is important to remain vigilant as to the dangers of pesticide exposure particularly during domestic use where the majority of exposures occur.

\section{Derivation and validation of a system for predicting mortality in acute paraquat poisoning mortality: Acute Paraquat Poisoning Mortality (APPM) Score}

\author{
Chih Chuan Lin and Chun-Kuei Chen \\ Emergency Medicine Department, Chang Gung Memorial Hospital, \\ Taipei, Taiwan
}

Objective: To develop and validate a scoring system to predict mortality in patients with acute paraquat poisoning.

Methods: Two datasets from two different hospitals were retrospectively reviewed and used for derivation and validation, respectively. Independent predictors were used from one dataset for derivation to form the scoring system, Acute Paraquat Poisoning Mortality score (APPM). APPM was then validated by the validation dataset. Serum paraquat concentration was not used as part of the model as it is time-consuming to obtain results and is not always available in in areas where paraquat poisoning is endemic. Instead, it was used as a comparator for APPM to test prediction efficiency.

Results: Patient numbers were 156 and 165 in the derivation and validation datasets, respectively. Both groups were similar in demographic characteristics, laboratory variables, severity and mortality $(72.73 \%$ versus $80.7 \%, p=0.20)$. Variables associated with mortality were age, body temperature, Glasgow Coma Sale $(\mathrm{GCS})$, respiratory rate, white blood cell count (WBC), creatinine, $\mathrm{pH}$, bicarbonate-, potassium, blood glucose and urine paraquat concentration. Three independent variables correlated to 28-day mortality were urine paraquat concentration $>10 \mathrm{ppm}$ (navy blue color), WBC $>13000 / \mu \mathrm{L}$ and blood glucose $>140 \mathrm{mg} / \mathrm{dL}$. Each $\beta$ coefficient was divided by 2 and rounded to the nearest integer. Patients were stratified by APPM into 4 risk groups with increasing mortality rates (low 7.1\%; moderate 52.2\%; high $76.2 \%$ and very high 100\%; score point 0 to 4 for each risk group, respectively). The mortality rates in the validation set were as follows: low $15.4 \%$, moderate $47.4 \%$, high $95.5 \%$ and very high $100 \%$. Both had similar mortality prediction efficiency. The area under the receiver operating characteristic (ROC) curve for the APPM score/validation set was 0.91 and 0.94 . The Hosmer-Lemeshow goodness of fit statistic of both sets was 1 for the model (i.e. the model has good fit). APPM score successfully stratified patients in the validation set into groups similar with APPM. Comparable AUC under the ROC curve for the APPM was found as APPM 0.9, 
plasma paraquat 0.95 and Severity Index of Paraquat Poisoning (SIPP) 0.95 ( $p=0.20$, and 0.29, respectively).

Conclusion: APPM could be used as an efficient tool to predict the mortality in patients with acute paraquat poisoning.

\section{An unusual case of organophosphate poisoning}

\author{
Aoife Doolan ${ }^{a}$, Nicola Cassidy ${ }^{\mathrm{b}}$ and Edel Duggan ${ }^{\mathrm{b}}$ \\ ${ }^{a}$ Department of Anaesthesia, Beaumont Hospital, Dublin, Ireland; \\ ${ }^{\mathrm{b}}$ National Poisons Information Centre, Beaumont Hospital, Dublin, \\ Ireland
}

Objective: Diazinon (dimpylate) is an organophosphate insecticide. Poisoning involves excessive cholinergic stimulation. Although cardiac symptoms occur, they are usually accompanied by neurological and respiratory symptoms. We describe an unusual case of accidental diazinon poisoning with predominantly cardiac features.

Case report: A 42-year-old farmer was working with diazinon (10\%) on a very warm day when he accidently ingested a mouthful which had been decanted into a lemonade bottle. He presented to hospital with nausea, abdominal pain and miosis, but was haemodynamically stable with a heart rate $82 \mathrm{bpm}$. While in the emergency department (ED), he had an episode of diarrhoea associated with bradycardia (40 bpm) followed by atrial fibrillation and a brief loss of consciousness. He was treated with magnesium $2 \mathrm{~g}$ and potassium chloride $40 \mathrm{mmol}$ and reverted to sinus rhythm. He had respiratory alkalosis $\left(\mathrm{pH} 7.48, \mathrm{PCO}_{2} 3.9, \mathrm{PO}_{2}\right.$ 11 , lactate $2.2 \mathrm{mmol} / \mathrm{L}$ ) but no increased secretions, bronchorrhea, rhinorrhea, myopathy, or slurred speech. His chest X-ray was clear. While in the ED, he had an episode of vomiting associated with profound bradycardia (20 bpm) followed by a second episode of unconsciousness. He was administered $600 \mu \mathrm{g}$ of atropine and subsequently transferred to the coronary care unit. He remained stable until 24 hours post-ingestion when he had further bradyarrhythmias followed by asystolic cardiac arrest. He responded to cardiopulmonary resuscitation, $600 \mu \mathrm{g}$ of atropine and had temporary pacing wires inserted. On transfer to intensive care, pralidoxime was administered and an infusion of atropine $120 \mu \mathrm{g} / \mathrm{h}$ started. An echocardiogram was normal. Plasma cholinesterase activity at 23 hours post-ingestion was $1257 \mathrm{U} / \mathrm{L}$ (reference 5320-12920), red blood cell cholinesterase activity was unavailable. He was intermittently pacemaker-dependent on day 2 and despite a low infusion rate of atropine, he developed symptoms suggestive of anticholinergic syndrome (dilated pupils, agitation, lower limb jerking). Atropine and pralidoxime were stopped and despite diazepam, he required sedation and intubation due to agitation. He was extubated the following day without sequelae but continued to have episodes of diarrhoea and intermittent bradycardia. Pralidoxime was restarted until his bradycardia resolved. Pacing wires were removed on day 6 . Plasma cholinesterase improved (2037 IU) on day 9 and he was discharged home with no evidence of intermediate syndrome or delayed neuropathy.

Conclusion: This case report demonstrates predominantly cardiac symptoms following diazinon poisoning that was managed with magnesium, atropine, pralidoxime and temporary pacing. Temporary pacing proved beneficial when atropine was discontinued secondary to iatrogenic anticholinergic syndrome.

\section{Paraquat poisoning in Taiwan: 13 years' experience}

\author{
Chun Kuei Chen ${ }^{\mathrm{a}}$ and Chih Chuan Lin ${ }^{\mathrm{b}}$ \\ ${ }^{a}$ Department of Emergency Medicine, Linkou Chang Gung \\ Memorial Hospital, Touyuan, Taiwan; 'binkou Chang Gung \\ Memorial Hospital, Touyuan, Taiwan
}

Objective: Paraquat is toxic with high mortality [1]. We would like to present the experience in Taiwan.

Methods: We retrospectively reviewed the data of patients with paraquat poisoning from 2003 to 2016 in two medical centers. Data on the blood paraquat concentration, treatment regimen, and outcome including mortality and pulmonary fibrosis were obtained.

Results: Overall 256 patients were included. Almost half of the patients had no underlying psychiatric disease $(47 \%, n=121)$. The mortality was $74.6 \%(191 / 256)$. The patients who died were more likely to be elderly, with increased initial heart rate and respiratory rate, and decreased body temperature and conscious level and they had dyspnoea (all $\mathrm{P}<0.05$ ). Regarding the laboratory data, higher white blood cell count (WBC), blood urea nitrogen (BUN), creatinine, $\mathrm{PaO}_{2}$, sodium, glucose, blood paraquat concentration, urine paraquat concentration and lower $\mathrm{pH}$, bicarbonate, $\mathrm{PaCO}_{2}$, and potassium were associated with higher mortality (all $p<0.05)$. Pulmonary fibrosis occurred in $10.2 \%(n=26)$ and three patients died due to pulmonary fibrosis. Using multivariate analysis, the blood paraquat concentration was the independent factor correlated with mortality. The odds rate was 1.99 (95\% confidence interval, 1.42-2.78) and the AUC of blood paraquat concentration to predict mortality was 0.9689 . The blood paraquat concentration to maximize the sensitivity and specificity was 1.52. We compared different treatment combinations including pulse therapy (cyclophosphamide plus methylprednisolone), acetylcysteine (NAC) plus methylprednisolone, NAC plus vitamin $C$, and vitamin $C$ plus methylprednisolone in two groups of different severity divided by blood paraquat concentration at $1.52 \mu \mathrm{g} /$ $\mathrm{mL}$. The results showed no difference among patients with paraquat concentration above 1.52. However, NAC plus methylprednisolone was associated with worse survival outcome among patients with paraquat concentration less than $1.52 \mu \mathrm{g} / \mathrm{mL}$. Pulse therapy [2] showed no benefit in overall or subgroup analysis. Also, in the survivors with pulmonary fibrosis, there was no difference in development of fibrosis between those given pulse therapy and those who did not receive it (all $n=23,7$ with pulse therapy, $p=0.44$ )

Conclusion: Paraquat poisoning is associated with a poor outcome. High blood paraquat concentration means high mortality, regardless of the treatment given. However, for patients with less severity (blood paraquat concentration $<1.52 \mu \mathrm{g} / \mathrm{mL}$ ), NAC plus methylprednisolone may be harmful.

\section{References}

[1] Senarathna L, Eddleston M, Wilks MF, et al. Prediction of outcome after paraquat poisoning by measurement of the plasma paraquat concentration. QJM. 2009;102:251-9.

[2] Lin JL, Leu ML, Liu YC, et al. A prospective clinical trial of pulse therapy with glucocorticoid and cyclophosphamide in moderate to severe paraquat-poisoned patients. Am J Respir Crit Care Med. 1999;159:357-60. 


\section{Author index by abstract number}

Aardal, Elisabeth ............................................................................. 188

Aaron, Cynthia .......................................................................................... 3

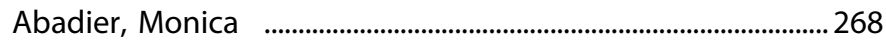

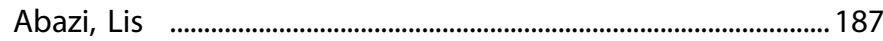

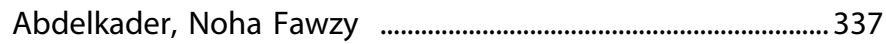

Abesamis, Michael .............................................................................42

Abouchedid, Rachelle ............................................................................. 9

Abraham, Siju V ...................................................................................... 238

Ackerman, John P ........................................................................... 151

Acquarone, Daniela ..............................................69, 106, 126, 305

Acquisto, Nicole ............................................................................... 199

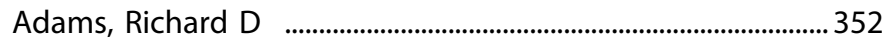

Aeberhard, Roger ......................................................................92

Afandiyev, Ismayil ..................................................................80, 204

Agarwal, Sumit ...................................................................................52

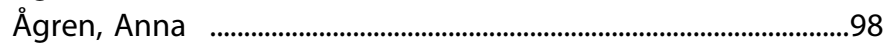

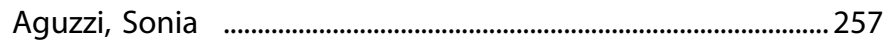

Ahlner, Johan ....................................................................... 175, 188

Ait-Daoud, Nassima .......................................................................... 194

Akpunonu, Peter ........................................................................... 335

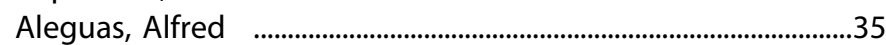

Almog, Yaniv ...................................................................................... 103

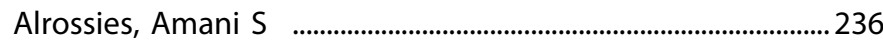

Alsufyani, Asaad I ..........................................................119, 136, 241

Angelats-Romero, Carlos Miguel ................................................ 278

Angerer, Verena ............................................................................ 123

Anniballi, Fabrizio ..............................................................................66

Anseeuw, Kurt ................................................................................ 2

Apicella, Andrea ............................................................................ 256

Arango Mathieu, Mireille ....................................................................43

Archer, John RH ....................................................................... 9, 236

Arias, Ana Sylvia .............................................................................90

Arif, Tara $216,217,218,219,220,221$

Arnold, Justin $29,177,329$

Arvidsson, Stefan .....91

Arya, Rita

341

Ascione, Serena

256

Assisi, Francesca .................................................................223, 247

Atgé, Benoît ................................................................................. 211

Atizaz, Hussain .......................................................................................... 112

Aubry, Lise .......................................................................................... 196

Aufderheide, Michaela .............................................................. 135

Auriacombe, Marc

Auwärter, Volker

Babić, Željka

Babu, Kavita M

Baccaro, Cecilia

Bach, Hannah

Bacis, Giuseppe .39

33,123

104,275

18,41

348

111,112

Baciu, Alexandru

$185,276,292$

251

Backus, Timothy $\mathrm{C}$
Badaras, Robertas

$.26,282,293$

Badea, Adina ............................................................................................ 186

Baeck, Marie-Madeleine ................................................................... 277

Bagnoli, Luca ............................................................................... 163

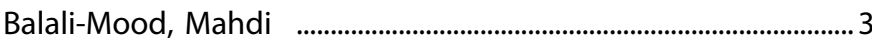

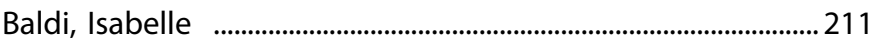

Baldvinsdottir, Helena Lindal ........................................66, 76, 330

Ballet, Steven ..................................................................................... 137

Balme, Kate .............................................................................................. 263

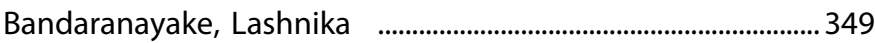

Banerji, Shireen ................................................................................... 300

Barari, Behjat ................................................................................... 224

Barasoain-Millán, Alberto ........................................................... 278

Barcelo Martin, Bernardino .................................................19, 148

Barlow, Nicola .......................................................................................58

Bartecka-Mino, Kinga .......................216, 217, 218, 219, 220, 221

Basanou, Eleni ................................................................................17

Basso, Gianluca ....................................................................................... 200

Bates, Nicola ................................................227, 228, 231, 232, 233

Batora, lgor ............................................................................................77

Baumann, Michael H ......................................................................... 237

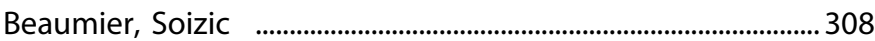

Becker, Therese ............................................................48, 182, 208

Bedussi, Francesca .............................................................................. 292

Begemann, Kathrin ........................................................... 126, 210

Bekjarovski, Niko ........................................................................ 104

Bellelli, Elena …................................................... 32, 260, 267, 270

Benabbas, Roshanak ................................................................... 328

Bennett, Kevin M ........................................................................... 136

Benowitz, Neal L .............................................................................. 109

Bentur, Yedidia _.................................................................... 103, 159

Bergese, llaria ....................................................................................... 257

Bertocchi, Silvia .................................................................................... 100

Bessire, Karine .............................................................................................94

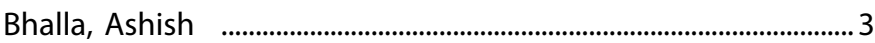

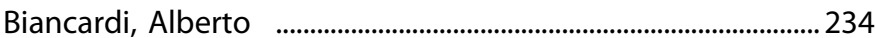

Biary, Rana .......................149, 164, 195, 258, 289, 301, 309, 325

Bihan, Kevin ................................................................................... 323

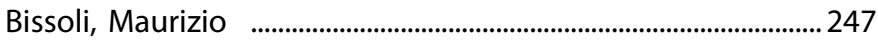

Bjelajac, Adrijana ............................................................................... 275

Bjornsson, Einar $\mathrm{S}$............................................................................. 330

Black, Joshua C ..................................................... 24, 25, 39, 315

Blackett, Tiffany ................................................................................ 227

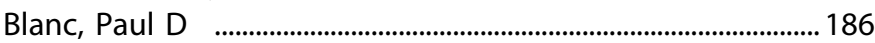

Blough, Bruce E ................................................................................... 237

Blumenberg, Adam .......................................................................... 328

Bøgevig, Søren .....................................................190, 198, 226, 302

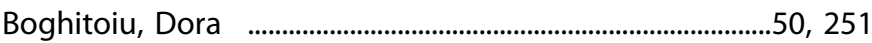

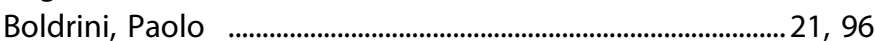

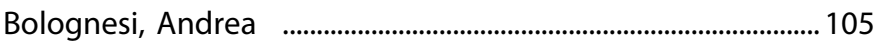

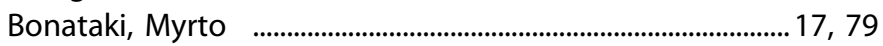


Bondone, Claudia

Bonney, Caitlin F $257,265,266$

Borchers, Maike

Borek, Heather

Boroughf, William J

Borrasca-Fernandes, Carla F

Boscaiu, Mihai

Bosetti, Francesca

Bottari, Gabriella

Boulamery, Audrey

Bourgue, Lucile

Bouthemy, Mathieu

Boyer, Edward

Bradberry, Sally M

$5,22,37,53$

$154,253,254,284,331,352$

Brambilla, Elena

Brambilla, William

Breindal, Torben

Breit, Andreas

Brekelmans, Pieter

Brekke, Mette

Brent, Jeffrey

Briggs, Nancy

Brogtrop, Jeroen

Bronstein, Alvin C

Brouste, Yanick

Brown, Jared A

Bruccoleri, Rebecca

Bruglieri, Giuseppe

Brusin, Konstantin

Brvar, Miran

$67,104,178,222$

Bucaretchi, Fabio

Buckley, Nicholas A

$3,63,129,165,183,240,242$,

$243,244,317,342$

Budenz, Beate . .75

Buisine, Anne ..30

Burmeister, Undine

319

Burnham, Randy

245

Burns, Michele

127

Buscaglia, Eleonora

$6,13,38,82,298,31$

Butera, Raffaella

Buylaert, Walter

Bvar, Miran

Cabie, Andre

Cagáňová, Blažena

Cairns, Rose

Camillerapp, Christophe

Campbell, Alexander

Campleman, Sharan

Canal Raffin, Mireille

Cano, Damon N

$85,276,292$

172

. .66

. .90

$31,77,104$

...63

137

. .337

. .336

201, 207, 211

Cantone, Damiano

339

Capleton, Alexander C

299

Carmellino, Virna

341

Carvalho Alves, Katia

$257,265,266$

Carvalho, Anna-Maria

332

... 149

Casavant, Marcel J

151

Casey, Patricia
Cassidy, Nicola

285,354

Castagno, Emanuele ........................................................265, 266

Castellan, Delphine ............................................................................ 323

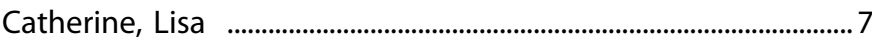

Cathro, Helen P ........................................................................136

Cecchetti, Corrado ................................................................................ 267

Celentano, Anna ...............................................192, 200, 247, 347

Cesaire, Raymond .................................................................................90

Ceschi, Alessandro .................................................................................16

Chaine, Manon .................................................................................. 155

Chan, Betty S .48, 129, 165, 182, 183, 208, 240, 242, 244, 342

Chan, Chi-Keung ................................................................................. 181

Chang, Tam Fei …........................................................................... 281

Chapman, Brittany P ............................................................18, 45

Charbatier, Cyrille .............................................................................. 166

Charlton, Jennifer R ........................................................................ 136

Charlton, Nathan P ...........................................................136, 241

Chary, Michael ................................................................................ 127

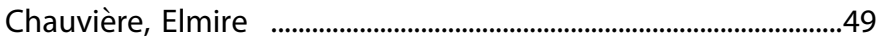

Chen, Chun-Kuei ...............................................................353, 355

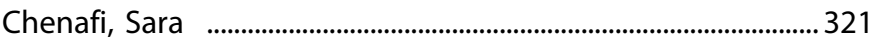

Cheng, Sonia …...................................................165, 183, 240, 342

Chevillard, Lucie ..........................................................121, 132, 137

Chiang, William ........................................................................................ 327

Chiara, Francesca …............................................... 82, 131, 192, 288

Chiew, Angela L .......................48, 129, 165, 182, 183, 208, 240,

$242,243,244,317,342$

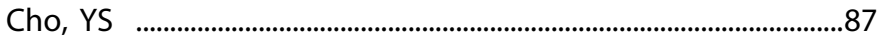

Choi, Kyoung Ho ................................................................................. 203

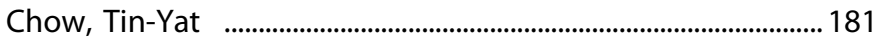

Christensen, Mikkel B ....................................................................... 226

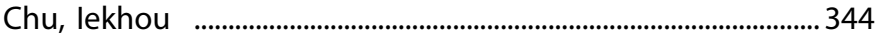

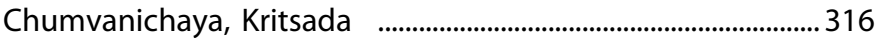

Chun, Byeongjo .........................................................................86, 87

Cirronis, Marco ....................................................................................... 246

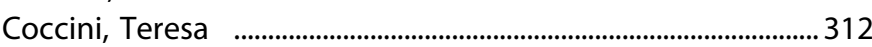

Colello, Diane ....................................................................................... 336

Compagnon, Patricia ........................................................................... 142

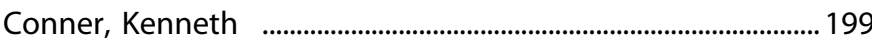

Cooper, Gillian A ...................................................................................57

Cooper, Jamie ................................................................................................44

Cordoba, Francisca ............................................................................ 156

Cormier, Monique ................................................................................. 179

Costagliola, Roberto .......................................................................... 192

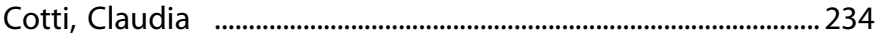

Coudouel, Hervé ........................................................................... 138

Coulson, James M ...............................................................................57

Crevani, Marta ..............32, 38, 82, 131, 209, 260, 271, 288, 312

Curtis, Amelia ........................................................................................

Da Silva Caçao, Oscar …………………………………....................... 211

D'Agostino, Maria ................................................................................. 276

Dalhoff, Kim P .......................................................................... 155, 302

Dargan, Paul I .......................3, 9, 15, 23, 24, 25, 39, 44, 124, 236

Dart, Richard C ..................................24, 25, 39, 62, 245, 269, 315

Davanzo, Franca ........................................105, 192, 200, 247, 347

Dawson, Andrew H ........................................................................63

Day, Rachael .......................................................................................... 284

De Capitani, Eduardo M ............................................................... 101

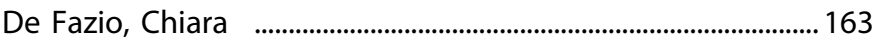


De Groot, Ronald

De Haro, Luc

De Lange, Dylan W

De Luca, Maia

De Olano, Jonathan

De Paepe, Peter

De Palma, Giuseppe

De Roos, Francis

De Vries, Irma

Dean, Danielle $S$

Dear, James W

Deatrick, K Barry

Degrandi, Colette

Deguigne, Marie

Deidda, Andrea

Dekker, Douwe

Del Vecchio, Amedeo

Delhotal Landes, Brigitte

Delhumeau, Sophie

Delogu, Mauro

Delva-Clark, Heather

Demenagas, Dimitrios

Dempsey, Delia A

Deronde, Kimberly A

Descamps, Anne-Marie K

Desel, Herbert

Deters, Michael

Deye, Nicolas

Di Giuseppe, Martina

Di Mita, Onorina

Di Pietro, Santi

Di Primo, Maria

Dieltiens, Greet

Dietrich, Robert

Dijkman, Marieke A

Dijkman, Wouter

Dimasi, Valeria

Dines, Alison M

Dobaja Borak, Mojca

Domangé, Bastien

Doolan, Aoife

Dorner-Schulmeister, Susanna

Doucet, Alexandre

Du Plessis, Catharina E

Dubé, Pierre-André

Ducint, Dominique

Duggan, Edel

Dunn, Michael

Durrani, Timur

Duthaler, Urs

Duval, Xavier

Ebbecke, Martin

Ebbehoej, Niels E

Ecolini, Anita

Eddleston, Michael

Edwards, Nick

Eitmontaite, Deima

Elamin, Muhammad EMO

Eleftheriou, Georgios
$12,66,97,215,286,308$

$4,147,150,229,230,252,340$

. .270

$195,296,327$

... 172

311

.113, 114

$51,55,95,141,147,150,252$

250

146

.279

69, 262

60,142

32,260

.252

.246

239,332

......60

.234

. .245

.. .79

... 109

... 136

$.78,172$

126,210

.....75

$47,157,239,332$

. .267

. .261

. .288

6, 297, 298

$\ldots . .2$

180

$95,229,230$

.... 340

200, 247

$. .9,15,124$

67,104

$12,97,215,286$

.....354

.221

. .117

....99

... 193

.211

$249,285,354$

36,44

...294

14,235

142

$126,269,277$

. .214

.... 1

$3,44,53,104$

228,233

.....26

$\ldots . .54,58,64,65,253,254,331$

$185,276,292$
Elek, István

$85,162,213$

El-Gowilly, Sahar ………………………………………………………..... 337

Ellis, Judson .............................................................................................. 304

Elnabarawy, Sherif Tarek ......................................................................337

Elorza, Miguel Angel ...............................................................19, 148

Eriksson, Frank ..................................................................................... 155

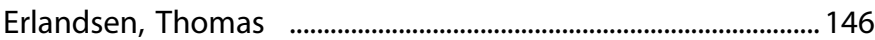

Erlangsen, Annette ...………………………………………………...... 155

Exadaktylos, Aristomenis K .................................................................16

Eyer, Florian ....................................................................69, 123, 126

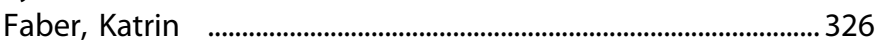

Fabre, Julian .................................................................................110, 166

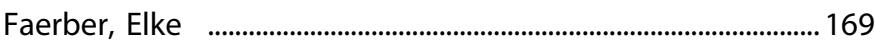

Faggionato, Elena ………………………………………………….... 234

Faraoni, Lorella ............................................................185, 276, 292

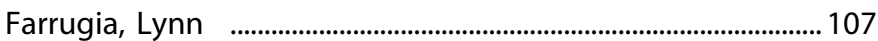

Federici, Tatiana ……………………………………………......32, 260

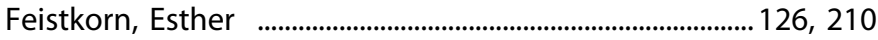

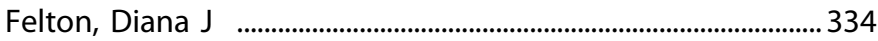

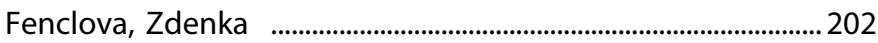

Ferrer Dufol, Ana .................................................................. 125, 156

Ferruzzi, Marcello ………………………………………….....247, 347

Figueroa, Magaly ……………………………………………………....43

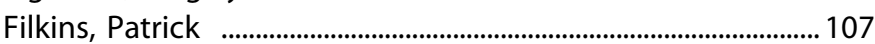

Finter, Johannes ..................................................................................... 139

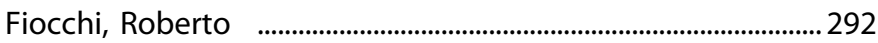

Florentin, Jonathan ............................................................................. 166

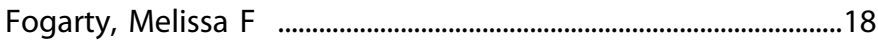

Fonseca, Maria Francina ...........................................................................39

Fontana, Riccardo .................................................................................. 145

Ford, Loretta ........................................................................................ 331

Forsberg, Sune ..................................................................................... 187

Fountas, Konstantinos ...........................................................................79

Francis, Arie P ...…………………………………………………….... 164

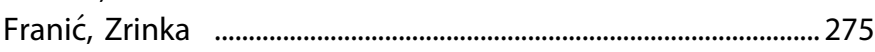

Frasnelli, Matteo ……………………………………………………....... 234

Fredriksson, Inga ................................................................................ 189

Friðdriksdottir, porbjörg Andrea ....................................................330

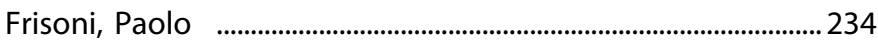

Fuchs, Joan ....................................................................................... 92, 94

Fuchs, Lior ................................................................................................. 103

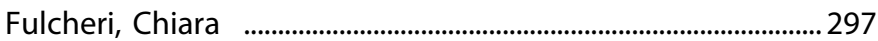

Fuster, Anne ....................................................................................... 157

Galante, Ori ..................................................................................... 103

Gallo, Mariapina ……………………………………..... 185, 276, 292

Gambassi, Francesco ……………………………..... 1, 34, 246, 348

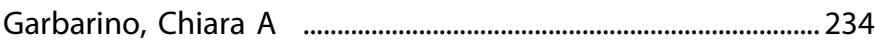

García Antich, Sandra ....................................................................... 153

Garcia Urdangarin, Agustin .............................................................. 125

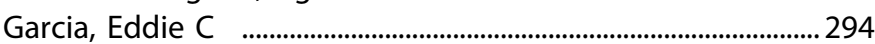

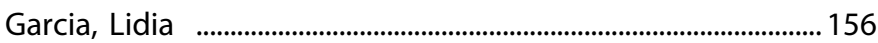

Garner, Alex ………………………………………………………..... 349

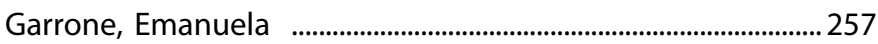

Garuti, Fabio .............................................................................1 192, 200

Gaudio, Rosa M .....................................................................163, 234

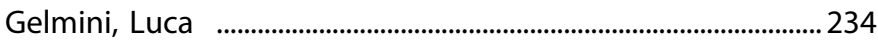

Genser, Dieter ........................................................ 66, 218, 219, 221

Genuini, Leonardo .............................................................................. 271

Georgatos, Joannhe ............................................................................. 247 
George, Nathan ... 5

Gerber, Bryan T 180

Gervilla Garcia, Elena

153

Ghazi, Ala

188

Giacomello, Rossella

192,200

Giampreti, Andrea

185, 276, 292

Giffin, Christine

Giraudon, Isabelle

Glaizal, Mathieu

.15

Glaser, Nina

$. .12,286$

Gohlsch, Katrin

126,210

Goldfine, Charlotte

.. 135

Gollmann, Mandy

Gomila Muñiz, Isabel

Gomila, Isabel

148

Gonzalez-Hernandez, Graciela

.....19

Goodnough, Robert

113,114

Gorman, Daniel P

186,294

Gorodetsky, Rachel

.. 241

Goslar, Tomaž

Gosselin, Sophie

199

Gottardo, Rossella

Gouda, Ahmed S

Gourlain, Hervé

Grange, G Fenix

Grassi, Maria Caterina

Graudins, Andis

222

$117,179,193$

.... 274

$30,157,320$

$49,108,121$

... 334

Graziani, Manuela

Green Vance, Emily

Greene, Shaun L

Gremo, Erik

Grenc, Damjan

Grimsrud, Marit M

Griswold, Matthew K

Grumann, Christina

Grundlingh, Johann

Gruzdyte, Laima

Guareschi, Marilena

$21,66,96,176$

$243,268,317$

.. .96

... 329

.243, 317

......98

Gudermann, Thomas

Gudjonsdottir, Gudborg A

Guerrini, Chiara

Gugelmann, Hallam

Gunja, Naren

Gutiérrez, José Maria

Habibi-Nodeh, Zahra

Hadjiangelis, Nicos

Halassy, Beata

Hamdi, Eya $67,104,178,222$

...28

$.18,107$

......33

. .44

.104

....39

.135

....76

192

109

Hammer, Paula EC

Hanston, Philippe

Hardy, Gareth

Harmouche, Elie

Harnett, James

Harris, Keith

Haschke, Manuel

Hassanian-Moghaddam, Hossein

Hatten, Benjamin W

Havel, Christopher

Hawkins, Leonard
Hawton, Keith

155

Haynes, Colleen M $24,25,39,315$

Heise, Craig W ....52

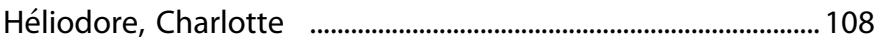

Henke, Des ............................................................................................65

Hentschel, Helmut ..................................................................................75

Herbots, Hanne

Hermanns-Clausen, Maren

$33,123,126,305,319$

Hernandez, Paula

158

Herr, Daniel L

. .255

Heyerdahl, Fridtjof

$15,28,124$

Hickle, Kelli

....41

Hilaire-Buys, Dominique

. .323

Hill, Simon L

$36,44,236$

Hines, Elizabeth Q

$.255,272,279$

Hlusicka, Jiri

134,212

Ho, Chumang

344

Hockenhull, Joanna

$23,24,25$

Hoegberg, Lotte CG

$84,190,198,226$

Hofer, Katharina E

262

Hoffman, Robert $\mathrm{S}$

$164,301,309,325$

Hoffmann, Sebastian ... 135

Höjer, Jonas

264,333

Holder-Koob, Elisabeth .................................................................... 169

Holland, Michael G .................................................................................. 143

Holstege, Benjamin J ............................................................................

Holstege, Christopher P ............... 71, 73, 88, 119, 140, 160, 161,

$194,225,248,280,324$

Holstege, Samuel C

$88,140,225$

Holze, Friederike

.235

Holzer, Angelika ........................................................................................69

Homar Amengual, Catalina ........................................................... 148

Hondebrink, Laura

Hovda, Knut Erik

$3,15,28,83,124$

Howland, Mary Ann

$258,325,327$

Hoyte, Christopher O ......................................................................... 300

Hruby, Sandra .................................................................................. 221

Hudson, Simon .......................................................................................... 9

Huiskens, Dave …………………………………………….......229, 230

Hultén, Peter ...............................................................................91, 333

Hume, Simon ............................................................................................... 168

Hunault, Claudine $C \quad$...............................................................................5

Hurt, Brenten R ..................................................................... 111, 112

Hussain, Atizaz ........................................................................................ 111

Hyslop, Stephen ...…………………………………………………..... 101

leong, Puii ...………………………………………………………….... 344

leri, Alessandra ................................................................ 1, 246, 348

Irena, Sollaku ............................................................................. 311

Isbister, Geoffrey K ....................................129, 242, 243, 244, 317

Isoardi, Katherine Z ...........................................................129, 250

Iwanicki, Janetta L ..........................................39, 62, 245, 269, 315

Jackson, Gillian …..............................................22, 56, 154, 284, 352

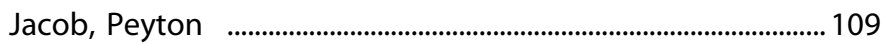

Jacobsen, Dag ………………………………………………….....3, 83

Jaffal, Karim ………...................................... 30, 128, 157, 196, 321

Jagpal, Pardeep S …………………………......... 64, 253, 254, 331

Jakab, Dóra O ………………………………………………………..... 213

James, David A ..........................................................................................

James, Laura P ................................................................................... 242 
Jarwani, Bhavash

Jeng, Amy

Jenkins, Valerie $\mathrm{R}$

Jimenez Lopez, Rafael

Johansen, Sys $S$

John, Harald

Jones, Dacia

Jones, Joseph $\mathrm{O}$

Jonsdottir, Freyja

Julin, Mikael

Jung, Hyun Ho

Just, Simone

Kabata, Piotr M

Kacer, Petr

Kaista, Maria

Kallel, Hatem

Kalostou, Angeliki

Kamps, Marlijn JA

Kan, Anne A

Kappen, Carolyn

Karabuva, Svjetlana

Karp, Galia

Kastanje, Ruth

Kataria, Himanshu

Kaukeinen, Kimberly

Kauth, Verena

Kazem, Yasser HRM

Kekla, Emilija

Khoury, Lana

Kim, Hong $\mathrm{K}$

King, Aleisha Jane

Kirby, Katharine A

Kishinevsky, Elena

Klatka, Michal

Klein-Schwartz, Wendy

Klumb, Wolfgang

Knudsen, Kai

Kolaczynska, Karolina E ............................................................. 237

Kolivand, Pirhossein ........................................................................ 224

Koller, Marianne

Kolovatsios, Dimitrios

Kolpach, Zuzana

Kopatic, Marissa

Korenke, G Christoph

Kovačić, Jelena

Koželj, Gordana

Krähenbühl, Stephan

Krakowiak, Anna

Kresanek, Jaroslav

Krischenowski, Olaf

Križaj, Igor

Krotulski, Alex J

Kruse, Heidi

Kubis, Nathalie

Kugelberg, Fredrik C

Kupferschmidt, Hugo

Kurtović, Tihana

Labat, Laurence

Lachireddy, Kishen
$.111,112$

153

190, 198

... 139

68,331

... 143

$.76,330$

......91

.203

. .69

66,104

.. 134

310

.. .90

17, 79

340

229,230

.. 143

... 178

173

$.70,104$

......44

199

...69

.337

231

159

$111,112,255,279$

206

.. 129

173

352

272,322

169

170

139

....17

104

160,280

277

275

222

10, 14, 133, 290

.. 104

...77

.135

178

...18

190

. .47

175,188

262,326

178

$. .7,30,108,116,132,196,239,320,321$

.63
Lagrotteria, James J

62,269

Lai, Jeffrey $\mathrm{T}$

$18,41,45$

Lakotta, Christian .............................................................................. 106

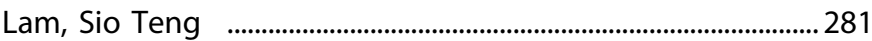

Lampinen, Terhi ...................................................................................59

Lang Balija, Maja ................................................................................ 178

Langlois, Hugo .................................................................... 117, 179

Lanni, Emanuela ........................................................................96, 176

Lanzi, Cecilia .................................................................................34, 348

Lao, Yvonne ...........................................................................................83

Laplanche, Jean-Louis …........................................................ 167, 239

Laskey, Dayne ......................................................................................... 107

Laubner, Gabija .................................................................................. 282

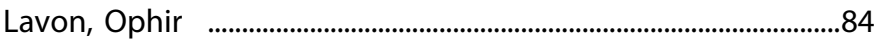

Lavry, Pierre .................................................................................... 295

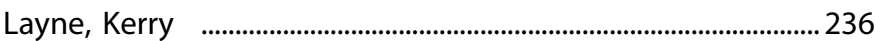

Lazzeri, Ilya J ....................................................................... 111, 112

Le Roux, Gaël ............................................................................60, 142

Leciñena, María A ...........................................................................19

Lee, Ho MR ...................................................................................58

Lee, Hyunjeong ............................................................................. 180

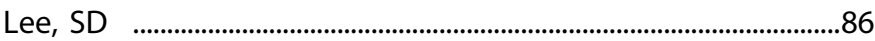

Legeay, Marion ...................................................................................60

Leonard, James B …...................................................272, 279, 322

Leong, Hoiip .............................................................................................. 344

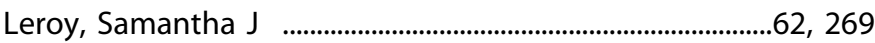

Levdov-Avital, Iris ........................................................................ 159

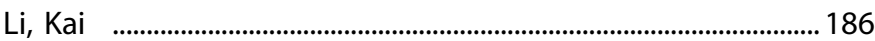

Liakoni, Evangelia ...........................................................16, 109, 124

Liechti, Matthias E ................ 10, 14, 16, 115, 122, 124, 235, 237

Lin, Chih Chuan .....................................................................353, 355

Lindeman, Erik ...........................91, 118, 158, 175, 188, 189, 338

Lippmann, Simone ......................................................................... 106

Lischkova, Lucie ........................................................134, 202, 212

Locatelli, Carlo A ........6, 13, 38, 66, 67, 82, 100, 131, 192, 209,

$234,274,288,297,298,299,311,312$

Lodrini, Giulia .......................................................................................

Logan, Barry K .......................................................................................18

Lombardi, Juliana ............................................................................. 179

Lonati, Davide 13, 32, 38, 66, 67, 192, 270, 274, 298, 311, 312

López-Ávila, Javier ................................................................................ 278

Luethi, Dino ….....................................................10, 14, 122, 237

Lukšić, Boris ............................................................................... 178

Luppi, Andrea ................................................................................... 234

Lupton, David J .................................................................22, 56, 284

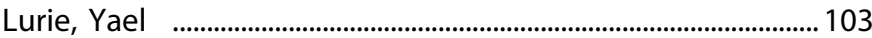

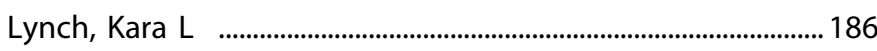

Lynch, Michael .........................................................................................

Macan, Jelena ............................................................................................. 275

Machold, Beate ........................................................................................8

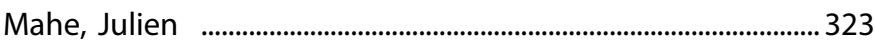

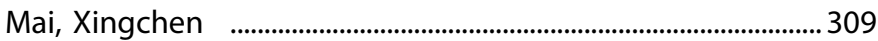

Malissin, Isabelle ........................ 47, 108, 116, 121, 157, 239, 332

Mallozzi Menegatti, Alessandra ........................................................ 163

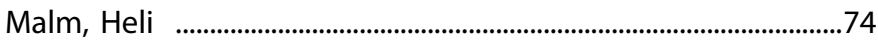

Mandujano Meneses, Daniela ......................................................43

Mangoura, Safwat A .......................................................................... 337

Manini, Alex F ....................................................................304, 350

Mannaioni, Guido …............................................... 1, 34, 246, 348 
Marano, Marco

$32,260,267,270,271$

Marcos, Josep

Margolin, Zachary R

62,269

Marino, Ryan

$8,40,42,303,345,346$

Marks, Carine J

....99

Marraffa, Jeanna M

143, 273

Martin, Charlotte

137

Martin, Didier

Martínez-Sánchez, Lidia ...umusume 278

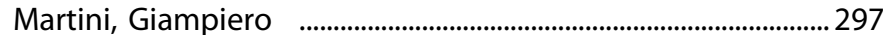

Martiñón Ríos, Rocío .........................................................................43

Mashlah, Anas

.327

Masini, Emanuela $1,246,348$

Masnada, Silvia .274

Massi, Paola .234

Mathieu, Laurence 138

Maystrova, Olha

Mazzariol, Sandro

Mazzeffi, Michael

Mazzucco, Viola

McCarty, Matthew

McMartin, Kenneth

McNulty, Richard

Mégarbane, Bruno

$3,7,30,47,49,62,90,108$

$110,116,121,128,132,137,157,166$,

$167,196,239,269,320,321,332$

Meggs, William J

27,180

Mehdaoui, Hossein

$90,110,166$

Meier, Jürg

.....92

Melara, Ilaria

131, 297

Melit, Ronald Jaison ................................................................ 238

Melvin, Krissy ........................................................................................ 300

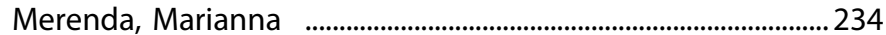

Merialdi, Giuseppe ......................................................................... 234

Messina, Genevieve H ......................................................................... 250

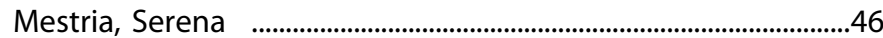

Mesturino, Alessia ................................................................................ 271

Metzsch, Carsten ................................................................................... 188

Meyer Zu Schwabedissen, Henriette E ......................................... 115

Meyer, Géraldine .................................................................................60

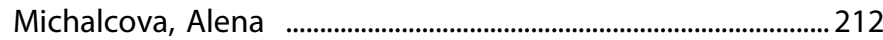

Michelsen, G Svanhvít ....................................................................76

Mifsud, Janet

337

Migueres, Hélène

157,320

Milanesi, Giovanni

247,347

Milella, Michele S

...96, 176

Miller, Susan N

27,180

Miller-Barmak, Adi N ....................................................................... 159

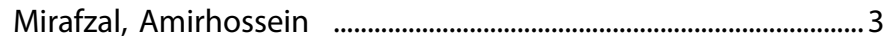

Mirkatouli, Nafiseh ............................................................................ 224

Mirzazade, Murad .....80

Missanelli, Andrea

348

Miu, Chi Fong

281

Moayedi, Siamak

111,112

Moed, Taleb

... 159

Moens, Jonas

66,78

Mohamedou, Moustapha

207

Mohammad, Mohammad

Möhle, Niklas
Molimard, Mathieu

207, 211

Molina-Cabañero, Juan Carlos ...................................................... 278

Montibeller, Marcello ............................................................................. 271

Moon, Jeong Mi ........................................................................8 86, 87

Mora, Pierre .................................................................... 157

Moran, Jeffrey H .......................................................................... 143

Morello, Francesca ….......................................................................... 100

Moreno, Emilia ……………………………….............................19

Moro, Paola ................................................................................. 247

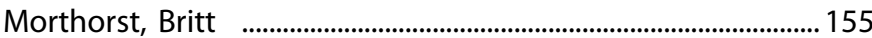

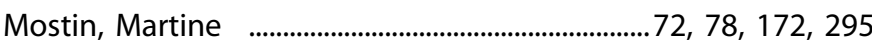

Moyns, Emma J .....................................................................................54

Mückter, Harald .......................................................................................... 135

Mueller, Laura ............................................................................................16

Mulder-Spijkerboer, Henneke N …............................................... 147

Müller, Daniel ..................................................................................... 326

Muller, Gert J ....................................................................................99

Murro, Vittoria ..................................................................................34

Musella, Giuseppe ............................................................................... 223

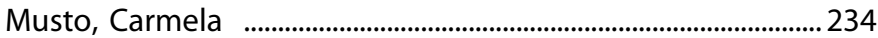

Myers, Katie ..................................................................................... 120

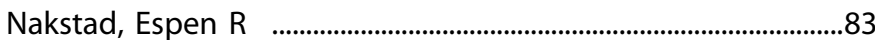

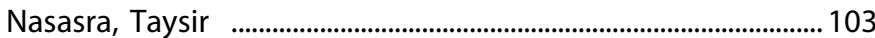

Navarro, Adeline ................................................................................... 138

Navratil, Tomas ….............................................................................. 212

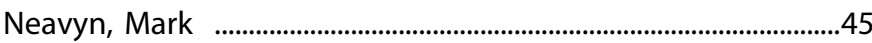

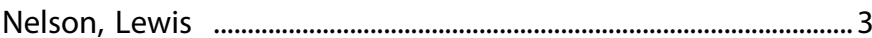

Neou, Polyxeni …..................................................................... 17, 66, 79

Neri, Margherita .................................................................................. 197

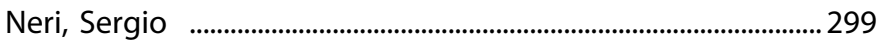

Neuman, Gal ......................................................................................... 159

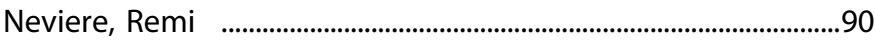

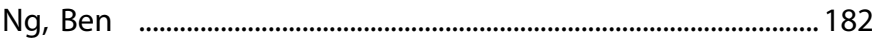

Ngo, Duc Anh ..............................71, 73, 119, 140, 160, 161, 194,

$230,248,280,324$

Nguyen, Philippe ....................................................................... 121

Nicolaescu, Radu ..........................................................................5

Nielsen, Trine ….............................................................................. 226

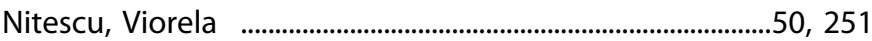

Nogue Xarau, Santiago ............................................................. 125

Nogué, Santiago ….........................................................................11, 156

Noori, Monica R .................................................................................. 307

Nordentoft, Merete ..................................................................... 155

Nordmark Grass, Johanna ......................... 98, 158, 175, 188, 259

Occupati, Brunella .............................................................................. 348

O'Connor, Feargal .......................................................................... 249

Oder, Mare .....................................................................................66, 70

Odoardi, Sara ….........................................................................................46

Oerlemans, Chris ......................................................................... 147

Officer, Jane ….............................................................................................44

Ohana, Dana ........................................................................................ 141

Oikonomou, Nikoleta ............................................................17, 79

Olita, Carla ...............................................................................32, 260

Olivotto, Sara …........................................................................................ 274

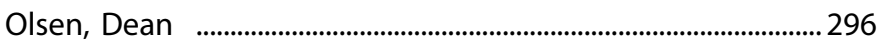

Olson, Kent ..................................................................................... 3

Ortega Perez, Juan .................................................................. 148, 153

Osinski, Karen ................................................................................... 146

Otrubova, Olga ................................................................................77 
Paasma, Raido

Page, Colin B

Paglia, Stefano

Palayer, M

Palmer, Charlotte

Palmiéri, Solène

Palmqvist, Dorte F

Pantaleoni, Roberto A

Pantini, Paolo

Pap, Csaba

Papa, Pietro

Papathanassiou, Vassiliki

Park, Jung Taek

Parker Cote, Jennifer L

Parra, Marina

Parris, Richard

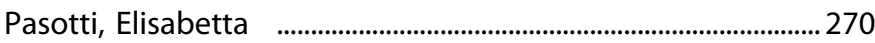

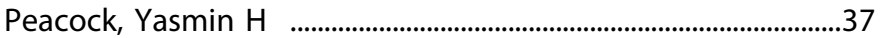

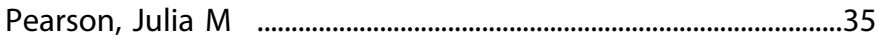

Pedersen, Ellen B

Pelclova, Daniela

$202,212,283$

Péloquin, Fannie

Peredy, Tamas

Perez Tuñon, Jorge G

Péron, Nicolas

Perrone, Jeanmarie

Perrone, Maria

Perrotta, Daniela

Persett, Per Sverre

Personne, Mark

Petersen, Tonny S

Petersson, Emma

Petrik, Brigita

Petrini, Stefano

Petrolini, Valeria M

Pettie, Janice

Phan, Hilda MH

Phelps, Jeremiah

Pichette, Vincent

$81,85,104,130,162$

$13,38,192$

203

7, 180

. .11

44

50

157,167

113,114

209

270

...28

61

198, 302

.. 259

... 282

197

Pierre, Sabrina

Pinamonti, Anna L

Pisani, Mara

Pispan, Wipada

Pistelli, Alessandra

Pizon, Anthony

Plačková, Silvia

Poirier, Vincent

Ponticiello, Eduardo

Pontrelli, Giuseppe $6,67,82,131,209,288,297,299$

Poon, Chester

Populo, Annuela

Porpiglia, Nadia M

Pozo, Oscar

Prasa, Dagmar

Pruvot, Valentin

Prybys, Katherine

Pucci, Mark

Puiguriguer Ferrando, Jordi

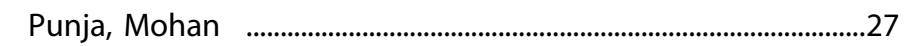

Quaranta, Alessandra _....................................................................... 163

Quarta, Brunella .................................................................................... 145

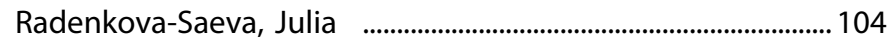

Rahmani Tzvi-Ran, Ilan ............................................................ 103

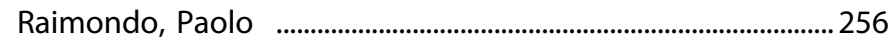

Rapp-Olsson, Malin ........................................................................... 245

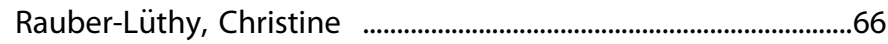

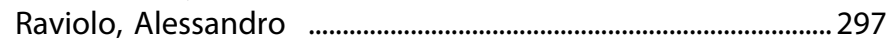

Rawson-Harris, Philip .................................................................... 231

Reale, Antonino ................................................................... 260

Rebutti, Ilaria ...................................................................................... 247

Rege, Saumitra ....................71, 73, 119, 140, 160, 194, 280, 324

Regenda, Marian ................................................................ 134, 212

Reichert, Cornelia .................................................................................... 169

Rejas Morras, Laura ....................................................................... 125

Renny, Madeline H ..............................................................258, 309

Repplinger, Daniel ......................................................................... 186

Resiere, Dabor ....................................................................90, 110, 166

Ress, Kirsty .........................................................................242, 244

Reuther, Lene ..................................................................................... 302

Reynoard, Julien ......................................................................... 12, 97

Reynolds, Kate M ............................................................................... 245

Ricci Frabattista, Angela ...................................................... 145, 163

Rice, Kenner C ................................................................................... 237

Richardson, Lynne D .............................................................304, 350

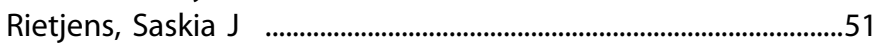

Rinker, John .......................................................................................... 171

Riou, Sybille ........................................................................................ 320

Risède, Patricia .................................................................. 132, 137

Rivera, Jessica V ....................................................................29, 177

Robben, Joris H ................................................................229, 230

Roberts, Darren .................................................................................... 3

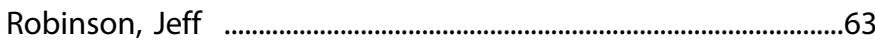

Rockhill, Karilynn ....................................................................................24

Rockwood, Gary ....................................................................................8

Roda, Elisa ........................................................................................... 312

Roelen, Chantal CJ ....................................................................... 141

Rogliano, Pierre-François .................................................132, 167

Ronchi, Anna .......................................................................... 311, 312

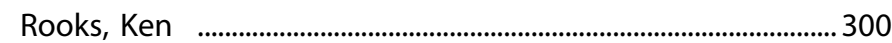

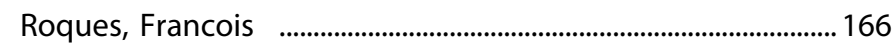

Rosa, Margherita ..............................................................256, 261

Roset Ferrer, Carolina .................................................................... 153

Rossi, Francesco P ........................................................32, 260, 270

Rotella, Joe A ................................................................. 168, 291

Rotulo, Angelo ..................................................................... 1, 34, 246

Rubini, Silva ...................................................................100, 234

Rudin, Deborah ................................................................... 133, 290

Ruggiero, Laura ................................................................................ 261

Ruiz Ruiz, Francisco ......................................................................... 125

Rushton, William F .............................................. 29, 171, 177, 329

Ruvado, Alexandra ..............................................................................90

Saathoff, Micaela E ............................................................................ 140

Sabatini, Diletta ...........................................................................21,96

Sahuquillo, Laura ..........................................................................19

Sakalauskaite, Greta .......................................................................... 293

Salama, Maisar .................................................................................. 159

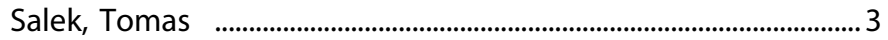


Salgado, Emilio . .11

Salimova, Maya ... 348

Salmonson, Helene

Salter, Mark

Sánchez, Bruno

Sandilands, Euan A

..5, 22 37, 53, 54, 56, 57, 58, 64, 65, $68,146,154,253,254,284,352$

Santos, Cynthia 120

Sarker, Abeed

113,114

Sauer, Oliver

$.69,169$

Saumitra, Rege

161, 248

Scanavacca, Paola

Scaravaggi, Giulia

Schaper, Andreas

Schenkel, Stephen

Scherbaum, Norbert

Schicchi, Azzurra

Schiel, Helmut

Schmid, Yasmin

Schmitt, Corinne

Scholz, Irene

Schrage, Norbert

..... 145

$6,13,38,82,298,299$

$69,269,277$

111,112

.....39

Schreiner, Ursula

Schult, Rachel

Scialanga, Barbara

Scoccimarro, Anthony

Seidel, Carola

Seif Eldin, Mohamed

Seifert, Steven A

Sein-Anand, Jacek

Sesana, Fabrizio

Sesana, Giovanni

Settimi, Laura

Severgnini, Paolo

Severtson, Geoff

Shao, Shirley

Shastry, Siri

Shin, $\mathrm{MH}$

Shulman, Joshua

Sidatt Cheikh El Moustaph, Mohamed

Sidlak, Alexander

Siegert, Markus

Sighinolfi, Davide

Signoretti, Susanna M

13, 192, 209, 274, 288, 299

.. 66, 216, 217, 221

$.16,124$

$12,97,215,286$

...16

138

220

... 199

267

$8,303,345,346$

$.69,126$

... 337

191, 339

... 104

$62,247,269,347$

200

105

223,247

62,269

......42

350

.. .86

Sikma, Maaike A

Simon, Nicolas

Simpson, Alexandra

$8,42,346$

207

...40

139

145

96,176

Sinert, Richard

Sipari, Emma

Siu, William

340

Sivilotti, Marco

Skafar, Mojca

Skagius, Ann-Sofi

Smith, Corey

Smith, Silas W

Smolinske, Susan C

Smollin, Craig G

Snook, Curtis $P$

Sofidiotou, Vassiliki $.12,97,215,286,308$

171

328

$165,183,342$

317

222

.. 333

182

164,306

191

186, 294

$. .76,330$
Soichot, Marion

$7,116,132,196,239,320,321$

Soininen, Leena . .59

Sommer, Michaela

.33, 123

Spiller, Henry A

151

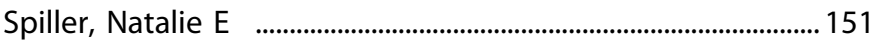

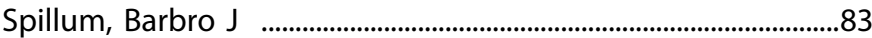

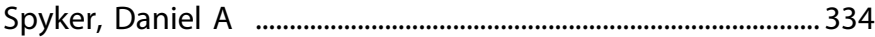

Sriapha, Charuwan ..............................................................93, 316

Stathakis, Paul .........................................................................242, 244

Stedtler, Uwe ..................................................................................69

Steger, Catherine ………………………………………………………... 296

Steinritz, Dirk .......................................................................................... 135

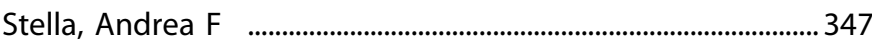

Stephen, Cindy ............................................................................. 263

Stewart, David ..........................................................................53, 352

Stigson, Michael ..................................................................................74

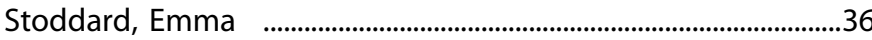

St-Onge, Maude ..................................................................................... 193

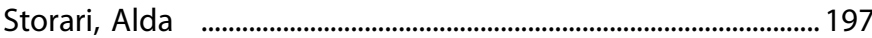

Strano Rossi, Sabina .............................................................46, 234

Stürer, Andreas

$69,126,169$

Stürzebecher, Anne ...........................................................33, 89

Su, Mark K .................................................149, 258, 287, 289, 301

Supervía, August ...................................................................................... 156

Sutterlin, Laetitia

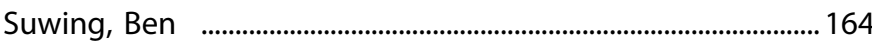

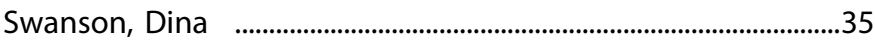

Symes, Emily ................................................................................................ 349

Syse, Victoria L ...............................................................................28

Tacquin, Camille ..............................................................................4

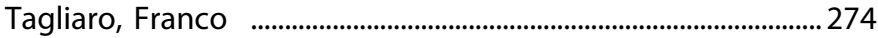

Tahmid, Farhan ............................................................................... 349

Talarico, Anna .........................................................163, 197, 234

Tallarico, Antonella ...................................................................... 145

Tan, Calvin J ............................................................................................. 287

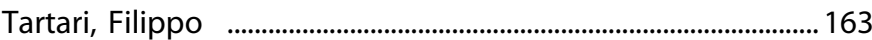

Taub, Emily S ...................................................................... 164, 306

Termälä, Anna-Mariia .......................................................................59

Tétreault, Camille ..............................................................117, 179

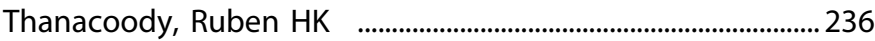

Thiermann, Horst ....................................................................... 135, 139

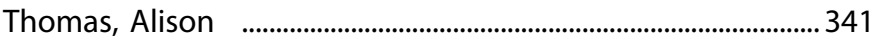

Thomas, Simon HL ...... 5, 22, 36, 37, 44, 53, 54, 56, 57, 58, 64, $65,68,154,236,253,254,284,331,352$

Thompson, John P ....5, 37, 53, 54, 56, 57, 58, 64, 65, 68, 154,
$253,254,284,341,352$

Thumtecho, Suthimon

256, 261

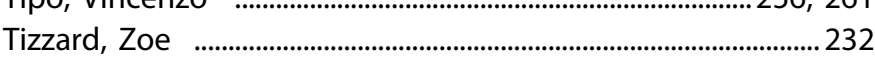

Tobback, Christina ....................................................................................72

To-Figueras, Jordi ..................................................................................11

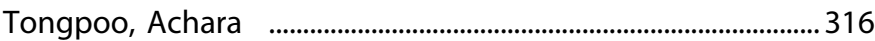

Torrents, Romain ..................................................12, 97, 215, 286

Touloupaki, Vassiliki ............................................................................17

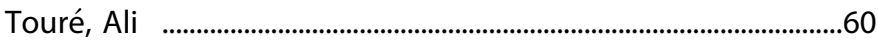

Trakulsrichai, Satariya ...................................................20, 93, 316

Travaglia, Angelo ..................................................................247, 347

Traversoni, Sara ...................................................................................... 348

Tremoloso, Federico ..................................................................... 266 
Tresoldi, Marco 209

Troiano, Annalisa

.265

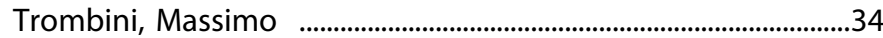

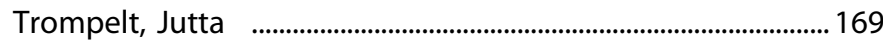

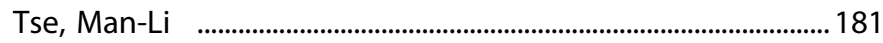

Tsoutsoulopoulos, Amelie .............................................................. 135

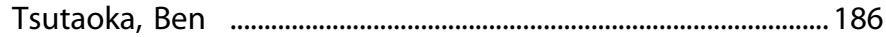

Turk, Rajka ................................................................................... 275

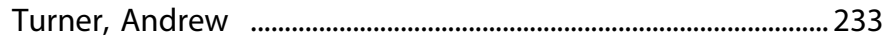

Tutdibi, Erol ..............................................................................69, 126

Udomsubpayakul, Umaporn ..................................................20, 316

Ulmeanu, Alexandru ........................................................................... 251

Ulmeanu, Coriolan ..................................................................50, 251

Urbán, Ildikó

314

Urbino, Antonio F

$257,265,266$

Valauskaite, Gabija

26,293

Vale, J Allister

$.58,284$

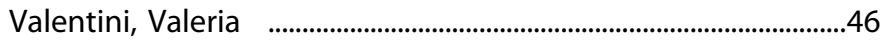

Vallersnes, Odd Martin ..................................................................28

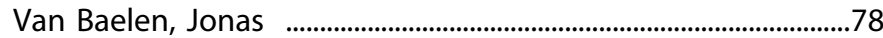

Van Hoof, Fabian KJ

Van Riel, Antoinette JHP

.. .51

Van Velzen, Agnes G

$141,147,252$

Vandijck, Dominique

$.55,147,150$

Varnai, Veda M

$.78,172$

Vasama, Justus

275

Vason, Milo . .74

Vecchio, Sarah 197

Vecellio, Elia

$. .82,131$

Venhuis, Bastiaan J

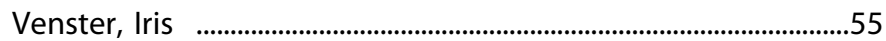

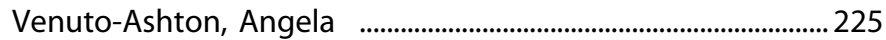

Verdun-Esquer, Catherine ………………........................................ 211

Vermena, Andrea .........................................................257, 265, 266

Veronesi, Caterina _......................................................................... 100

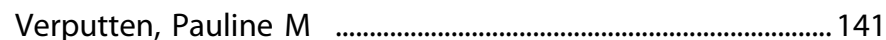

Vial, Thierry ........................................................................................... 323

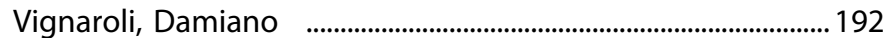

Villa, Antoine

$62,201,207,211,269$

Villalta, Maureen

.. .90

Villeneuve, Eric

Visser, Corine $\mathrm{C}$

117,179

147

Vizeli, Patrick

115,235

Vlckova, Stepanka

Vohra, Varun

202

Voicu, Sebastian
Von Fabeck, Katharina

$.12,308$

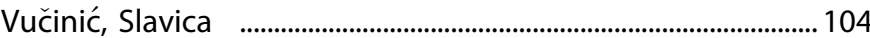

Walter, Melanie ................................................................................10

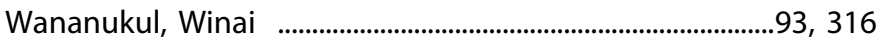

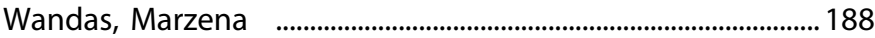

Wang, Josh J ...................................................................117, 149, 301

Warrick, Brandon J .............................................................................. 191

Waugh, Rebecca ML .....................................................................37

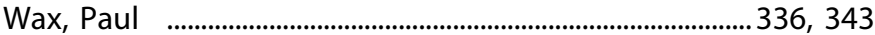

Weiler, Stefan .................................................................... 92, 94, 326

Weiner, Lindsay .................................................................................... 255

Westerbergh, Jenny ................................................................. 118, 264

White, Joanna C ............................................................................ 9

Whitman, Jeffrey ….............................................................................. 294

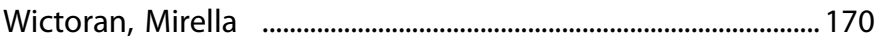

Wiegand, Timothy J ....................................................109, 199, 351

Wiener, Sage W .................................................................287, 328

Wijnands-Kleukers, Anja PG ...............................................252, 340

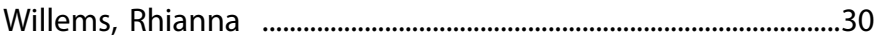

Williams, Hayley A .........................................................64, 65, 253

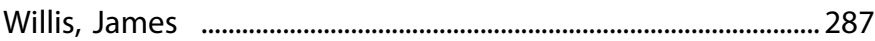

Wium, Cherylynn A ............................................................................99

Wong, Anselm .............................................168, 243, 268, 291, 317

Wong, Timothy .......................................................................................... 349

Wood, David M .........................9, 15, 23, 24, 25, 39, 44, 124, 236

Woolf, Alan D ................................................................................... 313

Wright, Matthew C .......................................................................... 337

Wu, Michael ................................................................................................. 294

Wylie, Carol J ...............................................................................63, 250

Yaker, May ................................................................................... 128

Yarema, Mark ...................................................................................... 317

Yates, Christopher ..........................................15, 19, 124, 148, 153

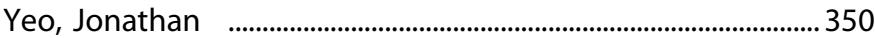

Yip, Wail Lam ....................................................................................... 144

Yu, Jenny ................................................................................................ 182

Zakharov, Sergey ............................................... 3, 66, 104, 134, 212

Zamani, Nasim ….............................................................................. 3, 224

Zammit, Mark L ................................................................................ 337

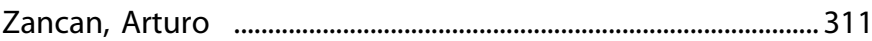

Zatloukal, Claudia

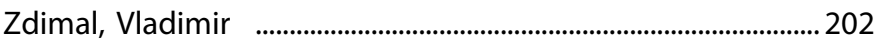

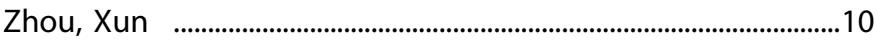

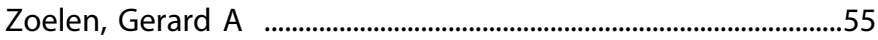

Zoniou, Spyridoula ...............................................................................79

Zoppellari, Roberto ......................................................145, 163, 197

Zotto, Alessandro …............................................................ 1, 34, 246 


\section{Keyword index by abstract number}

1,4-Butanediol

2,4-Dinitrophenol (DNP)

56,143

3,4-Methylenedioxymethamphetamine (MDMA)

3,4-Methylenedioxypyrovalerone (MDPV)

3-Methoxy-phencyclidine (3-MeO-PCP)

5-Fluorouracil (5-FU)

5-Hydroxytryptophan (5-HTP)

$\alpha$-Fluoro- $\beta$-alanine (FBAL)

Acetaminophen $158,191,236,242,243,268,302,317$

330,345

Acetylcysteine $191,236,243,317$

Acidosis, lactic .................................................................................. 328

Activated charcoal, single dose .....................................................54

Adder

53,89

Addiction

.....26

Adherence to advice

159

Adolescent

$43,151,160,251,257,303$

Adulteration $35,41,141$

Adverse drug reaction $260,274,297$

Agkistrodon contortrix

149,301

Airline 135

Air-liquid interface

146,346

Alcohol 109

Alcohol dehydrogenase

161,248

Alcohol intoxication 105

Alerting system .216

Aleurites moluccana 186

Alkalaemia ......52

Alkal 221

Allium ursinum 185,241

Alpha adrenoceptor agonist

18,22

Alprazolam

223

223, 247

Ambulance dispatch

Amdisen Score

165,240

Amisulpride 318

Amlodipine

175,188

Ammonia

282,286

Amphetamines

46,299

Anabolic steroid

50, 292

Anaemia

Anaemia, haemolytic

Angiotensin II receptor blocker 326

Anion gap acidosis

Anticholinergic syndrome 289

Anticholinesterase $193,215,338$

Anticoagulant rodenticide 193

Antidepressant drug ....35

Antidiabetic drug
Antidiarrhoeal drug 296

Antidote availability $\quad$...........77, 78, 79, 81, 82, 83, 162, 179, 184

Antidotes $.66,80,81,84,129,130,145,162,243,317$

Antiepileptic drugs

323,348

Antineoplastic drugs . .60

Antiretroviral drugs

207,211

Antivenom

Arrhythmia 298

Arsenic

$222,316,318$

Artificial intelligence ....................................................................... 127

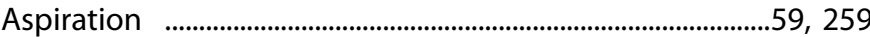

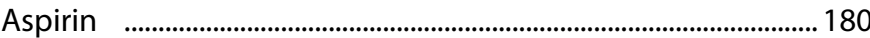

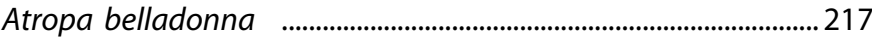

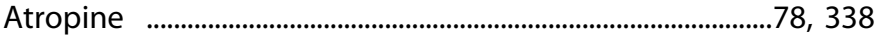

Atypical antipsychotic drugs ........................................................... 199

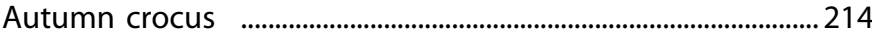

Azerbaijan ................................................................................80, 204

Azilsartan .................................................................................... 326

Azithromycin ................................................................................... 150

Baclofen ...........................................................................132, 274

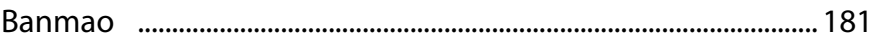

Barbiturate …................................................................................. 273

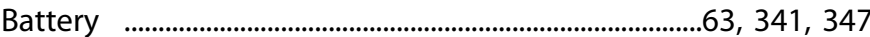

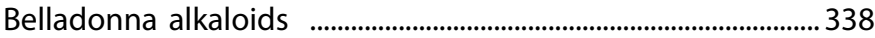

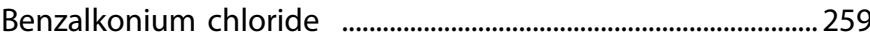

Benzodiazepine ….................................... 8, 9, 24, 25, 26, 276, 324

Beta-blockers ............................................................................ 110, 306

Bezoars …....................................................61, 173, 180, 198, 310

Big data ...........................................................................161, 248

Biological monitoring ...........................................................201, 207

Biomarker .............................86, 87, 134, 139, 168, 202, 242, 244

Bitis peringueyi ......................................................................................99

Bitis xeropaga ............................................................................................99

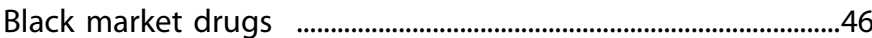

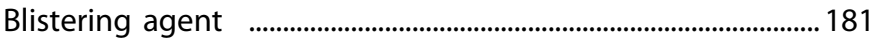

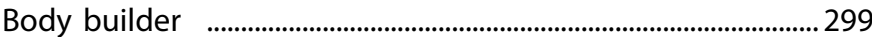

Body packer ................................................................................. 196

Body stuffer ....................................................................................... 196

Bone turnover ......................................................................................... 313

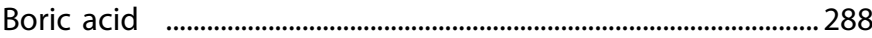

Bothrops asper ........................................................................................98

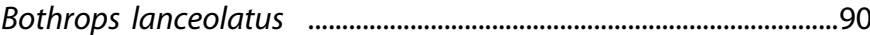

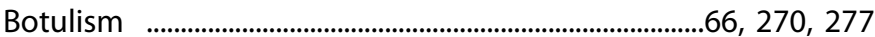

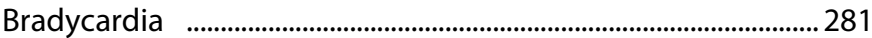

Breastfeeding …...................................................................................... 273

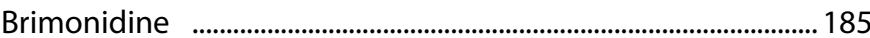

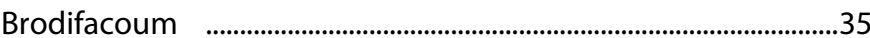

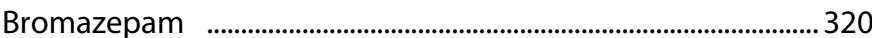

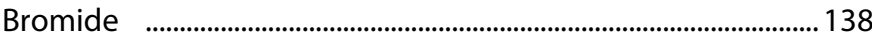

Brugmansia suaveolens ................................................................ 218 
Bufotoxin 316

Buprenorphine $194,301,351$

Bupropion 279

Butalbital 273

Button battery

$.63,341$

Butyrylcholinesterase (BChE)

139

Bystander naloxone

111

Calcium channel blocker $175,188,239,306$

Call duration

Camphor

Cannabinoids, synthetic

Cannabis

Cantharidin

Carbon monoxide

Cardiac glycosides

Cardiogenic shock

Cardiomyopathy

Cardiotoxicity

\begin{abstract}
mortality
\end{abstract}
$40,68,110,187,222,279,281,304$,

$306,316,318,354$

Cardiovascular mortality

110

Cathinones, synthetic 255

Cationic ferritin $10,13,36,42$

Centipede bite

Charcoal, single dose

Cheiracanthium punctorium

Chelation

Chemical biological radiological nuclear (CBRN) incidents 120

Chemical burn $210,282,305$

Chemical incident antidote supply

.... 4, 59, 69, 76, 120, 125, 134, 135,

Chemical poisoning

Chemical submission

203, 206, 210

Chemsex

Child ..........32, 63, 139, 159, 241, 249, 254, 256, 257, 258, 260, $261,262,263,265,266,267,268,269,270,271,272,275$,

$276,277,278,279,280,281,285,325$

Chloromethcathinone

Choosing Wisely

Chromatography

Chromium

203

Clarithromycin

260

Cleaning agents

Clenbuterol

Clomipramine

Clonidine

Clostridium botulinum

Clozapine

Coagulopathy

Cocaine

Cognitive enhancers

Colchichum autumnale

Colchicine

Colocynthis

Complement cascade

Complete blood count, predictive value

Complex regional pain syndrome

Compounded medicine

Computed tomography in poisoned patients
$76,210,266,275$

. .29

173

241,308

$66,270,277$ 199, 258, 309, 319

...94, 98, 103

$. .7,41,42,286$

.....23

.214

57, 67

...67

.. 134

. .87

.93
Consensus statement on methanol poisoning ............................... 3

Contamination of healthcare workers ......................................... 207

Continuing Professional Development ......................................... 337

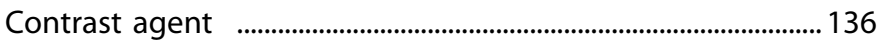

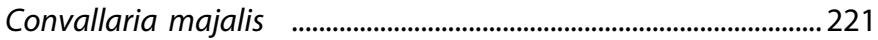

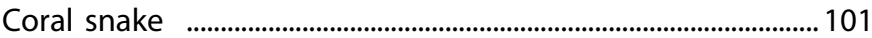

Corneal damage .............................................................................. 138

Corrosive ....................................................................209, 283, 286

Costs, healthcare ................................................................................ 172

Cough syrup ....................................................................................43

Counterfeit drugs ....................................................................................... 18

Crack .......................................................................................................... 7

Criminal poisoning ................................................. 88, 140, 148, 338

Crotalinae ........................................................................ 107, 177

Crotalus atrox ...........................................................................................91

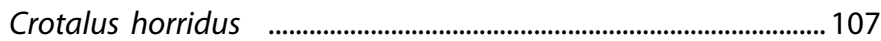

CULTEX® Radial Flow System …..................................................... 135

Cutaneous adverse reaction ............................................................ 297

Cyanide ...................................................................................... 2, 84, 88

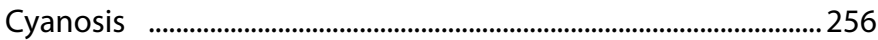

Dantrolene ....................................................................................... 117

Data linkage .....................................................................161, 248

Datura species …............................................................................... 218

Death .......................................35, 56, 88, 118, 143, 254, 322, 327

Delirium tremens .................................................................................. 346

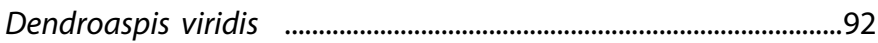

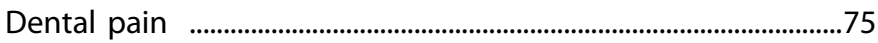

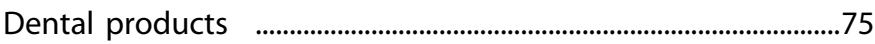

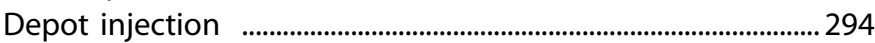

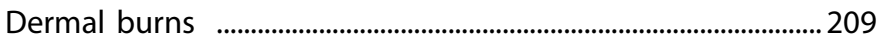

Desipramine ...................................................................................... 186

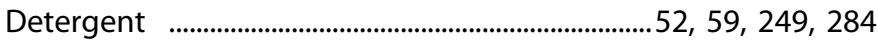

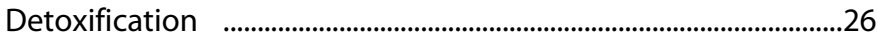

Dextromethorphan ............................................................31, 43

Diazinon ........................................................................... 139, 354

Dichloromethane ................................................................................ 192

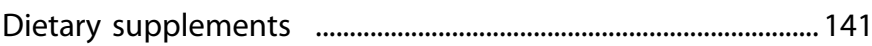

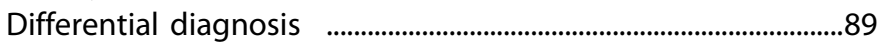

Digoxin .............................................129, 130, 281, 316, 325, 329

Digoxin assay ................................................................................. 329

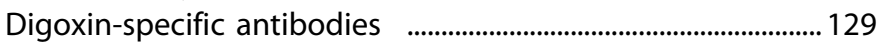

Dimethoate (dimpylate) ................................................................. 354

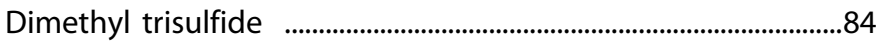

Dimpylate (dimethoate) .................................................................. 354

Diphenhydramine ........................................................43, 193, 195

Dipyrone .......................................................................133, 290

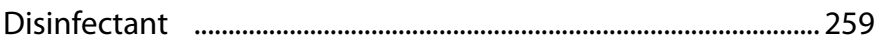

DMPS (2,3-dimercaptopropane-1-sulphonate, unithiol) .......314

DMSA (dimercaptosuccinic acid, succimer) ............................. 311

Dogs .........................100, 105, 227, 228, 229, 230, 231, 232, 233

Doping ............................................................................................... 299

Dosing error ....................................................................................57, 302

Drug induced liver injury (DILI) ...................................................... 299

Drug interaction ...............................................................27, 295, 306

Drug misuse ........................17, 22, 23, 24, 25, 30, 37, 39, 62, 315

Drug reactivation with eosinophilia and systemic symptoms

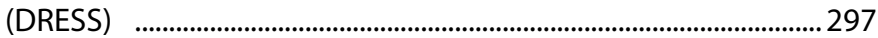

Dwarf adder (Bitis species) ..............................................................99

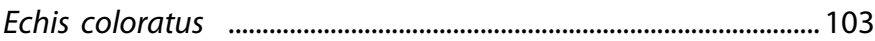


ECMO (extracorporeal membrane oxygenation) ........187, 195, $253,254,255,279,331$

Ecstasy $115,116,189,237,254$

Education 184,337

Elapidae ... 101

Elderly $157,165,319$

E-learning 337

Electrocardiogram (ECG) 182

Electroencephalogram (EEG)

Electronic prescription .......................................................................... 302

Elimination enhancement ...............................................................2239

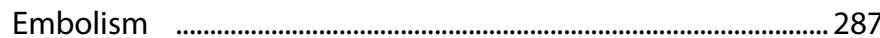

Enalapril 198

Encephalopathy 168

Endomorphin-2 peptide analogue

137

Endoscopy

173,180

Envenomation $53,89,90,91,92,94,95,96,98,99$ $101,103,104,107,176,177,178,200$, $238,246,267,271,339$

Epidemiology $.6,17,71,146,154,156,160,163$, $166,263,324$

Essential oils .... 168

Ethanol $47,49,172$

ETH-LAD (6-ethyl-6-nor-lysergic acid diethylamide) ................33

Ethylene glycol ......48

Etizolam .. .8

Eucalyptus oil ... 168

Euro-DEN Plus 15,124

European Article Number (EAN-Code) .....69

European Chemicals Agency (ECHA)

EVEIT (Ex vivo Eye Irritation Test) $1 . . .4$

Ex vivo Eye Irritation Test (EVEIT)

EXITOX study

Experimental studies $\ldots . .14,90,109,135,137,138,283$

Extended release drugs

Extracorporeal Life Support Organization (ELSO) 198,245

Extracorporeal membrane oxygenation (ECMO)

253, 254, 255, 279, 331

Extracorporeal treatment .............255 187,195 EXTRIP (EXtracorporeal TReatments In Poisoning) .240 Eye

\section{Fals}

Fasciotomy

Fatal $.35,56,88,118,143,254,322,327$

Favism $111,112,118,119,147$

Fentanyl

Fentanyl analogues $44,45,118$

Fertiliser ... 105

Fire ....85

Firelighter .285

First aid kit 149

Fish .311

Fish venom ...97

Flecainide

.332

Fluoxetine .303

Fomepizole 109

Food .229, 230

Food supplement ..142, 226

Foreign body
Forensic toxicology . .46

Fungi $223,224,247$

GABA analogues .......................................................................25, 315

Gabapentin ....................................................................................... 315

Gamma-butyrolactone (GBL) ……………………………………... 108

Gamma-hydroxybutyrate (GHB) ……………………….... 108, 109

Garlic .................................................................................................. 221

Gastric lavage .......................................................................................... 164

Gastrointestinal burns ....................................................................... 282

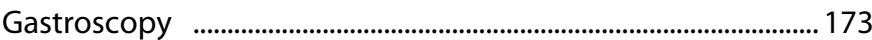

GBL (gamma-butyrolactone) ........................................................... 108

Gender differences .................................................................................2

Gentian (Gentiana lutea) ...................................................................220

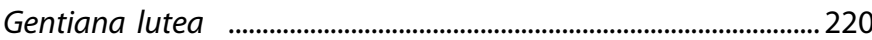

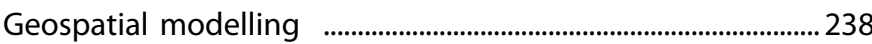

GHB (gamma-hydroxybutyrate) ..................................................... 108

Gingerbread ............................................................................2229, 230

Global Toxicosurveillance Network (GTNet) ................................62

Glucagon-like peptide ………………………………………………..... 291

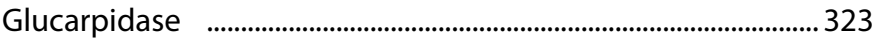

Glucose-6-phosphate dehydrogenase (G6PD) deficiency ..292

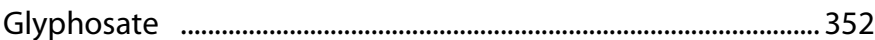

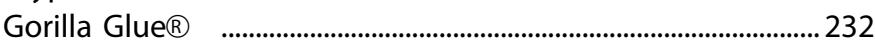

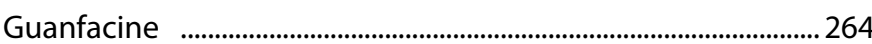

Gut decontamination .................................... 54, 164, 173, 180, 310

Haemodialysis .............................................................3, 167, 197, 208

Haemolytic anaemia ......................................................................... 292

Hallucinogen .......................................................................33, 218, 219

Healthcare costs .................................................................................... 172

Hearing aid battery ................................................................................. 347

Heatstroke ............................................................................................ 117

Heavy metals ......................................................311, 312, 313, 314

Hepatotoxicity ...........................10, 223, 224, 226, 242, 244, 299

Herbicide .......................................................................204, 353, 355

hERG channel blockade ..................................................................... 296

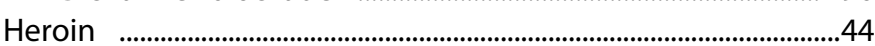

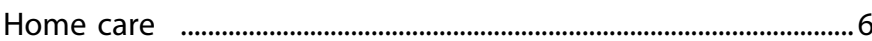

Homicide ..........................................................................................88, 140

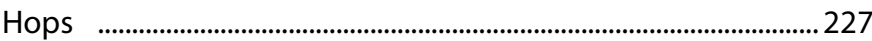

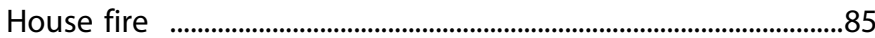

Household products ............................................125, 275, 285, 288

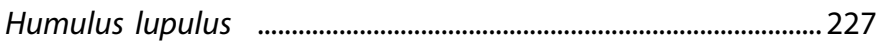

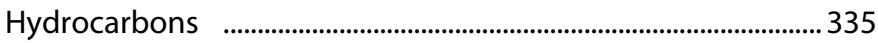

Hydrocodone …………………………………………………………....73

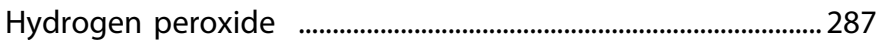

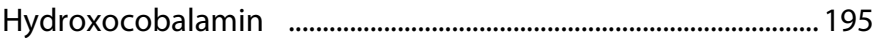

Hyperbaric oxygen therapy …………..........................192, 200, 287

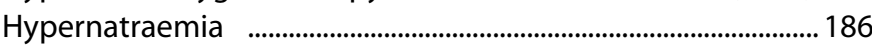

Hypersexual behaviour …………………………………………….....4

Hyperthermia ……………………………………………………........ 117

Hyponatraemic encephalopathy ………………………………...... 189

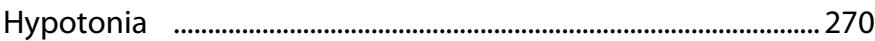

latrogenic medication error ….....................................301, 322, 345

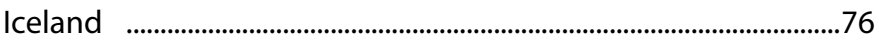

Illegal drug production ......................................................................340

Immunoassay ................................................................ 124, 144, 316

In vitro testing .................................................................90, 122, 135

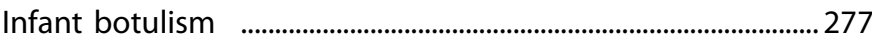

Inhalation exposure $\quad$...................................................202, 340, 344 
Injection, cyanide . .88

Insect repellents

Insulin therapy

126

Insulin therapy, high dose ......................................................... 188

Intensive Care Unit treatments ................................................ 163

Internet drugs

$23,46,418$

Intravenous immunoglobulin (IVIG)

Iron

$136,272,310$

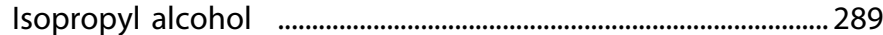

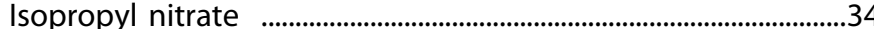

Kambô ritual ..................................................................106, 300

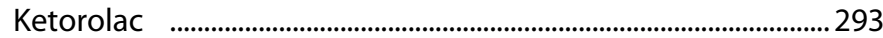

Kidney disease, chronic …............................................................ 136

Kidney injury

$86,103,167,293$

Kounis Syndrome

260

Kratom

226

Lacosamide

333

Lactate

. .49

Lactic acidosis

328

Laparotomic gastrostomy

310

Laundry detergent

Laundry detergent capsules 249,284

L-carnithine 121

Lead .313

Legal highs (recreational food)

Legislation

.....21

Lepiota brunneoincarnata

$123,146,155,272$

Levamisole .....41

Levetiracetam ... 169

Liquid laundry detergent capsules

249, 284

Liraglutide

... 291

Lithium $165,183,240,342$

Liver toxicity

224,330

Loperamide

37,296

Loxosceles rufescens

$96,200,246$

Loxoscelism

$.96,200,246$

LSD

14,235

Lupanine

215

Lupin

215

Lysergic acid diethylamide (LSD)

14,235

Macrolide antibiotics

150

Maculopathy

. .34

Magnesium

130

Magnetic putty

. .58

Malignant hyperthermia

.117

Marine envenomation

.. .97

MARS ${ }^{\circledR}$ (molecular adsorbent recirculating system) $\quad$............. 239

Mass intoxication

.213

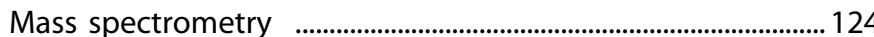

Mauritania

207

MDMA (3,4-methylenedioxymethamphetamine) .........115, 116,

$189,237,254$

MDPV (3,4-methylenedioxypyrovalerone)

13,237

Means restriction strategy

155

Media

$58,96,252$

Medical decision making

$.127,278$

Medical kit

Medical toxicology training

337,343

Medication error $\quad . . .67,150,190,258,265,302,308,322,347$
Mercury

311,312

Metabolism

.14, 237

Metal poisoning

$311,312,313,314$

Metamizole

133,290

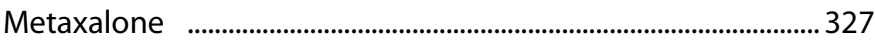

Meteorological modelling …........................................................... 238

Metformin

$166,197,328$

Methaemoglobinaemia

$.50,128,256$

Methanol

$3,134,340,344$

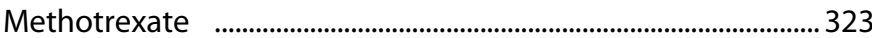

Methyl bromide .................................................................................. 208

Methylene blue …..........................................................128, 256, 295

Methylene chloride ........................................................................... 192

Methylenedioxypyrrolidinohexiophenone (MDPHP) ................36

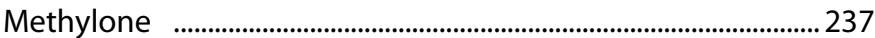

Methylphenidate ..................................................................23, 269

Methylthioninium chloride (methylene blue) ..... 128, 256, 295

Microsomal assay ...................................................................................14

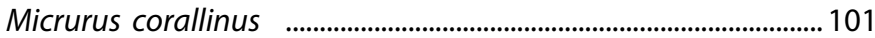

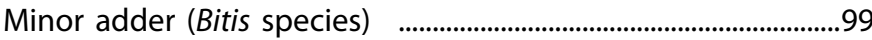

Mirabegron ............................................................................................. 228

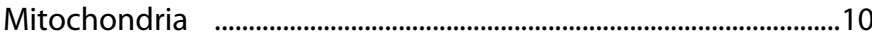

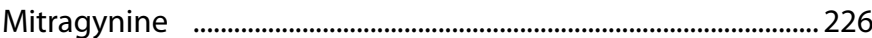

Modafinil ........................................................................................................22

Modified-release paracetamol ............................................................ 245

Monoamine transporters f............................................................. 237

Monographs, poison information ................................................55

Mortality ….................................30, 119, 156, 157, 304, 332, 353

Murder .................................................................................88, 140

Murine study ….............................................................................. 136

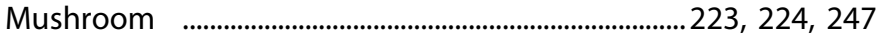

Myeloid differentiation .................................................................. 133

Myristica fragrans ............................................................................ 219

N-acetylcysteine ..................................................191, 236, 243, 317

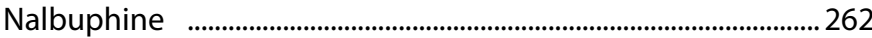

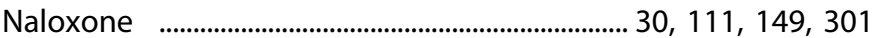

Nanoparticles ......................................................................202, 212

National Poison Data System (NPDS) ............ 73, 160, 241, 245,

272,280

National Serum Depot …..................................................................95

Neonate .............................................................. 67, 268, 273, 298

Neue-psychoaktive-Stoffe-Gesetz (NPSG, NPS Law) .............. 123

Neuroinflammation ................................................................... 134

Neuroleptic malignant syndrome .............................117, 294, 307

Neurotoxicity

$99,171,176,274,312$

Neutropenia

133,290

Neutrophil gelatinase-associated lipocalin (NGAL) ...................86

Nitrites

$.34,128$

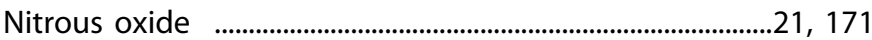

NMOBe

Non-convulsive status epilepticus ….................................................48

Non-medical use of drugs ............................................24, 25, 315

Non-steroidal anti-inflammatory drug (NSAID) ......................... 293

Novel psychoactive substances (NPS) ........... 10, 11, 13, 15, 19,

$31,33,118,122,123$

NPS (Novel psychoactive substances) ........... 10, 11, 13, 15, 19,

$31,33,118,122,123$

Nursing home . .59

Nutmeg 
Observation unit 350

Occupational exposure $201,202,204,209,210,212$

Ocretotide 188

Octodrine 141

Ocular exposure

$.52,138$

Oesophageal injury

283

Olanzapine 199

Ophthalmoplegia . .99

Opioid analgesics 147

Opioid dependence 351

Opioid receptor agonists 106,300

Opioids $18,30,38,44,45,71,73,111,112,113,114$,

$118,119,137,149,160,194,262,280,301,324$

Oral exposure at work

Organophosphate insecticide 139,354

Oxalates …..... 225

Oxidative stress 202

Oxycodone 147,160

Pacing .... 354

Paediatric $32,63,139,159,241,249,254,256,257$ $258,260,261,262,263,265,266,267,268,269,270$, $271,272,275,276,277,278,279,280,281,285,325$

Paliperidone 294

Paracetamol .158, 191, 236, 242, 243, 268, 302, 317, 330, 345

Paralytic shellfish toxins

233

Paraquat 353,355

Parathyroid surgery .295

Paroxetine 295

Particulate matter 334

Patient medical record documentation 250

Pesticide $126,139,234,352,353,354,355$

Pesticide surveillance $61,173,180,198,310$

Pharmacobezoar $. .38,115$

Pharmacogenetics

Pharmacokinetic/pharmacodynamic relationships ................. 121

Pharmacovigilance

19

Phencyclidine 106, 300

Phyllomedusa bicolor 199

Physostigmine 225

Phytolacca americana .. .94

Pit viper $.131,213,214,215,216,217,218,219,220,221$

Plants

$222,225,316$

Poison centre data ...... 5, 22, 37, 52, 54, 55, 57, 62, 63, 64, 65, 68, 70, 71, 75, 76, 82, 126, 194, 206, 252, 254, 269, 280

Poisons Centre advice adherence 250

Pokeweed 225

Poppers

$.34,128$

Potassium channel blockade ............................................................ 296

Pramipexole ............................................................................................... 190

Pregabalin .315

Pregnancy $. .74,348$

Pre-hospital treatment .....80

Prescription drug misuse . .39

Prescription opioids .....62

Prevention

Pre-workout supplements

Priapism

Product data
Product identification ..69

Prognosis $47,166,167,170,332,353$

Propranolol 29,65

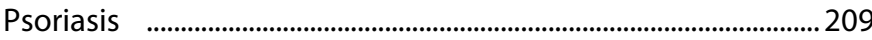

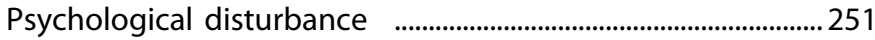

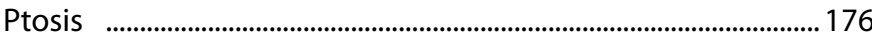

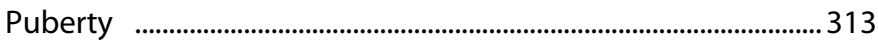

Public health $58,96,146,334$

Pulmonary toxicity 135

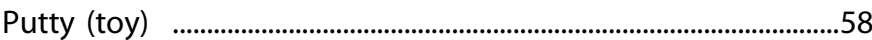

Pyoderma gangrenosum ......................................................................4

QRS interval ........................................................................................... 182

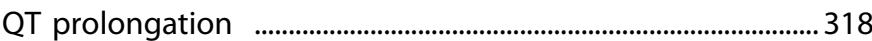

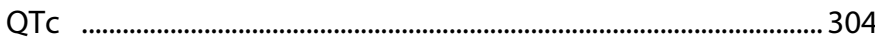

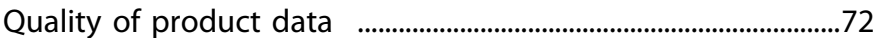

Quaternary ammonium compound .............................................. 259

Quetiapine ........................................................61, 144, 199, 321

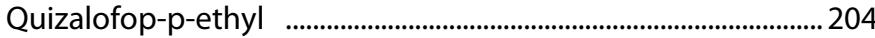

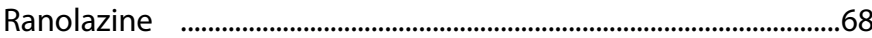

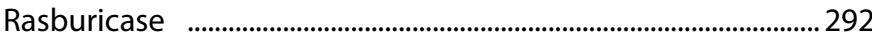

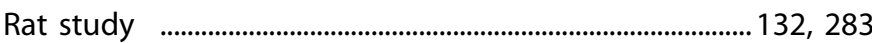

Rattlesnake ........................................................................................ 339

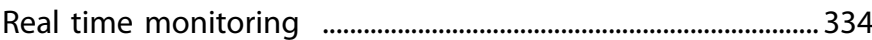

Recluse spider ….................................................................96, 200, 246

Recreational drugs .....................9, 11, 13, 15, 17, 18, 19, 20, 21 , $24,25,27,28,31,32,33,35,36,37,38,42,43,45$ $46,112,113,114,116,119,124,189,315,324$

Refractory shock .29

Regulation (EU) No 2017/452

.... 4

Renal failure

$86,103,167,293$

Repeat overdose

170

Researched Abuse, Diversion and Addiction-Related Surveillance (RADARS $®$ ) . .62

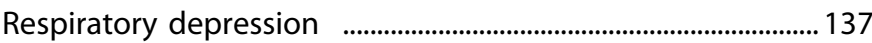

Retinoids ..............................................................................................

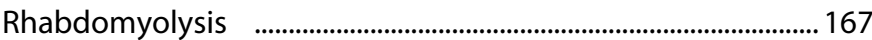

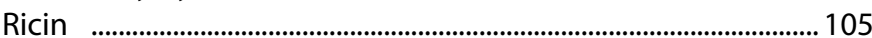

Rivastigmine ........................................................................................ 193

Rotational thromboelastometry (ROTEM $\left.{ }^{\circledR}\right)$..................................98

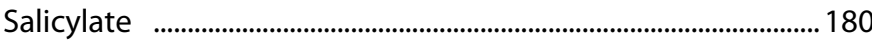

Sampling kit _......................................................................................... 211

Saturation gap …............................................................................. 256

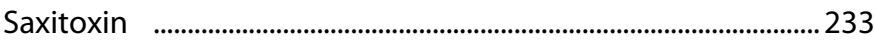

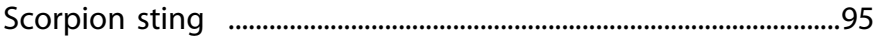

Seasonal effect on blood concentration …………………….....342

Seizure .................................................................47, 303, 333, 335

Selective serotonin re-uptake inhibitors (SSRI) ......................... 303

Serotonin syndrome ....................27, 45, 100, 116, 295, 231, 327

Serotonin system ................................................................................. 115

Sexual assault .......................................................................................... 148

Sexual stimulant ….............................................................................. 142

Shaman rituals ...................................................................106, 300

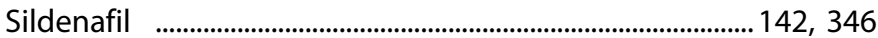

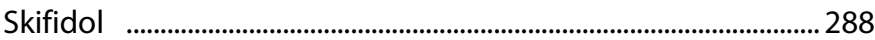

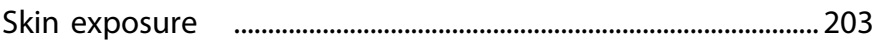

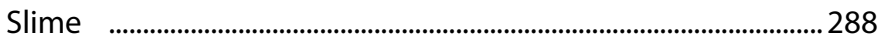

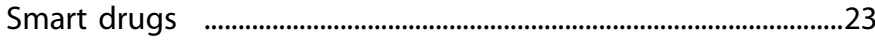

Smelling salts ........................................................................................ 282

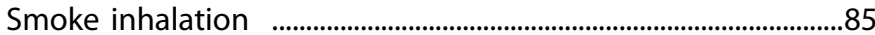


Smuggling, drug

Snake bite $53,89,90,91,92,94,95,98,99,101$

103, 104, 107, 176, 177, 178, 238, 267, 339

Social media

113,114

Sodium azide

252

Sodium bicarbonate

186, 304

Sodium channel blockade

296

Sodium nitrite

252

Spanish fly

181

Spanish Toxic Surveillance System (STSS)

Sparteine

Spider bites

$96,200,246,271$

Spirometry

$25,39,122$

Sting ....97

Stomatological preparations

.....75

Students

161, 248

Succimer (dimercaptosuccinic acid, DMSA)

311

Suicide

$71,151,153,252$

Suicide powder

252

Suicide prevention

Sulphur dioxide 334

Supratherapeutic overdose

268

Surface contamination

Surveillance $67,73,105,113,114$

Swine

Synthetic cannabinoids

Synthetic cathinones

Synthetic opioids

Takotsubo cardiomyopathy

Tardive dyskinesia

Taxus species

Telephone advice

$.55,64,250$

Teletoxicology

Temperature effect on blood concentration

$.35,123,261$

Teratology

Terrorism

Tetrahydrofuranyl fentanyl

Thailand

Thrombocytopenia

Thrombotic microangiopathy

Tolerance, drug
Torsades de pointes 318

TOXBASE

154,352

Toxicology Investigators Consortium (ToxIC) ........................... 336

Toy

$.58,288$

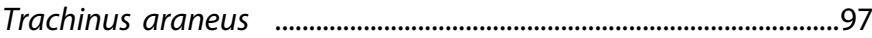

Training in toxicology ….....................................................337, 343

Transdermal intoxication .................................................................. 203

Transmission Electron Microscopy …………………………...... 212

Transporters, in vitro study ......................................................... 122

Tricyclic antidepressants ........................................144, 173, 182

Trimeresurus albolabris ..........................................................................91

Trimeresurus venustus .................................................................................99

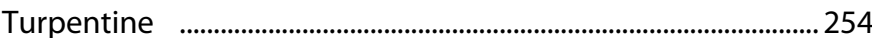

Twitter ...................................................................................... 113, 114

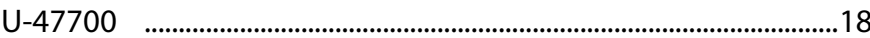

Unique Formula Identifier (UFI) ....................................................69

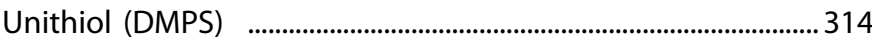

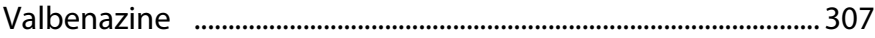

Valproic acid ................................................................................60, 121

Valpromide .............................................................................................60

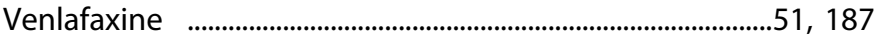

Ventricular assist device ................................................................. 309

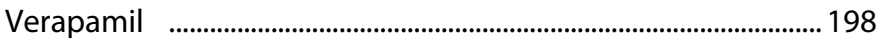

Veratrum album ....................................................................................... 220

Veterinary toxicology ................................100, 105, 228, 229, 230,

$231,232,233,234$

Video-assisted orogastric lavage ...................................................... 164

Vipera ammodytes ...............................................................104, 178

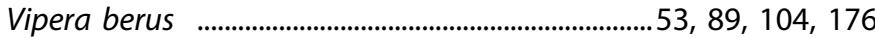

Vipera species ......................................................................104, 267

Vitamin B12 deficiency ................................................................. 171

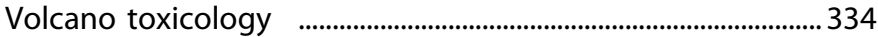

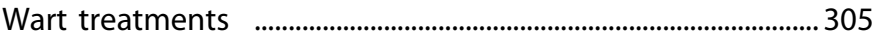

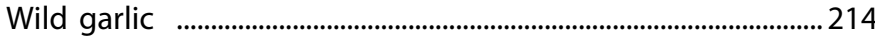

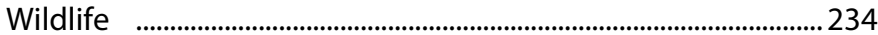

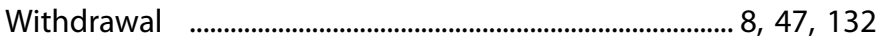

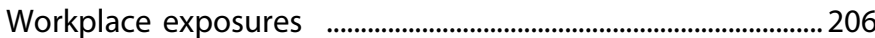

Xylitol ...................................................................................229, 230

Yew ..................................................................................................... 222

Z drugs ...................................................................................62, 269

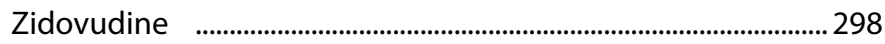

